\title{
SAMPLE DESCRIPTIONS AND SUMMARY LOGS \\ OF SELECTED WELLS \\ WITHIN THE HANFORD RESERVATION
}

(Four volumes)

VOLUME II

W. K. Summers

R. T. Hanson

W. K. Summers and Associates

Consultants

January 1977

This report was prepared as an account of work
sponsored by the United States Government Neither the
Unuted States nor the United States Department of
Energy, nor any of their employees, nor any of their
contractors, subcontractors, or their employees, makes
any warranty, express or implied, or assumes any legal
liabulity or responsibulty for the accuracy, completeness
or usefulness of any information, apparatus, product or
process disclosed, or represents that its use would not
mfrunge privately owned nghts

This report is based on work sponsored by Atlantic Richfield Hanford Company under United States. Energy Research and Development Administration Contract EY-76-C-06-2730. 


\section{DISCLAIMER}

This report was prepared as an account of work sponsored by an agency of the United States Government. Neither the United States Government nor any agency Thereof, nor any of their employees, makes any warranty, express or implied, or assumes any legal liability or responsibility for the accuracy, completeness, or usefulness of any information, apparatus, product, or process disclosed, or represents that its use would not infringe privately owned rights. Reference herein to any specific commercial product, process, or service by trade name, trademark, manufacturer, or otherwise does not necessarily constitute or imply its endorsement, recommendation, or favoring by the United States Government or any agency thereof. The views and opinions of authors expressed herein do not necessarily state or reflect those of the United States Government or any agency thereof. 


\section{DISCLAIMER}

Portions of this document may be illegible in electronic image products. Images are produced from the best available original document. 
$\frac{\text { Depth }}{\text { (ft.) }}$

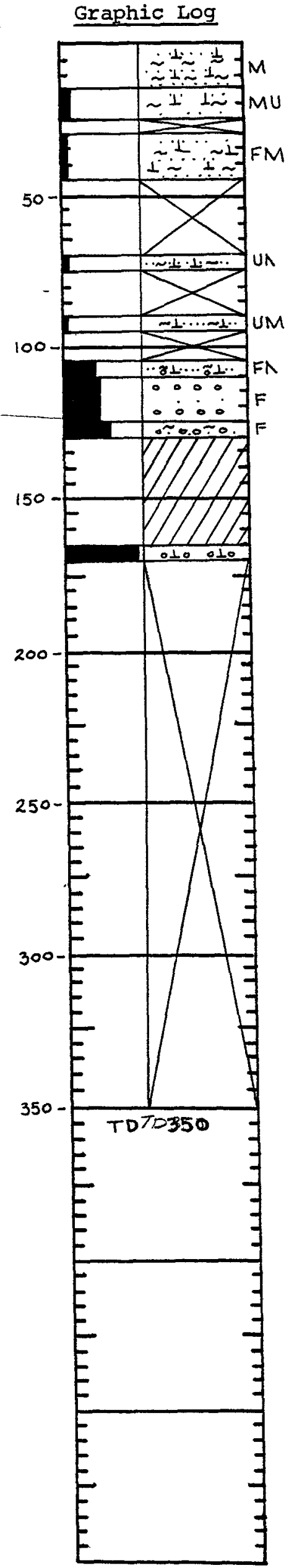

Sample Description

brownish buff

buff

buff

buff

butf

buff

greyish buff (salt \& pepper)

whitish buff; $A S H$ (?)

browrish black
Plant Coordinates

N 003000

$W 045000$

Altitude 503

Other Data used in

Interpretation

(1) Driller's Log

(2) $E \log$ 
ROCK CLASSIFICATION

DEPTH

(ET.)

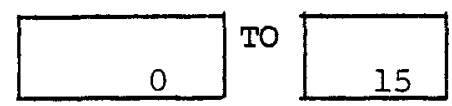

silty sand

\section{S SIZE RANGE SPHERICITY ROUNDNESS}

BASALT $\geq$ gravel sand
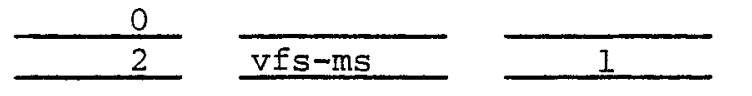

sb rnd

OTHER $\geq$ gravel sand
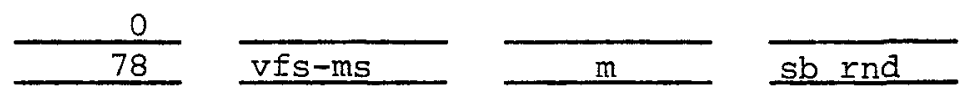

SILT AND CLAY 20

REMARKS

color Brownish Buff

HCL

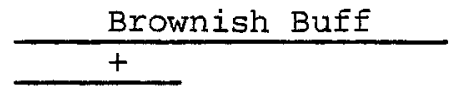

Micaceous

Immature

DEPTH

(FT.)

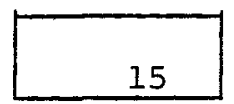

TO

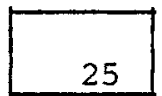

ROCK CLASSIFICATION

slightly silty sand

\section{\% SIZE RANGE SPHERICITY ROUNDNESS}

BASAIT $\geq \underset{\text { sand }}{\text { grave } 1}$
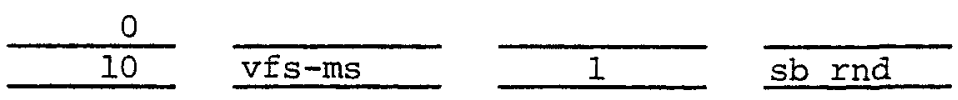

OTHER $\geq$ gravel
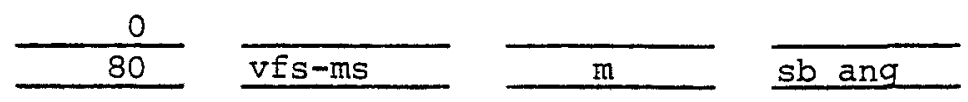

SILT AND CLAY 10

REMARKS

$$
\text { color }
$$

HCL

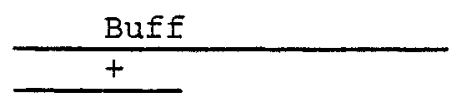

Micaceous

Unfrosted Quartz

Immature 


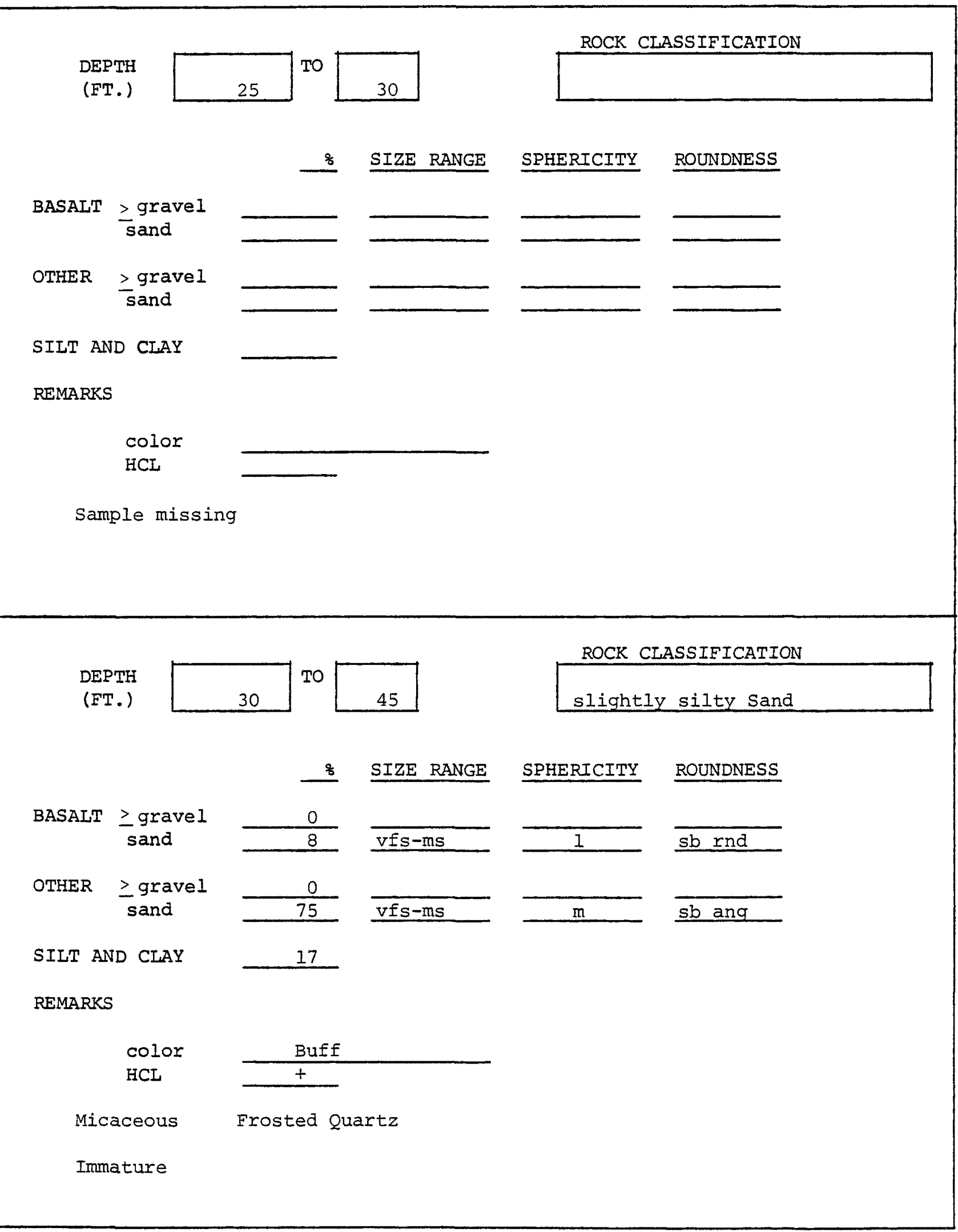




\section{DEPTH}

(FT.)

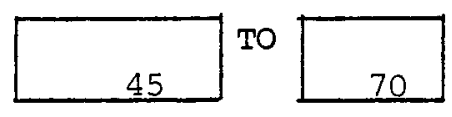

\% SIZE RANGE SPHERICITY ROUNDNESS

BASAIT $\geq$ gravel sand

OTHER $\geq$ gravel sand

SILT AND CLAY

REMARKS

color

HCL

Sample missing

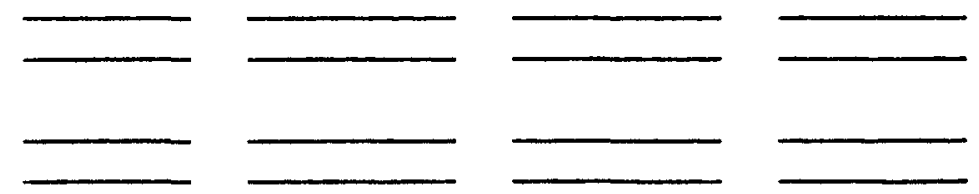

\section{REMARKS}

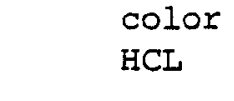

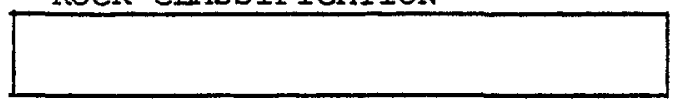
Sample missing

DEPTH

(FT.)

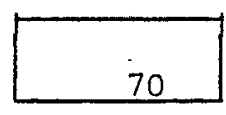

TO

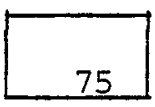

ROCK CLASSIFICATION

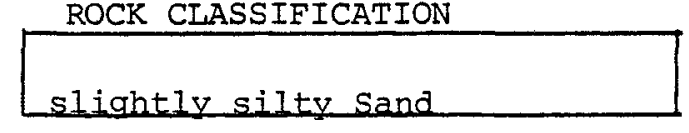

\% SIZE RANGE SPHERICITY ROUNDNESS BASALT $\underset{\text { sand }}{\text { gravel }}$
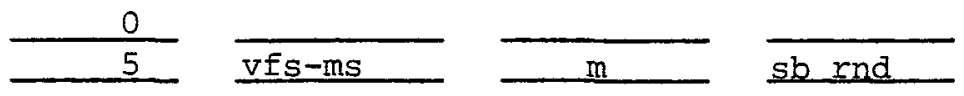
OTHER > gravel sand
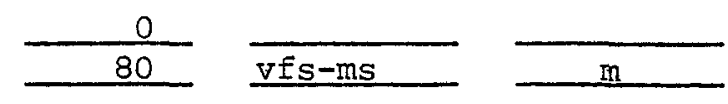

sbrnd

SIIT AND CLAY 15

REMARKS

$$
\begin{aligned}
& \text { color } \\
& \text { HCL }
\end{aligned}
$$

Buff

$+$

Slightly Micaceous Unfrosted Quartz

Immature 
ROCK CLASSIFICATION

\section{DEPTH}

(FT.)

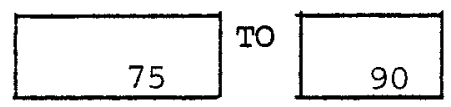

\% SIZE RANGE SPHERICITY ROUNDNESS

BASALT $\geq$ gravel sand

OTHER $\geq$ gravel sand

SIIT AND CIAY

REMARKS

color

$\mathrm{HCL}$

Sample missing
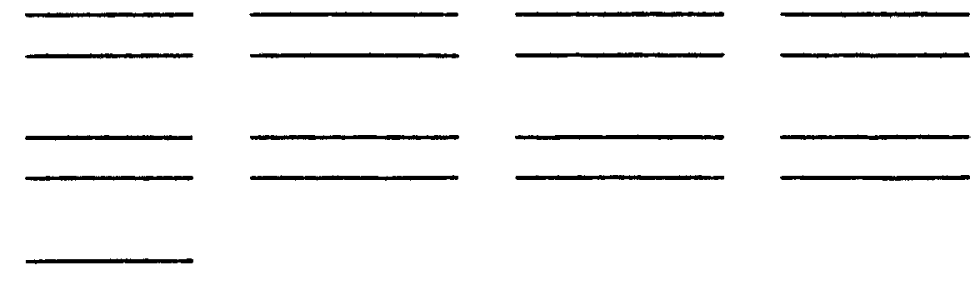

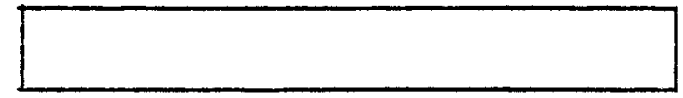


DEPTH
(FT.)

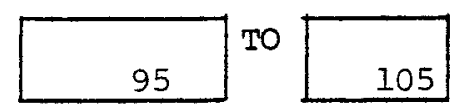

ROCK CLASSIFICATION

105

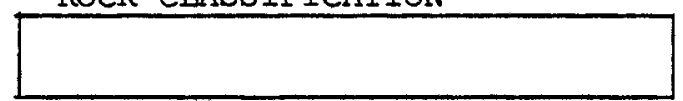

\% SIZE RANGE SPHERICITY ROUNDNESS

BASALT >gravel

sand

OTHER $\mathrm{P}$ gravel sand
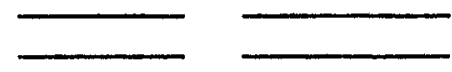

SILT AND CLAY

REMARKS

color

HCL

Sample missing

DEPTH

(FT.)

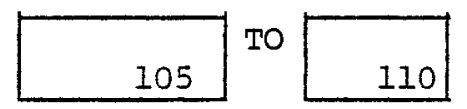

ROCK CLASSIFICATION

gravelly silty sand

S SIZE RANGE SPHERICITY ROUNDNESS

\begin{tabular}{|c|c|c|c|c|c|}
\hline \multirow[t]{2}{*}{ BASALT } & >gravel & $\frac{20}{20}$ & $\frac{v f p-c r p}{v f s-m s}$ & $\frac{m}{m}$ & rnd \\
\hline & & & & $\mathfrak{m}$ & $\mathrm{sb}$ rnd \\
\hline \multirow[t]{2}{*}{ OTHER } & > gravel & 10 & $v f p-c r p$ & $\mathrm{~m}$ & rnd \\
\hline & sand & 30 & vfs-ms & $\mathrm{m}$ & sb rnd \\
\hline
\end{tabular}

SIIT AND CLAY

20

REMARKS

color

HCL

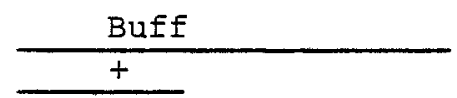

Slightly Micaceous

Frosted Quartz

Immature 
DEPTH

(FT.)

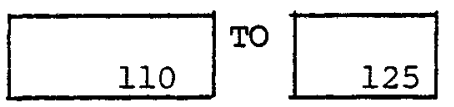

ROCK CIASSIFICATION

sandy Gravel

\section{SIZE RANGE SPHERICITY ROUNDNESS}

\begin{tabular}{|c|c|c|c|c|c|}
\hline \multirow[t]{2}{*}{ BASALT } & \multirow{2}{*}{$\begin{array}{l}\geq \text { gravel } \\
\text { sand }\end{array}$} & 30 & Vfp-crp & $\mathrm{m}$ & rnd \\
\hline & & 15 & vfs-vers & $\mathrm{m}$ & sb ang \\
\hline \multirow{2}{*}{ OTHER } & > gravel & 30 & $v f p-\approx r p$ & $\mathrm{~m}$ & rnd \\
\hline & sand & 25 & vfs-vers & $\bar{h}$ & sb rnd \\
\hline
\end{tabular}

SIIT AND CLAY

\section{0}

REMARKS

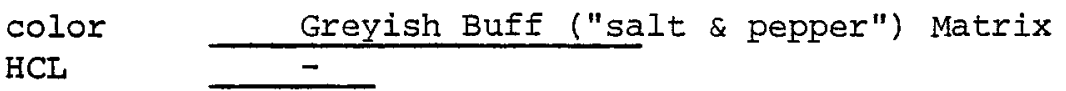

Frosted Quartz

Immature

DEPTH

(FT.)

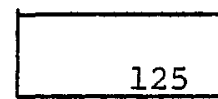

TO

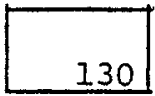

ROCK CIASSIFICATION

silty sandy Gravel

\section{SIZE RANGE SPHERICITY ROUNDNESS}

\begin{tabular}{|c|c|c|c|c|c|}
\hline BASALT & $\begin{array}{l}\geq \text { gravel } \\
\text { sand }\end{array}$ & $\begin{array}{l}50 \\
10 \\
\end{array}$ & $\frac{v f p-m p}{v f s-v c r s}$ & $\frac{\mathrm{m}}{\mathrm{m}}$ & $\begin{array}{l}\text { rnd } \\
\text { sb rnd }\end{array}$ \\
\hline OTHER & $\geq$ gravel & 20 & $v f p-m p$ & m & rnd \\
\hline & sand & 10 & vfs-vers & $h$ & sb rnd \\
\hline
\end{tabular}

SIIT AND CLAY 10

REMARKS

Color
HCL
Some Ash (?)
Immature




\begin{tabular}{|l|l|l|l|l|l|l|}
\hline $\begin{array}{l}\text { DEPTH } \\
\text { (FT.) }\end{array}$ & ROCK CLASSIFICATION \\
\hline 130 & Basalt Flow \\
\hline
\end{tabular}

\% SIZE RANGE SPHERICITY ROUNDNESS

BASAIT $\geq$ grave 1 sand

OTHER $\geq$ gravel sand

SILT AND CIAY

REMARKS
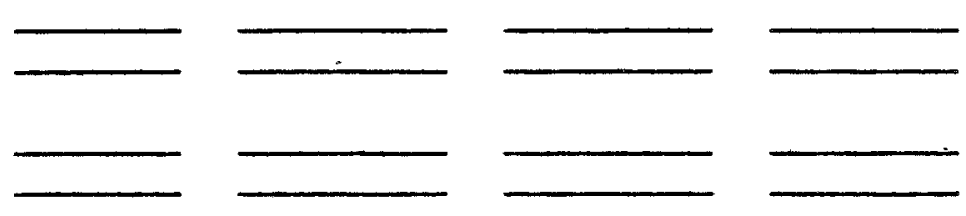

Color
HCL
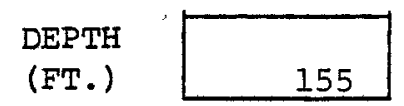

TO

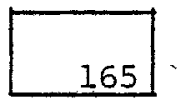

ROCK CLASSIFICATION

Basalt Flow or Boulder

\% SIZE RANGE SPHERICITY ROUNDNESS

BASALT >gravel sand

OTHER >gravel sand
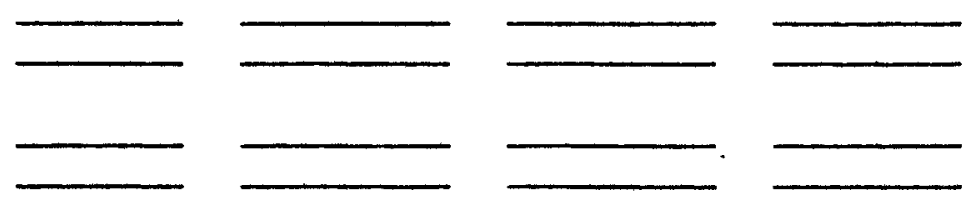

SILT AND CLAY

REMARKS

color

Reddish Black

HCL 


\section{DEPTH}

(FT.)

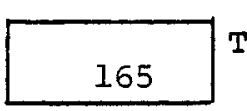

TO

170

ROCK CLASSIFICATION

Gravel

\section{\% SIZE RANGE SPHERICITY ROUNDNESS}

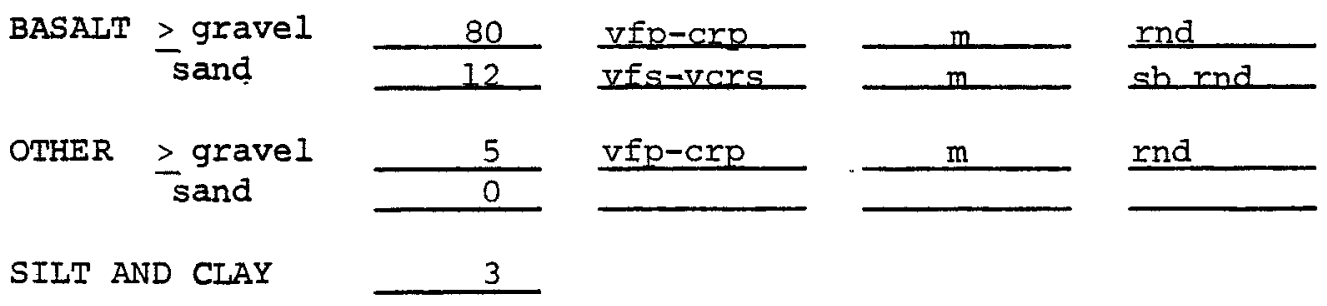

REMARKS

$\begin{array}{ll}\text { Color } & \text { Brownish Black } \\ \text { HCL } & +\end{array}$

Flow Layer fragments

Immature

\section{DEPTH}

(FT.)

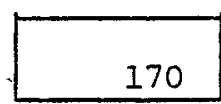

To

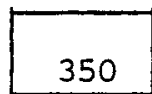

ROCK CIAASSIFICATION

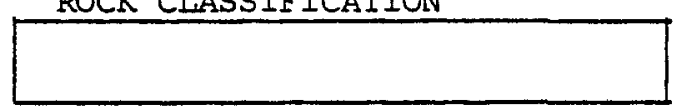

\section{SIZE RANGE SPHERICITY ROUNDNESS}

BASALT $\geq$ gravel sand

OTHER $\geq$ gravel sand
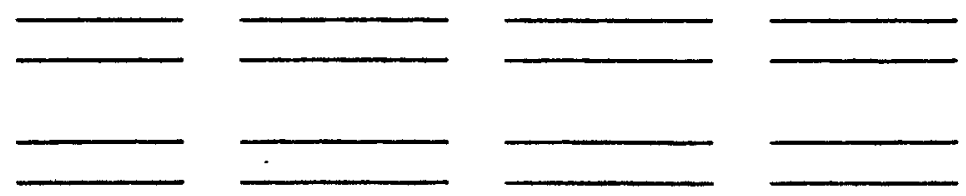

\section{SILT AND CLAY}

REMARKS

color

HCL

Sample missing

Total Drilled Depth reported as 350' 
$\frac{\text { Depth }}{\text { (ft. })}$

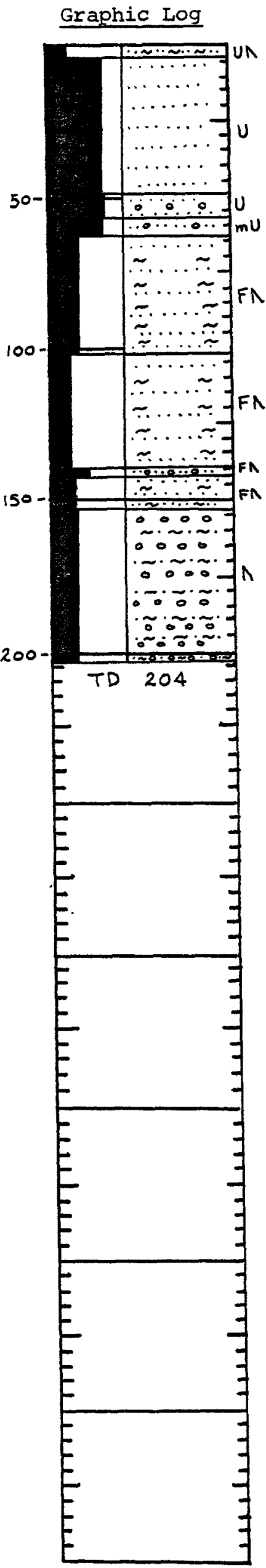

Sample Description

brown

greyish black

greyish black

greyish black

greyish buff

greyish buff

greyish butf

greyish buff

greyish buff
Plant Coordinates

$\mathrm{N} 007875$

$W 031800$

Altitude 552

Other Data used in Interpretation

(1) E Log 
ROCK CIASSIFICATION

DEPTH

(FT.)

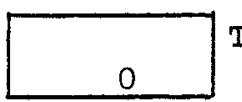

TO

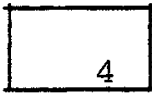

silty sand

\% SIZE RANGE SPHERICITY ROUNDNESS

BASALT $\geq$ gravel sand
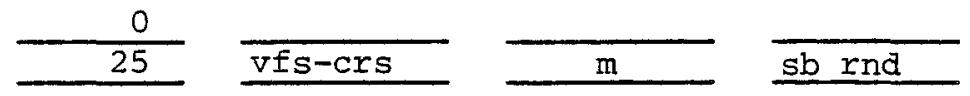

OTHER $\geq$ gravel sand
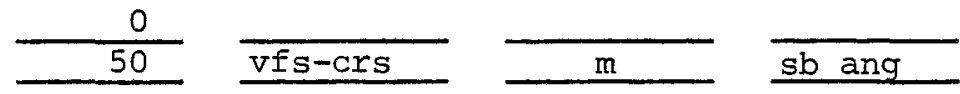

SILT AND CLAY 25

REMARKS

color Brown

Slightly Micaceous Unfrosted Quartz

Immature

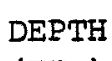

(FT.)

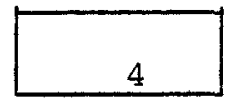

TO

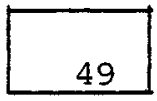

ROCK CLASSIFICATION

Sand

\% SIZE RANGE SPHERICITY ROUNDNESS

BASALT $\geq$ grave 1 sand

OTHER $\geq$ gravel sand

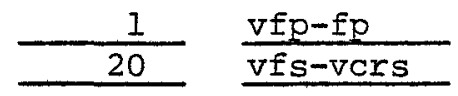

rnd sb rnd

rnd sb rnd

SILT AND CIAY

7

REMARKS

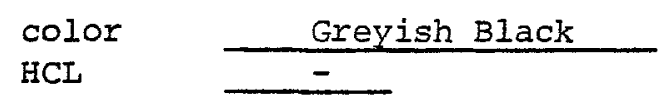

Unfrosted Quartz

Immature 


\section{DEPTH}

(FT.)

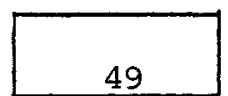

TO

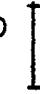

57

ROCK CLASSIFICATION

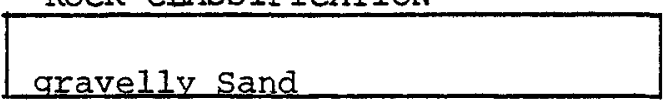

\section{\% SIZE RANGE SPHERICITY ROUNDNESS}

BASALT $\geq$ gravel sand
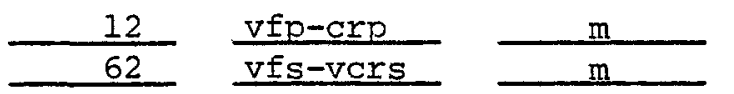

rnd

sb rnd

OTHER $\geq$ gravel sand
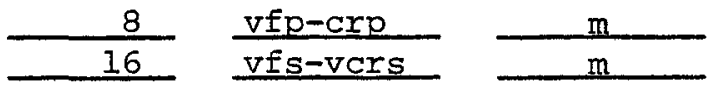

rnd sb rnd

SILT AND CLAY 2

REMARKS

color Greyish Black

HCL

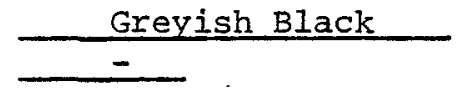

Unfrosted Quartz

Immature

DEPTH

(FT.)

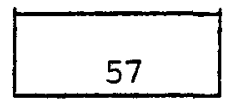

To

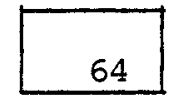

ROCK CLASSIFICATION

slightly gravelly sand

\section{\% SIZE RANGE SPHERICITY ROUNDNESS}

BASALT $\geq$ gravel sand
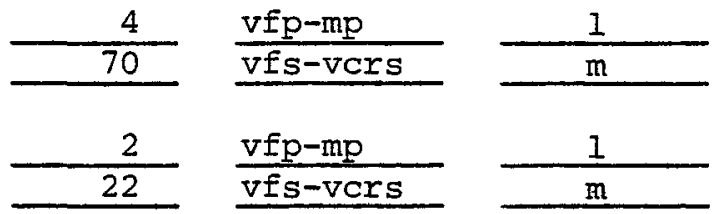

rnd

OTHER $\geq$ gravel sand

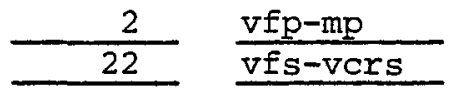

SILT AND CLAY

$$
2
$$

REMARKS

color

HCL Greyish Black ("salt \& pepper")

Unfrosted Quartz

Mature 
DEPTH

(FT.)

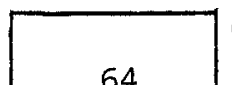

TO

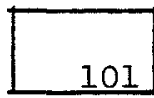

ROCK CLASSIFICATION

slightly silty sand

\section{$\frac{8}{3}$ SIZE RANGE SPHERICITY ROUNDNESS}

BASALT $\frac{\geq \text { gravel }}{\text { sand }}$

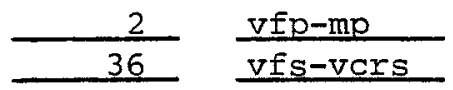

OTHER $\geq$ gravel sand

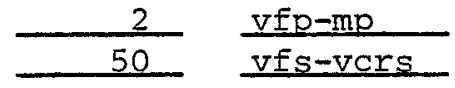

SILT AND CIAY 10

REMARKS

color

HCL

Greyish Buff $-$

Slightly Micaceous Frosted quartz

Immature

DEPTH

(FT.)

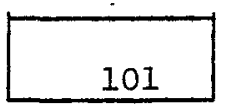

TO

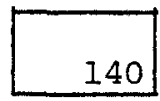

ROCK CLASSIFICATION

slightly silty sand

- 8 SIZE RANGE SPHERICITY ROUNDNESS

BASALT $\underset{\text { sand }}{\geq \text { gravel }}$

$\frac{2}{26} \quad \frac{v f p-f p}{v f s-v c r s}$

$\frac{1}{m} \quad \frac{\text { rnd }}{\mathrm{sb} \text { rnd }}$

OTHER $\geq$ grave1 sand

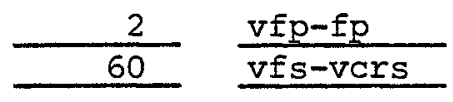

$\frac{1}{m} \frac{\text { rnd }}{\text { sb rnd }}$

SIIT AND CLAY 10

REMARKS

color Greyish Buff

HCL

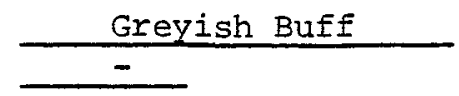

Slightly Micaceous Frosted Quartz

Immature 


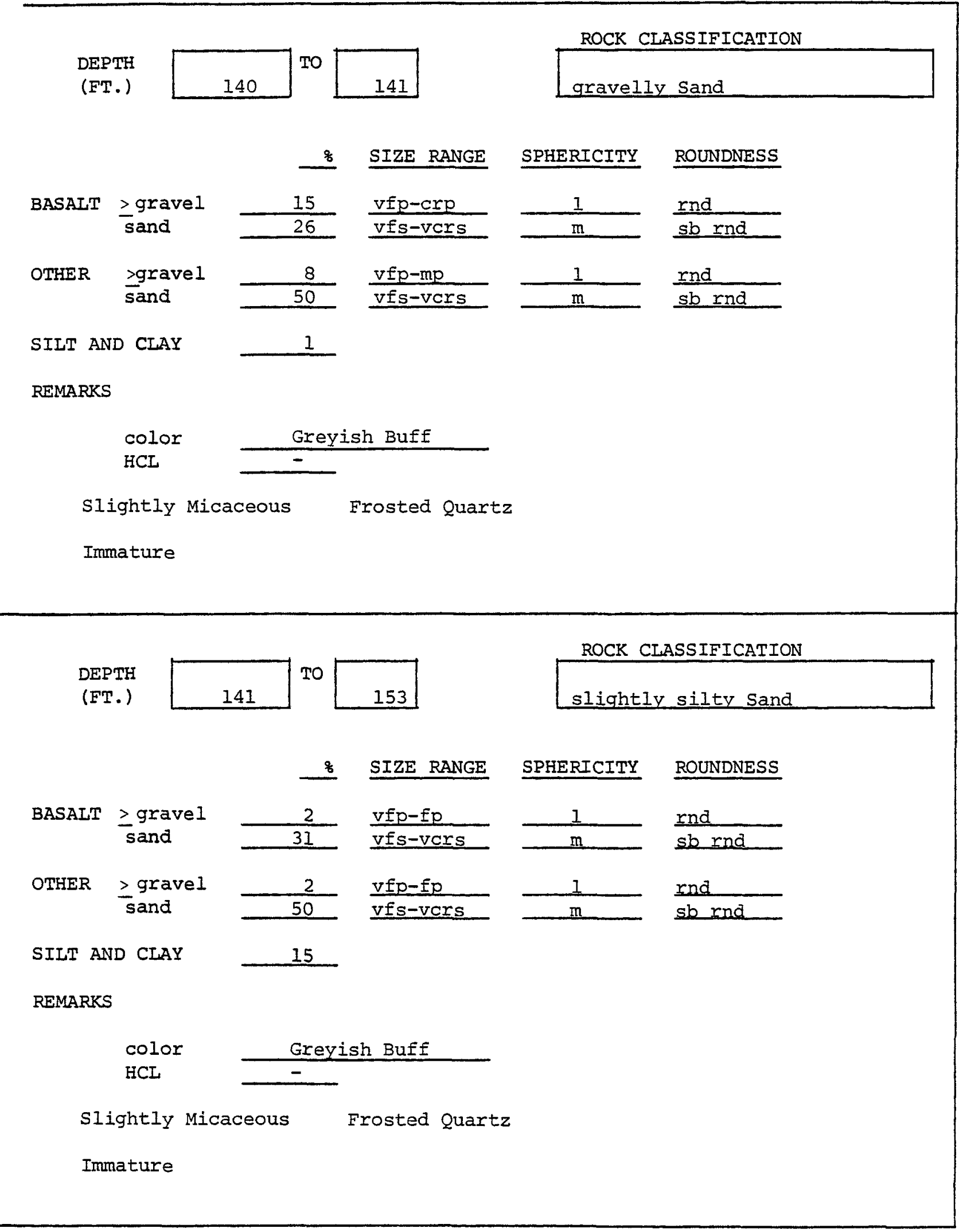


DEPTH
(FT.)

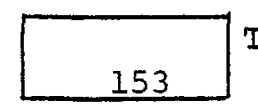

TO

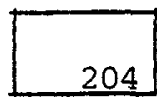

ROCK CLASSIFICATION

silty sandy Gravel

\% SIZE RANGE SPHERICITY ROUNDNESS

\begin{tabular}{|c|c|c|c|c|c|}
\hline BASALT & $\geq$ gravel & 14 & vfp-crp & m & rnd \\
\hline & sand & 20 & $v f_{S}$-vars & $\mathrm{m}$ & $\mathrm{sb} \times \mathrm{nd}$ \\
\hline OTHER & $\geq$ gravel & 19 & Vfp-crp & $\mathrm{m}$ & nnd \\
\hline & sand & 35 & vifs-vars & $\mathrm{m}$ & sb rnd \\
\hline
\end{tabular}

SIIT AND CLAY

REMARKS

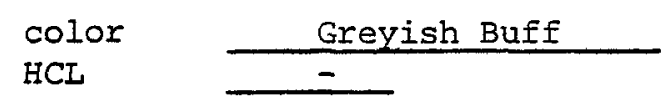

Slightly Micaceous

Immature

Total Drilled Depth reported as 204'

DEPTH

(FT.)

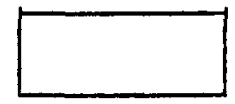

TO

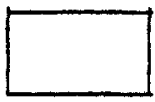

ROCK CLASSIFICATION

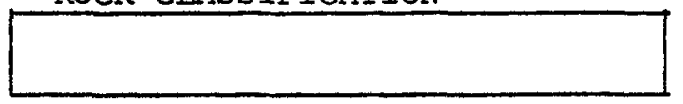

\% SIZE RANGE SPHERICITY ROUNDNESS

BASALT $\geq$ gravel sand

OTHER $\geq$ gravel sand

SILT AND CLAY

REMARKS

color

HCL
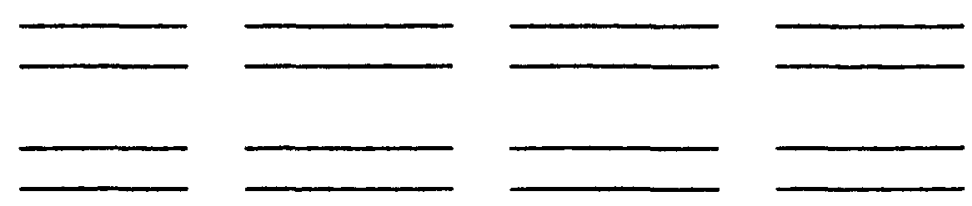
Depth (ft.)

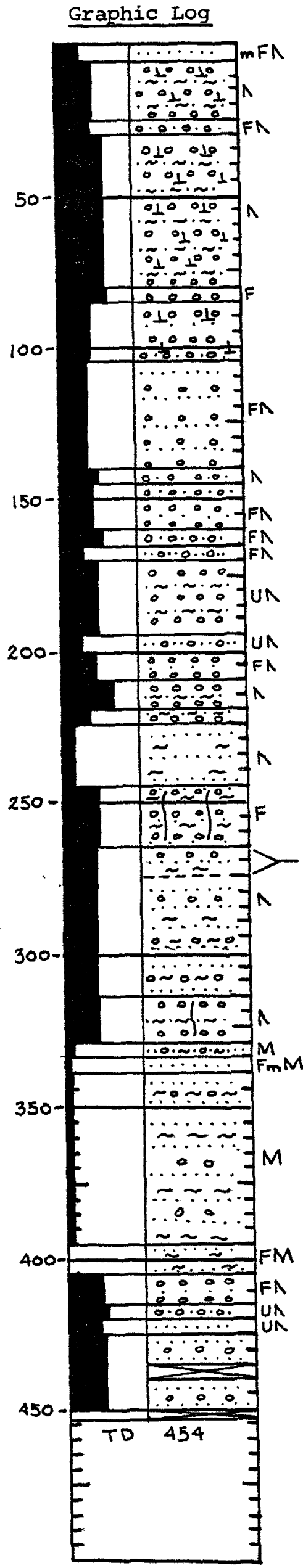

Sample Description

buff brown

greyish brown

greyish buff

brownish buff

brownish butf

Plant Coordinates

N 008577

E 002324

Altitude 416

Other Data used in Interpretation

Driller's Log

greyish brown

greyish brown

greyish brown

greyish buff

areyish brown

greyish brown

greyish brown

greyish brown

greyish brown

$\tan$

$\tan$

greyish brown; TRACE DIATOMITE (?)

TRACE OF DIATOMITE (?)

brownish $\tan$

greenish $\tan$

greenish tan; ASH PEBBLES

light brown; ASH PEBBLES

reddish light brown; ASH PEBBLES

brownish buff

brownish buff

greyish buff

grey 
DEPTH

(FT.)

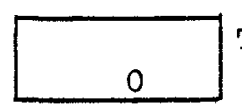

TO

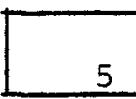

ROCK CIASSIFICATION

Sand

8 SIZE RANGE SPHERICITY ROUNDNESS

BASALT $\geq$ gravel sand
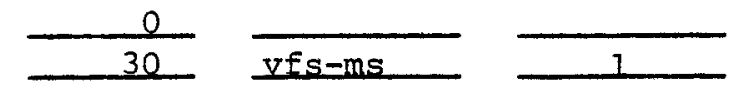

sb rnd

OTHER $\geq$ gravel
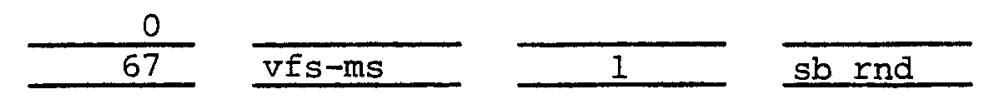

SIIT AND CIAY 3

REMARKS

\begin{tabular}{lll} 
Color & \multicolumn{1}{c}{ Buff Brown } \\
HCL & Frosted Quartz \\
lightly Micaceous &
\end{tabular}

Mature

DEPTH
(FT.)

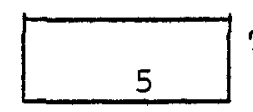

TO

25

ROCK CLASSIFICATION

silty sandy Gravel

\section{\% SIZE RANGE SPHERICITY ROUNDNESS}

BASAIT $\geq$ gravel
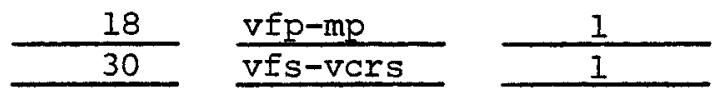

$\frac{\mathrm{sb} \text { rnd }}{\mathrm{sb} \text { rnd }}$

OTHER $\geq$ gravel
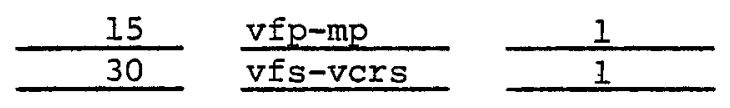

sb rnd

SIIT AND CIAY

7

REMARKS

$\begin{array}{ll}\text { color } & \text { Greyish Brown } \\ & +\end{array}$

Slightly Micaceous

Immature 


\section{DEPTH}

(ET.)

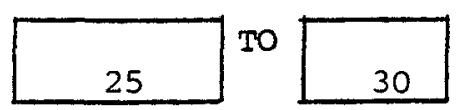

\% SIZE RANGE SPHERICITY ROUNDNESS

BASALT $\geq$ grave1 sand
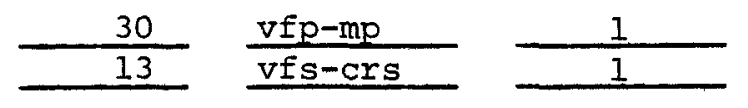

OTHER $\underset{\text { sand }}{\geq \text { gravel }}$

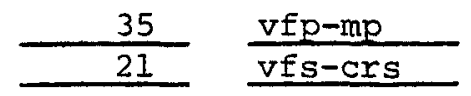

SILT AND CLAY

1

REMARKS

color

HCL

Greyish Buff

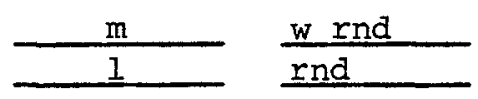

wrind

sandy Gravel

SIightly Micaceous Frosted Quartz

Immature
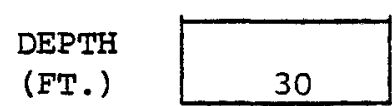

TO

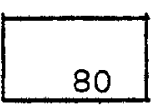

ROCK CLASSIFICATION 


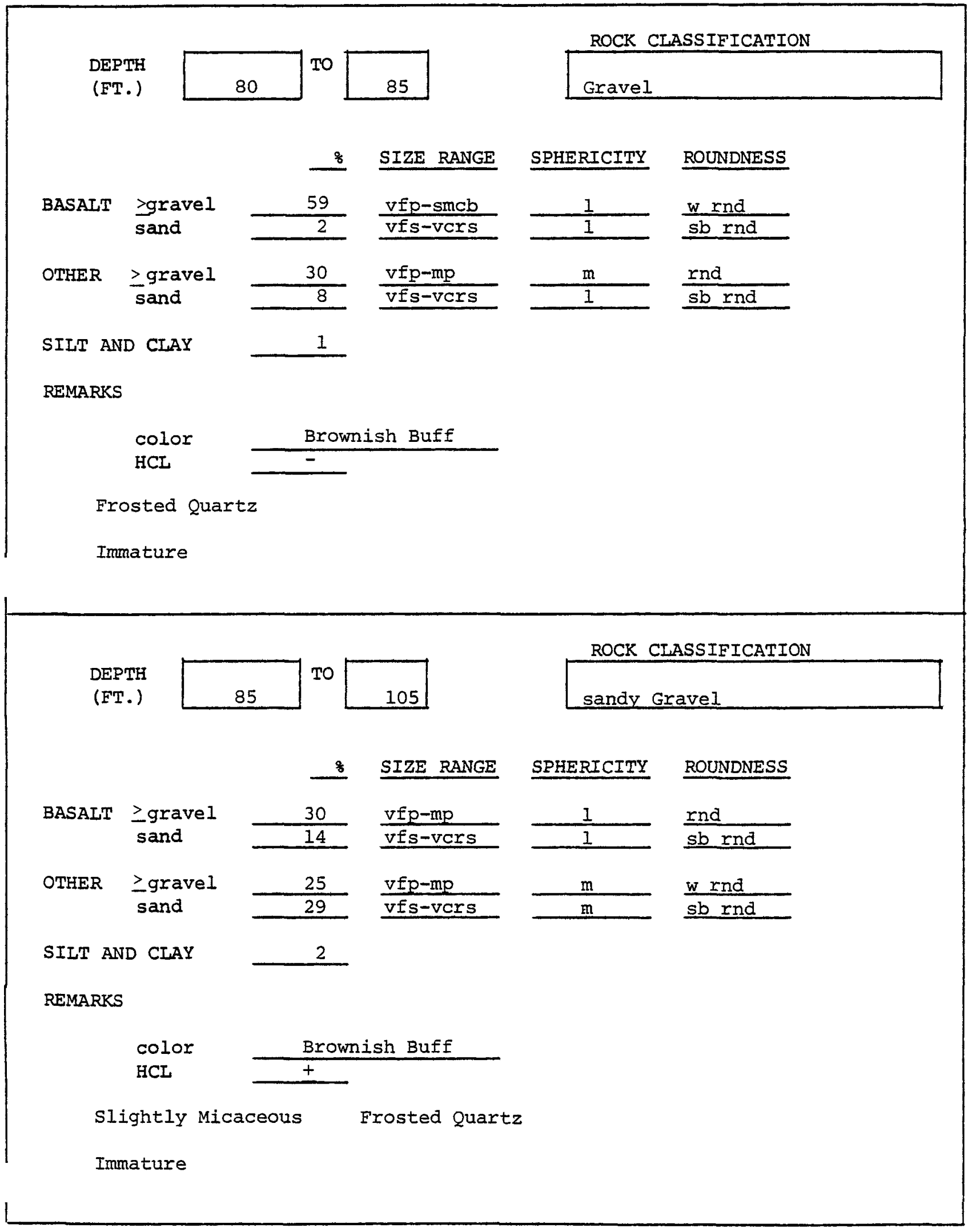




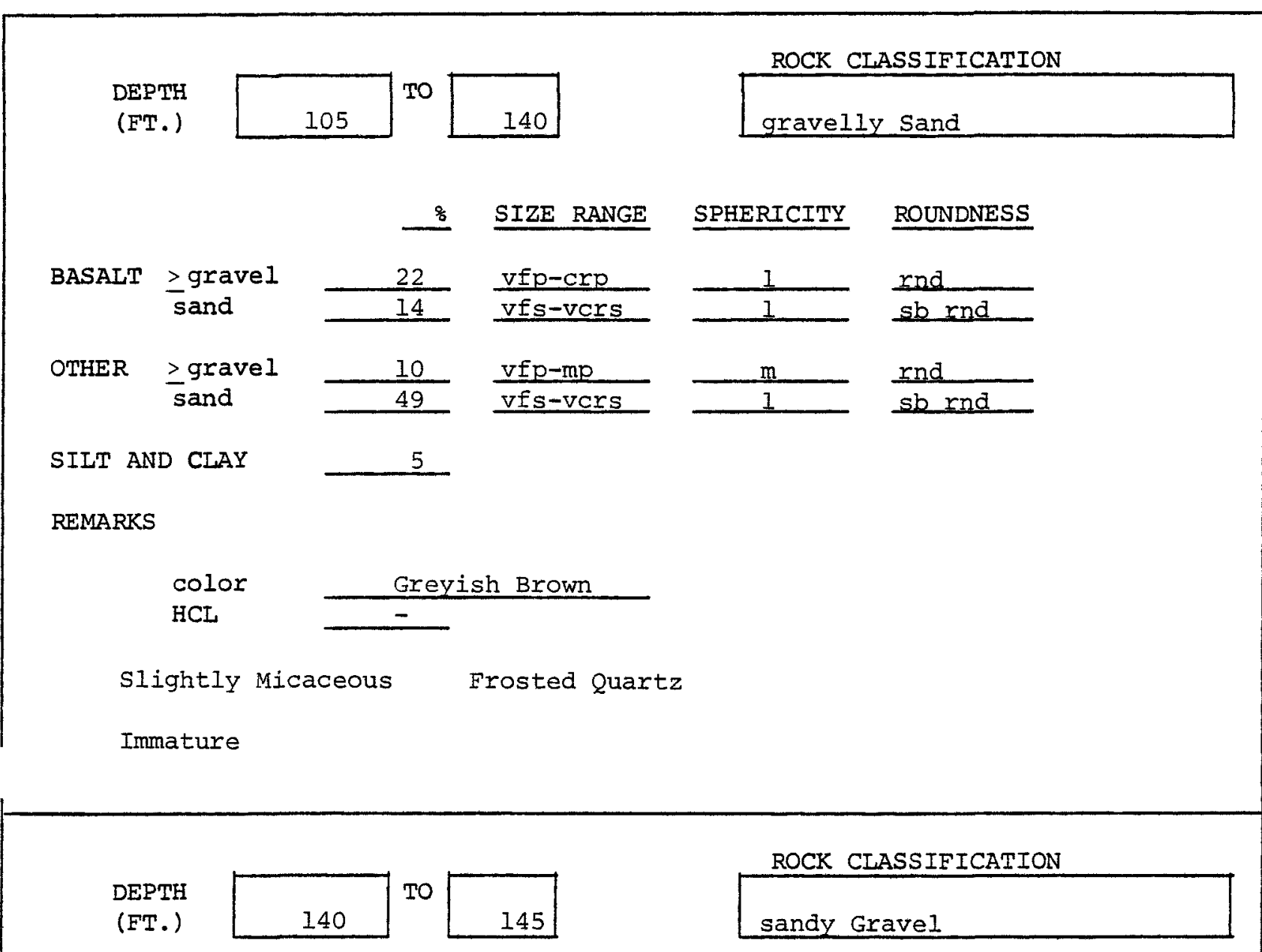

\% SIZE RANGE SPHERICITY ROUNDNESS

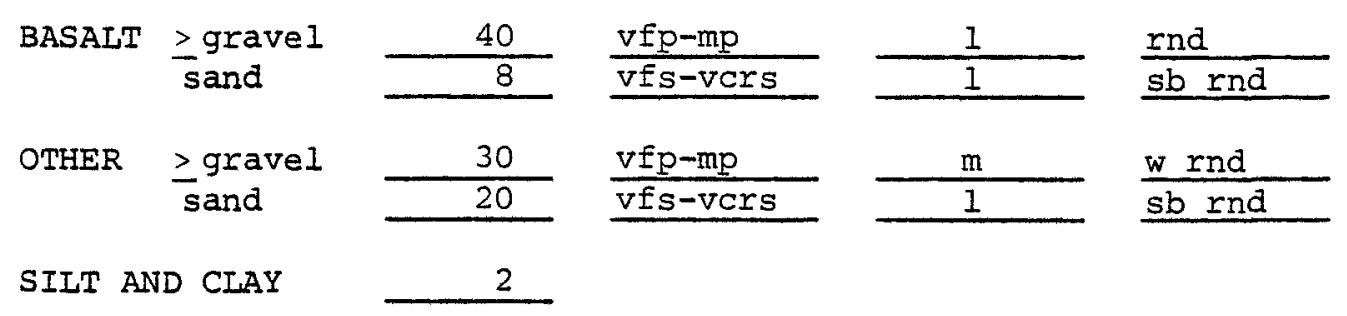

REMARKS

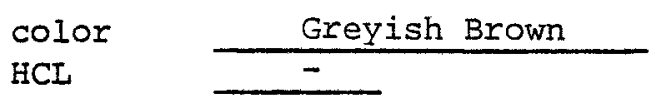

Slightly Micaceous

Immature 
DEPTH

(FT.)

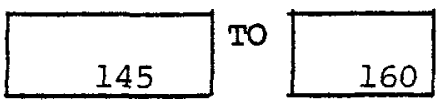

\section{ROCK CLASSIFICATION}

sandy Gravel

\section{SIZE RANGE SPHERICITY ROUNDNESS}

BASALT $\frac{\geq \text { graveI }}{\text { sand }}$

$\frac{27}{14} \frac{\text { vfp-crp }}{\text { vfs-vers }}$

OTHER

$$
\begin{aligned}
& \geq \text { gravel } \\
& \text { sand }
\end{aligned}
$$

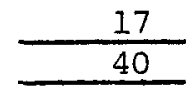
$\mathrm{vfp}-\mathrm{mp}$
vfs-vars

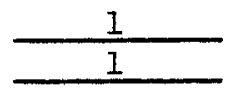

rnd

sb rnd

w rnd

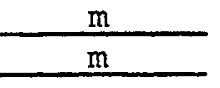

sb rnd

SILT AND CLAY

2

REMARKS

color

Greyish Brown

$\mathrm{HCL}$

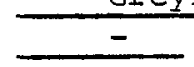

Slightly Micaceous Frosted Quartz

Immature

DEPTH

(FT.)

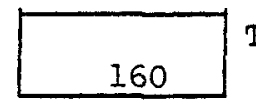

TO

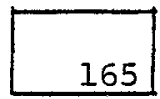

ROCK CLASSIFICATION

sandy Gravel

\section{- SIZE RANGE SPHERICITY ROUNDNESS}

BASALT

$$
\begin{aligned}
& \text { s gravel } \\
& \text { sand }
\end{aligned}
$$
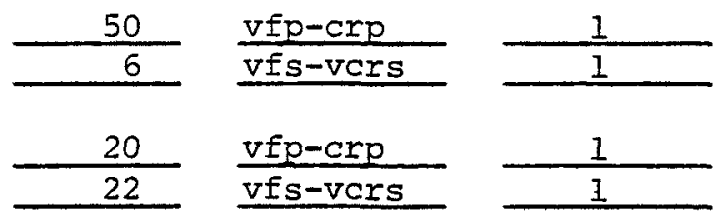

w rnd

OTHER $\geq$ gravel sand

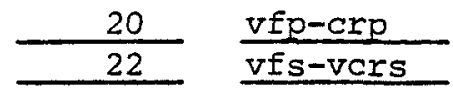

$\frac{w \text { rnd }}{\text { sb rnd }}$

SILT AND CLAY

2

REMARKS

color Greyish Buff

HCL

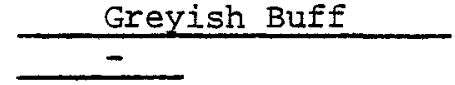

Slightly Micaceous Frosted quartz

Immature 
ROCK CIASSIFICATION

DEPTH

(FT.)

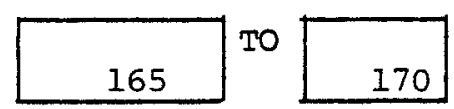

gravelly sand

\% SIZE RANGE SPHERICITY ROUNDNESS

BASALT >gravel sand
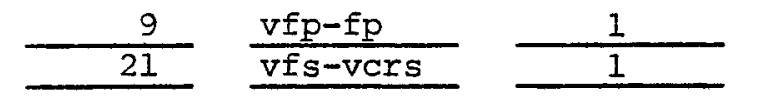

rnd

OTHER $\geq$ gravel sand
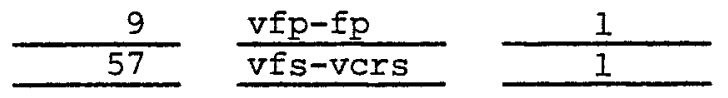

rnd

sb rnd

SIIT AND CIAY

4

REMARKS

color

HCL

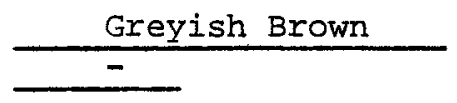

Slightly Micaceous

Frosted Quartz

Immature

\section{DEPTH}

(FT.)

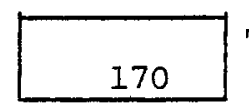

TO

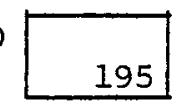

ROCK CIASSIFICATION

silty sandy Gravel

\section{S SIZE RANGE SPHERICITY ROUNDNESS}

\begin{tabular}{|c|c|c|c|c|c|}
\hline \multirow[t]{2}{*}{ BASAIT } & > gravel & 40 & $v f p-s m c b$ & 1 & $w$ rnd \\
\hline & sand & 10 & vfs-vcrs & 1 & sb rnd \\
\hline \multirow[t]{2}{*}{ OTHER } & > gravel & 20 & $v f p-s m c b$ & 1 & w rnd \\
\hline & sand & 26 & vfs-vcrs & 1 & sb rnd \\
\hline
\end{tabular}

SILT AND CLAY

4

REMARKS

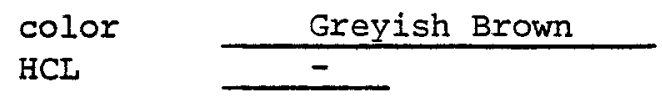

Slightly Micaceous Unfrosted Quartz

Immature 
DEPTH (FT.)

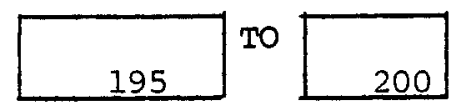

ROCK CLASSIFICATION

gravelly sand

\& SIZE RANGE SPHERICITY ROUNDNESS

BASALT $\geq$ gravel

$$
\text { sand }
$$
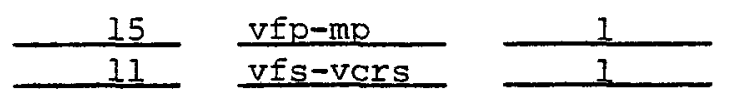

rnd

sb ang

OTHER $\underset{\substack{\text { sand } \\ \text { sand }}}{\text { grave }}$
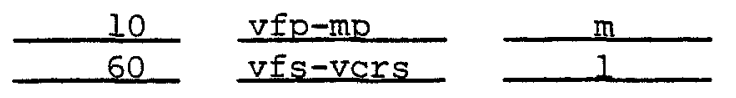

$\frac{\text { wrind }}{\text { sb rnd }}$

SILT AND CLAY

4

REMARKS

color

HCI

Grevish Brown

$-$

Slightly Micaceous Unfrosted quartz

Immature

DEPTH
(FT.)

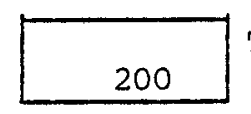

TO

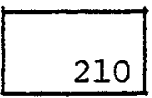

ROCK CLASSIFICATION

sandy Gravel

\% SIZE RANGE SPHERICITY ROUNDNESS BASALT $\underset{\text { sand }}{\geq \text { gravel }}$
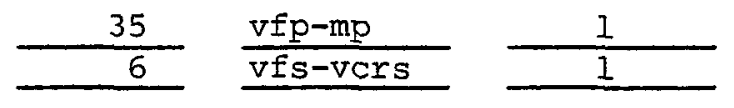

rnd

OTHER $\underset{\text { sand }}{\geq \text { gravel }}$
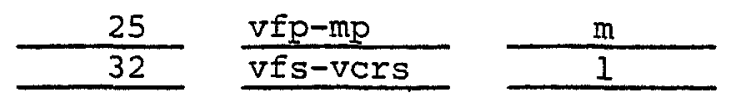

$\frac{w \text { rnd }}{\text { sb rnd }}$

SIIT AND CLAY 2

REMARKS

$$
\text { color }
$$

HCL

Greyish Brown

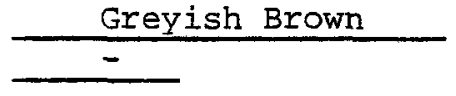

Slightly Micaceous

Frosted Quartz

Immature 


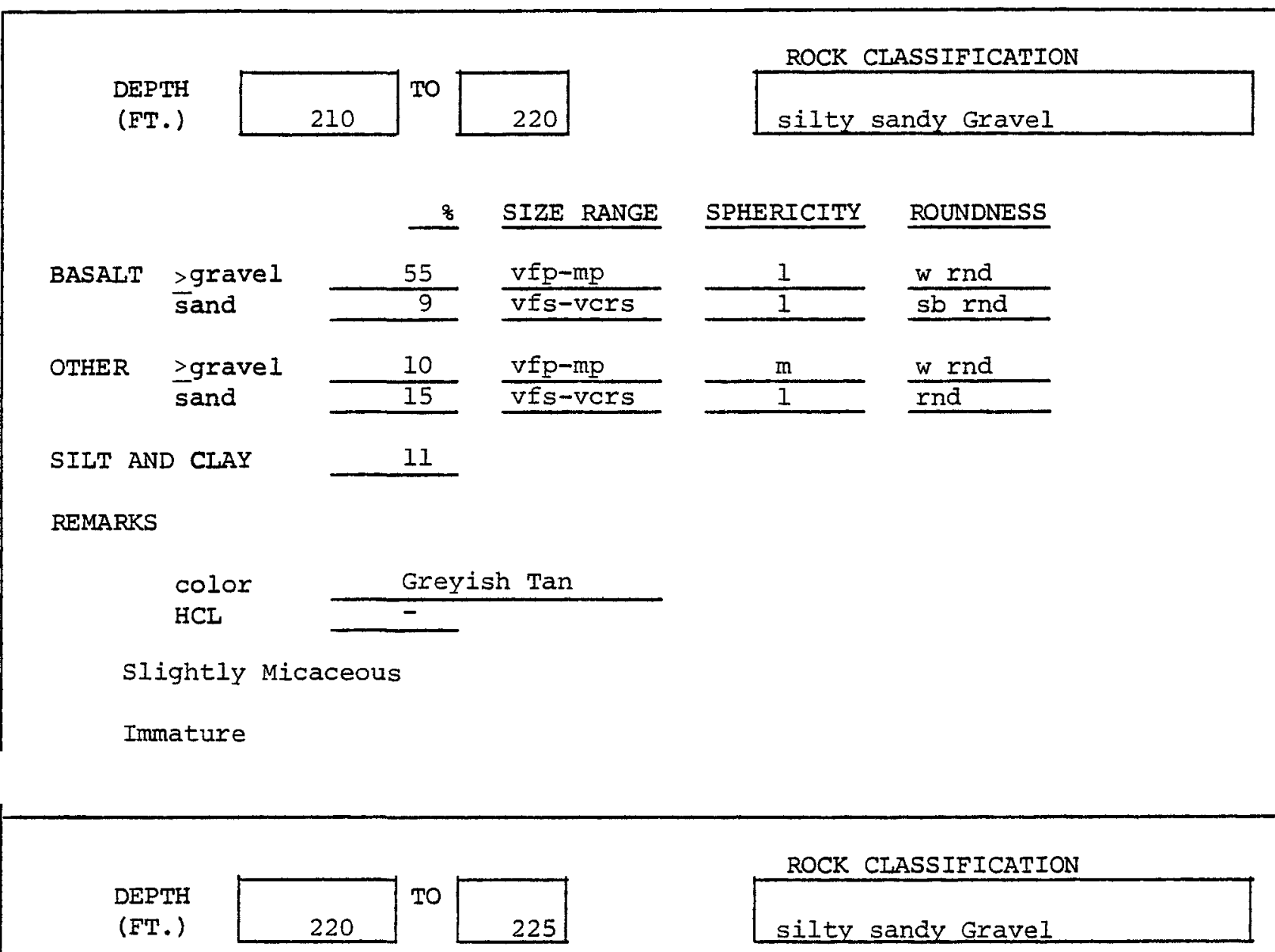

8 SIZE RANGE SPHERICITY ROUNDNESS

\begin{tabular}{|c|c|c|c|c|c|}
\hline \multirow[t]{2}{*}{ BASALT } & $>$ gravel & 25 & $v f p-m p$ & 1 & w rnd \\
\hline & sand & 9 & vfs-vers & 1 & $\mathrm{sb}$ rnd \\
\hline \multirow[t]{2}{*}{ OTHER } & Igravel & 15 & $v f p-m p$ & $\mathrm{~m}$ & w rnd \\
\hline & sand & 42 & vfs-vcrs & 1 & sb rnd \\
\hline SIIT AN & D CIAY & 9 & & & \\
\hline
\end{tabular}

REMARKS

color

HCI

Tan

Immature 
DEPTH

(FT.)

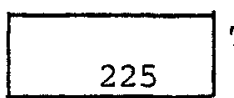

TO

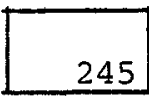

ROCK CLASSIFICATION

slightly silty sand

\section{\& SIZE RANGE SPHERICITY ROUNDNESS}

\begin{tabular}{|c|c|c|c|c|c|}
\hline BASALT & $\begin{array}{l}\text { > gravel } \\
\text { sand }\end{array}$ & $\frac{5}{8}$ & $\frac{v f p-f p}{v f s-c r s}$ & $\frac{1}{1}$ & $\frac{\text { rnd }}{\text { sb rnd }}$ \\
\hline OTHER & $>$ gravel & 0 & & & \\
\hline & sand & 77 & vfs-crs & $\mathrm{m}$ & sb rnd \\
\hline
\end{tabular}

REMARKS

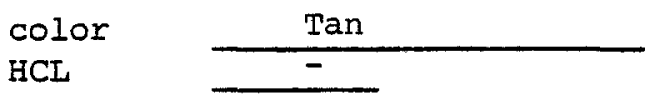

Slightly Micaceous

Immature

DEPTH

(FT.)

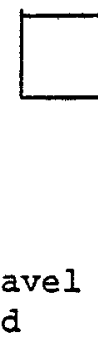

245

TO

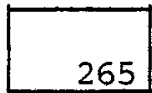

ROCK CLASSIFICATION

silty sandy Gravel

$\frac{\%}{3}$ SIZE RANGE SPHERICITY ROUNDNESS

BASALT $\begin{gathered}\geq \text { gravel } \\ \text { sand }\end{gathered}$
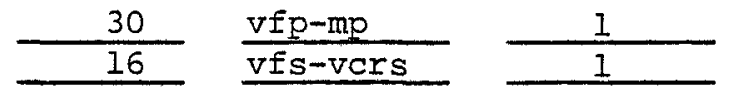

w rnd

OTHER $\underset{\text { sand }}{\geq \text { gravel }}$
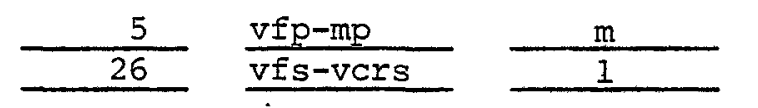

w rnd

SILT AND CLAY

$$
23
$$

REMARKS

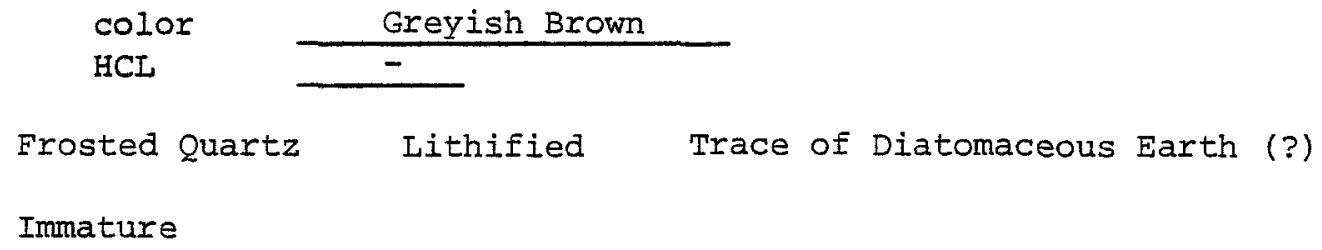



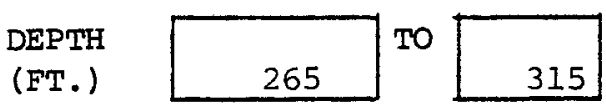

ROCK CLASSIFICATION

(FT.)

265

\section{\% SIZE RANGE SPHERICITY ROUNDNESS}

BASALT $\frac{\geq \text { gravel }}{\text { sand }}$
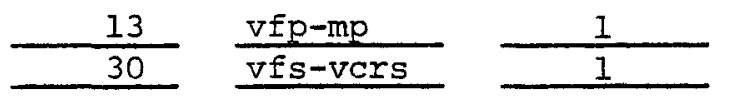

rnd
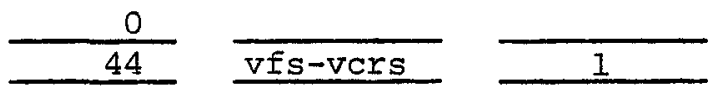

rnd

SIIT AND CIAY

13

REMARKS

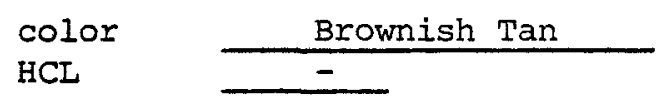

Slightly Micaceous Trace of Diatomaceous Earth (? between 265' \& 275')

Immature

DEPTH
(FT.)

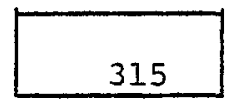

TO

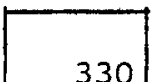

ROCK CLASSIFICATION

330

silty sandy Gravel

\section{SIZE RANGE SPHERICITY ROUNDNESS}

BASALT $\underset{\text { sand }}{\geq \text { gravel }}$
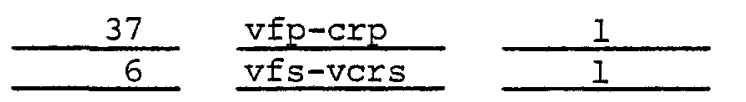

$\frac{\text { sb rnd }}{\text { sb rnd }}$

OTHER $\geq$ gravel
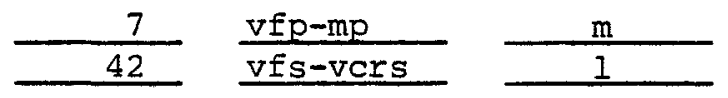

$\frac{w \text { rnd }}{\text { rnd }}$

SIIT AND CLAY

8

REMARKS

$$
\text { color }
$$

HCL.

Greenish Tan

Slightly Micaceous

Compacted

Immature 
ROCK CLASSIFICATION

DEPTH

(FT.)

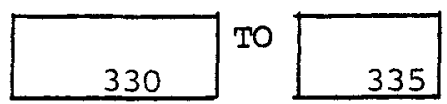

\& SIZE RANGE SPHERICITY ROUNDNESS

BASAIT > gravel sand
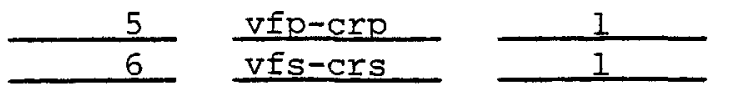

$\frac{\text { sb rnd }}{\text { sb rnd }}$

OTHER $\geq$ graveI
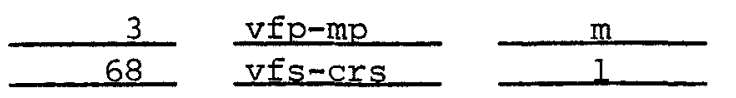

sb rnd

SILT AND CLAY

18

REMARKS

$$
\begin{aligned}
& \text { color } \\
& \text { HCL }
\end{aligned}
$$

Greenish Tan

Micaceous

Ash (pebbles present)

Immature

\section{DEPTH}

(ET.)

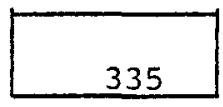

TO
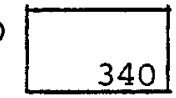

ROCK CIASSIFICATION

Sand

BASALT $\geq$ gravel sand
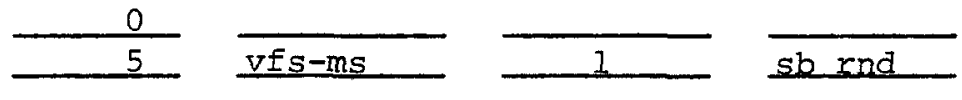

OTHER $\geq$ gravel sand
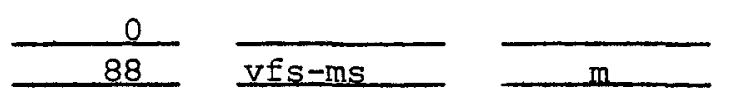

SILT AND CIAY

7

REMARKS

color
HCL

Micaceous

Mature
Light Brown

Ash (pebbles present)
Frosted Quartz (?) 


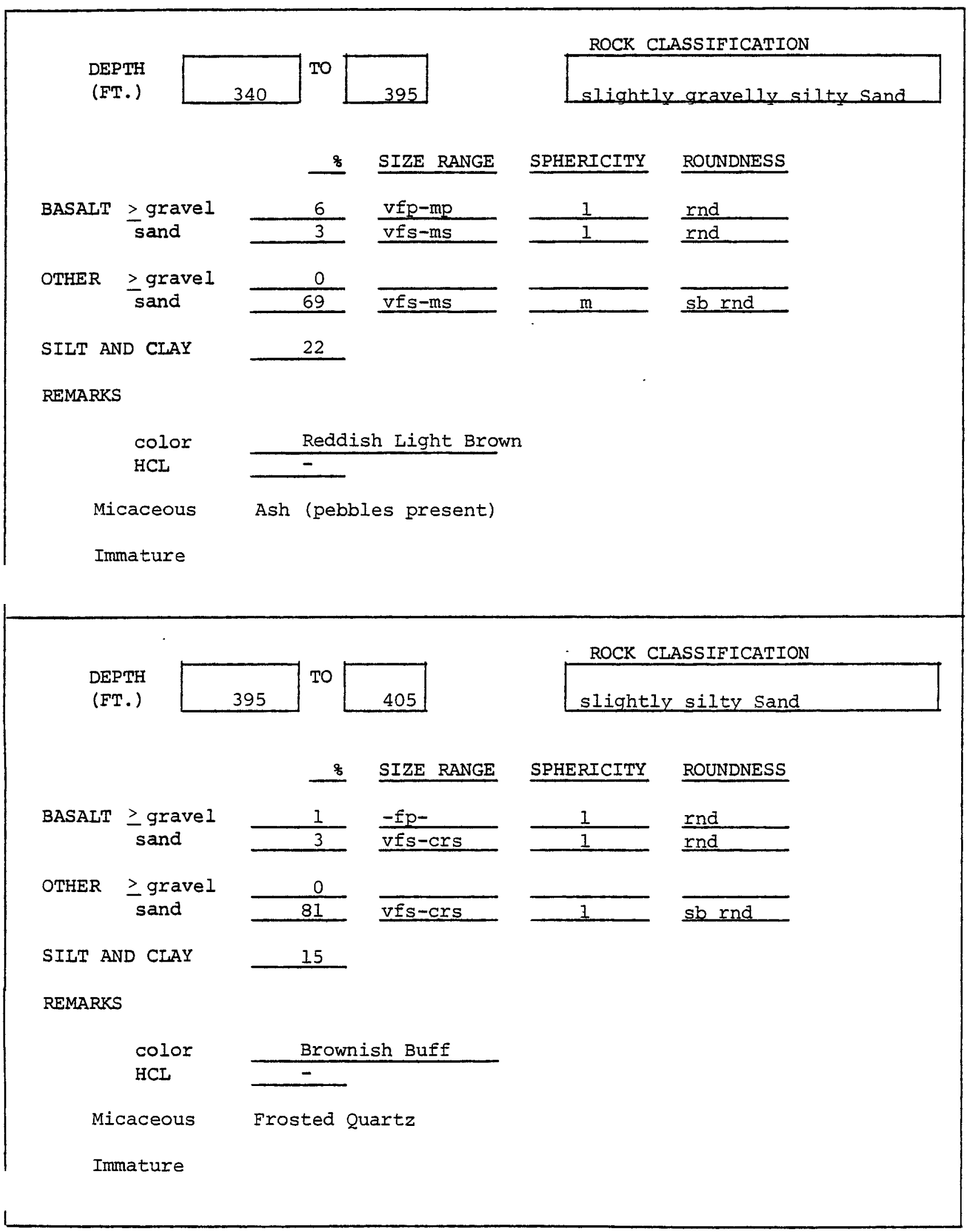

W. K. Summers \& Associates 


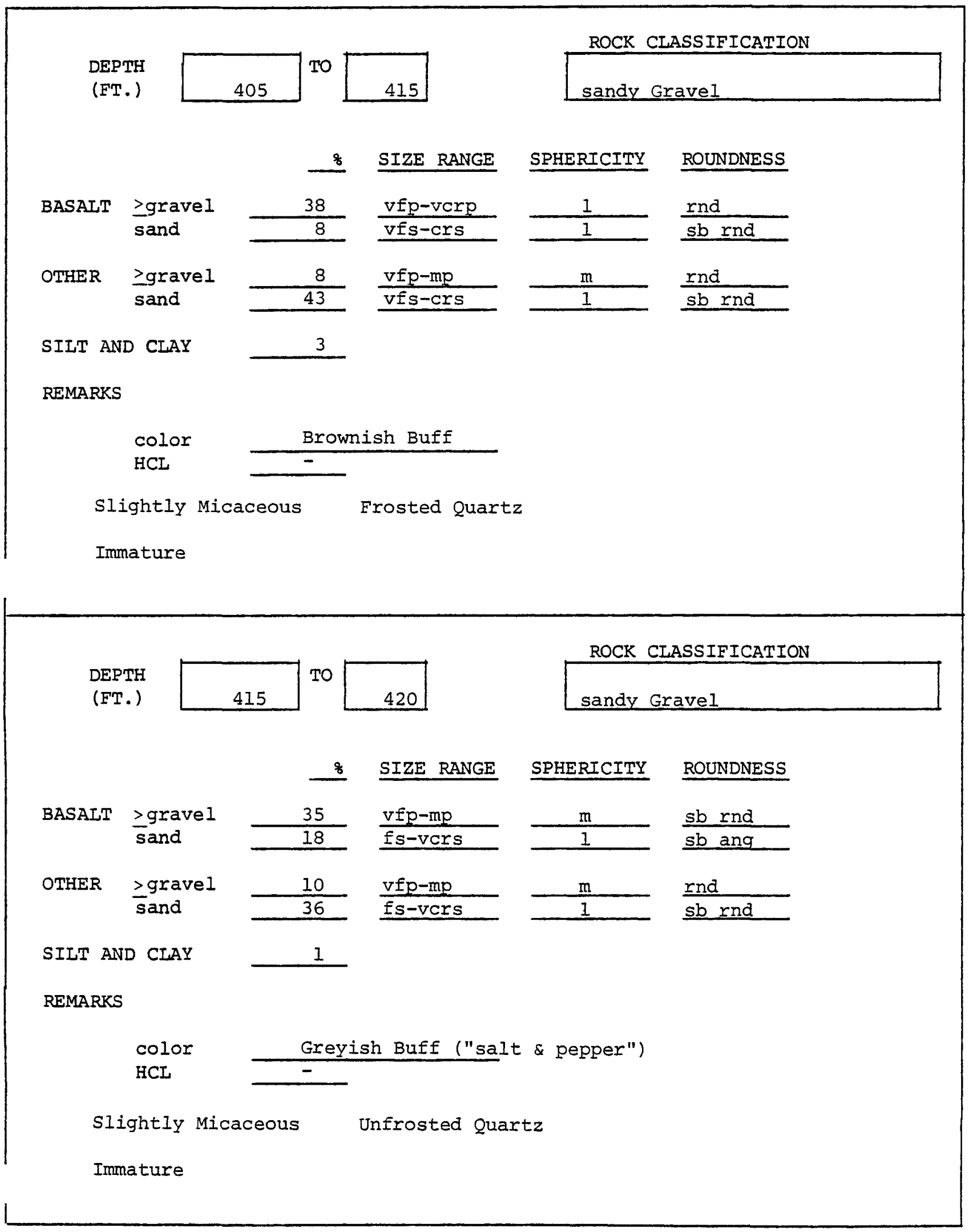




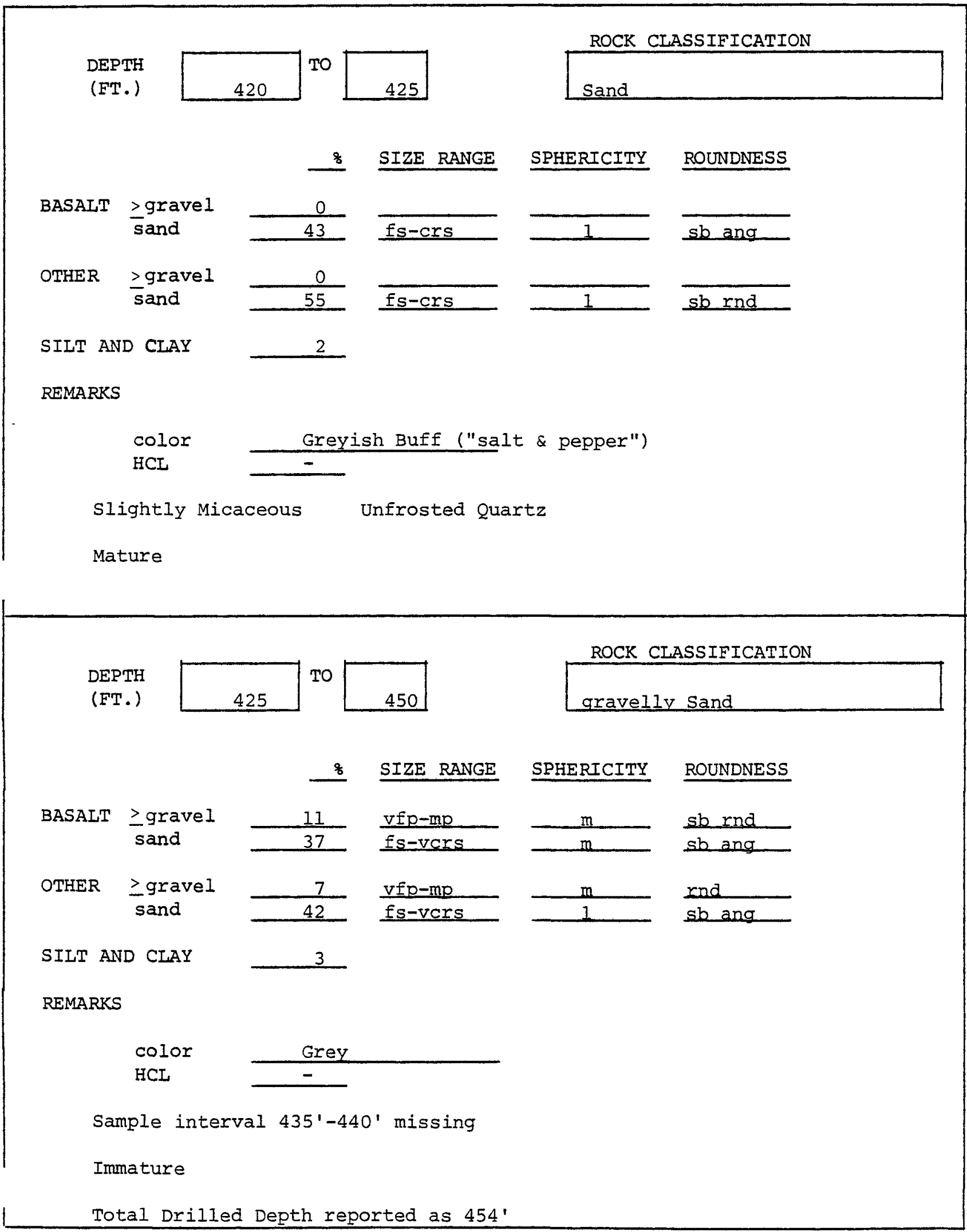




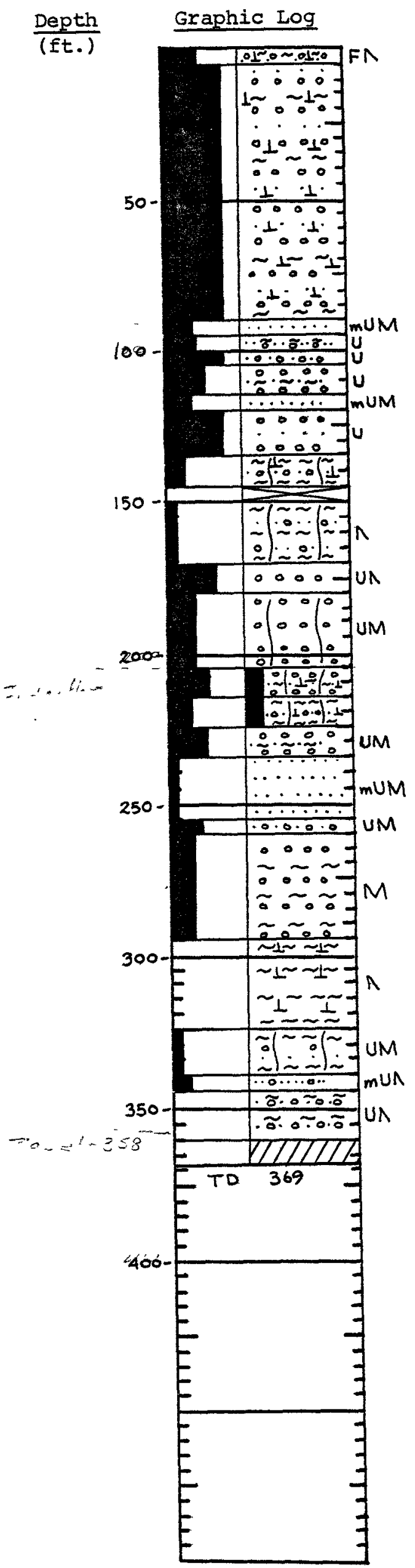

Sample Description

greyish brown

greyish black

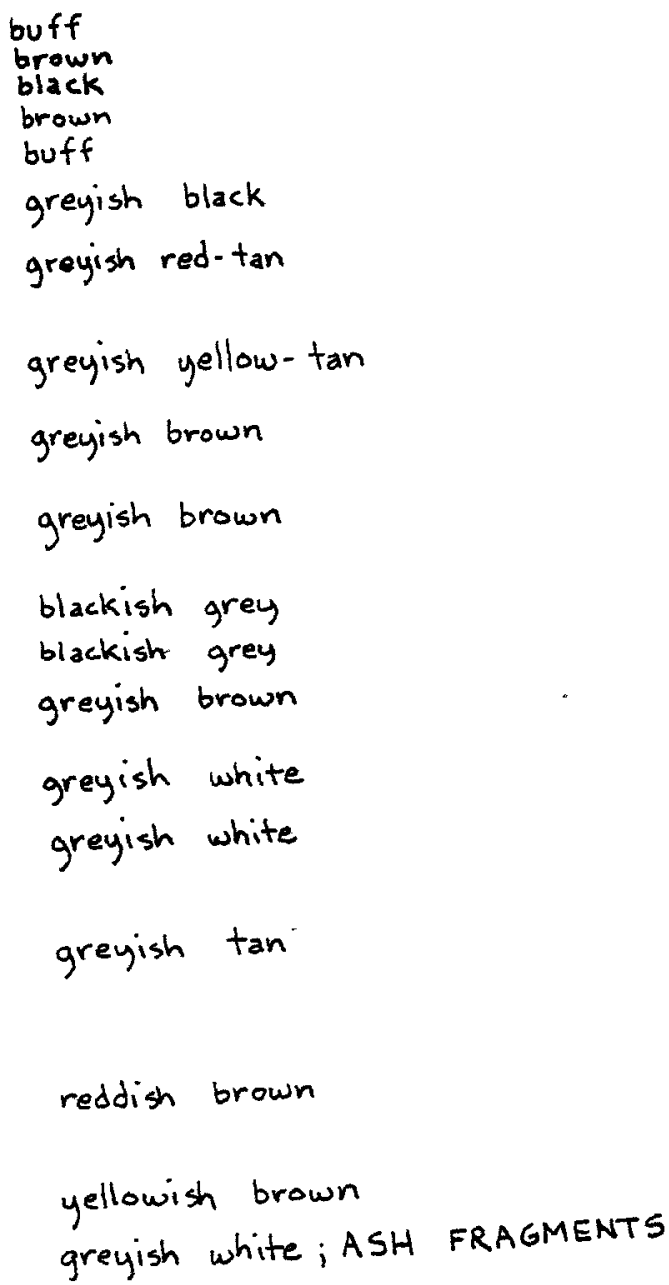

Nol0000

$\frac{E 012000}{\text { Altitude } 429}$




\section{DEPTH}

(FT.)

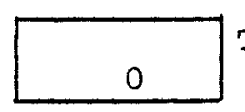

To

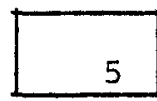

ROCK CLASSIFICATION

silty sandy Gravel

\% SIZE RANGE SPHERICITY ROUNDNESS

\begin{tabular}{|c|c|c|c|c|c|}
\hline \multirow[t]{2}{*}{ BASALT } & \multirow{2}{*}{$\begin{array}{l}\geq \text { gravel } \\
\text { sand }\end{array}$} & 30 & $v f p-s m c b$ & $I$ & $\mathrm{w}$ rnd \\
\hline & & 15 & vfs-vers & $\mathrm{m}$ & sb rnd \\
\hline \multirow[t]{2}{*}{ OTHER } & > gravel & 10 & $v f p-c r p$ & I & $w$ rnd \\
\hline & $\bar{s}$ and & 24 & vfs-vars & $h$ & sb ang \\
\hline
\end{tabular}

SILT AND CLAY

21

REMARKS

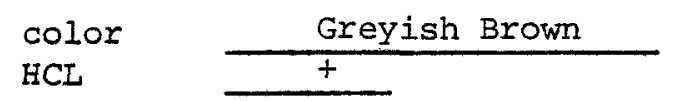

Slightly Micaceous Frosted quartz

Immature

\section{DEPTH}

(FT.)

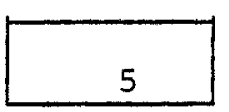

To

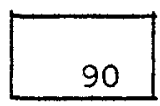

ROCK CLASSIFICATION

silty sandy Gravel

\section{SIZE RANGE SPHERICITY ROUNDNESS}

\begin{tabular}{|c|c|c|c|c|c|}
\hline BASALT & $\begin{array}{l}\geq \text { gravel } \\
\text { sand }\end{array}$ & $\frac{59}{20}$ & $\frac{\text { vfp-crp }}{\text { vfs-vers }}$ & $\frac{1}{m}$ & $\frac{\text { rnd }}{s b \text { rnd }}$ \\
\hline OTHER & $\geq$ gravel & 7 & $v f p-c r p$ & 1 & rnd \\
\hline & sand & 7 & vfs-vers & $\mathrm{h}$ & sb ang \\
\hline
\end{tabular}

SILT AND CLAY

7

REMARKS

color

HCL

Greyish Black

$+$

Immature 


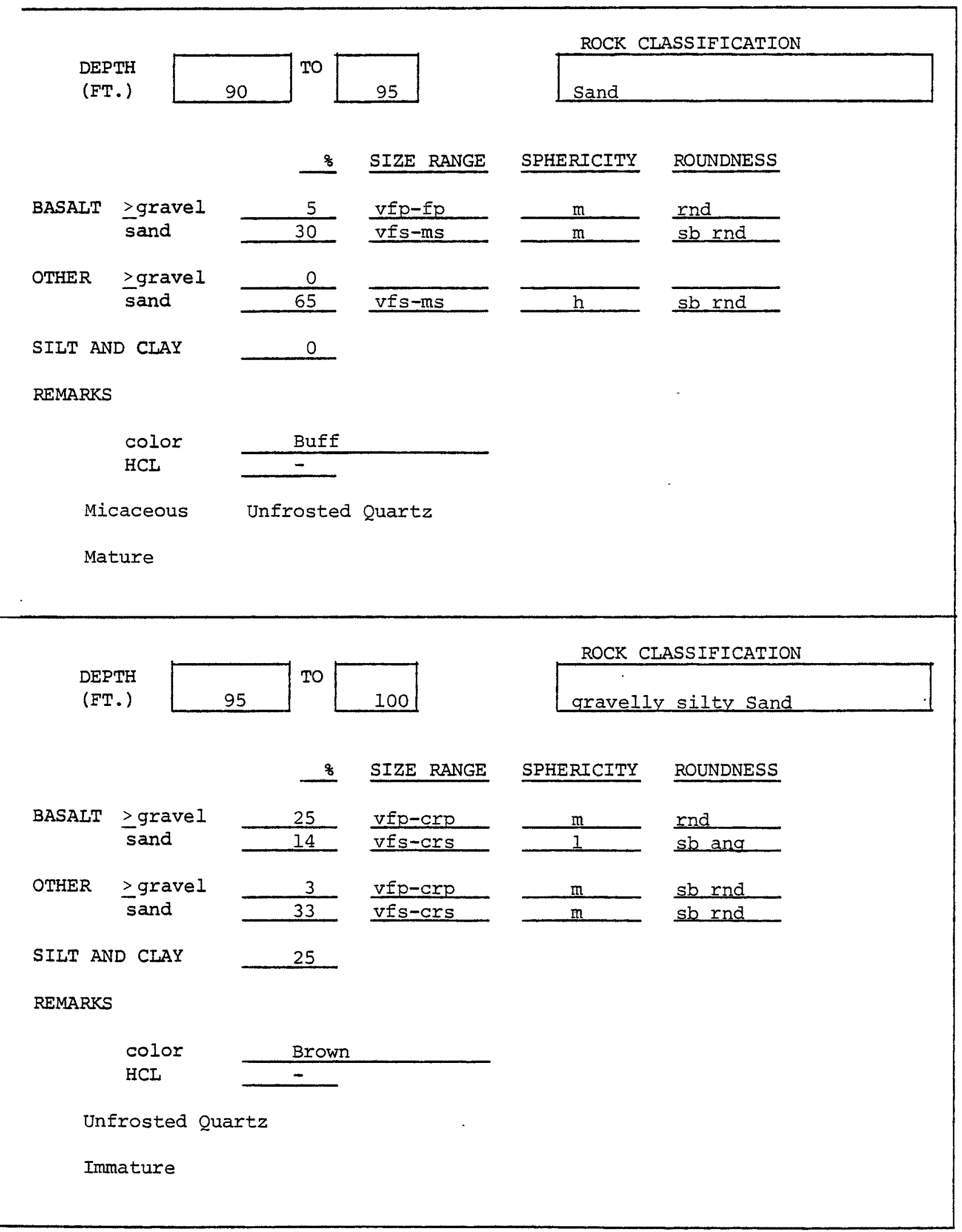




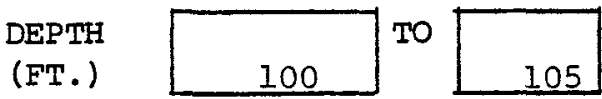

ROCK CIASSIFICATION

(FT.)

$100 \quad 105$

sandy Gravel

\& SIZE RANGE SPHERICITY ROUNDNESS

\begin{tabular}{|c|c|c|c|c|c|}
\hline \multirow[t]{2}{*}{ BASAIT } & >gravel & 70 & $\mathrm{vfp}-\mathrm{crp}$ & 1 & rnd \\
\hline & sand & 7 & VIs-vcrs & $\mathrm{m}$ & sb rnd \\
\hline \multirow[t]{2}{*}{ OTHER } & > gravel & 10 & $v \pm p-f p$ & 1 & rnd \\
\hline & sand & 12 & VIs-vcrs & $m$ & sb rnd \\
\hline
\end{tabular}

SIIT AND CIAY

1

REMARKS

color

Black

$\mathrm{HCL}$

$-$

Unfrosted Quartz

Immature

DEPTH
(ET.)

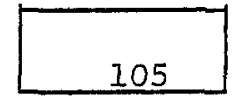

TO

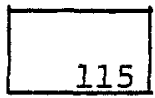

ROCK CIASSIFICATION

silty sandy Gravel

8 SIZE RANGE SPHERICITY ROUNDNESS

BASAIT $\geq$ gravel sand

OTHER $\geq$ gravel sand

SILT AND CIAY
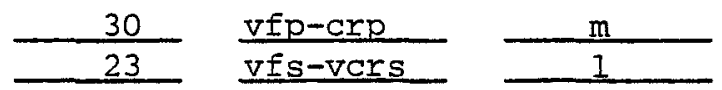

rnd

sp ang

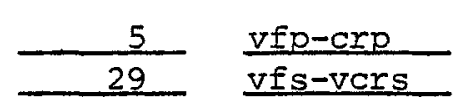

13

REMARKS

color

HCL

Brown

$-$

Unfrosted Quartz

Immature 


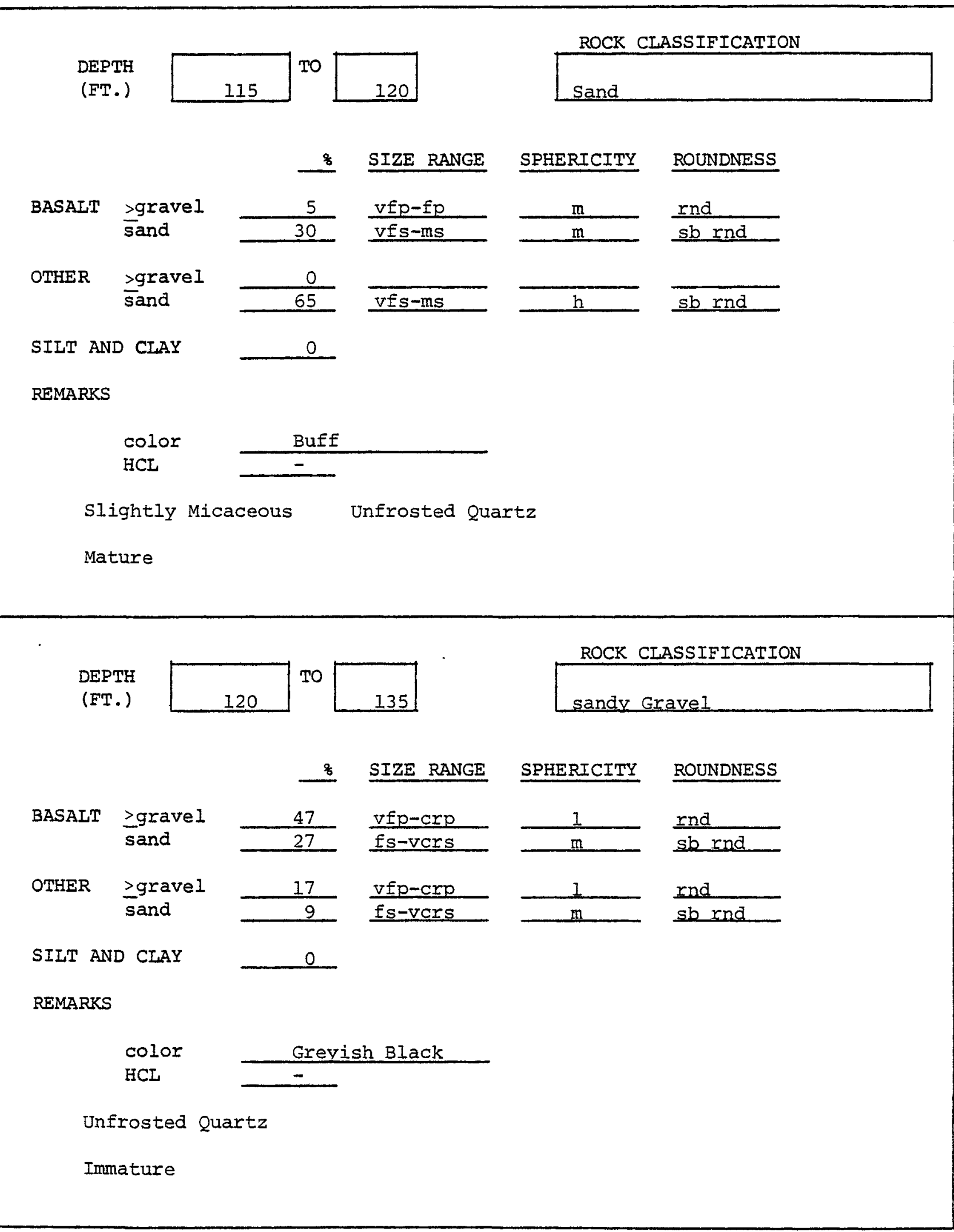




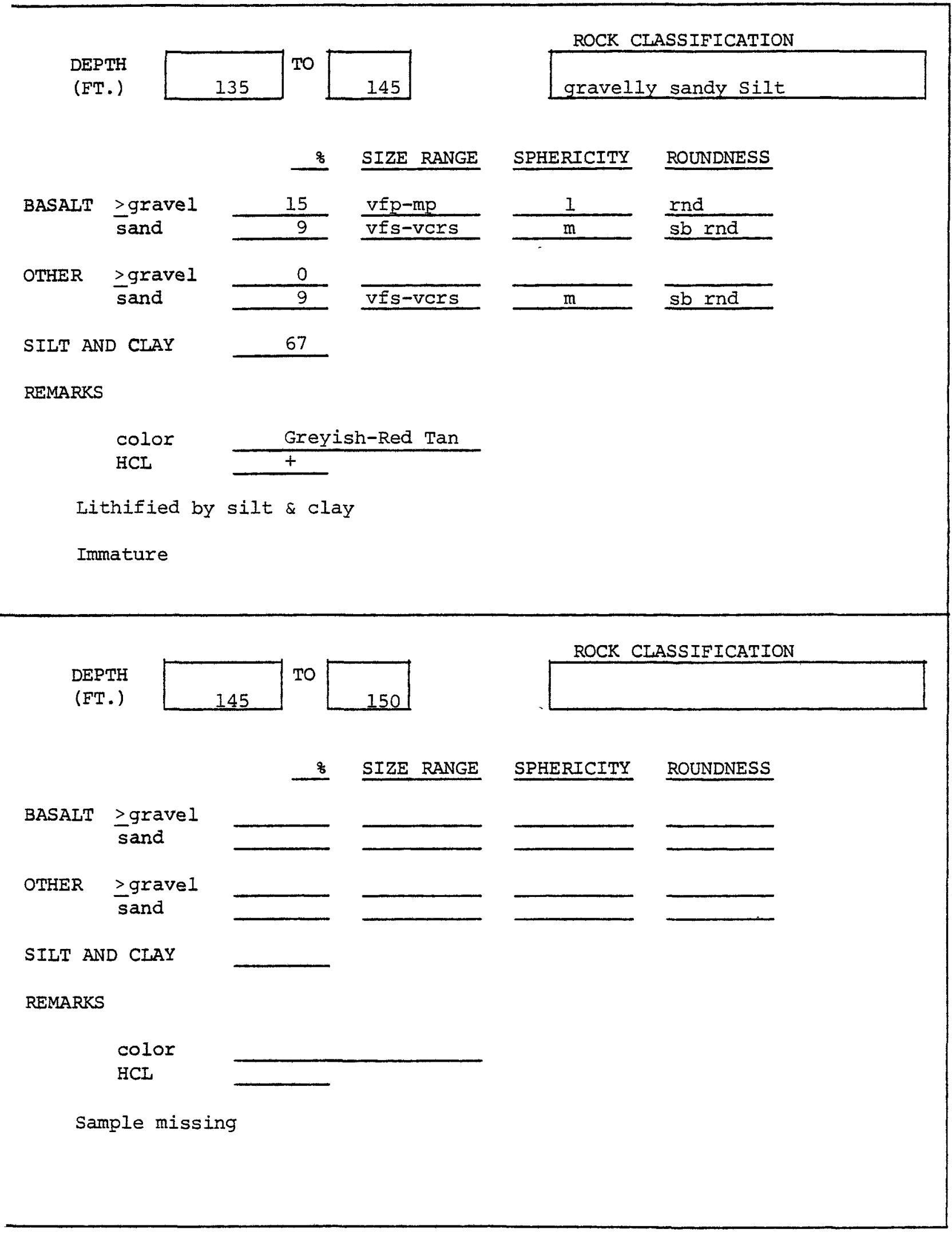




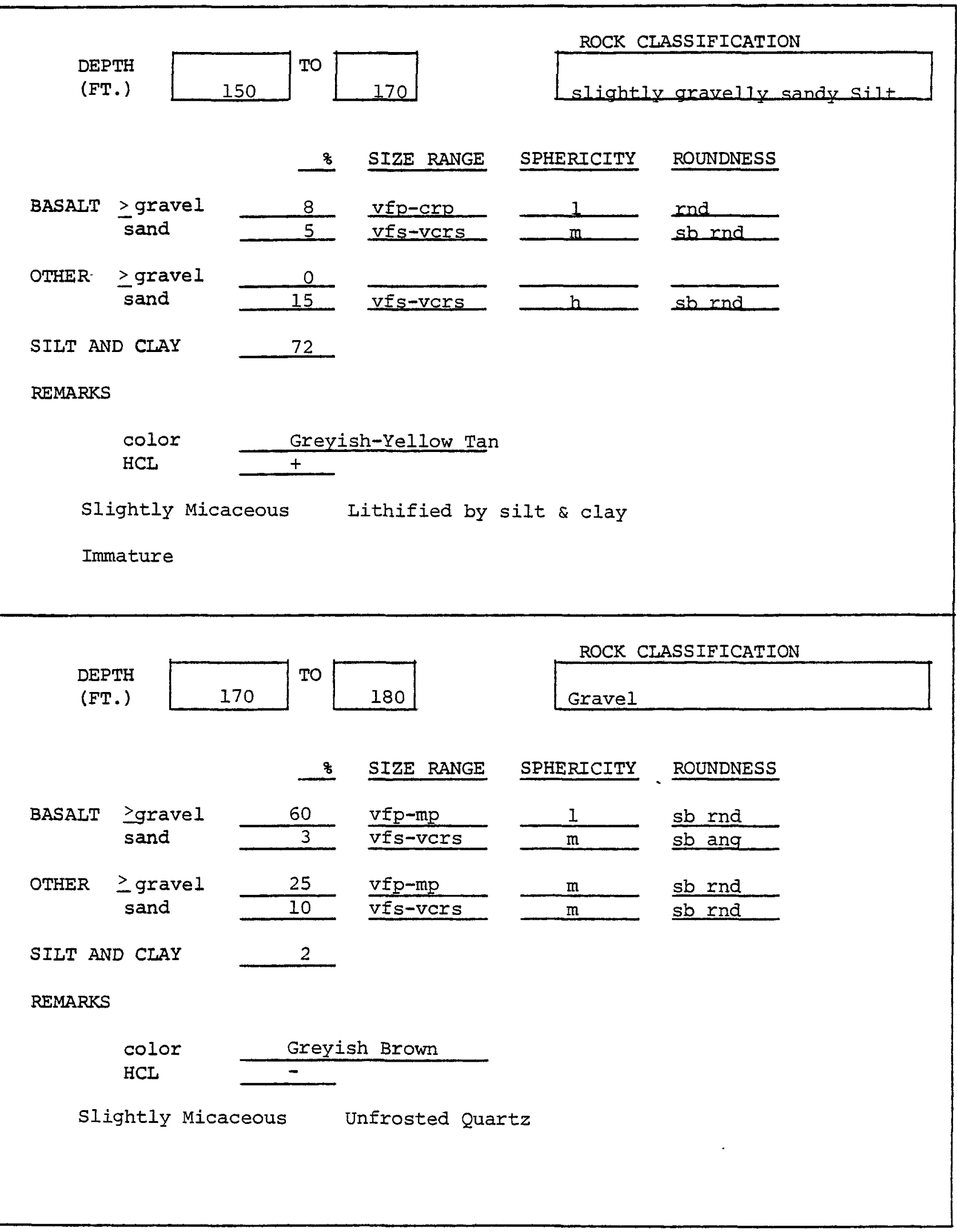




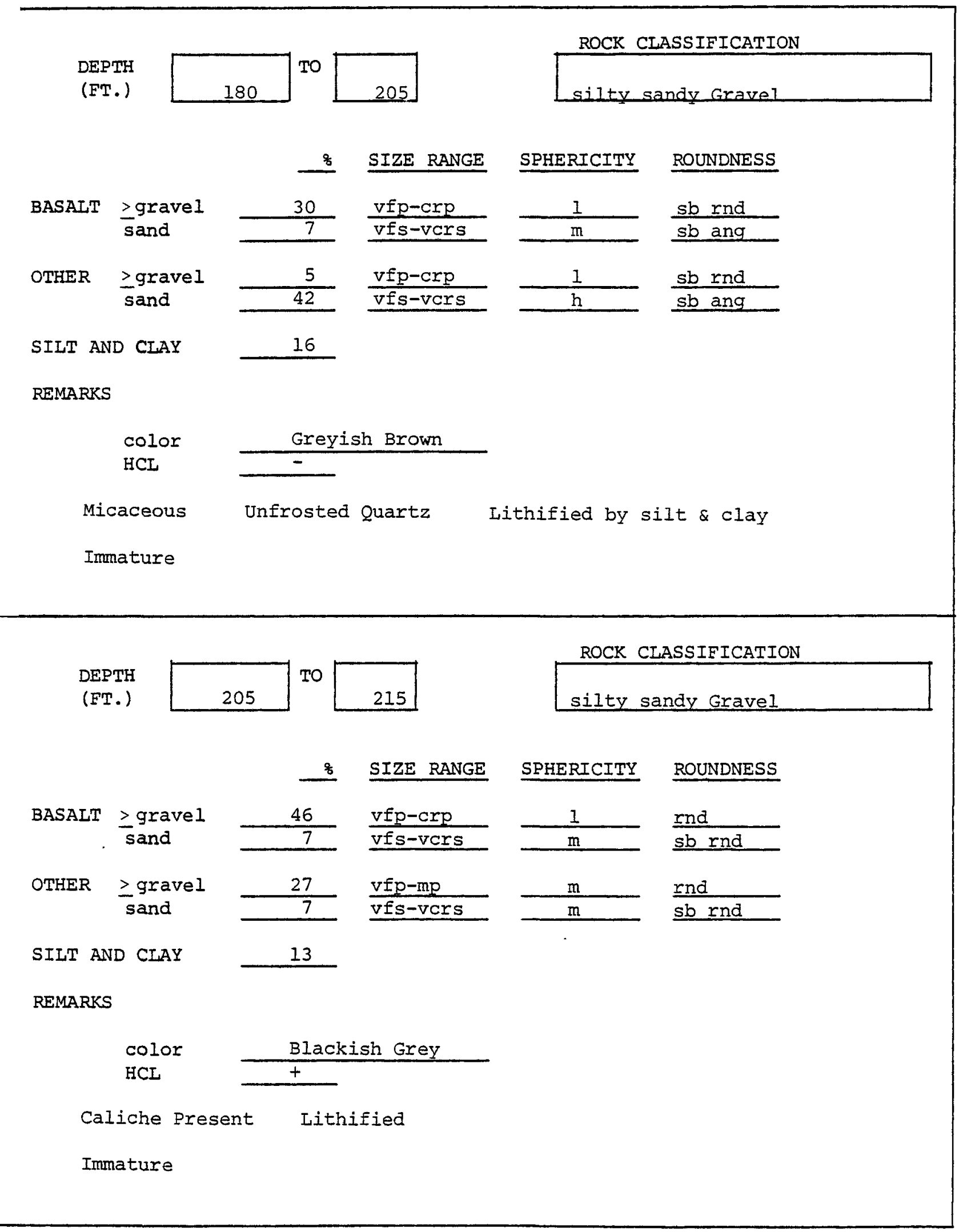


DEPTH
(FT.)

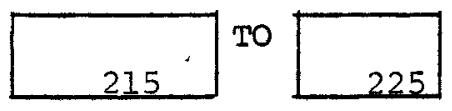

\% SIZE RANGE

SPHERICITY ROUNDNESS

BASALT $\geq$ gravel sand
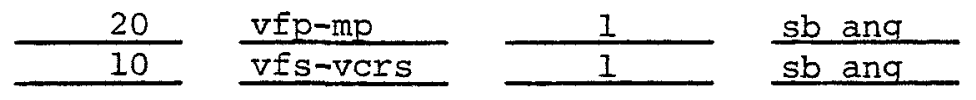

OTHER $\geq$ gravel sand
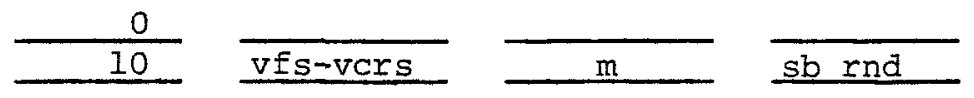

\section{0}

REMARKS

color

$\mathrm{HCL}$

Blackish Grey $+$

Caliche Present

Lithified
ROCK CIASSIFICATION

gravelly sandy Silt

Immature

DEPTH

(FT.)

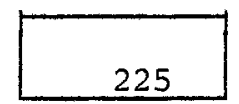

TO

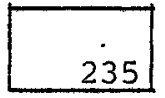

ROCK CLASSIFICATION

silty sandy Gravel

\section{\% SIZE RANGE SPHERICITY ROUNDNESS}

\begin{tabular}{|c|c|c|c|c|c|}
\hline BASALT & $\begin{array}{l}\text { >gravel } \\
\text { sand }\end{array}$ & $\frac{45}{5}$ & $\frac{v f p-m p}{v f s-v c r s}$ & $\frac{1}{m}$ & $\frac{\mathrm{sb} \text { ang }}{\text { sb ang }}$ \\
\hline OTHER & > gravel & 35 & $v f p-m p$ & 1 & sb rnd \\
\hline & sand & 12 & Vfs-vCrs & $\bar{m}$ & sb rnd \\
\hline
\end{tabular}

SIIT AND CLAY

REMARKS

color Greyish Brown

$\mathrm{HCL}$ $-$

Micaceous

Unfrosted quartz

Immature 


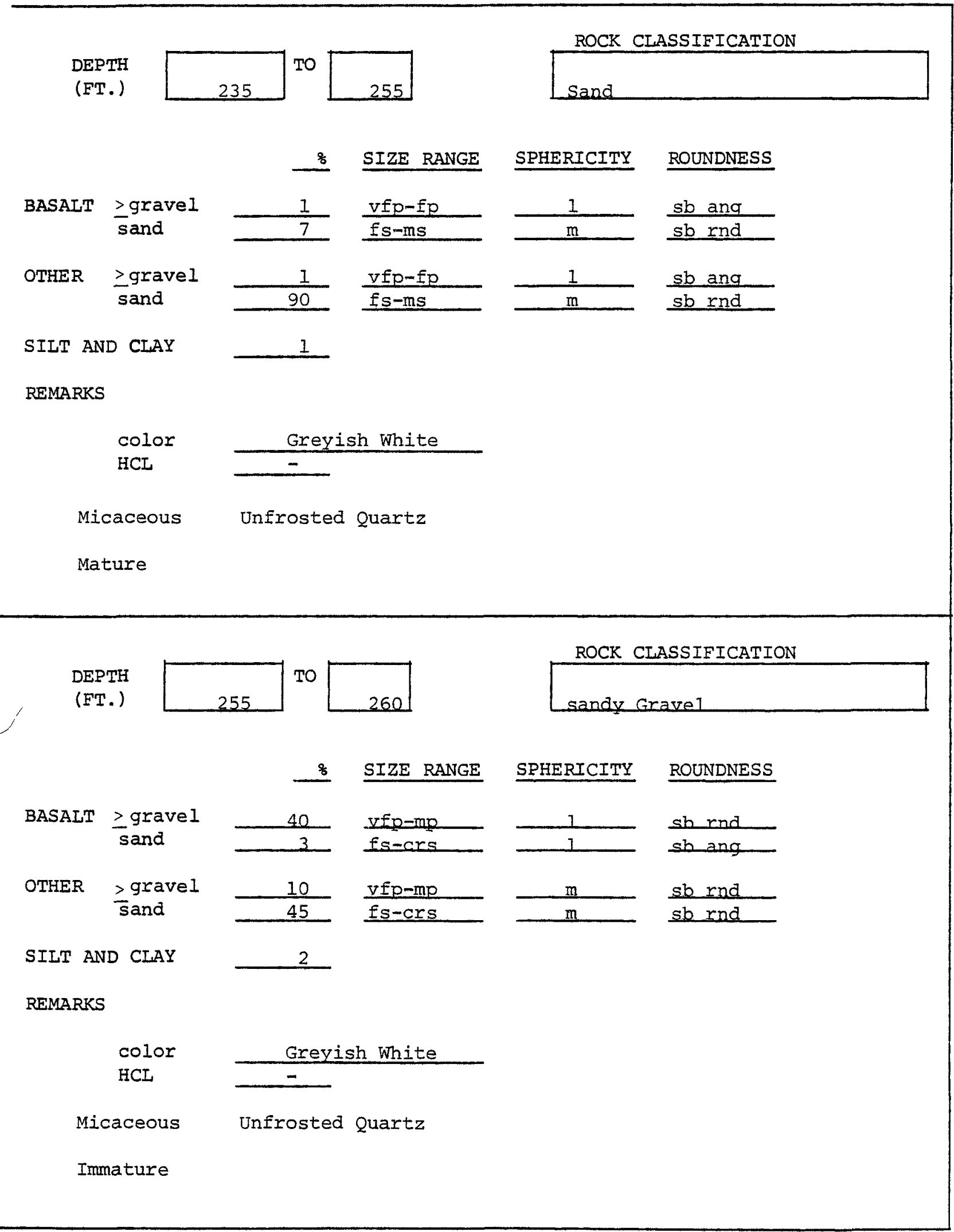


DEPTH
(FT.)

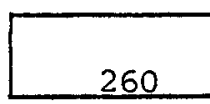

ro

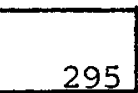

ROCK CLASSIEICATION

silty Gravel

\% SIZE RANGE SPHERICITY ROUNDNESS

BASALT $\geq$ gravel
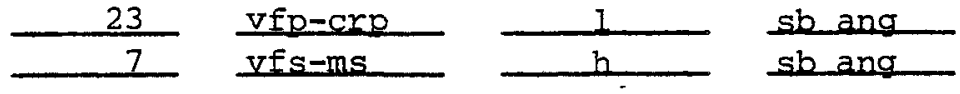

OTHER >gravel
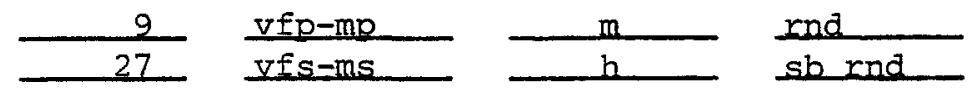

SILT AND CLAY 34

REMARKS

color

HCL Greyish Tan $-$

Micaceous

Immature

DEPTH
(FT.)

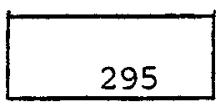

To

ROCK CLASSIFICATION

Silt

\% SIZE RANGE SPHERICITY ROUNDNESS BASALT $\frac{\geq \text { grave } 1}{\text { sand }}$

OTHER $\geq$ gravel sand

SIIT AND CIAY
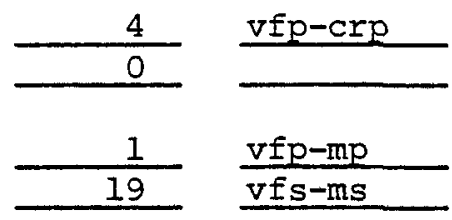

76

REMARKS

color

HCL

$\frac{\text { Reddish Brown }}{t}$

Slightly Micaceous

Immature
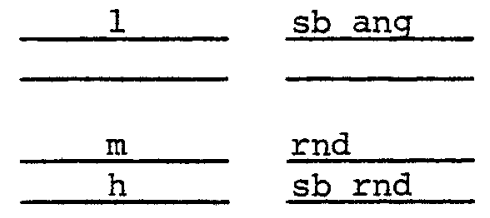

rnd sb rnd. 
DEPTH

(FT.)

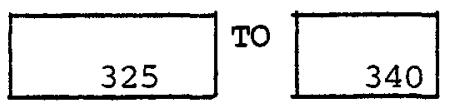

ROCK CLASSIFICATION

slightly gravelly sandy silt

\section{\% SIZE RANGE SPHERICITY ROUNDNESS}

BASALT $\underset{\text { sand }}{\geq \text { gravel }}$
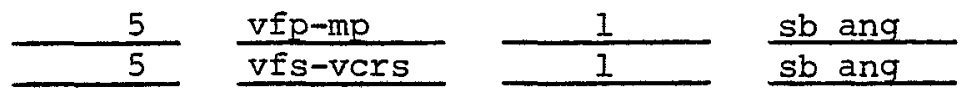

OTHER $\geq$ gravel
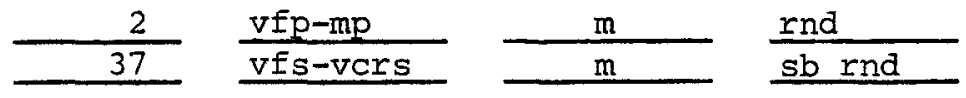

SIIT AND CLAY

REMARKS

color

HCL

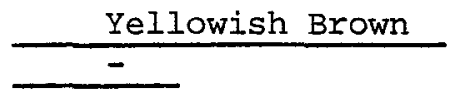

Micaceous

Unfrosted Quartz

Lithified by silt \& clay

Immature

\section{DEPTH \\ (FT.)}

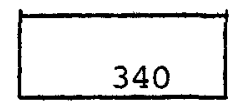

To

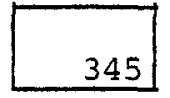

ROCK CLASSIFICATION

slightly gravelly sand

\section{\& SIZE RANGE SPHERICITY ROUNDNESS}

BASALT $\underset{\text { sand }}{\operatorname{gravel}}$
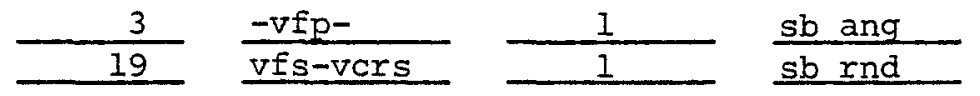

OTHER $\geq$ gravel
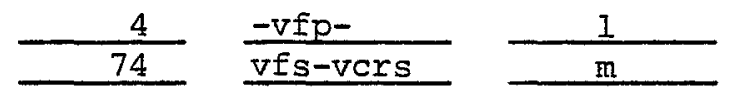

$\frac{\text { sb and }}{\text { sb rnd }}$

SILT AND CLAY

0

REMARKS

color

Greyish White

HCL

$-$

Slightly Micaceous

Ash (fragments present)

Unfrosted Quartz

Mature 


\section{DEPTH}

(FT.)
ROCK CLASSIFICATION

silty sandy Gravel

\section{\% SIZE RANGE SPHERICITY ROUNDNESS}

BASALT $\geq$ gravel
sand
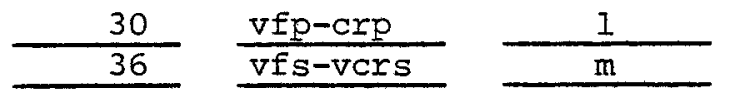
$\frac{\mathrm{sb} \text { rnd }}{\mathrm{sb} \text { ang }}$

OTHER $\geq$ gravel
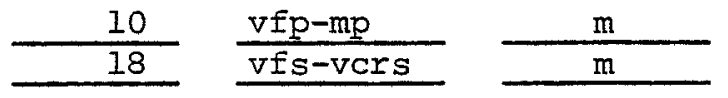

$\frac{\text { sb rnd }}{\text { sb rnd }}$

SIIT AND CLAY

6

REMARKS

color

HCL

Greyish Black

Slightly Micaceous Unfrosted quartz

Immature

DEPTH

(FT.)

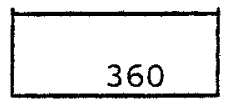

TO

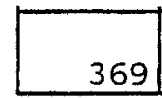

ROCK CIASSIFICATION

Grave1

\section{\& SIZE RANGE SPHERICITY ROUNDNESS}

BASAIT $\frac{\geq \text { gravel }}{\text { sand }}$
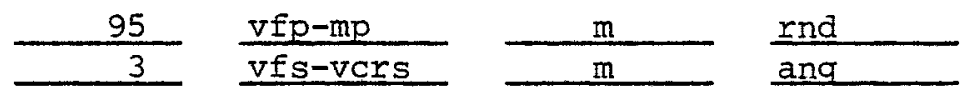

OTHER $\geq$ gravel
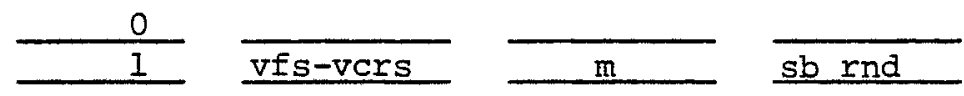

SIIT AND CIAY 1

REMARKS

$$
\begin{aligned}
& \text { color } \\
& \text { HCI }
\end{aligned}
$$

Black

$-$

Submature

Total Drilled Depth reported as 369' 
Depth

Graphic Log

(1)

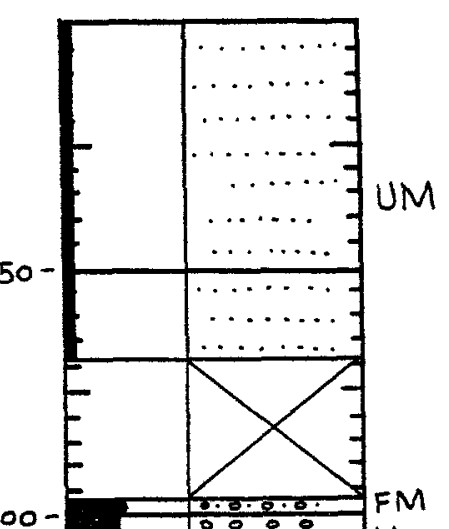$$
100
$$$$
\text { \%:20:-M }
$$$$
0000
$$$$
\text { - } 00.7 A
$$$$
\text { ....... }
$$$$
\because \ldots \therefore \text { JFM }
$$
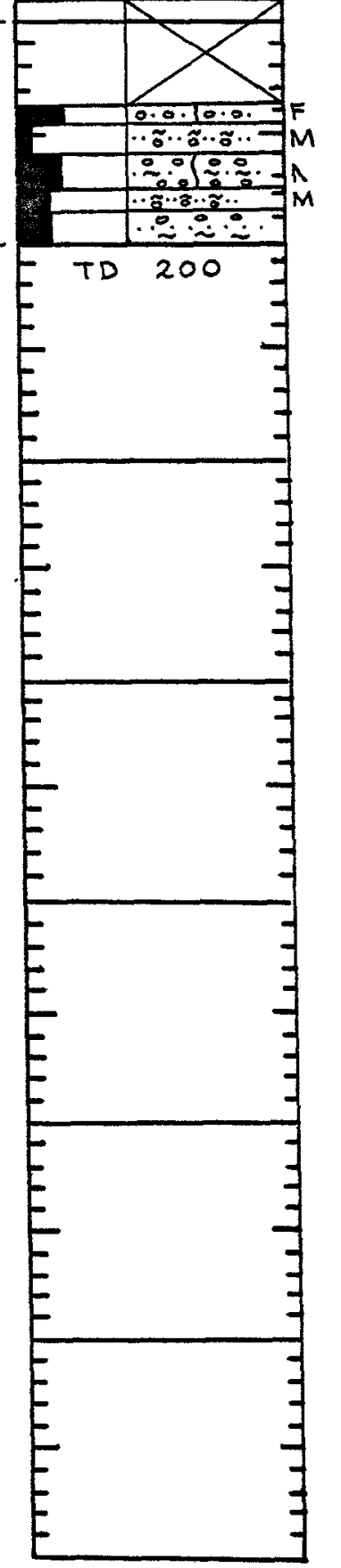

\section{Sample Description}

brownish buff

brownish buff

white- $\tan$

brownish $\tan$

brownish buff

yellowish brown

greyish brown

greyish tan

gritish tan; SLIGHTLY DIATOMACEOUS

whitish tan; DIATOMITE
Plant Coordinates

$N 010150$

$w 053550$

Altitude 514

Other Data used in Interpretation

(1) Driller's Log

(2) Descriptive Log fRom Newcomb, et al, 1972, p.59 


\section{DEPTH}

(FT.)

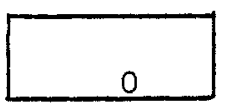

To

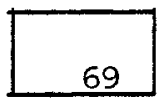

ROCK CLASSIFICATION

Sand

\section{\% SIZE RANGE SPHERICITY ROUNDNESS}

BASALT $\frac{\geq \text { gravel }}{\text { sand }}$

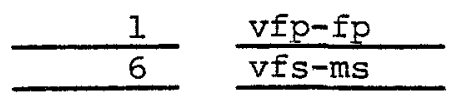

OTHER $\begin{gathered}\geq \text { gravel } \\ \text { sand }\end{gathered}$

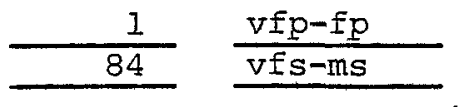

8

SILT AND CLAY

REMARKS

$$
\text { color }
$$

$\mathrm{HCL}$

Micaceous

Unfrosted Quartz

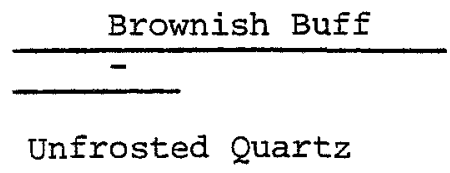

Mature

$\frac{\frac{1}{1}}{\frac{\text { sb rnd }}{\text { rnd }}} \frac{\text { sb rnd }}{1}$

ROCK CLASSIFICATION

DEPTH

(FT.)

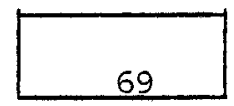

To

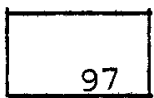

\% SIZE RANGE

BASALT $\geq$ gravel sand

OTHER $\geq$ gravel sand

SILT AND CLAY

REMARKS

color

HCL

Sample missing 
DEPTH
(FT.)

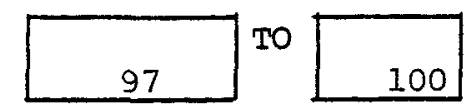

8 SIZE RANGE

SPHERICITY ROUNDNESS

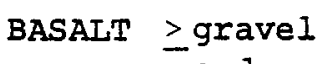
sand

OTHER $\geq$ gravel sand
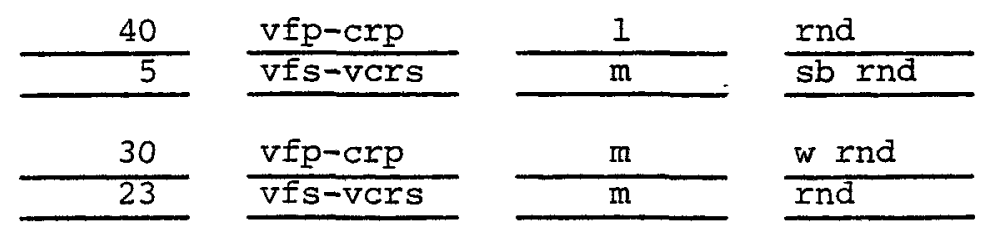

SIIT AND CLAY

REMARKS

$$
\begin{aligned}
& \text { color } \\
& \text { HCL }
\end{aligned}
$$

Micaceous

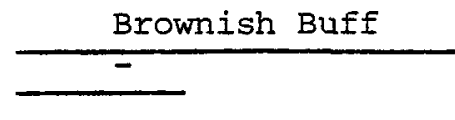

Frosted Quartz

Immature

\section{DEPTH}

(FT.)

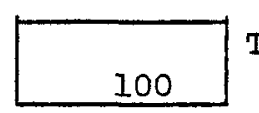

TO

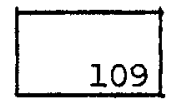

ROCK CLASSIFICATION

sandy Gravel 


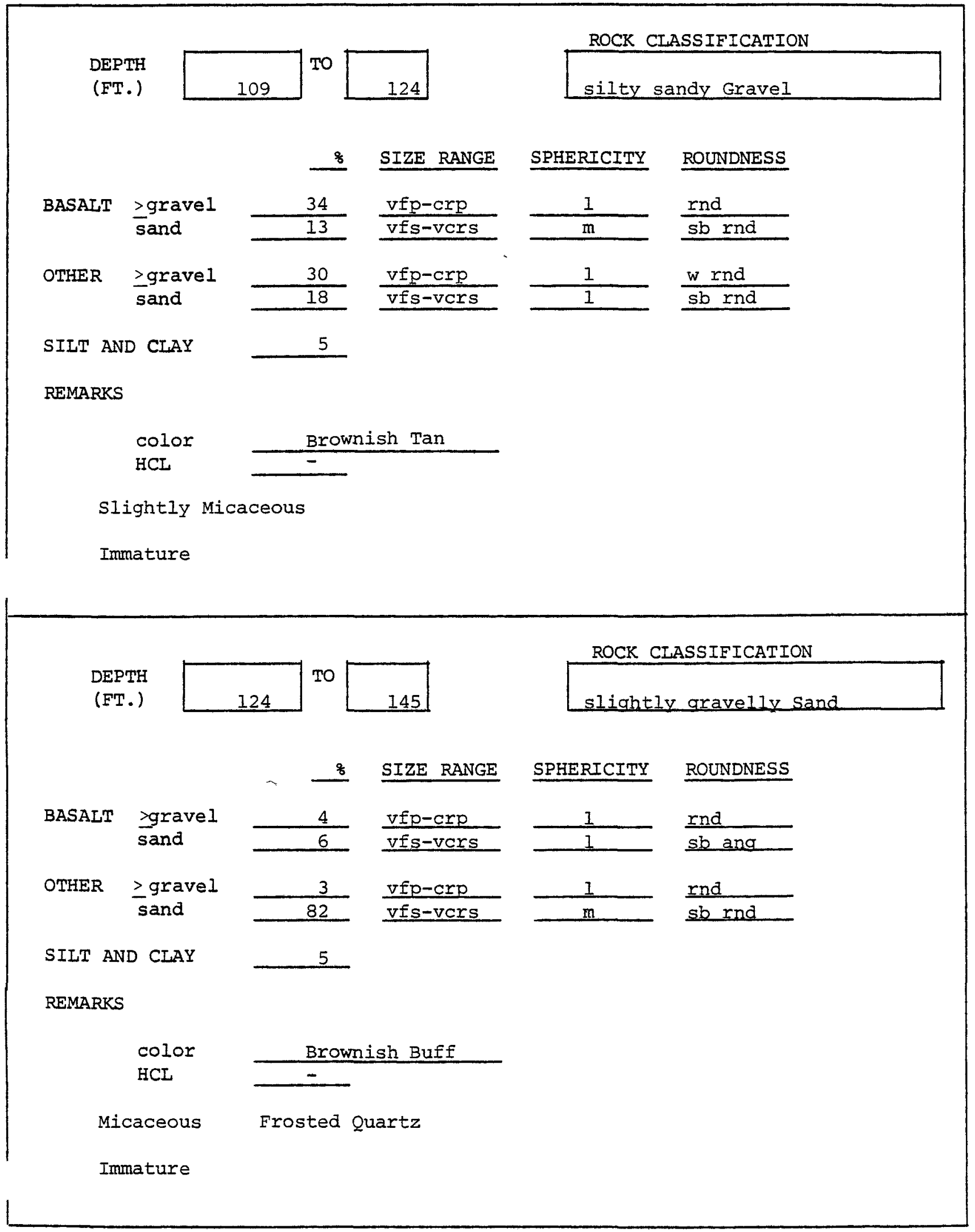

W. K. Summers \& Associates 


\section{DEPTH}

(FT.)

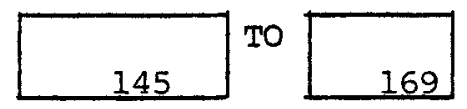

8 SIZE RANGE
ROCK CLASSIFICATION

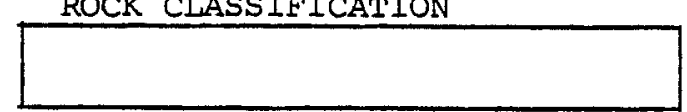

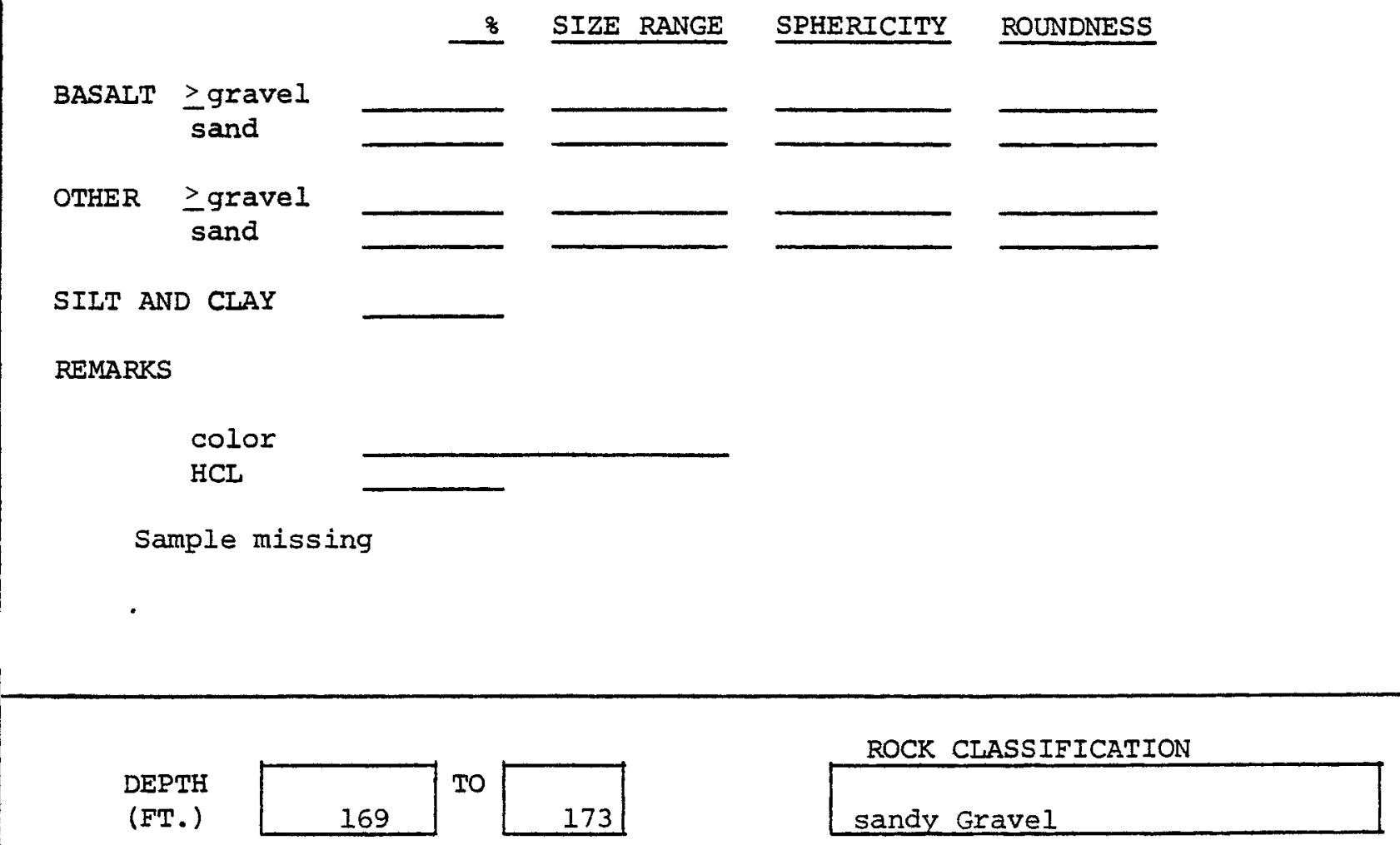

\section{\% SIZE RANGE SPHERICITY ROUNDNESS}

\begin{tabular}{|c|c|c|c|c|c|}
\hline BASALT & $\begin{array}{l}\text { Dgravel } \\
\text { sand }\end{array}$ & $\frac{33}{6}$ & $\frac{v f p-m p}{v f s-v c r s}$ & $\frac{1}{1}$ & $\frac{\text { rnd }}{\text { sb rnd }}$ \\
\hline OTHER & $\begin{array}{l}\geq \text { gravel } \\
\text { sand }\end{array}$ & $\frac{33}{26}$ & $\frac{v f p-m p}{v f s-v c r s}$ & $\frac{1}{1}$ & $\frac{\text { rnd }}{\text { sb rnd }}$ \\
\hline
\end{tabular}

SILT AND CLAY

REMARKS

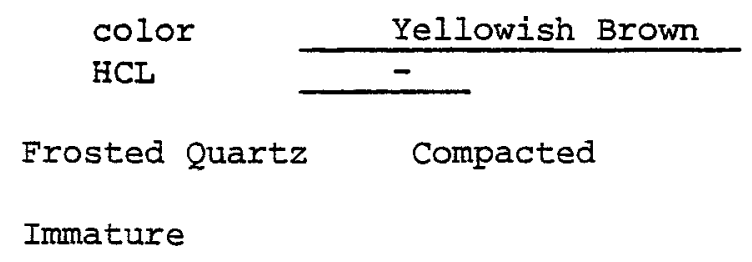




\section{DEPTH}

(FT.)

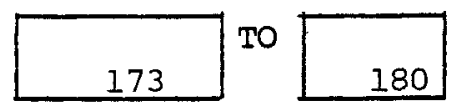

180
ROCK CLASSIFICATION

gravelly silty sand

\section{\% SIZE RANGE SPHERICITY ROUNDNESS}

BASALT $\underset{\text { sand }}{\text { gavel }}$

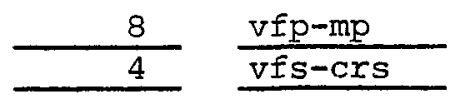

$\frac{1}{1} \quad \frac{\text { sb rnd }}{\text { sb rnd }}$

OTHER >gravel sand
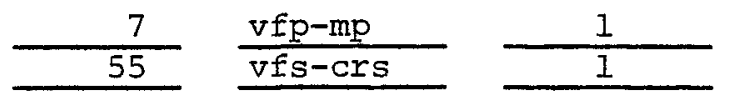

$$
\text { rnd }
$$

SIIT AND CLAY 26

REMARKS

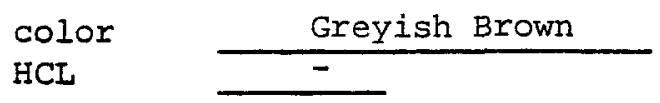

Micaceous

Immature

DEPTH

(ET.)

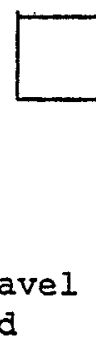

180
To

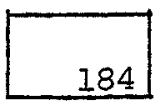

ROCK CLASSIFICATION

silty sandy Gravel

\section{\% SIZE RANGE SPHERICITY ROUNDNESS}

\begin{tabular}{|c|c|c|c|c|c|}
\hline BASALT & $\begin{array}{l}\text { > gravel } \\
\text { sand }\end{array}$ & $\frac{37}{3}$ & $\frac{\text { vfp-crp }}{\text { vfs-vers }}$ & $\frac{1}{1}$ & $\frac{\mathrm{sb} \text { rnd }}{\mathrm{sb} \text { rnd }}$ \\
\hline OTHER & > gravel & 29 & $v f p-c r p$ & 1 & rnd \\
\hline & sand & 26 & vfs-vers & 1 & rnd \\
\hline
\end{tabular}

SILT AND CLAY 5

REMARKS

color

HCL

Greyish Tan

Slightly Micaceous

Compacted (?)

Immature 

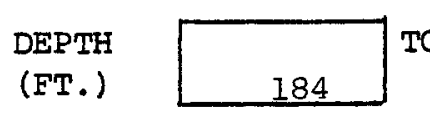

TO

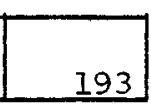

ROCK CTASSIFICATION

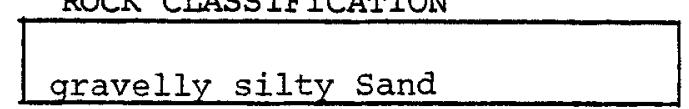

\section{\% SIZE RANGE SPHERICITY ROUNDNESS}

BASALT $\geq$ gravel
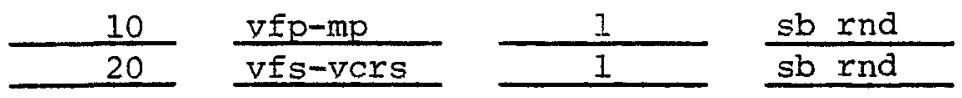

OTHER $\geq$ gravel sand
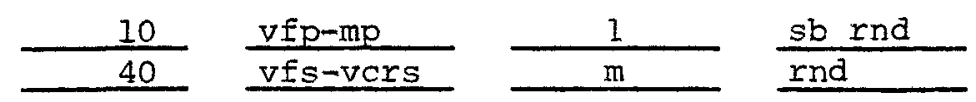

SILT AND CLAY 20

REMARKS

color

ish Tan

HCL

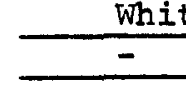

Slightly Diatomaceous

Micaceous

Immature

DEPTH

(ET.)

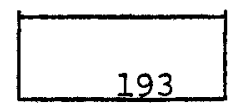

To

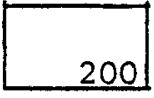

ROCK CLASSIFICATION

gravelly silty sand

\section{SIZE RANGE SPHERICITY ROUNDNESS}

BASALT $\geq$ gravel
sand
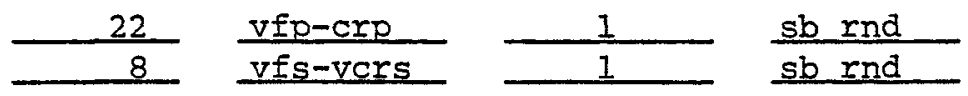

OTHER $\geq$ gravel sand
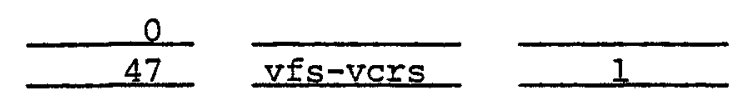

Ind

SILT AND CLAY 23

REMARKS

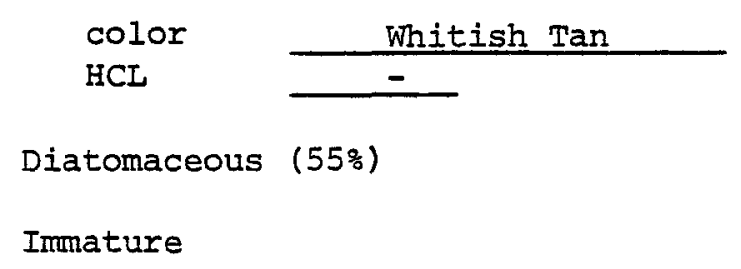

Total Drilled Depth reported as $200^{\prime}$ 


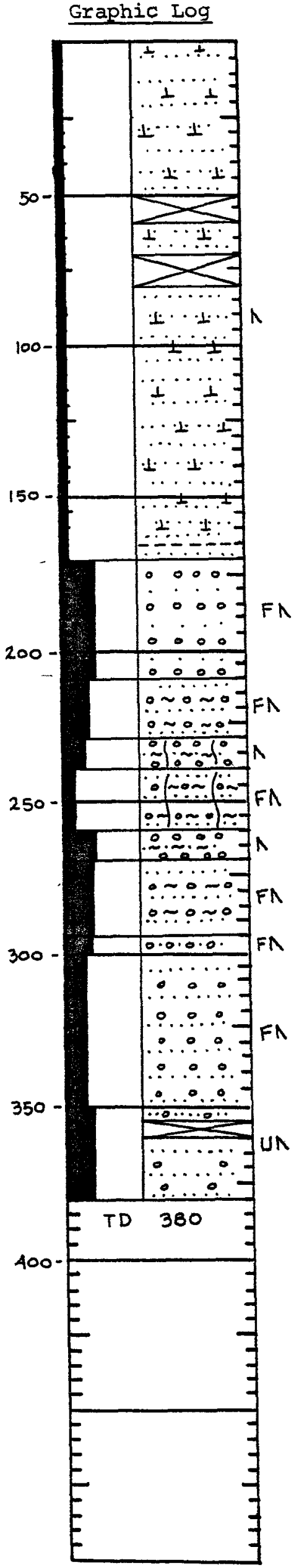

Sample Description

greyish tan-greyish brown

greyish brown

$$
\begin{aligned}
& \text { greyish tan } \\
& \text { greyish brown } \\
& \text { greyish tan } \\
& \text { greyish brown } \\
& \text { greyish brown } \\
& \text { greyish brown } \\
& \text { greyish buff }
\end{aligned}
$$

brownish grey
Plant Coordinates

No11159

$\frac{W 044960}{\text { Altitude } 576}$

Other Data used in Interpretation

(1) Driller's Log

(2) E - Log 
DEPTH

(FT.)

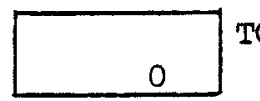

TO

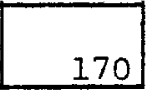

ROCK CLASSIFICATION

Sand

\section{\% SIZE RANGE SPHERICITY ROUNDNESS}

BASALT $\geq$ gravel sand
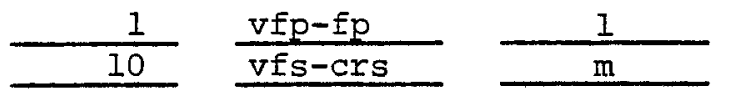

$\frac{\text { sb rnd }}{\text { rnd }}$

OTHER $\geq$ gravel
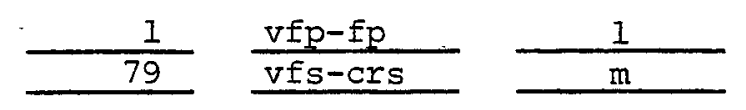

$\frac{\text { sb rnd }}{\text { sb rnd }}$

SIIT AND CLAY

9

REMARKS

color
HCL Greyish Tan-Greyish Brown

$+\left(0^{\prime}-165^{\prime}\right),-\left(165^{\prime}-170^{\prime}\right)$

Slightly Micaceous

Unfrosted Quartz

Sample intervals $50^{\prime}-60^{\prime} \& 70^{\prime}-80^{\prime}$ missing

Immature

\section{DEPTH}

(ET.)

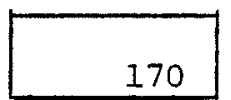

TO

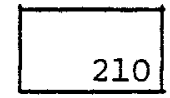

ROCK CIASSIFICATION

sandy Gravel

\section{\% SIZE RANGE SPHERICITY ROUNDNESS}

$$
\begin{gathered}
\text { BASALT } \\
\frac{\geq \text { gravel }}{\text { sand }}
\end{gathered}
$$

OTHER Igravel sand

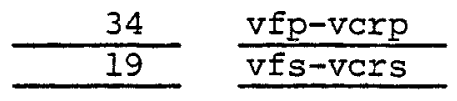

1

SILT AND CLAY

REMARKS Greyish Brown

HCI

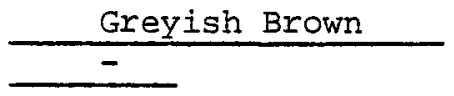

Slightly Micaceous Frosted Quartz

Immature 
DEPTH

(FT.)

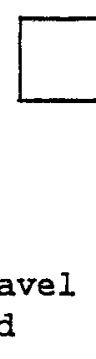

210

TO

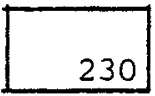

ROCK CLASSIFICATION

slightly silty gravelly sand

\section{$\frac{\circ}{3}$ SIZE RANGE SPHERICITY ROUNDNESS}

\begin{tabular}{|c|c|c|c|c|c|}
\hline \multirow[t]{2}{*}{ BASALT } & \multirow{2}{*}{$\begin{array}{l}\geq \text { gravel } \\
\text { sand }\end{array}$} & 15 & $v f p-c r p$ & $\mathrm{~m}$ & $w$ rnd \\
\hline & & 18 & vfs-vers & $\mathrm{m}$ & sb rnd \\
\hline \multirow{2}{*}{ OTHER } & > gravel & 15 & vfp-crp & $\mathrm{m}$ & $w$ rnd \\
\hline & $\bar{s}$ and & 45 & vfs-vers & $\mathrm{m}$ & sb rnd \\
\hline
\end{tabular}

\section{SIIT AND CLAY}

REMARKS

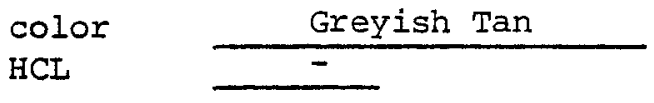

Slightly Micaceous Frosted Quartz

Immature

\section{DEPTH}

(FT.)

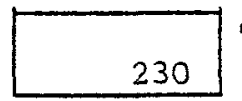

To

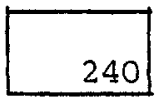

ROCK CLASSIFICATION

silty sandy Gravel

\% SIZE RANGE SPHERICITY ROUNDNESS

BASAIT $\underset{\text { sand }}{\text { savel }}$

$\frac{25}{5} \quad \frac{\text { vfp-crp }}{\text { vfs-vcrs }}$

OTHER Igravel sand

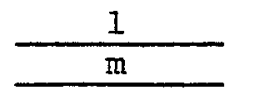

rnd

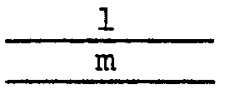

SILT AND CLAY 11

REMARKS

$$
\begin{aligned}
& \text { color } \\
& \text { HCL }
\end{aligned}
$$

Greyish Brown

Slightly Micaceous

Lithified (?)

Immature 


\section{DEPTH}

(FT.)

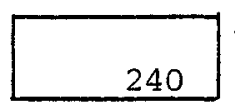

TO

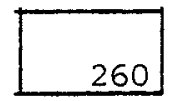

ROCK CIASSIFICATION

graveliy silty sand

\% SIZE RANGE SPHERICITY ROUNDNESS BASALT $\frac{\geq \text { gravel }}{\text { sand }}$
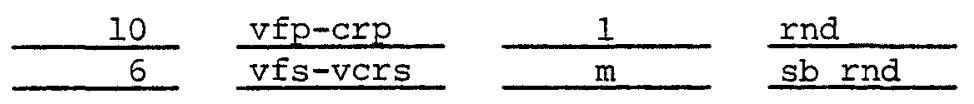

OTHER $\geq$ gravel

$\frac{9}{49} \quad \frac{v f p-c r p}{v f s-v \operatorname{crs}}$

$\frac{1}{m} \frac{w \text { rnd }}{\text { sb rnd }}$

SILT AND CLAY 26

REMARKS

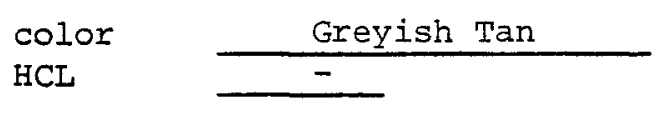

Slightly Micaceous Frosted quartz Lithified by silt \& clay Immature

\section{DEPTH}

(FT.)

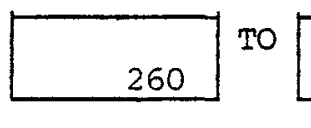

270
ROCK CIAASIFICATION

silty sandy Gravel

\section{\% SIZE RANGE SPHERICITY ROUNDNESS}

BASALT $\frac{\geq \text { gravel }}{\text { sand }}$
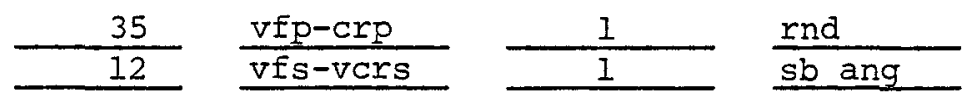

OTHER $\underset{\text { sand }}{\geq \text { gravel }}$
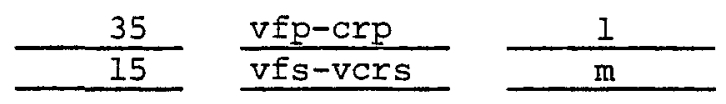

rnd sb rnd

SILT AND CILAY

3

REMARKS

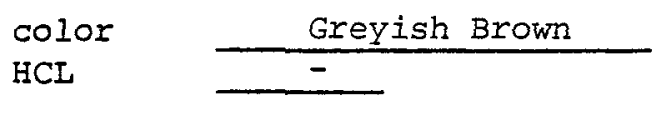

Slightly Micaceous

Immature 
DEPTH

(FT.)

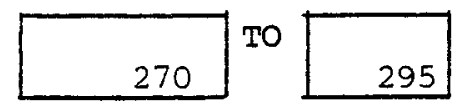

ROCK CLASSIFICATION

slightly silty gravelly sand

\section{\& SIZE RANGE SPHERICITY ROUNDNESS}

BASAIT $\geq$ gravel sand

OTHER $\geq$ gravel sand

SILT AND CLAY

REMARKS

color

HCL
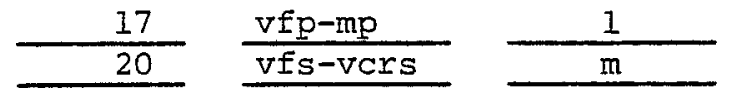

$\frac{\mathrm{w} \text { rnd }}{\text { sb rnd }}$

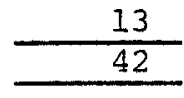

$\frac{\mathrm{vfp}-\mathrm{mp}}{\mathrm{vfs-vars}}$

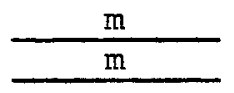

wrnd

rnd

Slightly Micaceous Frosted Quartz

Immature

DEPTH

(FT.)

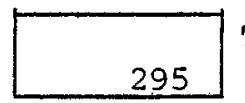

TO

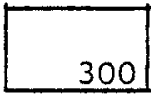

ROCK - CLASSIFICATION

sandy Gravel

S SIZE RANGE SPHERICITY ROUNDNESS

BASALT $\underset{\text { sand }}{\geq \text { gravel }}$
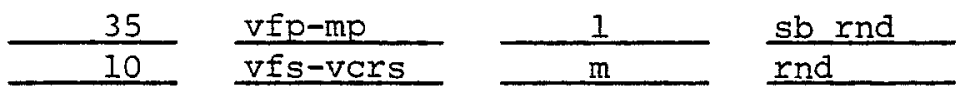

OTHER $\geq$ gravel sand
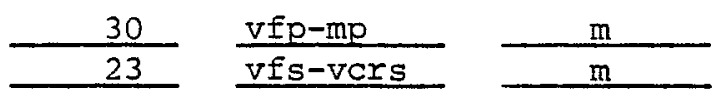

rnd

rnd

SILT AND CLAY 2

REMARKS

color

Greyish Brown

HCI

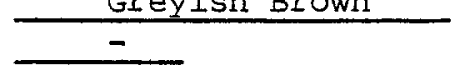

Slightly Micaceous

Frosted Quartz

Immature 


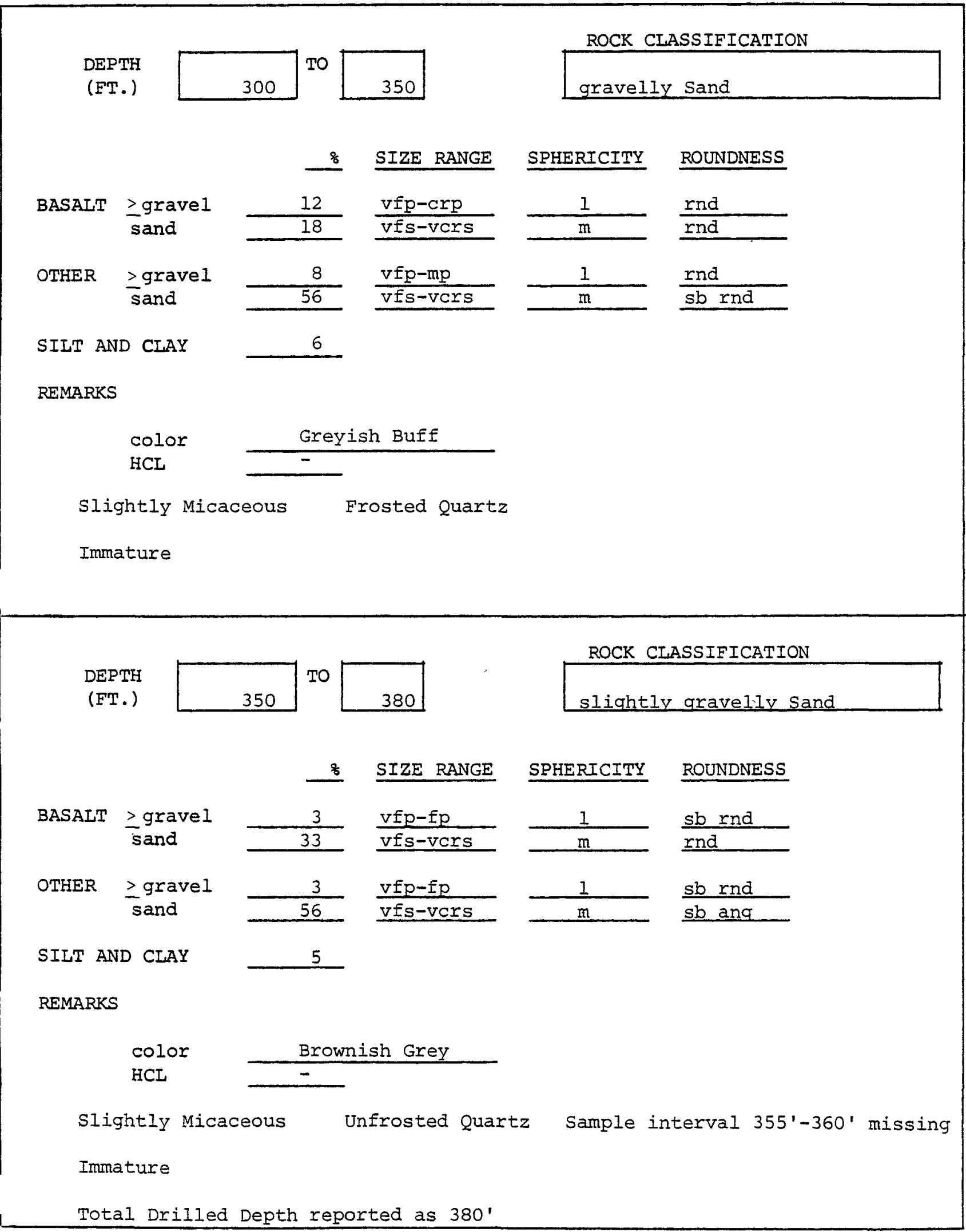


$\underline{\text { Depth }}$

(ft.)

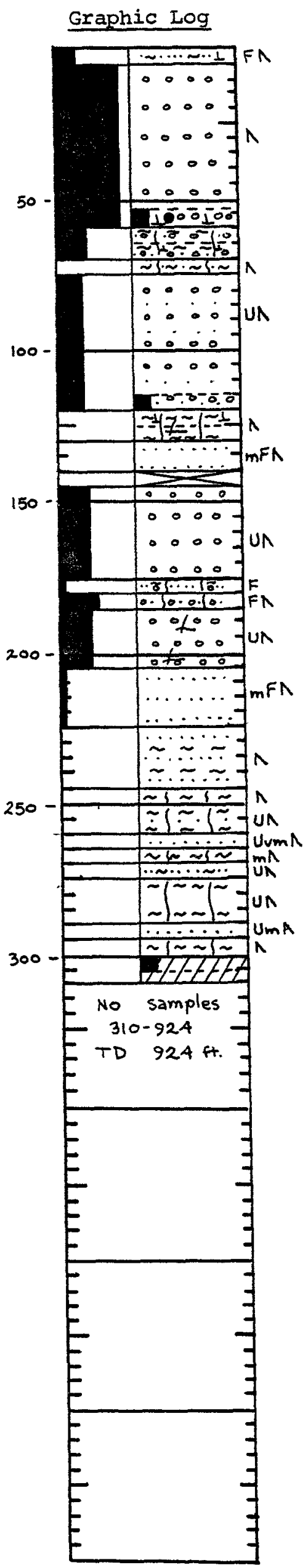

Sample Description

buff

blackish grey

buff brown

butf brown

blackish brown

blackish brown

tan buff; TRACE DIATOMITE (?)

greyish buff

greyish white: $15 \%$ DIATOMITE

greyish brown; TRACE DIATOMITE

greyish buff

$\tan$ buff

buff $\tan$

greenish buff

grey

buff grey

grey

grey

greyish buff

bluish grey

$\frac{\frac{\text { Plant Coordinates }}{N 015323}}{\text { E012713 }}$

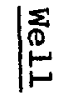

Other Data used in

Interpretation

$$
\begin{aligned}
& \text { (1) Partial Driller's (?) Log } \mid \frac{m}{\omega} \\
& \text { (2) E-Log } \\
& \text { (3) Rod Ledgerwood } \\
& \text { strip Log }
\end{aligned}
$$




\section{DEPTH \\ (FT.)}

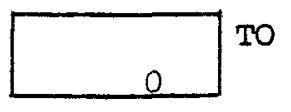

ROCK CLASSIFICATION

slightly silty sand

\section{\% SIZE RANGE SPHERICITY ROUNDNESS}

BASALT $\geq$ gravel
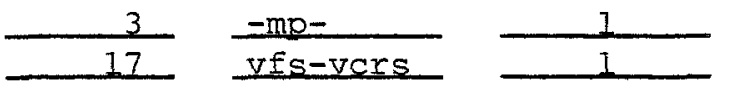

$$
\frac{\text { wrind }}{\text { ang }}
$$

OTHER >gravel
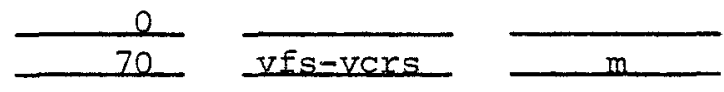

ang

SILT AND CLAY 10

REMARKS

color

HCL
Buff

$+$

Slightly Micaceous Frosted quartz

Inmature .

DEPTH

(FT.)

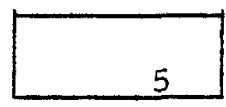

TO

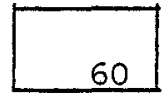

ROCK CLASSIEICATION

Grave1

\section{SIZE RANGE SPHERICITY ROUNDNESS}

BASAIT $\frac{\geq \text { gravel }}{\text { sand }}$
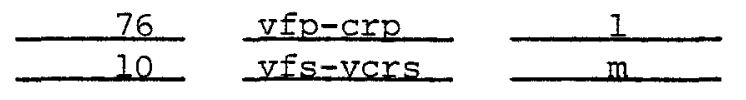

rnd

sb ang

OTHER $\underset{\text { sand }}{>\text { gravel }}$
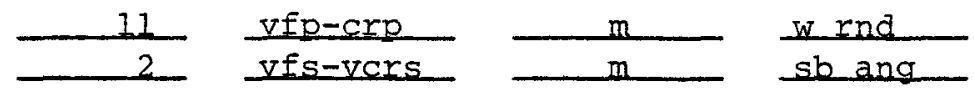

SILT AND CLAY

1

REMARKS

color

HCL

Blackish Grey

$-\left(5^{\prime}-55^{\prime}\right),+\left(55^{\prime}-60^{\prime}\right)$

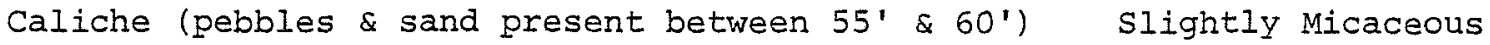
Immature 

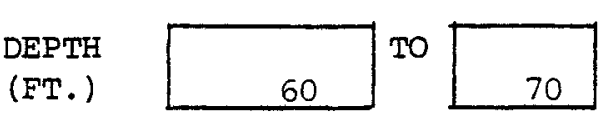

ROCK CIASSIFICATION

(FT.)

60

\& SIZE RANGE SPHERICITY ROUNDNESS

BASAIT $\geq$ grave 1

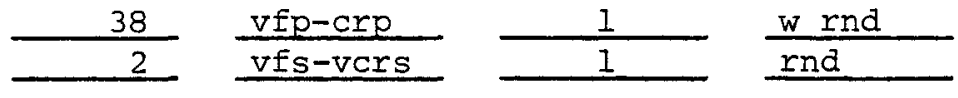

OTHER $\quad \frac{\text { grave } 1}{\text { sand }}$
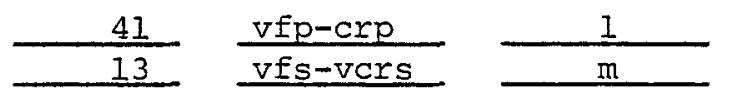

w rnd

6

SIIT AND CLAY

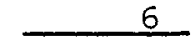

REMARKS
color
Buff Brown
HCL $-\left(60^{\prime}-65^{\prime}\right),+\left(65^{\prime}-70^{\prime}\right)$

Lithified by silt \& clay

Immature

$$
\begin{aligned}
& \text { DEPTH } \\
& \text { (ET.) }
\end{aligned}
$$

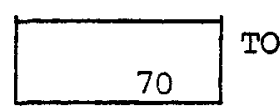

TO

75
ROCK CLASSIFICATION

sandy Silt

\section{\& SIZE RANGE SPHERICITY ROUNDNESS}

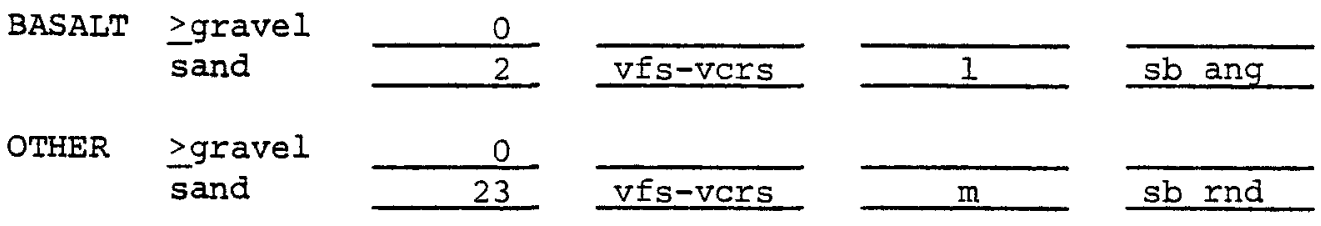

SIIT AND CIAY 75

REMARKS

$$
\operatorname{col} 0 x
$$

HCL

Buff Brown

Slightly Micaceous Iithified by silt \& clay

Immature 

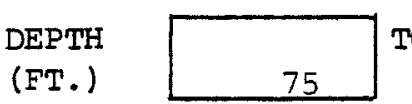

TO

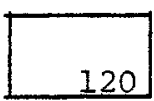

ROCK CLASSIFICATION

sandy Gravel

\section{\% SIZE RANGE SPHERICITY ROUNDNESS}

BASALT > gravel

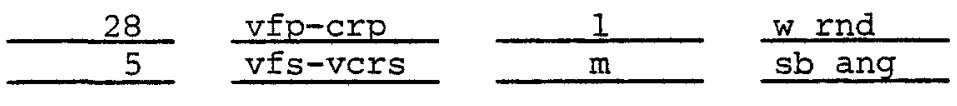

OTHER $\frac{\geq \text { gravel }}{\text { sand }}$
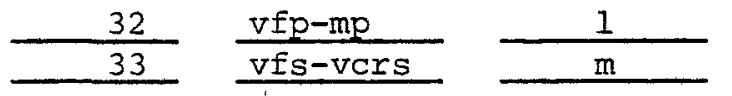

$\frac{w \text { rnd }}{\text { sb ang }}$

SILT AND CLAY

2

REMARKS

$$
\begin{aligned}
& \text { color } \\
& \text { HCI }
\end{aligned}
$$

Blackish Brown

$$
-\left(75^{\prime}-115^{\prime}\right),+\left(115^{\prime}-120^{\prime}\right)
$$

Caliche (fragments present between 115'\& 120')

Slightly Micaceous

Unfrosted Quartz

Immature
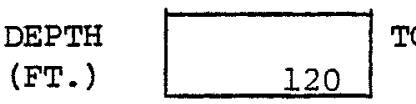

To

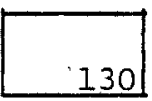

ROCK CLASSIFICATION

sandy silt

\section{\% SIZE RANGE SPHERICITY ROUNDNESS}

BASALT $\frac{\geq \text { gravel }}{\text { sand }}$
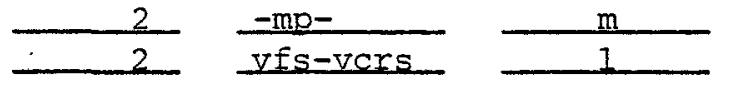

$\frac{\mathrm{sb} \text { rnd }}{\text { rnd }}$

OTHER

$$
\begin{aligned}
& \text { zgravel } \\
& \text { sand }
\end{aligned}
$$
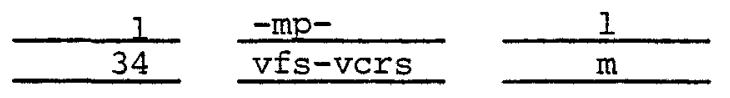

$\frac{\mathrm{w} \text { rnd }}{\text { sb ang }}$

SILT AND CLAY

61

REMARKS

$$
\text { color }
$$

HCL

Buff Brown $+\left(120^{\prime}-125^{\prime}\right),-\left(125^{\prime}-130^{\prime}\right)$

Slightly Micaceous

Slightly Gypsiferous

Unfrosted Quartz

Lithified by silt \& clay

Immature 

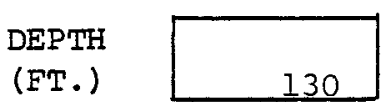

TO

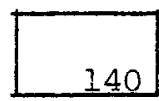

ROCK CLASSIFICATION

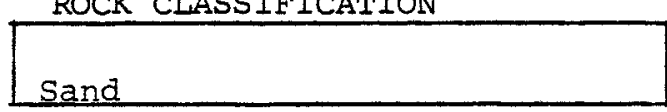

\section{SIZE RANGE SPHERICITY ROUNDNESS}

BASALT $\geq$ graveI sand
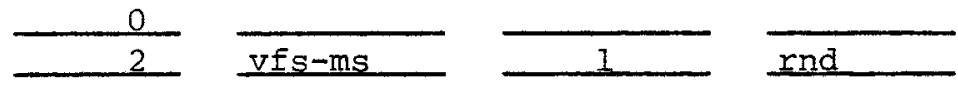

OTHER $\geq$ grave 1
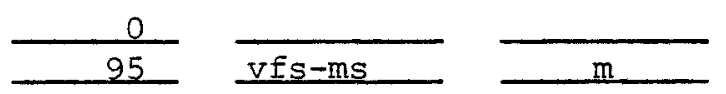

Ind

SILT AND CLAY 3

REMARKS

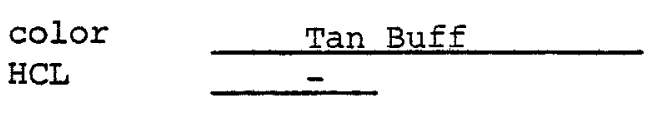

Slightly Diatomaceous (? sand)

Mature

ROCK CLASSIFICATION

\section{DEPTH}

(ET.)

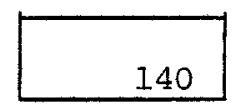

TO

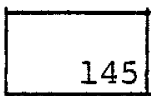

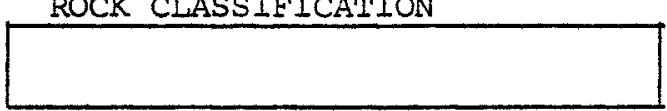

\section{$\frac{8}{5}$ SIZE RANGE SPHERICITY ROUNDNESS}

BASALT >gravel

$$
\text { sand }
$$
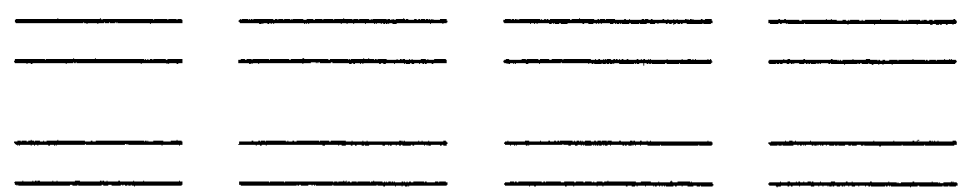

OTHER $\geq$ graveI sand

SILT AND CLAY

REMARKS

color

HCL

Sample missing 
DEPTH

(FT.)

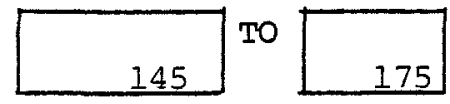

ROCK CLASSIFICATION

Grave I

\section{\% SIZE RANGE SPHERICITY ROUNDNESS}

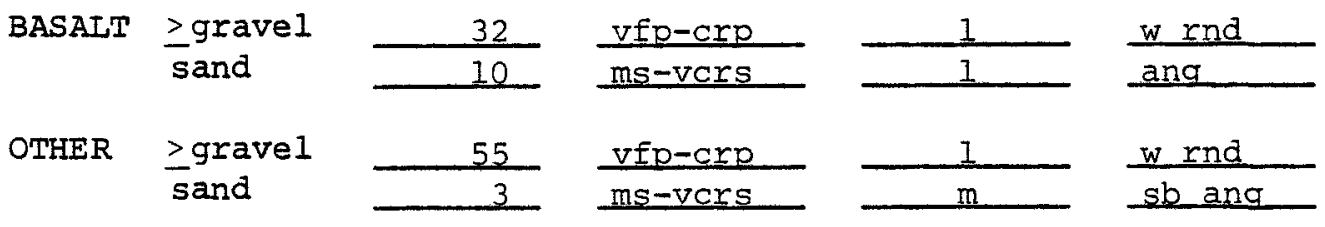

SIIT AND CLAY

0

REMARKS

color

Grevish Buff

HCL $-$

Slightly Micaceous Unfrosted Quartz

Immature

DEPTH

(FT.)

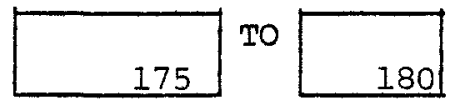

180
ROCK CIASSIFICATION

slightly silty slightly gravelly sand

8 SIZE RANGE SPHERICITY ROUNDNESS BASALT $\frac{\text { gravel }}{\text { sand }}$
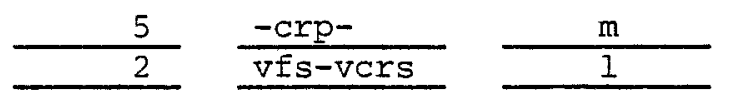

$\frac{\text { sb rnd }}{\text { ang }}$

OTHER $\geq$ gravel sand
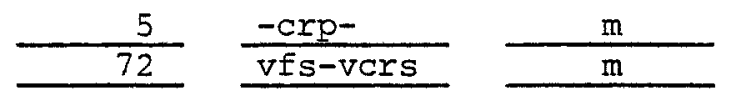

$$
\frac{\text { sb rnd }}{\text { sb ang }}
$$

SILT AND CLAY 16

REMARKS

color HCL

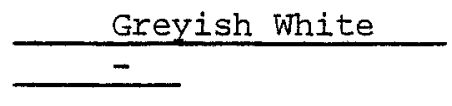

Diatomaceous (15\%, fragments, pebbles \& sand) Slightly Micaceous

Frosted Quartz Lithified (?)

Immature 


\section{DEPTH}

(FT.)

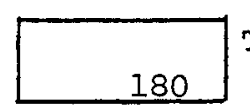

TO

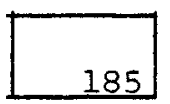

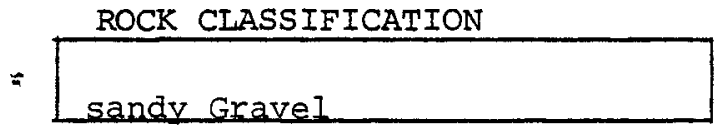

8 SIZE RANGE SPHERICITY ROUNDNESS

BASALT $\frac{\geq \text { gravel }}{\text { sand }}$
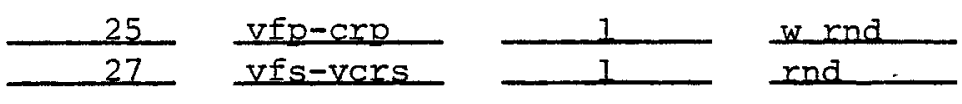

OTHER $\geq$ gravel
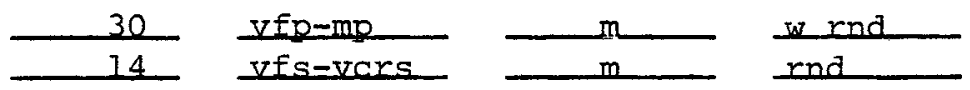

SIIT AND CLAY

REMARKS

color
HCL

Grevish Brown

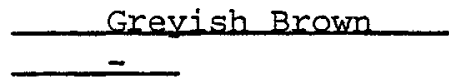

Slightly Diatomaceous (fragments, pebbles \& sand present)

Slightly Micaceous Frosted quartz Iithified

Immature

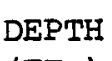

(FT.)

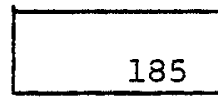

Tо

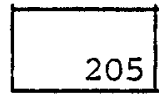

ROCK CLASSIFICATION

Gravel

\section{SIZE RANGE SPHERICITY ROUNDNESS}

\begin{tabular}{|c|c|c|c|c|c|}
\hline \multirow[t]{2}{*}{ BASALT } & zgravel & 39 & vfp-crp & 1 & $w$ rnd \\
\hline & sand & 4 & ms-vars & $\mathrm{m}$ & sb ang \\
\hline \multirow[t]{2}{*}{ OTHER } & zgravel & 42 & vfp-crp & 1 & $w$ rnd \\
\hline & sand & 15 & $\mathrm{~ms}-\mathrm{vcrs}$ & $\mathrm{m}$ & sb ang \\
\hline
\end{tabular}

SILT AND CLAY

REMARKS

color

Greyish Buff

HCL

Slightly Gypsiferous (pebbles \& sand present)

Slightly Micaceous

Unfrosted Quartz 

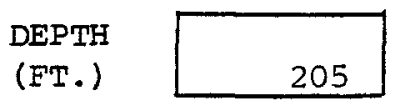

To

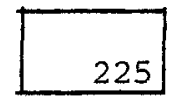

ROCK CLASSIFICATION

Sand
BASALT $\frac{\geq \text { grave } 1}{\text { sand }}$

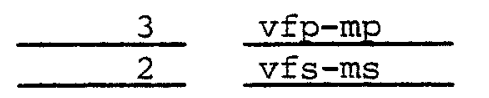
$\frac{1}{m} \frac{\text { sb rnd }}{\text { s.b rnd }}$
OTHER $\underset{\text { sand }}{\geq \text { gravel }}$
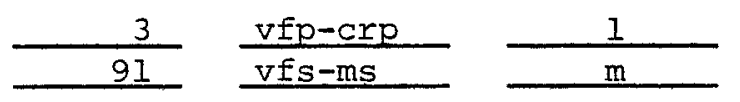
rnd

sb rnd

SILT AND CLAY

1

REMARKS

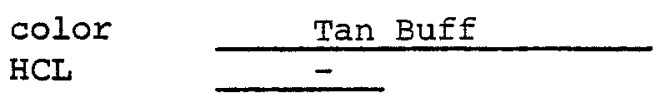

Slightly Micaceous Frosted quartz

Mature
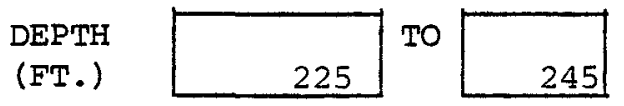

ROCK CLASSIFICATION

silty Sand

은 SIZE RANGE SPHERICITY ROUNDNESS

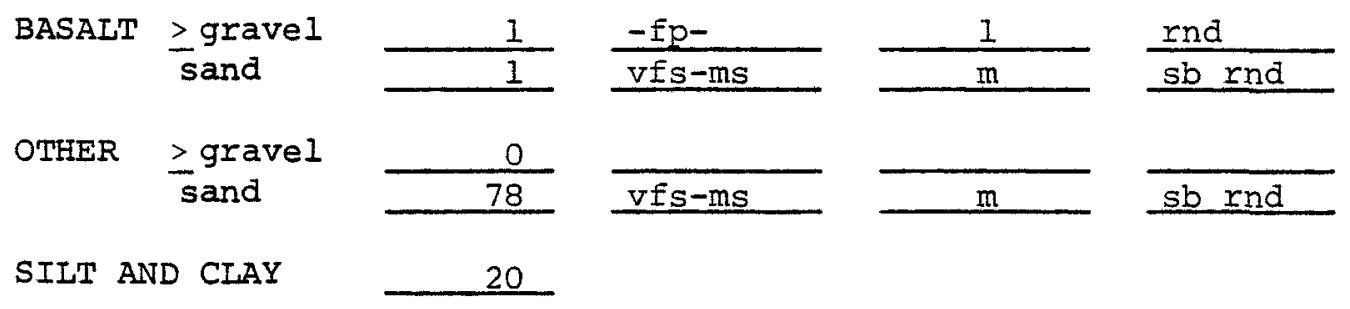

REMARKS

$\begin{array}{ll}\text { Color } & \text { Buff Tan } \\ \text { HCL } & -\end{array}$

Slightly Micaceous

Immature 


\section{DEPTH}

(FT.)

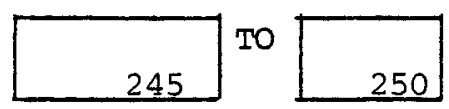

ROCK CLASSIFICATION

silt

\section{SIZE RANGE SPHERICITY ROUNDNESS}

BASALT $\underset{\substack{\text { sand } \\ \text { gravel }}}{ }$
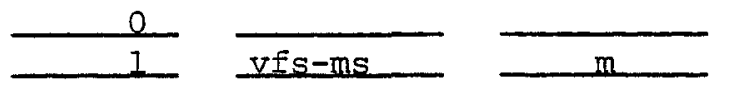

$\overline{s b \text { rnd }}$

OTHER $\geq$ gravel sand
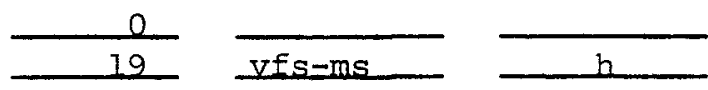

$\overline{\text { sbang }}$

SIIT AND CLAY

80

REMARKS

color

Greenish Buff

HCL

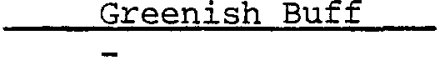

Slightly Micaceous

Lithified by silt \& clay

Imrnature .

\section{DEPTH}

(ET.)

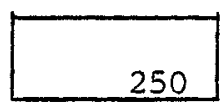

TO

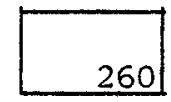

ROCK CIASSIFICATION

sandy silt

\section{S SIZE RANGE SPHERICITY ROUNDNESS}

BASALT >gravel sand
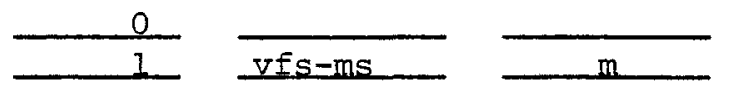

sbrad

OTHER $\geq$ gravel sand
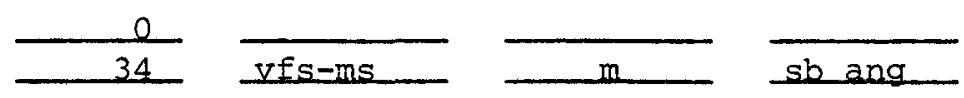

SILT AND CLAY 65

REMARKS

color
HCL

Grey

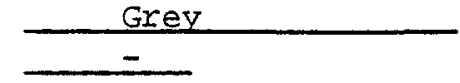

Slightly Micaceous

Unfrosted Quartz

Lithified by silt \& clay

Immature 

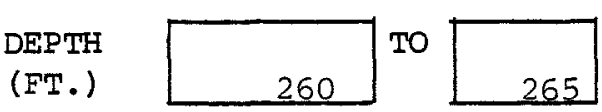

ROCK CLASSIFICATION

(FT.)

265

Sand

8 SIZE RANGE SPHERICITY ROUNDNESS

BASAIT $\geq$ gravel sand
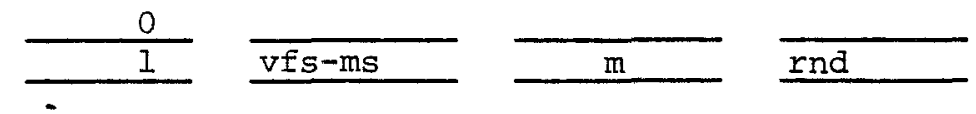

OTHER >gravel sand
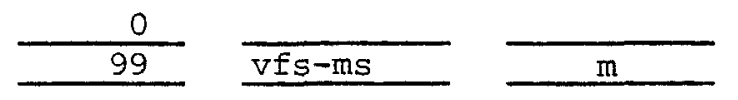

$\overline{\text { sb ang }}$

SIIT AND CIAY

0

\section{REMARKS}

color

HCL

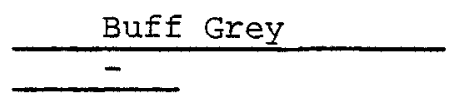

Slightly Micaceous

Unfrosted Quartz

Very Mature
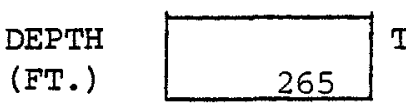

TO

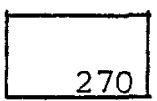

ROCK CIASSIFICATION

silt

\section{SIZE RANGE SPHERICITY ROUNDNESS}

BASALT >gravel sand
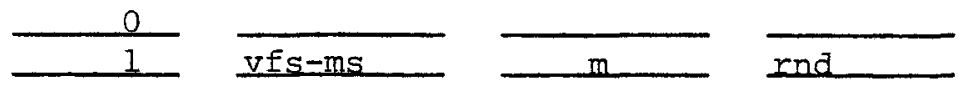

OTHER $\geq$ gravel sand
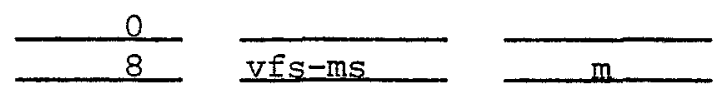

sb ang

SILT AND CIAYY 91

REMARKS

$$
\text { color }
$$

HCL

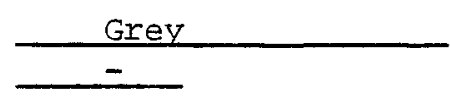

Slightly Micacenus

Lithified (?)

Mature 


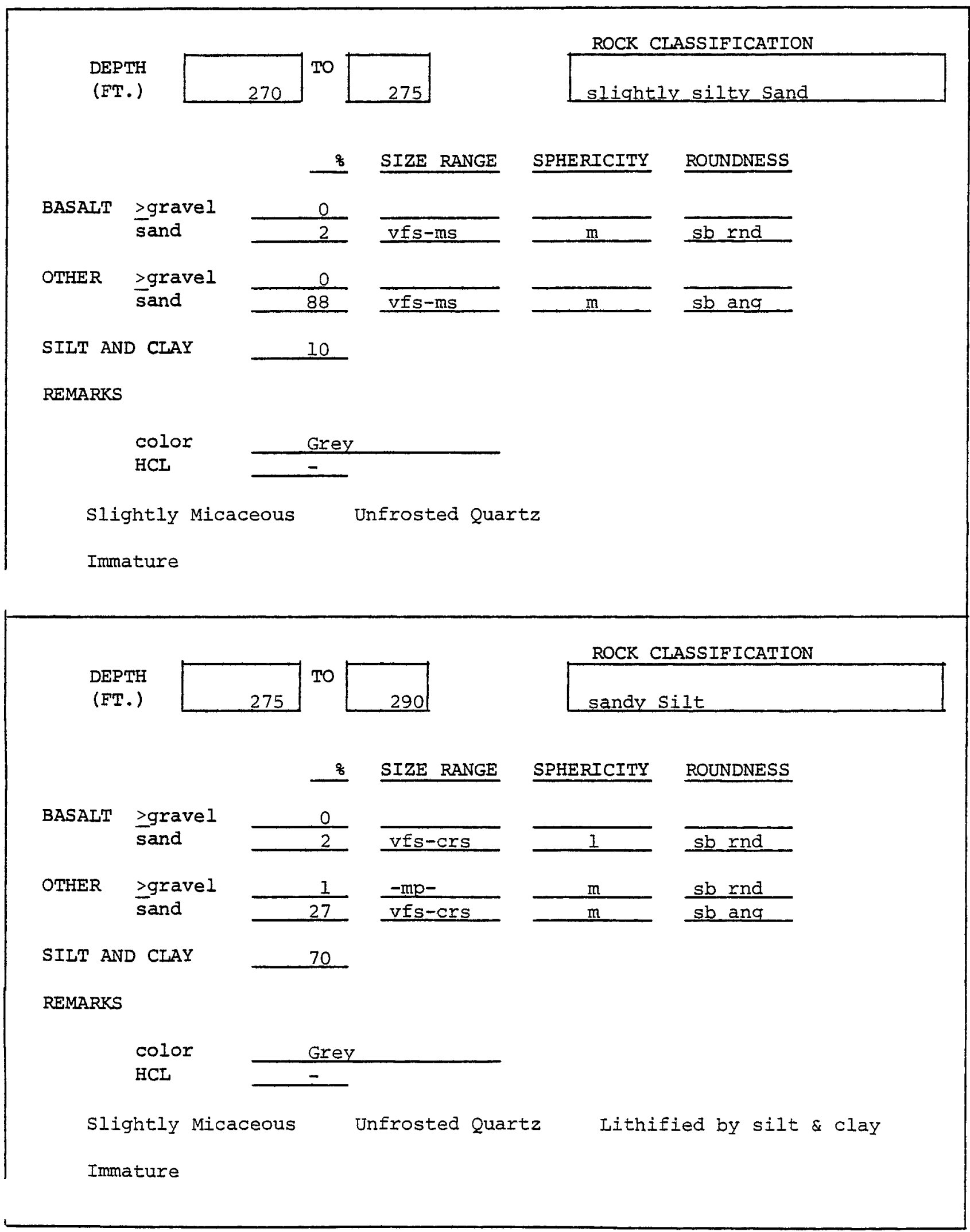

W. K. Summers \& Associates 


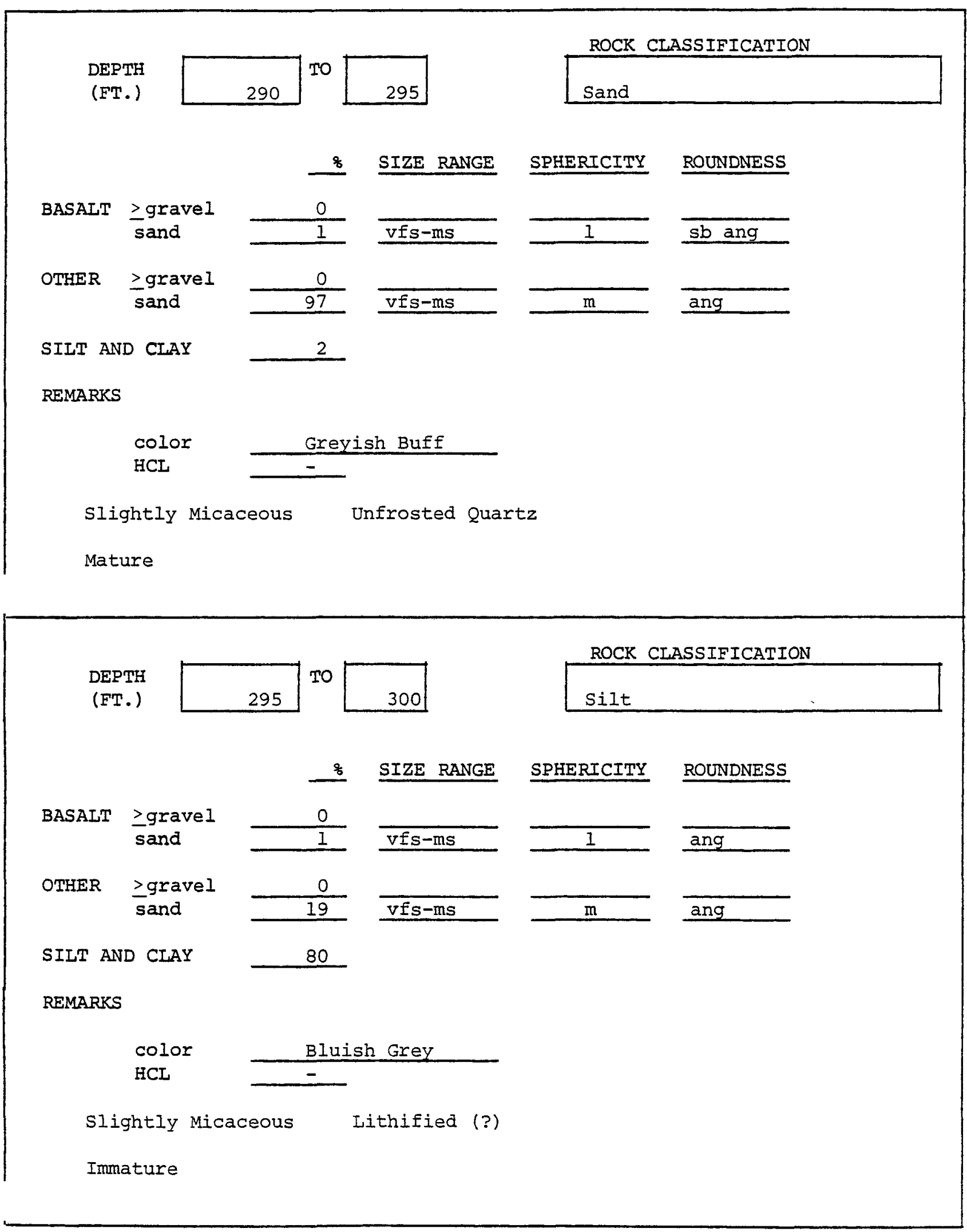




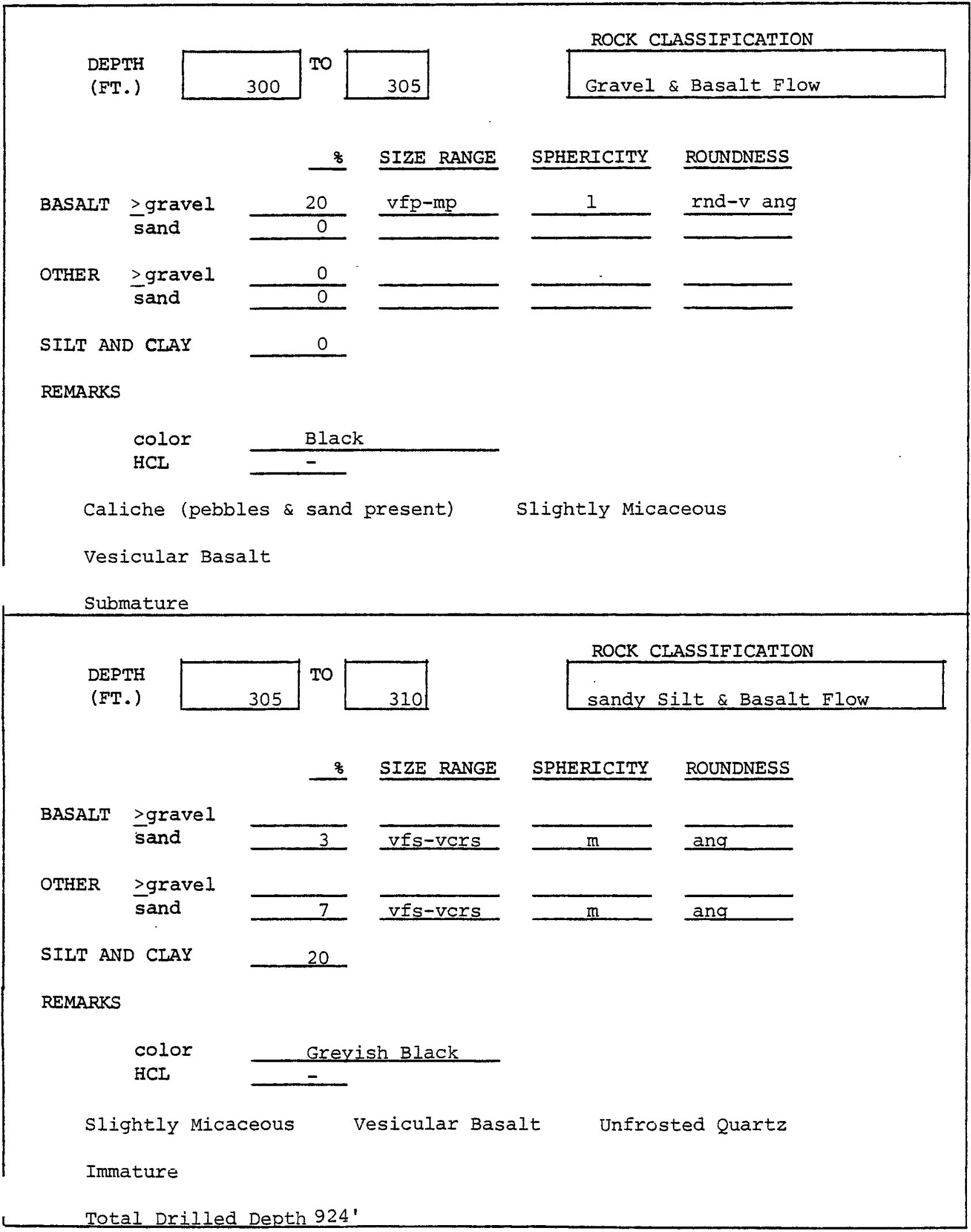

W. K. Summers \& Associates 


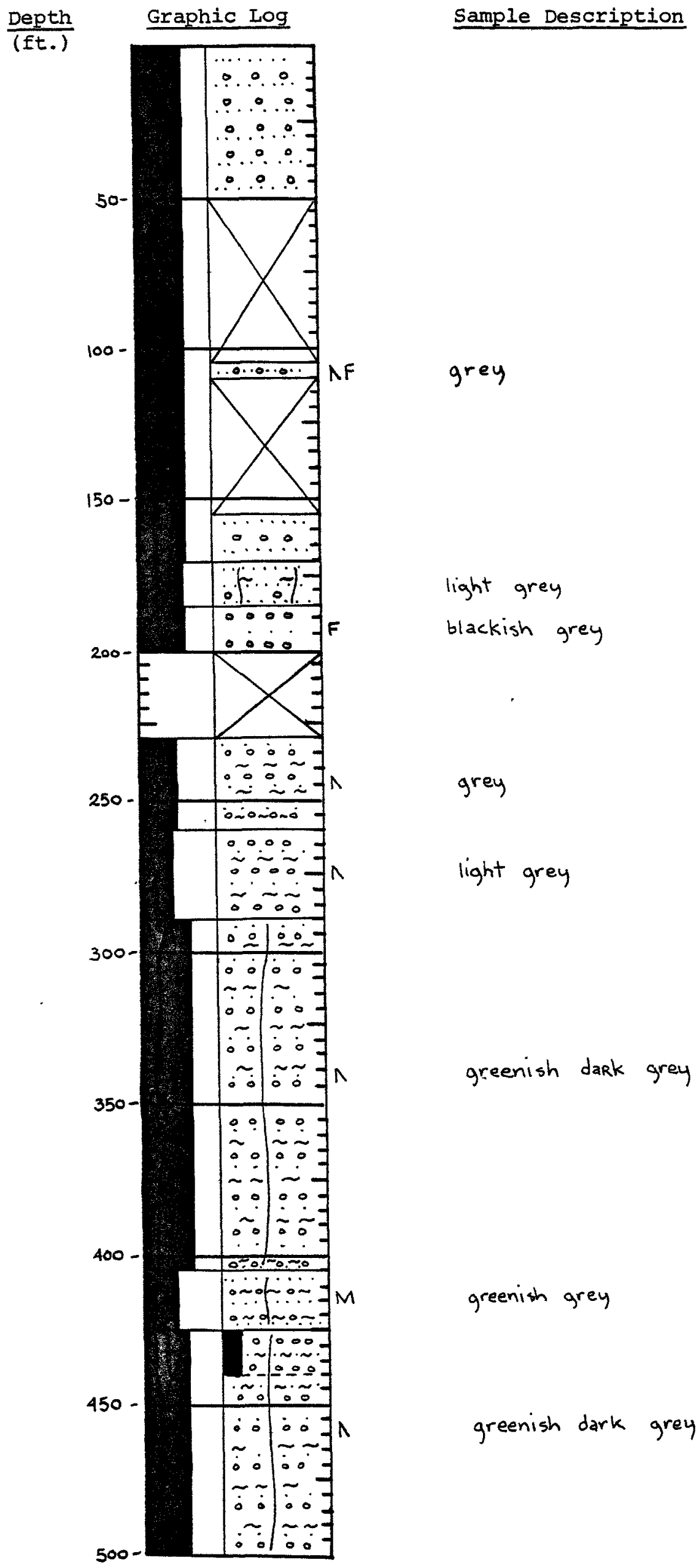

$\frac{\frac{P \text { lant Coordinates }}{N 014815}}{\frac{W 014963}{\text { Altitude } 545}}$

Other Data used in Interpretation

(1) Driller's Log

(2) E - Log

grey

light grey

blackish grey

grey

light grey

greenish dark grey

W. K. Summers \& Associates 
$\frac{\text { Depth }}{\text { (ft.) }}$

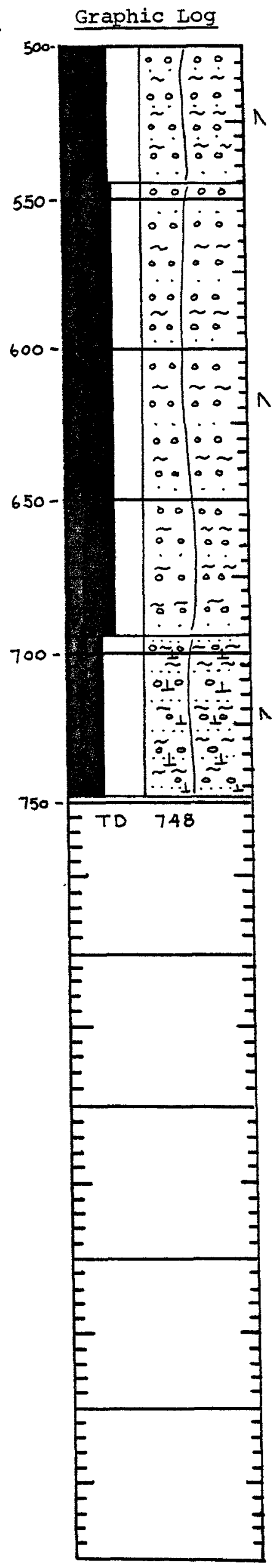

Sample Description

greenish dark grey

greenish dark grey
Plant Coordinates

$\mid \begin{aligned} & \Sigma \\ & \mathbb{D} \\ & \mathbb{1}\end{aligned}$

Altitude

Other Data used in Interpretation
10

in

$\left.\right|_{D} ^{\prime}$

$\stackrel{n}{0}$

greyish brown; ASH FRAGMENTS

W. K. Summers \& Associates 


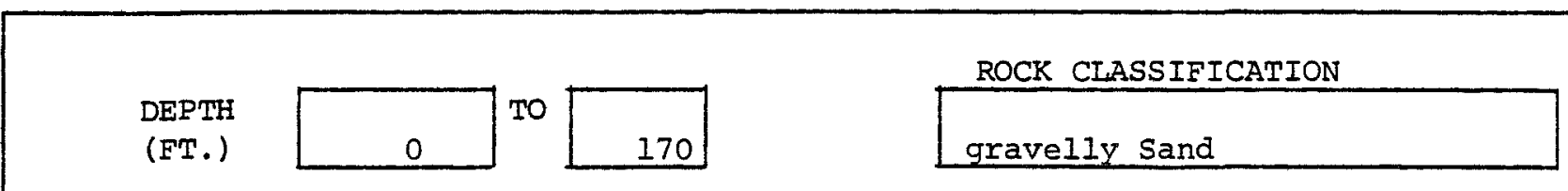

\% SIZE RANGE SPHERICITY ROUNDNESS

\begin{tabular}{|c|c|c|c|c|c|}
\hline BASAIT & $\geq$ gravel & $\frac{10}{56}$ & $v f p-c r p$ & $\frac{1}{7}$ & $\frac{\text { rnd }}{c h r n d}$ \\
\hline \multirow[t]{2}{*}{ OTHER } & > gravel & 10 & vfp-crp & 1 & rnd \\
\hline & sand & 21 & vfs-vers & $\mathrm{m}$ & sb ang \\
\hline
\end{tabular}

SILT AND CLAY 3

REMARKS

color

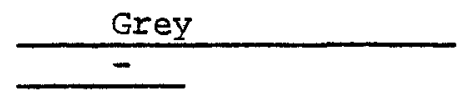

Slightly Micaceous Frosted Quartz

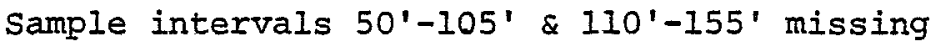

Immature

DEPTH

(FT.)

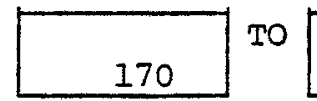

185
ROCK CLASSIFICATION

slightly silty slightly gravelly sand

\section{\% SIZE RANGE SPHERICITY ROUNDNESS}

BASALT

$$
\begin{aligned}
& \geq \text { gravel } \\
& \text { sand }
\end{aligned}
$$
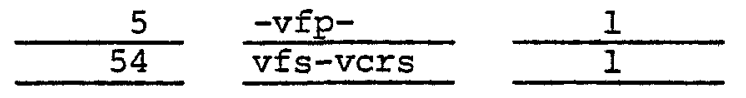

rnd

sb rnd

OTHER $\underset{\text { sand }}{\geq \text { gravel }}$
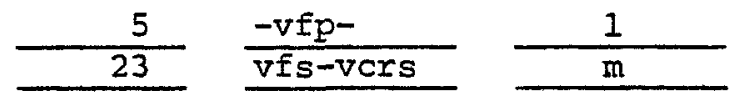

rnd

SILT AND CLAY

\section{3}

REMARKS

$$
\begin{aligned}
& \text { color } \\
& \text { HCL }
\end{aligned}
$$

Light Grey

Iithified by silt \& clay

Immature 


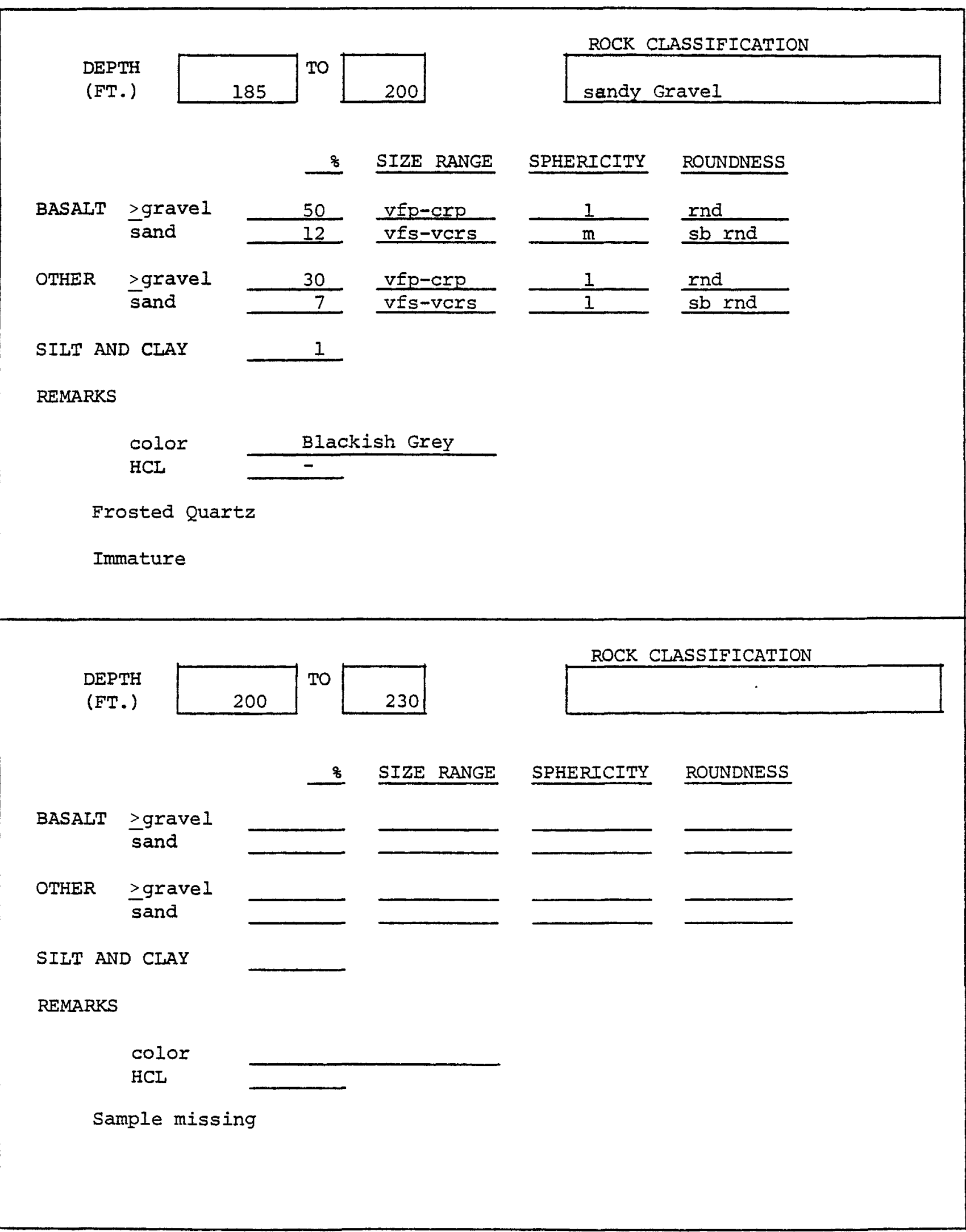


DEPTH

(FT.)

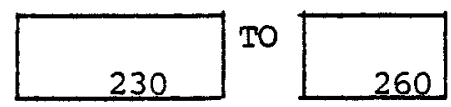

ROCK CIASSIFICATION

silty sandy Gravel

\section{\% SIZE RANGE SPHERICITY ROUNDNESS} BASALT $\underset{\text { sand }}{\text { gravel }}$
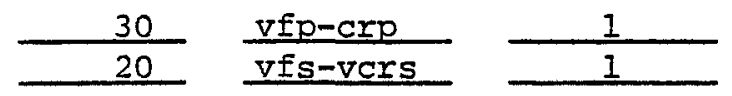

rnd

sb rnd

OTHER $\geq$ graveI

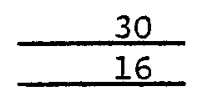

vfp-crp

SILT AND CLAY

4

REMARKS

color

Grey

$\mathrm{HCL}$

Slightly Micaceous

Immature

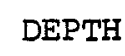

(FT.)

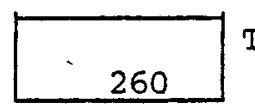

TO

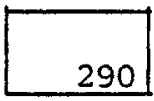

ROCK CLASSIFICATION

silty sandy Gravel

\section{SIZE RANGE SPHERICITY ROUNDNESS}

BASALT $\underset{\text { sand }}{\geq \text { gravel }}$
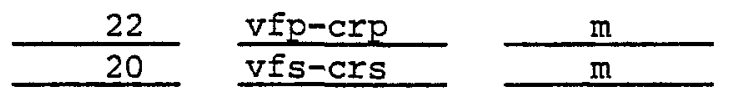

$\frac{\text { rnd }}{\text { sb rnd }}$

OTHER $\geq$ graveI

$\frac{22}{28} \frac{v f p-c r p}{v f s-c r s}$

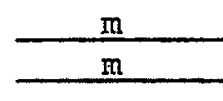

rnd

sb rnd

SILT AND CLAY

8

REMARKS

$$
\text { color }
$$

HCL

\section{Light Grey}

Slightly Micaceous

Immature 


\section{DEPTH}

(FT.)

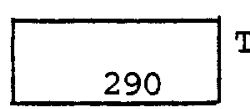

TO

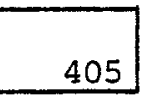

ROCK CLASSIFICATION

silty sandy Gravel

\section{\& SIZE RANGE SPHERICITY ROUNDNESS}

BASALT $\geq$ gravel

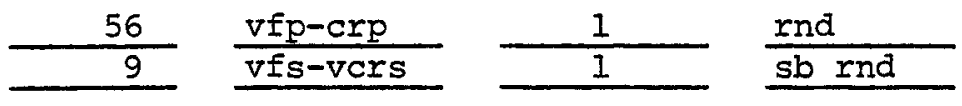

OTHER $\underset{\text { sand }}{\geq \text { gravel }}$
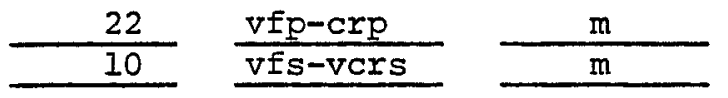

rnd

sb rnd

SILT AND CIAY

3

REMARKS

color
HCL

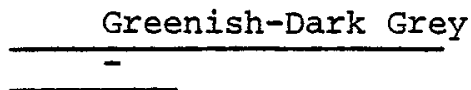

Slightly Micaceous

Compacted by silt, clay \& very fine sand

Immature

DEPTH

(FT.)

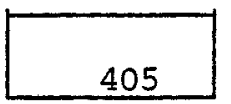

TO

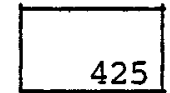

ROCK CLASSIEICATION

gravelly silty sand

\section{\& SIZE RANGE SPHERICITY ROUNDNESS}

\begin{tabular}{|c|c|c|c|c|c|}
\hline \multirow[t]{2}{*}{ BASALT } & >gravel & 15 & $v f p-m p$ & 1 & sb rnd \\
\hline & sand & 28 & vfs-vers & 1 & sb rnd \\
\hline \multirow[t]{2}{*}{ OTHER } & zgravel & 15 & $v f p-m p$ & 1 & sb rnd \\
\hline & sand & 28 & vfs-vcrs & m & sb rnd \\
\hline
\end{tabular}

SIIT AND CIAY

14

REMARKS

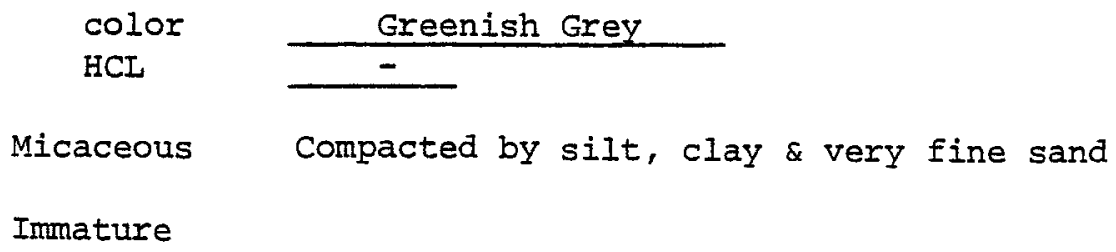


DEPTH

(FT.)
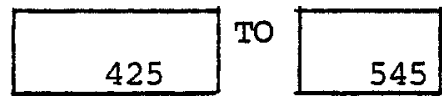

ROCK CLASSIFICATION

silty sandy Gravel

\section{$\%$ SIZE RANGE SPHERICITY ROUNDNESS}

BASALT $\geq$ gravel sand
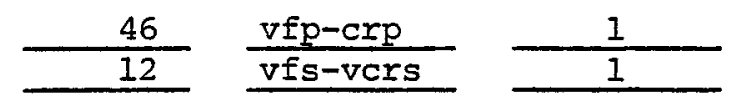

rnd

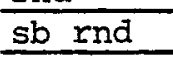
OTHER $\underset{\text { sand }}{\geq \text { gravel }}$

SIIT AND CLAY

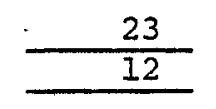

$\frac{\text { vfp-crp }}{\text { vfs-vers }}$

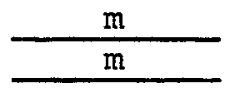
7

REMARKS

color

HCL Greenish-Dark Grey

Caliche (present between 425'\&440')

Slightly Micaceous

Compacted by silt, clay \& very fine sand

Immature

DEPTH

(FT.)

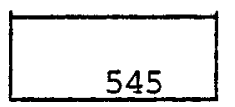

TO

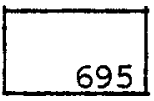

ROCK CIASSIFICATION

silty sandy Gravel

\section{\% SIZE RANGE SPHERICITY ROUNDNESS}

BASAIT $\begin{gathered}\geq \text { gravel } \\ \text { sand }\end{gathered}$
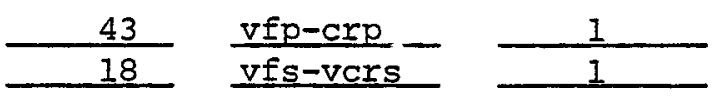

rnd

OTHER Igravel sand $\frac{v f p-c r p}{v f s-v a r s}$

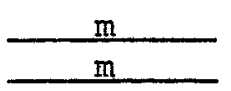

SILT AND CLAY 6

REMARKS

$$
\text { color }
$$

HCL Greenish-Dark Grey $-$

Slightly Micaceous Compacted by silt, clay \& very fine sand Immature 


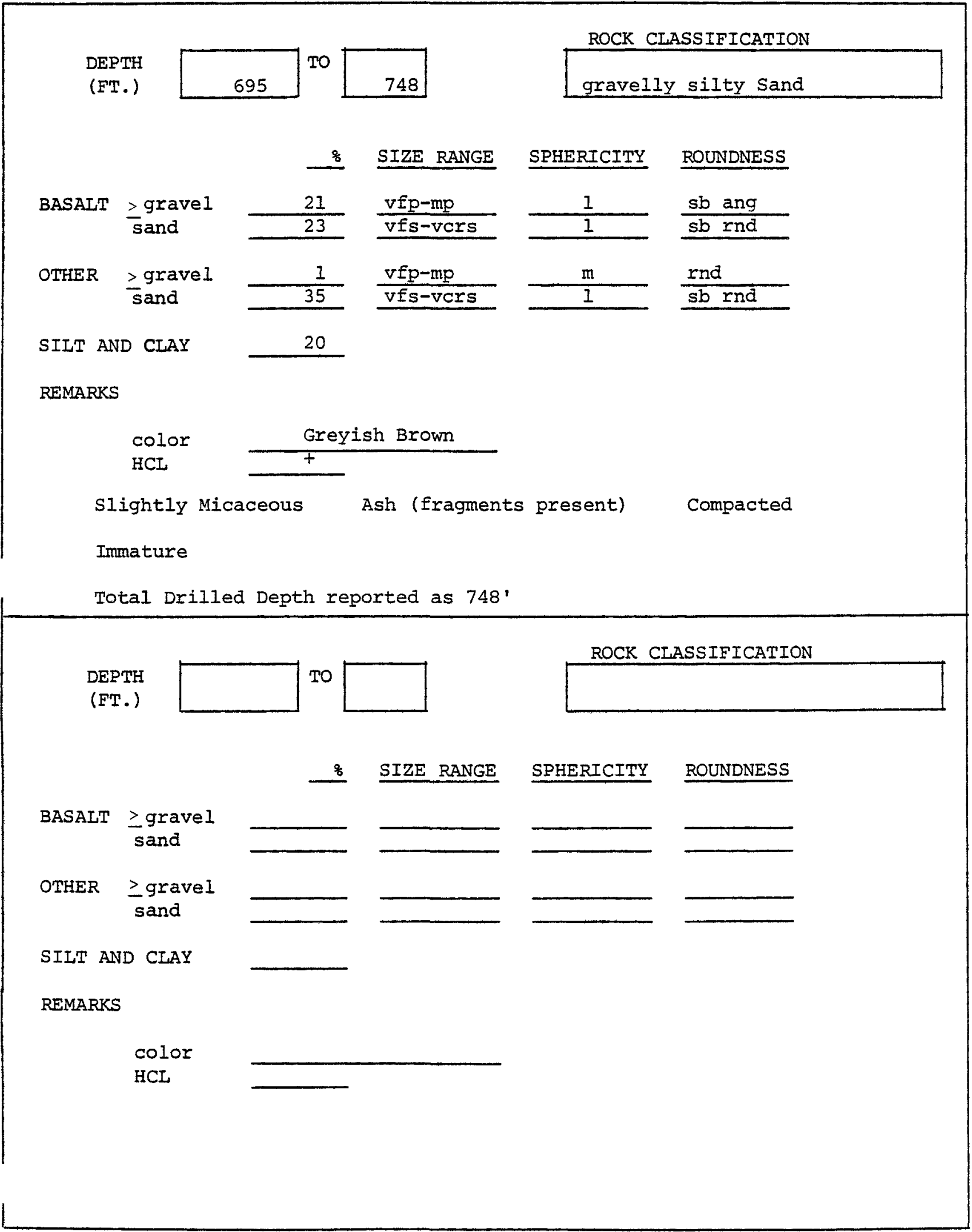


$\frac{\text { Depth }}{(f t .)}$
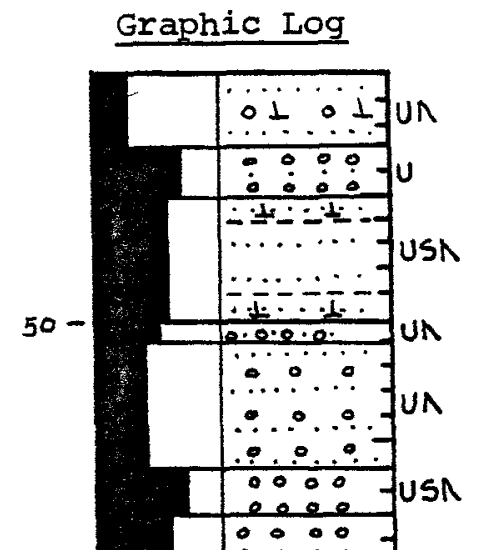

100

$$
250
$$
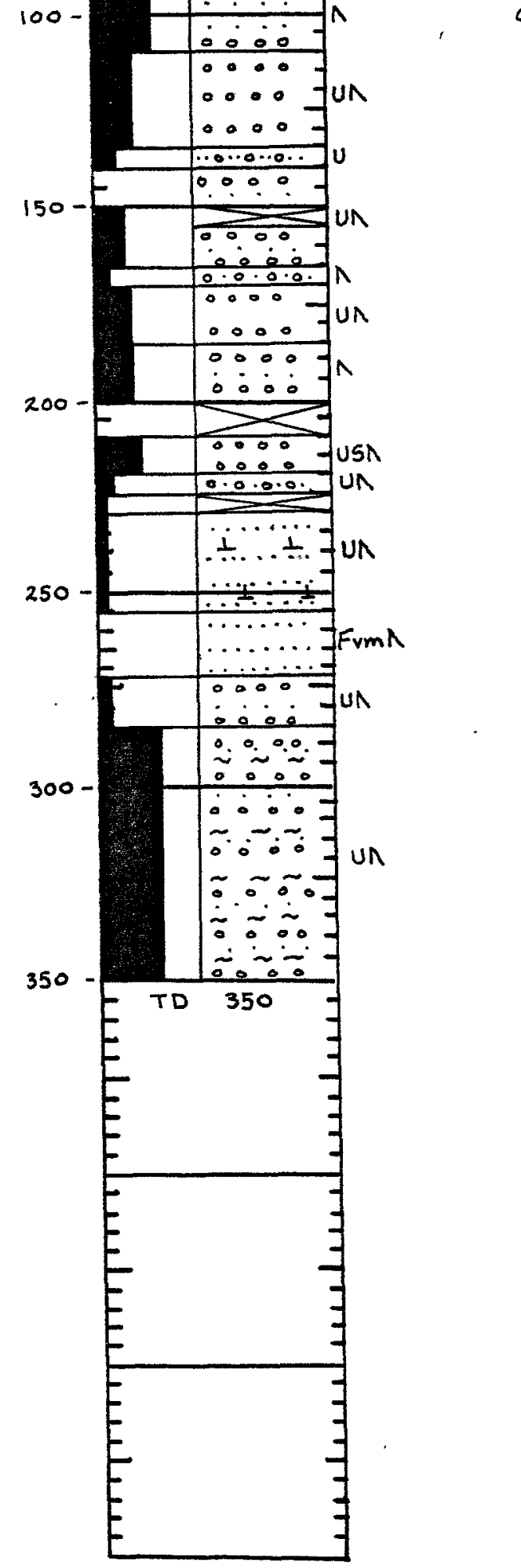

\section{Sample Description}

greyish buff

greyish black

blackish grey (salt \& pepper)

greyish black

butf grey

black

greyish brown

greyish buff

greyish buff

greyish buff

white

greyish buff

greyish brown

greyish buff
buff

tannish Buff

white

buff

brownish grey
Plant Coordinates

No15602

$\frac{W 026370}{\text { Altitude } 522}$

Other Data used in Interpretation

Driller's Log 


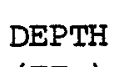

(FT.)

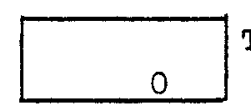

TO

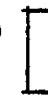

ROCK CLASSIFICATION

slightly gravelly sand

\section{SIZE RANGE SPHERICITY ROUNDNESS}

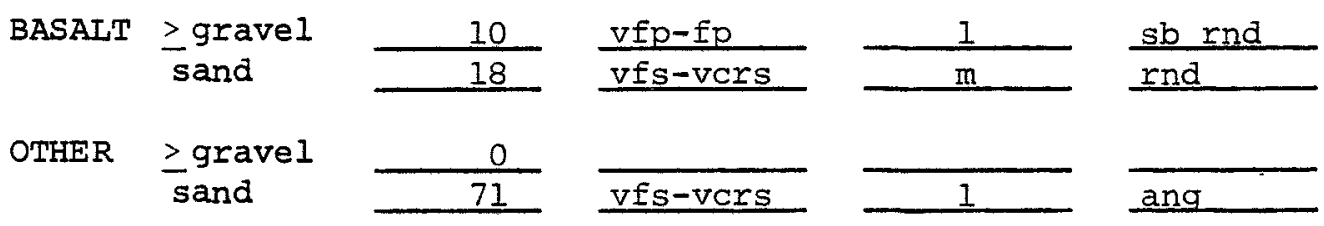

SIIT AND CLAY

1

REMARKS

color

Greyish Buff

HCL $+$

Slightly Micaceous Unfrosted Quartz

Immature

DEPTH

(FT.)

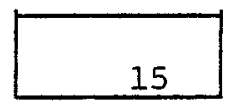

TO

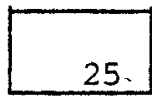

ROCK CLASSIFICATION

sandy Gravel

\section{$\underline{8}$ SIZE RANGE SPHERICITY ROUNDNESS}

BASAIT $\geq$ gravel sand

$\frac{53}{16}$
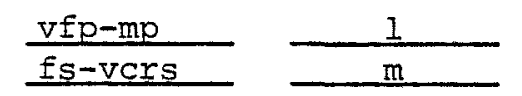

rnd

OTHER Igravel

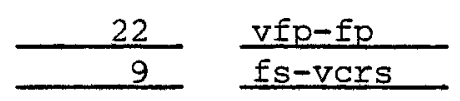

rnd

sb ang

SILT AND CLAY

0

REMARKS

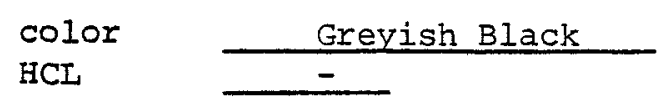

Unfrosted Quartz

Immature 
DEPTH

(FT.)

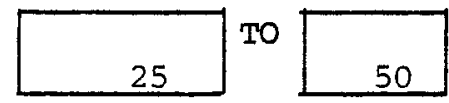

ROCK CIASSIFICATION

Sand

\section{S SIZE RANGE SPHERICITY ROUNDNESS}

BASALT >gravel sand OTHER $\frac{\text { >gravel }}{\text { sand }}$

SILT AND CLAY

REMARKS

color

HCL
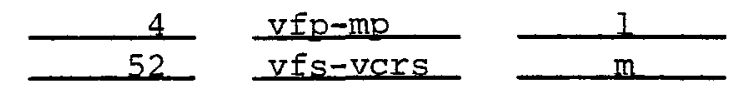

$\frac{\text { sb rnd }}{\text { ang }}$
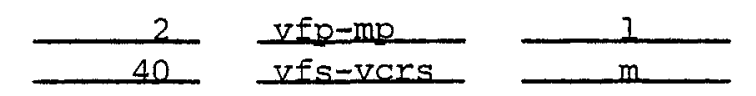

Slightly Micaceous Unfrosted Quartz

Submature

DEPTH

(FT.)

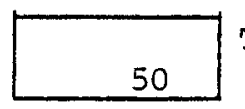

To

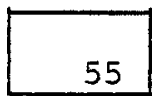

ROCK CLASSIFICATION

sandy Gravel

\% SIZE RANGE SPHERICITY ROUNDNESS

\begin{tabular}{|c|c|c|c|c|c|}
\hline BASALT & >gravel & $\frac{30}{20}$ & $v f p-m p$ & $\frac{1}{m}$ & rnd \\
\hline OTHER & $>$ gravel & 30 & $v f p-m p$ & 1 & rnd \\
\hline & sand & 20 & fs-vers & m & sb ang \\
\hline
\end{tabular}

SILT AND CLAY

0

REMARKS

color

HCI

Greyish Black

Slightly Micaceous

Unfrosted Quartz

Immature 


\section{DEPTH \\ (ET.)}

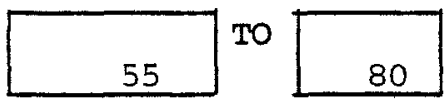

ROCK CIASSIFICATION

gravelly sand

\% SIZE RANGE SPHERICITY ROUNDNESS

BASALT $\geq$ gravel sand

OTHER $\geq$ gravel. sand

SILT AND CLAY

REMARKS Buff Grey HCL
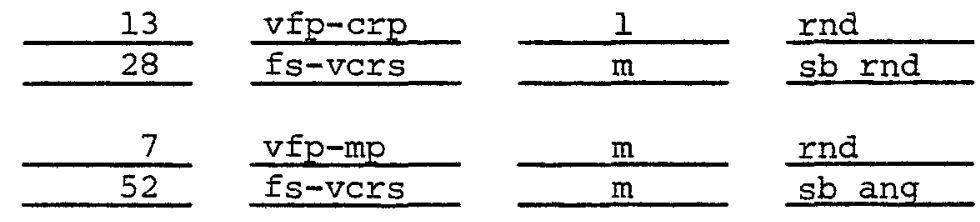

rnd sb ang

Slightly Micaceous Unfrosted Quartz

Immature

DEPTH

(ET.)

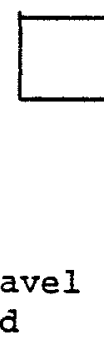

80

TO

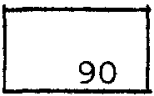

ROCK CLASSIFICATION

Gravel

\% SIZE RANGE SPHERICITY ROUNDNESS

BASALT $\geq$ gravel
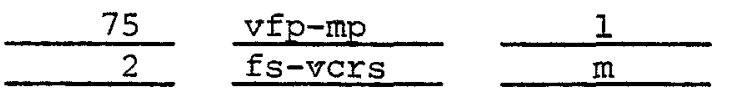

$\frac{\text { sb rnd }}{\text { sb rnd }}$

OTHER $\frac{\text { g gravel }}{\text { sand }}$
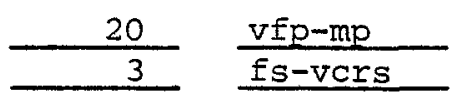

1

rnd

SILT AND CLAY

0

REMARKS

$$
\text { color }
$$

HCL

Black

Slightly Micaceous

Unfrosted Quartz

Submature 
DEPTH
(FT.)

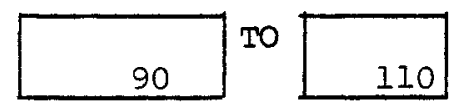

ROCK CLASSIFICATION

sandy Gravel

\section{S SIZE RANGE SPHERICITY ROUNDNESS} BASAIT $\underset{\text { sand }}{\text { gravel }}$ OTHER $\begin{aligned} & \geq \text { graveI } \\ & \text { sand }\end{aligned}$

SILT AND CLAY

REMARKS

color

$\mathrm{HCL}$
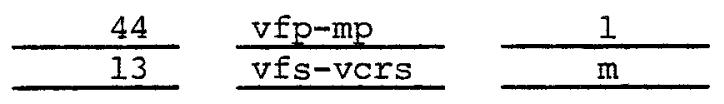

rnd

sb rnd
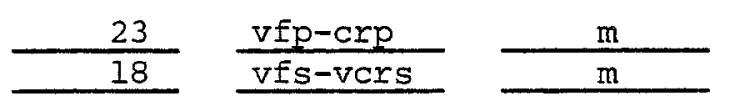

rnd

Slightly Micaceous

Immature

DEPTH
(FT.)

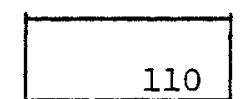

TO

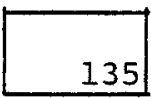

ROCK CLASSIFICATION

Gravel

8 SIZE RANGE SPHERICITY ROUNDNESS

BASALT >gravel
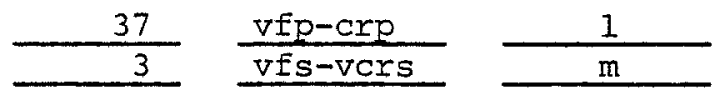

wnd

OTHER $\quad \frac{\text { gravel }}{\text { sand }}$
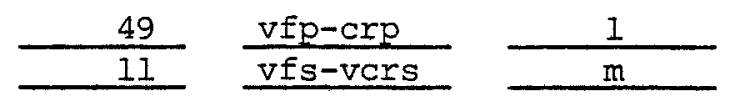

\begin{tabular}{l} 
w rnd \\
\hline ang
\end{tabular}

SILT AND CLAY 0

REMARKS

$$
\text { color }
$$

HCL

Greyish Buff

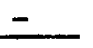

Slightly Micaceous Unfrosted quartz

Immature 
DEPTH

(FT.)

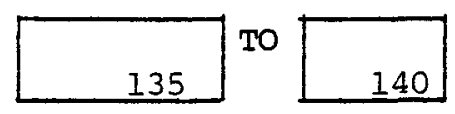

\section{ROCK CLASSIFICATION}

grave $11 y$ sand

\section{\& SIZE RANGE SPHERICITY ROUNDNESS}

BASAIT $\underset{\text { sand }}{\geq \text { gravel }}$
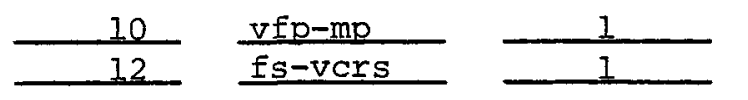

$\frac{\mathrm{w} \text { rnd }}{\text { sb ang }}$

OTHER >gravel
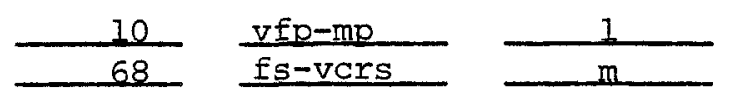

$\frac{\text { w rnd }}{\text { ang }}$

\section{SILT AND CLAY}

0

REMARKS

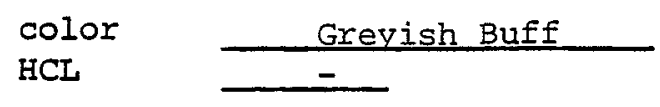

Unfrosted Quartz

Immature

\section{DEPTH}

(ET.)

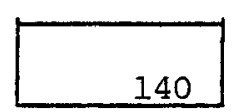

TO

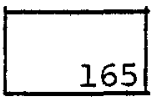

ROCK CLASSIFICATION

sandy Gravel

\section{\% SIZE RANGE SPHERICITY ROUNDNESS}

\begin{tabular}{|c|c|c|c|c|c|}
\hline \multirow[t]{2}{*}{ BASALT } & \multirow{2}{*}{$\begin{array}{l}\text { Zgravel } \\
\text { sand }\end{array}$} & 28 & $\mathrm{vfp}-\mathrm{crp}$ & 1 & w rnd \\
\hline & & 2 & vfs-vers & $\mathrm{m}$ & rnd \\
\hline \multirow[t]{2}{*}{ OTHER } & >gravel & 49 & $v f p-c r p$ & 1 & $w$ rnd \\
\hline & sand & 21 & vfs-vars & $m$ & ang \\
\hline
\end{tabular}

SILT AND CLAY

0

REMARKS

color
HCL

Grevish Buff

Slightly Micaceous Unfrosted Quartz

Sample interval $150^{\prime}-155^{\prime}$ missing

Immature 


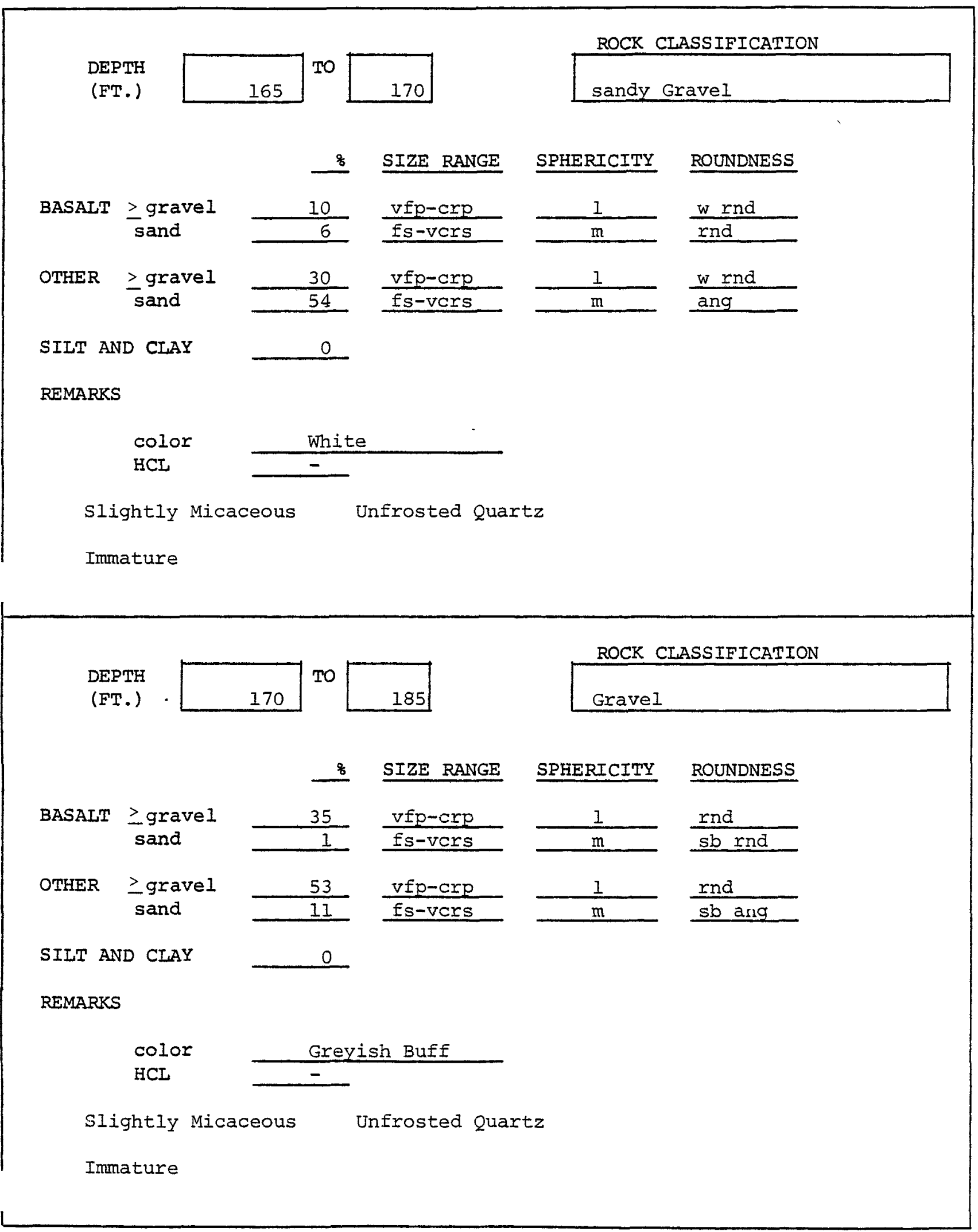




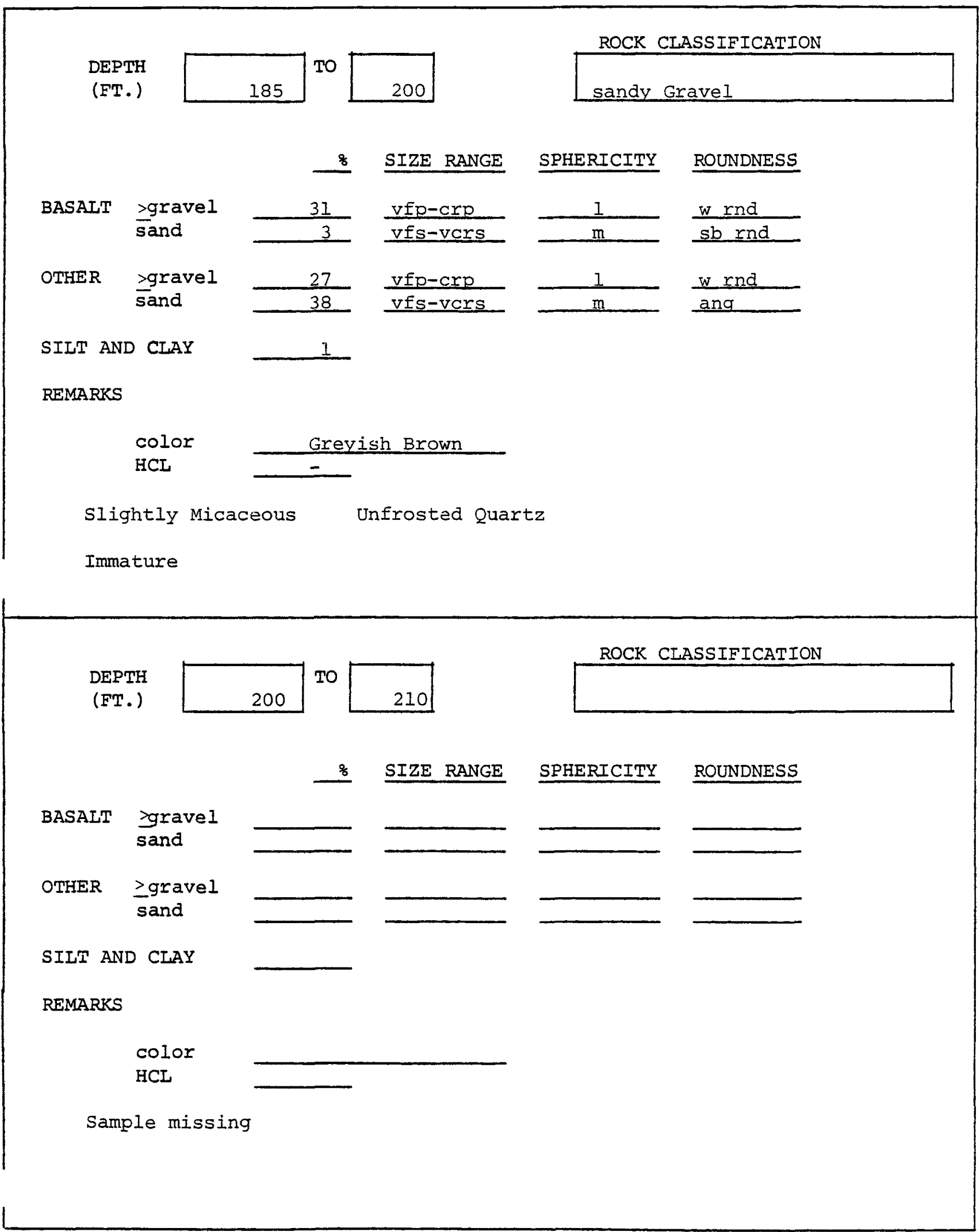

W. K. Summers \& Assoclates 


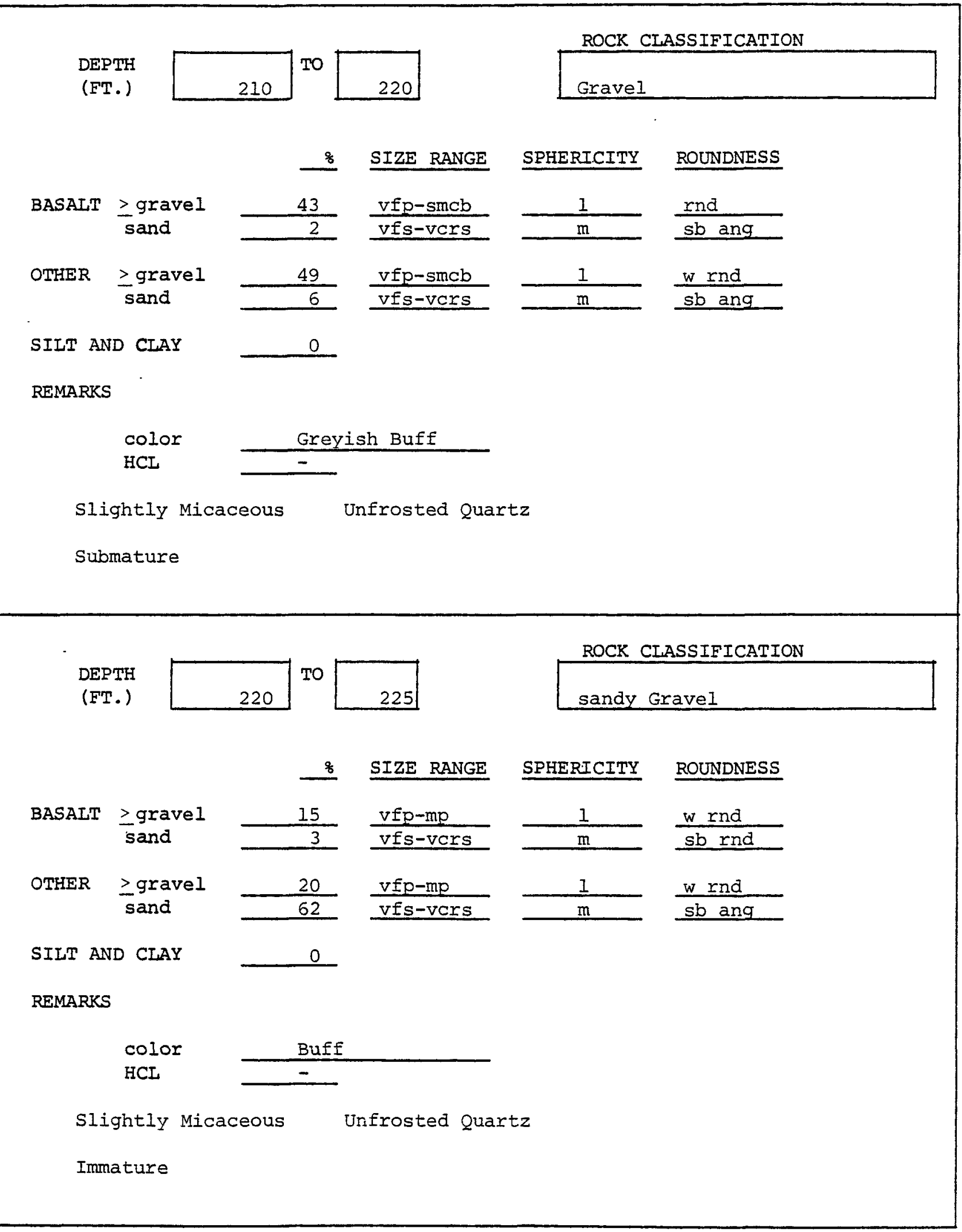

W. K. Summers \& Associates 
DEPTH

(FT.)

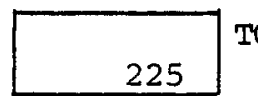

O

230

\% SIZE RANGE SPHERICITY ROUNDNESS

BASAIT >gravel sand

OTHER >gravel sand

SIIT AND CIAY

REMARKS

$$
\text { color }
$$$$
\text { HCL }
$$

Sample missing

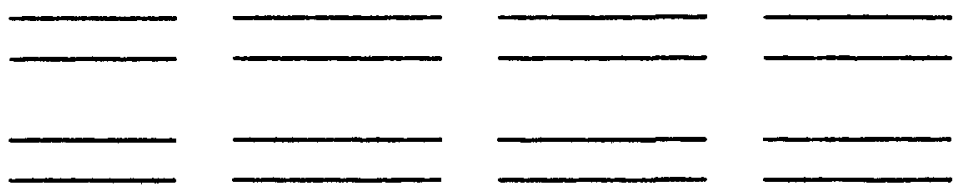

ROCK CIASSIFICATION

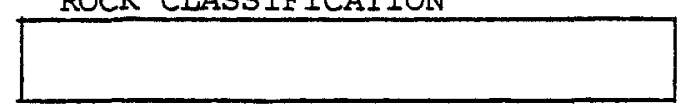




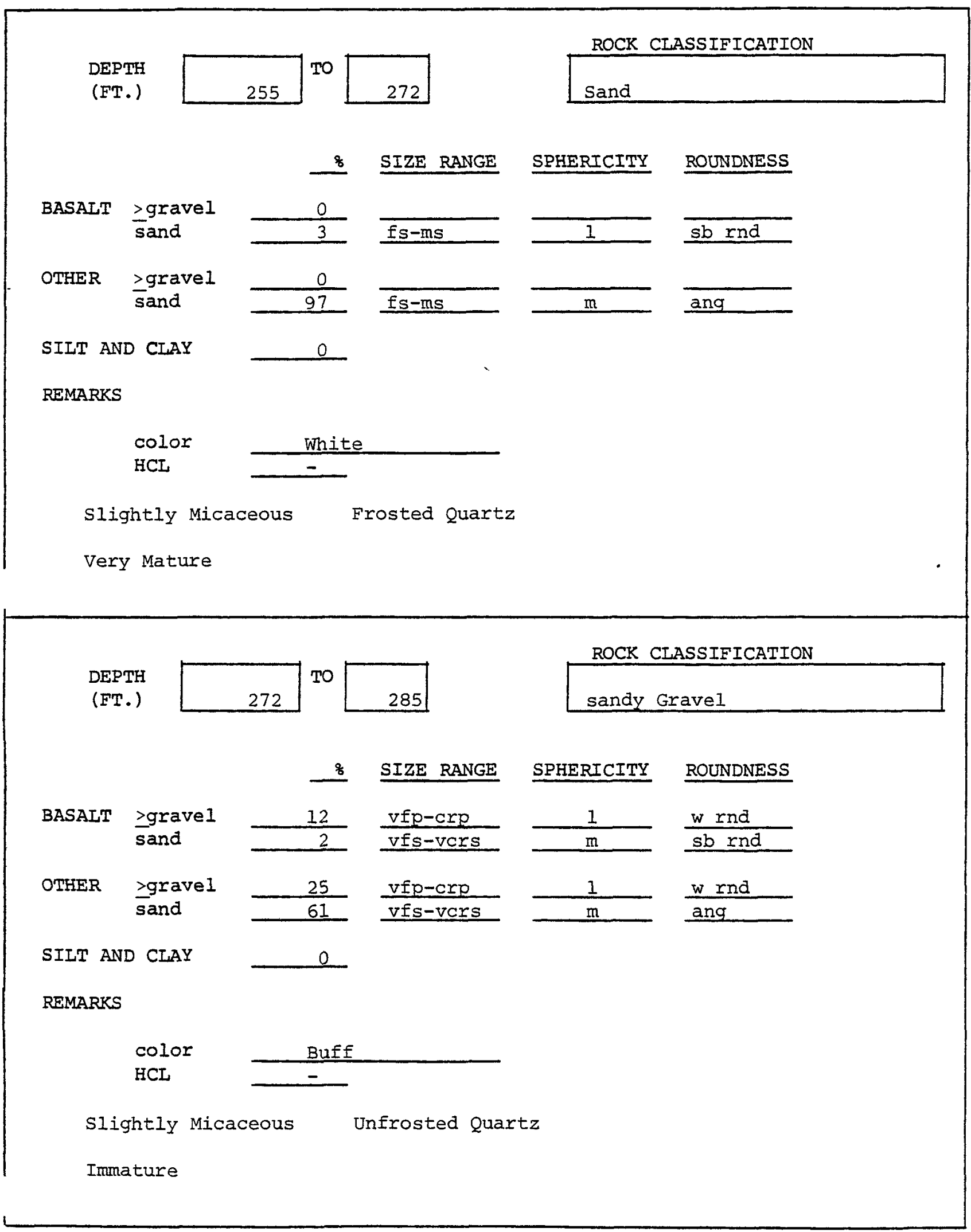

W. K. Summers \& Associates 


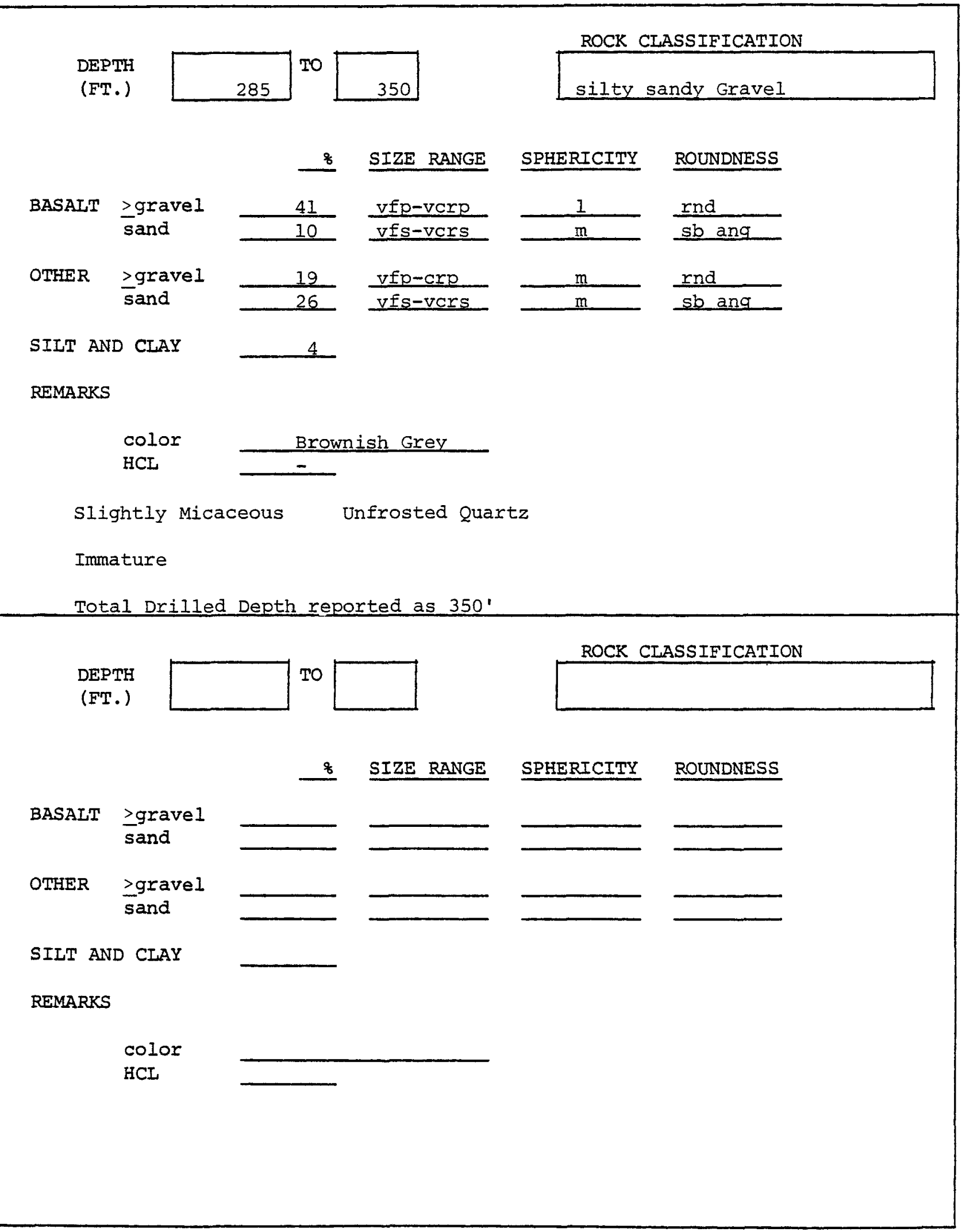

W. K. Summers \& Associates 


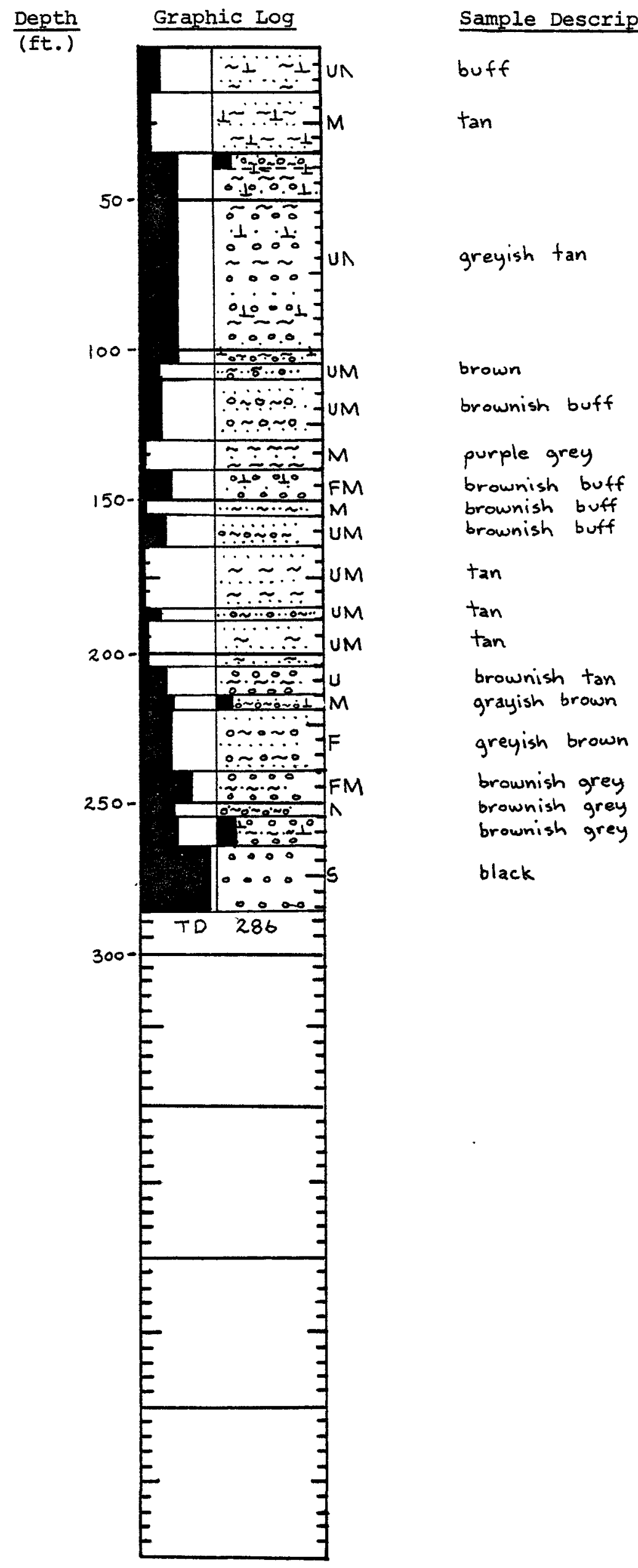

Plant Coordinates

N017000

W007000

Altitude 563

Other Data used in

Interpretation

Driller's Log
purple grey
brownish buff
brownish buff
$\tan$
brownish $\tan$
grown
greyish brown
brownish grey
brownish grey
black




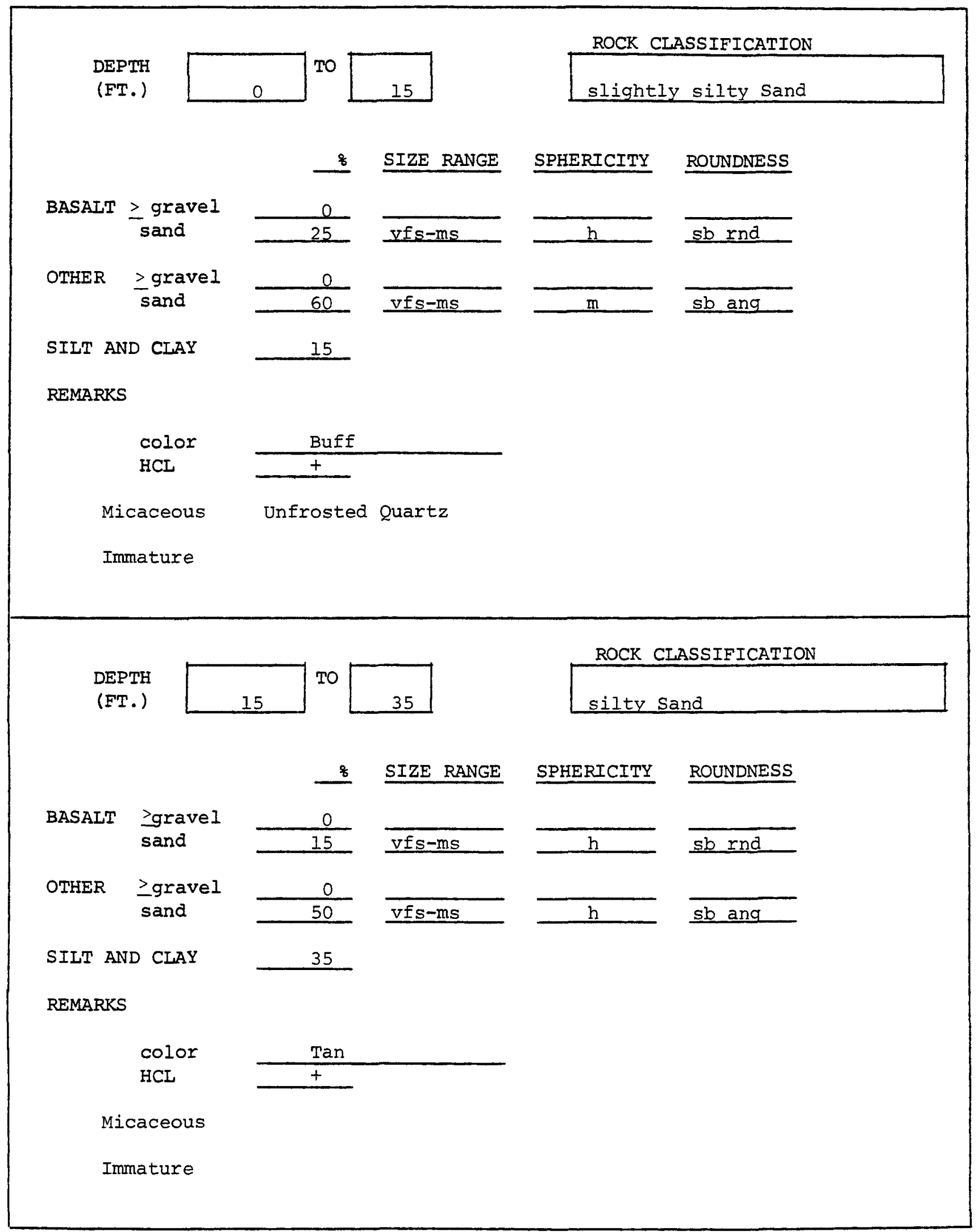




\section{DEPTH}

(FT.)

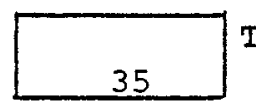

To

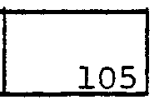

105
ROCK CLASSIFICATION

silty sandy Gravel

\section{SIZE RANGE SPHERICITY ROUNDNESS}

BASAIT $\underset{\text { sand }}{\geq \text { gravel }}$
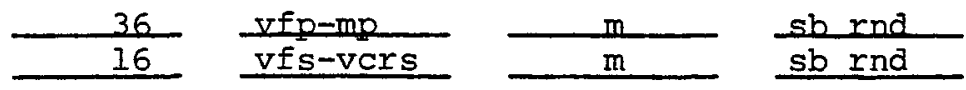

OTHER $\geq$ gravel sand

$\frac{v f p-m p}{v f s-v \operatorname{crs}}$

$\frac{m}{h} \frac{\text { sb rnd }}{\text { sb rnd }}$

SIIT AND CLAY 12

REMARKS

$$
\text { color }
$$
HCI

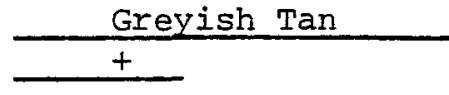

Caliche (present between $35^{\prime} \& 40^{\prime}$ )

Slightly Micaceous

Unfrosted Quartz

Immature

DEPTH

(FT.)

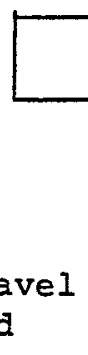

105
TO

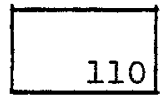

ROCK CLASSIFICATION

slightly silty gravelly sand

\section{SIZE RANGE SPHERICITY ROUNDNESS}

$$
\text { BASALT } \underset{\text { sand }}{\geq \text { gravel }}
$$

OTHER $\geq$ gravel sand
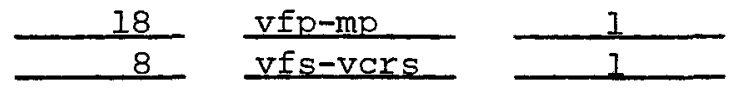

Ind sbrand

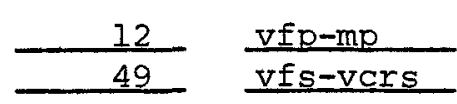

SILT AND CLAY 13

REMARKS

color

HCL

Brown

Micaceous

Unfrosted Quartz

Immature 


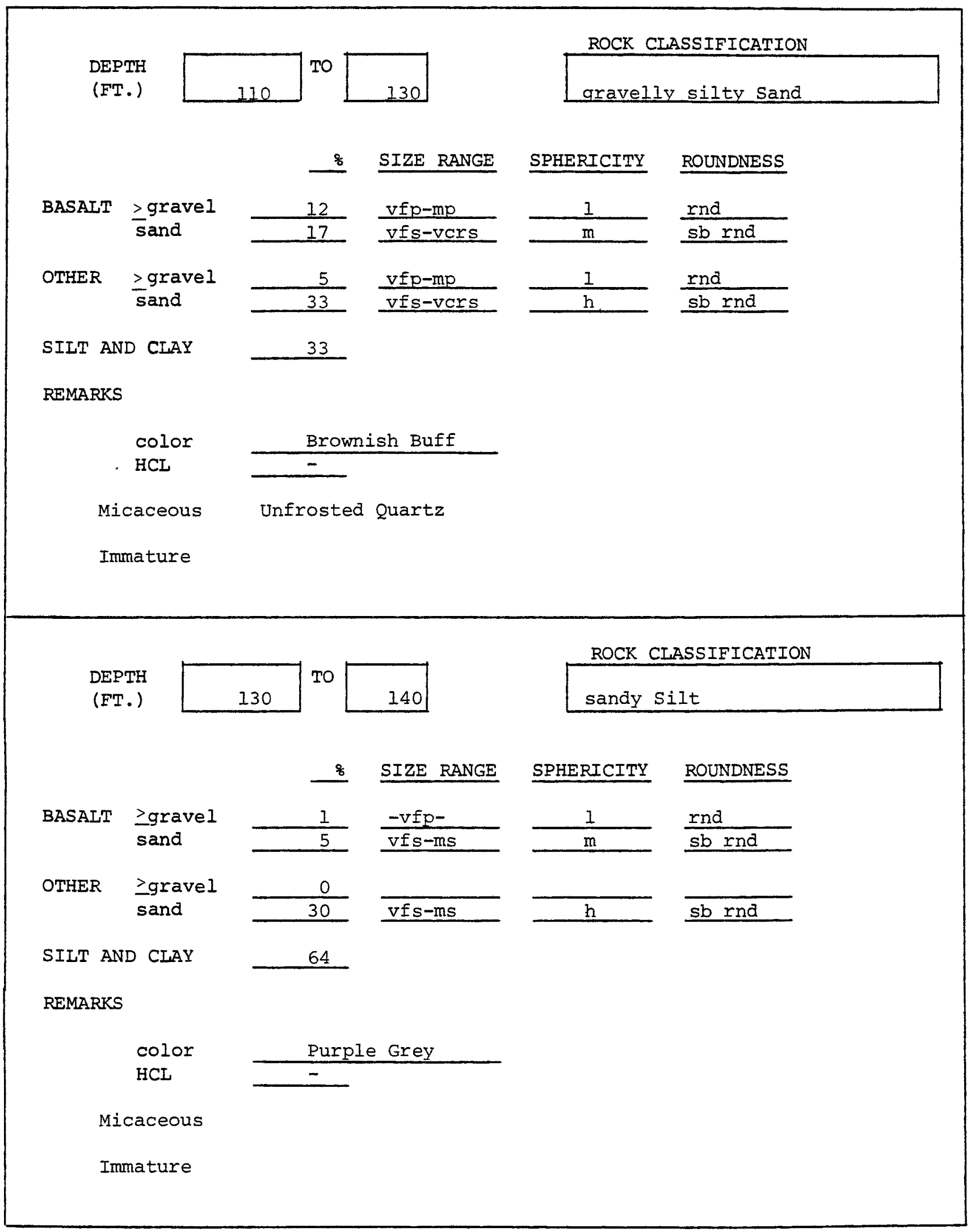




\section{DEPTH}

(FT.)

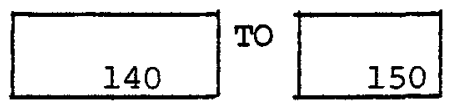

SIZE RANGE

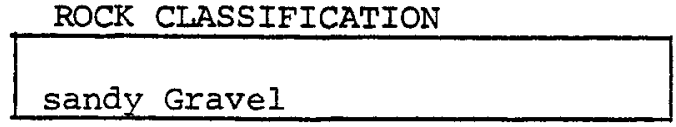

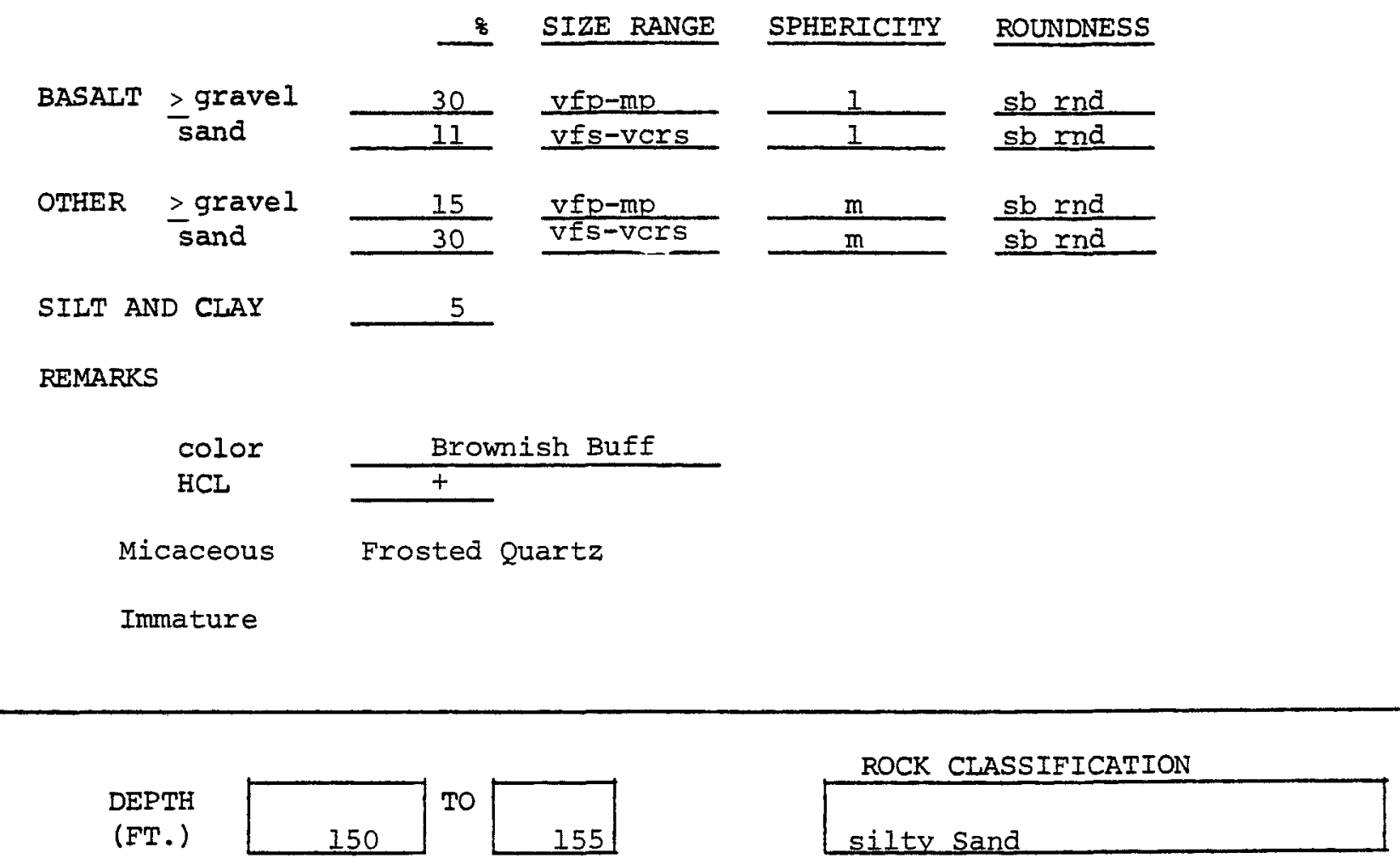

\section{SIZE RANGE SPHERICITY ROUNDNESS}
BASALT $\geq$ gravel sand
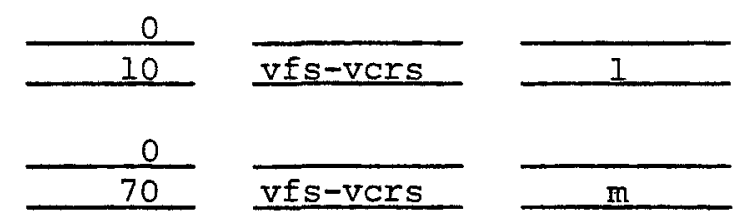

sbrna

OTHER Igravel
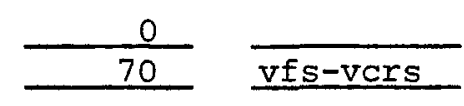

sb ang

SILT AND CLAY 20

REMARKS

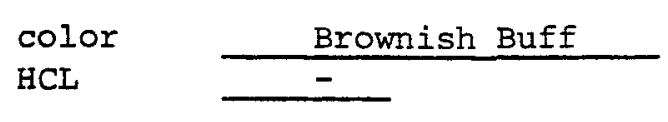

Micaceous

Immature 


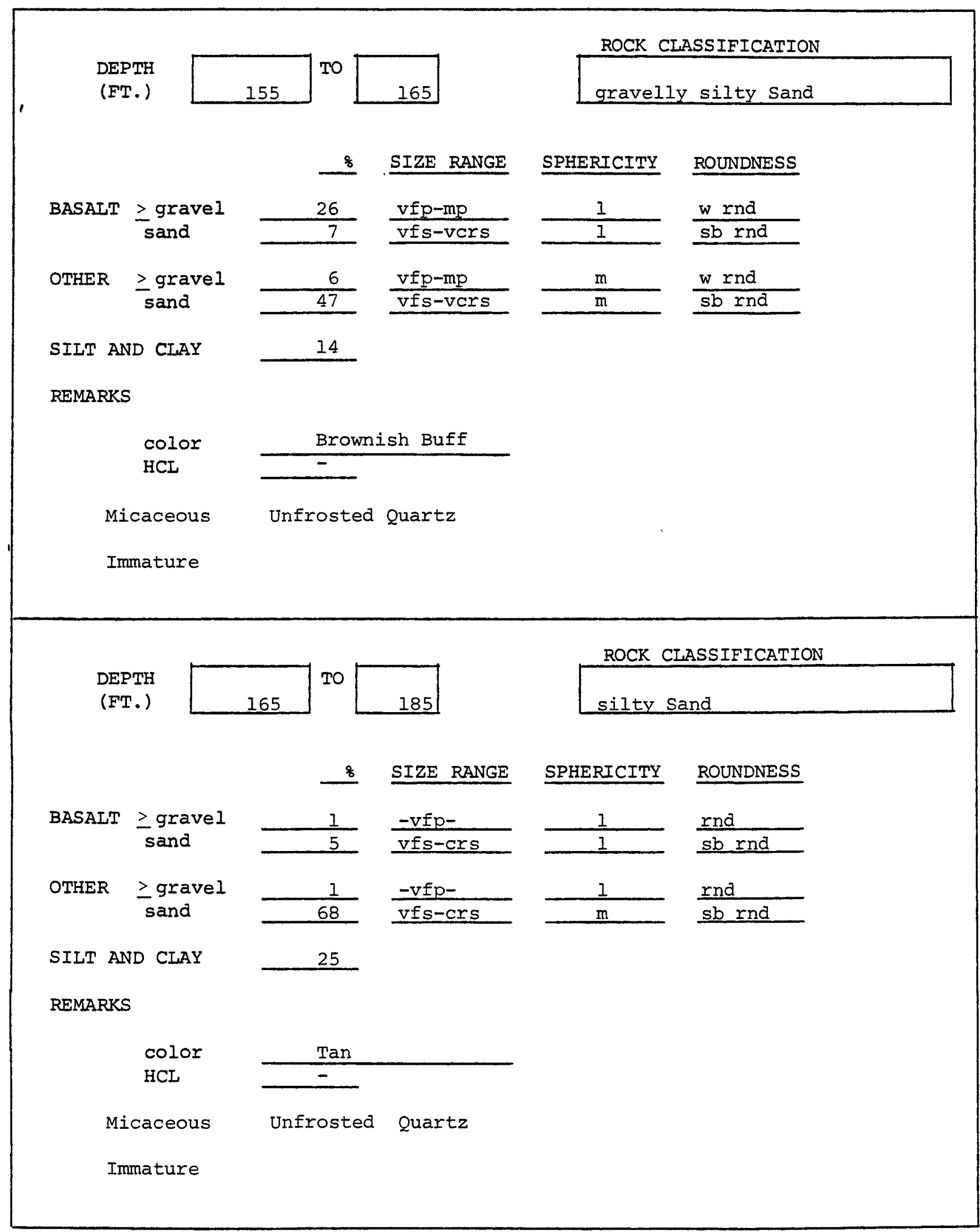

W. K. Summers \& Associates 


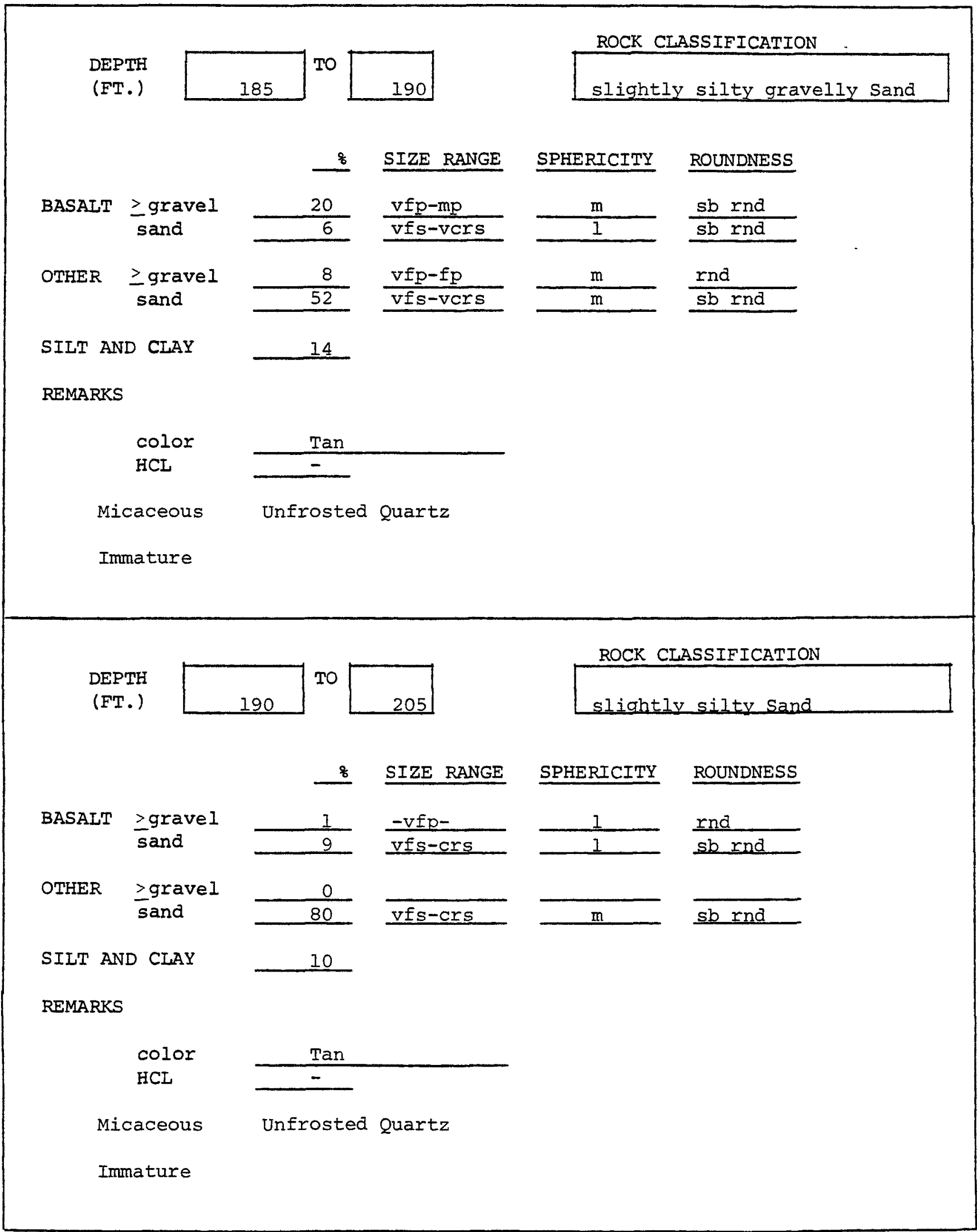




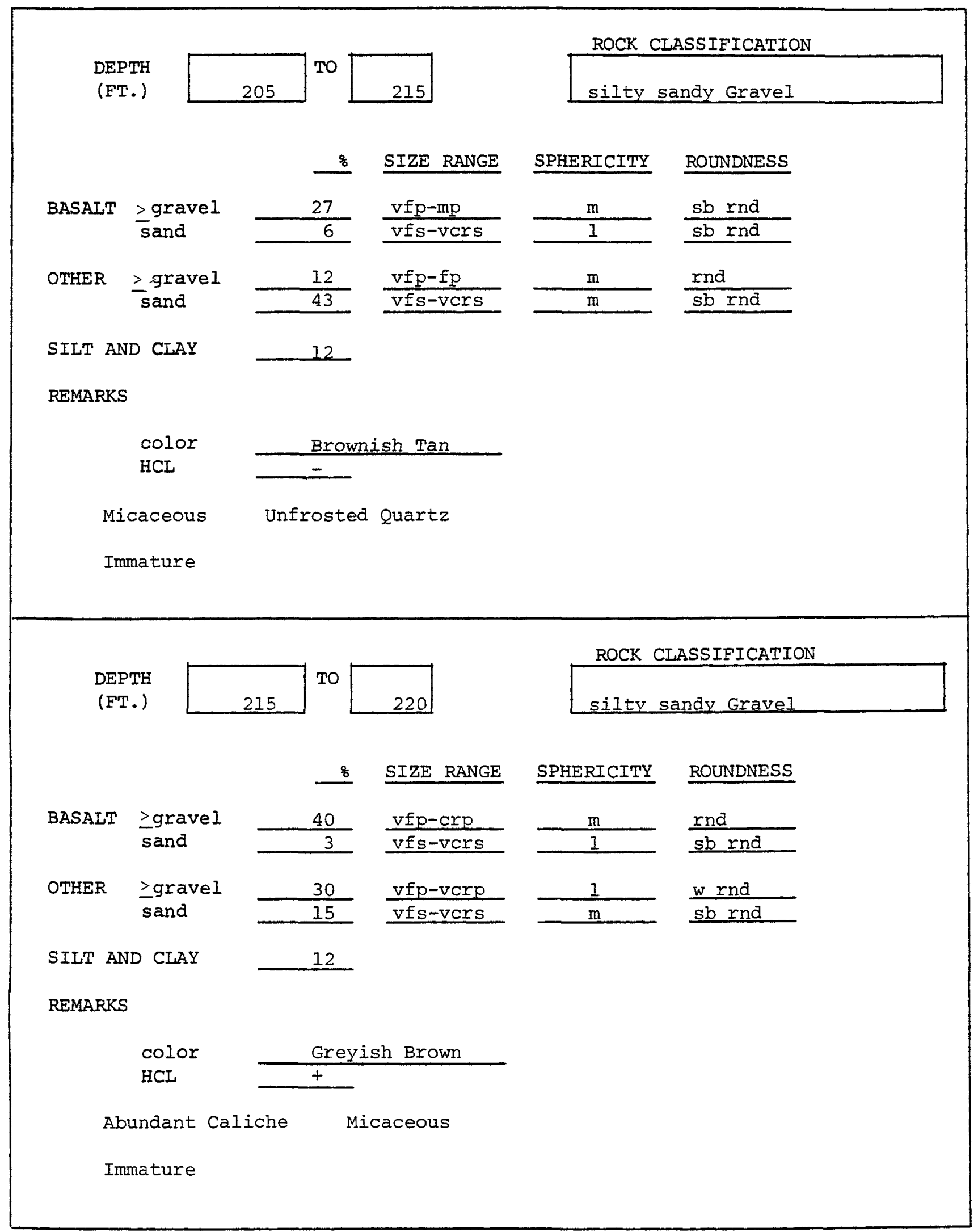




\section{DEPTH}

(FT.)

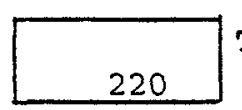

TO

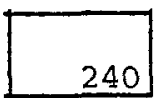

ROCK CLASSIFICATION

slightly silty gravelly sand

\section{\% SIZE RANGE SPHERICITY ROUNDNESS}

BASALT $\underset{\substack{\geq \text { gravel } \\ \text { sand }}}{ }$
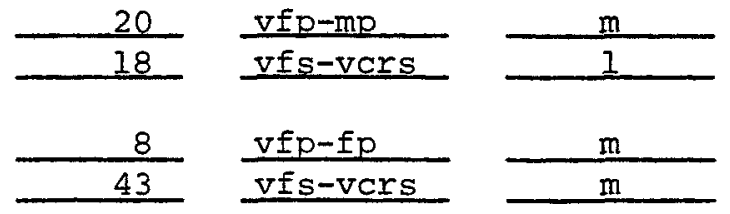

$\frac{\mathrm{sb} \text { rnd }}{\mathrm{sb} \text { rnd }}$

OTHER Igravel sand 11

SILT AND CLAY

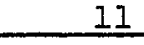

REMARKS
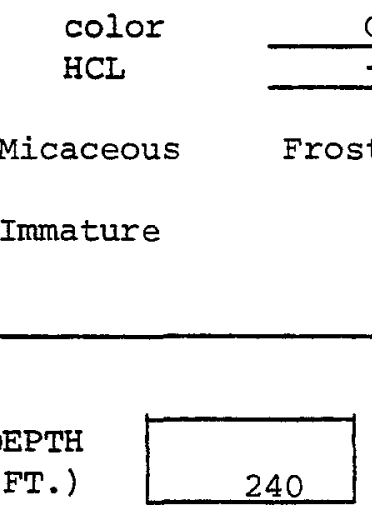

Greyish Brown

Micaceous

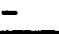

Immature

DEPTH

(FT.)

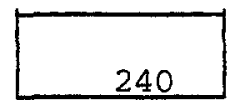

TO

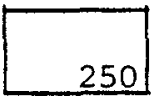

ROCK CLASSIFICATION

silty sandy Gravel

\section{\% SIZE RANGE SPHERICITY ROUNDNESS}

BASAIT

$$
\begin{aligned}
& \geq \text { gravel } \\
& \text { sand }
\end{aligned}
$$

OTHER $\geq$ gravel sand
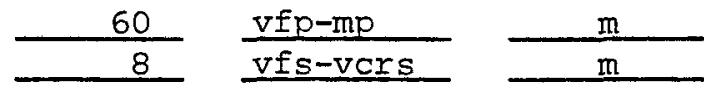

rnd
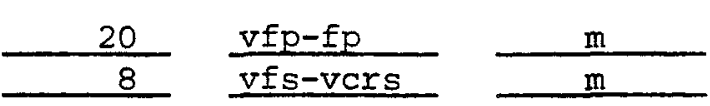

rnd sb rnd

SILT AND CLAY

\section{4}

REMARKS

color

HCL

Brownish Grey

Micaceous

Frosted Quartz

Immature 


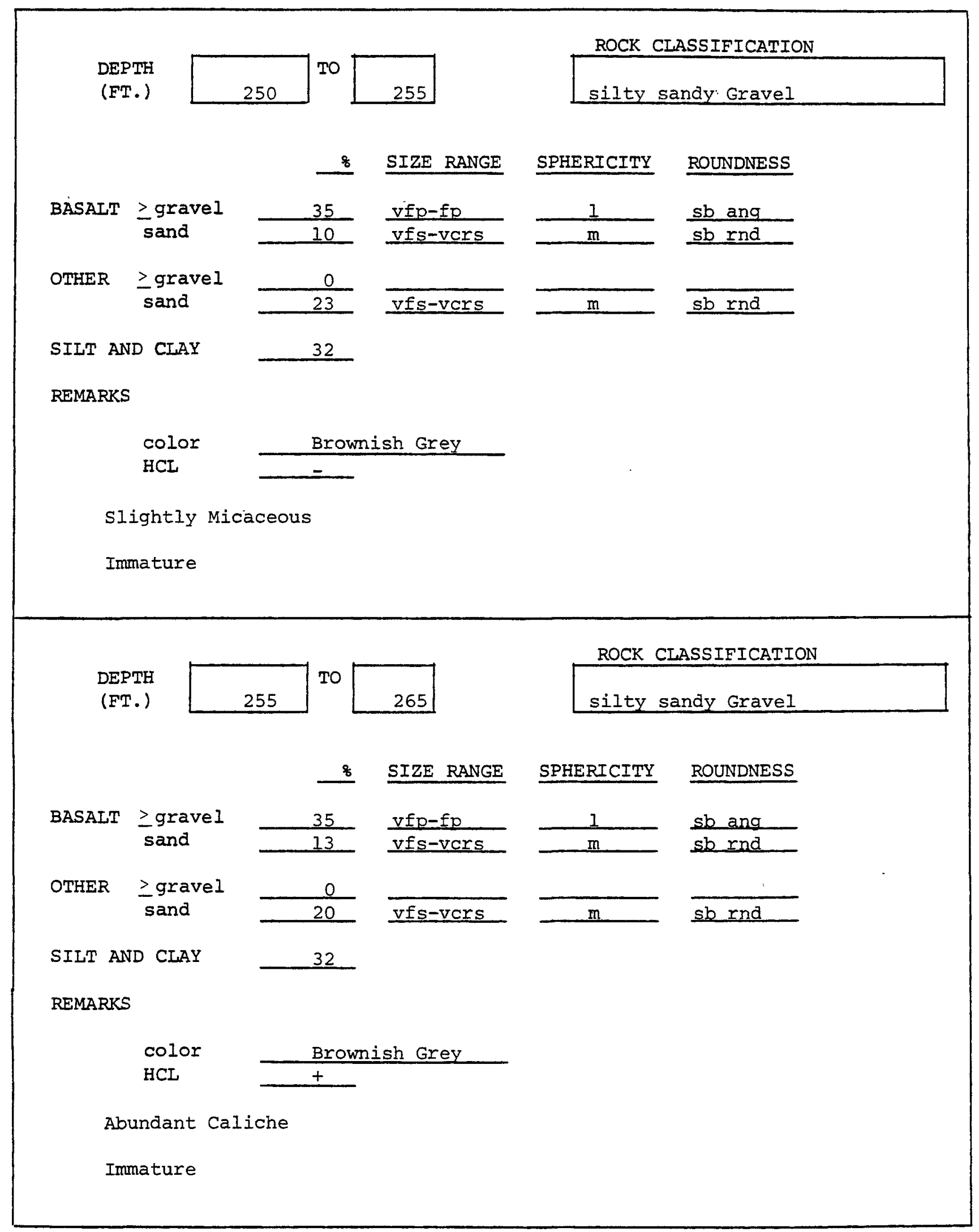




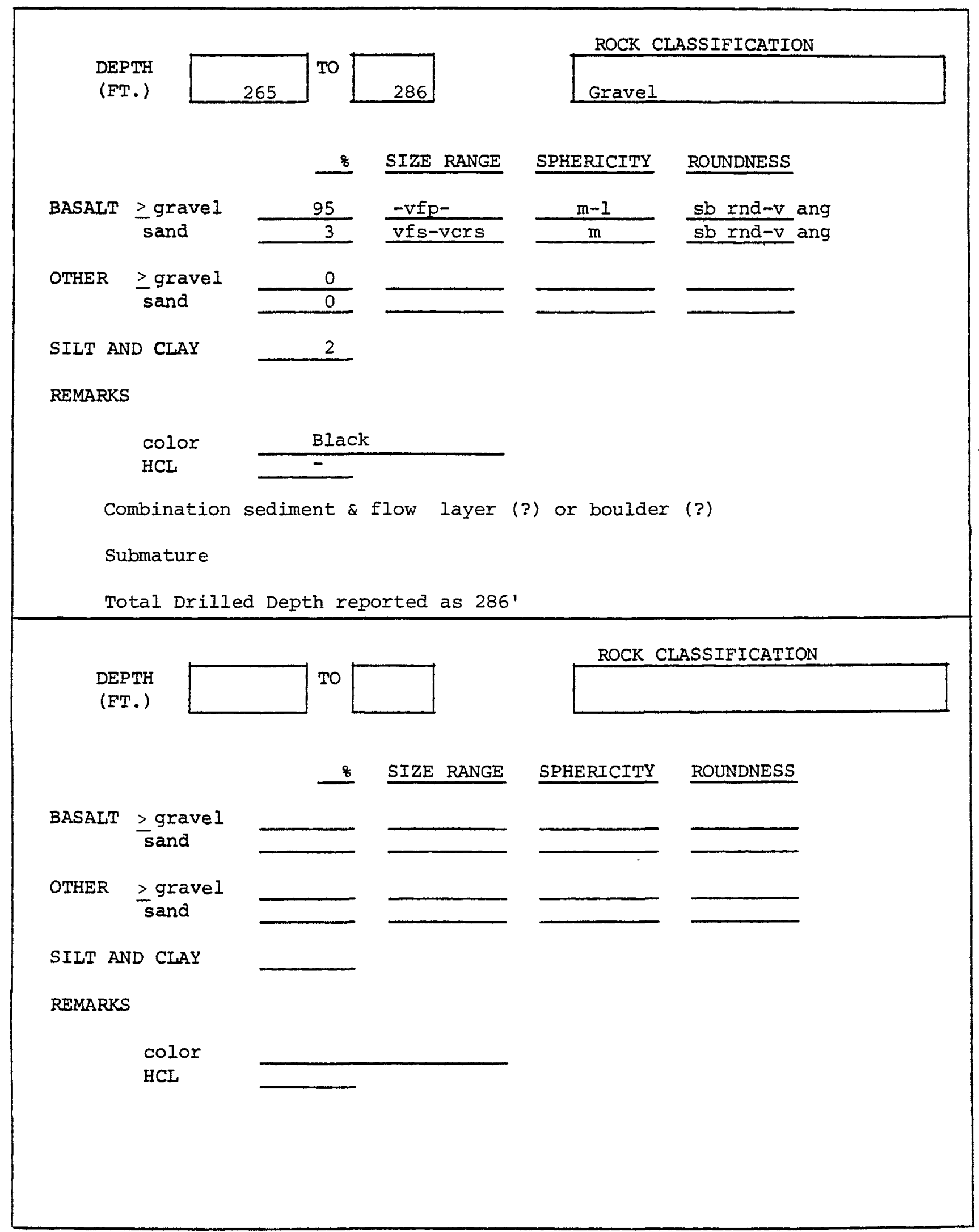




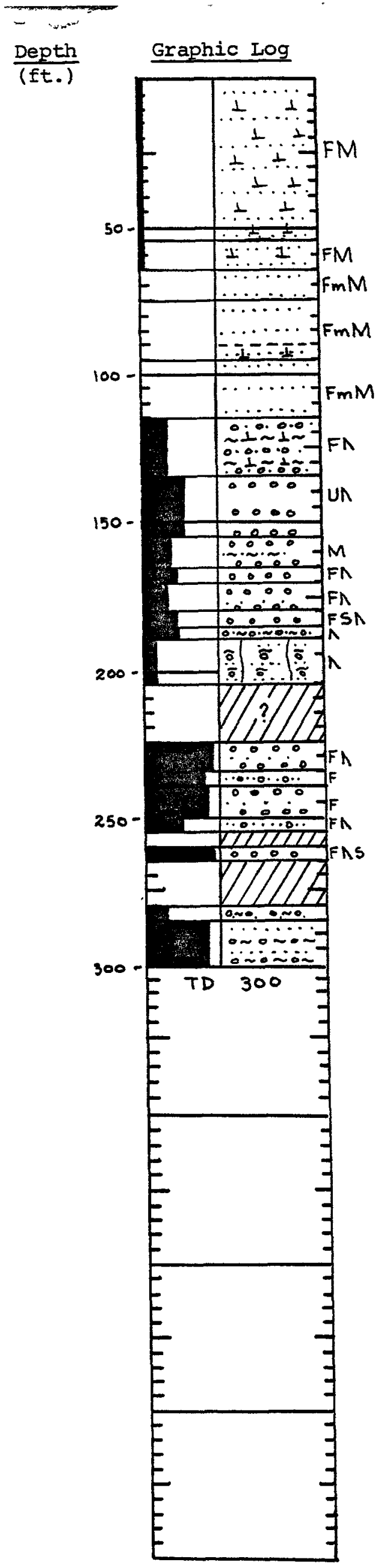

Sample Description

brownish buff -buff

brownish buff buff

brownish buff

brownish buff-buff

greyish buff

buff grey

buff brown

greyish brown

greyish brown

greyish black

buff brown

brown

abundant scoriaceous

black; abundant scoriaceous

greyish black; ASH

greyish black

greyish buff

greyish black

brownish black

brownish black
Plant Coordinates

$N 018953$

$W 058260$

Altitude 571

Other Data used in

绻

Interpretation

10

$1 \frac{1}{6}$

Driller's Log

$\lim _{\infty}^{1}$

W. K. Summers \& Associates 


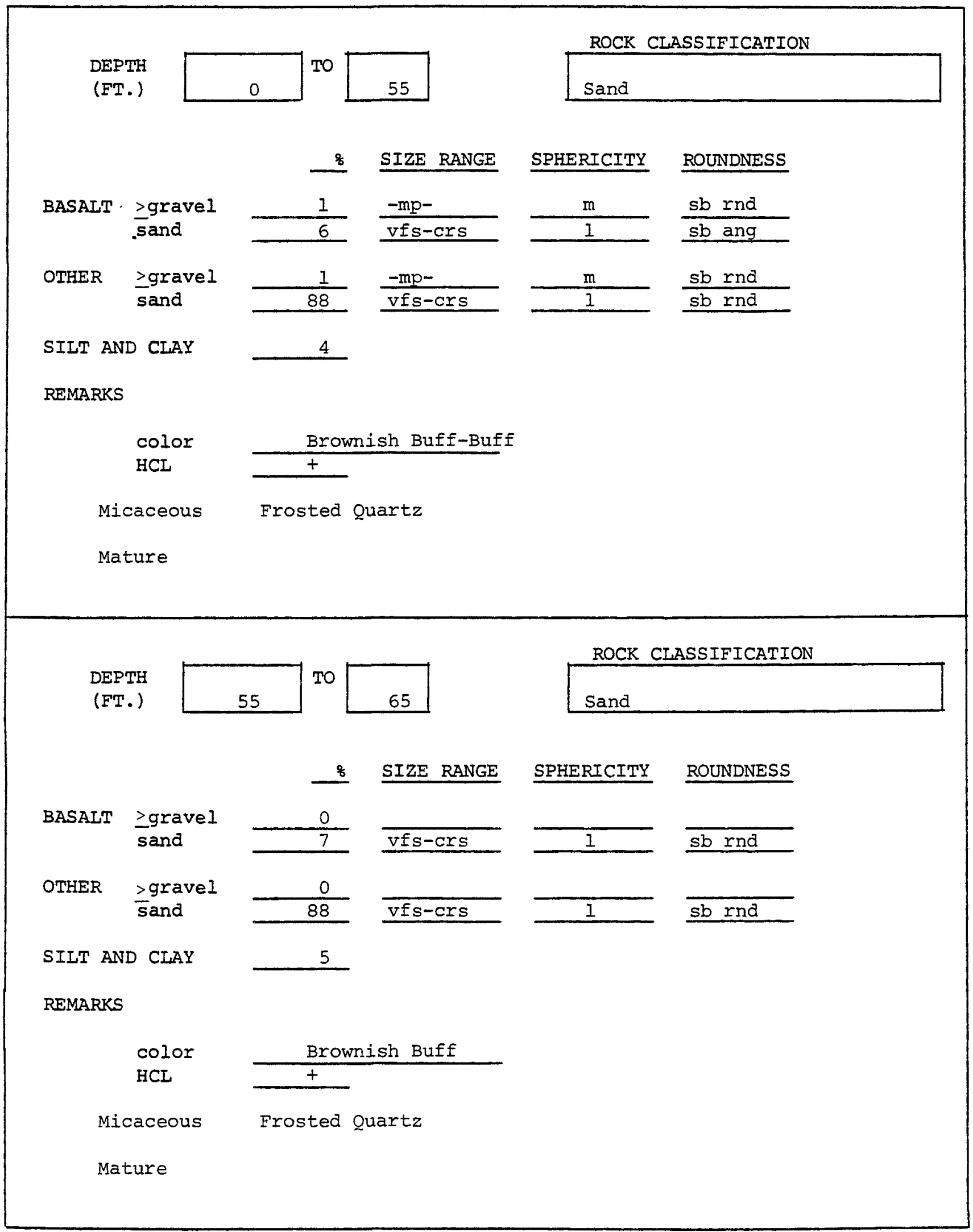




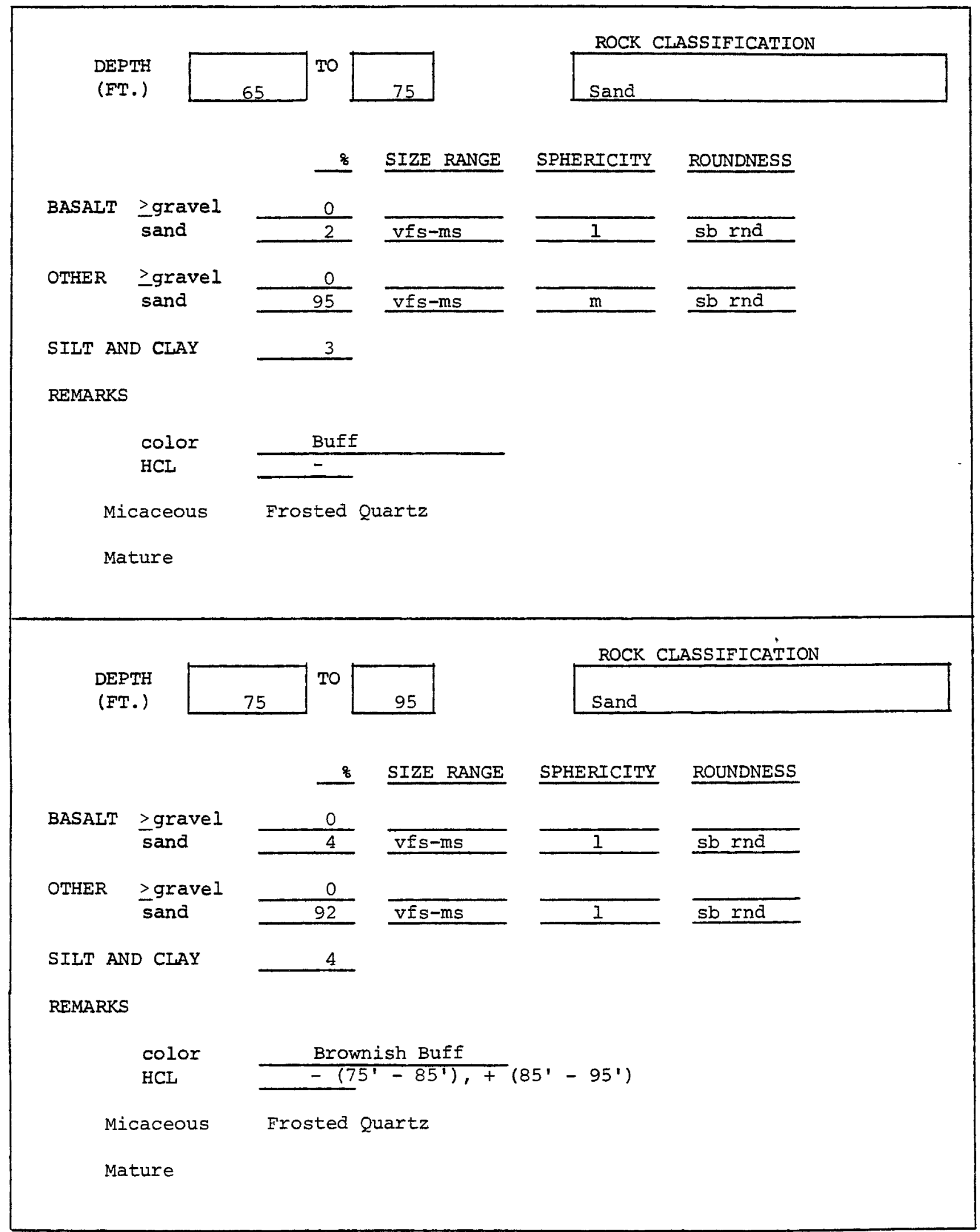




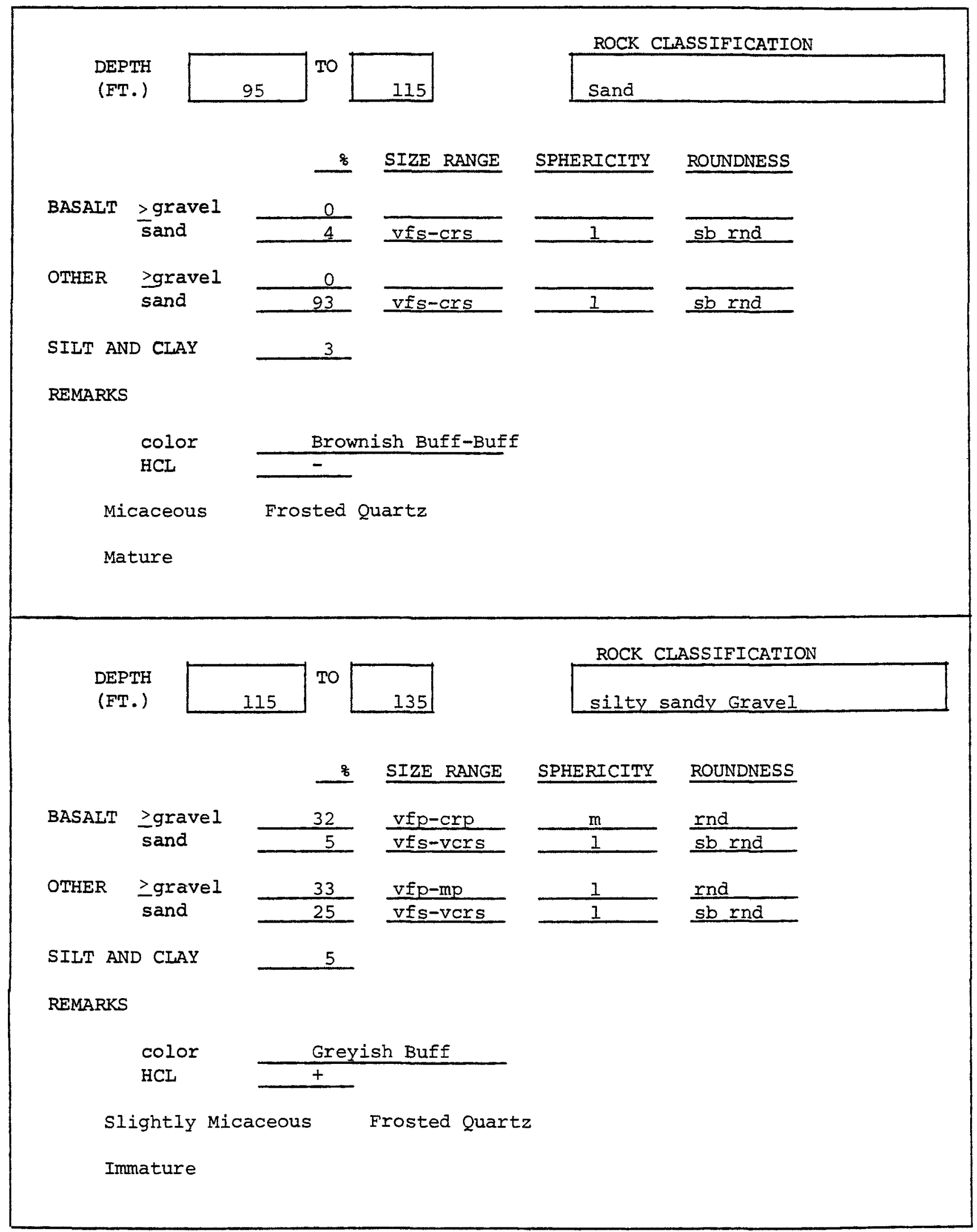




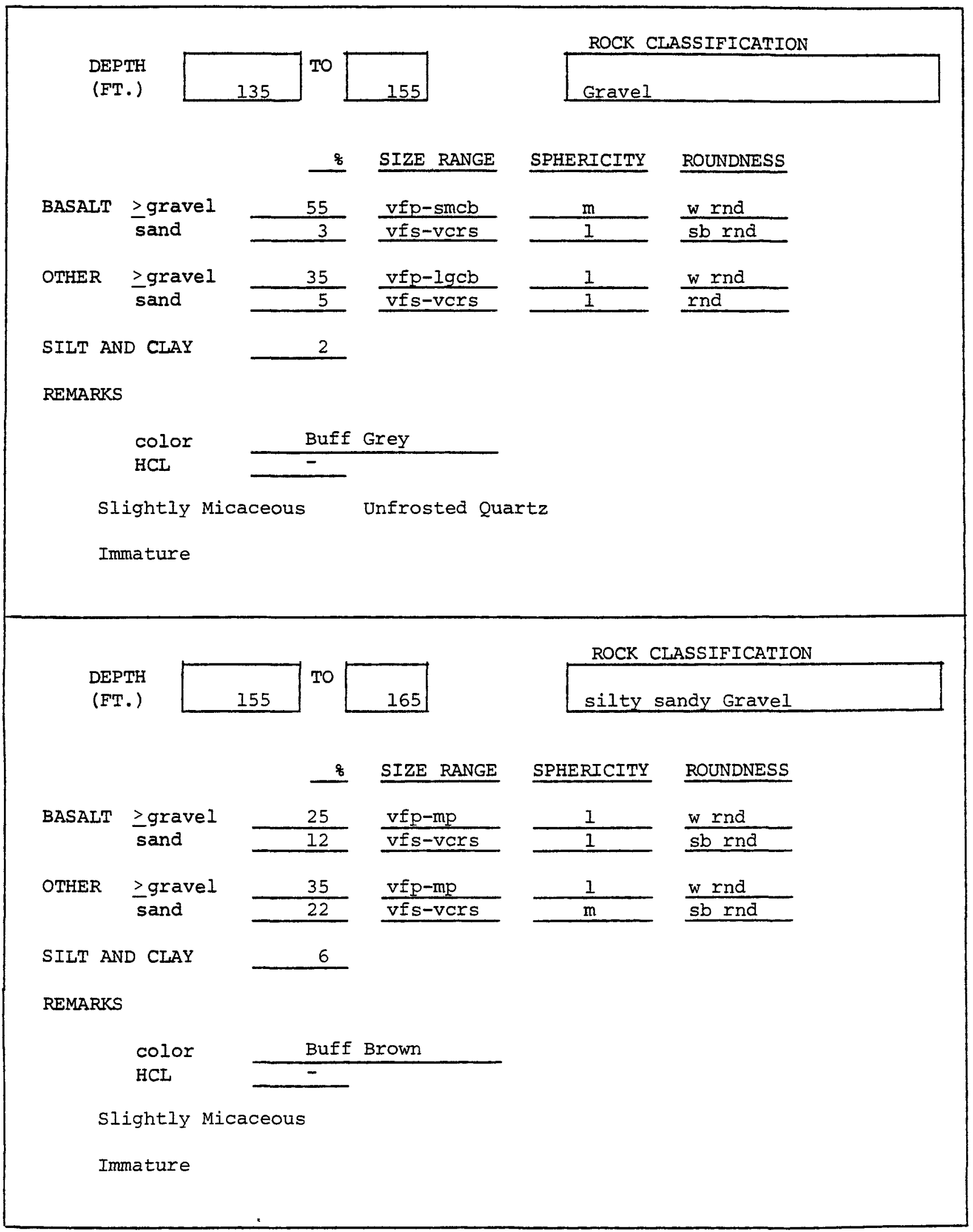


DEPTH

(FT.)

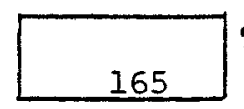

TO

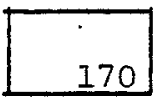

\section{ROCK CIASSIFICATION}

Gravel

\section{\% SIZE RANGE SPHERICITY ROUNDNESS}

BASALT >gravel sand

OTHER $\geq$ gravel sand

SIIT AND CLAY

REMARKS

$\frac{1}{1}$

w rnd
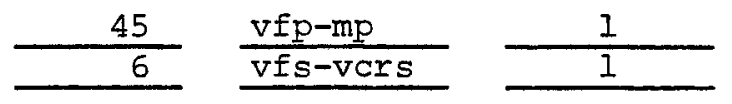

w rnd

1

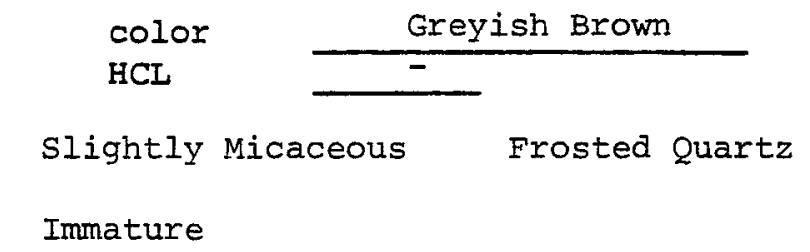

DEPTH

(FT.)

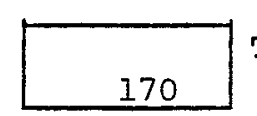

TO

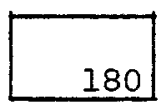

ROCK CIASSIFICATION

sandy Gravel

\& SIZE RANGE SPHERICITY ROUNDNESS

\begin{tabular}{|c|c|c|c|c|c|}
\hline BASAIT & $\begin{array}{l}\text { zgravel } \\
\text { sand }\end{array}$ & $\frac{20}{11}$ & $\frac{v f p-c r p}{v f s-v c r s}$ & $\frac{1}{1}$ & $\frac{w \text { rnd }}{\text { sb rnd }}$ \\
\hline OTHER & $\begin{array}{l}\text { >gravel } \\
\text { sand }\end{array}$ & $\frac{25}{41}$ & $\frac{v f p-c r p}{v f s-v c r s}$ & $\frac{1}{m}$ & $\frac{w \text { rnd }}{s b \text { rnd }}$ \\
\hline
\end{tabular}

SILT AND CIAY 3

REMARKS

\begin{tabular}{lll} 
HCL & \multicolumn{1}{c}{ Greyish Brown } \\
Slightly Micaceous $\quad$ Frosted Quartz
\end{tabular}




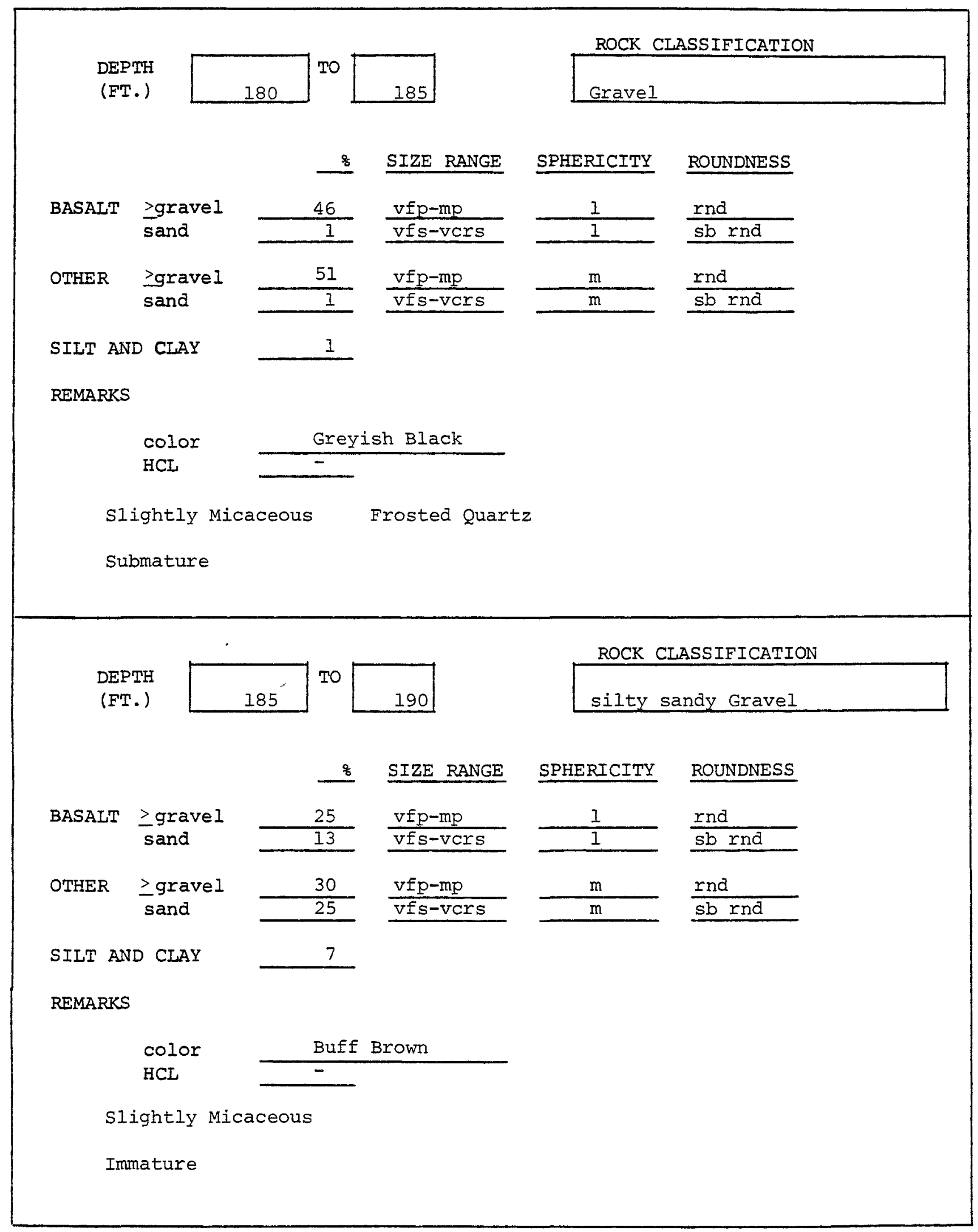




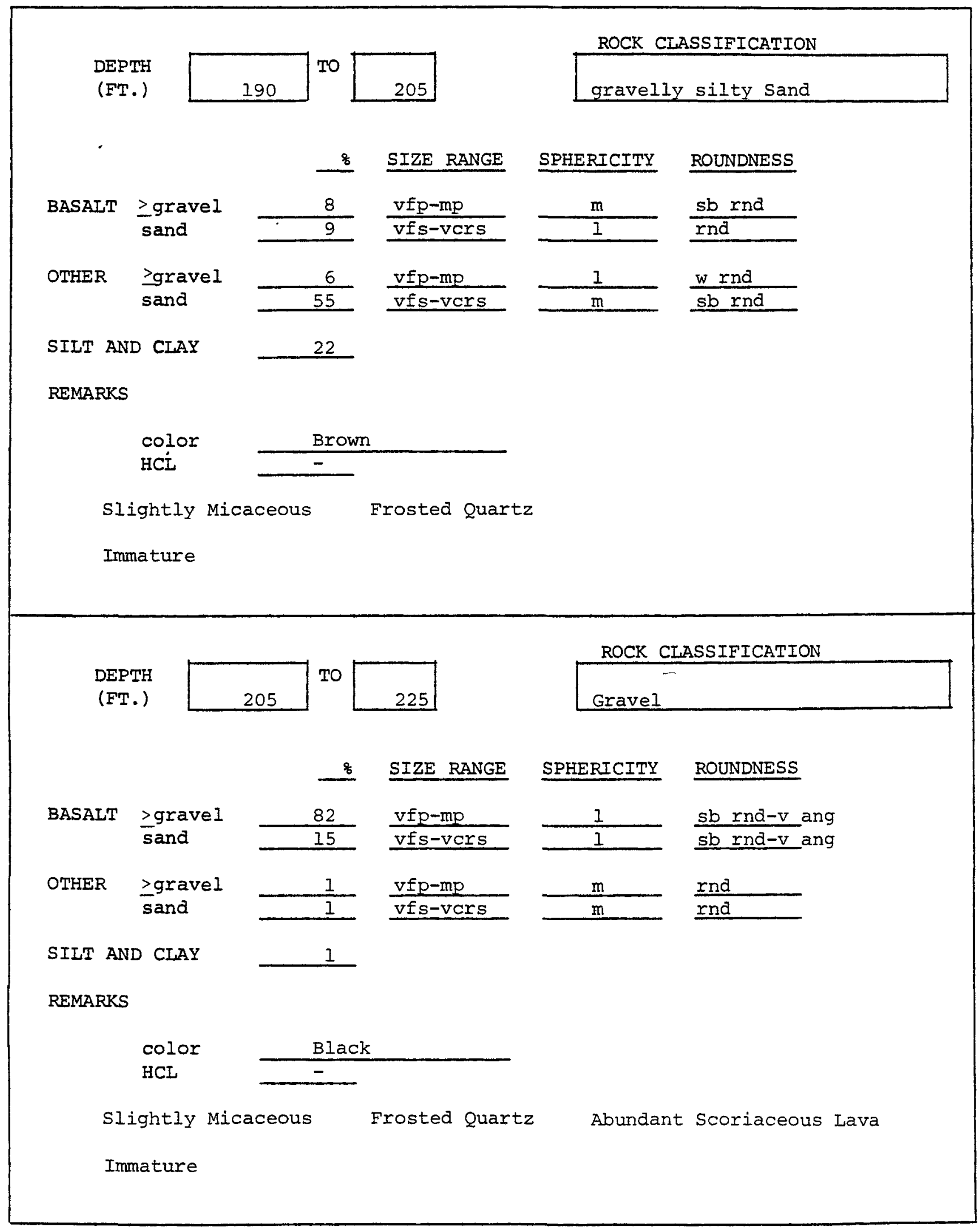

W. K. Summers \& Associates 


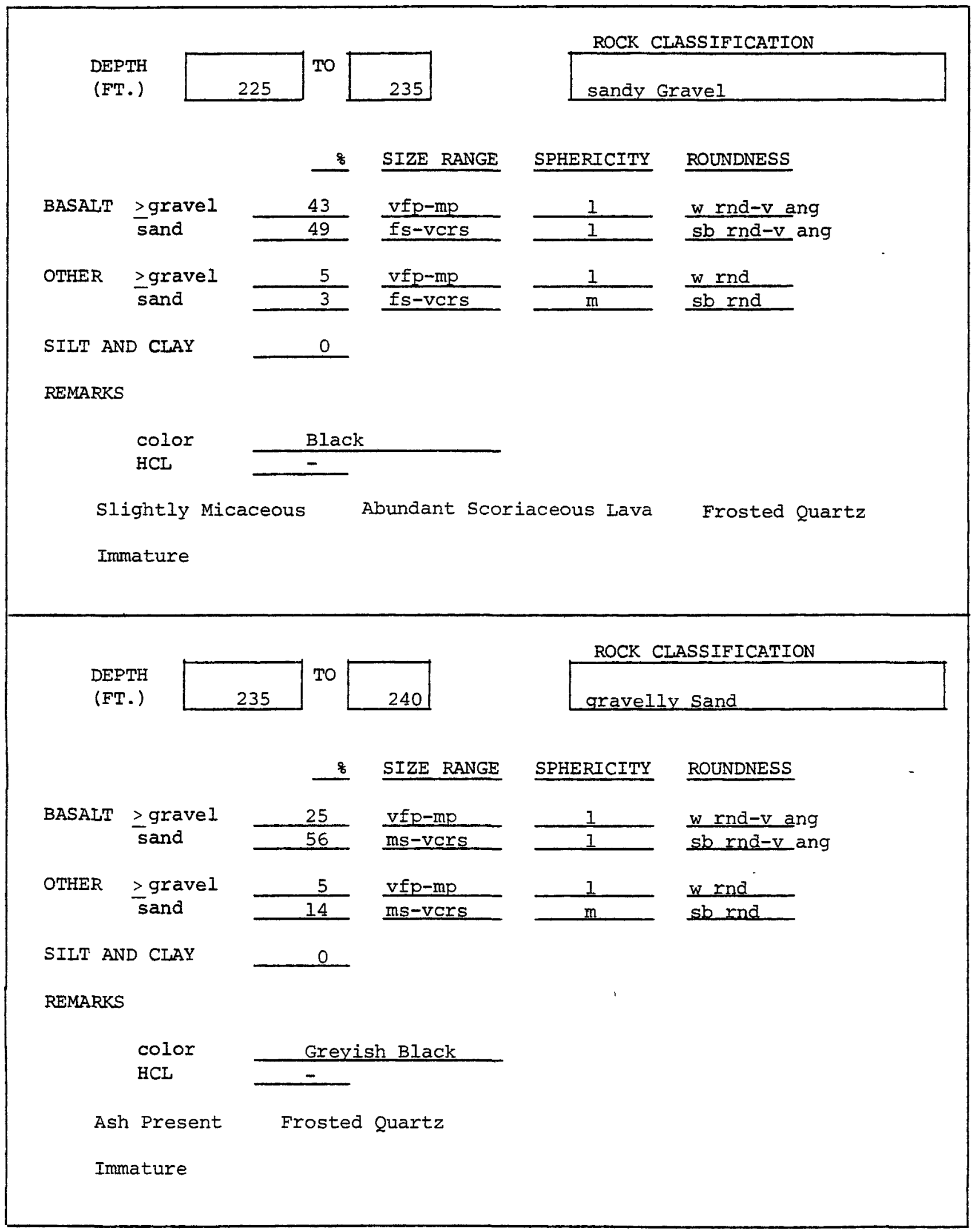




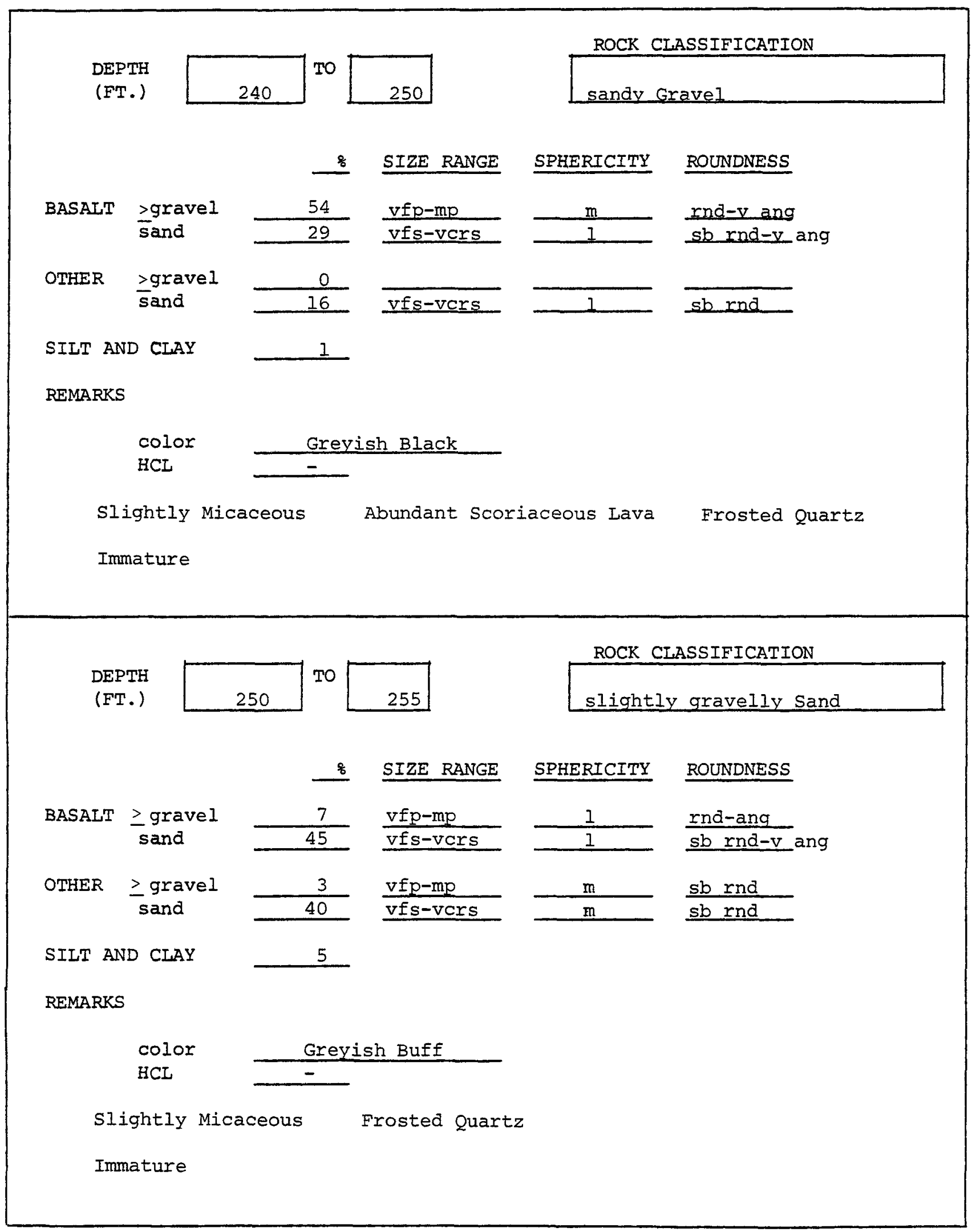




\section{DEPTH}

(FT.)

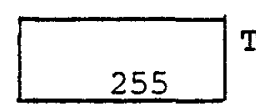

TO

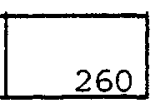

ROCK CLASSIEICATION

Flow Iayer

\section{\% SIZE RANGE SPHERICITY ROUNDNESS}

BASALT $\geq$ gravel sand
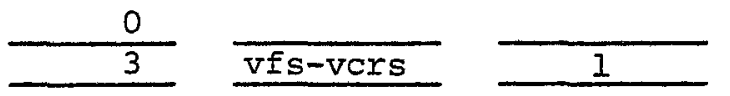

$\overline{\text { sb rnd-v }}$ ang

OTHER $\geq$ gravel sand
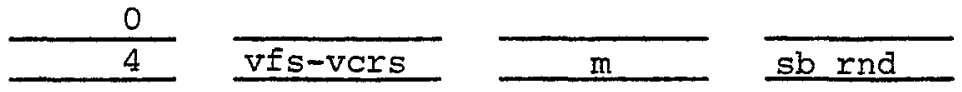

SILT AND CLAY 1

REMARKS

color

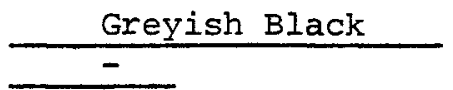

Slightly Micaceous Scoriaceous Flow Layer with sediments (92\%)

Frosted Quartz

DEPTH

(FT.)

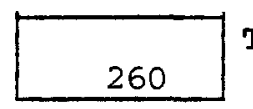

TO

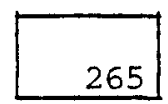

ROCK CLASSIFICATION

Gravel

\section{\% SIZE RANGE SPHERICITY ROUNDNESS}

BASAIT

$$
\begin{aligned}
& \geq \text { gravel } \\
& \text { sand }
\end{aligned}
$$
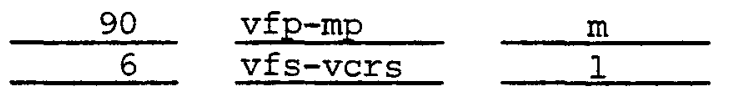

sb ang-ang

sb rnd-v ang

OTHER Igravel
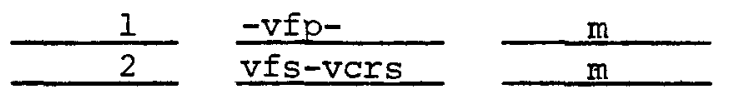

sb rnd

sb rnd

\section{SIIT AND CLAY}

1

REMARKS

$$
\underset{\mathrm{HCL}}{\operatorname{col}} \stackrel{\text { Greyish Black }}{\text { Slightly Micaceous }} \text { Frosted Quartz }
$$

Submature 

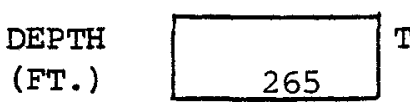

TO

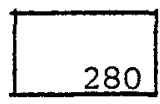

\% SIZE RANGE

\section{SPHERICITY ROUNDNESS}

BASAIT $\frac{\geq \text { gravel }}{\text { sand }}$
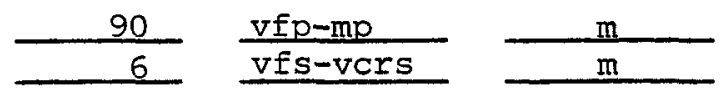

sb ang-ang

OTHER $\quad$ gravel sand
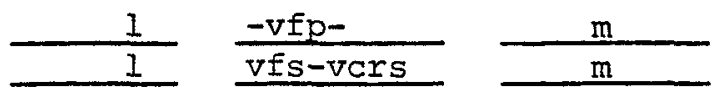

SILT AND CIAY

2

REMARKS

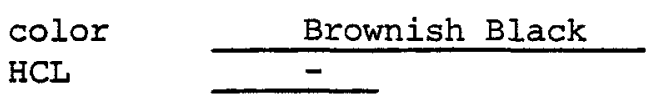

Frosted Quartz

Immature
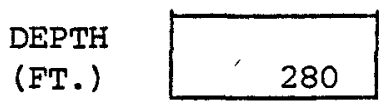

TO

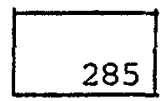

ROCK CLASSIFICATION

slightly silty sandy Gravel

\% SIZE RANGE SPHERICITY ROUNDNESS BASALT $\underset{\text { sand }}{\text { savel }}$
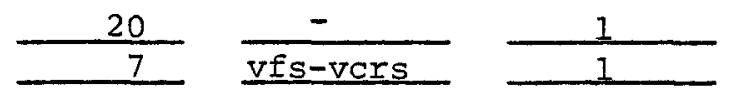

sb rnd-vang

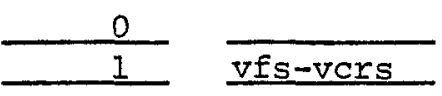

sb rad $-v$ ang

OTHER $\geq$ gravel sand

2

\section{SILT AND CIAAY}

REMARKS

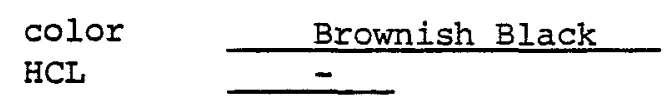

$70 \%$ Vesicular Flow Layer with sediments

Immature 
DEPTH
(ET.)

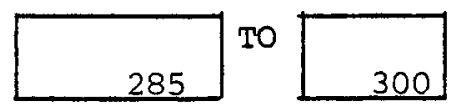

\% SIZE RANGE SPHERICITY ROUNDNESS

BASAIT > gravel sand
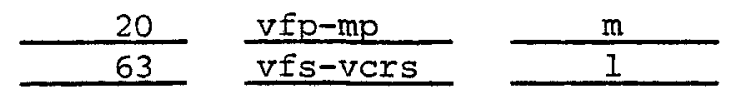

sb ang

sb rnd $-v$ ang

OTHER $\geq$ grave 1 sand

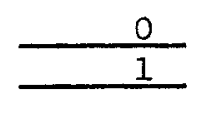

vfs-vers 16

SILT AND CIAY

REMARKS

$$
\begin{aligned}
& \text { color } \\
& \text { HCL }
\end{aligned}
$$

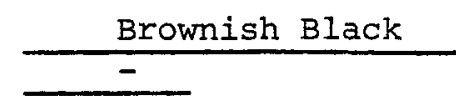

Immature

Total Drilled Depth reported as 300'
ROCK CLASSIFICATION

gravelly silty sand 
Depth

(ft.)

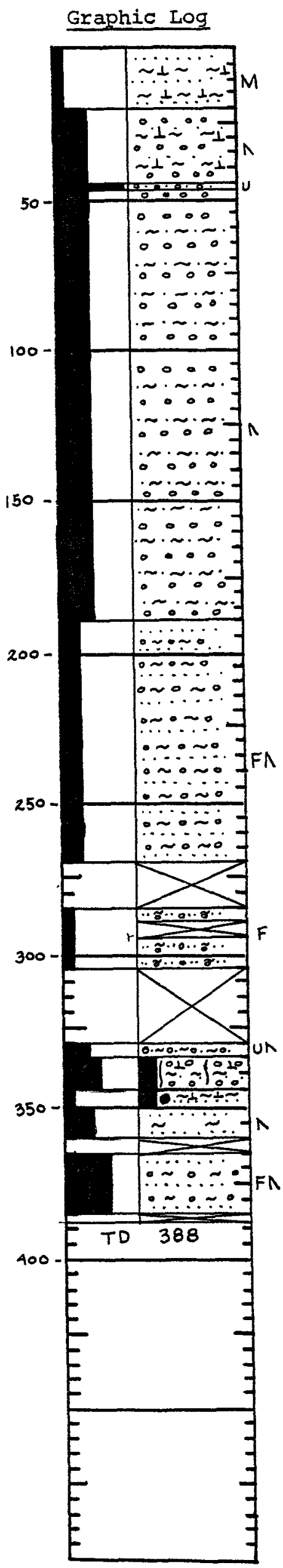

Sample Description

$\tan$

$\tan$

black

greyish $\tan$

brownish $\tan$

brownish $\tan$

$$
\begin{aligned}
& \text { brownish tan } \\
& \text { greyish tan } \\
& \text { black grey } \\
& \text { greyish tan } \\
& \text { greyish brown } \\
& \text { greyish brown }
\end{aligned}
$$

Plant Coordinates

$\mathrm{N} 019185$

$\frac{w 087736}{\text { Altitude } 642}$

Other Data used in Interpretation

$$
\text { Driller's Log }
$$


DEPTH

(ET.)

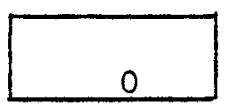

TO

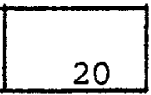

ROCK CLASSIFICATION

silty Sand

\% SIZE RANGE SPHERICITY ROUNDNESS

BASALT $\underset{\substack{\text { sand } \\ \text { gravel }}}{\text { gand }}$
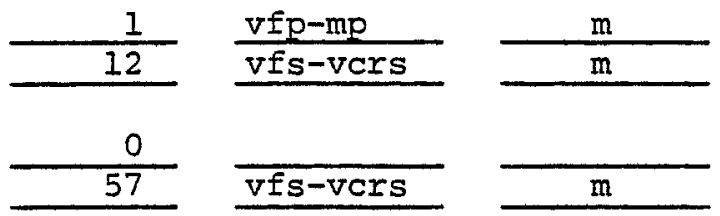

rnd

OTHER $\geq$ gravel

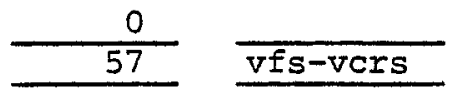

rnd

SIIT AND CLAY

30

REMARKS

$$
\text { color }
$$

$\mathrm{HCL}$

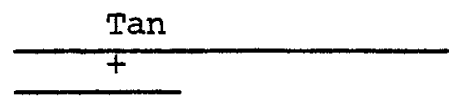

Micaceous

Immature

DEPTH

(FT.)

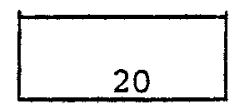

TO

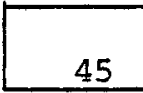

ROCK CIASSIFICATION

silty sandy Gravel

SIZE RANGE SPHERICITY ROUNDNESS

BASALT $\geq$ gravel
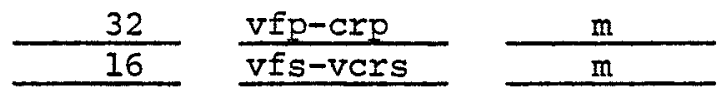

sb rnd rnd

OTHER $\underset{\text { sand }}{\geq \text { gravel }}$

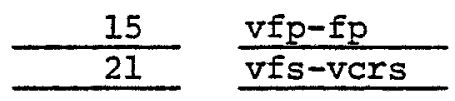

1

sb rnd

m rnd

SILT AND CLAY

16

REMARKS

color

$\frac{\operatorname{Tan}}{t}$

Slightly Micaceous

Immature 
DEPTH
(FT.)

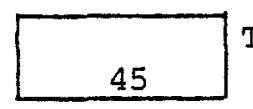

TO

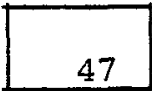

ROCK CLASSIFICATION

sandy Gravel Basalt-Flow

\section{S SIZE RANGE SPHERICITY ROUNDNESS}

BASALT > gravel sand

OTHER Igravel sand

SILT AND CLAY

REMARKS
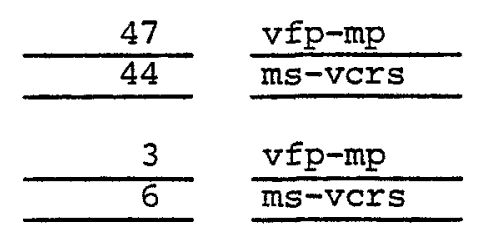

0

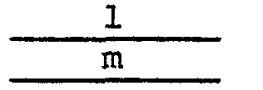

rnd-v ang

sb rnd-ang

$\frac{1}{h} \frac{\text { wrnd }}{\text { rnd }}$

color

HCL

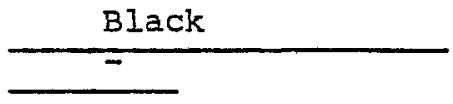

Unfrosted quartz

Immature

\section{DEPTH}

(FT.)

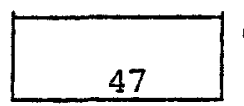

TO

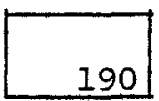

ROCK CLASSIFICATION

silty sandy Gravel

\section{\& SIZE RANGE SPHERICITY ROUNDNESS}

BASALT $\underset{\text { sand }}{ }$
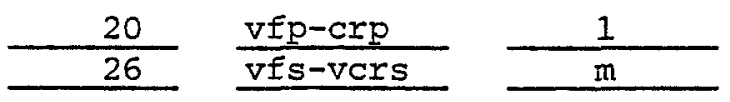

$\frac{\text { sb rnd }}{\text { sb rnd }}$

OTHER

zgravel

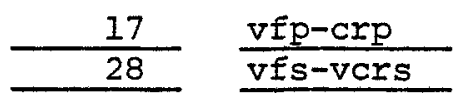

$\frac{1}{\mathrm{~m}}$

rnd sand

\section{9}

SILT AND CIAY

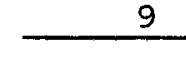

\section{REMARKS}

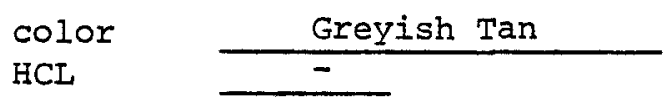

Slightly Micaceous

Immature 
ROCK CLASSIFICATION

DEPTH

(FT.)

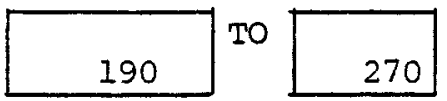

slightly silty gravelly sand

\% SIZE RANGE SPHERICITY ROUNDNESS

BASAIT $\frac{\geq \text { gravel }}{\text { sand }}$
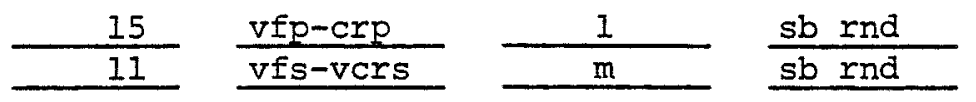

OTHER $\geq$ gravel

$\frac{15}{52} \frac{v f p-c r p}{v f s-v c r s}$

$\frac{1}{m} \frac{\text { sb rnd }}{\text { rnd }}$

SILT AND CLAY

7

REMARKS

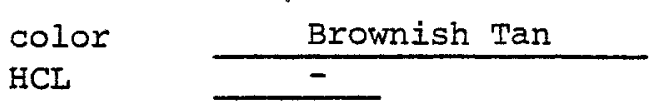

Slightly Micaceous Frosted Quartz

Immature

DEPTH
(FT.)

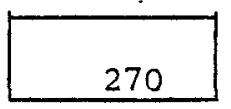

TO

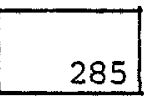

ROCK CIASSIFICATION

$\%$ SIZE RANGE SPHERICITY ROUNDNESS

BASALT $\underset{\text { sand }}{\text { savel }}$

OTHER $\geq$ gravel sand

SILT AND CLAY

REMARKS

color

HCL

Sample missing 


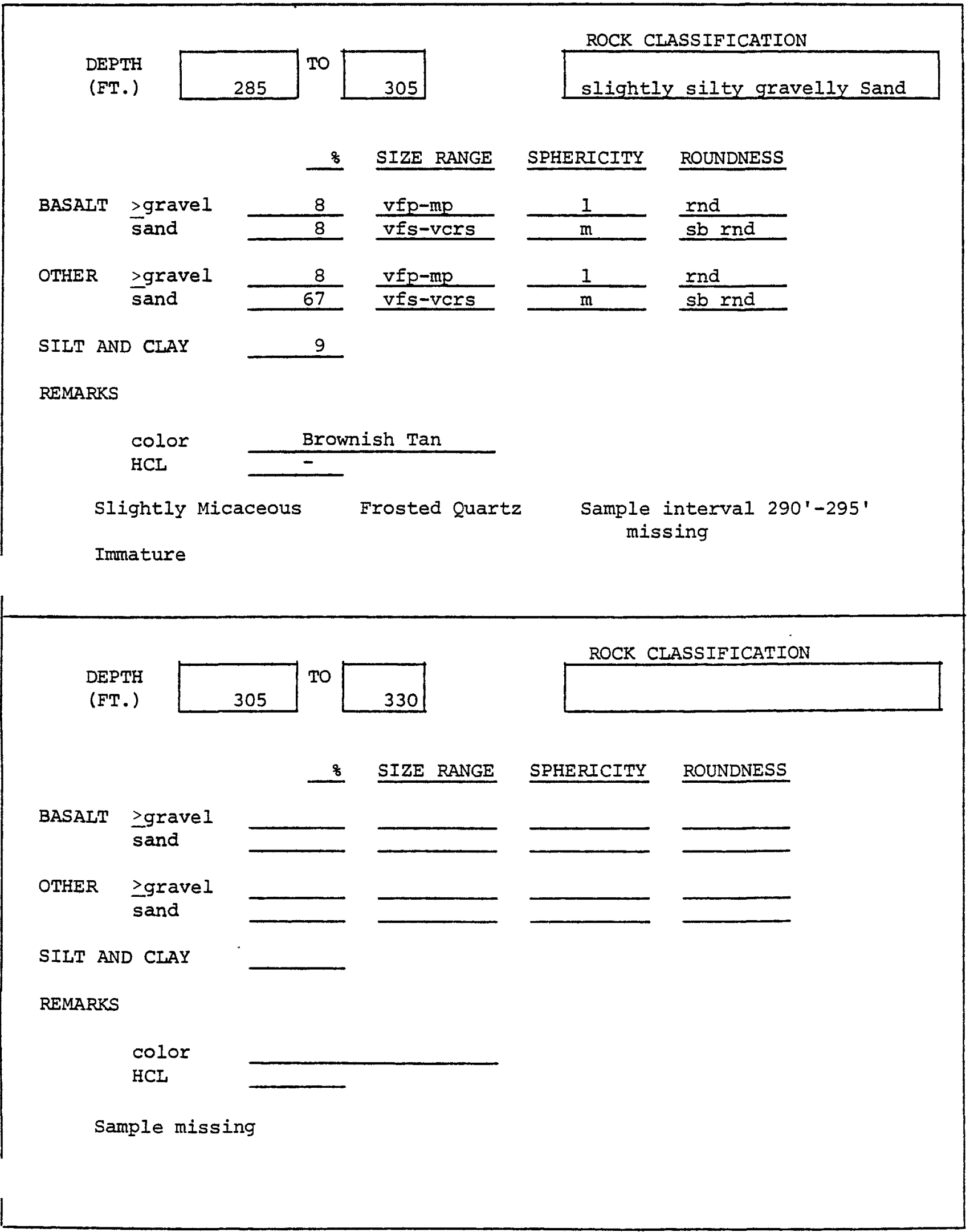


DEPTH

(FT.)

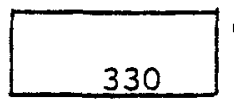

TO

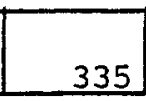

ROCK CLASSIFICATION

silty sandy Grave1

\section{\% SIZE RANGE SPHERICITY ROUNDNESS}

BASALT $\underset{\substack{\geq \text { gravel } \\ \text { sand }}}{ }$
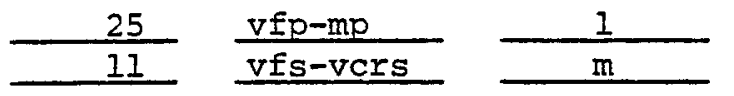

$\frac{w \text { rnd }}{\text { sb rnd }}$

OTHER $\geq$ gravel sand
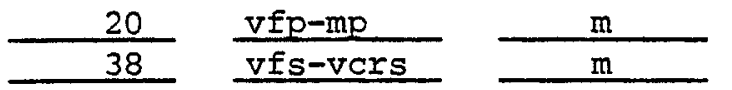

w rnd

rnd

SIIT AND CLAY 6

REMARKS

$$
\begin{aligned}
& \text { color } \\
& \text { HCL }
\end{aligned}
$$

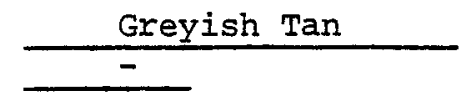

Slightly Micaceous

Unfrosted Quartz

Immature

(FT.)

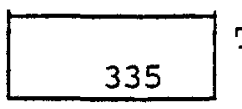

TO

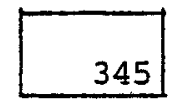

ROCK CLASSIFICATION

silty sandy Gravel

\% SIZE RANGE SPHERICITY ROUNDNESS

BASAIT $\geq$ gravel
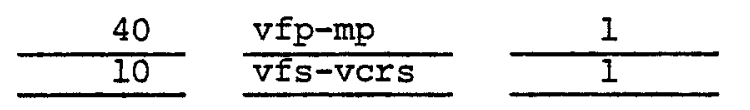

w rnd

sand
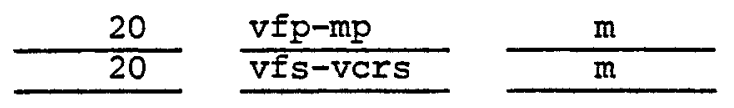

sb rnd

OTHER Igravel sand 10

SILT AND CLAY

10

REMARKS

$$
\begin{aligned}
& \text { color } \\
& \text { HCL }
\end{aligned}
$$

Blackish Grey

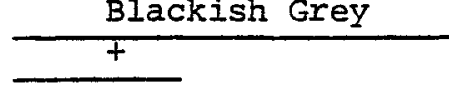

Abundant Caliche (30\%, fragments)

Immature 


\section{DEPTH}

(FT.)

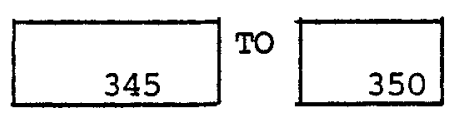

ROCK CLASSIFICATION

silty Sand

\section{\% SIZE RANGE SPHERICITY ROUNDNESS}

BASALT $\underset{\text { gravel }}{\text { sand }}$
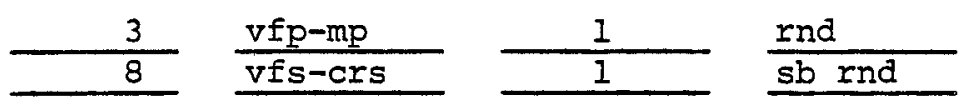

OTHER >graveI sand
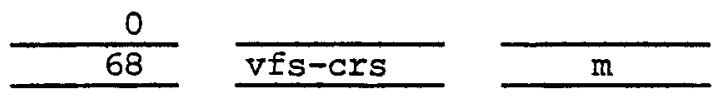

rnd

SILT AND CLAY

\section{$2 I$}

REMARKS

$$
\text { color }
$$

HCL

Greyish Tan

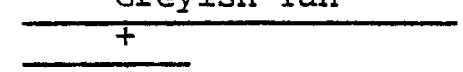

Caliche (pebbles \& sand present)

Imnature

DEPTH

(FT.)

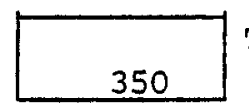

TO

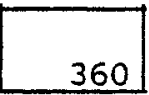

ROCK CLASSIFICATION

slightly silty sand

\% SIZE RANGE SPHERICITY ROUNDNESS

BASALT >gravel sand

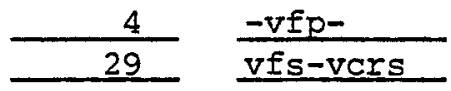

OTHER $\geq$ gravel sand
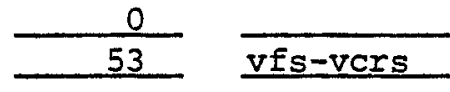

SIIT AND CLAY 14

REMARKS

color Greyish Brown

HCL

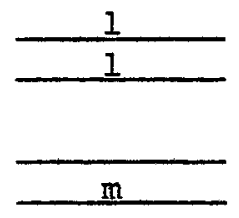

$\frac{\text { sb and }}{\text { sb rnd }}$

Ind

Slightly Micaceous

Immature 


\section{DEPTH}

(ET.)

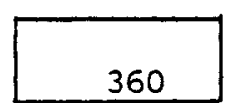

TO

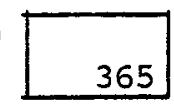

ROCK CLASSIFICATION

\section{\% SIZE RANGE SPHERICITY ROUNDNESS}

BASAIT $\underset{\text { sand }}{>\text { gravel }}$

OTHER >gravel sand

SILT AND CIAY

REMARKS

$$
\text { color }
$$$$
\text { HCL }
$$

Sample missing
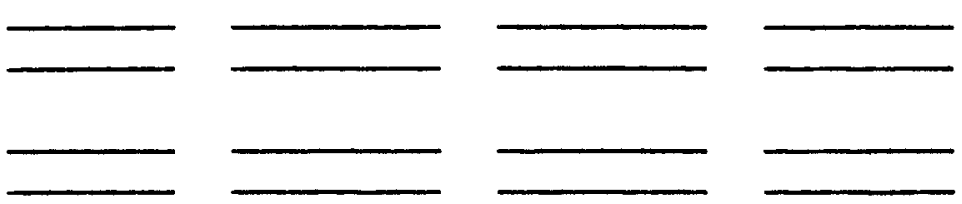

REMARKS

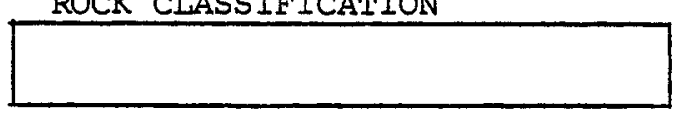


ROCK CLASSIFICATION

DEPTH

(ET.)

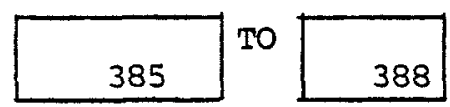

\% SIZE RANGE SPHERICITY ROUNDNESS

BASALT >gravel sand

OTHER $\geq$ gravel sand

SILT AND CIAY

REMARKS

$$
\text { color }
$$$$
\mathrm{HCL}
$$

Sample missing

Total Drilled Depth reported as $388^{\prime}$

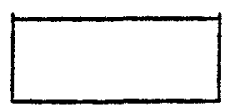

TO

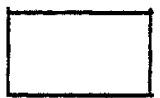

ROCK CLASSIEICATION

(FT.)

\& SIZE RANGE SPHERICITY ROUNDNESS

BASALT $\geq$ gravel sand

OTHER > gravel $\bar{s}$ and
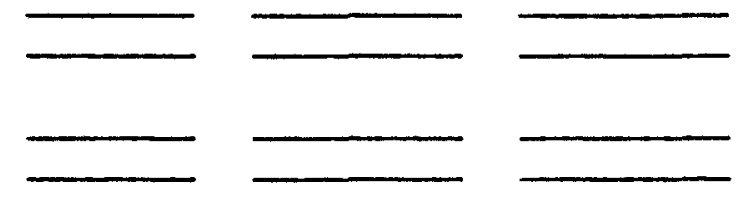

SIIT AND CIAY

REMARKS

color

HCL

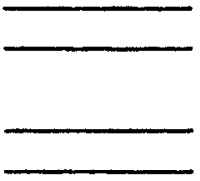




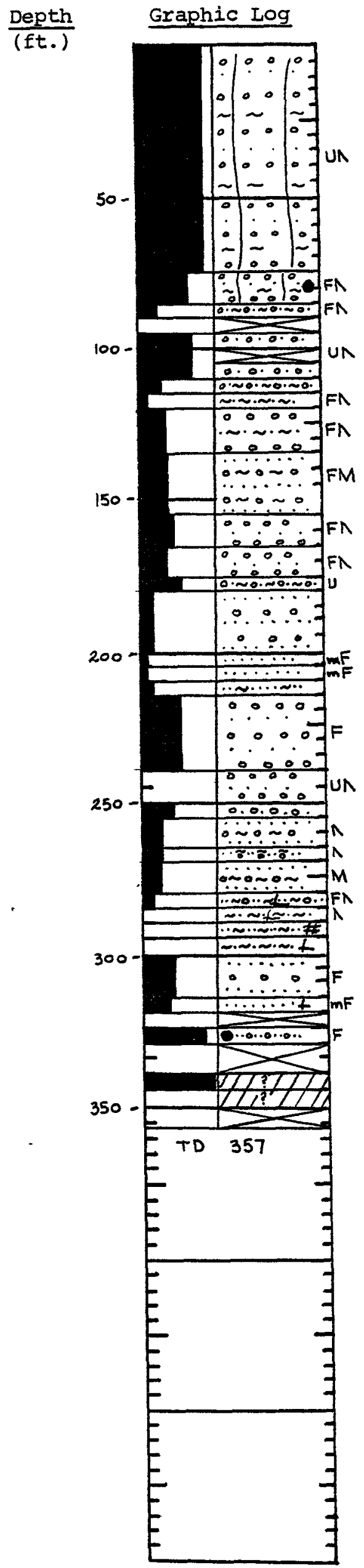

Sample Description

goiden butf

light brown

blackish grey

greyish white

greyish tan

light brown

greyish white

greyish buff

greyish $\tan$

greyish buff

buff white; TRACE DIATOMITE

buff: TRACE DIATOMITE

whitish buff; DIATOMITE

greysh

buff grey

greyish brown

greyish buff

greyish white

reddish brown

light brown

brownish grey

light bro

grey

greyish butf

greyish buff

black
Plant Coordinates

NO20304

$\frac{E 012017}{\text { Altitude } 435}$

Other Data used in Interpretation 


\section{DEPTH}

(FT.)

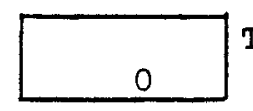

To

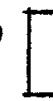

\section{5}

ROCK CLASSIFICATION

silty sandy Gravel

\section{SIZE RANGE SPHERICITY ROUNDNESS}

BASALT $\underset{\text { sand }}{\geq \text { gravel }}$
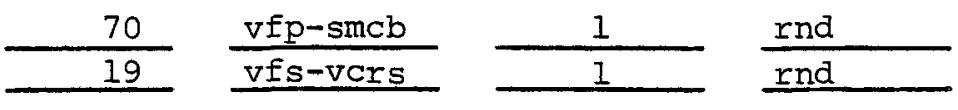

OTHER >gravel sand
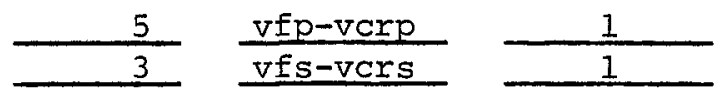

$\frac{w \text { rnd }}{\text { wrnd }}$

SILT AND CLAY 3

REMARKS

$$
\text { color }
$$

HCL

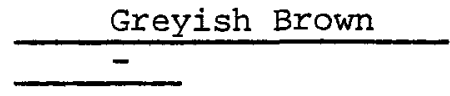

Slightly Micaceous

Unfrosted Quartz

Lithified by silt \& clay

- Immature

DEPTH

(FT.)

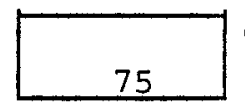

TO

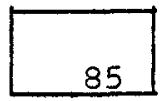

ROCK CLASSIFICATION

silty sandy Gravel

\section{\% SIZE RANGE SPHERICITY ROUNDNESS}

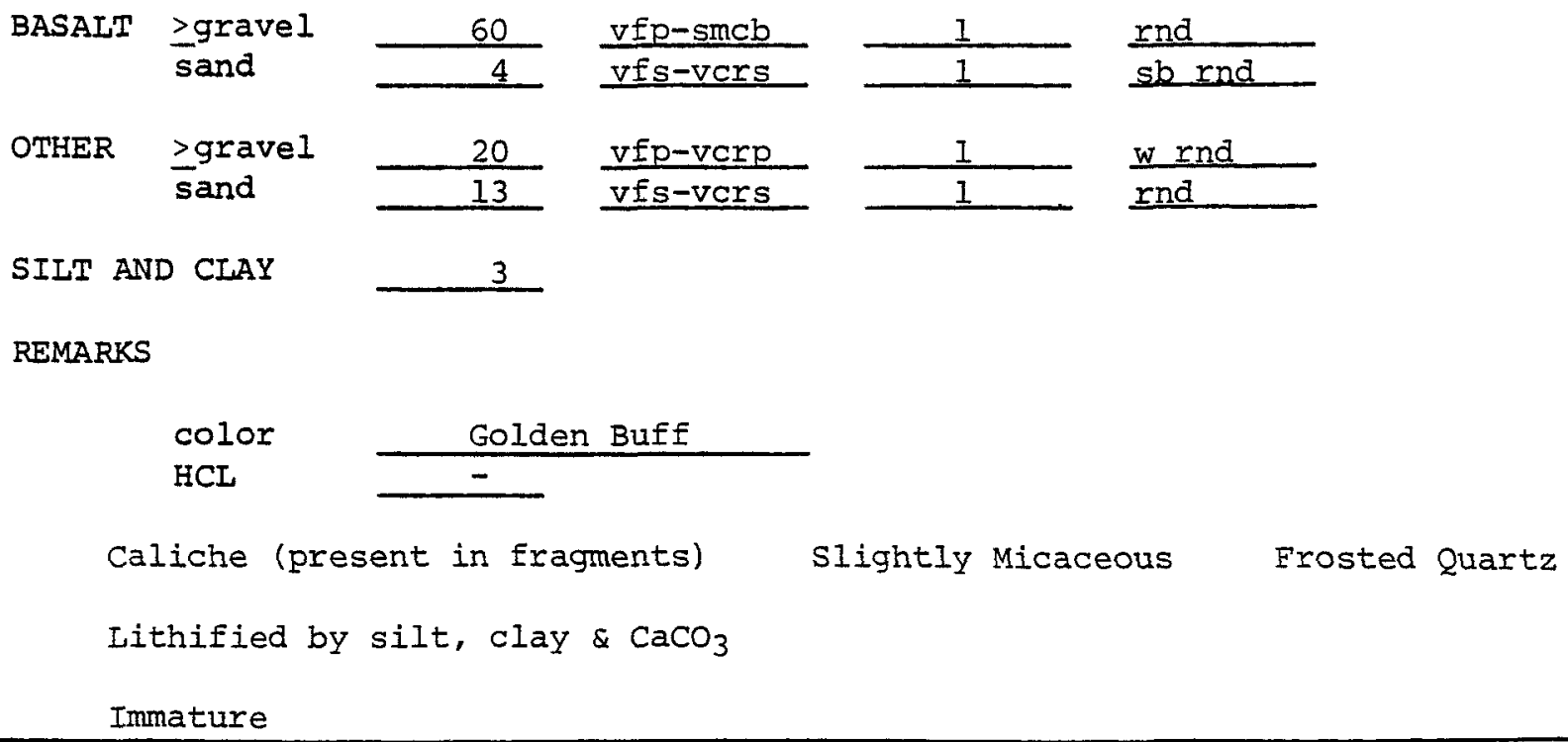

REMARKS

color Golden Buff

HCL

Caliche (present in fragments)

Iithified by silt, clay \& $\mathrm{CaCO}_{3}$

Immature 


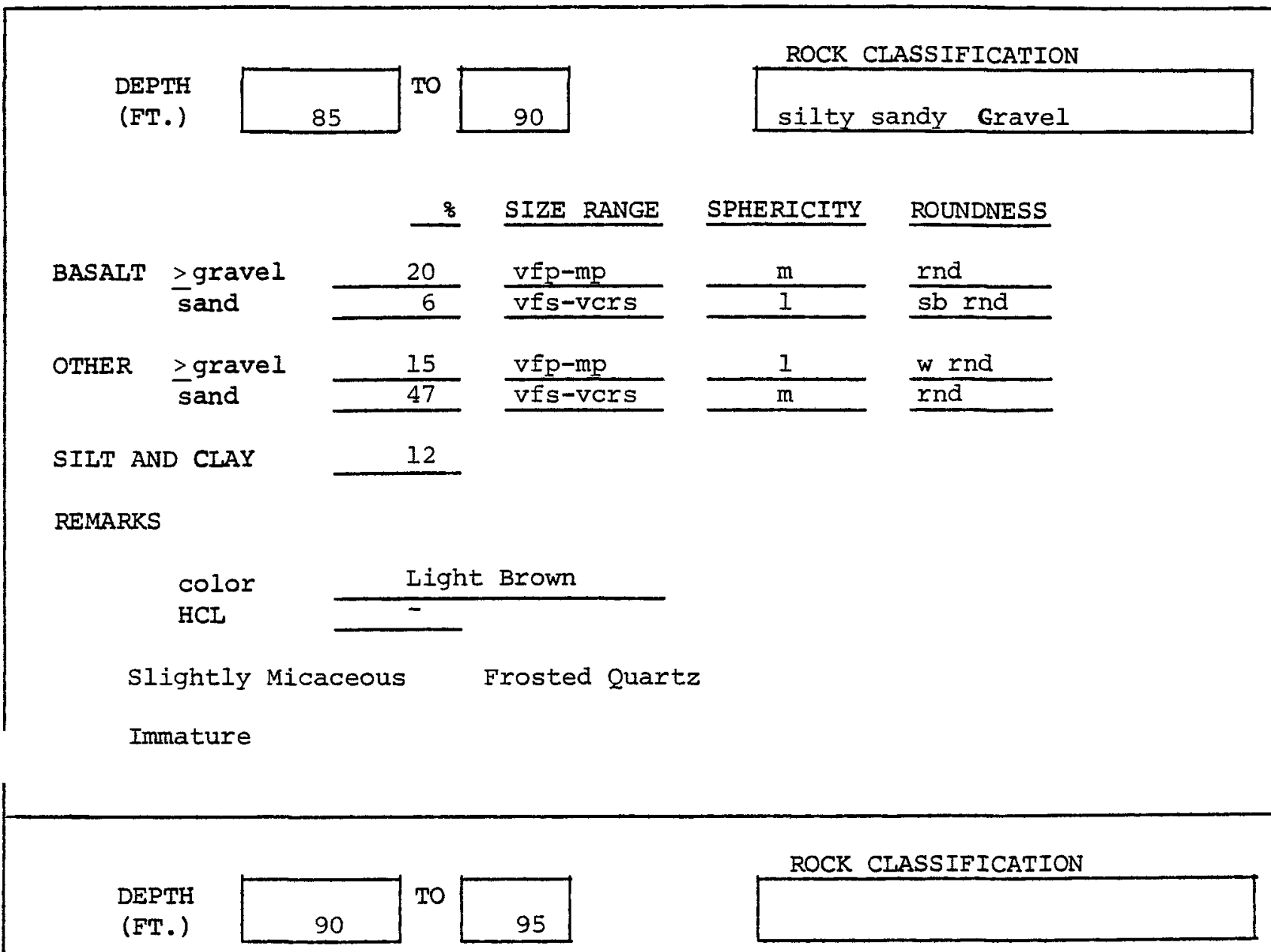

\% SIZE RANGE SPHERICITY ROUNDNESS

BASALT $\geq$ gravel

OTHER $\begin{aligned} & \geq \text { gravel } \\ & \text { sand }\end{aligned}$
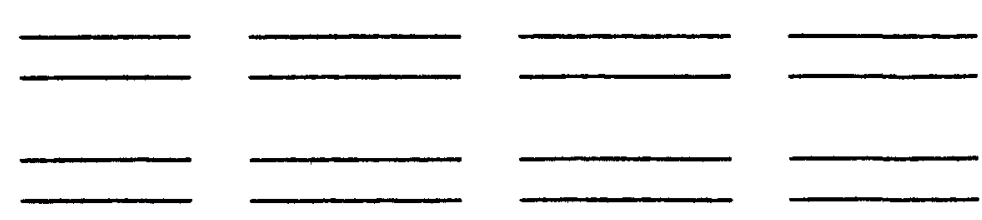

SILT AND CLAY

REMARKS

color

$\mathrm{HCL}$

Sample missing 
DEPTH

(FT.)

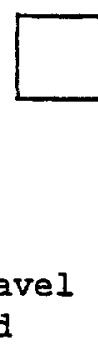

95

TO

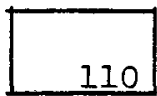
sand
ROCK CIASSIFICATION

sandy Gravel

\section{\% SIZE RANGE SPHERICITY ROUNDNESS}

BASALT > gravel
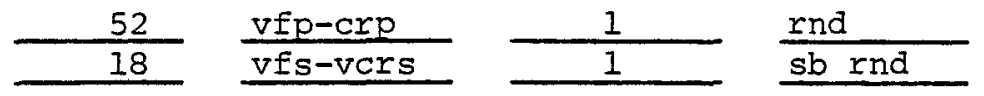

OTHER $\geq$ gravel
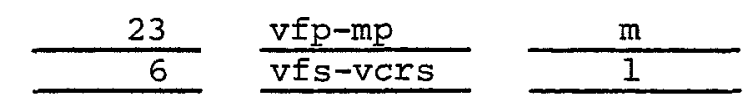

$\underline{\text { wnd }}$

SIIT AND CLAY 1

REMARKS

color

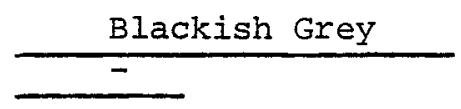

Slightly Micaceous Unfrosted quartz

Sample interval 100'-105' missing

Immature

DEPTH

(FT.)

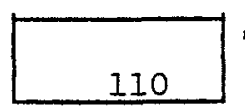

TO

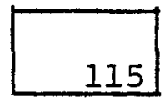

ROCK CLASSIFICATION

silty sandy Gravel

\section{\% SIZE RANGE SPHERICITY ROUNDNESS}

BASALT $\frac{\text { sravel }}{\text { sand }}$
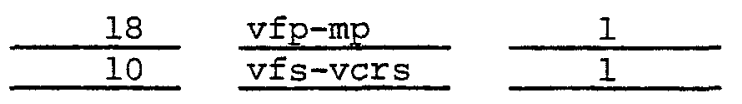

rnd

$\frac{18}{32} \frac{\text { vfp-mp }}{\text { vfs-vers }}$

$\frac{1}{m}$

OTHER $\geq$ gravel sand

SILT AND CLAY

22

REMARKS

color

HCL Greyish White $-$

Immature 
DEPTH
(FT.)

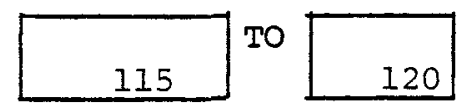

\& SIZE RANGE SPHERICITY ROUNDNESS
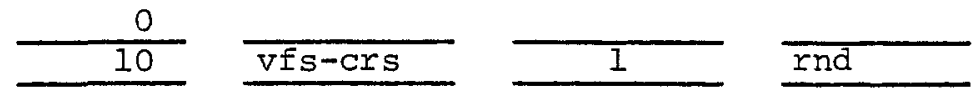

OTHER $\geq$ gravel sand
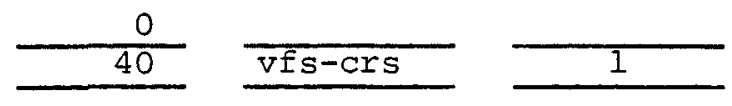

rnd

SILT AND CLAY

\section{0}

REMARKS

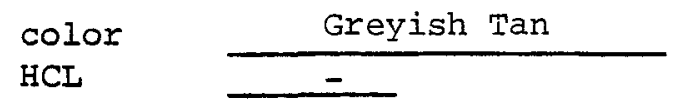

Slightly Micaceous Frosted Quartz

Immature

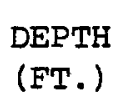

DEPTH
(FT.)

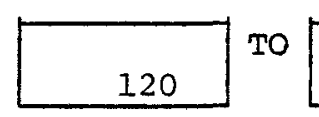

135
ROCK CLASSIEICATION

silty sandy Gravel

\% SIZE RANGE SPHERICITY ROUNDNESS

BASALT $\frac{\geq \text { gravel }}{\text { sand }}$

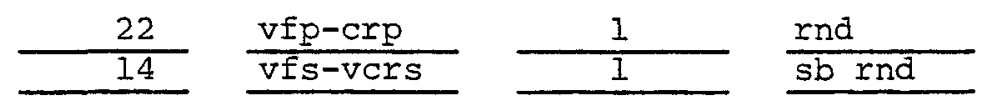

OTHER

zgravel

$\frac{18}{36} \quad \frac{v f p-c r p}{v f s-v c r s}$

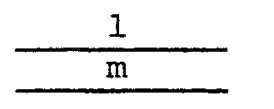

rnd

SILT AND CLAY

10

REMARKS

$$
\begin{aligned}
& \underset{\text { Color }}{\text { HCL }} \text { Slightly Micaceous } \quad \text { Frosted Quartz } \\
& \text { Immature }
\end{aligned}
$$


ROCK CLASSIFICATION

DEPTH

(FT.)

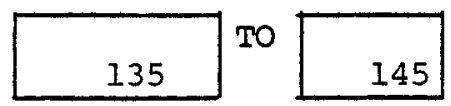

sandy Gravel

\section{\% SIZE RANGE SPHERICITY ROUNDNESS}

BASALT $>$ gravel sand
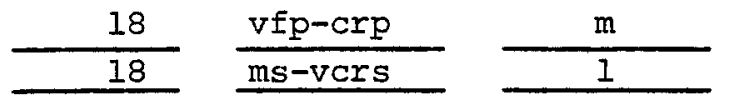

$\frac{\text { sb rnd }}{\text { sb ang }}$

OTHER $\geq$ gravel
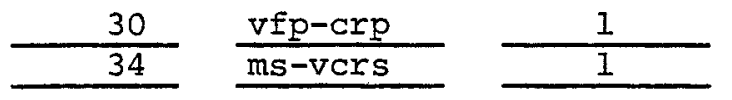

$\frac{\text { w rnd }}{\text { sb rnd }}$

SILT AND CLAY

0

REMARKS

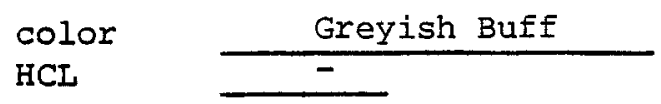

Slightly Micaceous Frosted quartz

Immature

DEPTH

(FT.)

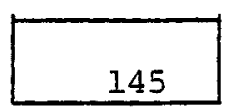

TO

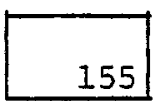

ROCK CLASSIFICATION

slightly silty gravelly sand

? SIZE RANGE SPHERICITY ROUNDNESS

\begin{tabular}{|c|c|c|c|c|c|}
\hline BASALT & $\begin{array}{l}\geq \text { gravel } \\
\text { sand }\end{array}$ & $\frac{13}{16}$ & $\frac{v f p-m p}{v f s-v c r s}$ & $\frac{\mathrm{m}}{1}$ & $\frac{\text { sb rnd }}{\text { sb rnd }}$ \\
\hline OTHER & $\begin{array}{l}\geq \text { gravel } \\
\text { sand }\end{array}$ & $\frac{13}{48}$ & $\frac{v f p-m p}{v f s-v c r s}$ & $\frac{m}{h}$ & $\frac{\text { sb rnd }}{\text { rnd }}$ \\
\hline
\end{tabular}

REMARKS

$\begin{array}{ll}\text { color } & \frac{\text { Greyish White }}{\text { HCI }} \\ \text { Micaceous } & \text { Frosted Quartz } \\ \text { Immature } & \end{array}$




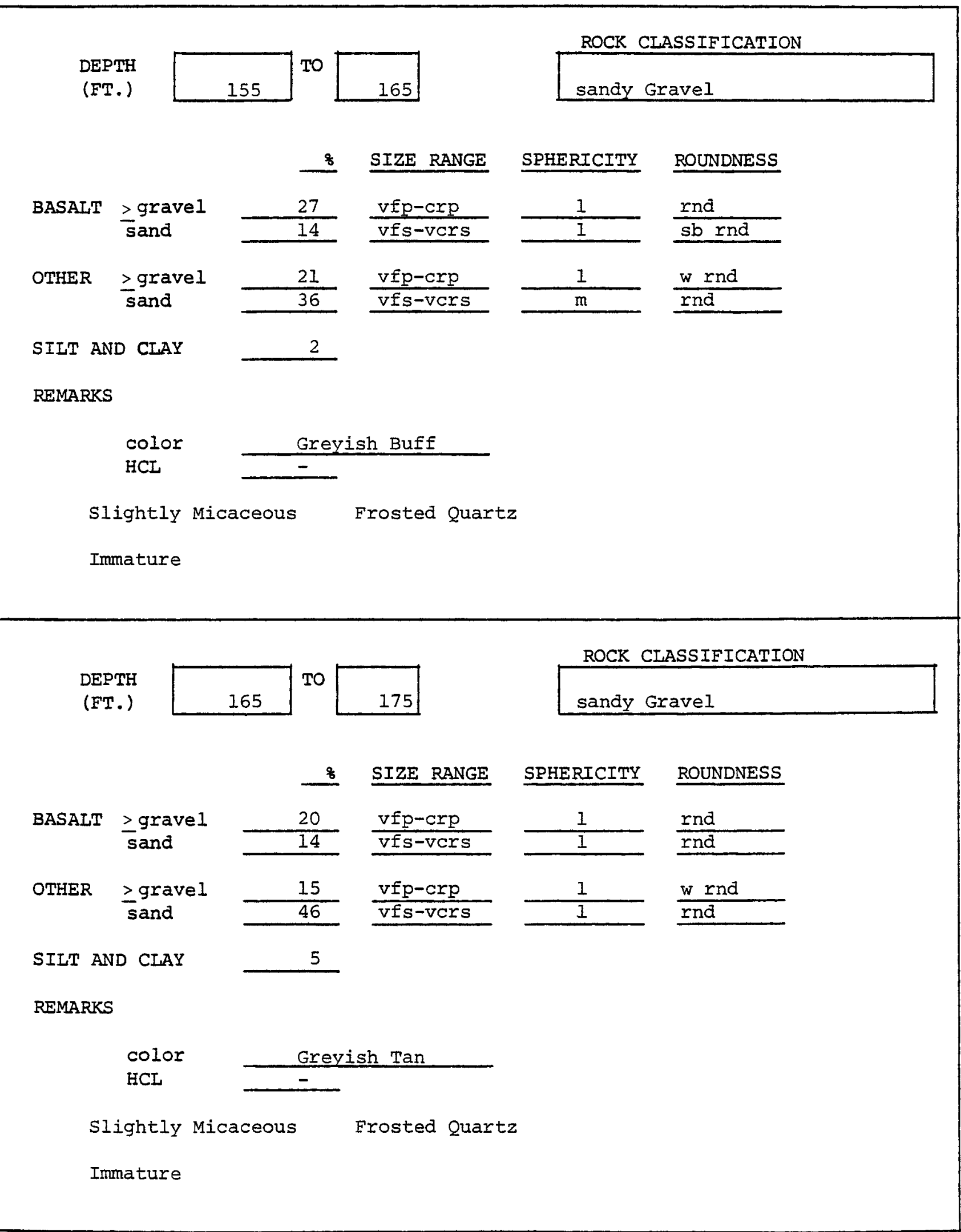




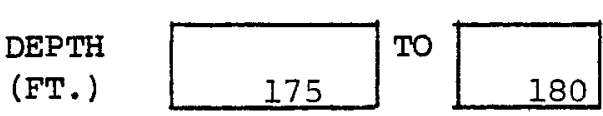

ROCK CLASSIFICATION

(FT.)

175

180

silty sandy Gravel

\section{$\%$ SIZE RANGE SPHERICITY ROUNDNESS}

BASALT $\underset{\substack{\geq \text { gravel } \\ \text { sand }}}{\text { gand }}$
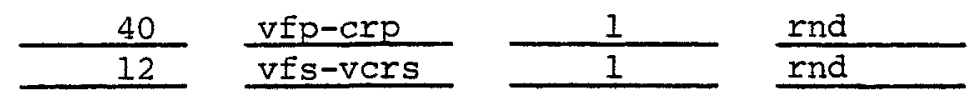

OTHER $\geq$ gravel
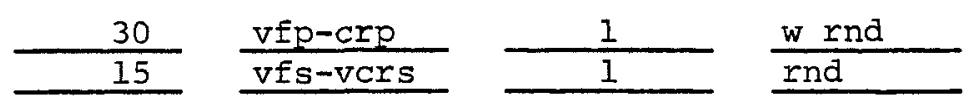

SILT AND CLAY

3

REMARKS

color

$\mathrm{HCL}$

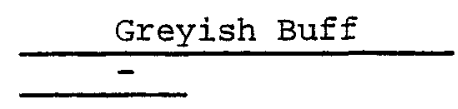

Unfrosted Quartz

Immature
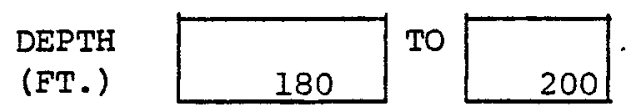

ROCK CLASSIFICATION

(FT.)

180

200

gravelly sand

\section{\& SIZE RANGE SPHERICITY ROUNDNESS}

BASALT $\underset{\text { sand }}{\geq \text { gravel }}$
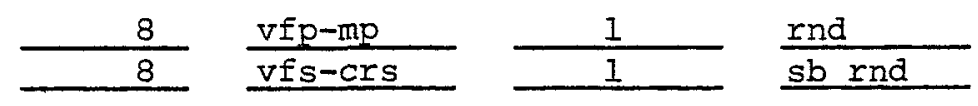

OTHER $\underset{\substack{\text { sand } \\ \text { gravel }}}{\text { OTh }}$
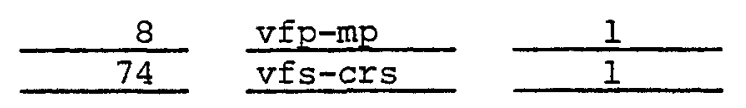

rnd

SILT AND CLAY

\section{2}

REMARKS

color

Buff White

HCL

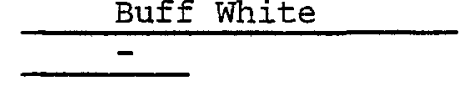

Slightly Diatomaceous

Frosted Quartz

Immature 


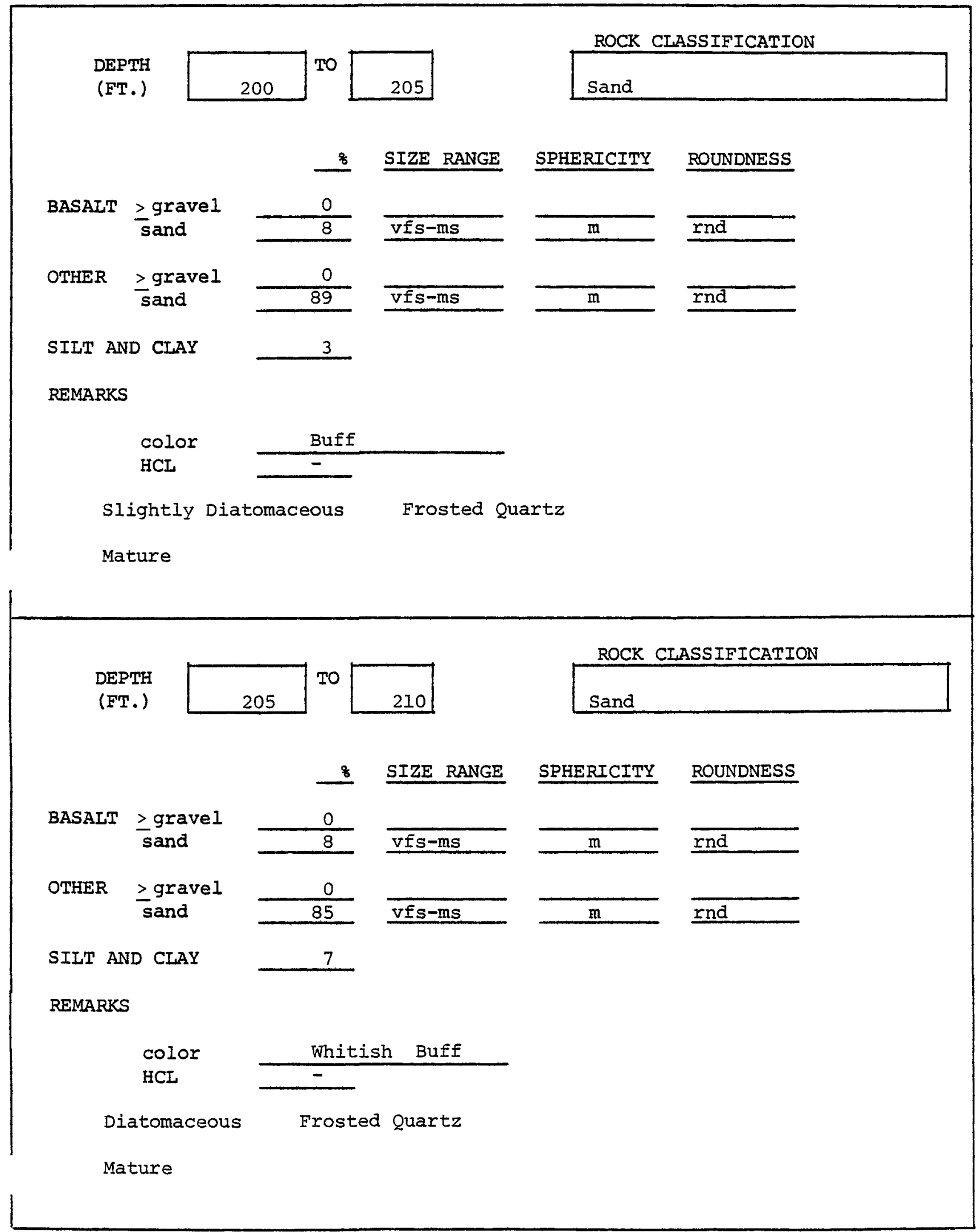




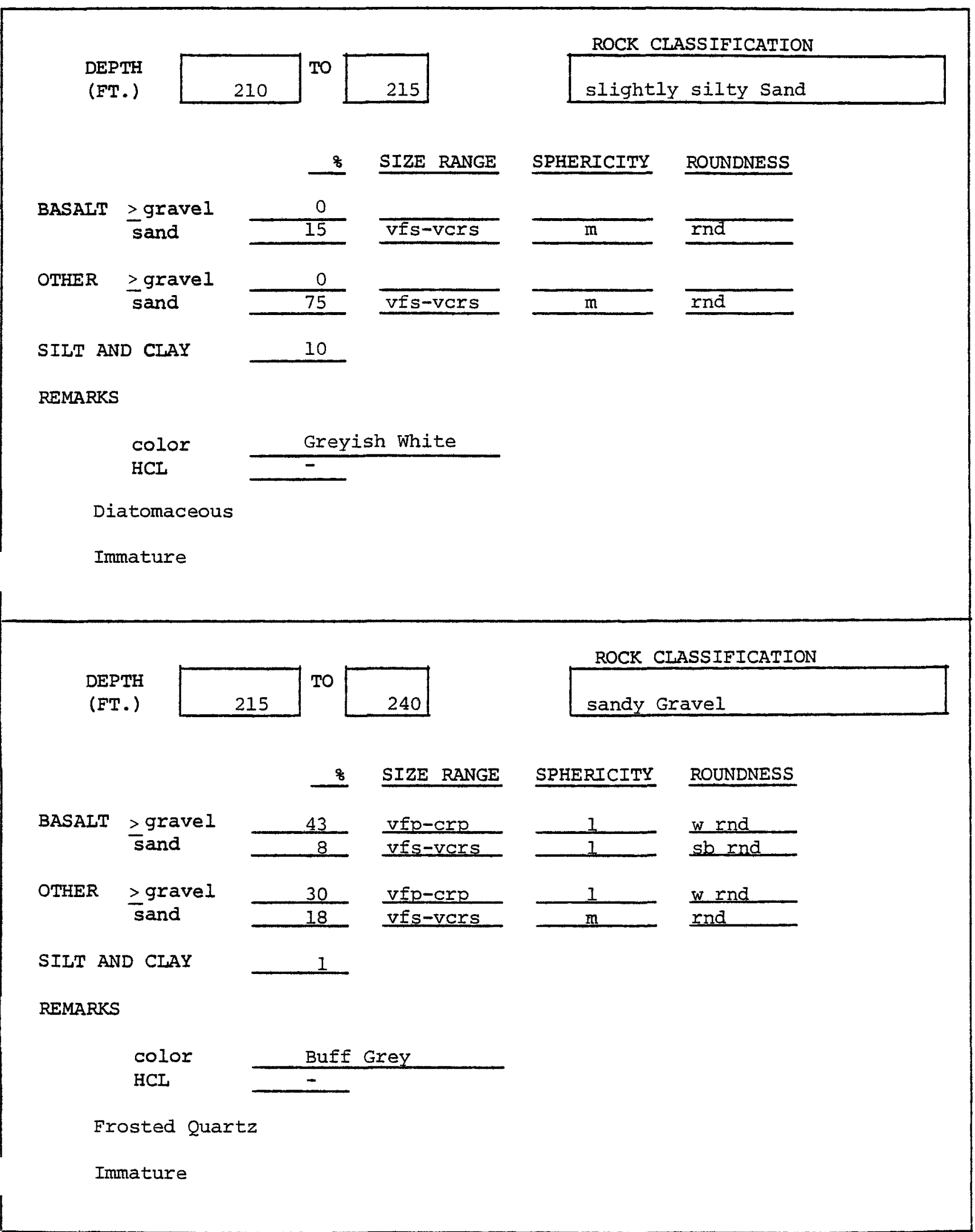




$$
\begin{aligned}
& \text { DEPTH } \\
& \text { (FT.) }
\end{aligned}
$$

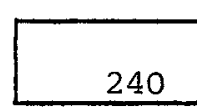

To

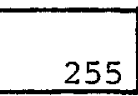

ROCK CIASSIFICATION

sandy Gravel

S SIZE RANGE SPHERICITY ROUNDNESS

BASALT $\geq$ gravel sand OTHER $\frac{>\text { grave } 1}{\text { sand }}$

SIIT AND CIAY
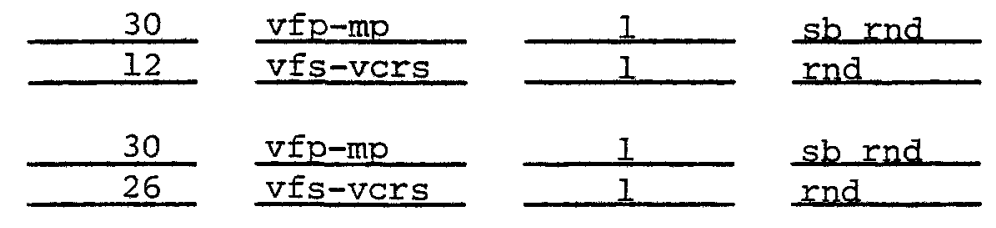

$\frac{\text { vfp-mp }}{\text { vfs-vers }}$

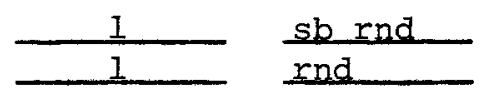

REMARKS

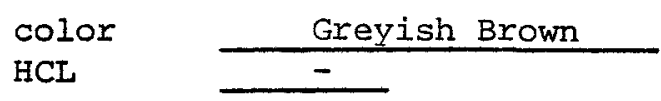

Slightly Micaceous Unfrosted Quartz

Immature

DEPTH

(FT.)

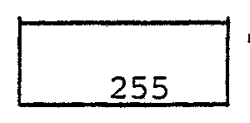

TO

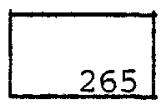

ROCK CLASSIFICATION

slightly silty grave $1 \mathrm{y}$ sand

\section{\% SIZE RANGE SPHERICITY ROUNDNESS}

BASALT

$$
\begin{aligned}
& \geq \text { gravel } \\
& \text { sand }
\end{aligned}
$$
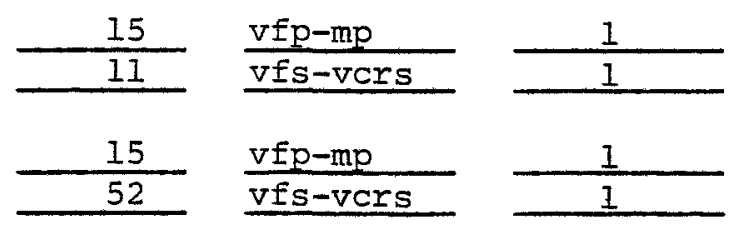

OTHER $\geq$ gravel sand
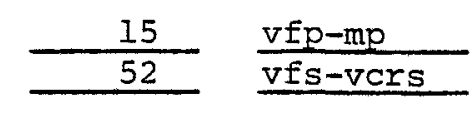

7

SIIT AND CIAY

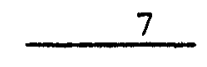

REMARKS

$$
\begin{aligned}
& \text { color } \\
& \text { HCL }
\end{aligned}
$$

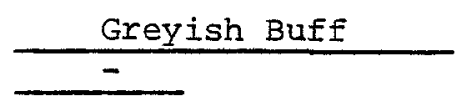

Slightly Micaceous

Immature 
DEPTH

(FT.)

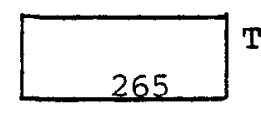

To

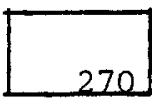

ROCK CLASSIFICATION

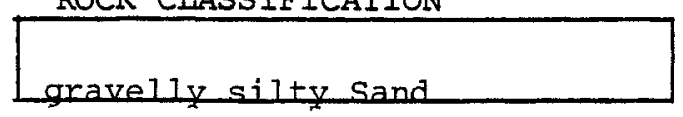

\section{\& SIZE RANGE SPHERICITY ROUNDNESS}

BASALT $\geq$ gravel
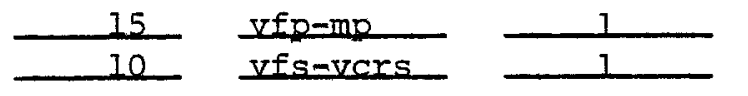

$\frac{\text { sb_ang }}{\text { end }}$

OTHER $\underset{\text { sand }}{\geq \text { gravel }}$
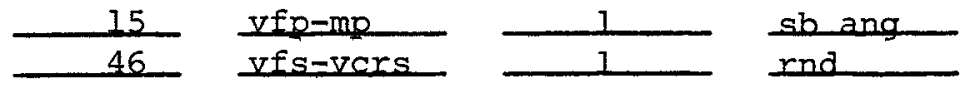

SIIT AND CLAY 14

REMARKS

color

HCL Grevish White

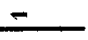

Slightly Micaceous

Immature

DEPTH

(FT.)

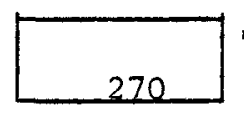

TO

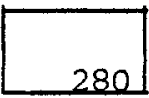

ROCK CIASSIFICATION

gravelly silty sand

\% SIZE RANGE SPHERICITY ROUNDNESS

BASALT $\geq$ grave1
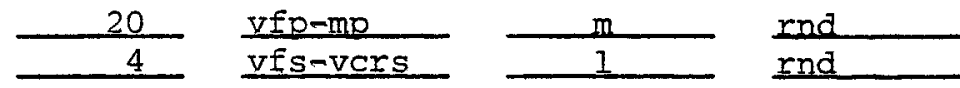

OTHER $\geq$ gravel sand
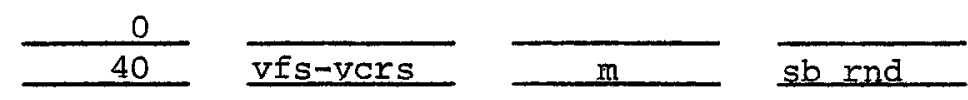

SILT AND CLAY 36

REMARKS

color Reddish Brown

HCL -

Micaceous

Immature 
DEPTH

(FT.)

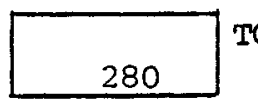

TO

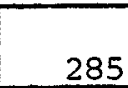

ROCK CLASSIFICATION

slightly gravelly silty sand

\section{SIZE RANGE SPHERICITY ROUNDNESS}

BASALT >gravel sand
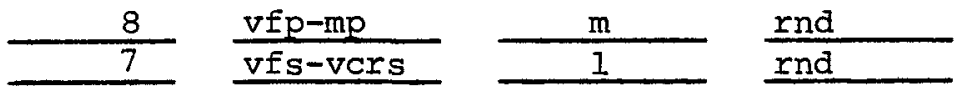

OTHER $\quad$ gravel
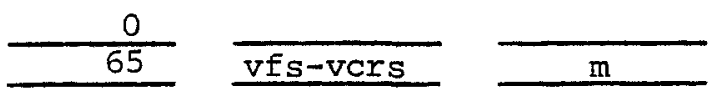

sb rnd

SIIT AND CIAY

20

REMARKS

$$
\begin{aligned}
& \text { color } \\
& \mathrm{HCL}
\end{aligned}
$$

Light Brown

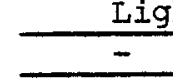

SIightIy Micaceous

Frosted Quartz

Immature

DEPTH
(FT.)

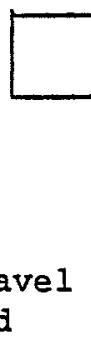

TO

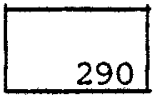

ROCK CIASSIFICATION

sandy silt

\% SIZE RANGE SPHERICITY ROUNDNESS

BASALT $\geq$ grave 1
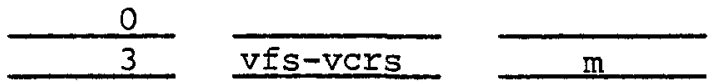

rind

OTHER $\geq$ gravel
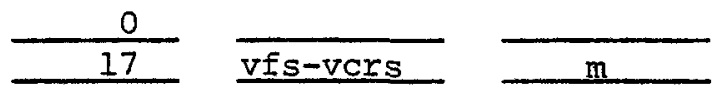

rnd

SIIT AND CIAY

80

REMARKS

color

Brownish Grey

HCL -

Slightly Micaceous

Slightly Gypsiferous

Immature 


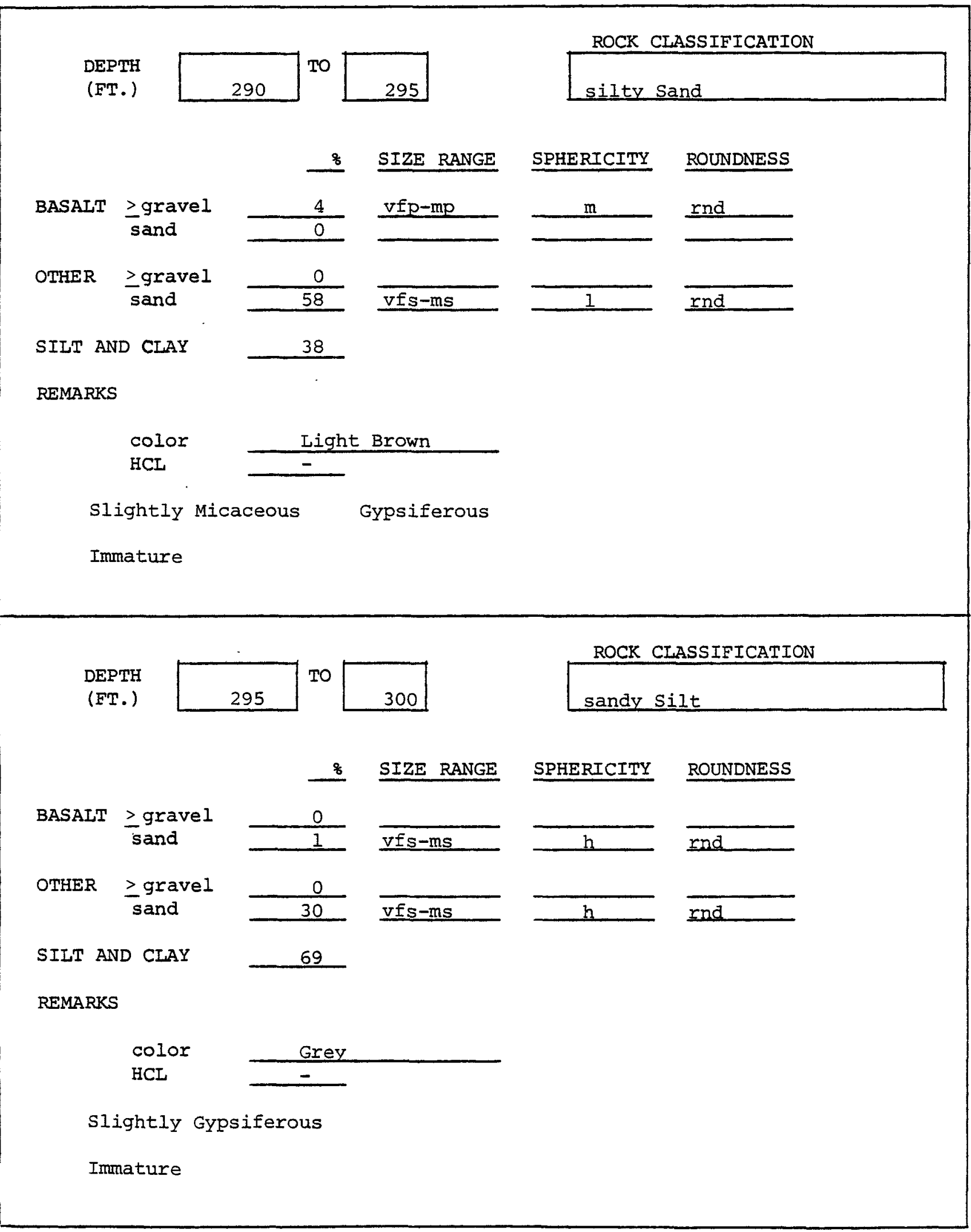

W. K. Summers \& Associates 
DEPTH

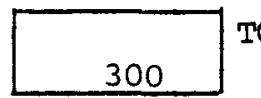

TO

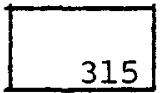

ROCK CIASSIFICATION

gravelly sand

\section{\% SIZE RANGE SPHERICITY ROUNDNESS}

\begin{tabular}{|c|c|c|c|c|c|}
\hline \multirow[t]{2}{*}{ BASALT } & \multirow{2}{*}{$\begin{array}{l}\text { >gravel } \\
\text { sand }\end{array}$} & 17 & $v f p-m p$ & 1 & $\mathrm{sb}$ rnd \\
\hline & & 29 & vfs-vers & $m$ & rnd \\
\hline \multirow[t]{2}{*}{ OTHER } & \multirow{2}{*}{$\begin{array}{l}\text { >gravel } \\
\text { sand }\end{array}$} & 0 & & & \\
\hline & & 53 & vfs-vers & m & rnd \\
\hline
\end{tabular}

SIIT AND CLAY

1

REMARKS

color Greyish Buff

HCL $-$

Frosted Quartz

Immature

DEPTH

(ET.)

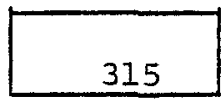

TO

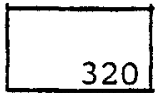

ROCK CIASSIFICATION

Sand

\% SIZE RANGE SPHERICITY ROUNDNESS

BASALT $\frac{\geq \text { grave } 1}{\text { sand }}$
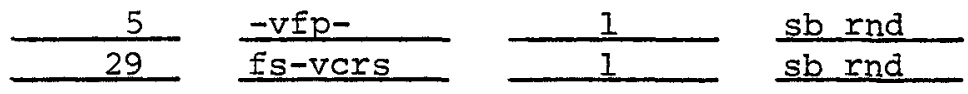

OTHER >gravel
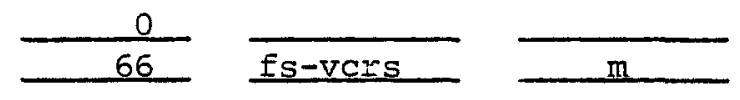

rnd

SILT AND CIAY

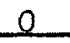

REMARKS

color Greyish Buff

$\mathrm{HCL}$

$-$

Slightly Gypsiferous Frosted Quartz

Mature 
DEPTH

(FT.)

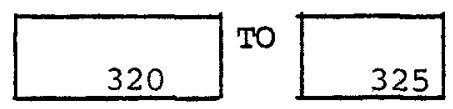

\section{SIZE RANGE SPHERICITY ROUNDNESS}

BASAIT >gravel sand

OTHER $\quad \frac{\text { gravel }}{\text { sand }}$

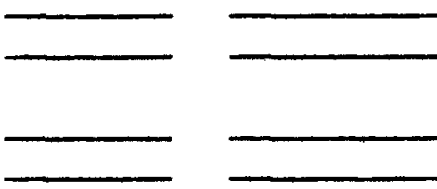

SILT AND CIAY

REMARKS

color

$\mathrm{HCL}$

Sample missing

DEPTH

(ET.)

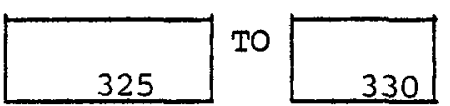

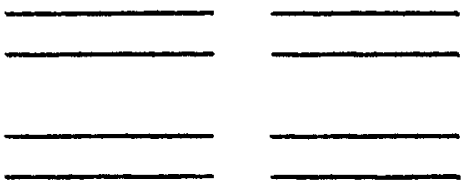

ROCK CLASSIFICATION

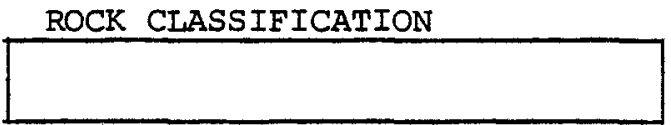

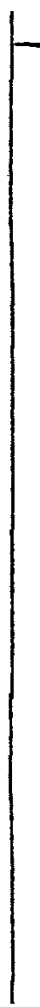

\section{SIZE RANGE SPHERICITY ROUNDNESS}

\begin{tabular}{|c|c|c|c|c|c|}
\hline BASALT & $\begin{array}{l}\text { >gravel } \\
\text { sand }\end{array}$ & $\frac{17}{64}$ & $\frac{v E p-m p}{f S-v c r s}$ & $\frac{1}{1}$ & $\frac{\mathrm{sb} \text { ang }}{\mathrm{sb} \text { rnd }}$ \\
\hline OTHER & >gravel & 3 & $\mathrm{v} F \mathrm{p}-\mathrm{mp}$ & $m$ & $w$ rnd \\
\hline & sand & 16 & $f_{S-v c r s}$ & 1 & rnd \\
\hline
\end{tabular}

SILT AND CLAY

0

REMARKS

$$
\begin{aligned}
& \text { color } \\
& \text { HCL }
\end{aligned}
$$
Black

Caliche (pebbles present) Frosted quartz Immature 
DEPTH

(FT.)

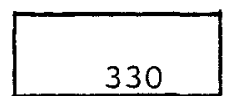

TO

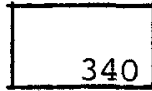

340

\section{ROCK CLASSIFICATION}

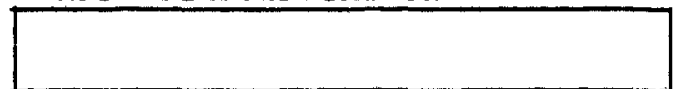

\% SIZE RANGE SPHERICITY ROUNDNESS

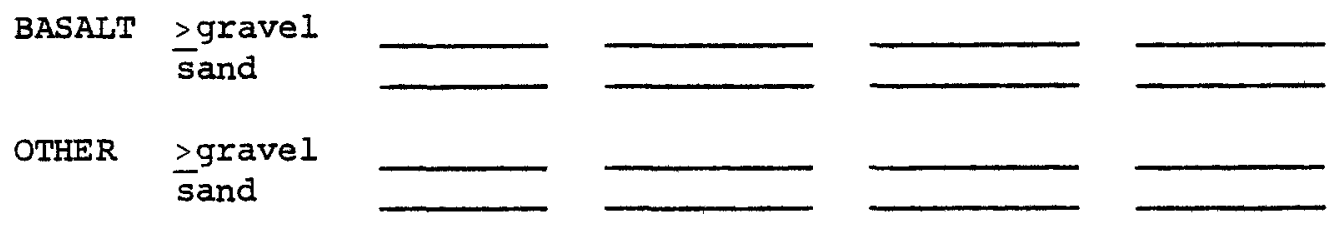

SIIT AND CIAY

REMARKS

color

HCL

Sample missing

DEPTH

(FT.)

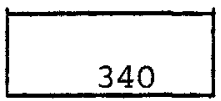

TO

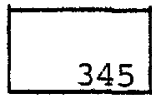

ROCK CLASSIFICATION

Basalt?

\& SIZE RANGE SPHERICITY ROUNDNESS

BASAIT $\geq$ gravel
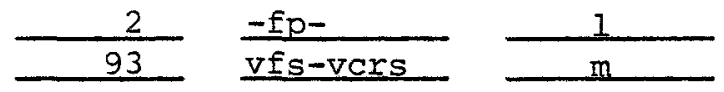

$\frac{\text { sb rnd }}{\text { sb rnd }}$

OTHER 2 gravel
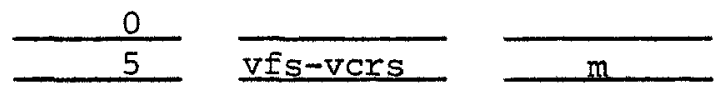

rnd

SILT AND CLAY 0

REMARKS

color

HCL Greyish Black

Frosted Quartz 


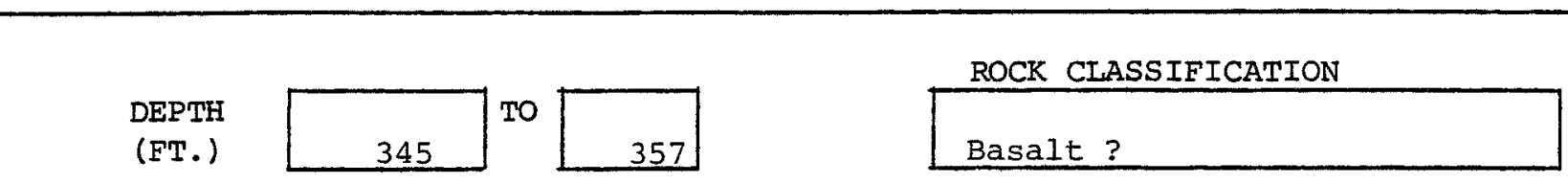

8 SIZE RANGE SPHERICITY ROUNDNESS

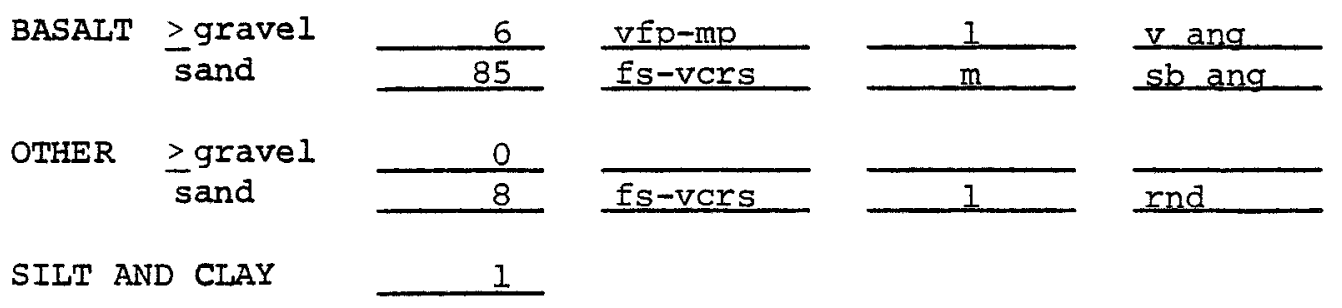

REMARKS

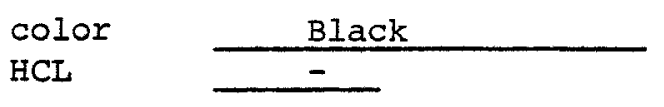

Frosted Quartz Sample interval 350'-357' missing

Total Drilled Depth reported as $357^{\circ}$

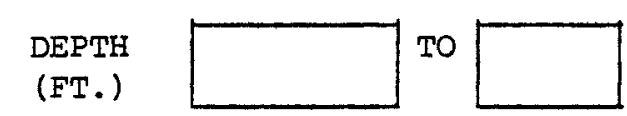

ROCK CIAASSIFICATION

\& SIZE RANGE SPHERICITY ROUNDNESS

BASALT $\frac{\geq \text { grave } 1}{\text { sand }}$
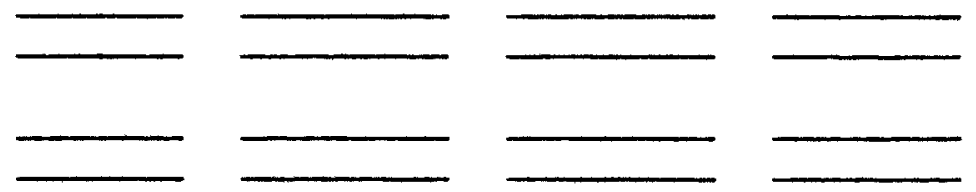

SILT AND CIAAY

REMARKS

color

HCL 


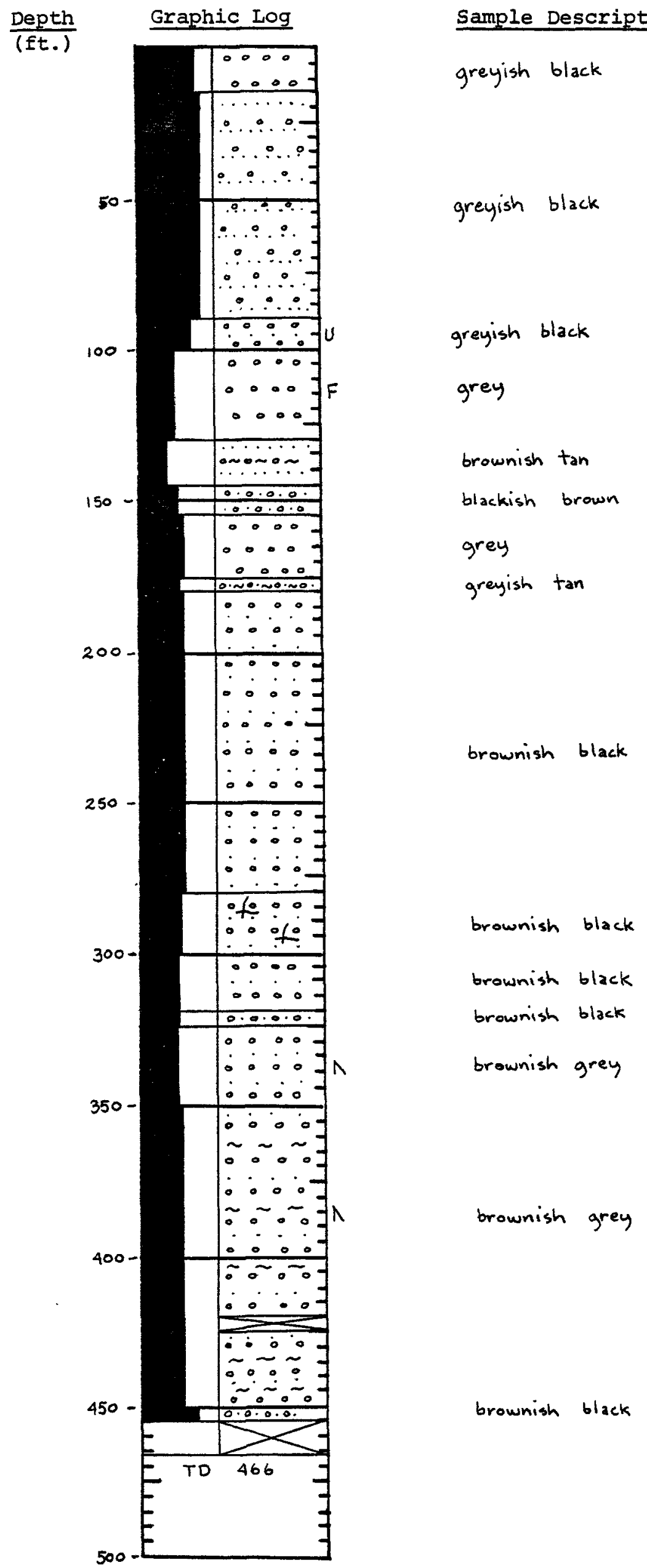

Plant Coordinates

$\frac{N 020008}{\frac{E 04755}{\text { Altitude } 465}}$

Other Data used in Interpretation

$$
\text { Driller's Log }
$$

greyish black

grey

brownish $\tan$

blackish brown

grey

greyish tan

brownish black

brownish black

brownish black

brownish black

brownish grey

brownish grey 
DEPTH

(FT.)

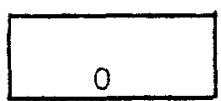

TO

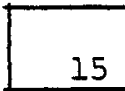

ROCK CLASSIFICATION

Grave1

S SIZE RANGE SPHERICITY ROUNDNESS

\begin{tabular}{|c|c|c|c|c|c|}
\hline BASALT & $\begin{array}{l}\geq \text { gravel } \\
\text { sand }\end{array}$ & $\frac{60}{15}$ & $\frac{v f p-c r p}{m s-v c r s}$ & $\frac{1}{1}$ & $\frac{w \text { rnd }}{\text { rnd }}$ \\
\hline OTHER & $\begin{array}{l}\geq \text { gravel } \\
\text { sand }\end{array}$ & $\frac{23}{2}$ & $\frac{v f p-c r p}{m s-v c r s}$ & $\frac{1}{m}$ & $\frac{w \text { rnd }}{\text { rnd }}$ \\
\hline
\end{tabular}

SILT AND CLAY

0

REMARKS

$\begin{array}{ll}\text { Color } & \text { Greyish Black } \\ & -\end{array}$

Immature

DEPTH

(FT.)

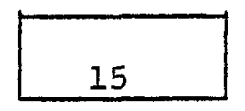

TO

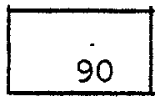

ROCK CIASSIFICATION

gravelly sand

\section{\% SIZE RANGE SPHERICITY ROUNDNESS}

\begin{tabular}{|c|c|c|c|c|c|}
\hline \multirow[t]{2}{*}{ BASALT } & \multirow{2}{*}{$\begin{array}{l}\text { >gravel } \\
\text { sand }\end{array}$} & 12 & vfp-crp & 1 & rnd \\
\hline & & 68 & $\mathrm{~ms}-\mathrm{vcrs}$ & 1 & rnd \\
\hline \multirow[t]{2}{*}{ OTHER } & $\geq$ gravel & 8 & $v f p-c r p$ & 1 & rnd \\
\hline & sand & 12 & ms-vars & 1 & rnd \\
\hline
\end{tabular}

SIIT AND CLAY

0

REMARKS

color Greyish Black ("salt \& pepper") HCL -

Immature 


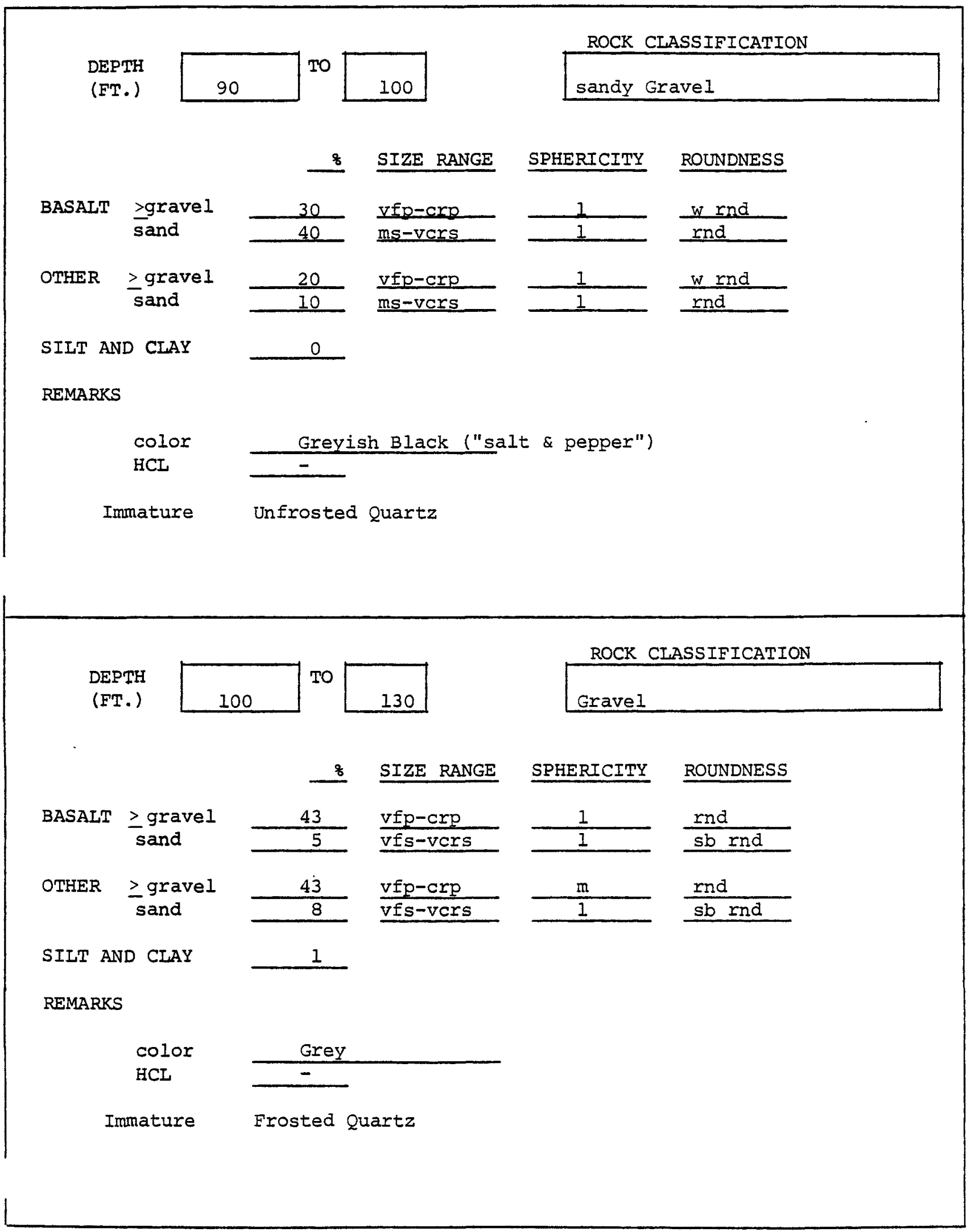


DEPTH

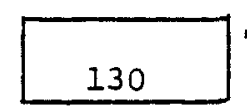
To 145
ROCK CLASSIFICATION

gravelly silty sand

\section{SIZE RANGE SPHERICITY ROUNDNESS}

BASALT $\geq$ gravel sand

OTHER $\geq$ gravel sand

SILT AND CIAY

REMARKS

color

HCL
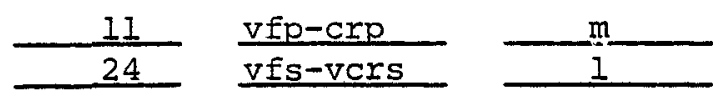

rnd
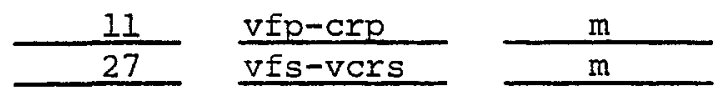

rnd

Immature

\section{DEPTH}

(FT.)

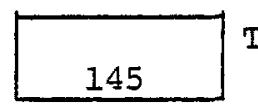

TO

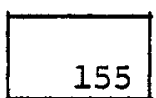

ROCK CIASSIFICATION

sandy Gravel

\section{\% SIZE RANGE SPHERICITY ROUNDNESS}

BASALT $\geq$ gravel

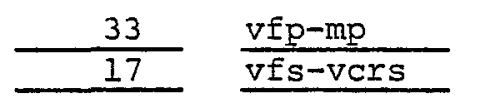

$\frac{1}{1} \frac{\text { rnd }}{\text { sb rnd }}$

OTHER $\geq$ grave1 sand

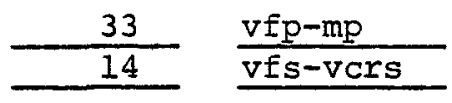

$\frac{1}{1} \frac{\text { rnd }}{\text { rnd }}$

SILT AND CIAY 3

REMARKS

$$
\text { color }
$$

HCL

Blackish Brown

Immature 
DEPTH
(FT.)

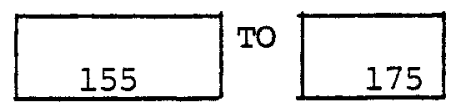

ROCK CIASSIFICATION

$155 \quad 175$

Grave 1

\section{\% SIZE RANGE SPHERICITY ROUNDNESS}

BASALT $\geq$ gravel sand
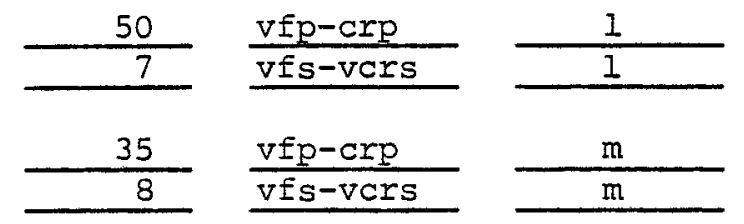

$\frac{\mathrm{sb} \text { rnd }}{\mathrm{sb} \text { rnd }}$

OTHER $\geq$ gravel

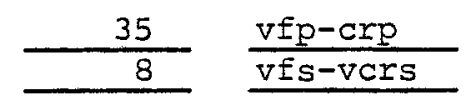

rnd

SIIT AND CLAY

0

REMARKS

$$
\begin{aligned}
& \text { color } \\
& \text { HCL }
\end{aligned}
$$

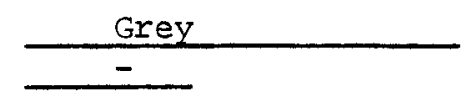

Immature

$$
\begin{aligned}
& \text { DEPTH } \\
& \text { (FT.) }
\end{aligned}
$$

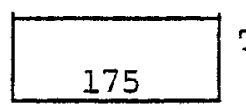

TO

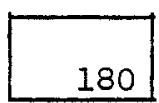

ROCK CLASSIFICATION

silty sandy Gravel

\section{\% SIZE RANGE SPHERICITY ROUNDNESS}

BASALT $\frac{\text { zgravel }}{\text { sand }}$
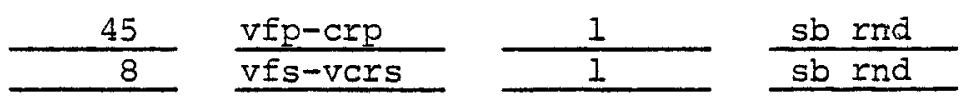

OTHER $\geq$ gravel
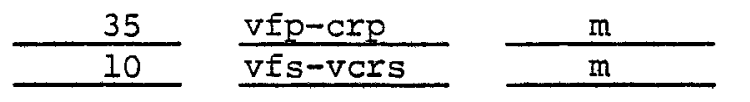

$$
\begin{aligned}
& \text { rnd } \\
& \text { sb rnd }
\end{aligned}
$$

SILT AND CLAY

2

REMARKS

$$
\text { color }
$$

HCL

Greyish Tan

Immature 
DEPTH

(FT.)

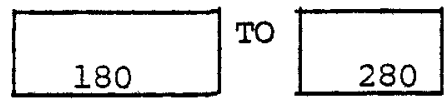

ROCK CIASSIFICATION

sandy Gravel.

\section{\% SIZE RANGE SPHERICITY ROUNDNESS}

\begin{tabular}{|c|c|c|c|c|c|}
\hline BASALT & $\begin{array}{l}\text { 2 gravel } \\
\text { sand }\end{array}$ & $\frac{44}{14}$ & $\begin{array}{l}\mathrm{vfp}-\mathrm{crp} \\
\mathrm{vfs}-\mathrm{vcrs}\end{array}$ & $\frac{1}{1}$ & $\begin{array}{l}\text { sb rnd } \\
\text { sb rnd }\end{array}$ \\
\hline OTHER & $\begin{array}{l}\geq \text { gravel } \\
\text { sand }\end{array}$ & 30 & $\frac{v f p-c r p}{v f s-v c r s}$ & $\underline{m}$ & rnd \\
\hline
\end{tabular}

SILT AND CIAY

REMARKS

color
$\mathrm{HCL}$

Immature

\section{DEPTH}

(ET.)

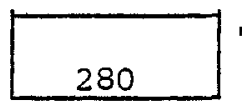

TO

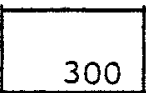

ROCK CLASSIFICATION

sandy Gravel

\section{\% SIZE RANGE SPHERICITY ROUNDNESS}

BASALT $\frac{\geq \text { gravel }}{\text { sand }}$
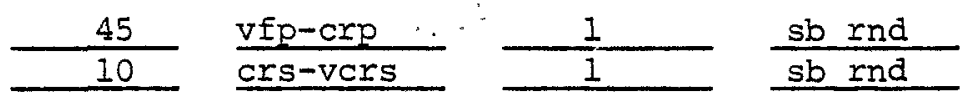

OTHER $\geq$ gravel $\frac{35}{10} \frac{\text { vfp-crp }}{\text { crs-vers }}$

$\frac{m}{I} \frac{\text { rnd }}{\text { sb rnd }}$

SILT AND CLAY 0

REMARKS

color

HCL Brownish Black

$-$

Slightly Gypsiferous

Immature 


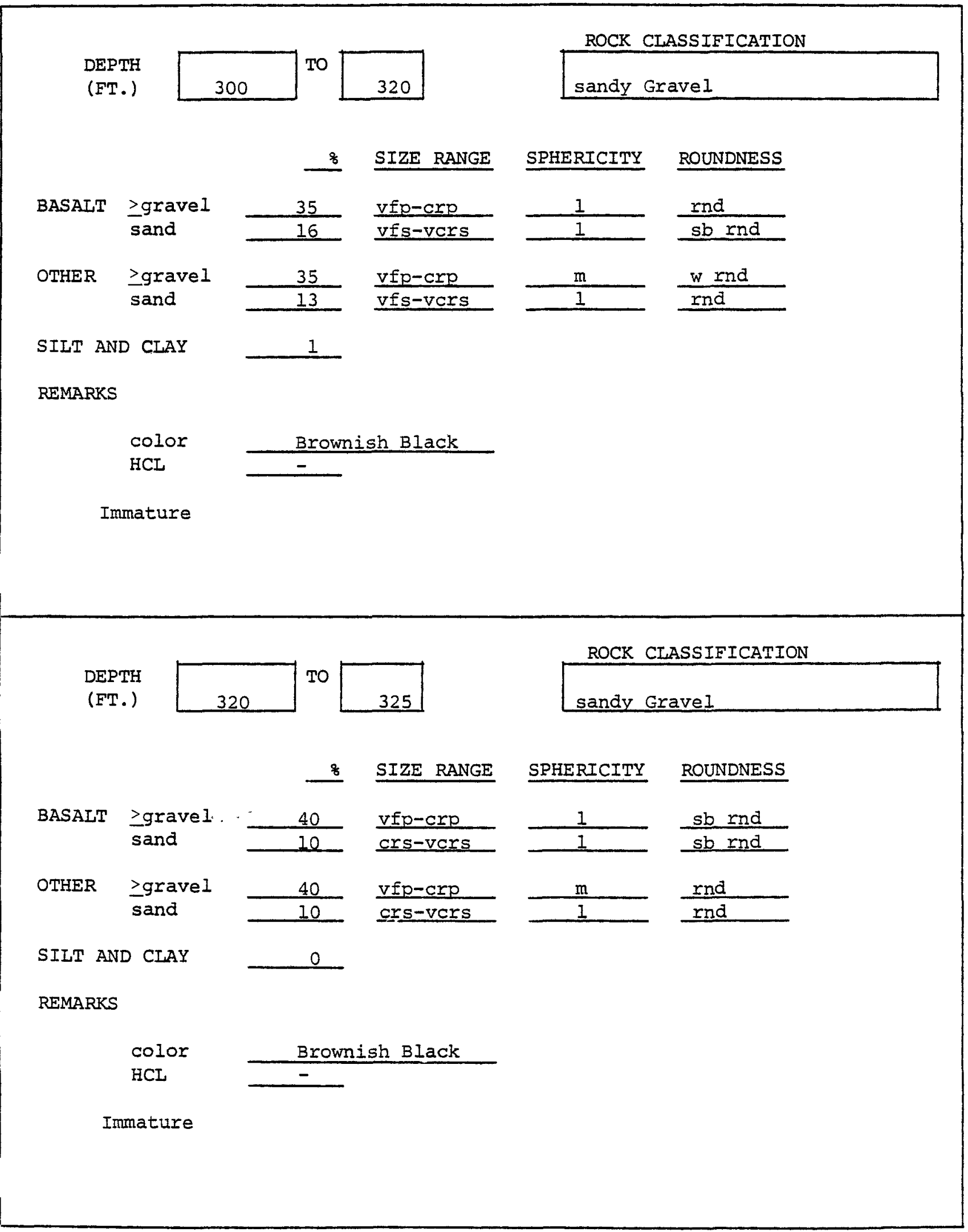




\section{DEPTH}

(FT.)

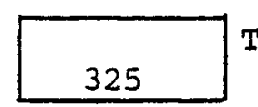

TO

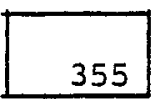

\section{ROCK CLASSIFICATION}

sandy Gravel

\section{\% SIZE RANGE SPHERICITY ROUNDNESS}

\begin{tabular}{|c|c|c|c|c|c|}
\hline BASALT & $>$ gravel & 40 & vfp-crp & $\mathrm{m}$ & sb rnd \\
\hline & sand & 11 & crs-vcrs & 1 & $\mathrm{sb}$ rnd \\
\hline OTHER & $>$ gravel & 40 & $v f p-c r p$ & $\mathfrak{m}$ & sb rnd \\
\hline & sand & 9 & crs-vcrs & 1 & rnd \\
\hline SILT ANI & D CLAY & 0 & & & \\
\hline
\end{tabular}

REMARKS

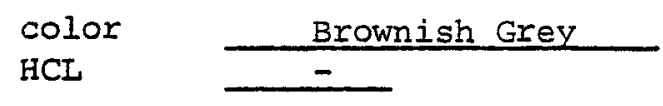

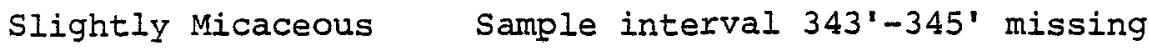

Immature

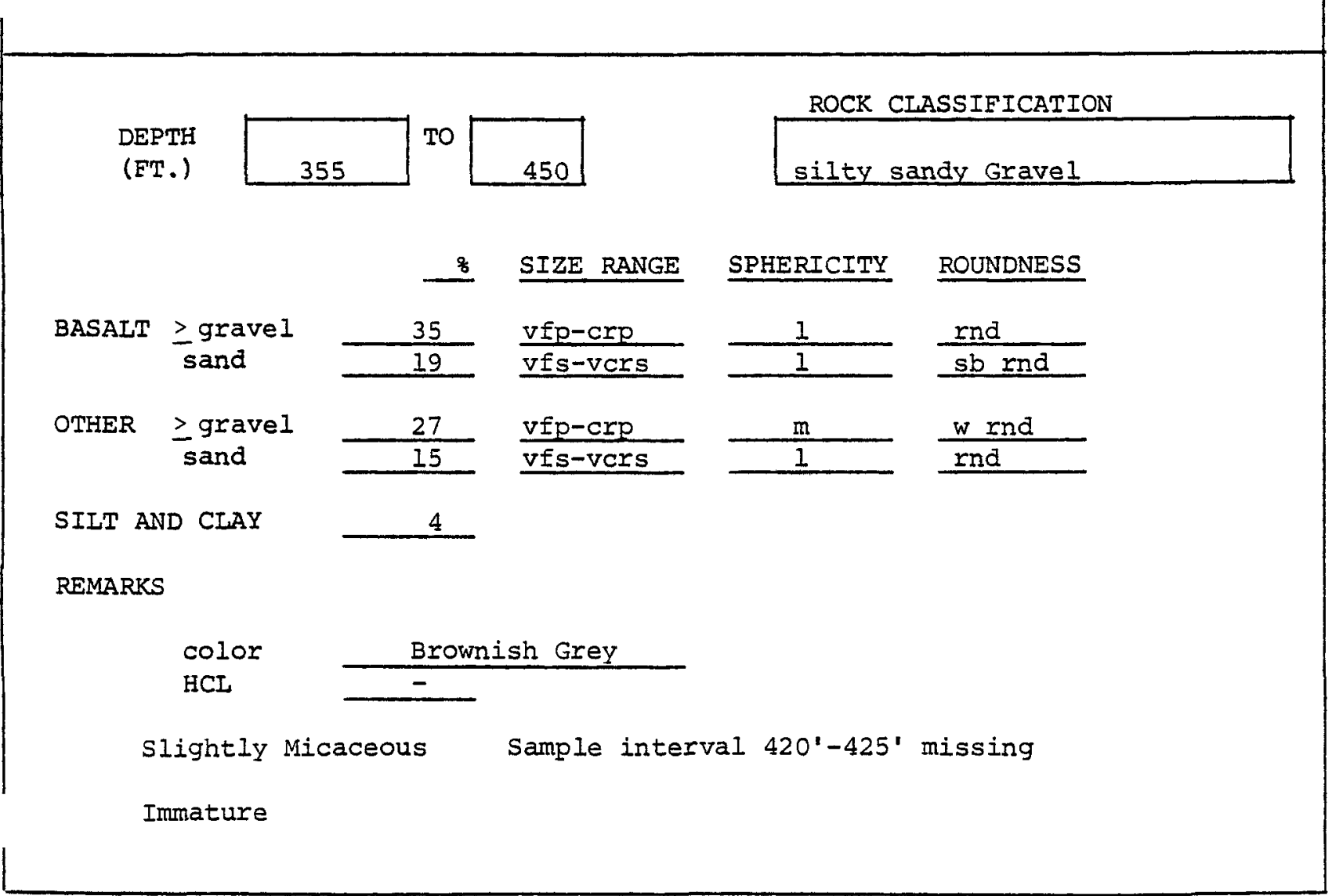


DEPTH

(FT.)

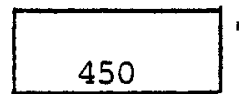

TO

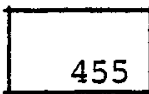

ROCK CIASSIFICATION

sandy Gravel

\section{SIZE RANGE SPHERICITY ROUNDNESS}

BASALT >gravel sand
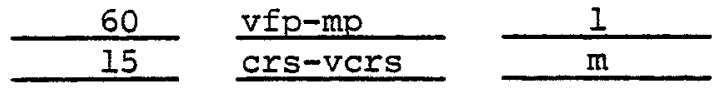

rnd

OTHER $\geq$ gravel
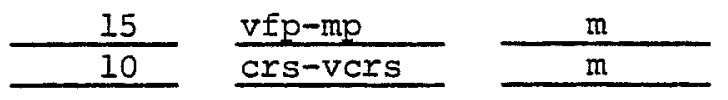

rnd

SIIT AND CLAY

0

REMARKS

color Brownish Black

HCL $-$

Immature

\section{DEPTH}

(FT.)

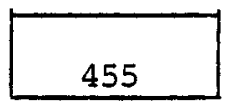

TO

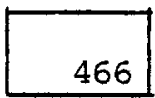

ROCK CLASSIFICATION

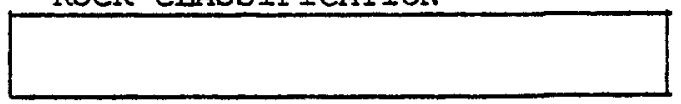

\section{\& SIZE RANGE SPHERICITY ROUNDNESS}

BASALT $\underset{\substack{\text { sand } \\ \text { grave }}}{\text { sand }}$
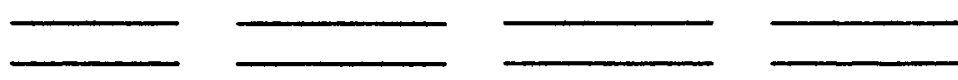
OTHER $\geq$ gravel sand
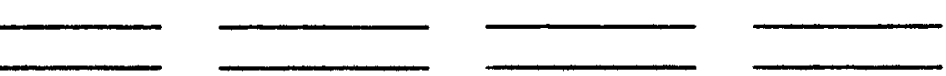

SILT AND CIAY

REMARKS

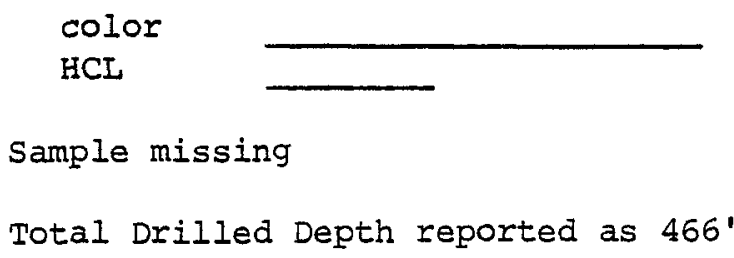


$\frac{\text { Depth }}{\text { (ft.) }}$

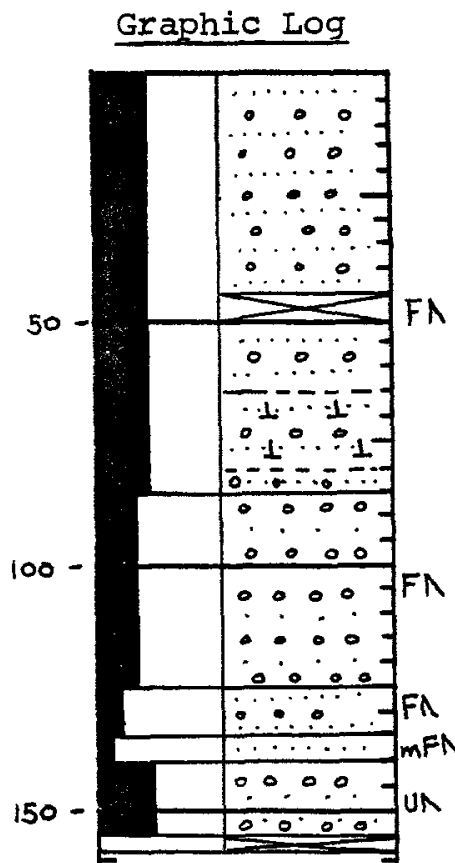

TD 158

$E$

$E$

E

$E$

$E$

$E$

$E$

$E$

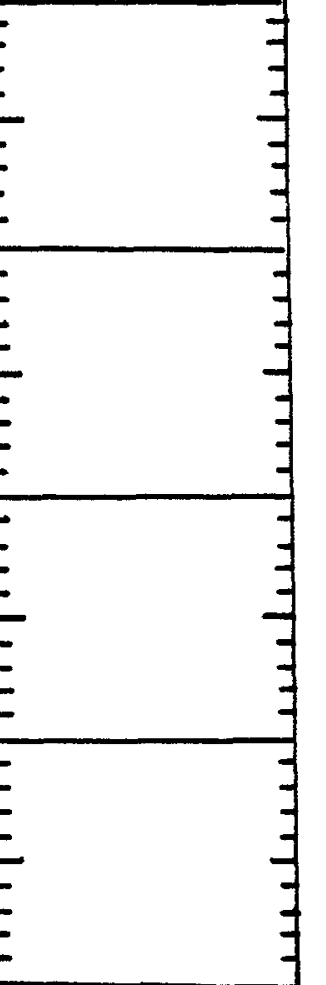

Sample Description

brownish grey-greyish brown

greyish $\tan$

tanned buff

greyish butf; ASH FRAGMENTS

tanned grey
Plant Coordinates

$\frac{N 020389}{W 020390} \frac{1504}{\text { Altitude_504 }}$

Other Data used in Interpretation

(1) Driller's Log

(2) Descriptive Log from Newcomb, et al, $1972, p .68$ 
DEPTH

(FT.)

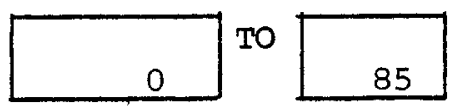

ROCK CLASSIFICATION

grave11y Sand

\section{\& SIZE RANGE SPHERICITY ROUNDNESS}

BASALT $\frac{\geq \text { gravel }}{\text { sand }}$
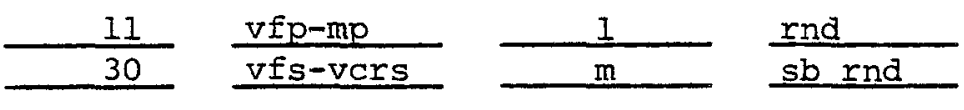

OTHER $\geq$ gravel sand
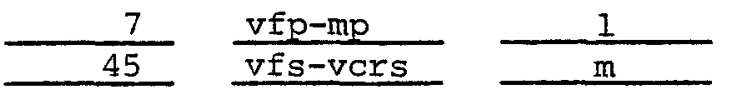

rnd

SIIT AND CLAY

7

REMARKS
color
Brownish Grey-Greyish Brown
HCL
$-\left(0^{\prime}-65^{\prime}\right),+\left(65^{\prime}-80^{\prime}\right),-\left(80^{\prime}-85^{\prime}\right)$

Slightly Micaceous

Frosted Quartz

Sample interval $45^{\prime}-50^{\prime}$ missing

Immature
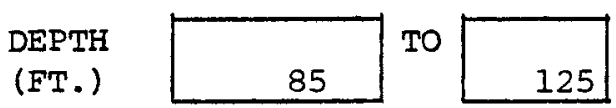

ROCK CIASSIFICATION

sandy Gravel

\section{\% SIZE RANGE SPHERICITY ROUNDNESS}

BASALT $\frac{\substack{\text { gravel } \\ \text { sand }}}{}$
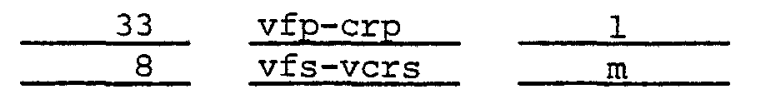

W rnd

OTHER

$$
\begin{aligned}
& \geq \text { gravel } \\
& \text { sand }
\end{aligned}
$$
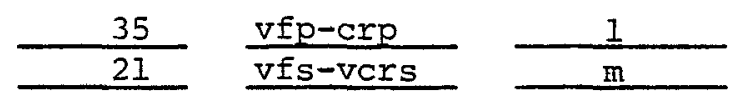

wrind

rnd

SILT AND CLAY

\section{3}

REMARKS

$$
\text { color }
$$

$\mathrm{HCL}$

Greyish Tan

Slightly Micaceous Frosted quartz

Immature 


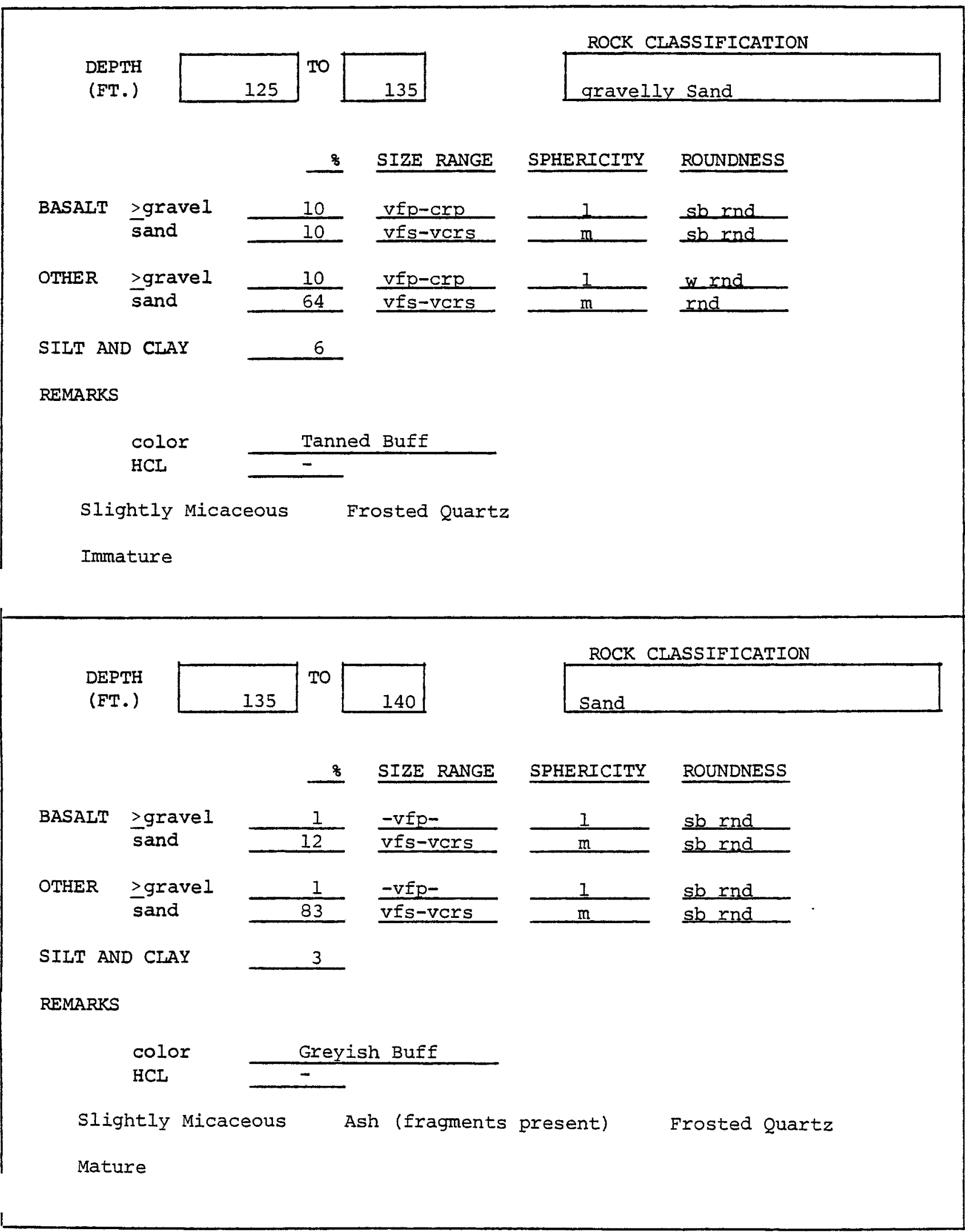




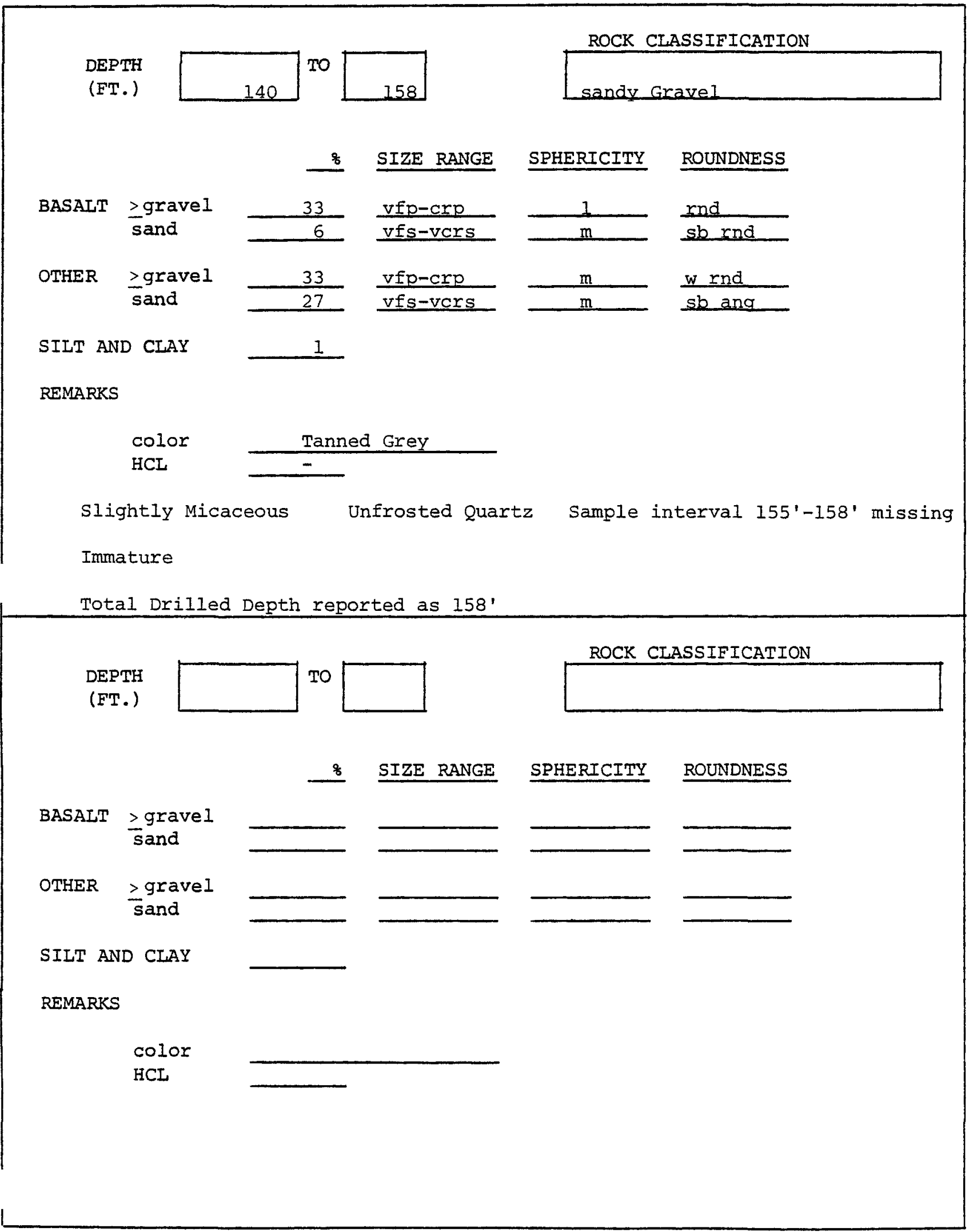




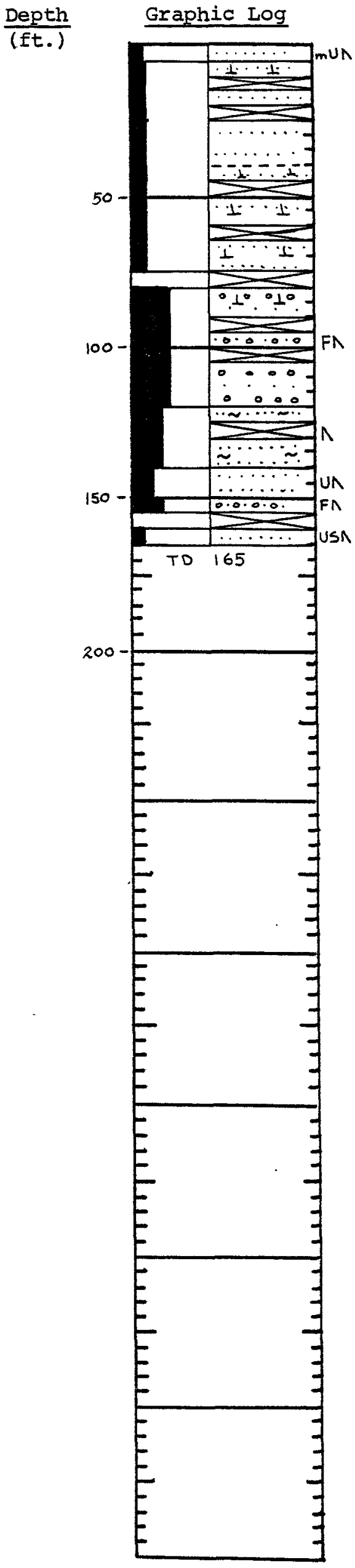

Sample Description

buff

buff grey

greyish buff-greyish tan

greyish $\tan$

buff grey

buff grey

greyish buff
Plant Coordinates

N023809

$\frac{W 033315}{\text { Altitude } 522}$

Other Data used in Interpretation

(1) Driller's Log

(2) $E-\log$

(3) Sieve analyses in MeHenry, 1957, p.36 


\section{ROCK CLASSIFICATION}

DEPTH

(FT.)

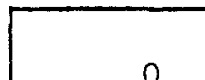

TO

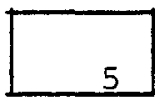

5

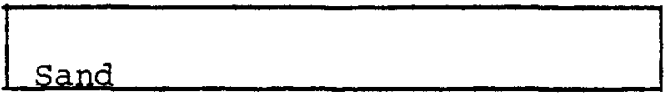

\section{\% SIZE RANGE SPHERICITY ROUNDNESS}

BASALT $\geq$ gravel sand
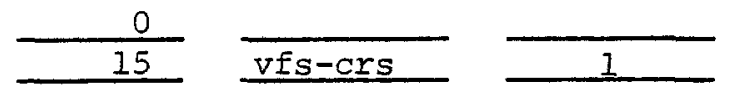

sb rnd

OTHER Igravel
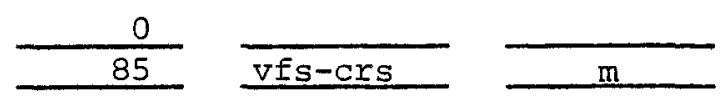

sb ang

SILT AND CLAY

\section{0}

REMARKS

color

Buff

$\mathrm{HCL}$

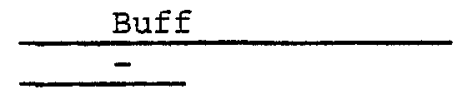

Slightly Micaceous

Unfrosted quartz

Mature

DEPTH

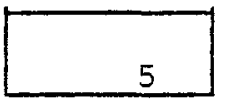

To

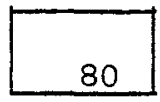

ROCK CLASSIFICATION

(FT.)

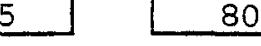

Sand

\% SIZE RANGE SPHERICITY ROUNDNESS

BASALT $\frac{\geq \text { grave } 1}{\text { sand }}$

$\frac{2}{2 I} \quad \frac{v f p-\operatorname{crp}}{\mathrm{vfs}-\mathrm{vers}}$

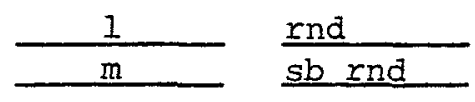

OTHER $\geq$ gravel
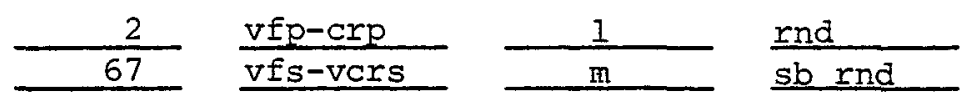

SILT AND CLAY

8

REMARKS

color

Buff Grey

HCL

$$
+\left(5^{\prime}-10^{\prime}\right),-\left(10^{\prime}-40^{\prime}\right),+\left(40^{\prime}-80^{\prime}\right)
$$

\section{Slightly Micaceous Frosted Quartz}

Sample intervals $10^{\prime}-15^{\prime}, 20^{\prime}-25^{\prime}, 45^{\prime}-50^{\prime}, 60^{\prime}-65^{\prime}$ \& $75^{\prime}-80^{\prime}$ missing Immature 
DEPTH

(FT.)

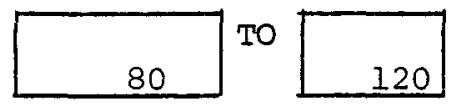

ROCK CLASSIFICATION

sandy Grave 1

\section{SIZE RANGE SPHERICITY ROUNDNESS}

\begin{tabular}{|c|c|c|c|c|c|}
\hline BASALT & $\begin{array}{l}\text { >gravel } \\
\text { sand }\end{array}$ & $\frac{36}{16}$ & $\frac{v f p-c r p}{v f s-v c r s}$ & $\frac{m}{m}$ & $\begin{array}{l}\text { sb rnd } \\
\text { sb ang }\end{array}$ \\
\hline OTHER & $\begin{array}{l}\geq \text { gravel } \\
\text { sand }\end{array}$ & $\frac{15}{30}$ & $\frac{v f p-c r p}{v f s-v c r s}$ & $\frac{m}{m}$ & $\frac{\text { rnd }}{s b \text { rnd }}$ \\
\hline
\end{tabular}

SIIT AND CLAY

3

REMARKS

color

Greyish Buff-Greyish Tan

HCL $+\left(80^{\prime}-90^{\prime}\right),-\left(90^{\prime}-120^{\prime}\right)$

Slightly Micaceous

Frosted Quartz

Sample intervals 90'-95',

Immature

100'-105' missing

\section{ITmature}

DEPTH

(FT.)

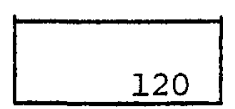

TO

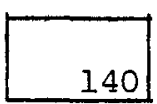

ROCK CIASSIFICATION

slightIy silty sand

\section{\% SIZE RANGE SPHERICITY ROUNDNESS}

\begin{tabular}{|c|c|c|c|c|c|}
\hline \multirow[t]{2}{*}{ BASALT } & \multirow{2}{*}{$\begin{array}{l}\geq \text { gravel } \\
\text { sand }\end{array}$} & 2 & $v f p-m p$ & 1 & rnd \\
\hline & & 38 & $v f s-v c r s_{1}$ & m & sb rnd \\
\hline \multirow[t]{2}{*}{ OTHER } & $\geq$ gravel & 2 & $v f p-m p$ & 1 & rnd \\
\hline & sand & 48 & ves-vars & $\mathrm{m}$ & $\mathrm{sb} \mathrm{rnd}$ \\
\hline
\end{tabular}

SIIT AND CIAY 10

REMARKS

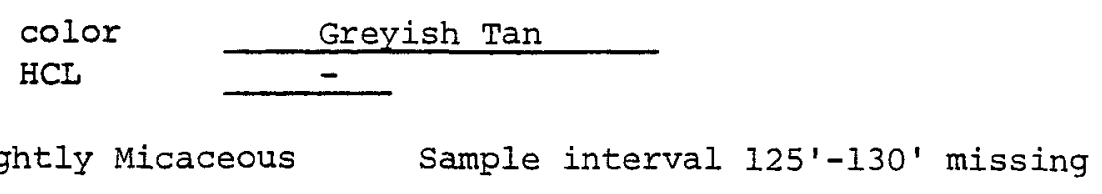


ROCK CLASSIFICATION

DEPTH

(FT.)

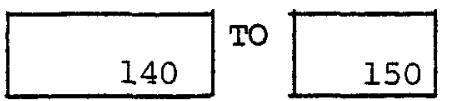

8 SIZE RANGE SPHERICITY ROUNDNESS

BASAIT $\underset{\text { sand }}{\geq \text { gravel }}$
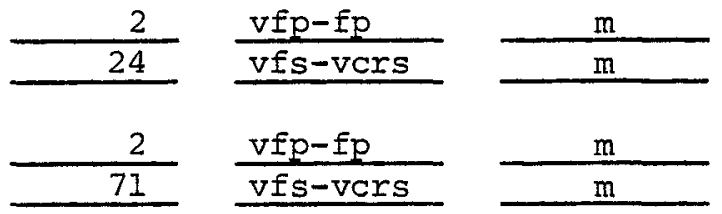

sb rnd

sb ang

OTHER $\geq$ gravel sand

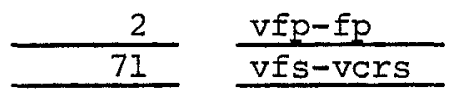

$\frac{\text { sb rnd }}{\text { sb ang }}$

Sand

SILT AND CIAY 1

REMARKS

color
HCI

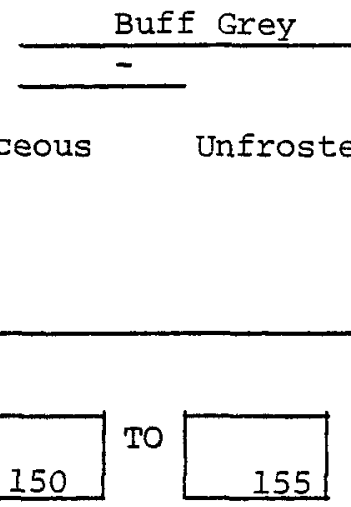

ROCK CLASSIFICATION

DEPTH

(EP.)

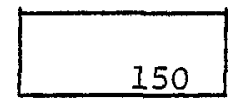

To

155

Slightly Micaceous Unfrosted quartz

Submature

\% SIZE RANGE SPHERICITY ROUNDNESS

\begin{tabular}{|c|c|c|c|c|c|}
\hline \multirow[t]{2}{*}{ BASAIT } & $\geq$ gravel & 20 & VEp-crp & 1 & $w$ rnd \\
\hline & sand & 21 & vfs-vers & $\mathrm{m}$ & sb rnd \\
\hline \multirow[t]{2}{*}{ OTHER } & $\geq$ gravel & 25 & $v f p-c r p$ & $\mathrm{~m}$ & w rnd \\
\hline & sand & 33 & vfs-vers & $\mathrm{m}$ & sb rnd \\
\hline
\end{tabular}

SIIT AND CLAY

1

REMARKS

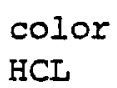

Buff Grey

HCI

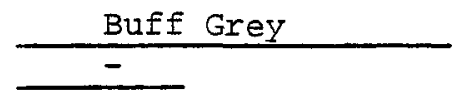

Slightly Micaceous

Frosted Quartz

Immature 
DEPTH

(FT.)

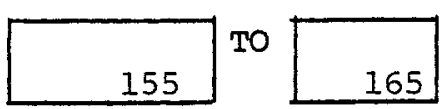

ROCK CLASSIFICATION

Sand

\section{\% SIZE RANGE SPHERICITY ROUNDNESS}

BASALT $\geq$ gravel
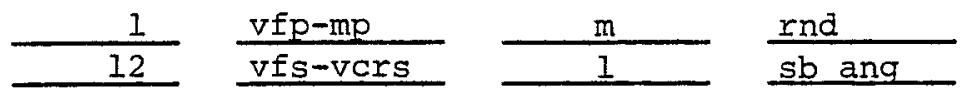

OTHER $\underset{\text { sand }}{\geq \text { gravel }}$
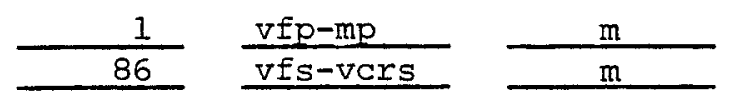

rnd

SIIT AND CLAY 0

REMARKS

$$
\begin{aligned}
& \text { color } \\
& \text { HCL }
\end{aligned}
$$

Greyish Buff

Slightly Micaceous

Unfrosted Quartz

Sample interval $155^{\prime}-160^{\prime}$ missing

Submature

Total Drilled Depth reported as $165^{\prime}$

DEPTH
(FT.)

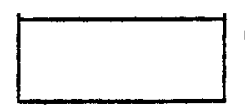

TO

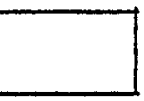

ROCK CIASSIFICATION

\section{\& SIZE RANGE SPHERICITY ROUNDNESS}

BASALT $\geq$ gravel

sand

OTHER $\geq$ grave1 sand
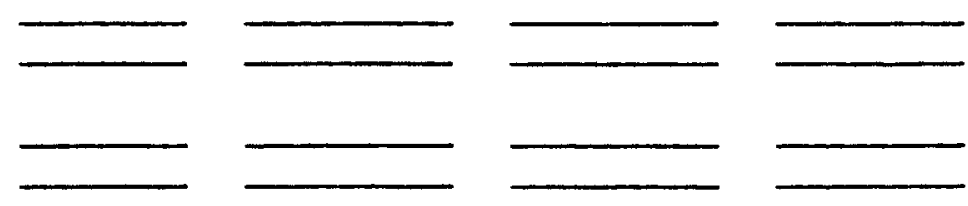

SILT AND CLAY

REMARKS

color

$\mathrm{HCL}$ 


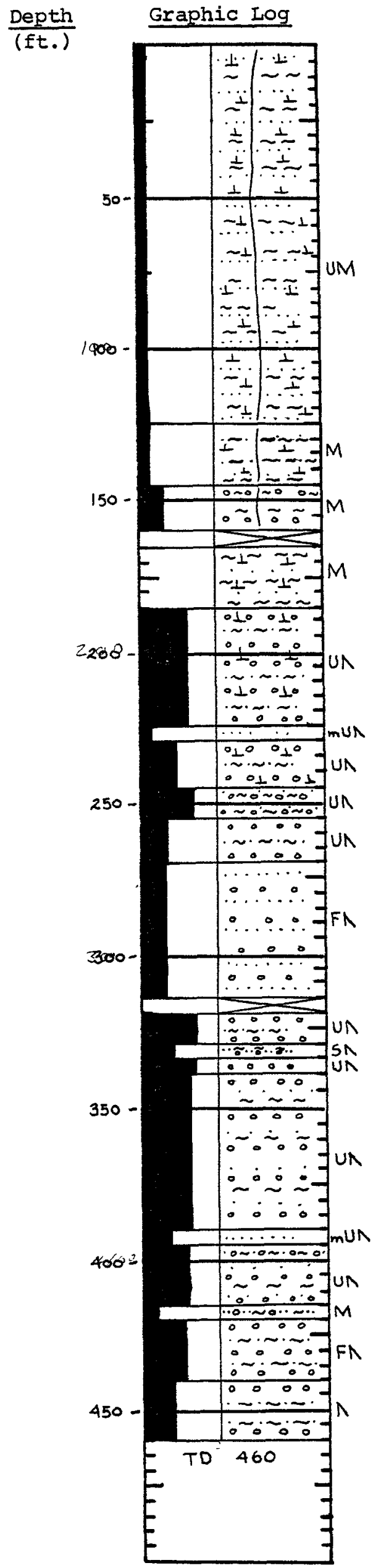

Sample Description

brownish buff

brownish $\tan$

brownish $\tan$

brownish $\tan$

greyish buff

buff

greyish butf

greyish buff

yellowish brown

yellowish buff

greyish buff

greyish buff

buft grey

greyish butf

greyish buff

greyish buff

greyish buff

greyish buff

greyish buff
Plant Coordinates

N 025258

$\frac{W 070080}{\text { Altitude } 628}$

Other Data used in Interpretation

(1) Descriptive log from $\left.\right|_{0} ^{1}$ Newcomb, et al , 1972, p.67-8

(2) Driller's Log

(3) Sieve analyses in MeHenry, 1957, 0.38 
DEPTH
(FT.)

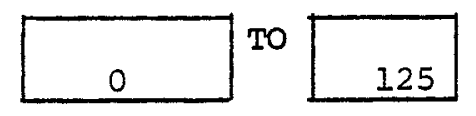

8 SIZE RANGE SPHERICITY ROUNDNESS

BASALT $\geq$ gravel sand

OTHER $\geq$ grave 1 sand

SILT AND CLAY
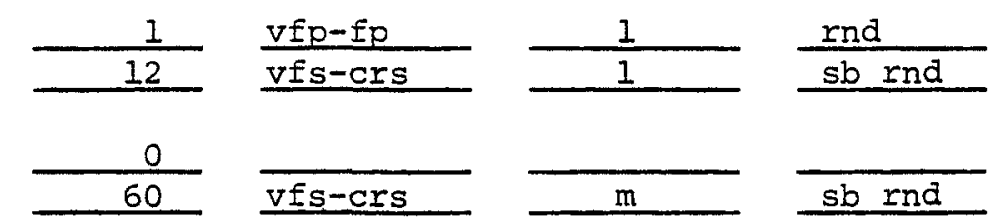

27

REMARKS

$$
\begin{aligned}
& \text { color } \\
& \text { HCL }
\end{aligned}
$$

Micaceous

Immature
ROCK CLASSIFICATION

silty sand

\section{REMARKS}

$\begin{array}{cl}\text { Color } & \frac{\text { Brownish Buff }}{+} \\ \text { Micaceous } & \text { Unfrosted Quartz }\end{array}$

compacted (?)

Imature
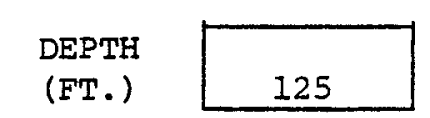

TO

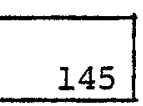

ROCK CLASSIFICATION

\% SIZE RANGE SPHERICITY ROUNDNESS
BASALT >gravel sand
OTHER >gravel sand
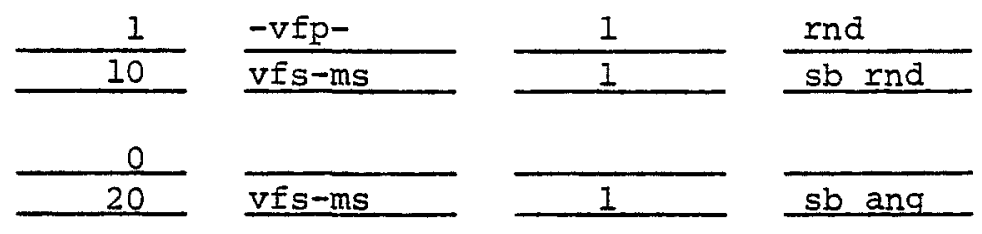

sb ang

SIIT AND CLAY 69

REMARKS

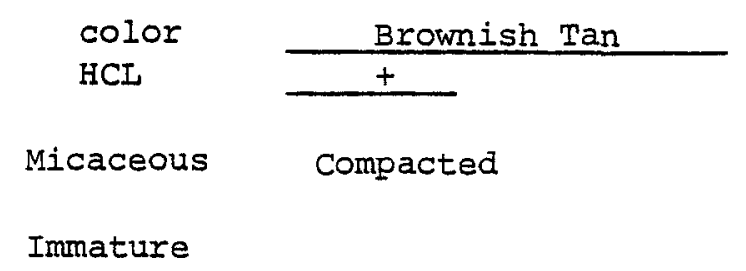




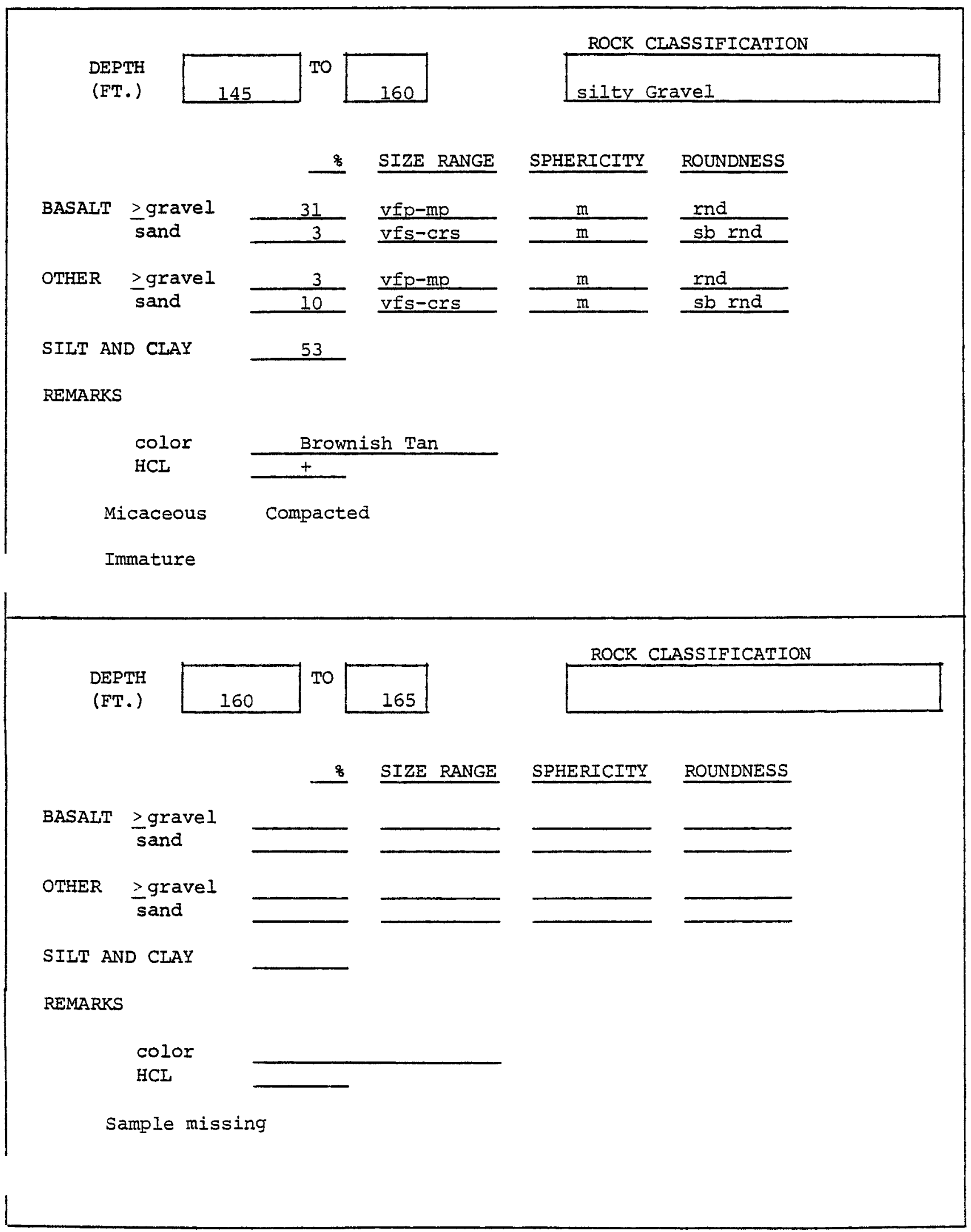


DEPTH

(ET.)

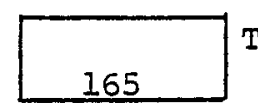

TO

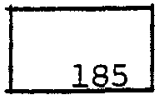

ROCK CIASSIFICATION

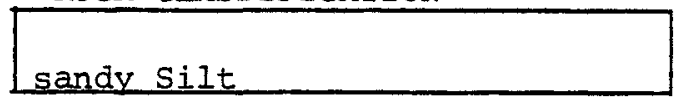

\section{SIZE RANGE SPHERICITY ROUNDNESS}

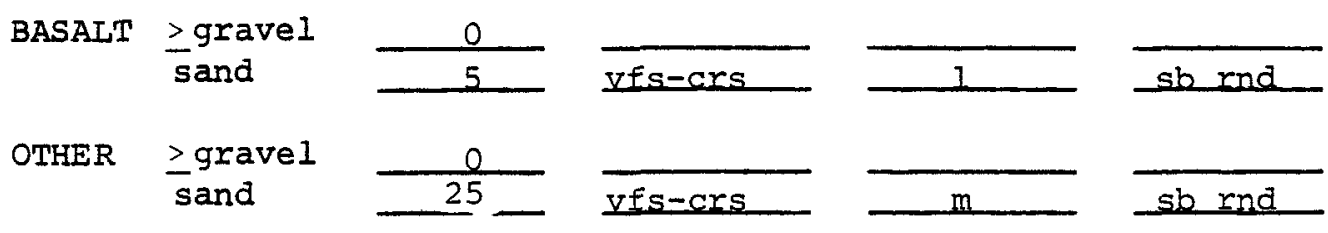

SILT AND CLAY

70

REMARKS

color

HCL

Brownish Tan

Micaceous

Immature

DEPTH

(FT.)

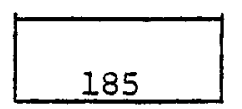

TO

225
ROCK CLASSIFICATION

silty sandy Gravel

官 SIZE RANGE SPHERICITY ROUNDNESS

BASALT $\geq \underset{\text { gravel }}{\text { sand }}$
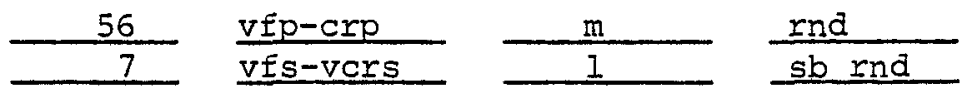

OTHER $\underset{\text { sand }}{\geq \text { gravel }}$
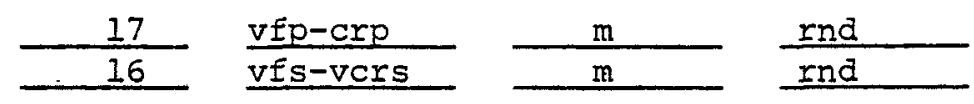

SILT AND CLAY

4

REMARKS

$$
\text { color }
$$

HCL

Greyish Buff

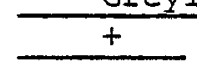

Slightly Micaceous

Unfrosted Quartz

Immature 


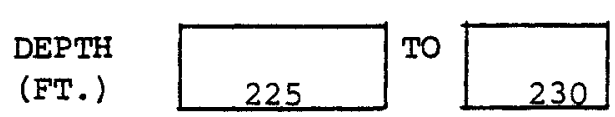

ROCK CLASSIFICATION

(FT.)

225

230

Sand

\% SIZE RANGE SPHERICITY ROUNDNESS

BASALT $\geq$ gravel sand
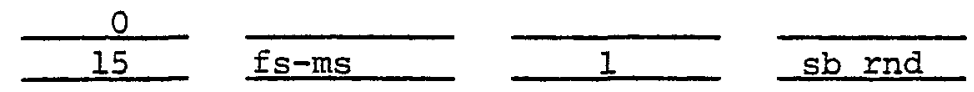

OTHER $\geq$ gravel
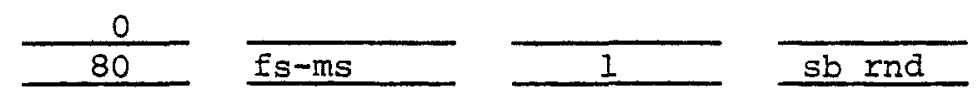

SILT AND CIAYY

REMARKS

color

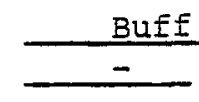

Slightly Micaceous

Unfrosted Quartz

Mature

\section{DEPTH}

(FT.)

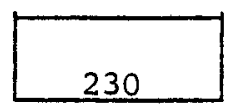

To

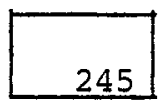

ROCK CLASSIFICATION

silty sandy Grave 1

\section{\% SIZE RANGE SPHERICITY ROUNDNESS}

BASAIT $\frac{\text { >gravel }}{\text { sand }}$
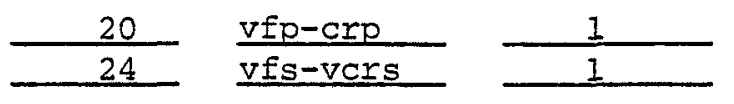

rnd

OTHER $\geq$ gravel sand

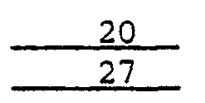

$\frac{v f p-c r p}{v f s-v c r s}$

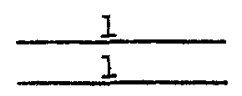

SIIT AND CLAY 9

REMARKS

$$
\text { color }
$$

HCL

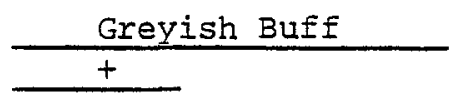

Slightly Micaceous

Unfrosted Quartz

Immature 


\section{DEPTH}

(FT.)

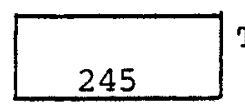

TO

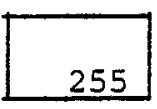

\section{ROCK CLASSIFICATION}

\section{silty sandy Gravel}

\section{\% SIZE RANGE SPHERICITY ROUNDNESS}

BASALT $\underset{\text { sand }}{\geq \text { grave } 1}$

OTHER $\geq$ gravel sand

SIIT AND CLAY
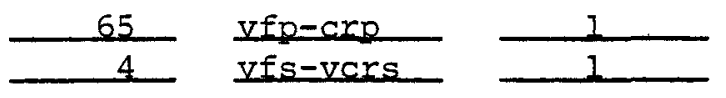

$\frac{\text { rnd }}{\text { sb rnd }}$
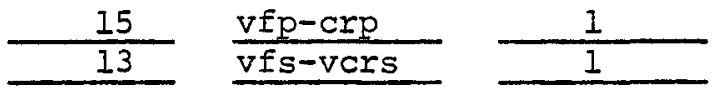

REMARKS

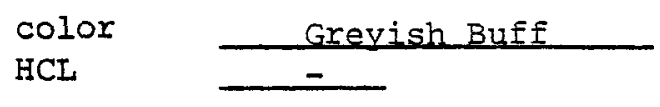

Slightly Micaceous Unfrosted quartz

Immature

DEPTH

(FT.)

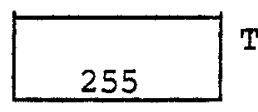

To

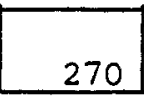

ROCK CLASSIFICATION

silty sandy Gravel

\section{\% SIZE RANGE SPHERICITY ROUNDNESS} BASALT $\underset{\substack{\text { sand } \\ \text { save } 1}}{\text { gand }}$ OTHER $\underset{\text { sand }}{\geq \text { gravel }}$
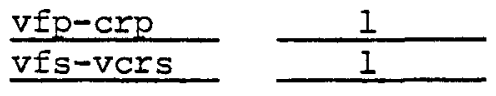

rnd

sb ang

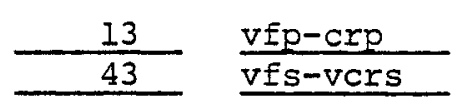

9

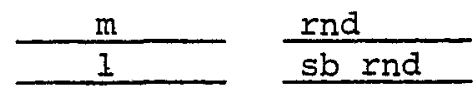

SILT AND CLAY

REMARKS

color

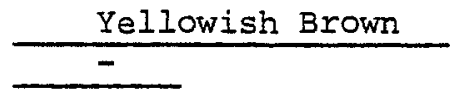

Slightly Micaceous

Unfrosted Quartz

Immature 
DEPTH
(FT. )

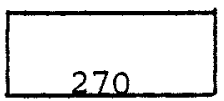

To

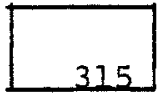

ROCK CLASSIFICATION

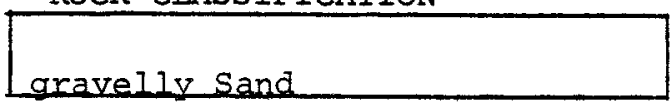

\section{\% SIZE RANGE SPHERICITY ROUNDNESS}

BASALT $\underset{\text { sand }}{\geq \text { gravel }}$
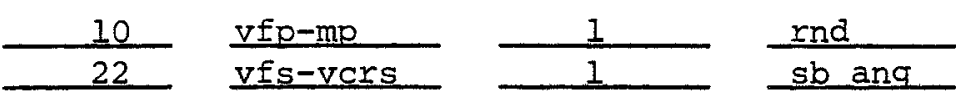

OTHER $\geq$ gravel sand
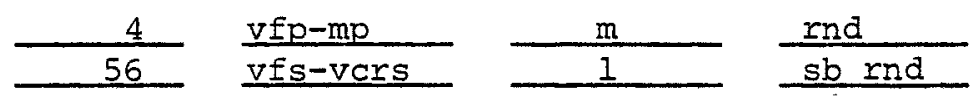

SIIT AND CLAY

8

REMARKS

color

HCL

Yellowish Buff

$-$

Slightly Micaceous Frosted Quartz

Immature

ROCK CLASSIFICATION

DEPTH

(FT.)
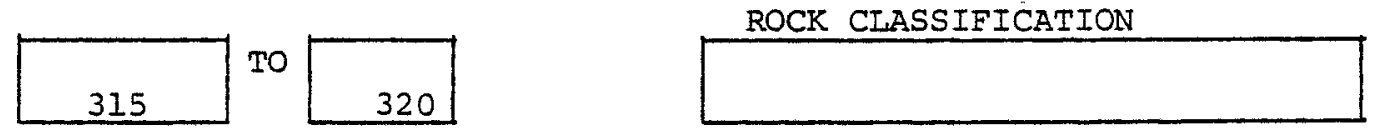

\% SIZE RANGE SPHERICITY ROUNDNESS

BASALT $\underset{\text { sand }}{\geq \text { gravel }}$

OTHER $\underset{\text { sand }}{\geq \text { gravel }}$

SILT AND CLAY

REMARKS

color

HCL

Sample missing 


$$
\text { DEPTH }
$$

(FT.)

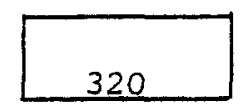

TO

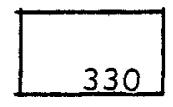

ROCK CLASSIFICATION

silty sandy Gravel

\& SIZE RANGE SPHERICITY ROUNDNESS

\begin{tabular}{|c|c|c|c|c|c|}
\hline BASALT & $\geq$ gravel & 60 & vfp-mp & $\frac{1}{1}$ & sb rnd \\
\hline OTHER & $\geq$ gravel & 5 & $\mathrm{v} f \mathrm{p}-\mathrm{mp}$ & $\mathrm{m}$ & rnd \\
\hline & sand & 19 & vfs-vcrs & 1 & rnd \\
\hline
\end{tabular}

SILT AND CLAY

REMARKS

Color
$\mathrm{HCL}$

Slightly Micaceous Unfrosted quartz

Immature

DEPTH

(FT.)

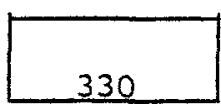

TO

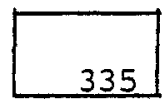

ROCK CLASSIFICATION

Gravelly silty sand

SIZE RANGE SPHERICITY ROUNDNESS

BASALT $\geq$ gravel
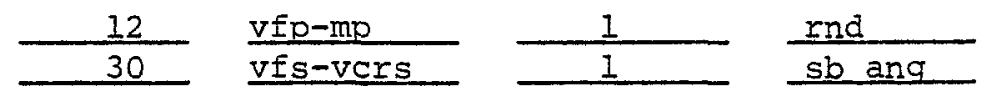

OTHER $\quad \frac{\text { gravel }}{\text { sand }}$
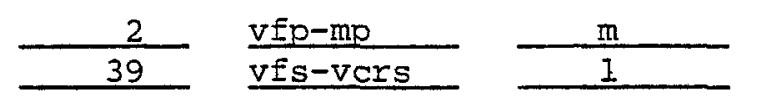

rnd

SILT AND CLAY 17

REMARKS

color

HCL Grevish Buff $-$

Slightly Micaceous

Immature 


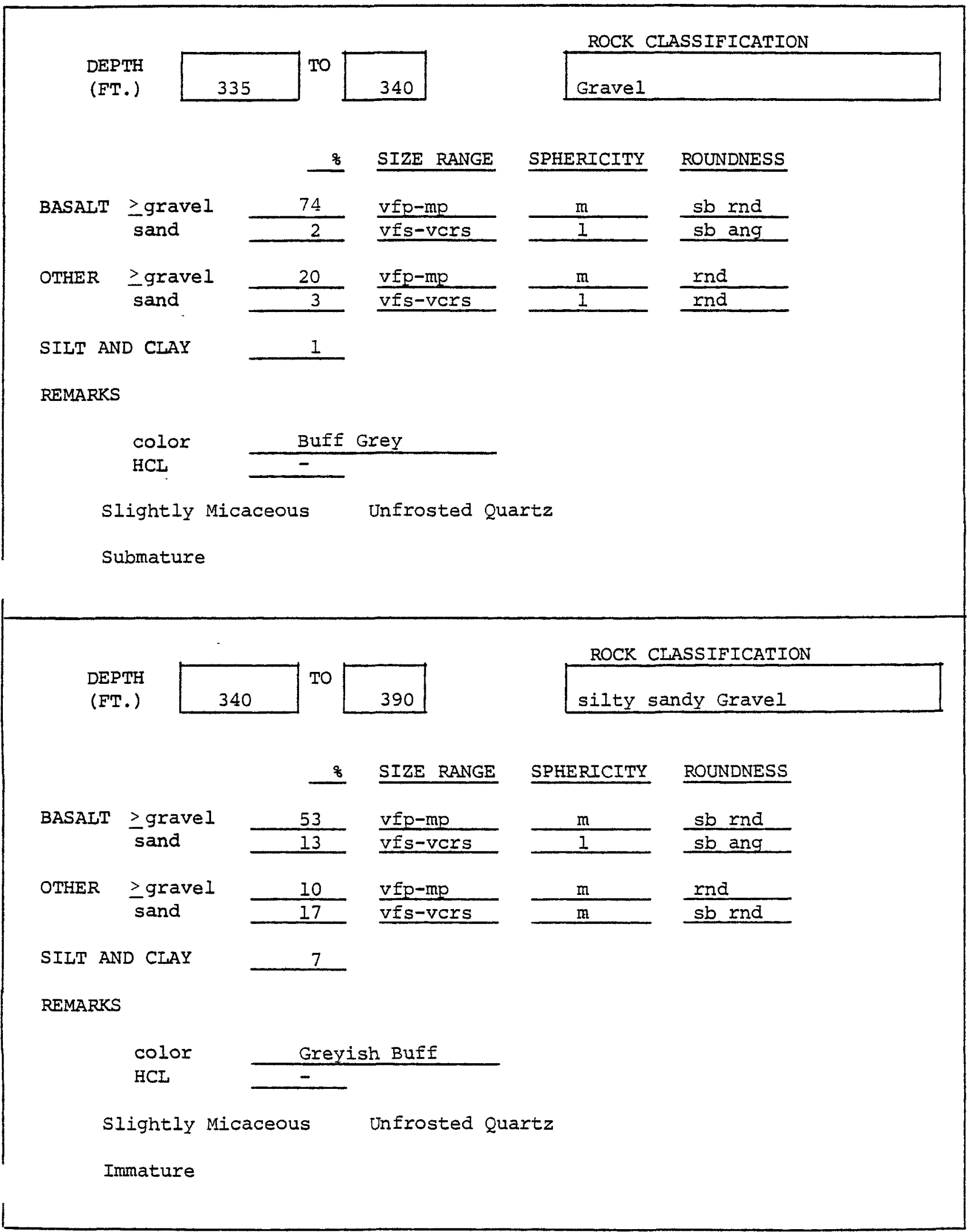


WELI NUMBER

DEPTH

(FT.)

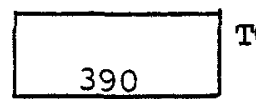

TO

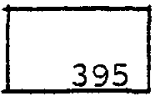

ROCK CLASSIFICATION

Sand

\section{\% SIZE RANGE SPHERICITY ROUNDNESS}

BASALT $\geq$ gravel sand
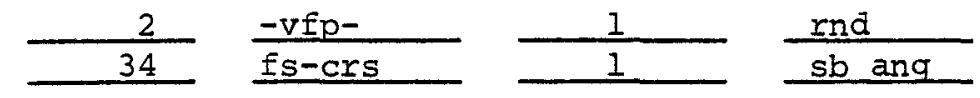

OTHER $\geq$ gravel
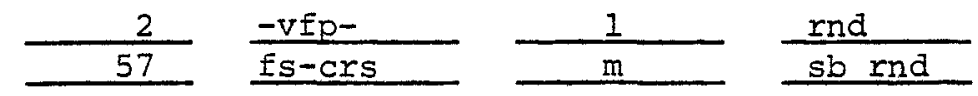

SILT AND CLAY 5

REMARKS

color

$\mathrm{HCL}$ Greyish Buff

Slightly Micaceous Unfrosted Quartz

Mature

\section{DEPTH}

(FT.)

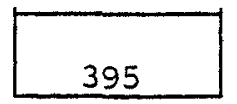

TO

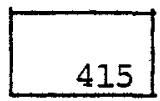

ROCK CLASSIFICATION

silty sandy Gravel

\section{\% SIZE RANGE SPHERICITY ROUNDNESS}

BASALT $\underset{\substack{\text { sand } \\ \text { gavel }}}{\text { gand }}$
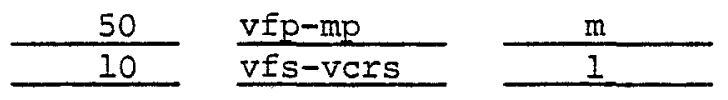

$\frac{\text { sb rnd }}{\text { sb ang }}$

OTHER $\geq$ gravel sand
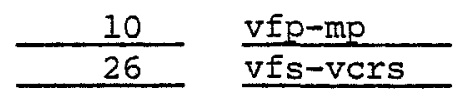

$$
\frac{\text { rnd }}{\text { sb rnd }}
$$

SILT AND CLAY

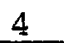

REMARKS

$$
\text { color }
$$

HCL

Greyish Buff

$\longrightarrow$

Slightly Micaceous

Unfrosted Quartz

Immature 
DEPTH

(FT.)

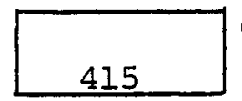

TO

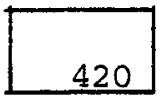

slightly silty slightly gravelly sand

\% SIZE RANGE SPHERICITY ROUNDNESS

BASALT $\frac{\geq \text { gravel }}{\text { sand }}$
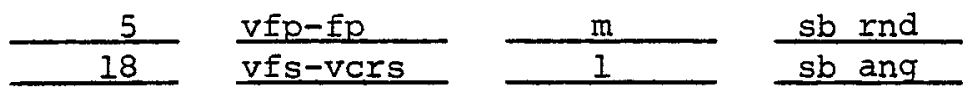

OTHER $\geq$ gravel sand

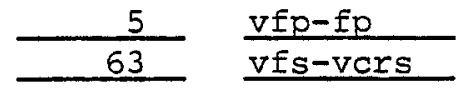

$\frac{m}{1} \frac{\text { sb rnd }}{\text { sb rnd }}$

SIIT AND CIAY

9

REMARKS

color Greyish Buff

HCL $-$

Micaceous

Immature

DEPTH

(FT.)

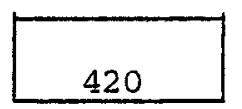

TO

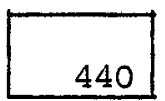

ROCK CLASSIFICATION

silty sandy Gravel

\section{SIZE RANGE SPHERICITY ROUNDNESS}

BASALT $\underset{\text { sand }}{\text { gravel }}$
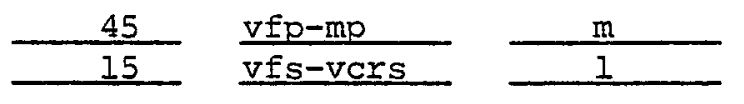

$\mathrm{sb}$ rnd

OTHER $\underset{\text { sand }}{\text { gravel }}$
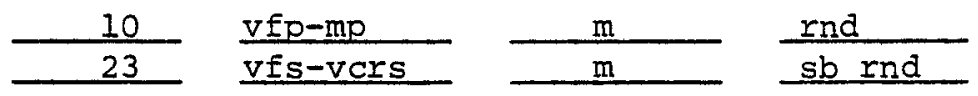

SILT AND CLAY

7

REMARKS

color

HCL

Greyish Buff

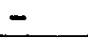

Slightly Micaceous Frosted quartz

Immature 
ROCK CLASSIFICATION

DEPTH

(FT.)

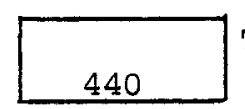
TO

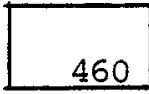

ROCK CLASSIFICAIION

silty sandy Gravel.

\section{\% SIZE RANGE NPHERICITY ROUNDNESS}

BASAIT $\frac{\geq \text { gravel }}{\text { sand }}$

$\frac{17}{23} \quad \frac{v f p-f p}{v f s-v e r s}$

$\frac{m}{1}$

$\frac{\text { sb rnd }}{\text { sb ang }}$

OTHER $\geq$ grave1 sand
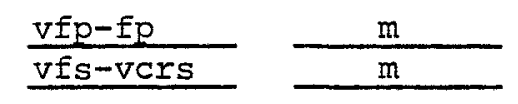

$\frac{\mathrm{sb} \text { rnd }}{\mathrm{sb} \text { rnd }}$

SIIT AND CLAY 7

REMARKS

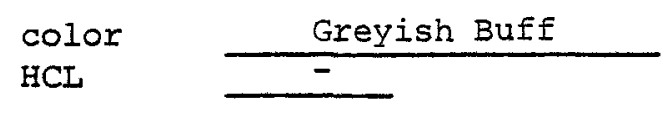

Slightly Micaceous

Immature

Total Drilled Depth reported as $460^{\prime}$
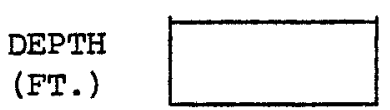

TO

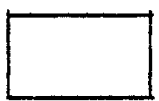

\% SIZE RANGE

BASALT $\geq$ gravel sand

OTHER $\geq$ gravel sand

SILT AND CLAY

REMARKS

color

HCL

\section{SPHERICITY ROUNDNESS}

ROCK CLASSIFICATION

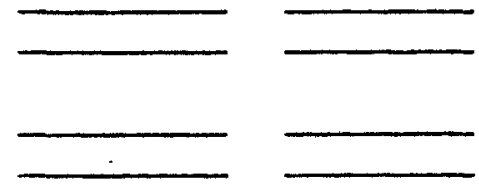


Sample Description

brownish butf

buff brown

buff brown

greyish brown buff

light grey

\section{ASH FRAGMENTS}

brownish black

greyish black

greyish $\tan$

whitish grey
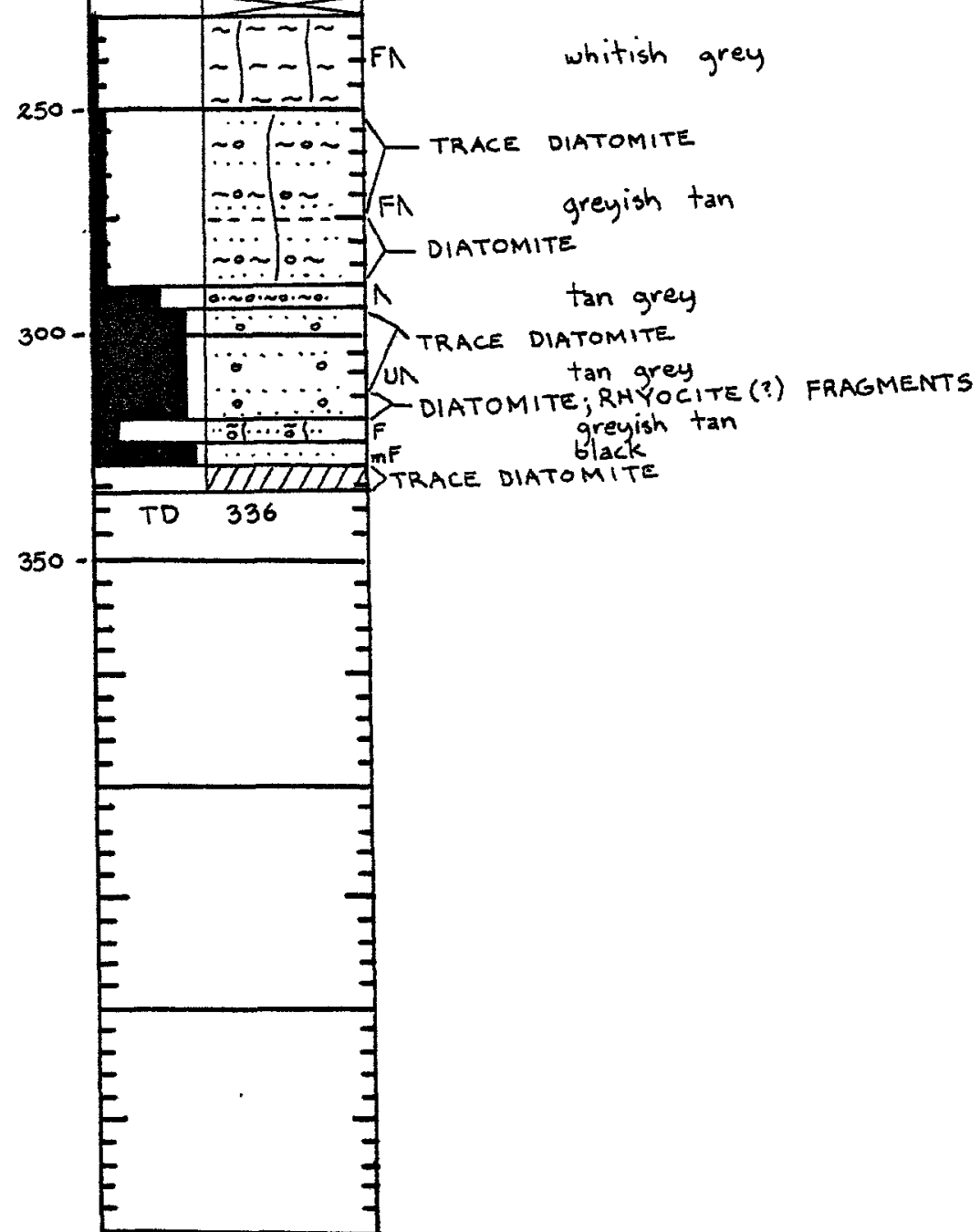

Plant Coordinates

N 025080

$\frac{W 079512}{\text { Altitude } 614}$

Other Data used in

Interpretation

(1) Descriptive Log from $\left.\right|^{1}$ Newcomb, et al, $1972, p .65$

(2):Driller's Log 
DEPTH

(FT.)

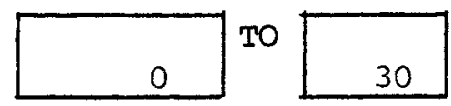

ROCK CLASSIFICATION

slightly silty sand

\section{$\%$ SIZE RANGE SPHERICITY ROUNDNESS}

\begin{tabular}{|c|c|c|c|c|c|}
\hline \multirow[t]{2}{*}{ BASALT } & $\geq$ gravel & 2 & $\mathrm{v} f \mathrm{p}-\mathrm{mp}$ & 1 & rnd \\
\hline & sand & 5 & vfs-vers & $\mathrm{m}$ & rnd \\
\hline \multirow[t]{2}{*}{ OTHER } & >gravel & 1 & $v f p$-mp & $\mathrm{m}$ & rnd \\
\hline & sand & 78 & vfs-vers & $\bar{m}$ & sb ang \\
\hline
\end{tabular}

SILT AND CLAY 14

REMARKS

color HCL Brownish Buff

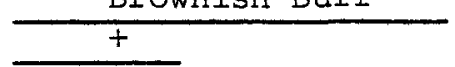

Slightly Micaceous Unfrosted Quartz

Immature

DEPTH

(FT.)

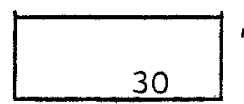
TO

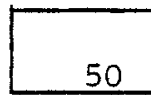

ROCK CLASSIFICATION

gravelly Sand

8 SIZE RANGE SPHERICITY ROUNDNESS

\begin{tabular}{|c|c|c|c|c|c|}
\hline BASALT & $\begin{array}{l}\geq \text { gravel } \\
\text { sand }\end{array}$ & $\frac{17}{15}$ & $\frac{v f p-c r p}{v f s-v c r s}$ & $\frac{1}{1}$ & $\frac{r n d}{s b r n d}$ \\
\hline OTHER & $\geq$ gravel & 9 & $v \mp p-m p$ & 1 & $w$ rnd \\
\hline & sand & 52 & vfs-vers & $\mathrm{m}$ & sb rnd \\
\hline
\end{tabular}

SILT AND CLAY

7

REMARKS

color

HCL

Buff Brown $+$

Caliche (pebbles present between $30^{\prime} \& 40^{\prime}$, sand)

Slightly Micaceous

Unfrosted Quartz

Immature

W. K. Summers \& Associdtes 


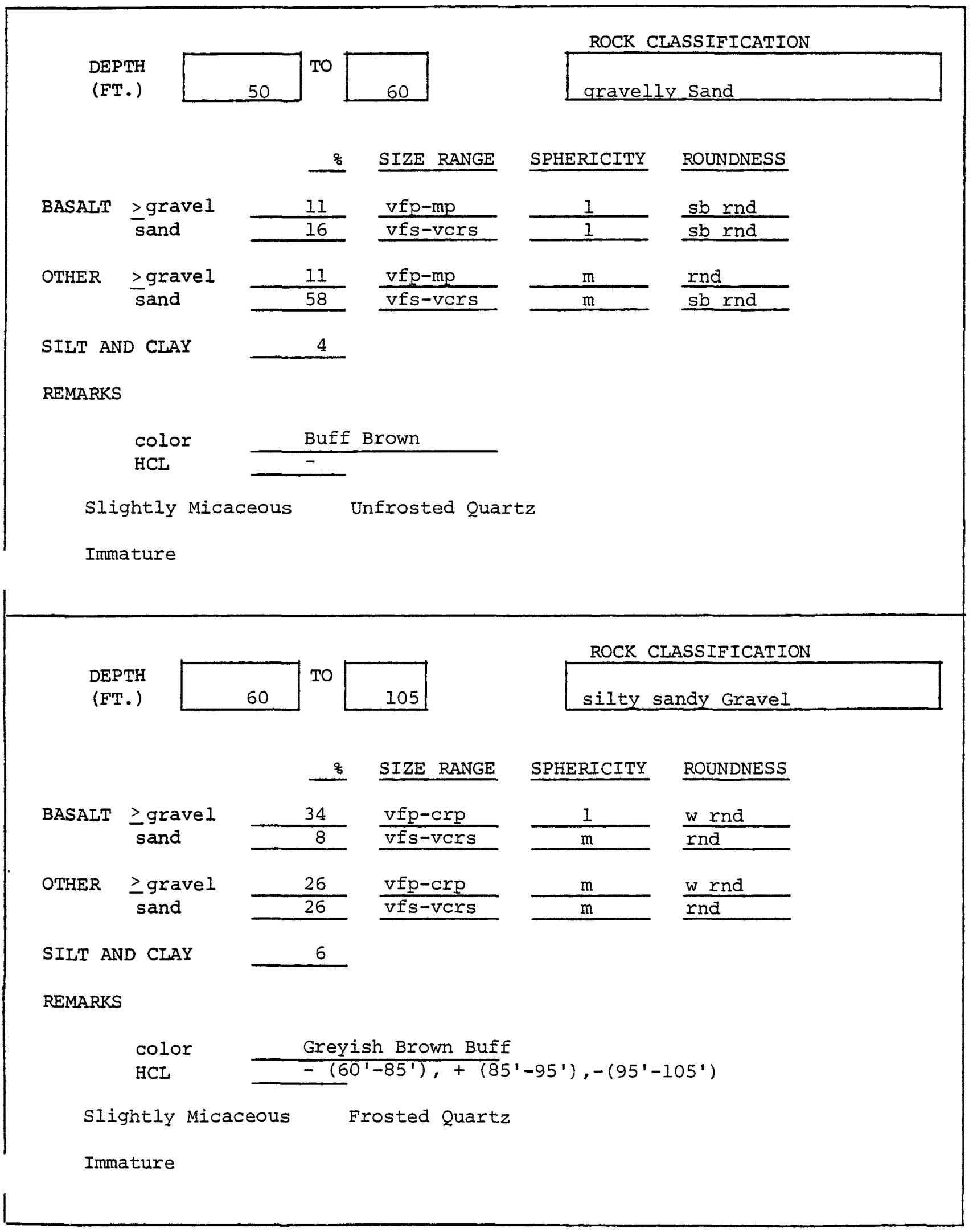

W. K. Summers \& Associates 


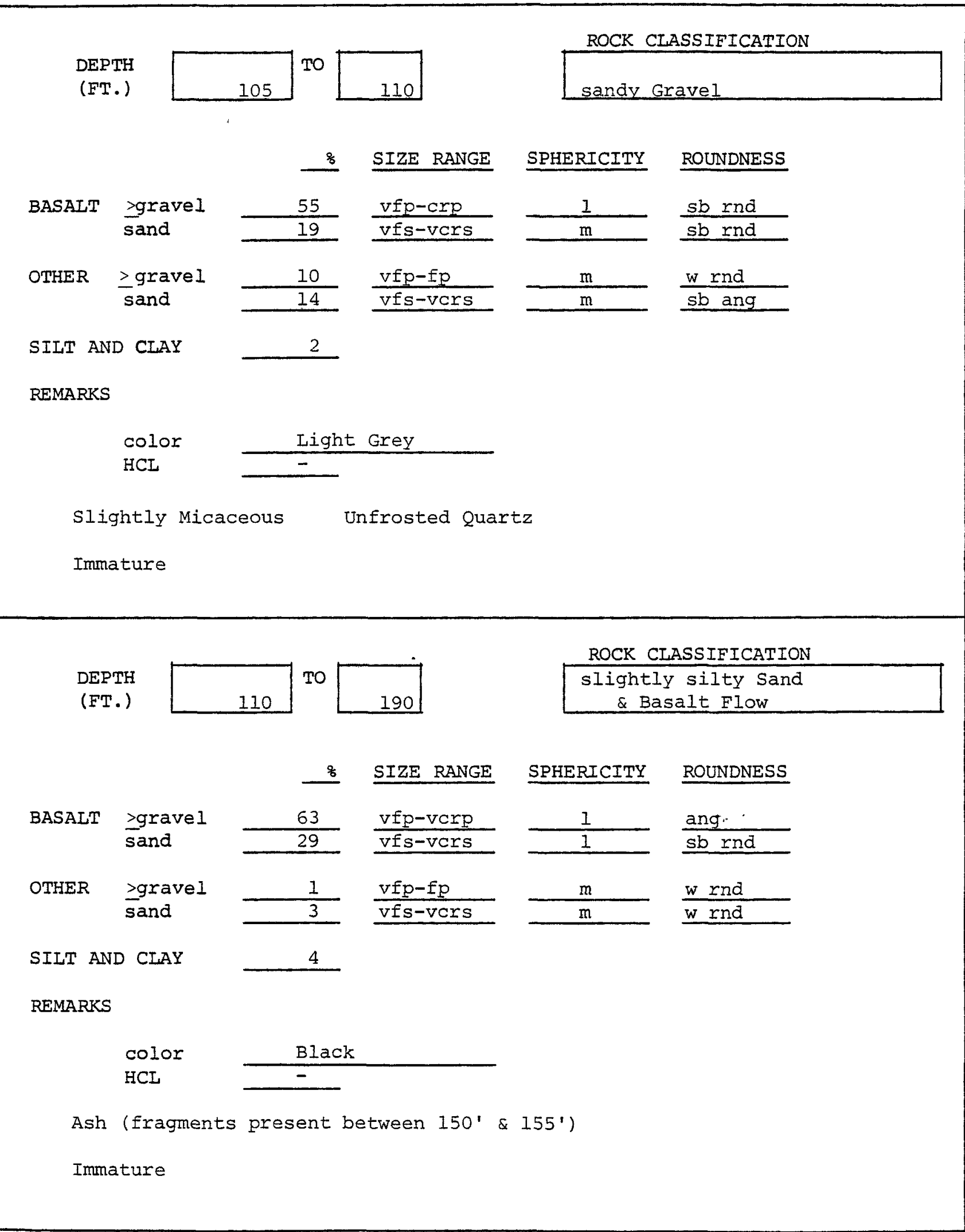




\section{ROCK CLASSIFICATION}

DEPTH

(FT.)

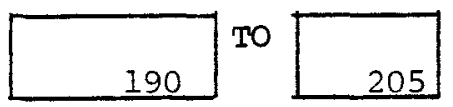

205

\section{SIZE RANGE SPHERICITY ROUNDNESS}

BASALT $\geq$ gravel
sand
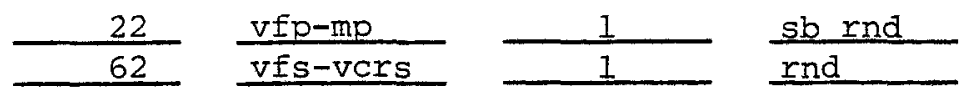

OTHER >gravel sand
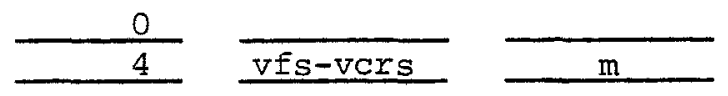

$\overline{\mathrm{w} \text { rnd }}$

SILT AND CLAY

\section{2}

REMARKS

color HCL Brownish Black

Immature

\section{DEPTH \\ (FT.)}

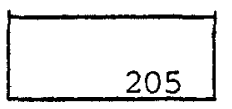

TO

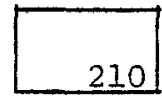

ROCK CLASSIFICATION

silty sandy Grave 1

\section{\% SIZE RANGE SPHERICITY ROUNDNESS}

BASALT

$\geq$ gravel

65 vfp-mp. sand
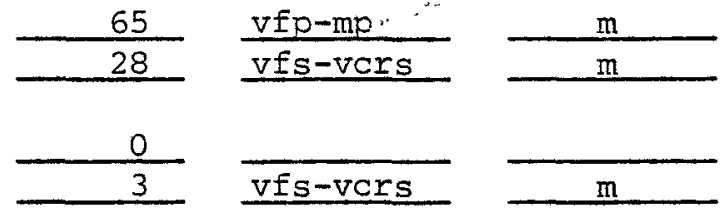

sb rnd

sb and

OTHER Igravel

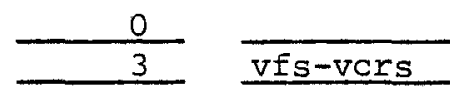

4

SIIT AND CLAY

$\underline{4}$

REMARKS

color Greyish Black

$\mathrm{HCL}$

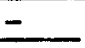

Immature 


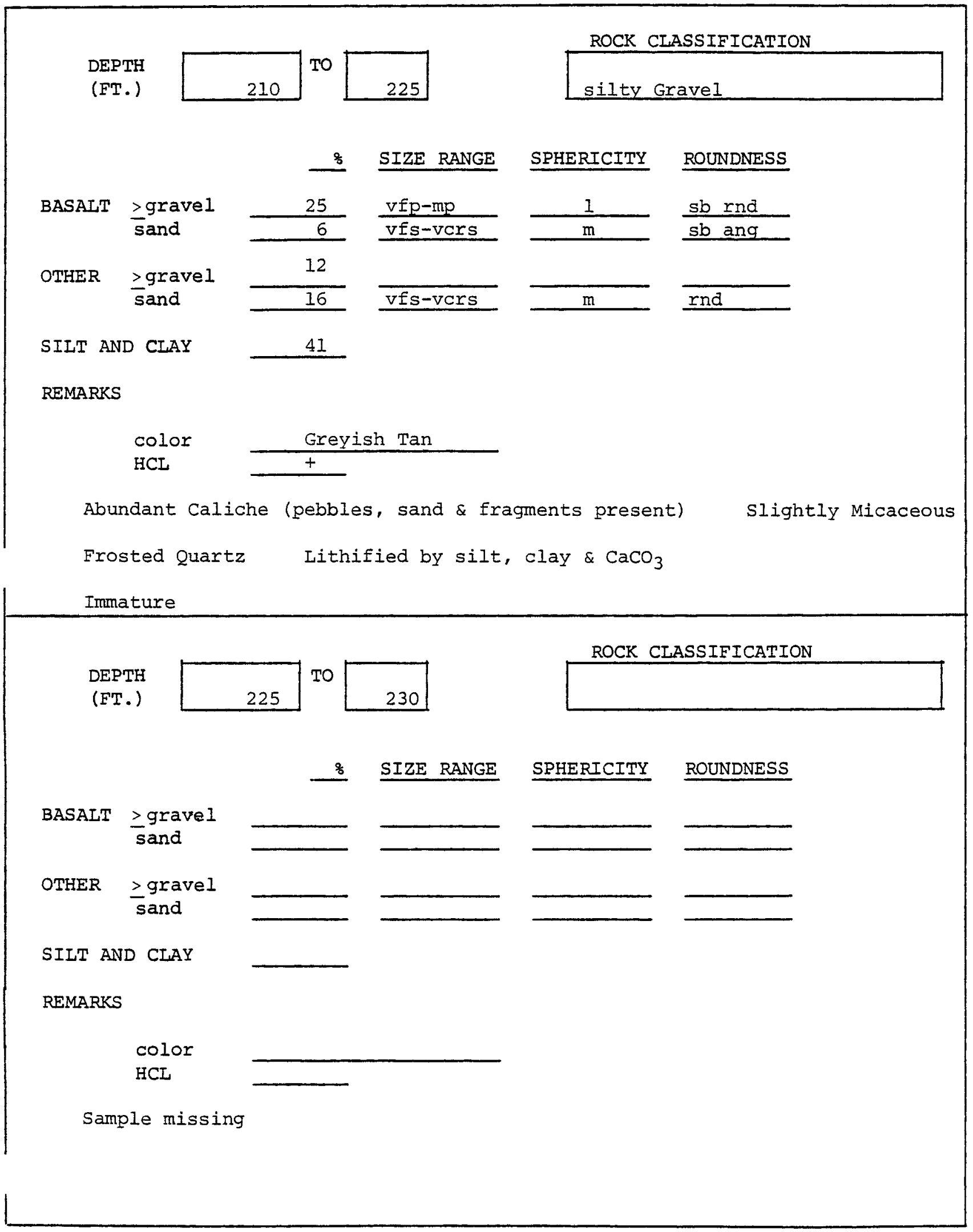




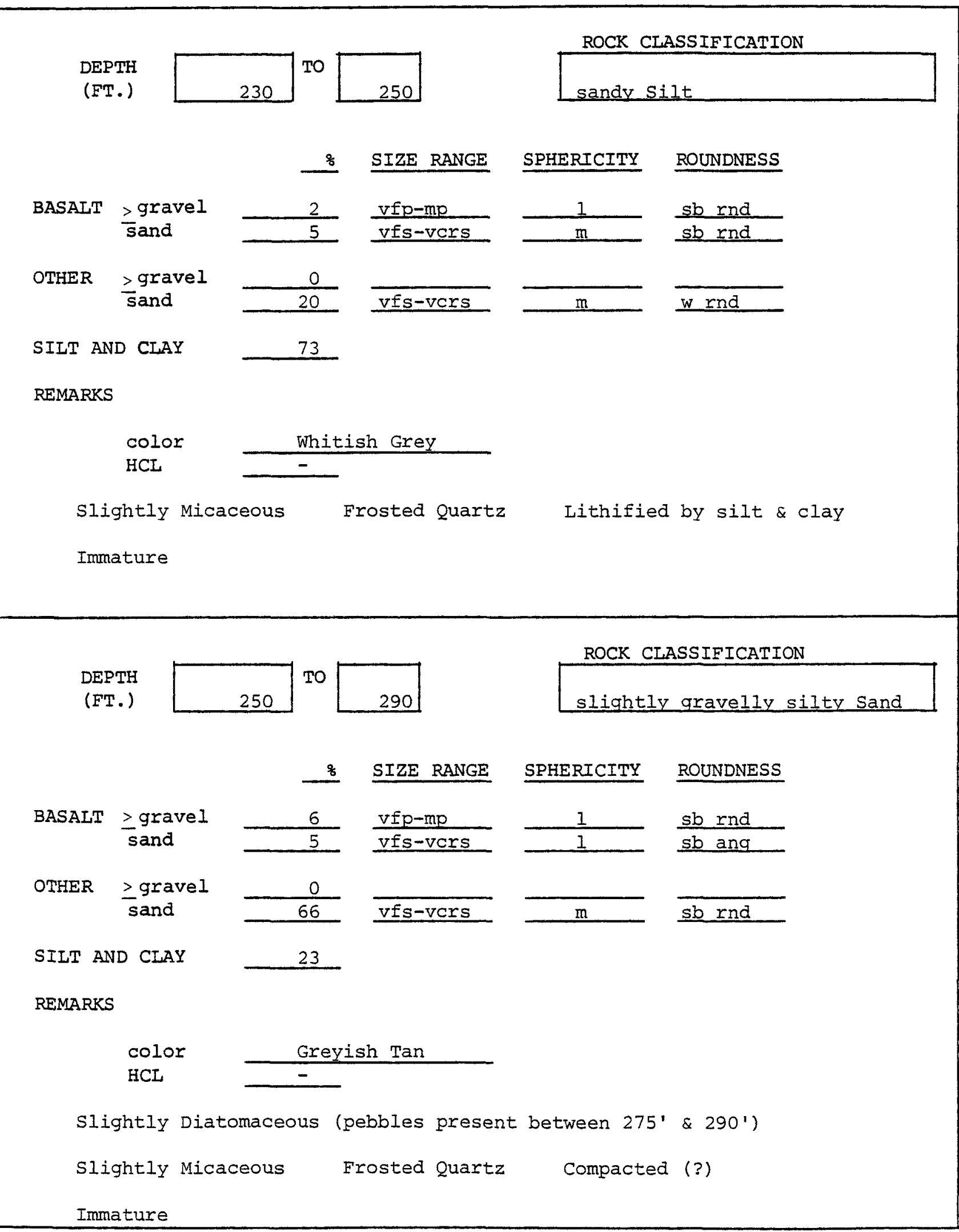


DEPTH

(FT.)

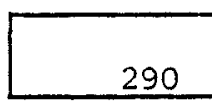

TO

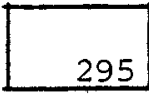

ROCK CLASSIFICATION

silty sandy Gravel

\& SIZE RANGE SPHERICITY ROUNDNESS

BASALT $\geq$ gravel sand

$\frac{40}{2 I} \frac{v f p-m p}{v i s-v e r s}-\frac{m}{m} \frac{\text { sb rnd }}{\text { sb rnd }}$

OTHER > gravel sand
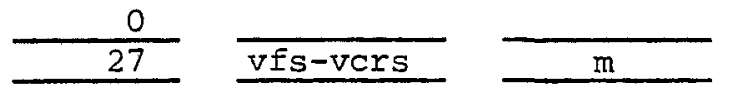
sb rnd

SILT AND CLAY 12

REMARKS

Color
HCL

SlightIy Micaceous

Immature

DEPTH

(FT.)

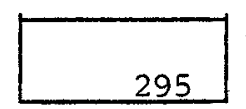

TO

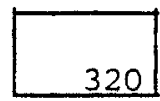

ROCK CIASSIFICATION

slightly gravelly sand

8 SIZE RANGE SPHERICITY ROUNDNESS

BASALT
$\frac{\text { sand }}{\text { sanel }}$
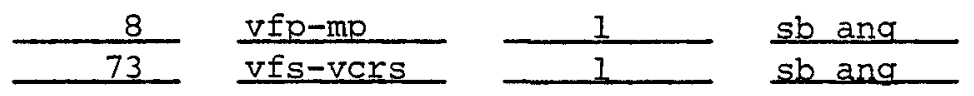

OTHER $\geq$ gravel
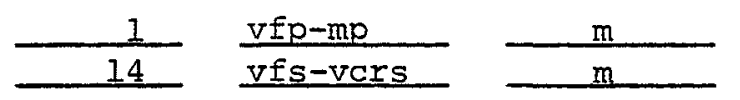

sb rnd sand 4

SILT AND CLAY

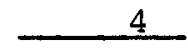

REMARKS

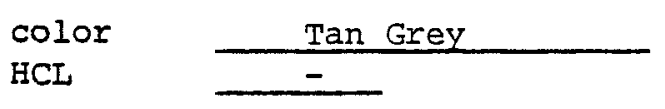

Slightly Diatomaceous (pebbles present between $315^{\prime} \& 320^{\prime}$, sand)

Slightly Micaceous Rhyolite (? fragments present) Unfrosted Quartz

Immature 

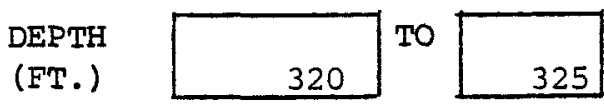

\section{\% SIZE RANGE}

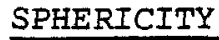

ROUNDNESS

BASAIT $\underset{\text { sand }}{\geq \text { gravel }}$
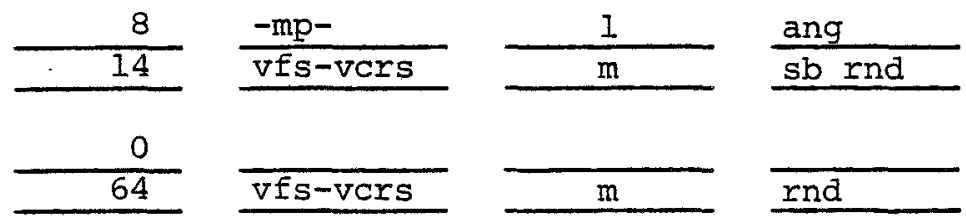

OTHER $\geq$ gravel sand 14

SIIT AND CLAY

REMARKS

$$
\text { color }
$$
HCL

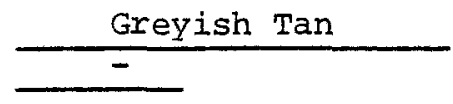

Frosted Quartz

Iithified by silt \& clay

Immature

DEPTH

(FT.)

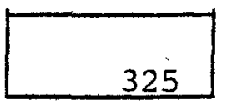

TO

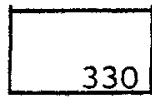

ROCK CLASSIFICATION

slightly silty slightly gravelly sand

(n)

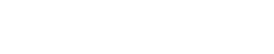

\section{\% SIZE RANGE SPHERICITY ROUNDNESS}

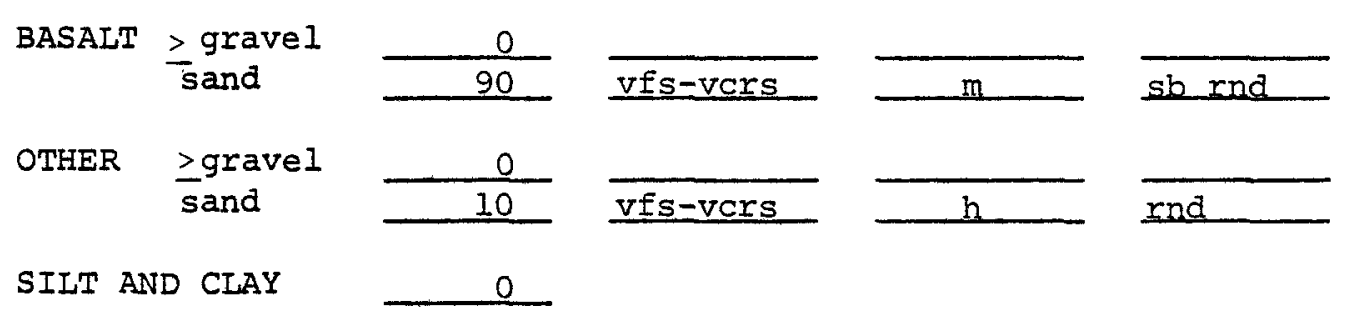

REMARKS

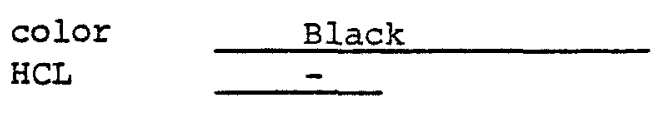

Frosted Quartz

Mature 
DEPTH

(FT.)

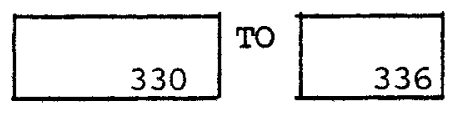

ROCK CLASSIFICATION

Sand \& Basalt Flow

\% SIZE RANGE SPHERICITY ROUNDNESS

BASALT $\geq$ gravel sand
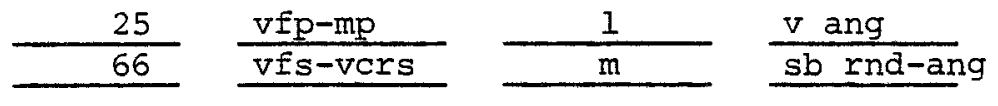

OTHER $\geq$ gravel sand
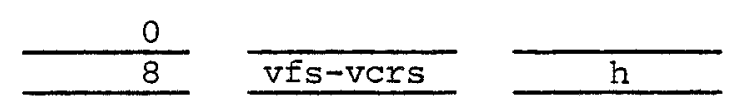

sb ang

SILT AND CLAY 1

REMARKS

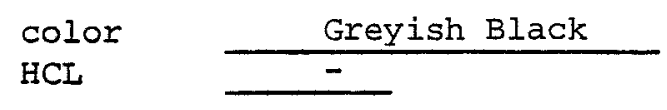

Slightly Diatomaceous (fragments present) Unfrosted Quartz

Immature

Total Drilled Depth reported as 336'

DEPTH

(FT.)

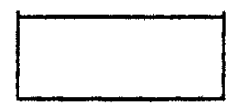

$\mathrm{TO}$

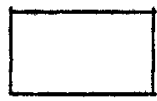

\& SIZE RANGE SPHERICITY ROUNDNESS
ROCK CIAASSIFICATION

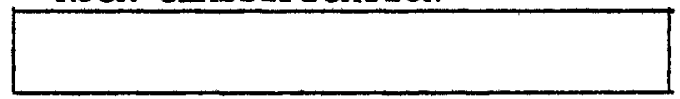
BASAIT $\frac{>\text { gravel }}{\text { sand }}$

OTHER $\geq$ gravel sand

SILT AND CLAY

REMARKS

color

$\mathrm{HCL}$
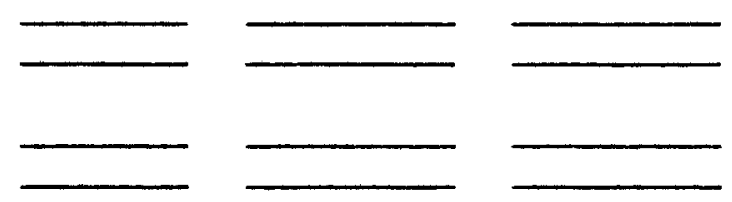


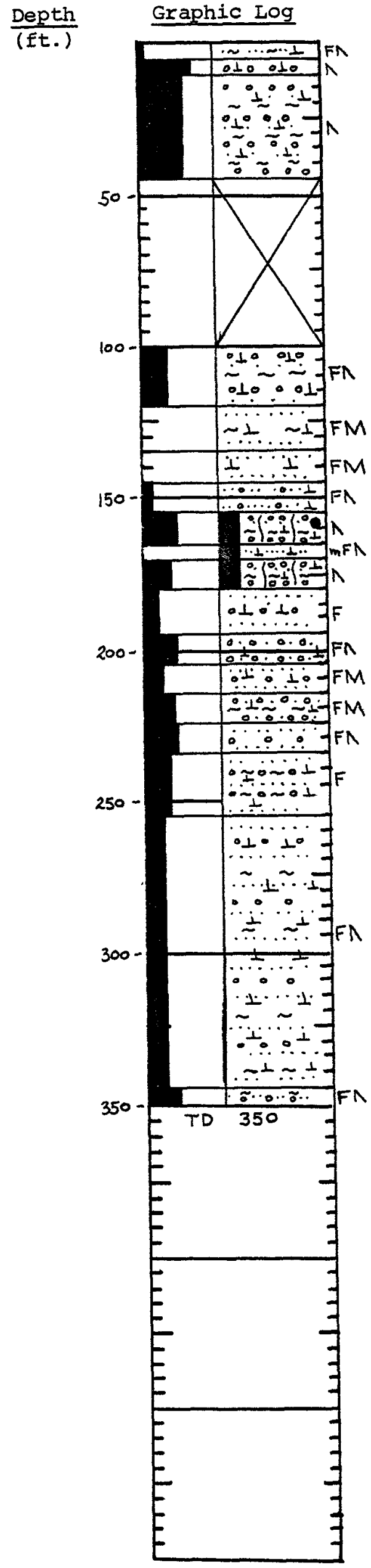

Sample Description

buff

$\tan$

$\tan$

Other Data used in Interpretation

(1) Driller's Log

(2) E-Logs

Plant Coordinates

N 025665

W 014554

Altitude 441 $\tan$

brownish buff

butf

buff

greenish grey

buff

greenish grey

greyish buff

brownish buff

brownish buff

brownish buff

brownish butf

greyish tan

greyish tan

light grey 
DEPTH

(FT.)
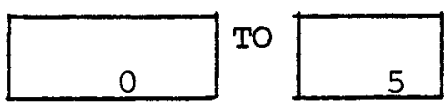

ROCK CLASSIFICATION

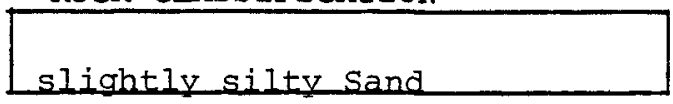

\& SIZE RANGE SPHERICITY ROUNDNESS

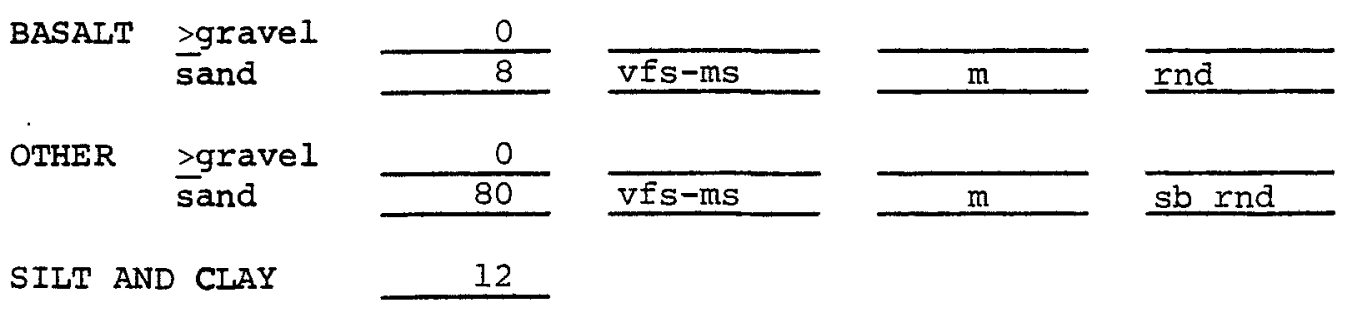

REMARKS

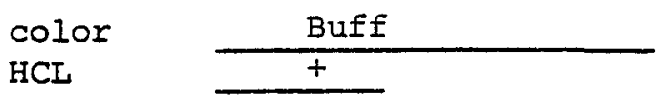

Slightly Micaceous Frosted Quartz

Immature

DEPTH

(FT.)

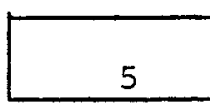

TO

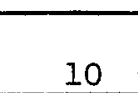

ROCK CLASSIFICATION

Grave1

\% SIZE RANGE SPHERICITY ROUNDNESS

BASALT >gravel
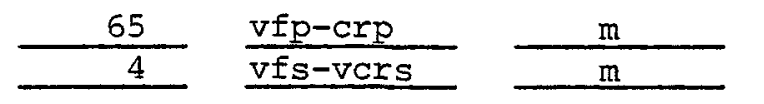

rnd

OTHER $\frac{\text { zgravel }}{\text { sand }}$
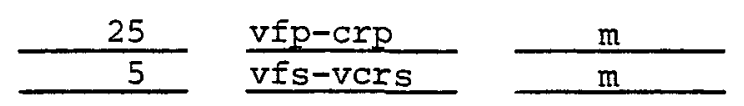

rnd

SILT AND CLAY

1

REMARKS

color

HCL

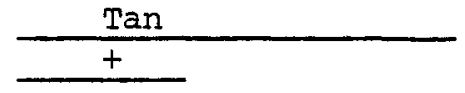

Slightly Micaceous

Immature 
ROCK CIASSIFICATION

DEPTH

(FT.)

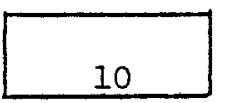

TO

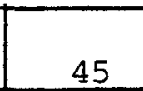

\section{silty sandy Gravel}

\section{\% SIZE RANGE SPHERICITY ROUNDNESS} BASALT $\underset{\text { sand }}{\geq \text { gravel }}$

OTHER $\quad$ gravel sand
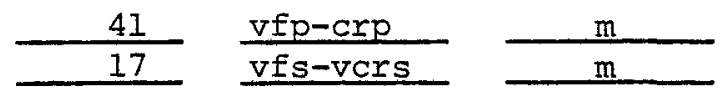

rnd
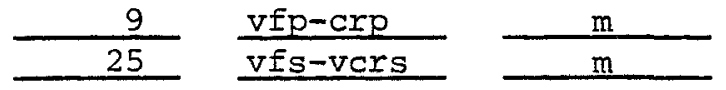

SILT AND CIAY

\section{8}

REMARKS

color

HCL

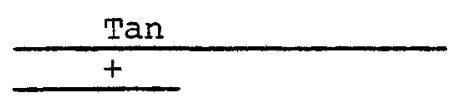

Slightly Micaceous

Immature

ROCK CIASSIFICATION

DEPTH

(ET.)

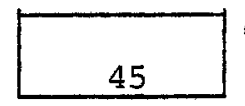

BASAIT $\geq$ gravel sand

OTHER Igravel sand

SIIT AND CIAY

REMARKS

color

HCL

Sample missing
TO

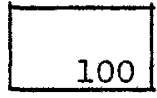

SIZE RANGE SPHERICITY ROUNDNESS

$\longrightarrow$
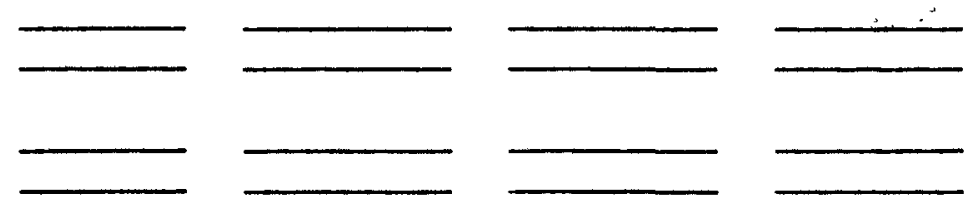
DEPTH
(FT.)

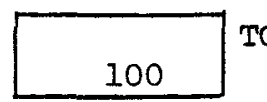

TO

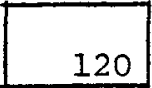

ROCK CIASSIFICATION

silty sandy Gravel

\section{\% SIZE RANGE SPHERICITY ROUNDNESS}

\begin{tabular}{|c|c|c|c|c|c|}
\hline \multirow[t]{2}{*}{ BASALT } & \multirow{2}{*}{$\begin{array}{l}\geq \text { gravel } \\
\text { sand }\end{array}$} & 28 & vfp-crp & m & rnd \\
\hline & & 7 & vfs-vers & $\mathrm{m}$ & $\mathrm{sb}$ rnd \\
\hline \multirow[t]{2}{*}{ OTHER } & \multirow{2}{*}{$\begin{array}{l}\geq \text { gravel } \\
\text { sand }\end{array}$} & 4 & vfp-crp & $\mathrm{m}$ & rnd \\
\hline & & 44 & vfs-vers & $\mathrm{m}$ & rnd \\
\hline
\end{tabular}

SILT AND CLAY 17

REMARKS

color

HCL

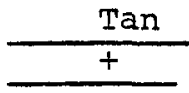

Slightly Micaceous

Frosted Quartz

Immature

DEPTH

(FT.)

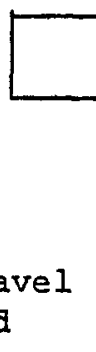

120

To

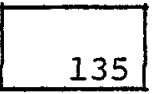

ROCK CLASSIFICATION

slightly silty sand

\% SIZE RANGE SPHERICITY ROUNDNESS

BASAIT $\underset{\text { sand }}{\geq \text { gravel }}$
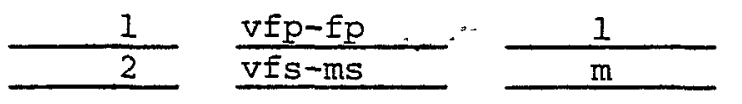

sb rnd

OTHER $\geq$ gravel
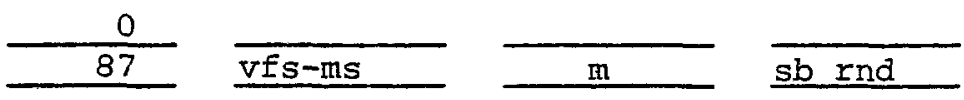

SILT AND CLAY 10

REMARKS

$$
\text { color }
$$

HCL

Brownish Buff

Micaceous

Frosted Quartz

Immature 


\section{DEPTH}

(FT.)

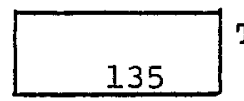
To

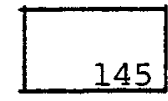

ROCK CIASSIFICATION

Sand

\section{SIZE RANGE SPHERICITY ROUNDNESS}

BASALT $\geq$ grave 1 sand
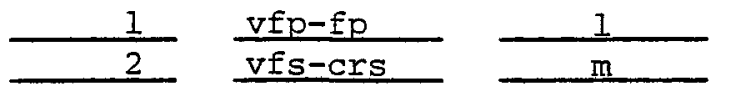

sb rnd.

OTHER $\geq$ graveI
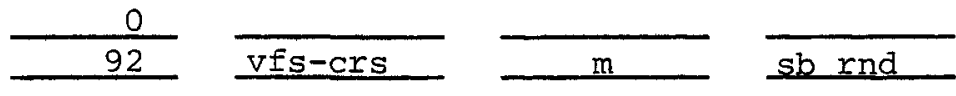

SILT AND CLAY 5

REMARKS

color
HCL

Micaceous

Mature
Buff

$+$

\section{Frosted Quartz}

DEPTH

(FT.)

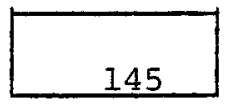

To

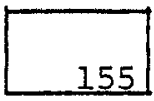

ROCK CLASSIFICATION

slightly gravelly sand

\% SIZE RANGE SPHERICITY ROUNDNESS

BASALT

$$
\begin{aligned}
& \text { >gravel. } \\
& \text { sand }
\end{aligned}
$$

$\frac{4}{9}$
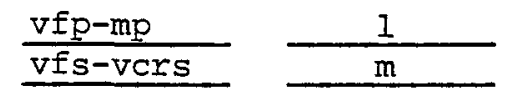

rnd

OTHER >gravel

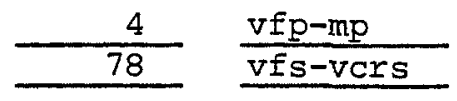

$\frac{1}{\mathrm{~m}}$

rnd

sb rnd

SILT AND CLAY

5

REMARKS

color

Buff

HCL

$\frac{\text { Buff }}{+}$

Slightly Micaceous

Frosted Quartz

Immature 


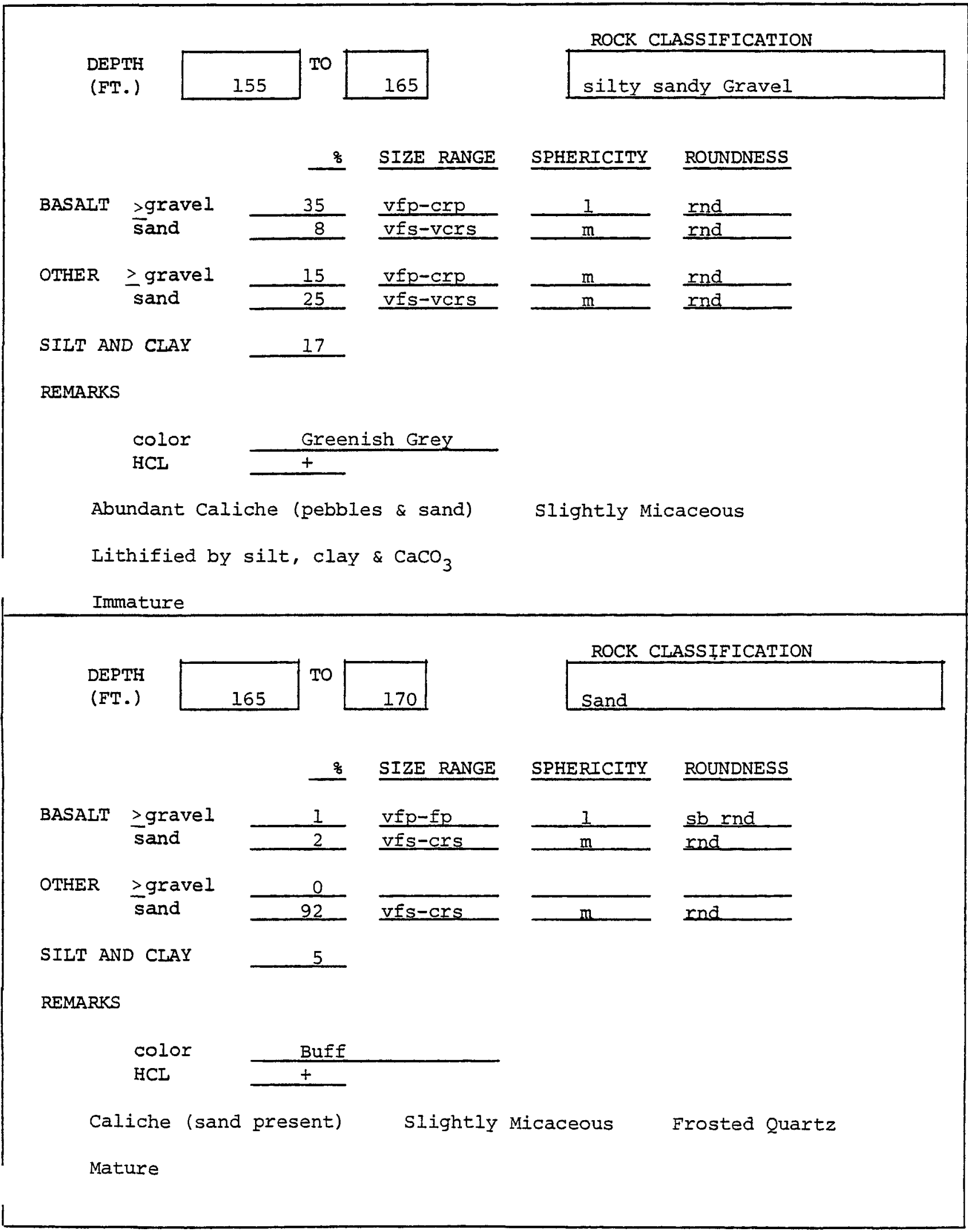


DEPTH

(ET.)

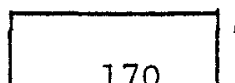

TO

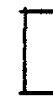

ROCK CIASSIFICATION

silty sandy Gravel

\section{- SIZE RANGE SPHERICITY ROUNDNESS}

BASALT > gravel sand

OTHER $\geq$ grave1 sand

SIIT AND CLAY

REMARKS

color

HCL

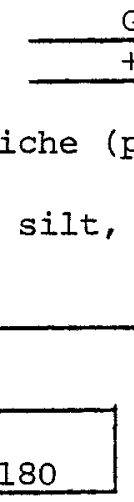

Greenish Grey

$+$

Abundant Caliche (pebbles \& sand)

Slightly Micaceous

Lithified by silt, clay $\& \mathrm{CaCO}_{3}$

Immature
DEPTH

(FT.)

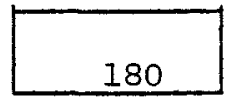

TO

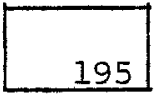

ROCK CIASSIFICATION

gravel1y sand

\section{SIZE RANGE SPHERICITY ROUNDNESS}

BASALT

$\geq$ gravel sand

$6 \quad \mathrm{vfp}-\mathrm{f}$

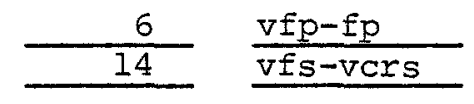

\begin{tabular}{c}
$\frac{\mathrm{m}}{\mathrm{l}}$ \\
\hline $\mathrm{m}$ \\
\hline $\mathrm{m}$
\end{tabular}

$\frac{\text { sb rnd }}{\text { sb rnd }}$

OTHER $\geq$ gravel

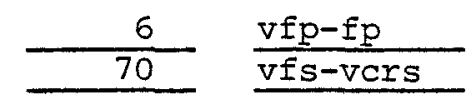

sb rnd

sb rnd

SILT AND CIAY

4

REMARKS

$\begin{array}{ll}\text { color } & \text { Greyish Buff } \\ \text { HCL } & +\end{array}$

Frosted Quartz

Immature 


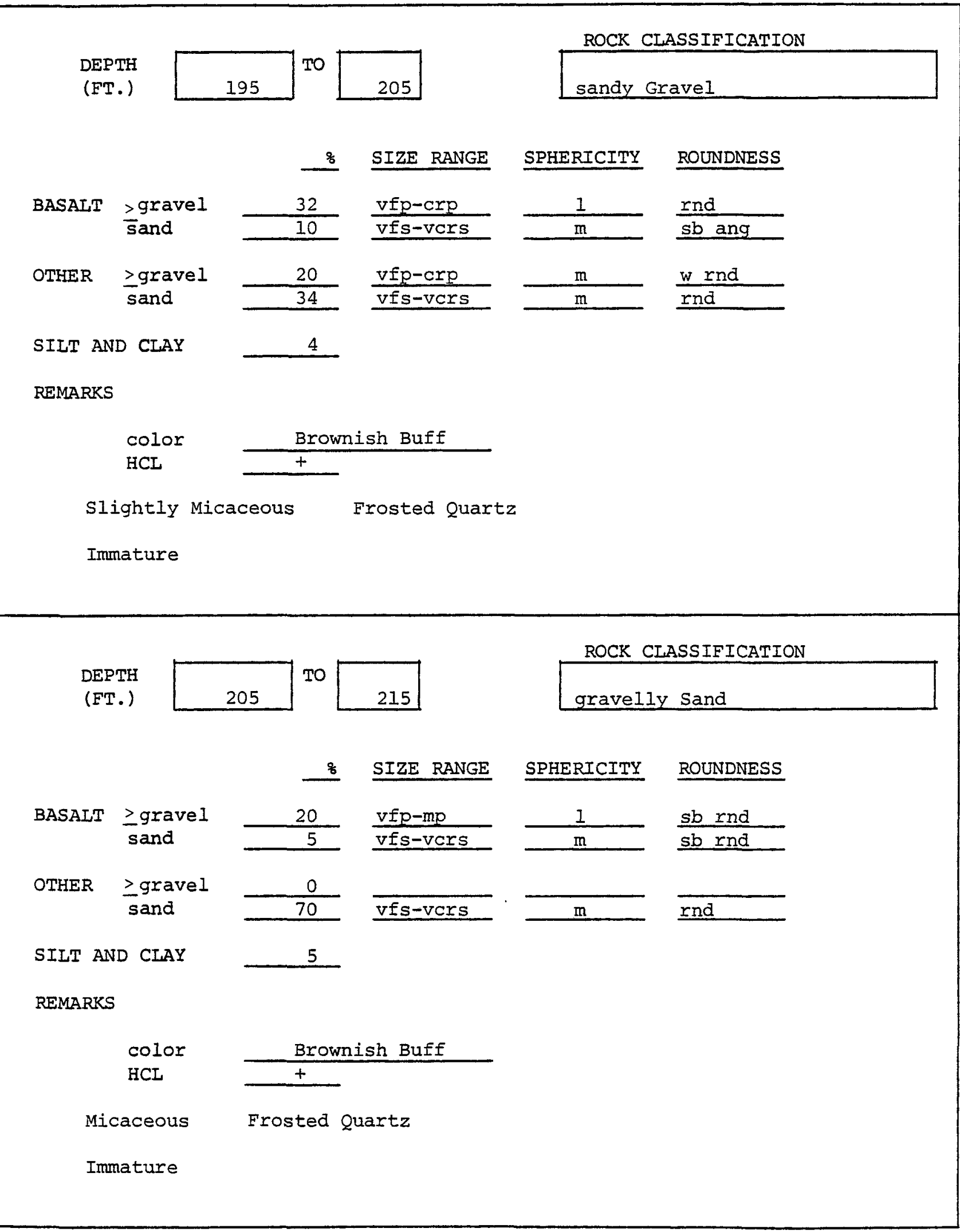

W. K. Summers \& Associates 
DEPTH

(FT.)

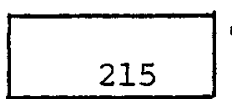

TO

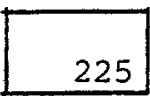

225
ROCK CLASSIFICATION

silty sandy Gravel

\section{SIZE RANGE SPHERICITY ROUNDNESS}

\begin{tabular}{|c|c|c|c|c|c|}
\hline BASALT & $\begin{array}{l}\geq \text { gravel } \\
\text { sand }\end{array}$ & $\frac{33}{8}$ & $\frac{v f p-m p}{v f s-v c r s}$ & $\frac{1}{m}$ & sb rnd \\
\hline OTHER & $\geq$ gravel & $\frac{12}{41}$ & $\frac{v f p-c r p}{v f s-v c r s}$ & $\frac{1}{m}$ & $\frac{\mathrm{sb} \text { rnd }}{\mathrm{rnd}}$ \\
\hline
\end{tabular}

SILT AND CLAY

\section{6}

REMARKS

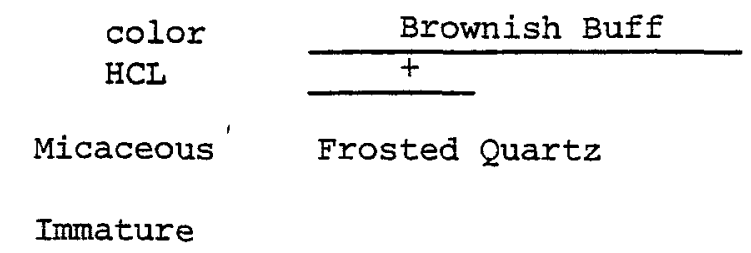

DEPTH

(FT.)

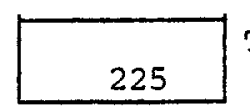

TO

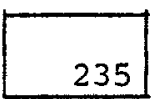

ROCK CLASSIFICATION

gravel1y Sand

S SIZE RANGE SPHERICITY ROUNDNESS

BASALT $\begin{aligned} & \geq \text { gravel } \\ & \text { sand }\end{aligned}$
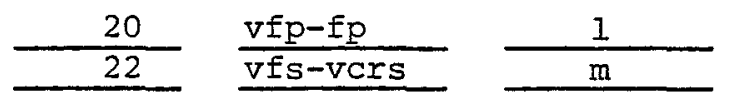

sb rnd
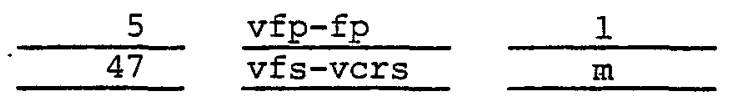

sb rnd

OTHER $\frac{\geq \text { grave1 }}{\text { sand }}$

6

SILT AND CLAY

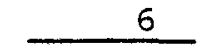

REMARKS

color

Brownish Buff

$\mathrm{HCL}$

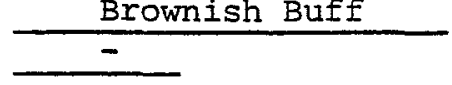

Slightly Micaceous Frosted quartz

Immature 


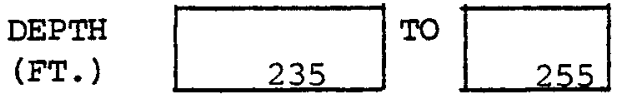

\section{SIZE RANGE SPHERICITY ROUNDNESS}
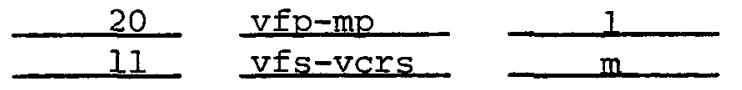

OTHER $\geq$ gravel sand
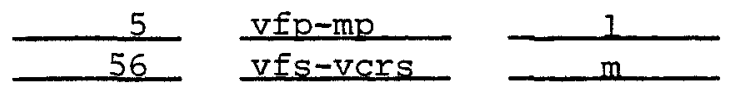

SILT AND CLAY 8

REMARKS

color HCL Greyish Tan $+$

Slightly Micaceous Frosted Quartz

Immature

DEPTH
(FT.)

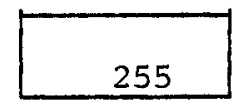

TO

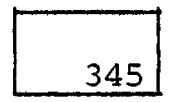

ROCK CLASSIFICATION

slightly silty gravelly sand

8 SIZE RANGE SPHERICITY ROUNDNESS

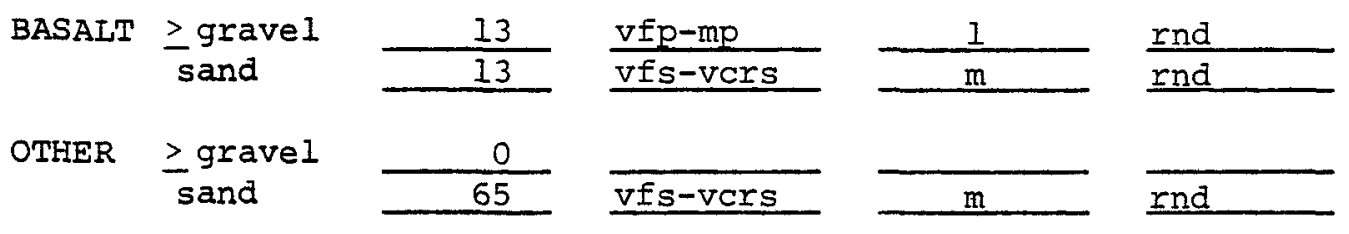

SIIT AND CLAY

9

REMARKS

color Greyish Tan

HCI

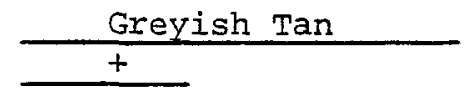

Slightly Micaceous

Frosted Quartz

Immature 
ROCK CLASSIFICATION

DEPTH

(ET.)

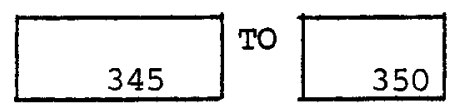

ROCK CIASSIFICAIION

slightly silty gravelly sand

\section{\% SIZE RANGE SPHERICITY ROUNDNESS}

BASAIT $\frac{\geq \text { gravel }}{\text { sand }}$
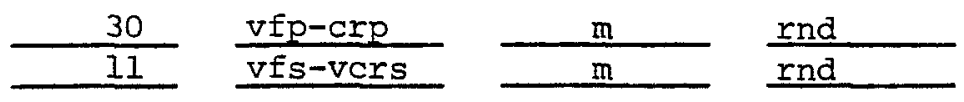

OTHER $\geq$ gravel
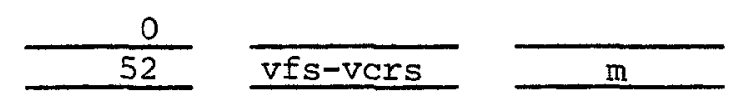

rnd

SILT AND CLAY

REMARKS

color
HCI

Iight Grey

Slightly Micaceous

Frosted Quartz

Immature

Total Drilled Depth reported as $350^{\prime}$

DEPTH

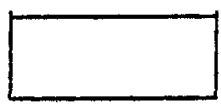

TO

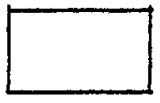

ROCK CLASSIFICATION

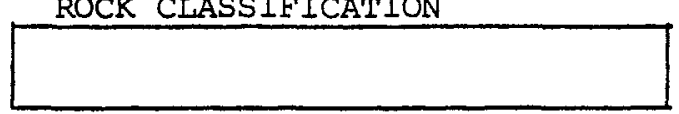

\% SIZE RANGE SPHERICITY ROUNDNESS

BASALT $\geq$ gravel sand

OTHER Igravel sand

SILT AND CLAY

REMARKS

color

HCL
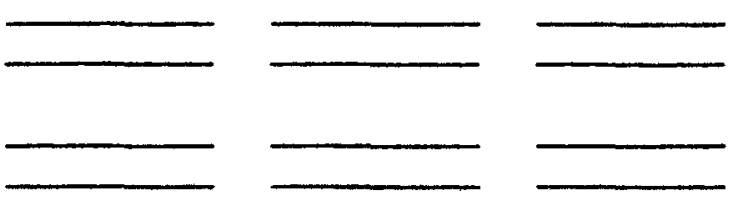


\section{0}

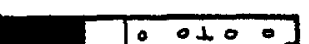

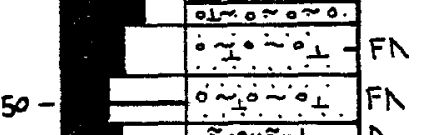$$
10
$$

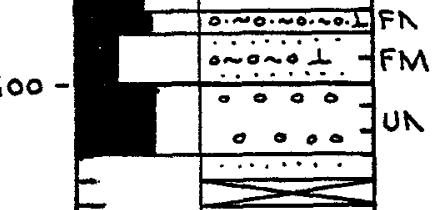

Sample Description

brownish black

greyish buff

brownish buff greyish buff

greyish buff

greyish butf greyish buff brownish grey

butf

brownish buff

greyish brown

greyish brown

brown

$\tan$

brown

brown

greyish brown

greenish grey

greyish brown yellowish tan greyish brown yellowish tan

greyish brown

yellowish $\tan$ greyish brown blackish brown blackish brown blackish brown
Plant Coordinates

$\frac{N 027027}{\text { W008281 }}$

Other Data used in Interpretation

(1) Driller's Log

(2) E- Logs

.


Depth

Graphic Log

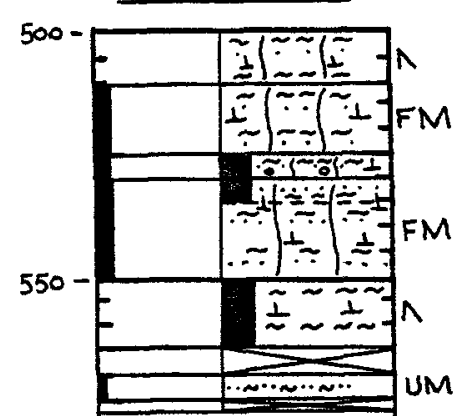

UM
Sample Description

blackish brown

blackish brown

blackish brown

blackish brown

greyish brown - brown

greyish brown

\section{Plant Coordinates}

Altitude

Other Data used in Interpretation 
DEPTH

(FT.)

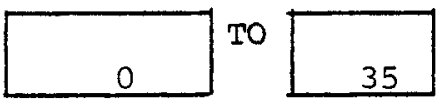

ROCK CLASSIFICATION

silty sandy Gravel

\section{$\because$ SIZE RANGE SPHERICITY ROUNDNESS} BASALT $\frac{\geq \text { graveI }}{\text { sand }}$ OTHER $\frac{\geq \text { gravel }}{\text { sand }}$

SILT AND CLAY
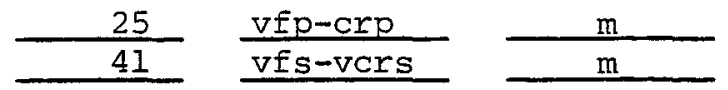

$\frac{\text { sb rnd }}{\text { sb ang }}$

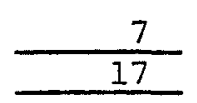

$\frac{\text { vfp-mp }}{\text { vfs-vers }}$

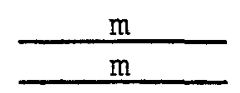

rnd

sb rnd

REMARKS

color

HCL

Brownish Black

$+$

Sample interval $25^{\prime}-30^{\prime}$ missing

Immature

DEPTH

(FT.)

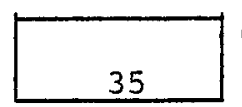

To

45
ROCK CIAASSIFICATION

slightly silty gravelly sand

\% SIZE RANGE SPHERICITY ROUNDNESS

BASALT $\underset{\substack{\text { sand } \\ \text { gravel }}}{ }$

OTHER $\geq$ gravel sand

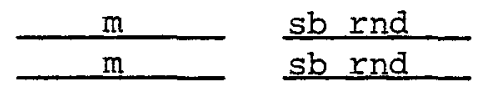

sb rnd sb rnd

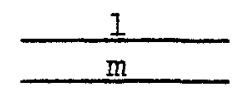

SIIT AND CLAY

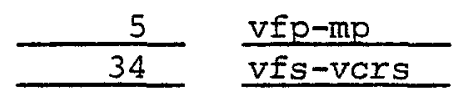
9

REMARKS

color HCL Greyish Buff $+$

Slightly Micaceous Frosted Quartz

Immature 


\section{DEPTH \\ (ET.)}

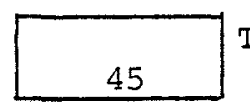

TO

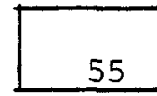

ROCK CLASSIFICATION

slightly silty gravelly sand

\section{SIZE RANGE SPHERICITY ROUNDNESS}

BASALT $\geq$ gravel sand

OTHER $\geq$ gravel sand

$\frac{10}{26}$

$\frac{\mathrm{vfp}-\mathrm{mp}}{\mathrm{vfs- \operatorname {crs }}}$

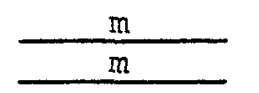

$\frac{\text { sb rnd }}{\text { sb rnd }}$

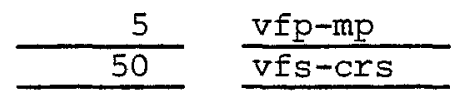

$\frac{1}{\mathrm{~h}} \frac{\text { sb rnd }}{\text { s.b rnd }}$

SILT AND CLAY 9

REMARKS

color HCL

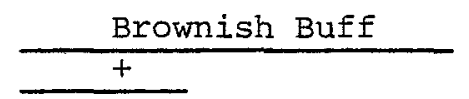

Slightly Micaceous Frosted Quartz

Immature

DEPTH

(FT.)

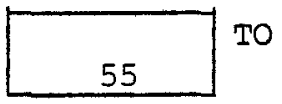

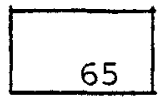

ROCK CIASSIFICATION

slightly silty gravelly Sand

SIZE RANGE SPHERICITY ROUNDNESS

BASALT $\underset{\text { sand }}{\geq \text { gravel }}$

$\frac{20}{23} \frac{\mathrm{vfp}-\mathrm{crp}}{\mathrm{vfs-vers}}-\frac{\mathrm{m}}{\mathrm{m}} \frac{\mathrm{sb} \mathrm{rnd}}{\mathrm{sb} \mathrm{rnd}}$

OTHER Igravel sand
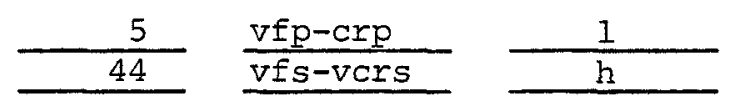

$\frac{\text { sb rnd }}{\text { sb rnd }}$

SIIT AND CLAY

8

REMARKS

color

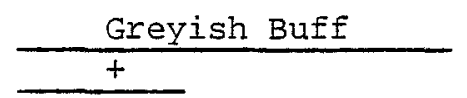

Slightly Micaceous

Immature 

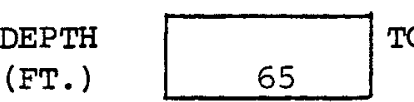

TO

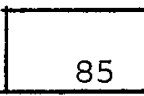

ROCK CLASSIFICATION

silty sandy Gravel

\section{\% SIZE RANGE SPHERICITY ROUNDNESS}

BASALT $\geq$ gravel
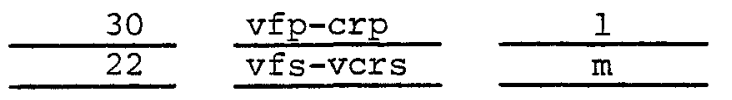

rnd

sand
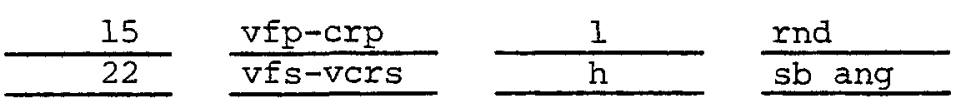

OTHER $\geq$ gravel sand

\section{1}

SILT AND CLAY

REMARKS

color

HCL

Greyish Buff

$-$

Slightly Micaceous Unfrosted Quartz

Immature

DEPTH

(FT.)

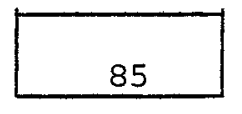

TO

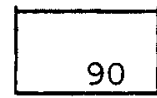

ROCK CLASSIFICATION

silty sandy Grave1

을 SIZE RANGE SPHERICITY ROUNDNESS

BASALT $\geq$ gravel sand

OTHER $\geq$ gravel sand

SILT AND CLAY
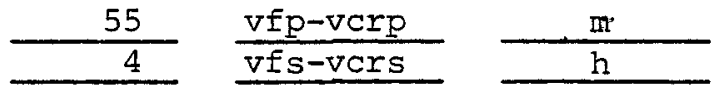

shd

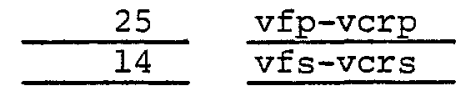

$\frac{\mathrm{m}}{\mathrm{h}}$

rnd

2

REMARKS

color

HCL

Greyish Buff

Slightly Micaceous

Frosted Quartz

Immature 


\section{DEPTH}

(FT.)

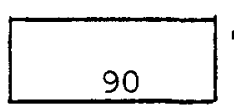

TO

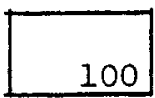

ROCK CLASSIFICATION

slightly silty gravelly sand

\section{\% SIZE RANGE SPHERICITY ROUNDNESS}

BASALT
$\begin{aligned} & \text { sand } \\ & \text { savel }\end{aligned}$
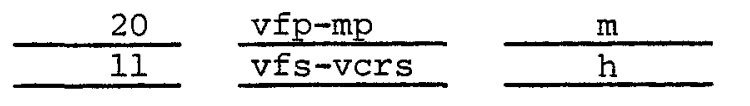

$\frac{\text { rnd }}{\text { sb rnd }}$

OTHER $\quad \underset{\text { sand }}{\text { gravel }}$
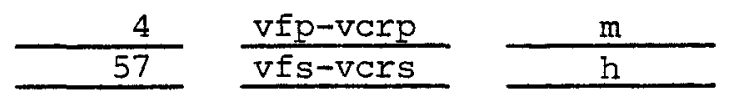

sb ang

SILT AND CLAY

8

REMARKS

$$
\text { color }
$$

HCL

Greyish Buff

Micaceous

Frosted Quartz

Immature
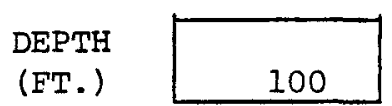

To

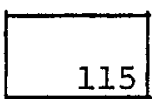

ROCK CLASSIFICATION

Grave1

S SIZE RANGE SPHERICITY ROUNDNESS

BASALT $\geq$ gravel
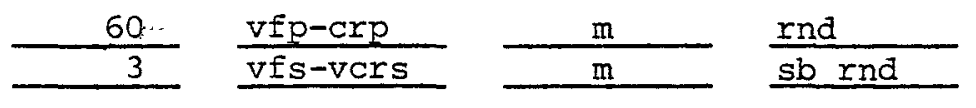

OTHER $\frac{\geq \text { gravel }}{\text { sand }}$
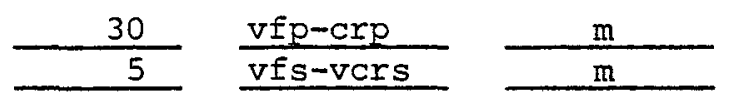

rnd

SILT AND CLAY

2

REMARKS

color

HCL

Brownish Grey

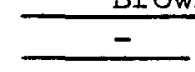

Slightly Micaceous

Unfrosted Quartz

Immature 
DEPTH (FT.)

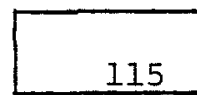

TO

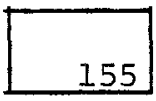

\section{ROCK CLASSIFICATION}

Sand

\section{은 SIZE RANGE SPHERICITY ROUNDNESS}

BASALT $\underset{\text { sand }}{\geq \text { gravel }}$

OTHER I gravel sand
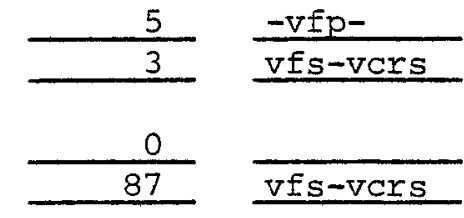

SILT AND CIAY

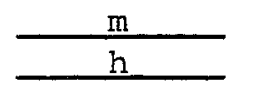

rnd

sb rnd

REMARKS

color

Buff

Micaceous

Unfrosted Quartz

Sample interval 120'-125' missing

Immature

DEPTH

(FT.)

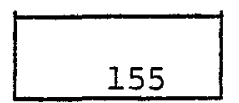

TO

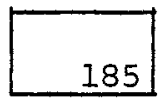

ROCK CLASSIFICATION

slightly silty sand

\section{\% SIZE RANGE SPHERICITY ROUNDNESS}

BASALT $\underset{\text { sand }}{\operatorname{gravel}}$
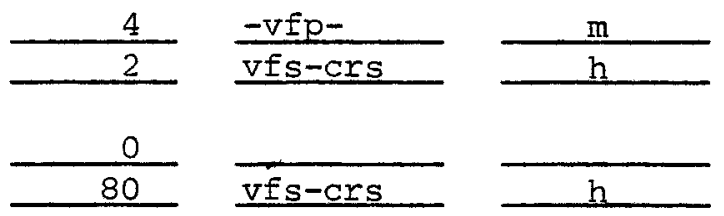

rnd

sb rnd

OTHER >gravel sand

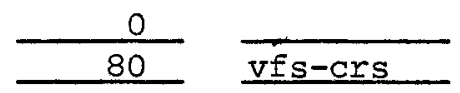

ang

SILT AND CIAY 14

REMARKS

color Brownish Buff

HCL $+$

Slightly Micaceous Frosted quartz

Immature 


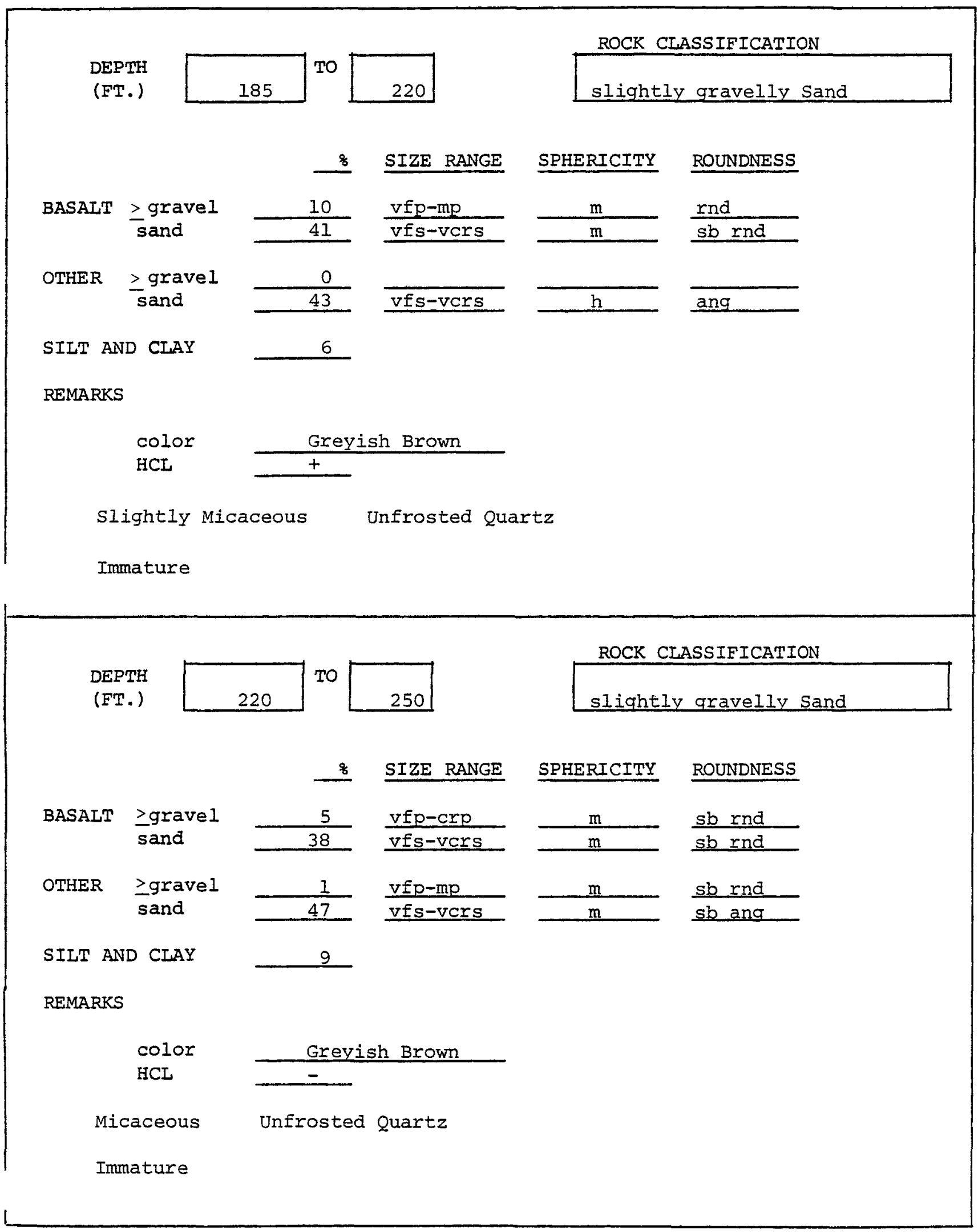




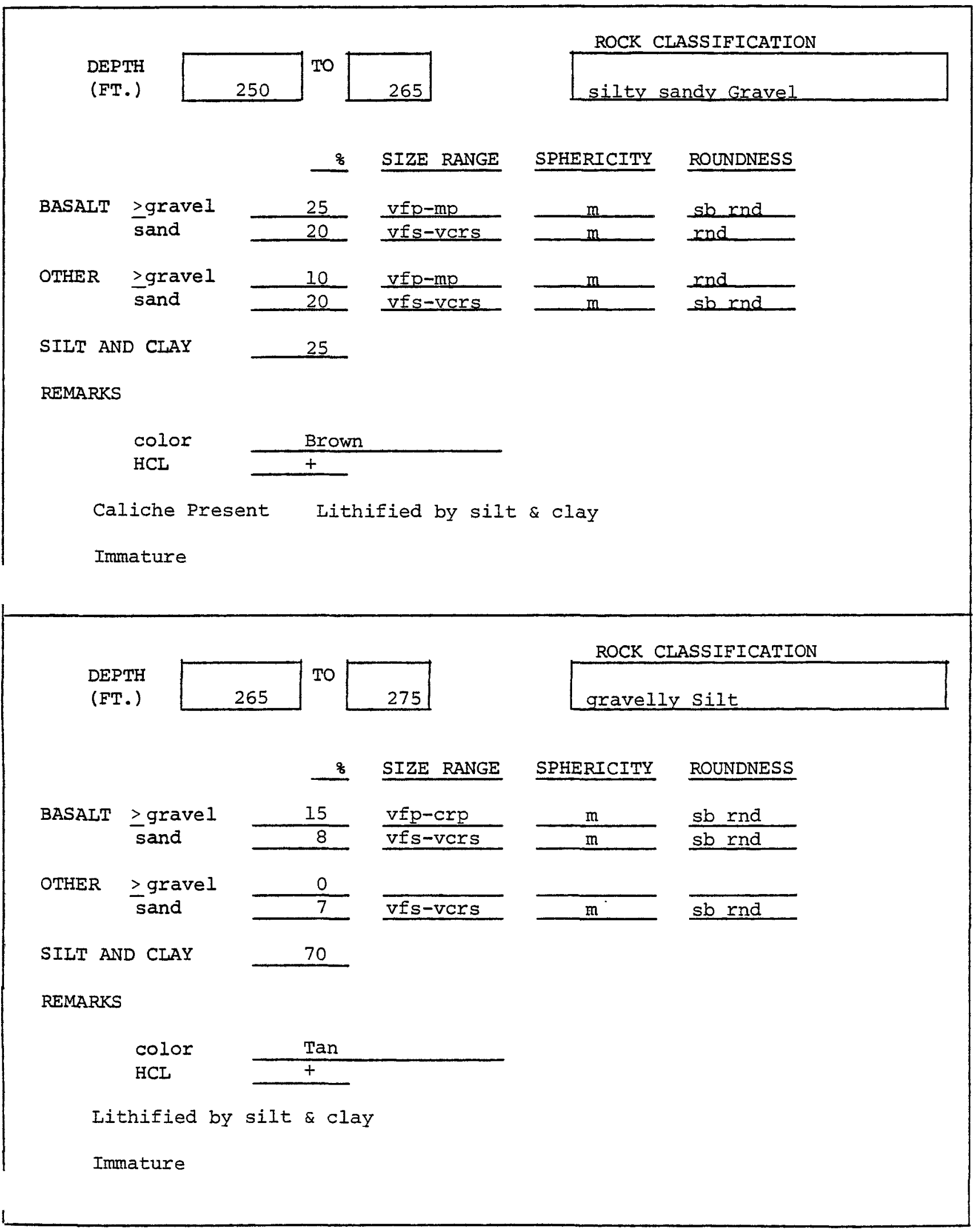


DEPTH

(FT.)

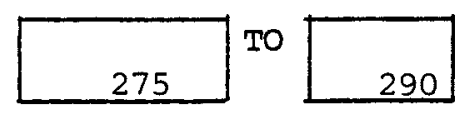

\& SIZE RANGE SPHERICITY ROUNDNESS
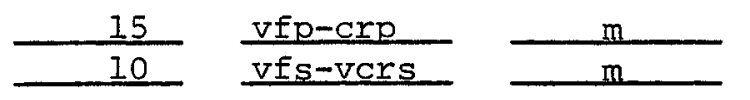

sb rad

ROCK CLASSIFICATION

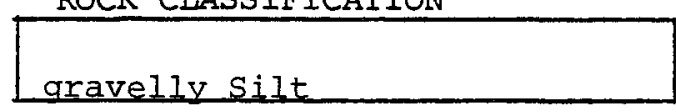

OTHER >gravel sand

SIIT AND CLAY

REMARKS
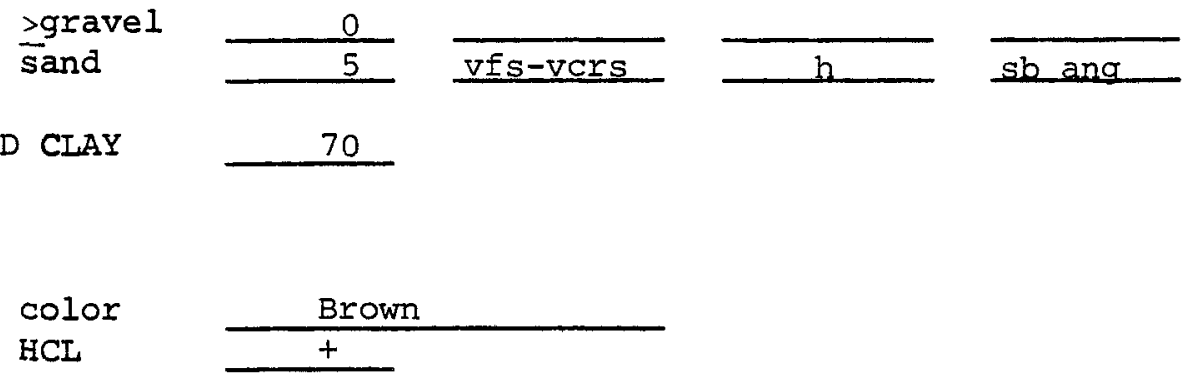

Slightly Micaceous Iithified by silt \& clay

Immature

\section{DEPTH}

(ET. )

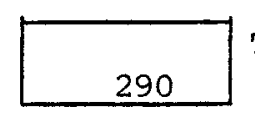

TO

320
ROCK CLASSIFICATION

silty sand

8 SIZE RANGE SPHERICITY ROUNDNESS

BASALT $\underset{\text { sand }}{\text { >gravel }}$

OTHER >gravel sand

SIIT AND CLAY

REMARKS

$\begin{array}{cl}\text { Color } & \frac{\text { Brown }}{\text { HCL }} \\ { } } & \text { Unfrosted Quartz } \\ \text { Immature } & \end{array}$




\section{DEPTH}

(FT.)
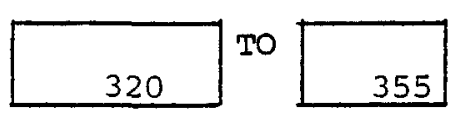

S SIZE RANGE SPHERICITY ROUNDNESS

BASALT $\frac{\geq \text { gravel }}{\text { sand }}$
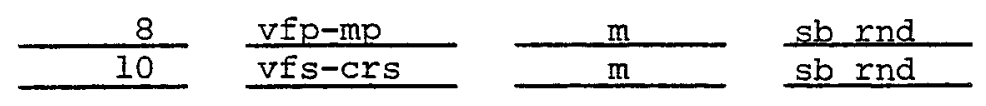

OTHER $\geq$ gravel sand
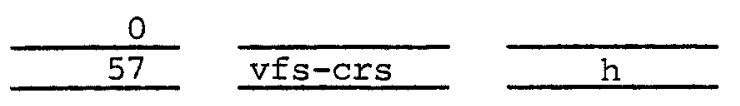

SILT AND CLAY

25

REMARKS

color

HCI

Micaceous

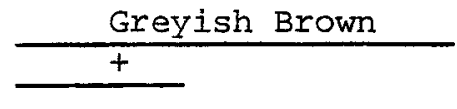

Immature
ROCK CLASSIFICATION

slightly gravelly silty sand 


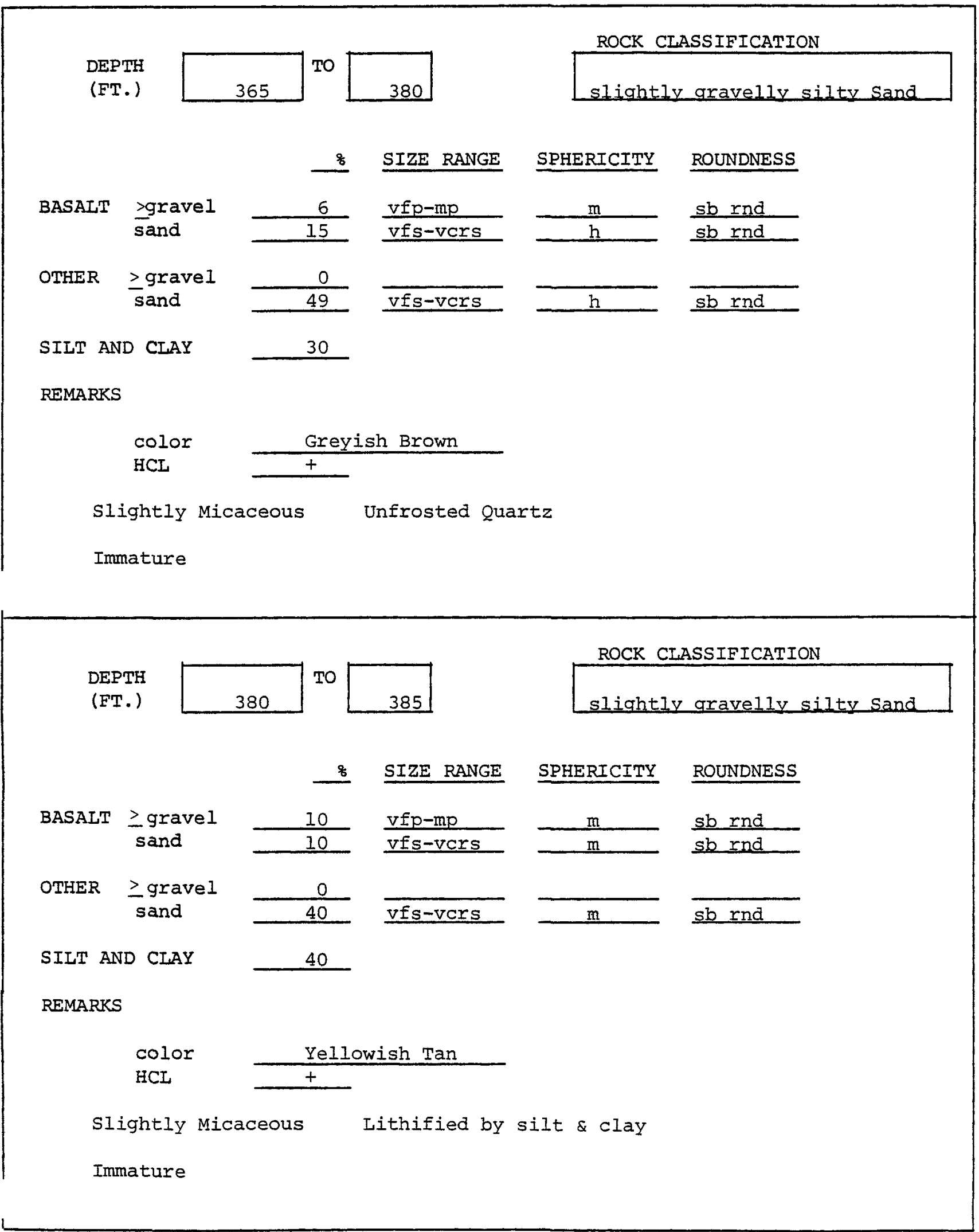




\section{DEPTH}

(FT.)

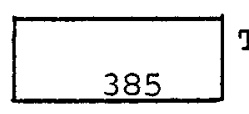

TO

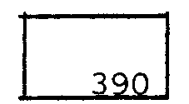

ROCK CLASSIFICATION

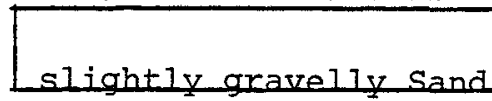

\section{\% SIZE RANGE SPHERICITY ROUNDNESS}

BASALT >gravel
sand
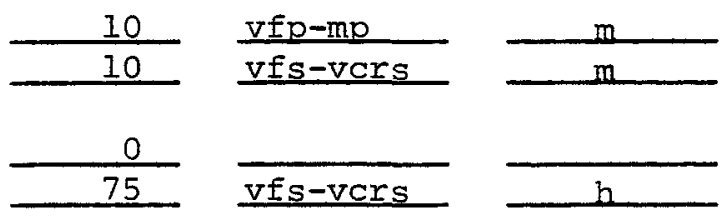

$$
\frac{\text { sh rnd }}{\text { sh_rnd }}
$$

OTHER $\geq$ gravel sand

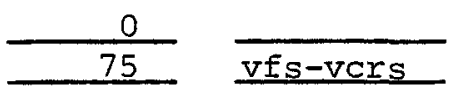

sbrnd

SIIT AND CLAY 5

REMARKS

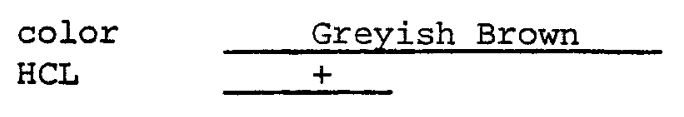

Slightly Micaceous Frosted quartz

Immature
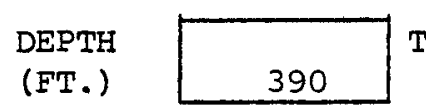

TO

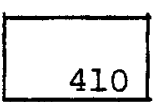

ROCK CLASSIFICATION

silty sand

\section{\% SIZE RANGE SPHERICITY ROUNDNESS}

BASALT zgravel
sand

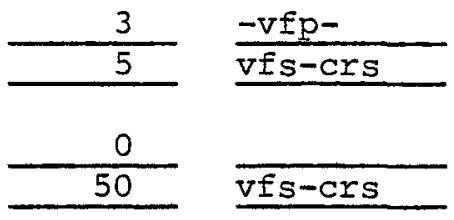

42

SILT AND CLAY

REMARKS

$$
\text { color }
$$

HCL

Micaceous

Frosted Quartz

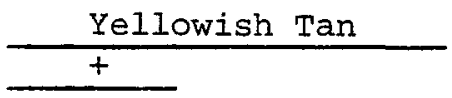

Immature

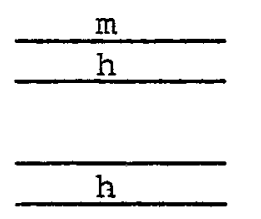

sb rnd

sb rnd

$\overline{\text { sb rnd }}$ 


\section{DEPTH}

(FT.)

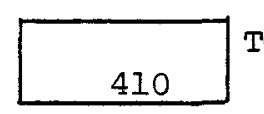

TO

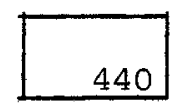

ROCK CIASSIFICATION

slightly gravelly silty sand

SIZE RANGE SPHERICITY ROUNDNESS BASALT $\frac{\geq \text { gravel }}{\text { sand }}$
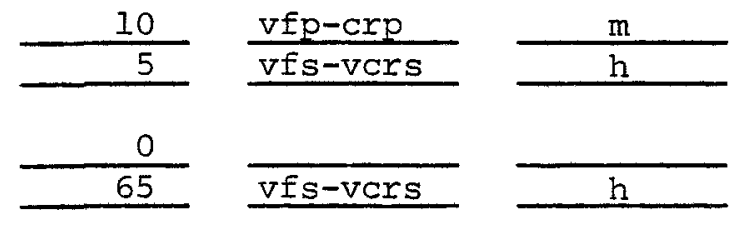

$\frac{\text { sb ang }}{\text { sb rnd }}$

OTHER $\frac{\geq \text { gravel }}{\text { sand }}$

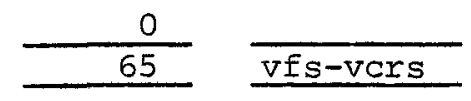

20

SILT AND CLAY

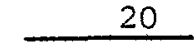

REMARKS

$$
\text { color }
$$

HCL

Greyish Brown

Micaceous

Frosted Quartz

Immature
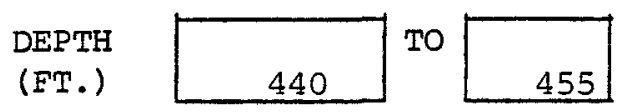

ROCK CLASSIFICATION

slightly qravelly silty sand

\% SIZE RANGE SPHERICITY ROUNDNESS

BASALT $\geq$ gravel

$$
\text { sand. }
$$
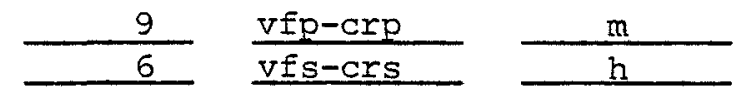

$\frac{\text { sb ang }}{\text { sb rnd }}$

OTHER $\geq$ gravel
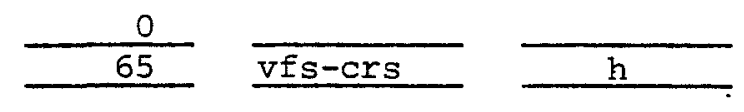

sb rnd

SILT AND CIAY 20

REMARKS

\begin{tabular}{ll}
\multicolumn{1}{c}{$\begin{array}{c}\text { HCL } \\
\text { Micaceous }\end{array}$} & $\frac{\text { Yellowish Tan }}{+}$ \\
Frosted Quartz
\end{tabular}


DEPTH
(FT.)

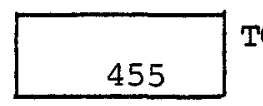

To

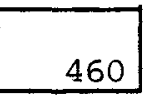

ROCK CLASSIFICATION

slightly silty sand

\section{\% SIZE RANGE SPHERICITY ROUNDNESS}

BASALT >gravel sand
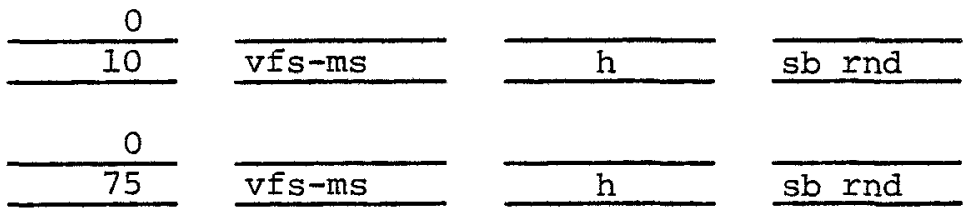

OTHER $\geq$ gravel

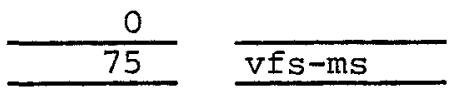

SILT AND CLAY 15

REMARKS

color

HCL

Greyish Brown

Micaceous

Frosted Quartz

Immature

DEPTH
(FT.)

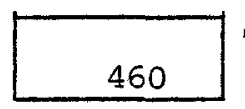

TO

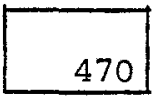

ROCK CLASSIFICATION

gravelly sandy silt

S SIZE RANGE SPHERICITY ROUNDNESS

BASALT $\geq \frac{\text { gravel }}{\text { sand }}$
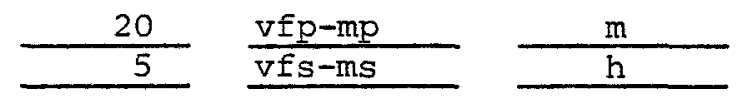

$\frac{\text { rnd }}{\text { sb rnd }}$

OTHER >gravel

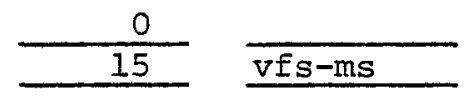

h

sb rnd

SILT AND CLAY

60

REMARKS

color

HCL

Blackish Brown

$+$

Slightly Micaceous

Lithified by silt \& clay

Imnature 


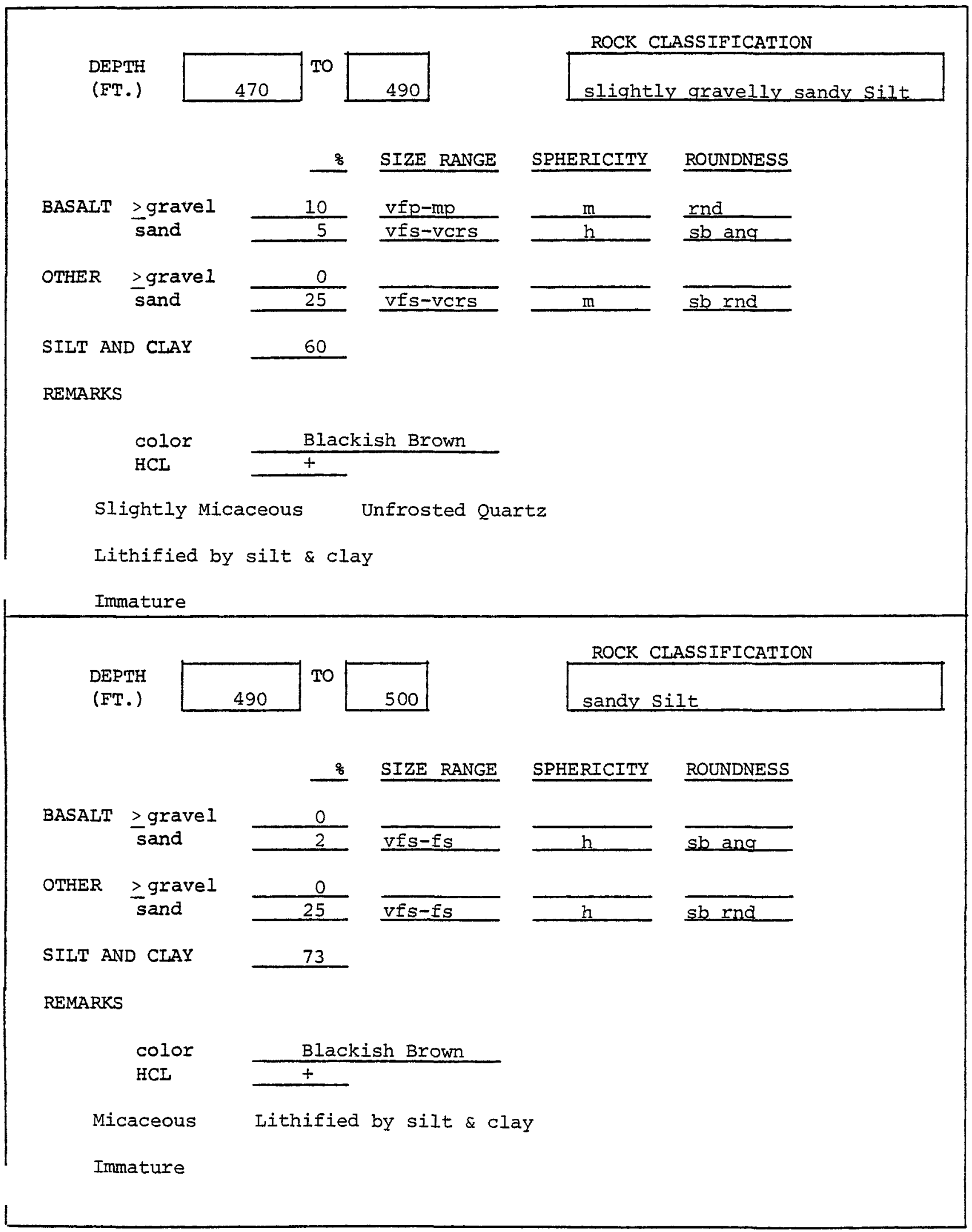

W. K. Summers \& Associates 


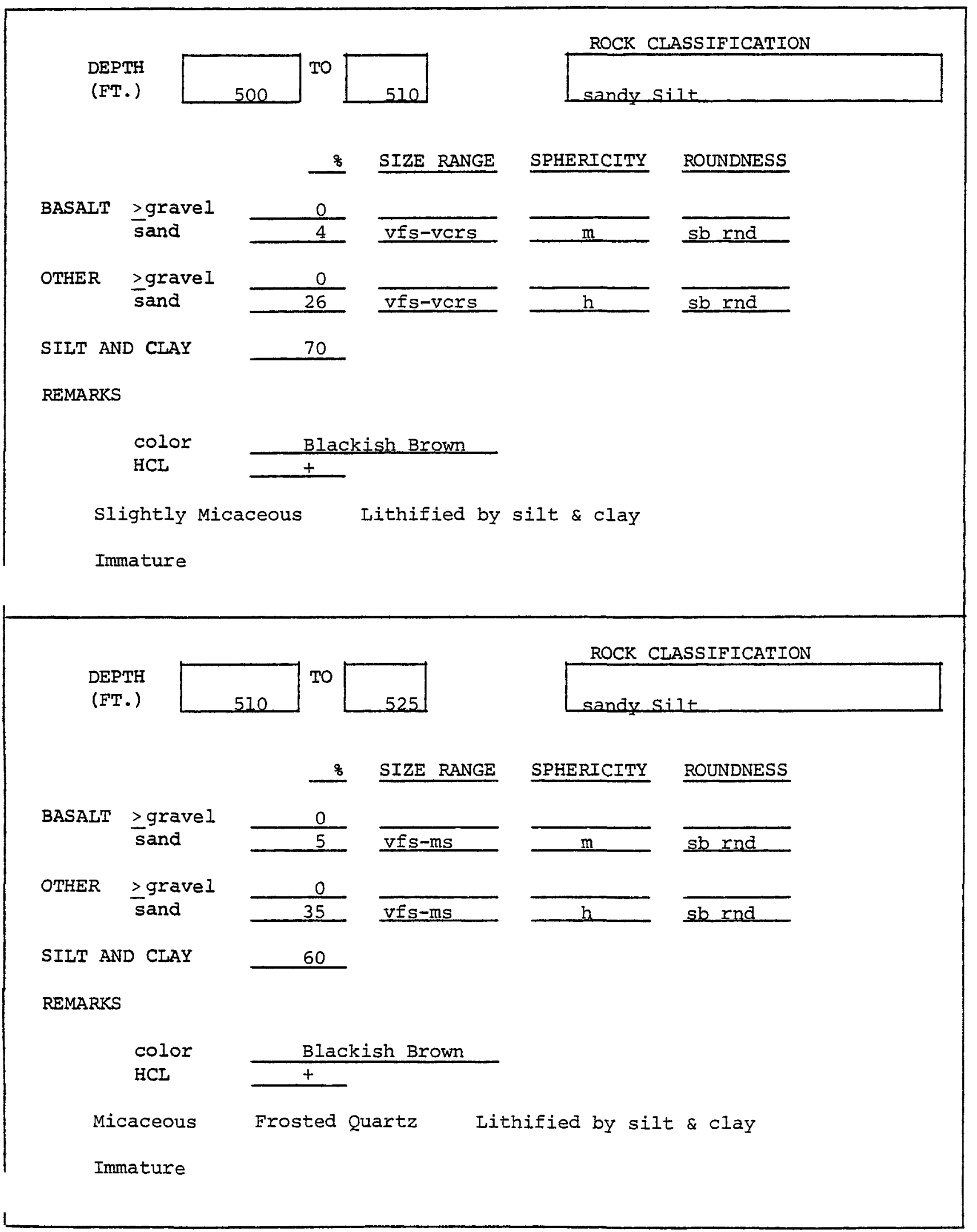

W. K. Summers \& Associates 


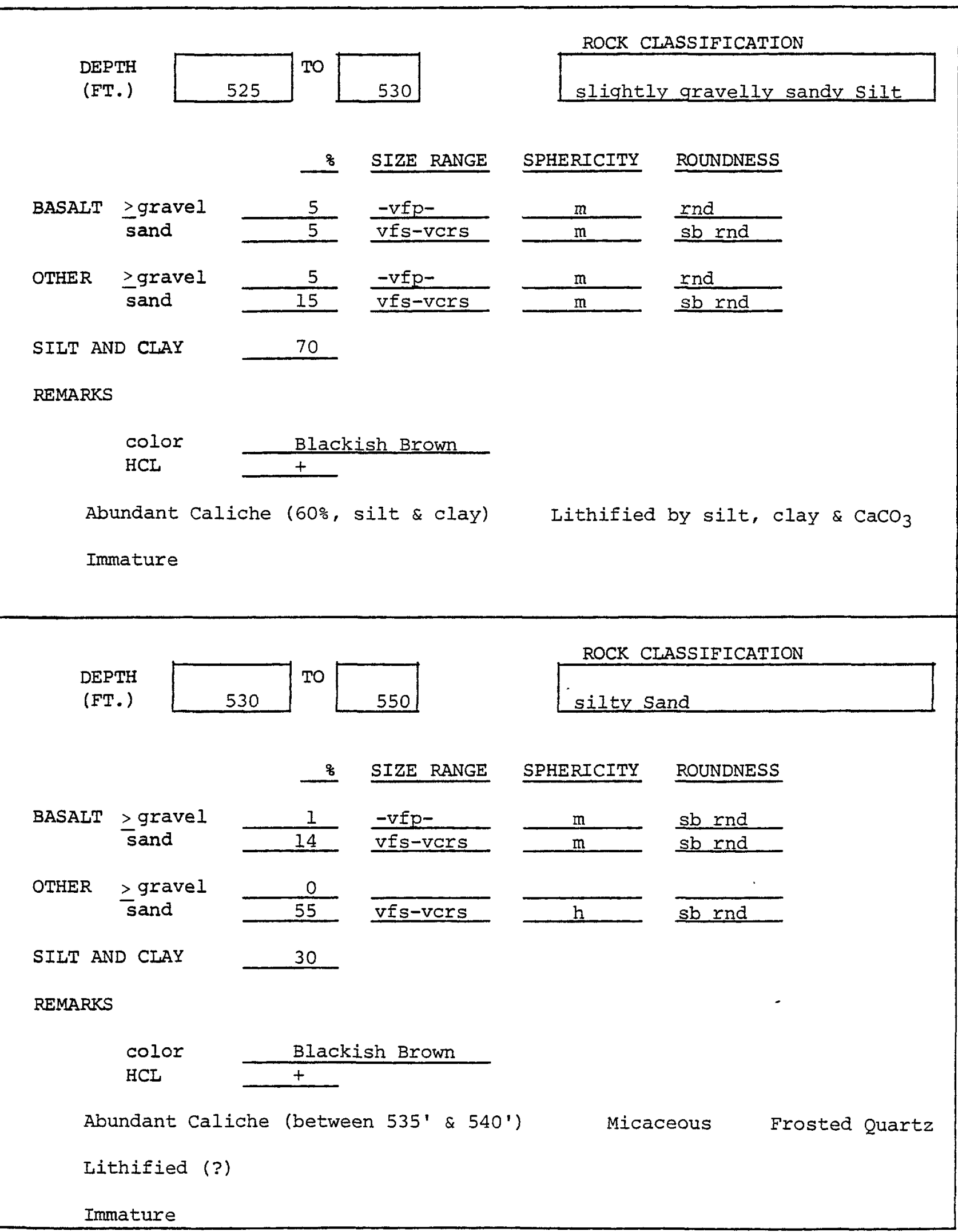

W. K. Summers \& Associates 


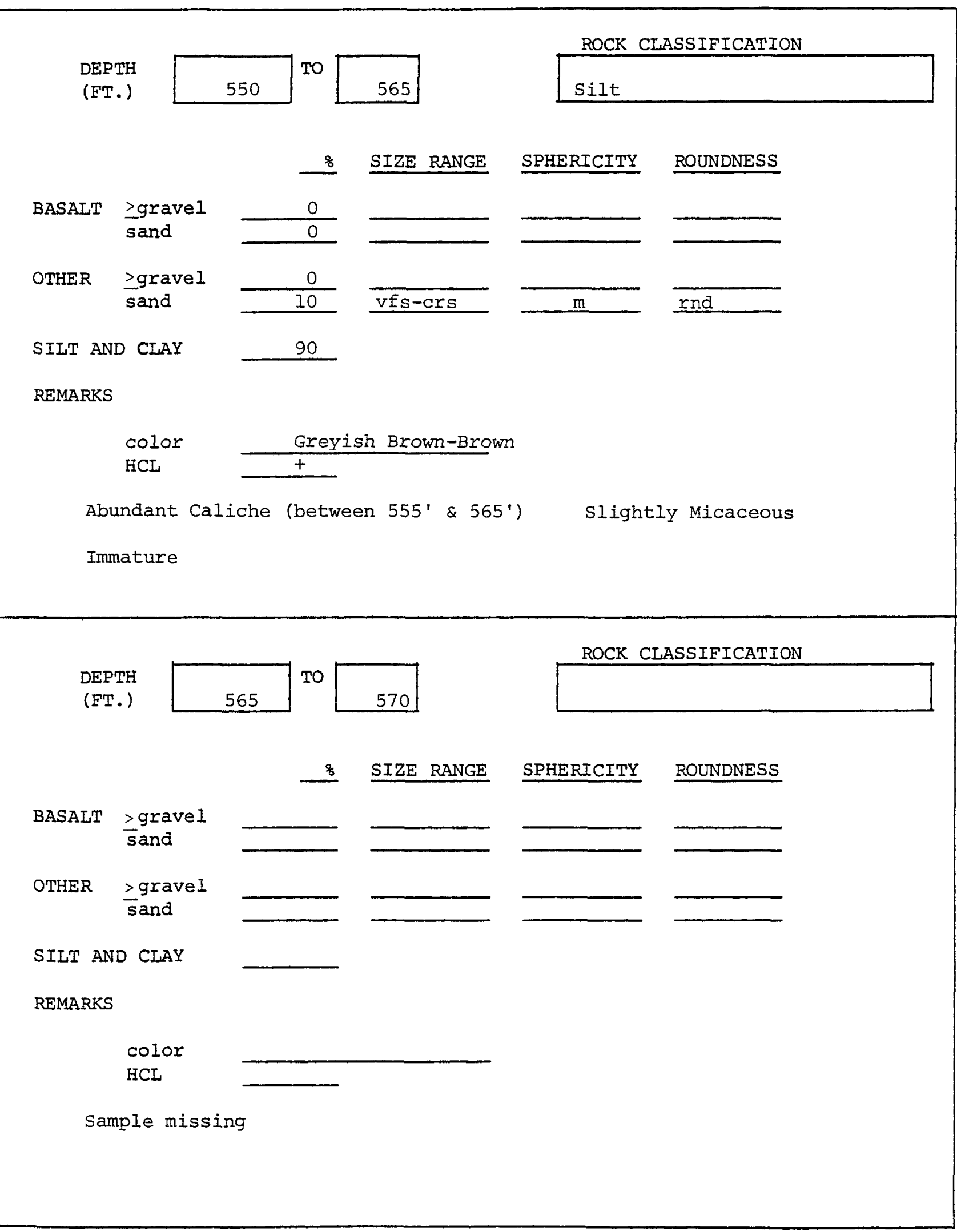

W. K. Summers \& Assoctates 


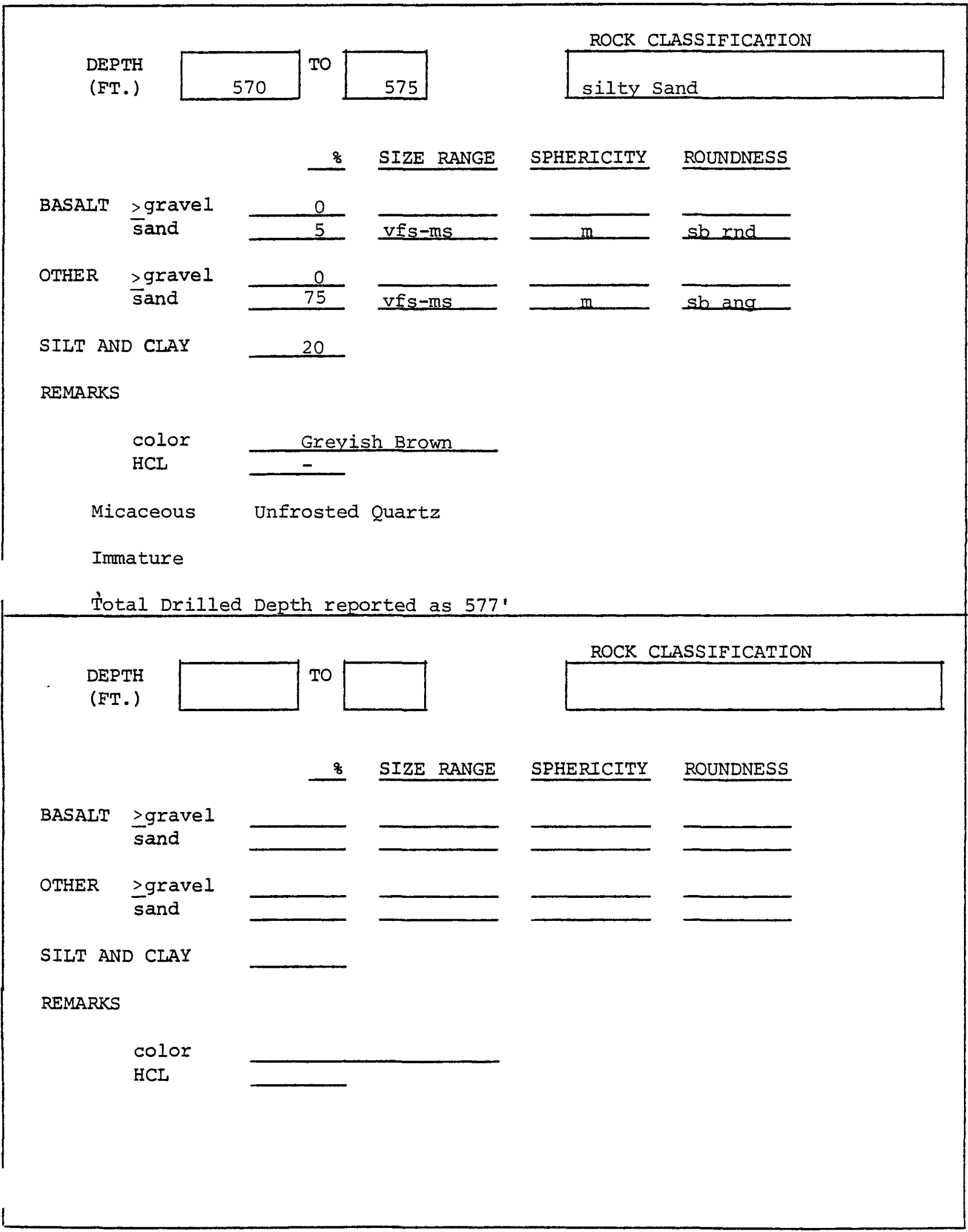


Depth (ft.)

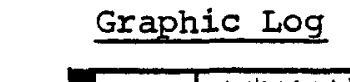

$-\because \div-\div=1$

Sample Description

greyish $\tan$

greyish buff

greyish $\tan$

greyish buff

greyish buff

greyish buff

greyish butf

butf grey

greyish tan

grey greyish buff

greyish buff

greyish $\tan$

greyish $\tan$

greyish $\tan$

greyish butf

greyish buff

greyish tan

greyish $\tan$

greyish brown

tan.

greyish tan

greyish tan

greyish $\tan$

greyish tan

yellowish $\tan$

grey

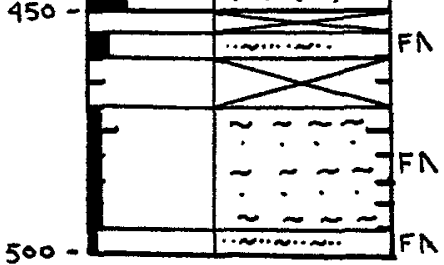

yellowish tan

Plant coordinates

$N 028400$

W 051525

Altitude 683

Other Data used in Interpretation

Driller's Log 


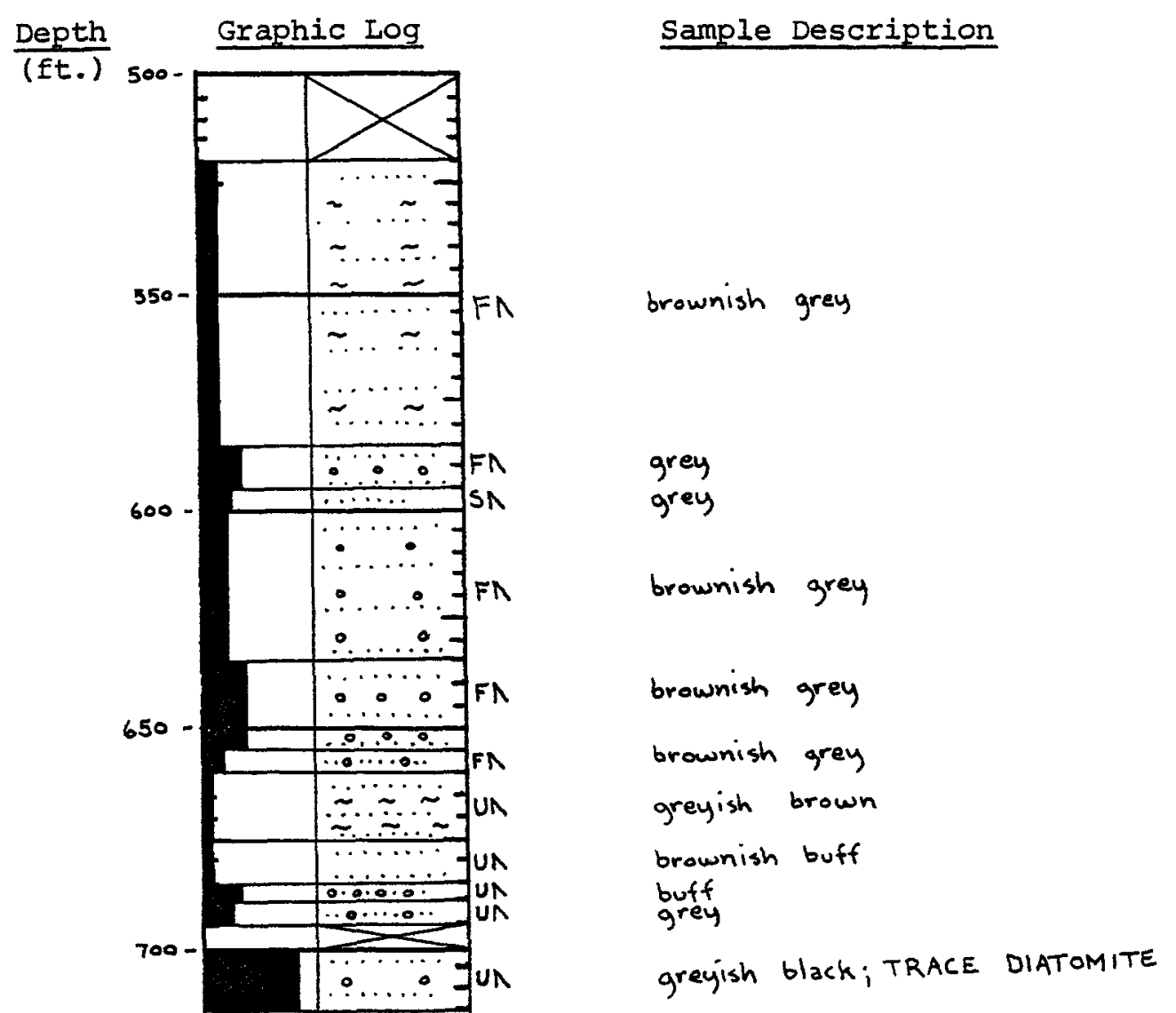

Plant Coordinates

Other Data used in Interpretation

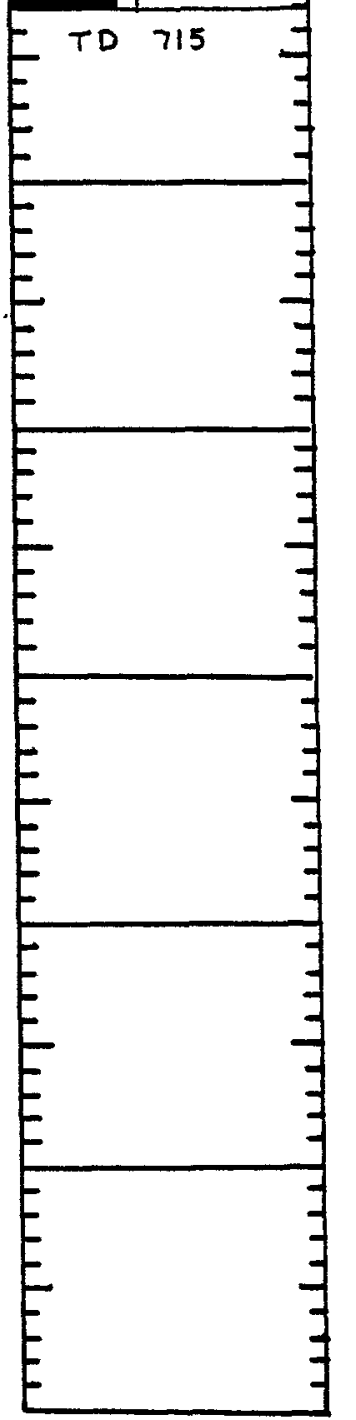




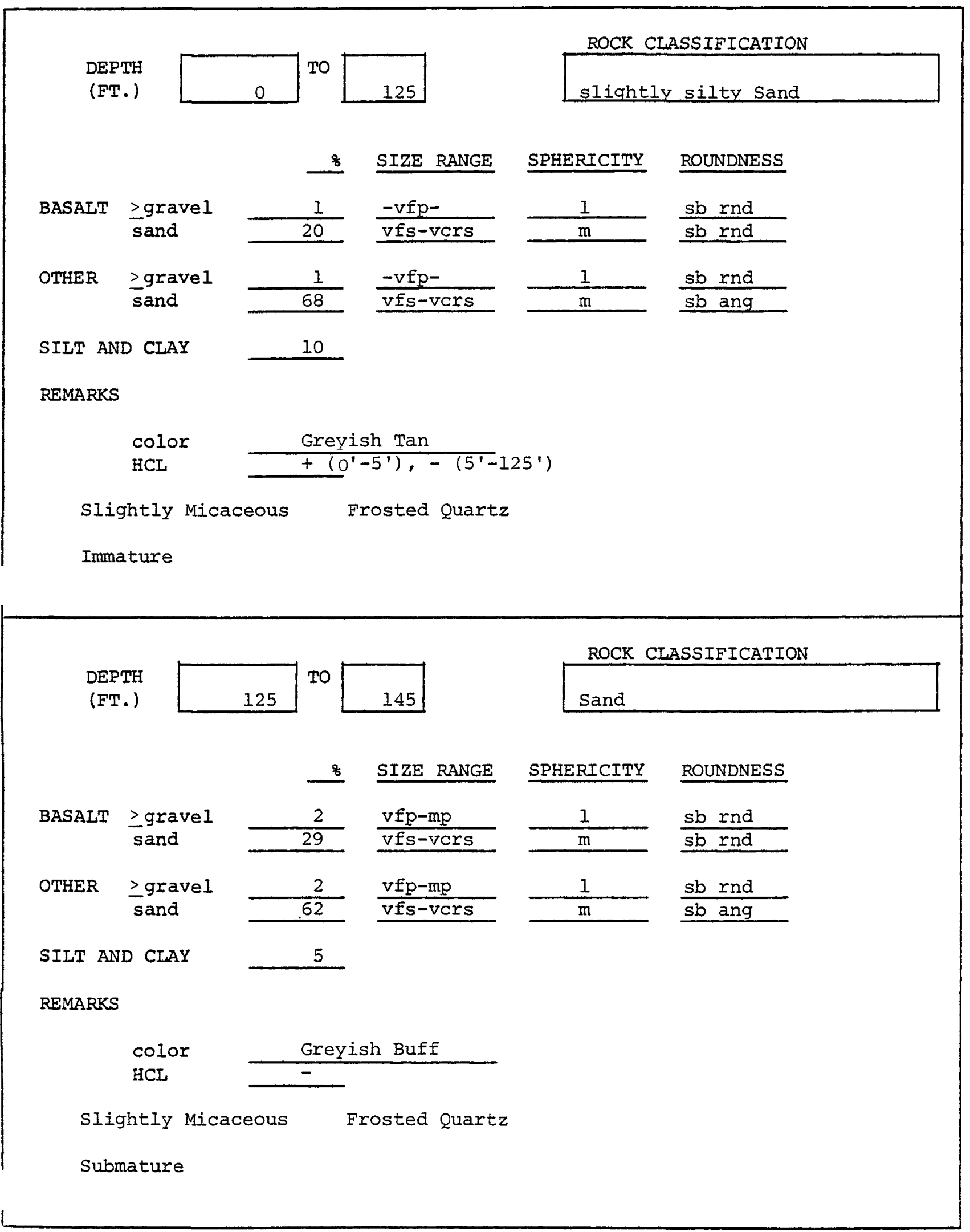




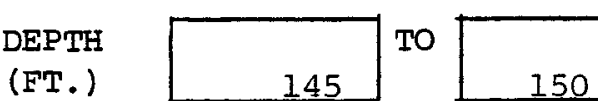

ROCK CLASSIFICATION

(FT.)

145

150

Sand

\% SIZE RANGE SPHERTCITY ROUNDNESS

BASALT $\geq$ gravel sand
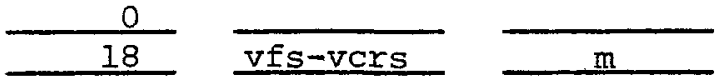

rnd

OTHER IgraveI
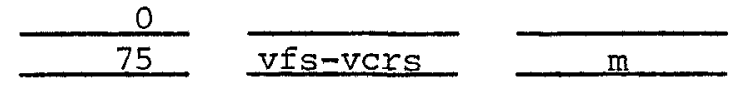

sb ang

SILT AND CIAAY

7

REMARKS

color

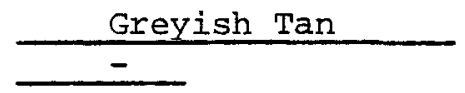

Slightly Micaceous Frosted Quartz

Submature

DEPTH
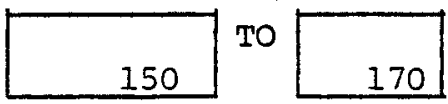

ROCK CLASSIFICATION

(FT.)

을 SIZE RANGE SPHERICITY ROUNDNESS

BASAIT $\frac{\geq \text { gravel }}{\text { sand }}$
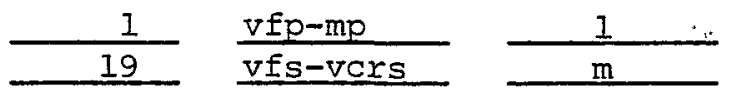

s.b rnd

OTHER $\geq$ gravel
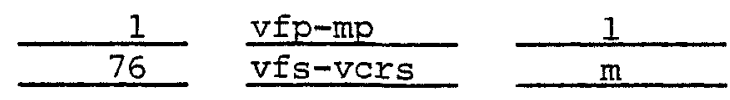

$\frac{\mathrm{sb} \text { rnd }}{\mathrm{sb} \text { rnd }}$

SILT AND CLAY

3

REMARKS

color

Greyish Buff

HCL

$-$

Slightly Micaceous Frosted quartz

Submature 


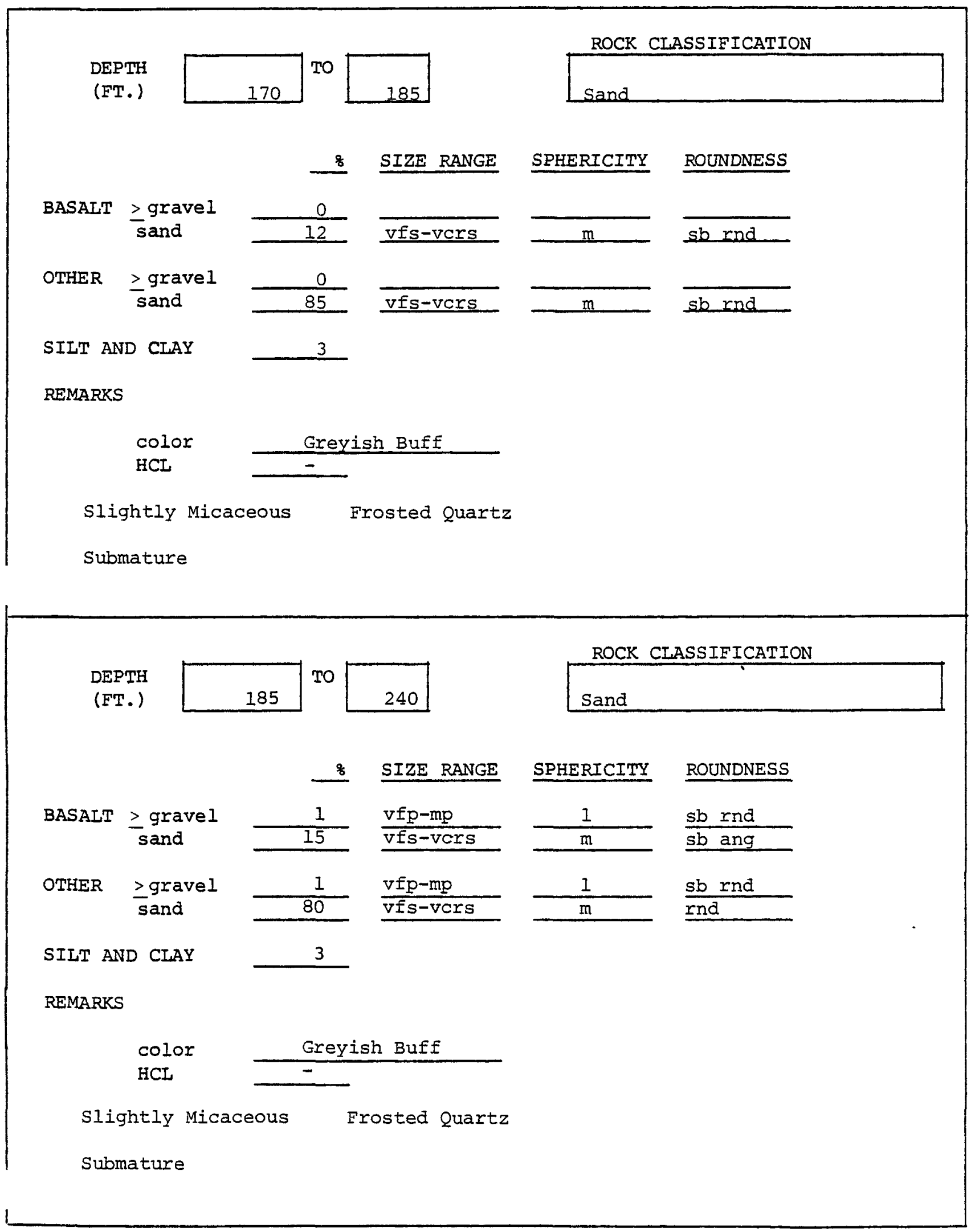

W. K. Summers \& Associates 
DEPTH
(FT.)

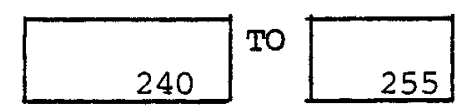

ROCK CLASSIFICATION

240

255

slightly gravelly sand

SIZE RANGE SPHERICITY ROUNDNESS

BASALT > gravel

$$
\text { sand }
$$

5 vfp-mp

OTHER $\underset{\frac{\geq \text { gravel }}{\text { sand }}}{\text { OTH }}$
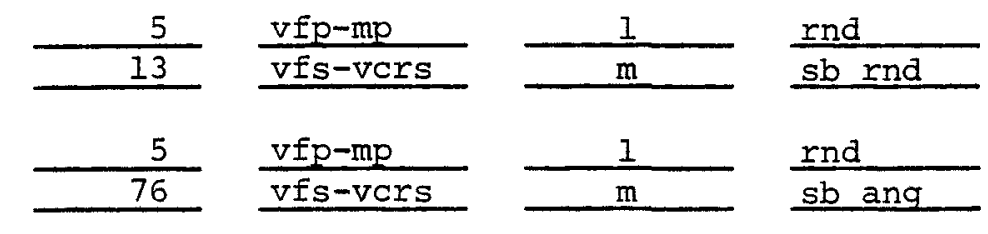

SILT AND CLAY

1

REMARKS

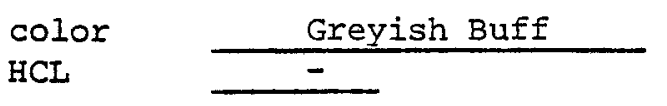

Unfrosted Quartz

Slightly Micaceous

Immature

DEPTH
(FT.)

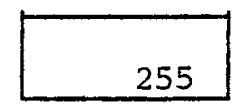

To

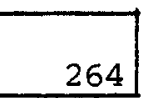

ROCK CLASSIFICATION

sandy Gravel

\section{\% SIZE RANGE SPHERICITY ROUNDNESS}

\begin{tabular}{|c|c|c|c|c|c|}
\hline BASALT & $\begin{array}{l}\text { >gravel } \\
\text { sand }\end{array}$ & $\frac{21}{11}$ & $\frac{v f p-c r p}{v f s-v c r s}$ & $\frac{1}{1}$ & $\frac{w \text { rnd }}{\text { sb ang }}$ \\
\hline \multirow[t]{2}{*}{ OTHER } & >gravel & 25 & $v f p-c r p$ & 1 & w rnd \\
\hline & sand & 42 & $v f s-v c r s$ & m & sb ang \\
\hline
\end{tabular}

SILT AND CLAY

1

REMARKS

color

Buff Grey

HCL

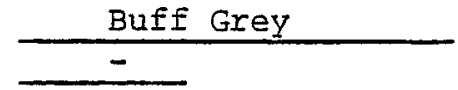

Slightly Micaceous Unfrosted Quartz

Immature 


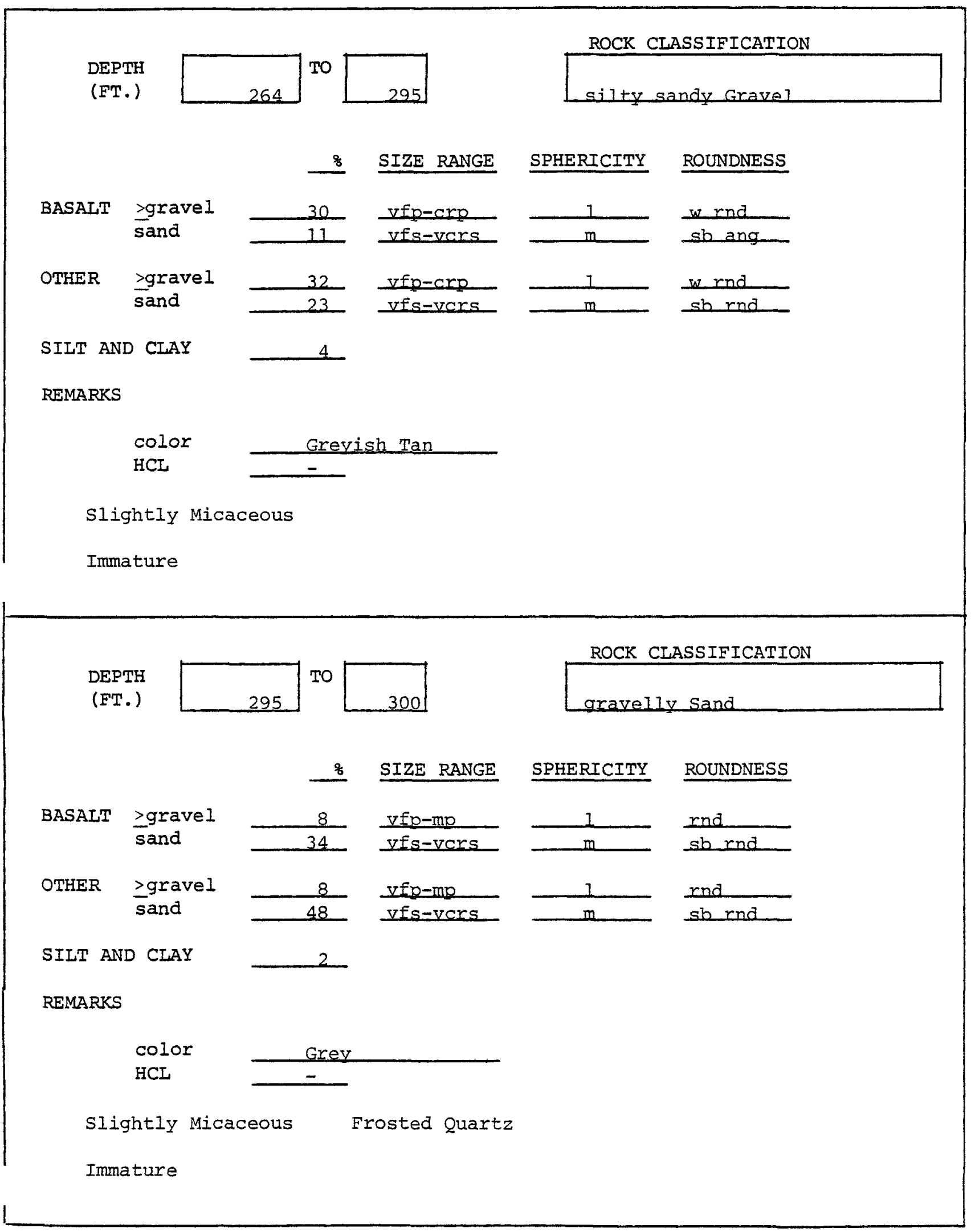




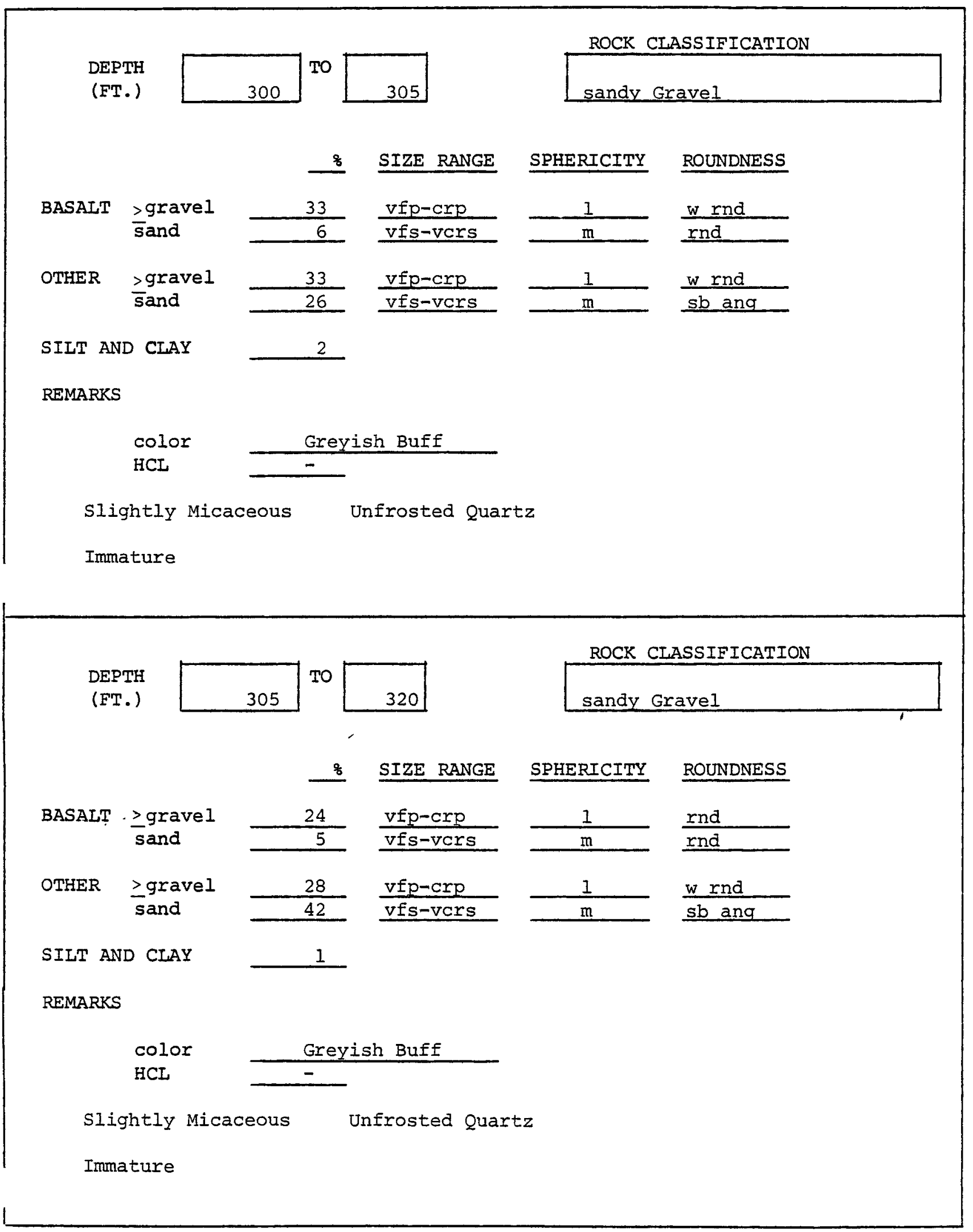




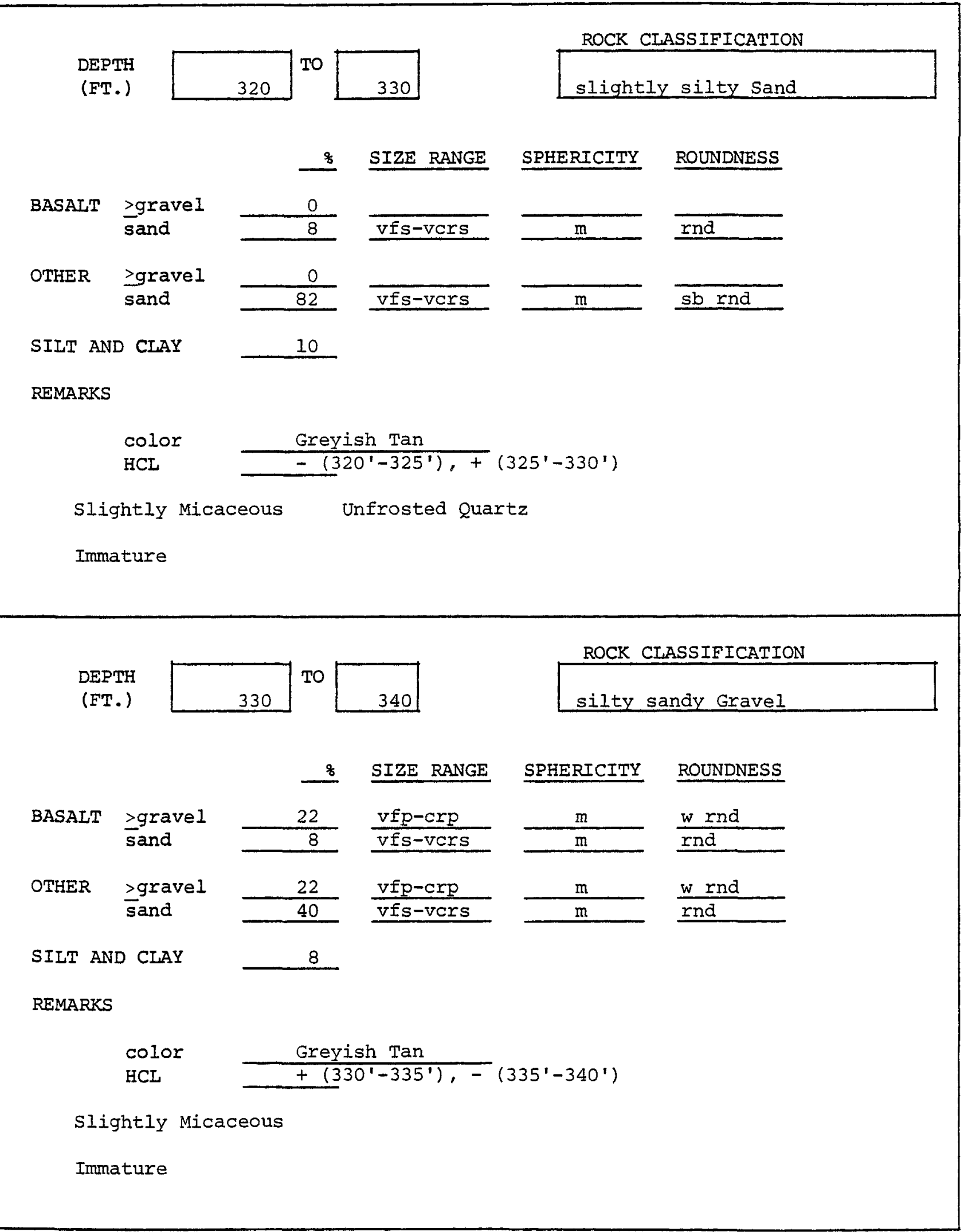




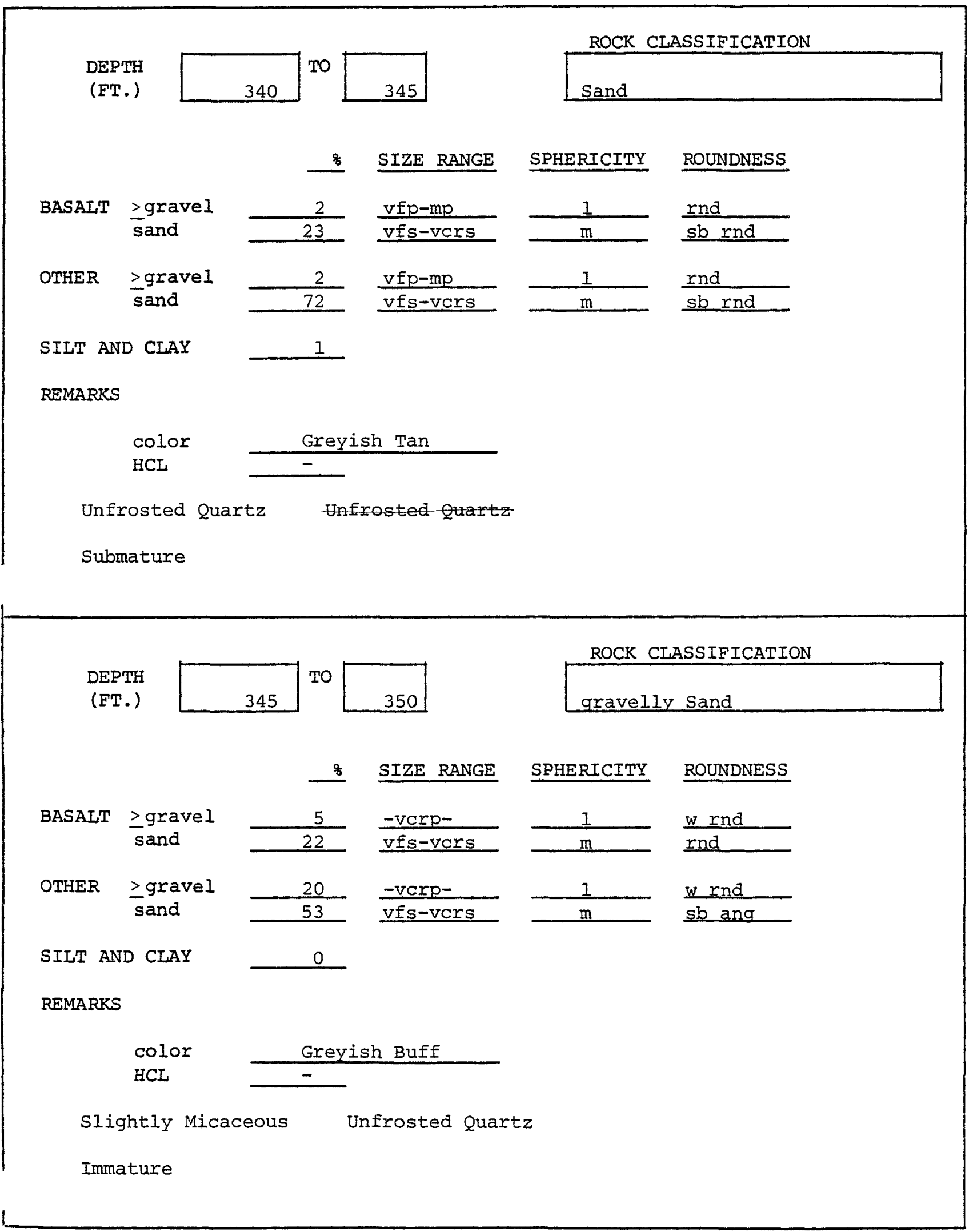


DEPTH

(FT.)

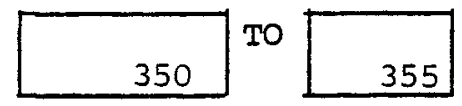

8 SIZE RANGE

SPHERICITY ROUNDNESS

BASALT
$\underset{\text { sand }}{\text { gravel }}$

$\frac{20}{6} \frac{v f p-c r p}{v f s-v \operatorname{crs}}$

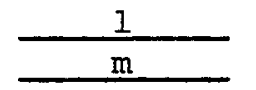

$\frac{\mathrm{w} \text { rnd }}{\text { sb rnd }}$

OTHER $\geq$ gravel
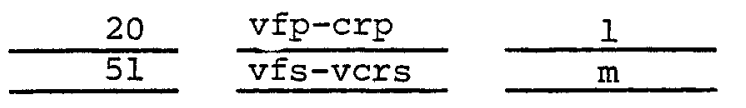

w rnd

sb rnd

SILT AND CLAY

3

REMARKS

color

HCL

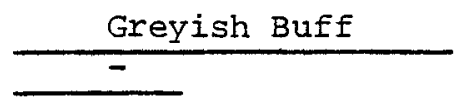

Slightly Micaceous

Frosted Quartz

Immature

DEPTH

(FT.)

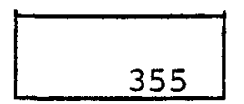

TO

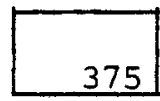

ROCK CLASSIFICATION

slightly silty slightly gravelly sand

\section{SIZE RANGE SPHERICITY ROUNDNESS}

BASALT $\underset{\substack{\geq \text { graveI } \\ \text { sand }}}{ }$
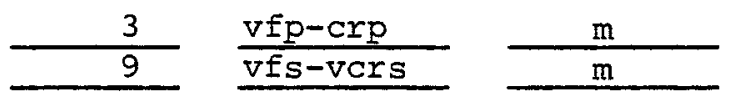

$\mathrm{w}$ rnd

sb rnd

OTHER $\geq$ gravel sand
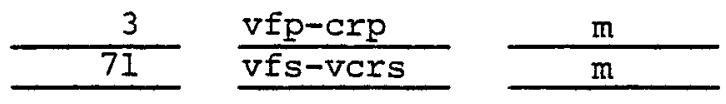

wrind

sb rnd

SILT AND CLAY 14

REMARKS

$$
\begin{aligned}
& \text { color } \\
& \text { HCL }
\end{aligned}
$$

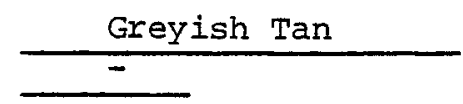

Slightly Micaceous

Frosted Quartz

Sample interval 360'-365' missing

Immature 


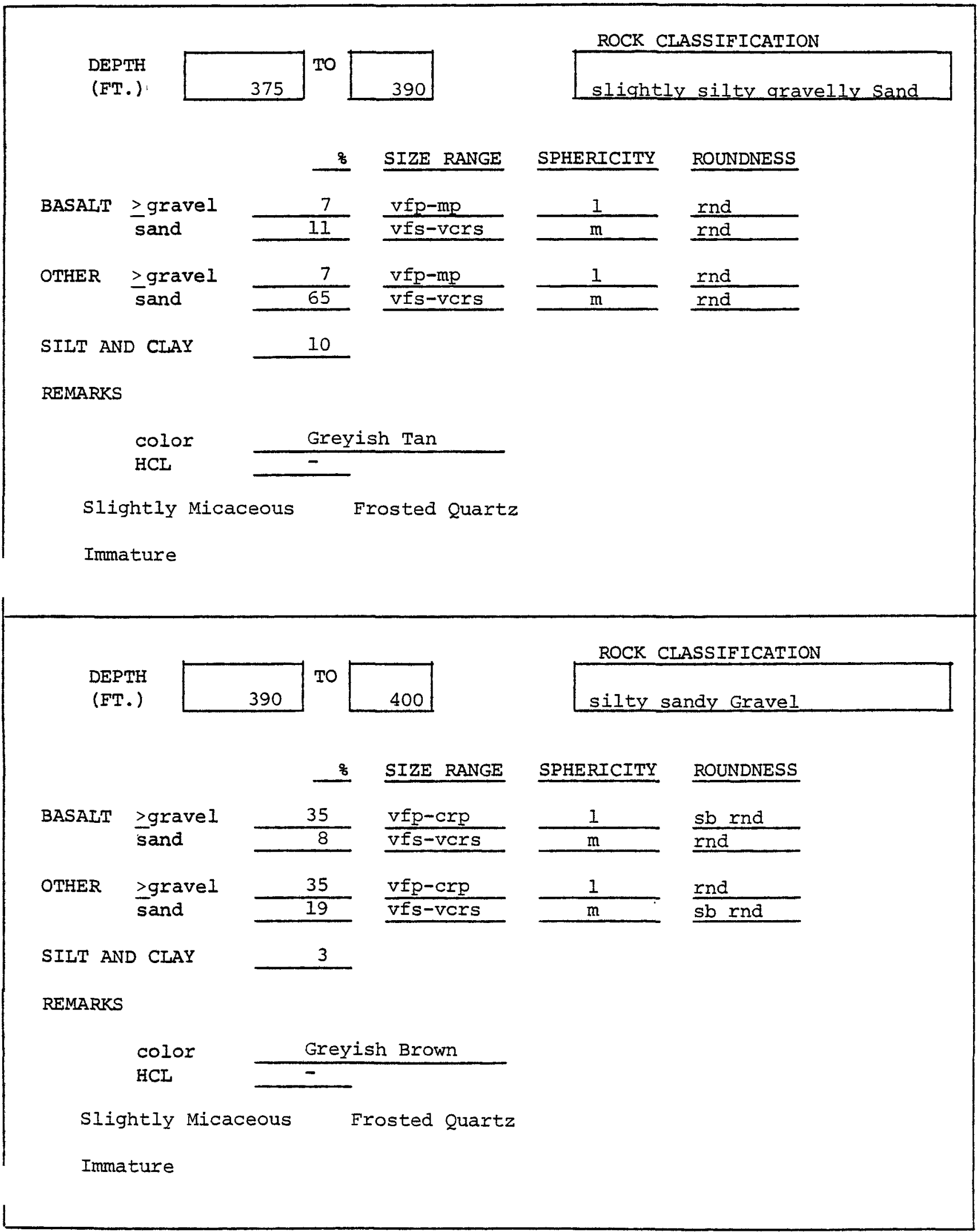


DEPTH

(FT.)

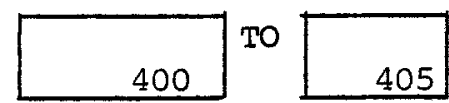

ROCK CLASSIFICATION

Silt

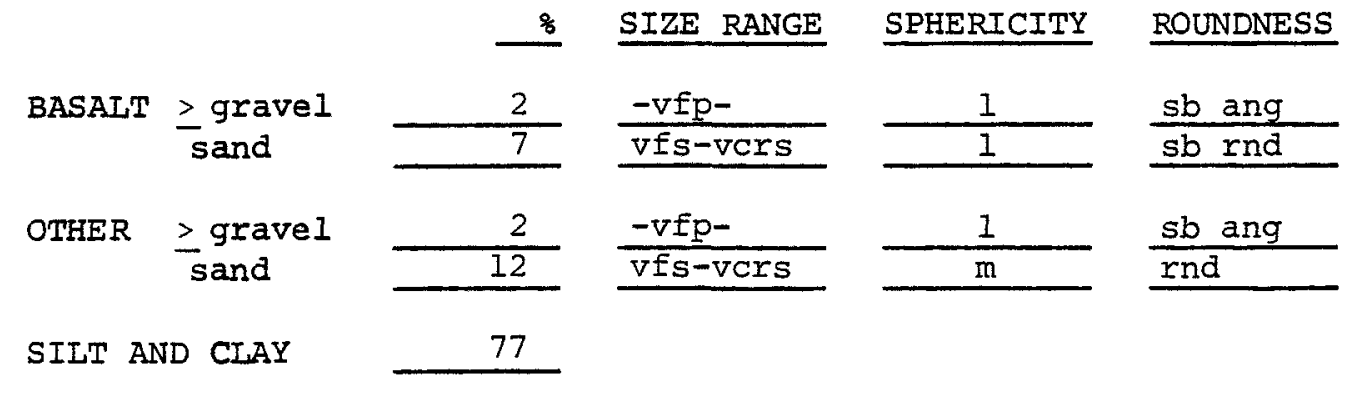

REMARKS

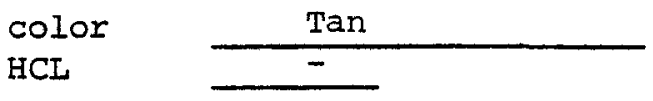

Slightly Micaceous frosted quartz

Immature

\section{DEPTH}

(ET.)

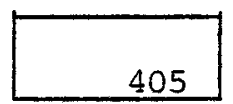

TO

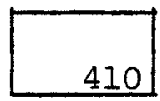

ROCK CIASSIFICATION

gravelly silty sand

\section{\% SIZE RANGE SPHERICITY ROUNDNESS}

\begin{tabular}{|c|c|c|c|c|c|}
\hline BASALT & $\geq$ gravel & 10 & $v f p-f p$ & 1 & sb rnd \\
\hline & sand & 12 & vfs-vers & $\mathrm{m}$ & Ind \\
\hline OTHER & $\geq$ gravel & 10 & $\nabla f p-f p$ & 1 & $\mathrm{sb}$ rnd \\
\hline & sand & 52 & $\mathrm{VfS}-\mathrm{vCrS}$ & $\mathrm{m}$ & rnd \\
\hline
\end{tabular}

SILT AND CLAY 16

REMARKS

color Greyish Tan

HCL

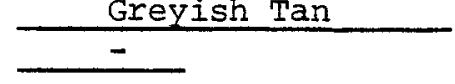

Slightly Micaceous Frosted Quartz

Immature 


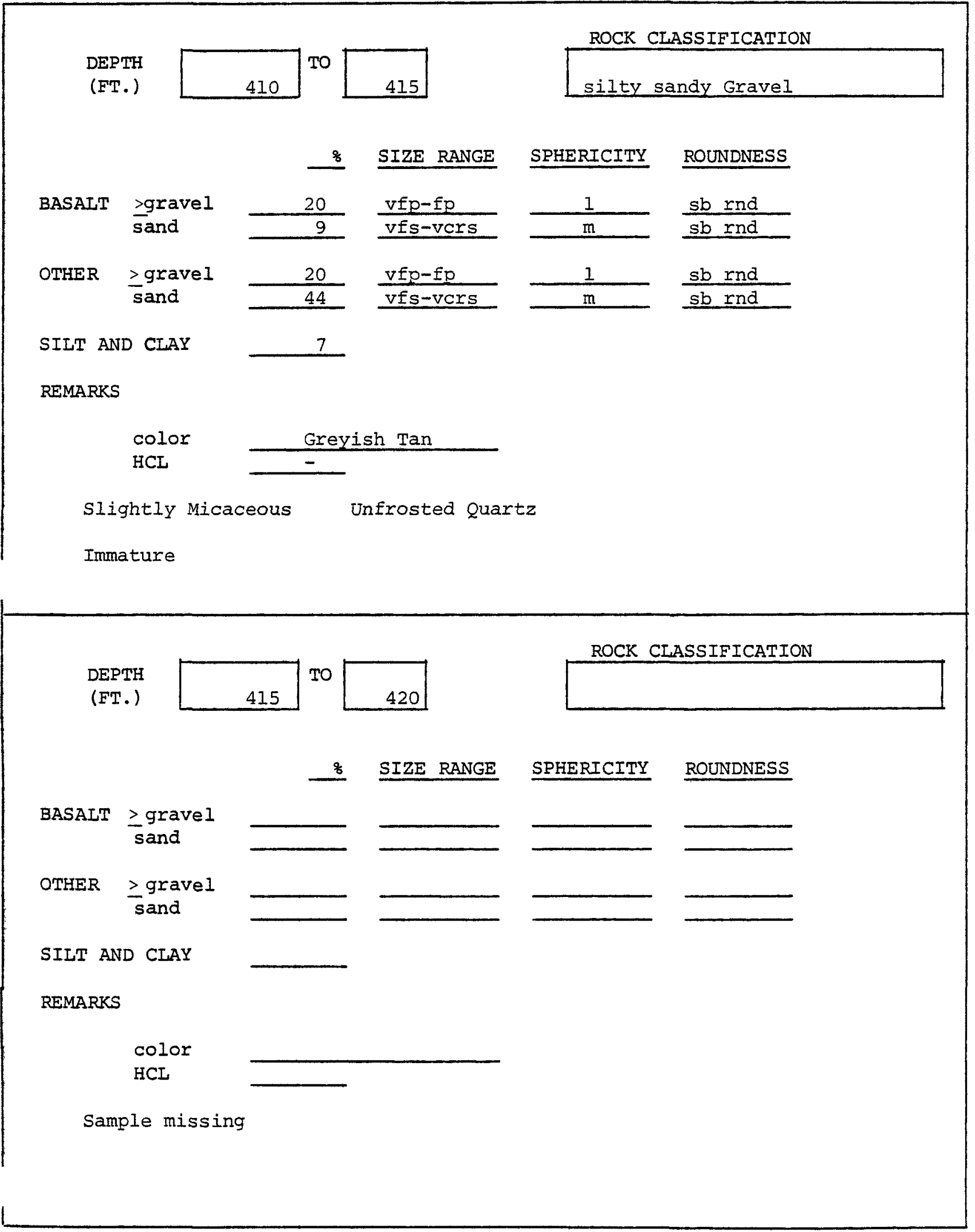


DEPTH
(FT.)

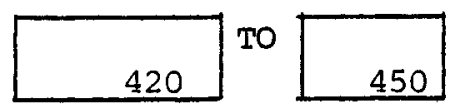

ROCK CIASSIFICATION

420

450

silty sandy Gravel

\section{\& SIZE RANGE SPHERICITY ROUNDNESS}

BASAIT $>$ gravel sand

OTHER > gravel sand

SIIT AND CLAY
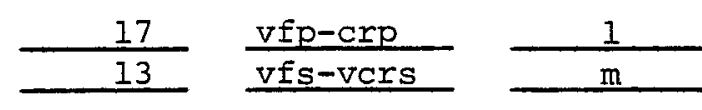

$\frac{\text { rnd }}{\text { sb rnd }}$
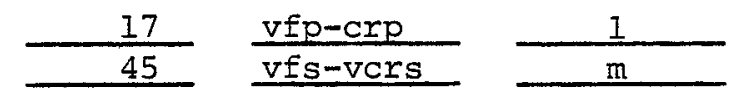

rnd

\section{8}

REMARKS

$$
\begin{aligned}
& \text { color } \\
& \text { HCL }
\end{aligned}
$$

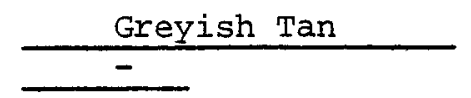

Slightly Micaceous

Frosted Quartz

Immature

\section{DEPTH \\ (FT.)}

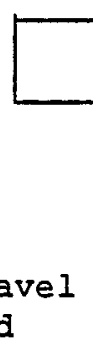

BASALT $\geq$ grave 1
sand

OTHER zgravel
sand

SILT AND CIAY

REMARKS color Sample missing
ROCK CLASSIFICATION

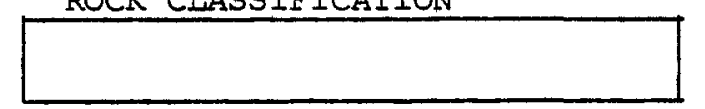

\% SIZE RANGE SPHERICITY ROUNDNESS

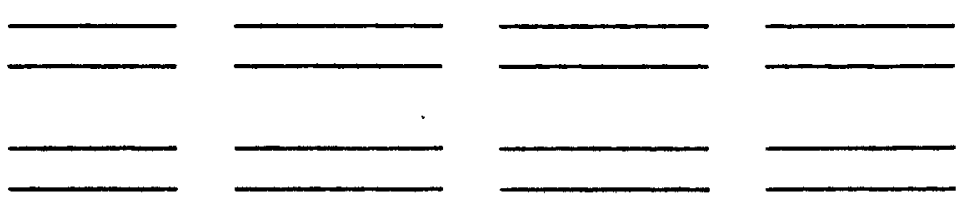




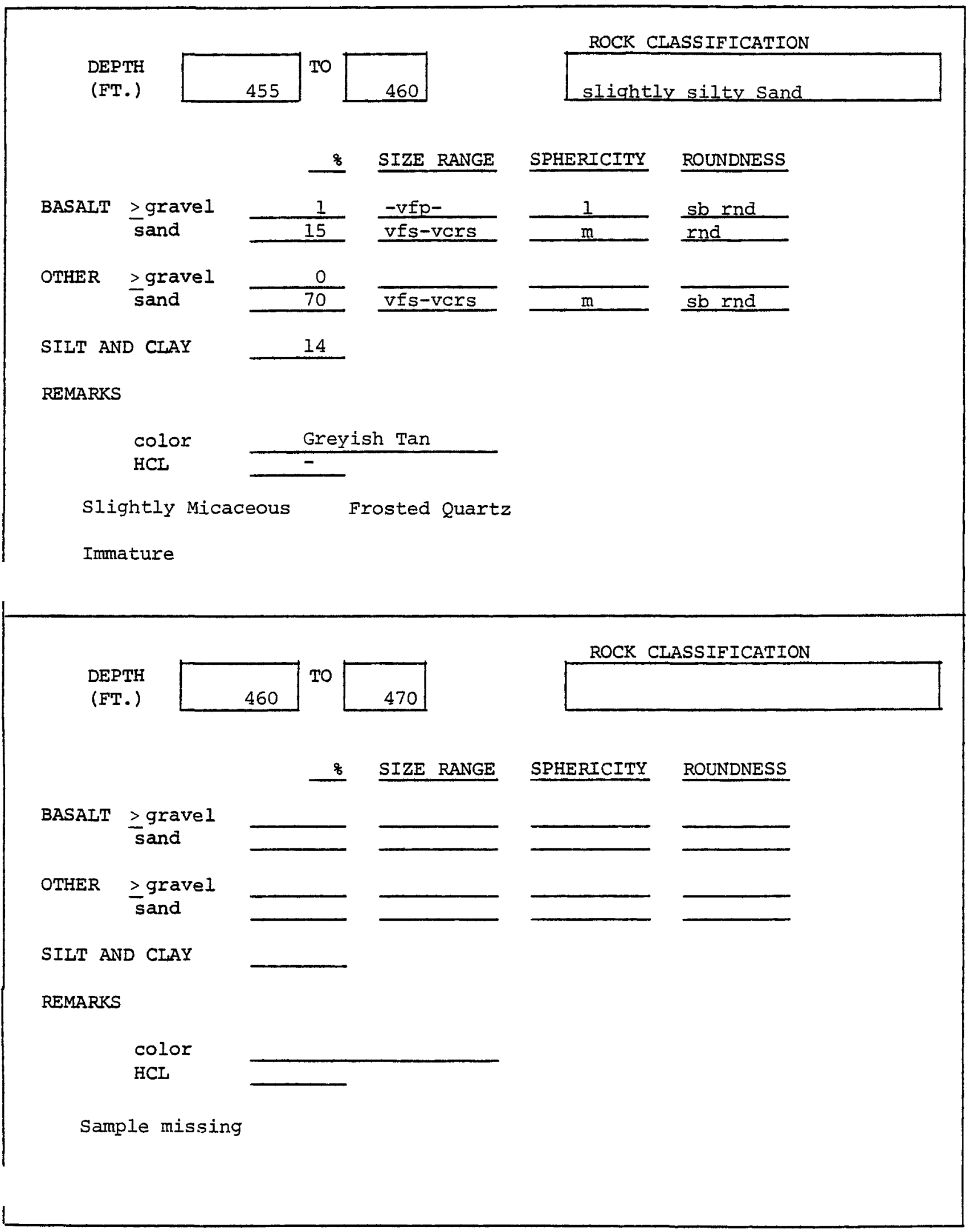


DEPTH

(FT.)

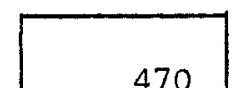

$\mathrm{TO}$

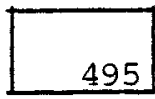

ROCK CIASSIFICATION

sandy silt

\section{S SIZE RANGE SPHERICITY ROUNDNESS}

BASAIT > graveI sand
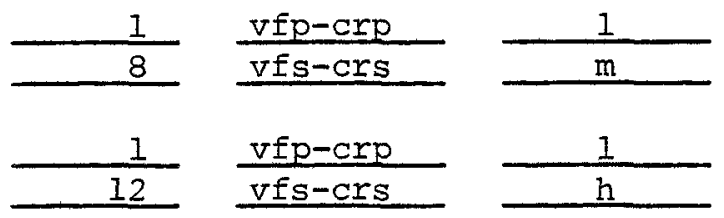

rnd

sb rnd

OTHER >gravel sand

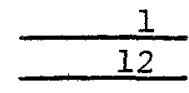

$\frac{v f p-c r p}{v f s-c r s}$ 78

SILT AND CIAY

REMARKS

color

HCI

Yellowish Tan

Slightly Micaceous Frosted Quartz

Immature

\section{DEPTH}

(FT.)

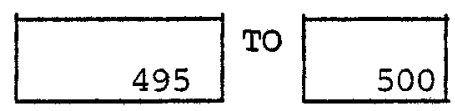

ROCK CIASSIFICATION

silty Sand

\% SIZE RANGE SPHERICITY ROUNDNESS

BASALT >gravel sand
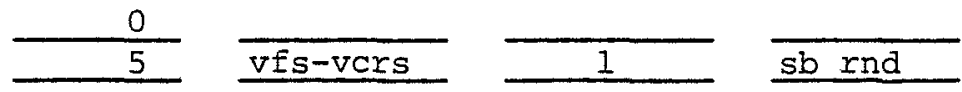

OTHER >graveI sand

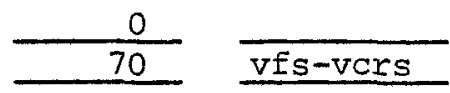
25

SILT AND CLAY

REMARKS

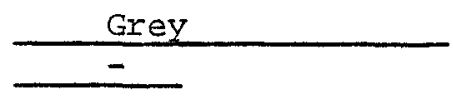

Slightly Micaceous Frosted quartz

Immature 
DEPTH
(FT.)

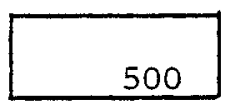

TO

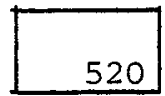

\% SIZE RANGE

\section{SPHERICITY}

ROUNDNESS

BASALT > gravel sand

OTHER Igravel sand

\section{SILT AND CLAY}

REMARKS

color HCL

Sample missing
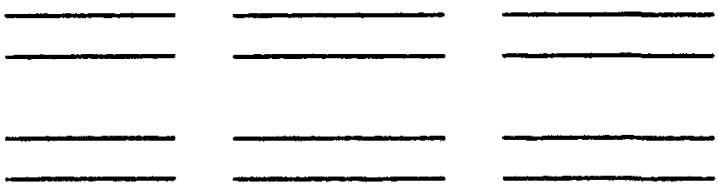

ROCK CLASSIFICATION

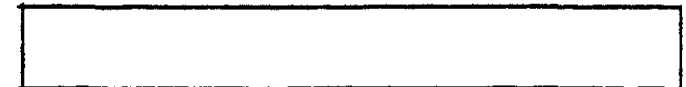




\section{DEPTH \\ (FT.)}

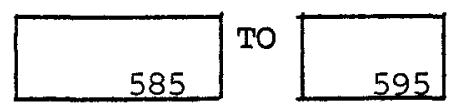

ROCK CLASSIFICATION

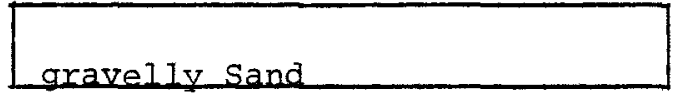

\section{S SIZE RANGE SPHERICITY ROUNDNESS}

\begin{tabular}{|c|c|c|c|c|c|}
\hline BASALT & $\begin{array}{l}\text { >gravel } \\
\text { sand }\end{array}$ & $\frac{14}{22}$ & $\frac{v f p-c r p}{v f s-v c r s}$ & $\frac{1}{m}$ & $\frac{r n d}{r n d}$ \\
\hline OTHER & >gravel & 13 & vfp-crp & 1 & rnd \\
\hline & sand & 46 & vfs-vers & $\mathrm{h}$ & rnd \\
\hline
\end{tabular}

SILT AND CLAY

5

REMARKS

color

HCL

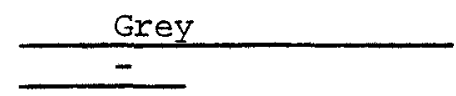

Slightly Micaceous Frosted quartz

Imnature

DEPTH

(FT.)

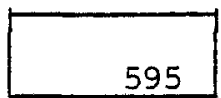

TO

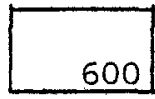

ROCK CLASSIFICATION

Sand

\section{? SIZE RANGE SPHERICITY ROUNDNESS} BASALT $\frac{\geq \text { gravel }}{\text { sand }}$
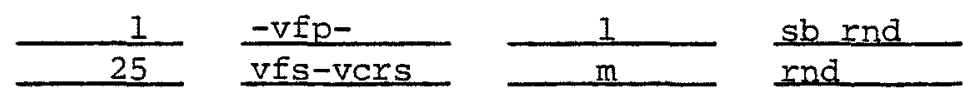

OTHER Igravel
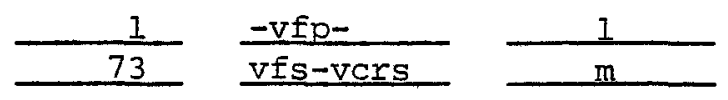

$\frac{\text { sb rnd }}{\text { sb rnd }}$

SILT AND CLAY

0

REMARKS

color

HCL

$\frac{\text { Grey }}{-}$

Slightly Micaceous

Submature 
DEPTH

(FT.)

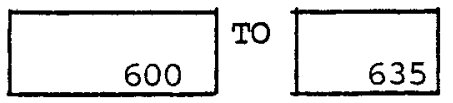

ROCK CLASSIFICATION

slightly gravelly sand

\% SIZE RANGE SPHERICITY ROUNDNESS

BASALT $\geq$ gravel sand
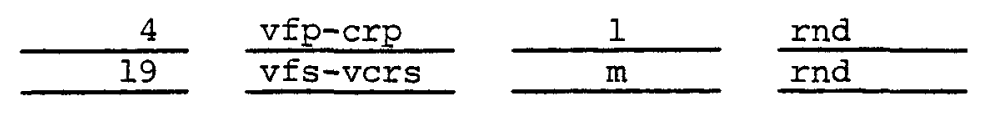

OTHER $\geq$ gravel

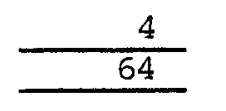

$\frac{v f p-c r p}{v f s-v c r s}$

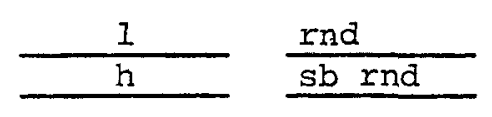

SILT AND CLAY 9

REMARKS

color HCL

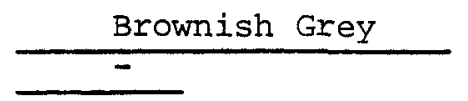

Slightly Micaceous Frosted Quartz

Immature

DEPTH

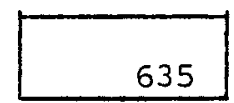

TO

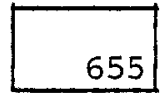

ROCK CLASSIFICATION

gravelly sand

S SIZE RANGE SPHERICITY ROUNDNESS

\begin{tabular}{|c|c|c|c|c|c|}
\hline BASALT & $\begin{array}{l}\text { >gravel } \\
\text { sand }\end{array}$ & $\frac{13}{26}$ & $\frac{v f p-m p}{v f s-v c r s}$ & $\frac{1}{m}$ & $\frac{\text { sb rnd }}{\text { sb rnd }}$ \\
\hline OTHER & $\begin{array}{l}\text { zgravel } \\
\text { sand }\end{array}$ & $\frac{13}{41}$ & $\frac{v f p-m p}{v f s-v c r s}$ & $\frac{1}{m}$ & $\frac{\mathrm{sb}}{\mathrm{sb}} \mathrm{rnd}$ \\
\hline
\end{tabular}

SIIT AND CLAY

REMARKS

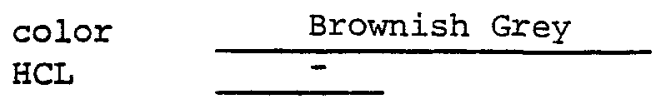

Slightly Micaceous Frosted Quartz

Immature 
ROCK CLASSIFICATION

DEPTH

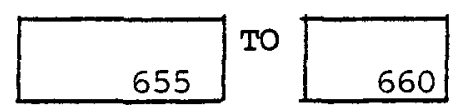

660 slightly gravelly sand

\section{\% SIZE RANGE SPHERICITY ROUNDNESS}

BASALT >gravel sand

$\frac{5}{13} \frac{v f p-c r p}{v f s-c r s}$

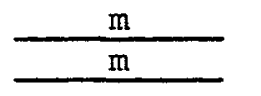

$\frac{\mathrm{w} \text { rnd }}{\text { sb rnd }}$

OTHER $\geq$ gravel
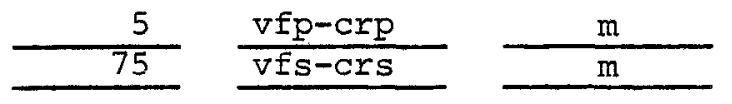

wrind

SILT AND CLAY

2

REMARKS

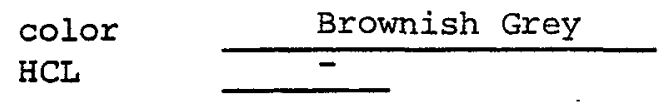

Slightly Micaceous Frosted quartz

Immature

\section{DEPTH}

(FT.)

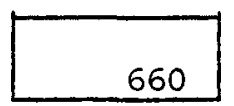

TO

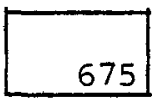

ROCK CLASSIFICATION

silty sand

\section{\% SIZE RANGE SPHERICITY ROUNDNESS}

$$
\text { BASALT } \underset{\text { sand }}{\geq \text { graveI }}
$$

OTHER $\geq$ gravel sand

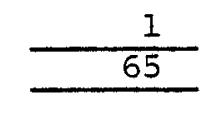

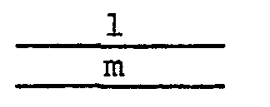

$\frac{\mathrm{sb} \text { rnd }}{\mathrm{sb} \text { rnd }}$

sb rnd

SIIT AND CIAY

25

REMARKS

$$
\begin{aligned}
& \text { color } \\
& \text { HCL }
\end{aligned}
$$

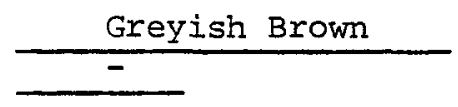

Slightly Micaceous

Unfrosted Quartz

Immature 


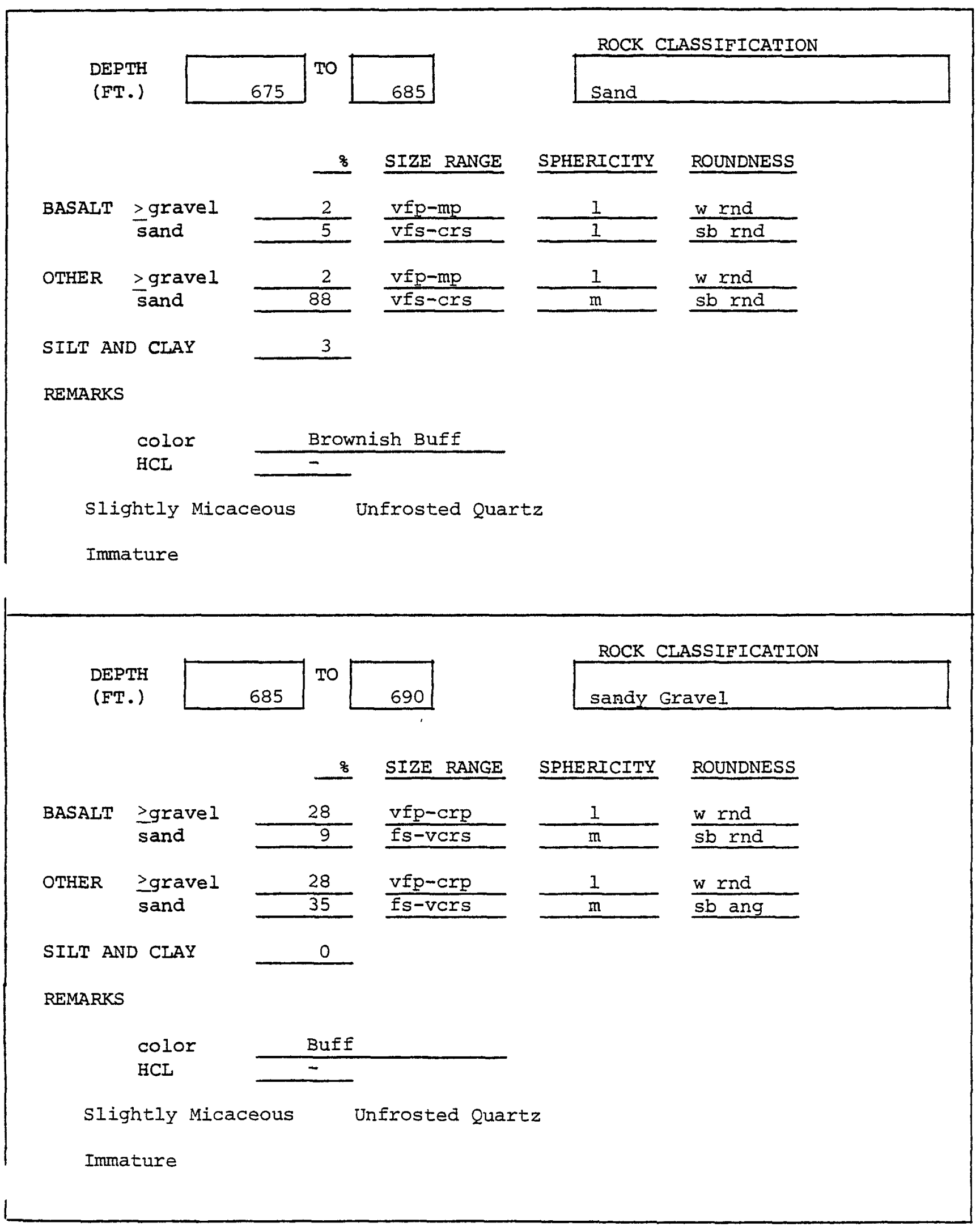


DEPTH

(FT.)

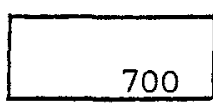

TO

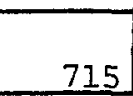

ROCK CLASSIFICATION

slightly gravelly sand

\% SIZE RANGE SPHERICITY ROUNDNESS

BASALT >gravel sand

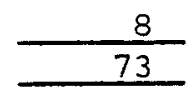

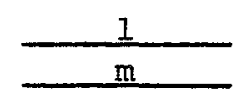

$$
\frac{\text { sb rnd }}{\text { sb rnd }}
$$

OTHER $\underset{\text { sand }}{\text { gravel }}$
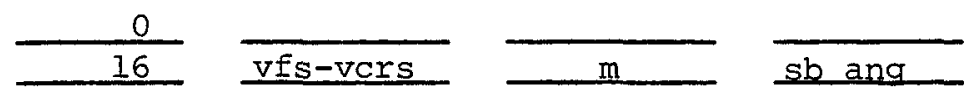

SILT AND CLAY 3

REMARKS

$$
\text { color }
$$
HCL

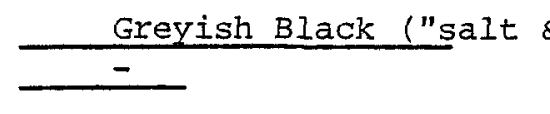

\& pepper")

Slightly Diatomaceous (? pebbles \& sand)

Slightly Micaceous

Unfrosted Quartz Immature

Total Drilled Depth reported as $715^{\prime}$

DEPTH
(FT.)

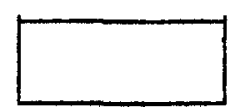

TO

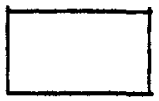

ROCK CLASSIFICATION

\section{\% SIZE RANGE SPHERICITY ROUNDNESS}

BASALT $\geq$ gravel sand

OTHER Zgravel sand

SILT AND CLAY

REMARKS

color HCI
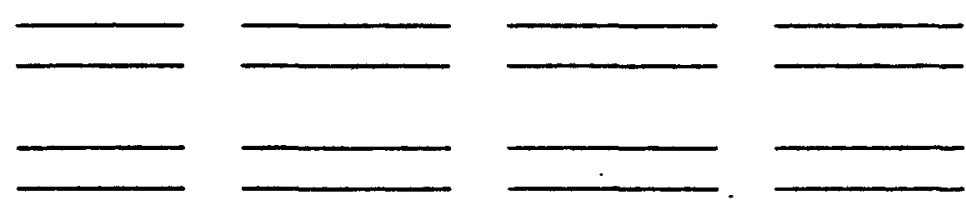


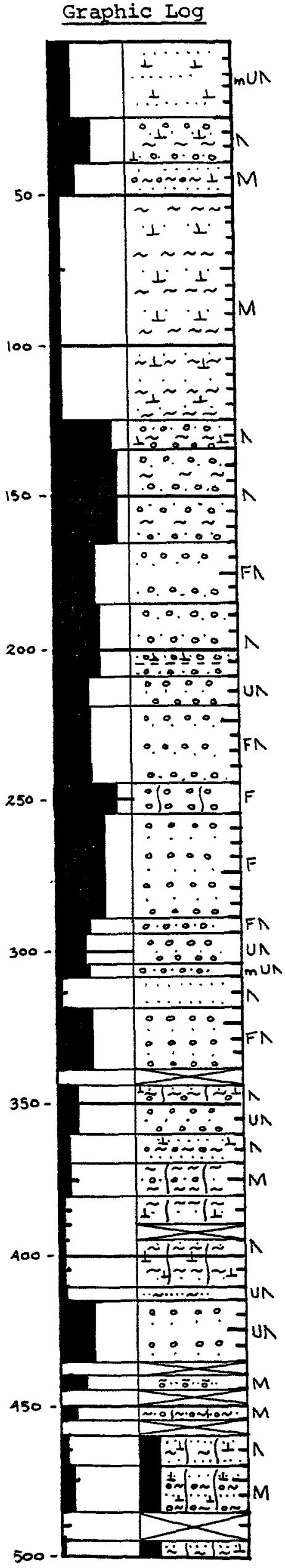

Sample Description

grey

greyish tan

greyish $\tan$

brownish $\tan$

greyish brown

brownish grey

brownish grey

buff grey

buff

greyish buff

brownish grey

greyish buff

greyish butf

greyish buff

greyish buff

buff

greyish buff

$\tan$

buff

brownish $\tan$

brownish $\tan$

$\tan$

buff

buff grey

greyish tan

light brown

whitish brown

greyish $\tan$

whitish tan
Plant Coordinates

N029000

$\frac{W 078000}{\text { Altitude } 645}$

Other Data used in Interpretation

Driller's Log 
Depth (ft.)
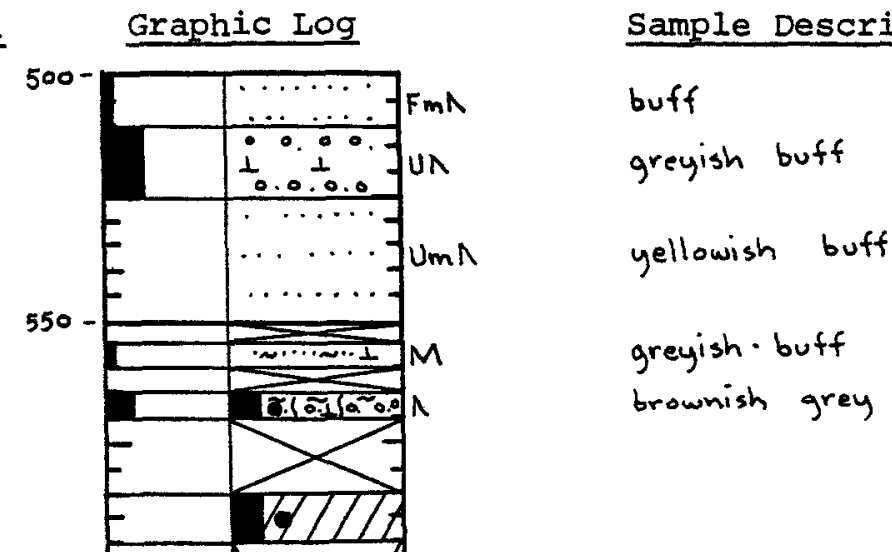

Plant Coordinates

Altitude

Other Data used in

Interpretation

60
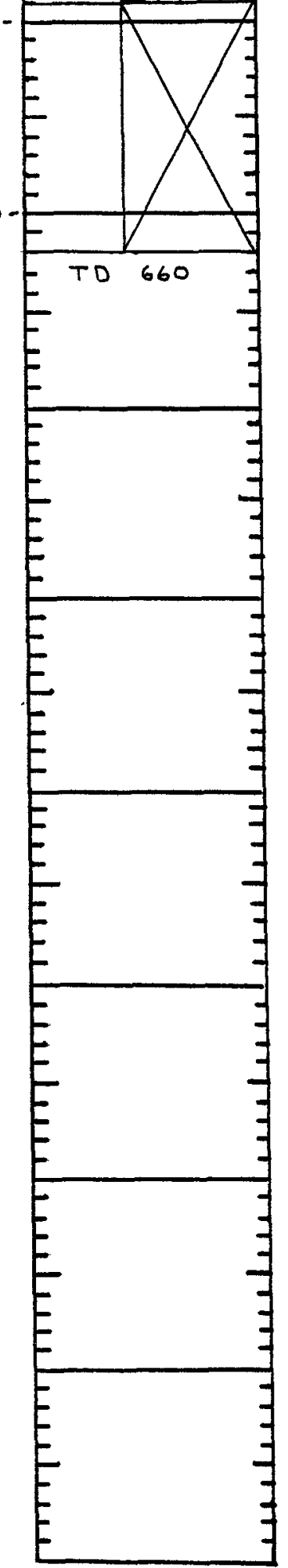


\section{DEPTH}

(FT.)

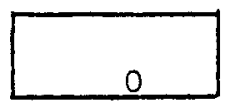

TO

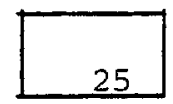

ROCK CLASSIFICATION

Sand

8 SIZE RANGE SPHERICITY ROUNDNESS

BASAIT $\frac{\geq \text { gravel }}{\text { sand }}$
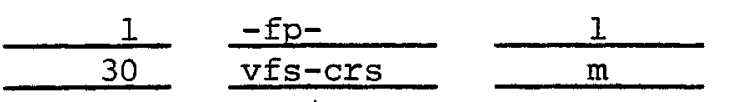

$\frac{\text { sb rnd }}{\text { sb rnd }}$

OTHER >gravel

sand
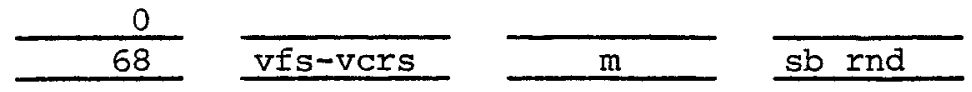

SILT AND CLAY

1

REMARKS

color

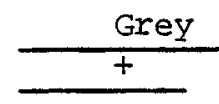

Slightly Micaceous

Unfrosted Quartz

Mature

DEPTH
(FT.)

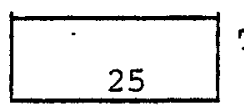

To

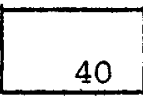

ROCK CLASSIFICATION

silty sandy Gravel

\section{\% SIZE RANGE SPHERICITY ROUNDNESS}

BASALT $\frac{\text { zgravel }}{\text { sand }}$
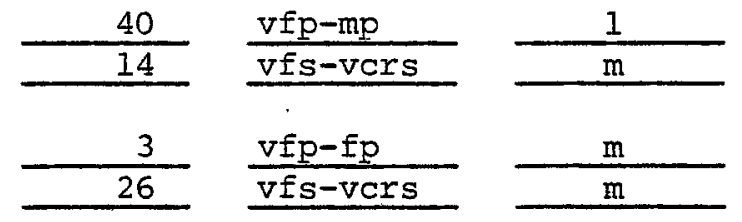
sb rn
rnd

OTHER $\geq$ gravel

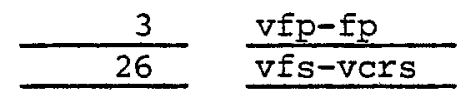

sb rnd sand

SILT AND CLAY

17

REMARKS

$\begin{array}{ll}\text { Color } & \text { Greyish Tan } \\ \text { HCL } & +\end{array}$

Slightly Micaceous

Immature 
ROCK CIASSIFICATION

DEPTH

(FT.)

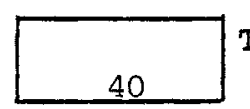

TO

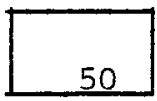

gravelly silty sand

\section{\% SIZE RANGE SPHERICITY ROUNDNESS}

BASALT $\begin{aligned} & \text { >gravel } \\ & \text { sand }\end{aligned}$

OTHER $\geq$ gravel sand

SILT AND CLAY

REMARKS
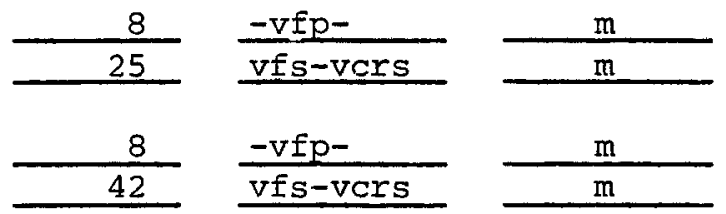

vfs-vcrs sb rnd

sb rnd

\section{7}

rnd

color

HCL

Greyish Tan

Micaceous

Immature

DEPTH

(FT.)

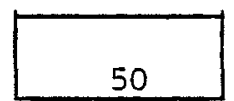

TO

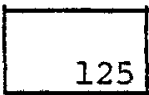

ROCK CLASSIFICATION

sandy Silt

\section{SIZE RANGE SPHERICITY ROUNDNESS}

\begin{tabular}{|c|c|c|c|c|c|}
\hline BASALT & $\begin{array}{l}\text { Pgravel } \\
\text { sand }\end{array}$ & $\frac{1}{10}$ & $\frac{-v f p-}{v f s-c r s}$ & $\frac{m}{m}$ & $\frac{\text { sb rnd }}{r n d}$ \\
\hline OTHER & Igravel & 0 & & & \\
\hline & sand & 30 & $\mathrm{vfs}-\mathrm{crs}$ & $\mathrm{m}$ & rnd \\
\hline SIIT AN & D CLAYY & 59 & & & \\
\hline
\end{tabular}

REMARKS

$\begin{array}{ll}\text { Color } & \text { Brownish Tan } \\ \text { HCL } & t\end{array}$

Micaceous

Immature 


\section{DEPTH}

(FT.)

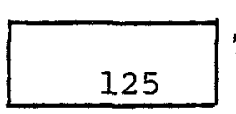

TO

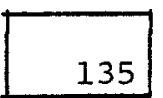

ROCK CIASSIFICATION

silty sandy Gravel

\section{\& SIZE RANGE SPHERICITY ROUNDNESS}

\begin{tabular}{|c|c|c|c|c|c|}
\hline BASALT & $\begin{array}{l}\text { >gravel } \\
\text { sand }\end{array}$ & $\frac{40}{37}$ & $\begin{array}{l}v f p-m p \\
v f s-v c r s\end{array}$ & $\frac{m}{m}$ & $\frac{\text { sb rnd }}{r n d}$ \\
\hline OTHER & $\begin{array}{l}\text { >gravel } \\
\text { sand }\end{array}$ & $\frac{3}{11}$ & $\frac{v f p-f p}{v f s-v e r s}$ & $\frac{m}{m}$ & $\frac{\text { rnd }}{\text { rnd }}$ \\
\hline
\end{tabular}

SILT AND CLAY

REMARKS

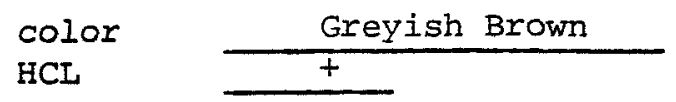

Slightly Micaceous

Immature

DEPTH

(FT.)

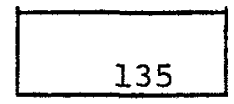

TO

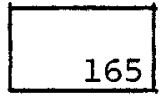

165
ROCK CIASSIFICATION

silty sandy Gravel

\& SIZE RANGE SPHERICITY ROUNDNESS

\begin{tabular}{|c|c|c|c|c|c|}
\hline \multirow[t]{2}{*}{ BASALT } & >gravel & 52 & $v f p-m p$ & $\mathrm{~m}$ & sb rnd \\
\hline & sand & 32 & vis-vers & $\mathrm{m}$ & rnd \\
\hline \multirow[t]{2}{*}{ OTHER } & I gravel & 8 & vfp-crp & $\mathrm{m}$ & rnd \\
\hline & sand & 4 & vfs-vers & $m$ & rnd \\
\hline
\end{tabular}

SILT AND CLAY

4

REMARKS

$\begin{array}{ll}\text { Color } & \text { Brownish Grey } \\ \text { HCL } & -\end{array}$

Slightly Micaceous

Immature 


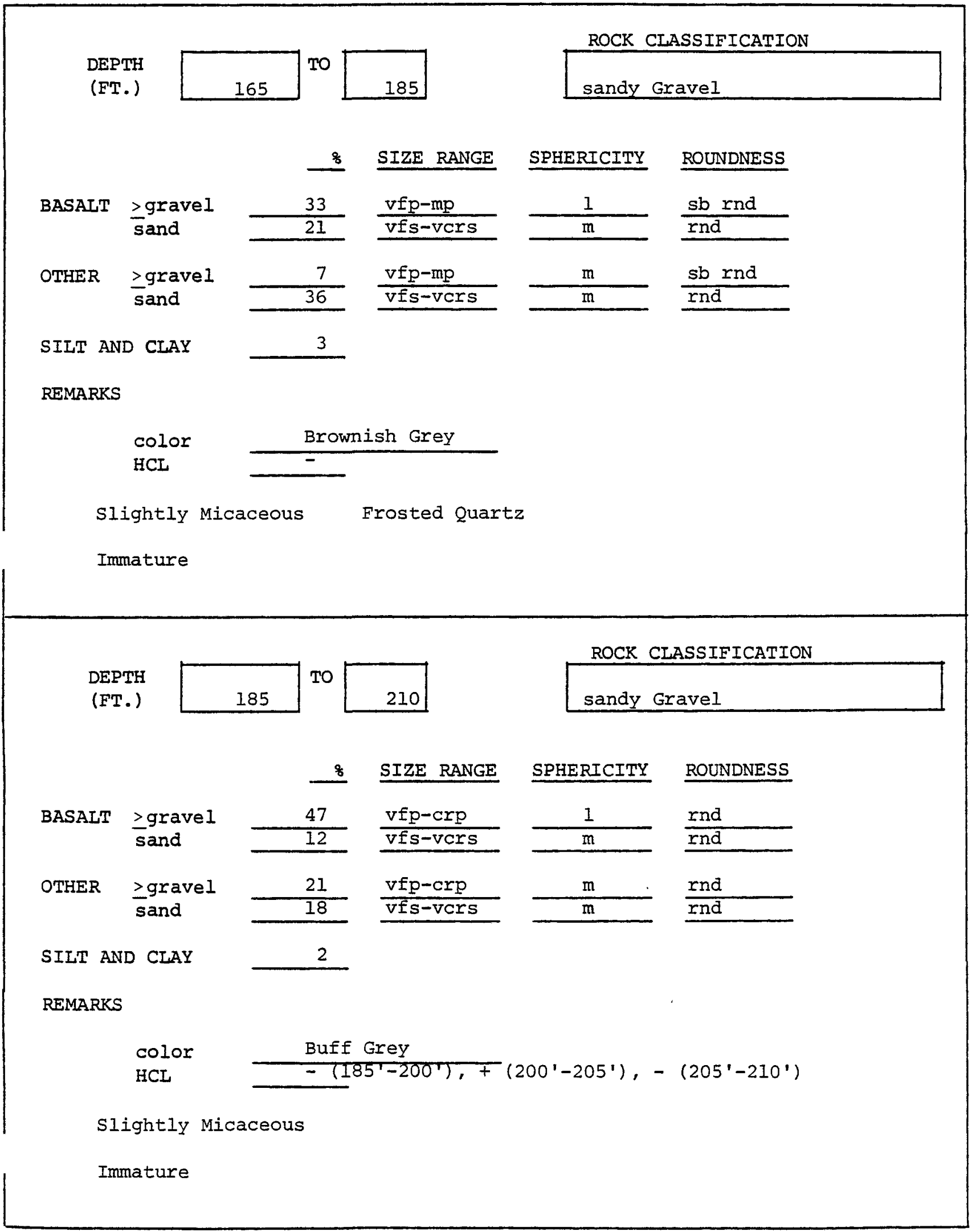




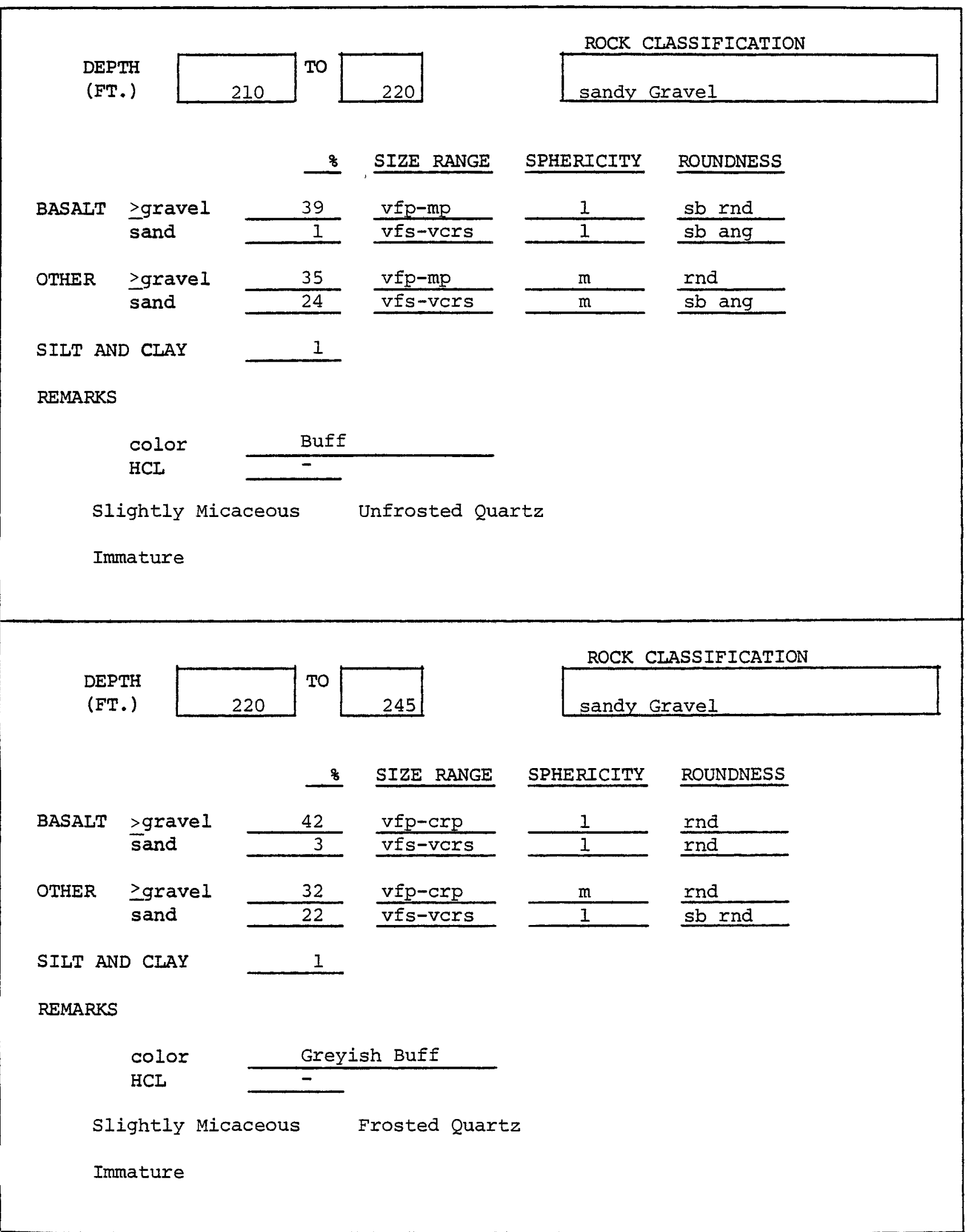




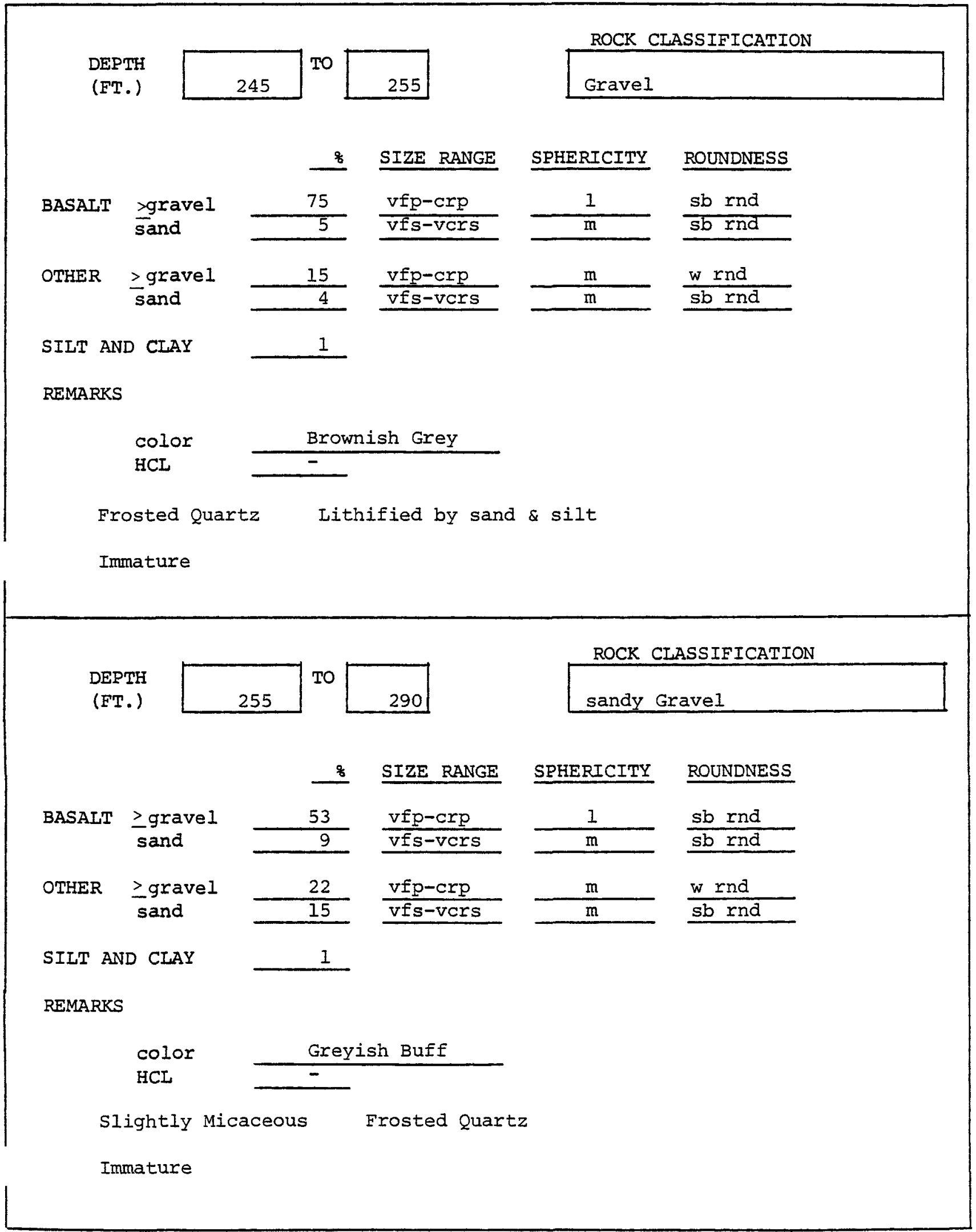




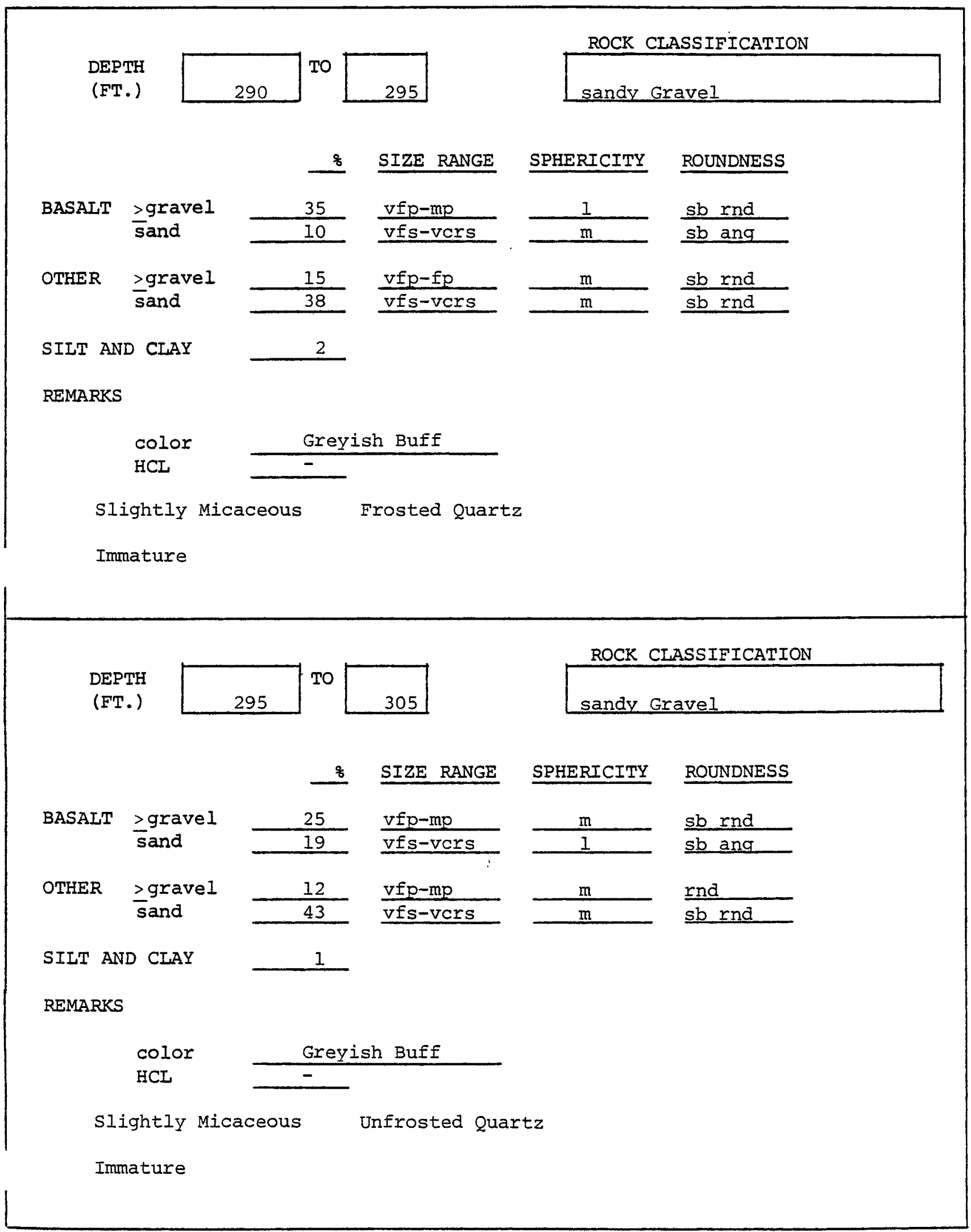


DEPTH
(ET.)

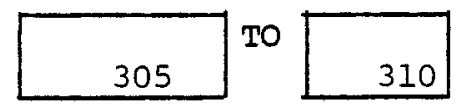

ROCK CLASSIFICATION

sandy Gravel

8 SIZE RANGE SPHERICITY ROUNDNESS

BASALT > gravel sand
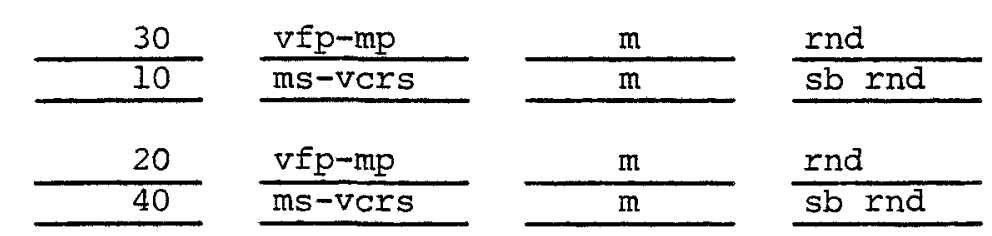

OTHER $\geq$ gravel
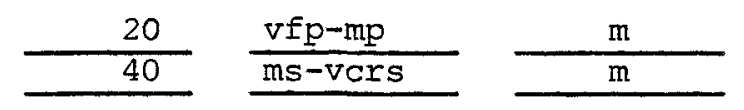

sb rnd

SILT AND CLAY

0

REMARKS

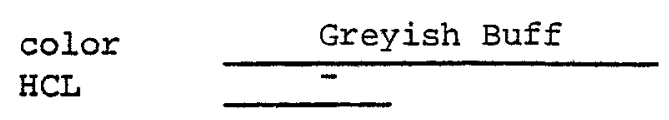

Slightly Micaceous Unfrosted quartz

Immature

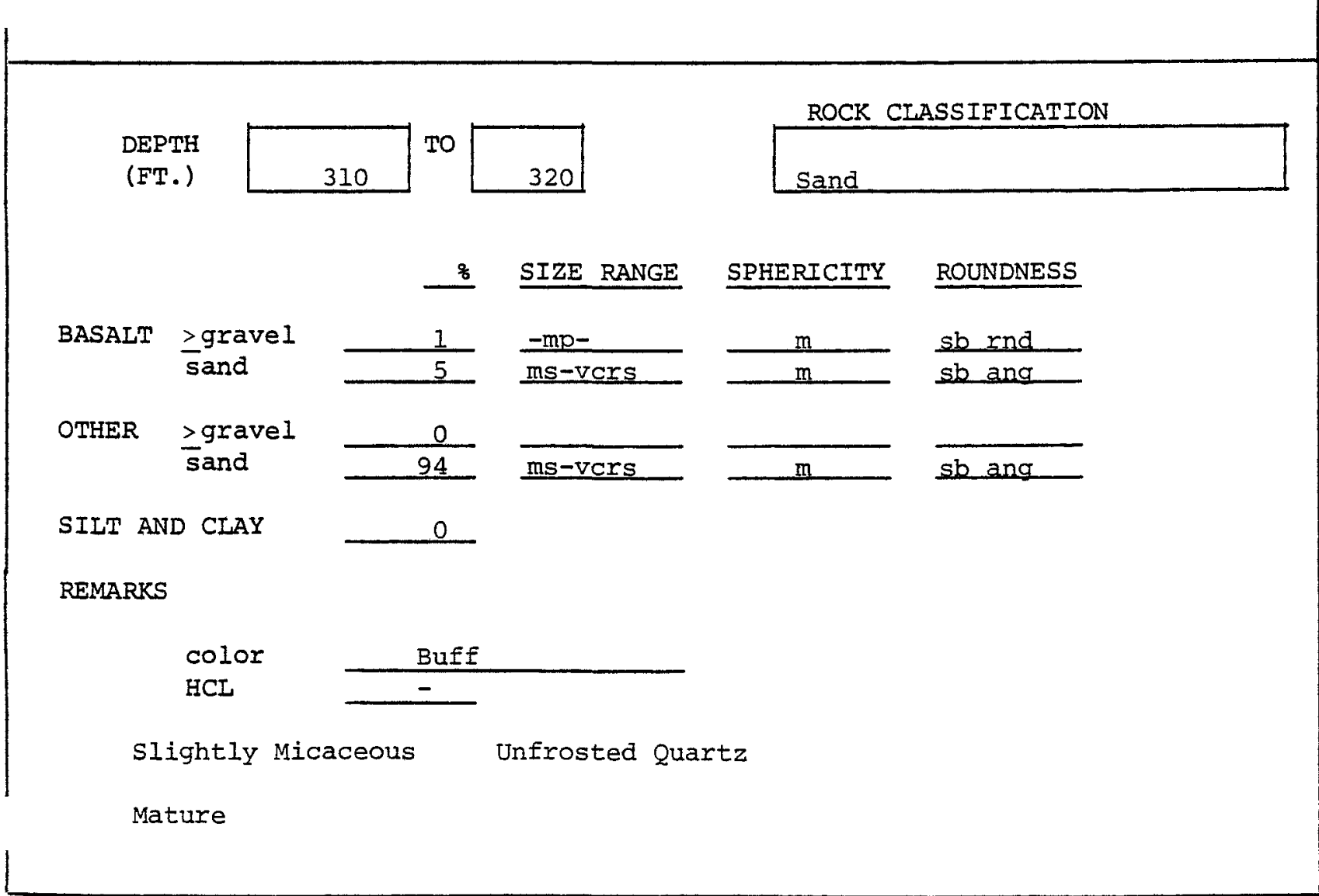




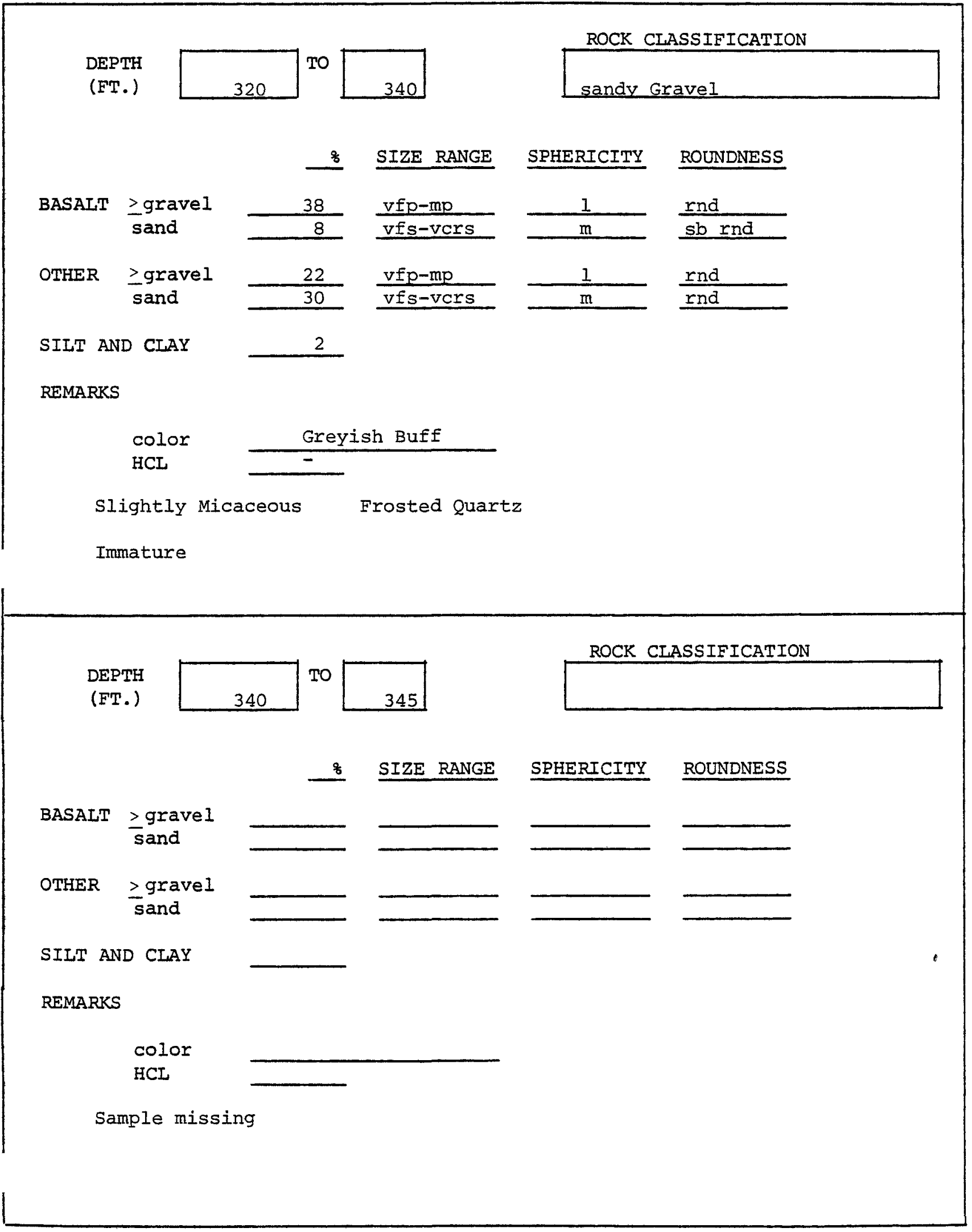


DEPTH
(FT.)

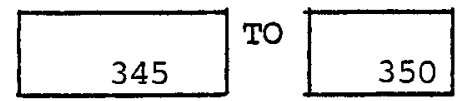

ROCK CLASSIFICATION

gravelly sandy silt

\section{s SIZE RANGE SPHERICITY ROUNDNESS}

BASAIT $\frac{\geq \text { gravel }}{\text { sand }}$
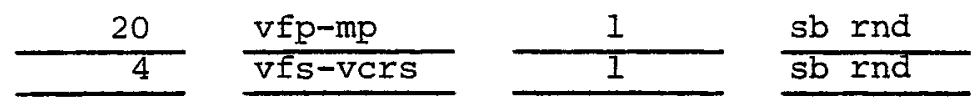

OTHER $\geq$ gravel sand
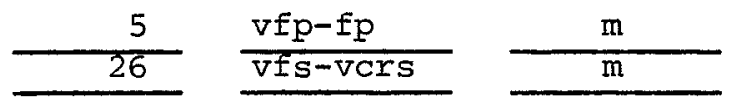

rnd

rnd

SILT AND CIAY

45

REMARKS

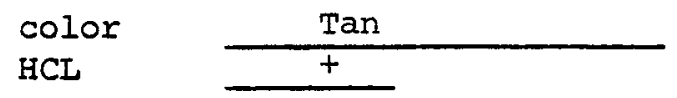

Slightly Micaceous Lithified by silt, clay \& $\mathrm{CaCO}_{3}$

Immature

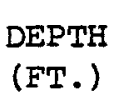

DEPTH
(ET.)

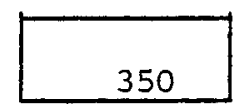

TO

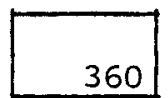

ROCK CIASSIFICATION

sandy Gravel

\section{SIZE RANGE SPHERICITY ROUNDNESS}

BASALT $\frac{\geq \text { gravel }}{\text { sand }}$

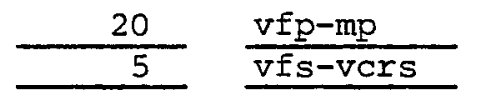

$\frac{1}{\mathrm{~m}} \quad \underline{\text { rnd }}$

OTHER $\underset{\text { sand }}{\text { gravel }}$
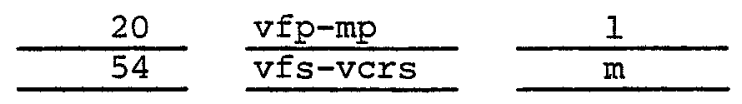

rnd

sand

1

SILT AND CLAY

REMARKS

$$
\text { color }
$$

HCL

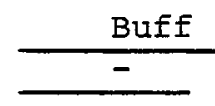

Slightly Micaceous

Unfrosted Quartz

Immature 
ROCK CIASSIFICATION

DEPTH

(FT.)

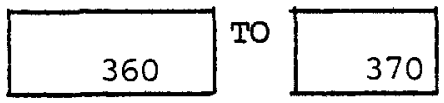

\% SIZE RANGE SPHERICITY ROUNDNESS

BASALT $\underset{\text { sand }}{\geq \text { gravel }}$

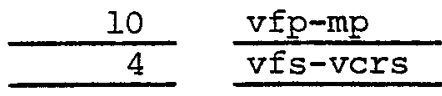

$\frac{1}{1} \quad \frac{\text { rnd }}{\text { sb rnd }}$

OTHER $\geq$ graveI sand
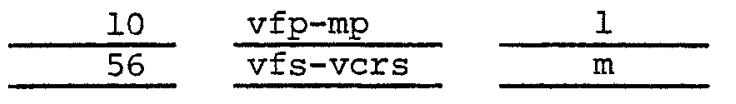

rnd

SIIT AND CLAY

20

REMARKS

color

Brownish Tan

$\mathrm{HCL}$

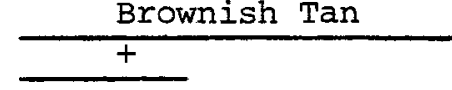

Slightly Micaceous

Immature

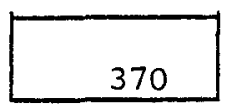

TO

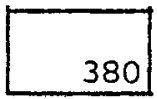

ROCK CLASSIFICATION

(FT.)

\section{\% SIZE RANGE SPHERICITY ROUNDNESS}

BASALT $\geq$ gravel

$$
\text { sand }
$$

OTHER Igravel sand

SILT AND CLAY

REMARKS

color

HCI

Micaceous

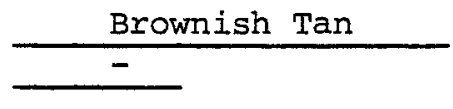

Lithified by silt \& clay 


\section{DEPTH}

(FT.)

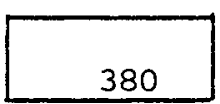

TO

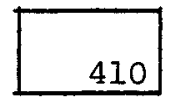

ROCK CLASSIFICATION

sandy silt

S SIZE RANGE SPHERICITY ROUNDNESS

BASALT $\geq$ gravel sand
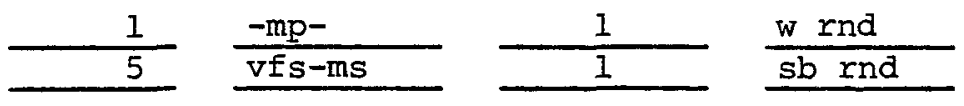

OTHER >gravel sand
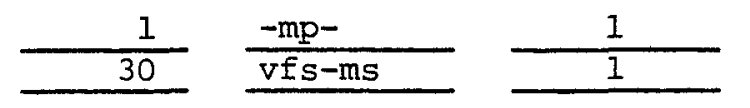

$\frac{w \text { rnd }}{\text { rnd }}$

SIIT AND CLAY

63

REMARKS

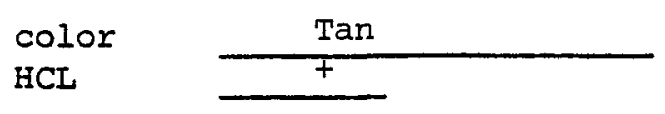

Slightly Micaceous Lithified by silt, clay \& $\mathrm{CaCO}_{3}$

Sample interval 390'-395' missing

Immature

DEPTH

(FT.)

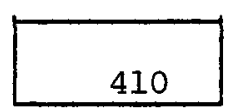

TO

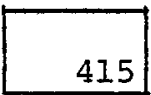

ROCK CLASSIEICATION

slightly silty sand

\% SIZE RANGE SPHERICITY ROUNDNESS

BASALT $\geq$ gravel sand
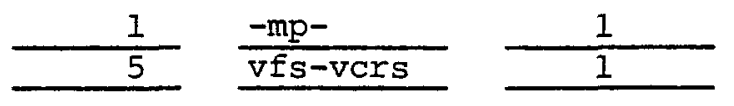

$\frac{w \text { rnd }}{\text { sb ang }}$

OTHER $\geq$ gravel
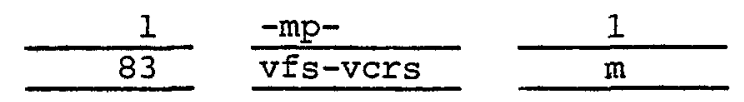

$\frac{w \text { rnd }}{\text { sb ang }}$

SILT AND CLAY 10

REMARKS

color

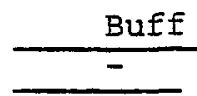

Slightly Micaceous Unfrosted Quartz

Immature 
DEPTH

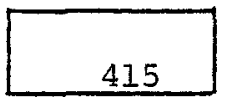

TO

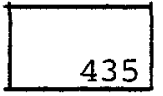

ROCK CIASSIFICATION

sandy Gravel

\% SIZE RANGE SPHERICITY ROUNDNESS

\begin{tabular}{|c|c|c|c|c|c|}
\hline \multirow[t]{2}{*}{ BASALT } & \multirow{2}{*}{$\begin{array}{l}\geq \text { gravel } \\
\text { sand }\end{array}$} & 37 & $v f p-m p$ & 1 & $w$ rnd \\
\hline & & 5 & $\mathrm{vfs-vCrs}$ & $m$ & sb ang \\
\hline \multirow[t]{2}{*}{ OTHER } & \multirow{2}{*}{$\begin{array}{l}\geq \text { gravel } \\
\text { sand }\end{array}$} & 22 & $v f p-m p$ & 1 & w rnd \\
\hline & & 35 & $v f s-v c r s$ & $\mathrm{~m}$ & sb rnd \\
\hline
\end{tabular}

SIIT AND CLAY 1

REMARKS
color
Buff Grey
HCL

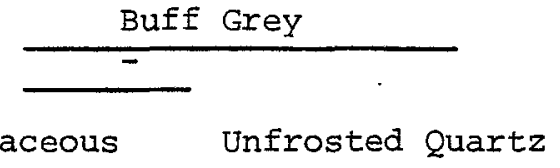
Slightly Micaceous
Unfrosted Quartz

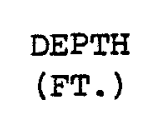

DEPTH
(FT.)

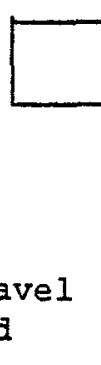

OTHER

$\geq$ gravel sand

SILT AND CLAY

REMARKS

color

HCL

Sample missing
ROCK CLASSIFICATION

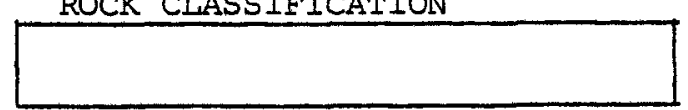

S SIZE RANGE SPHERICITY ROUNDNESS
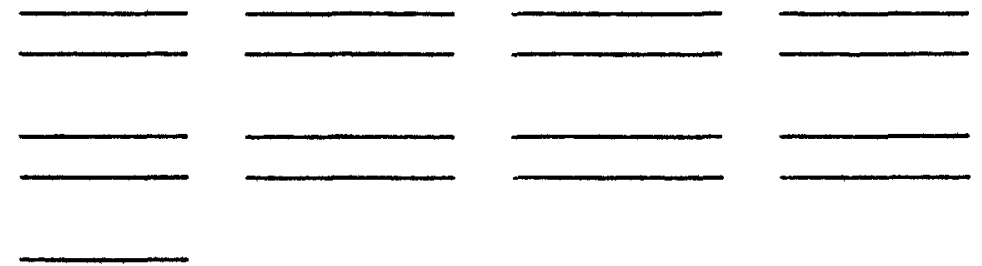

440 


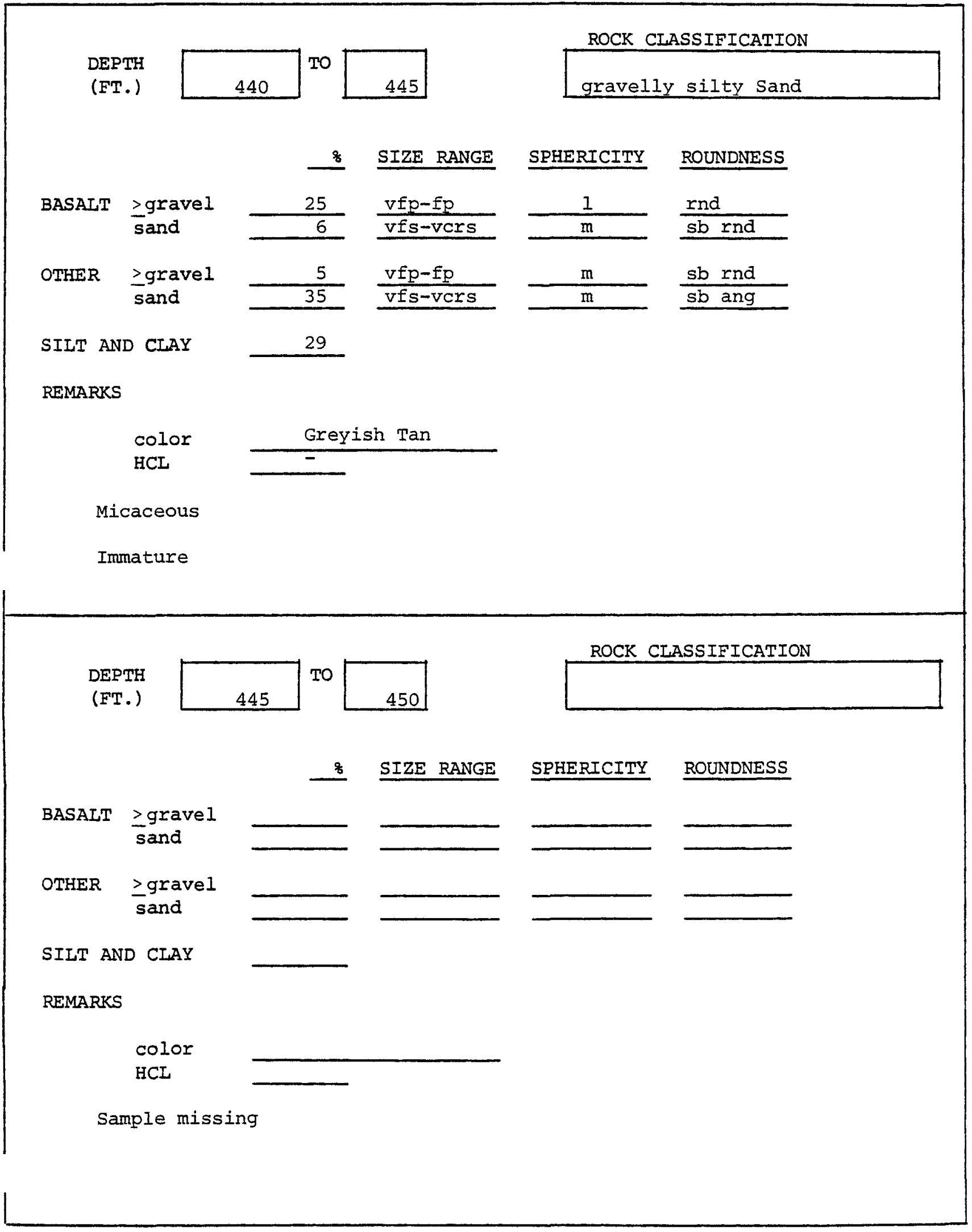

W. K. Summers \& Associates 


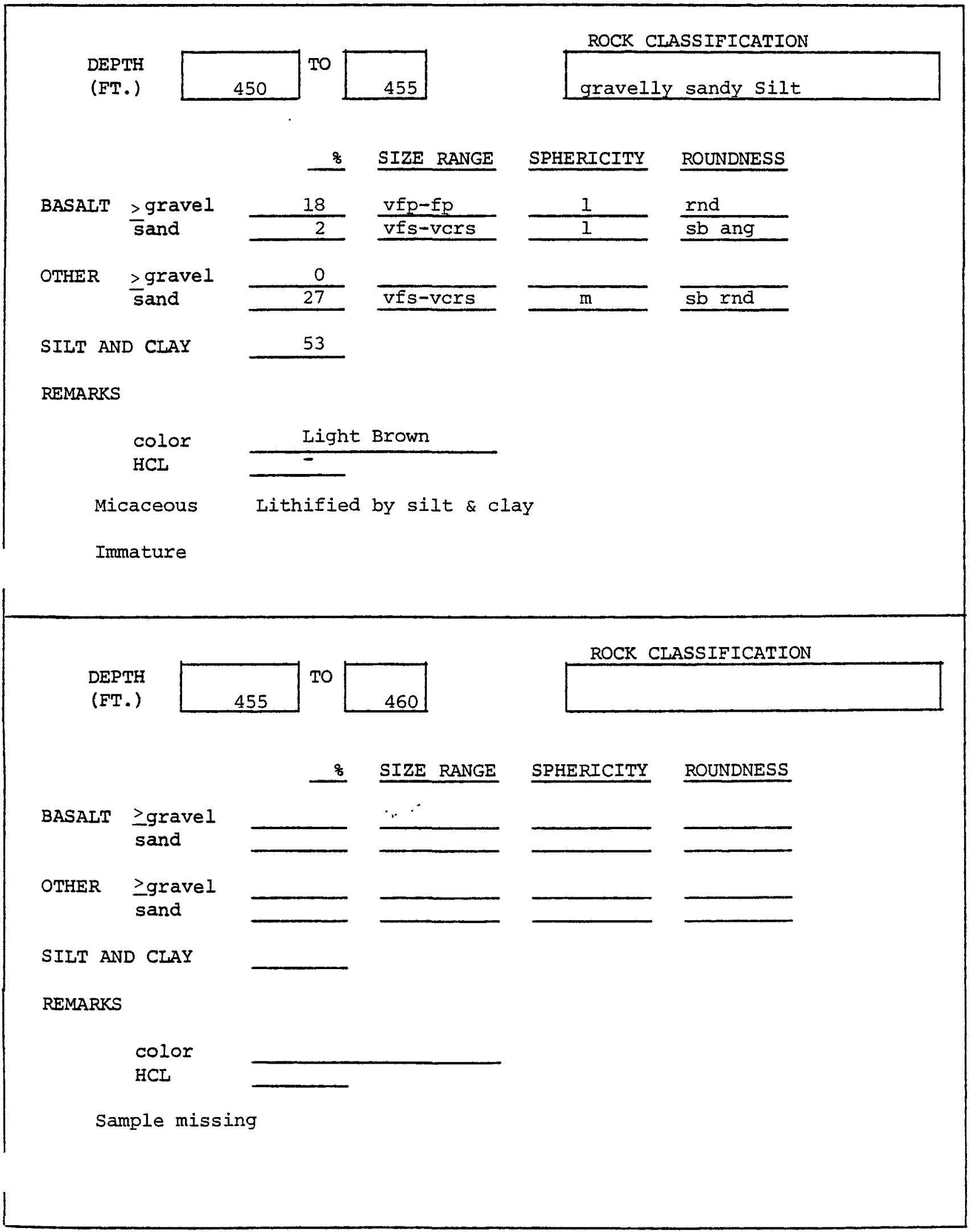




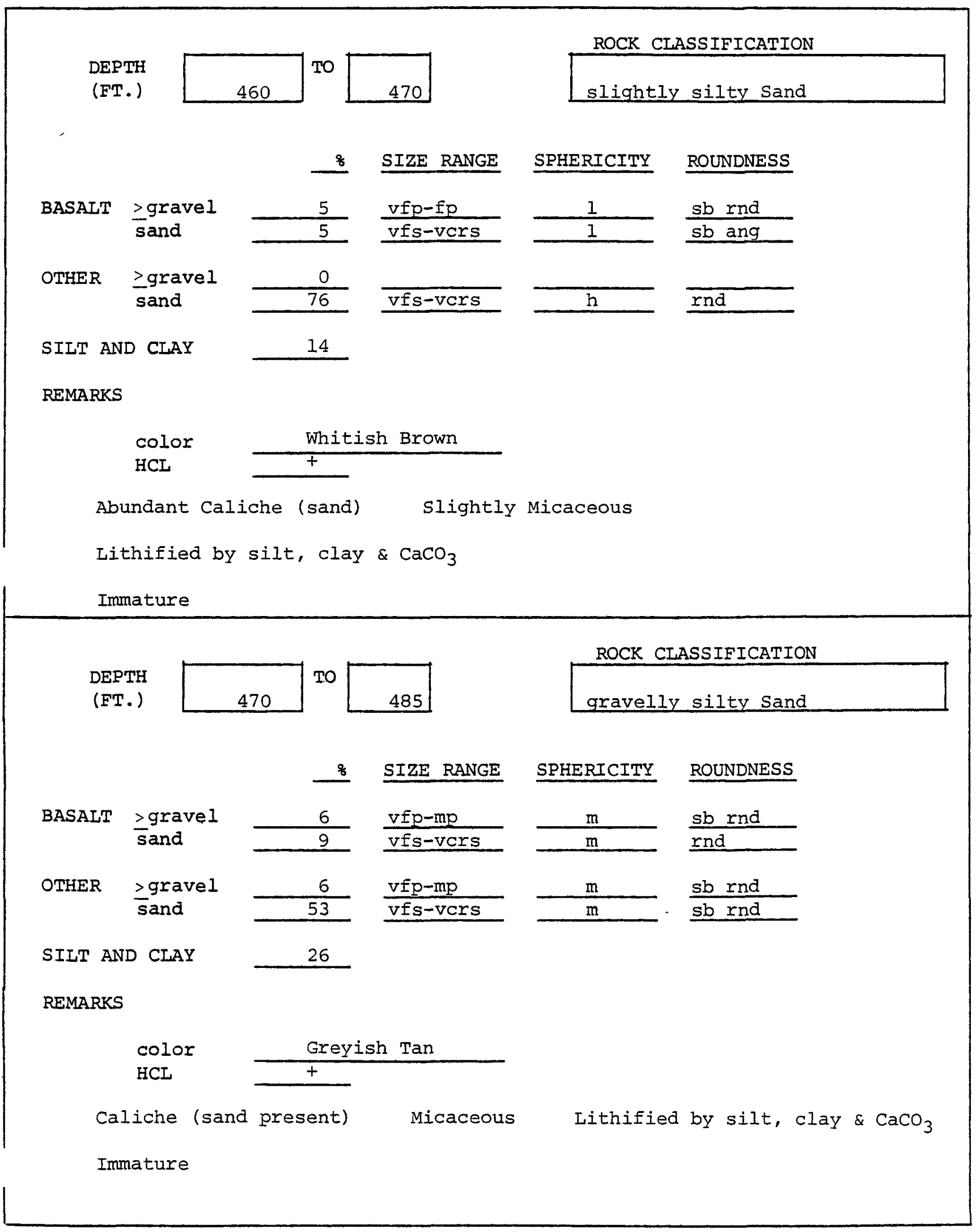



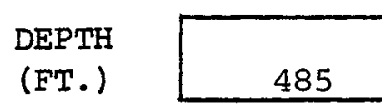

TO

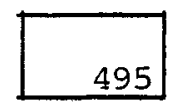

\% SIZE RANGE SPHERICITY ROUNDNESS

BASALT $\geq$ gravel sand

OTHER $\quad \frac{\text { gravel }}{\text { sand }}$

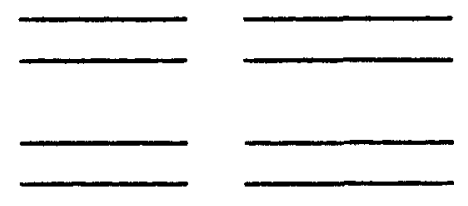

SILT AND CLAY

REMARKS

color

HCL

Sample missing

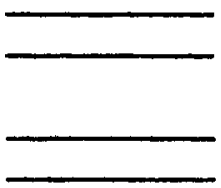

ROCK CLASSIFICATION

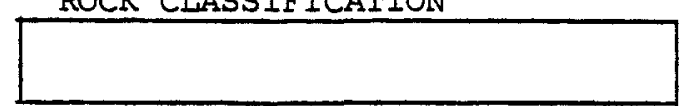

DEPTH

(FT.)

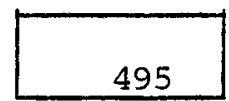

TO

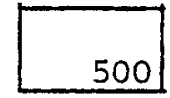

ROCK CIASSIFICATION

silty sand

\section{\& SIZE RANGE SPHERICITY ROUNDNESS}

BASALT $\geq$ gravel
sand
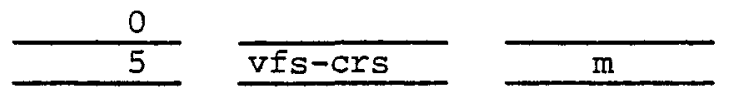

$$
\text { rnd }
$$

OTHER Igravel sand
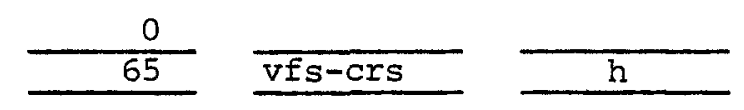

sb rnd

SILT AND CLAY 30

REMARKS

$$
\begin{array}{ll}
\text { Color } & \text { Whitish Tan } \\
\text { HCL } & +
\end{array}
$$

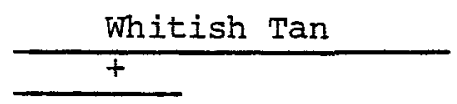

Abundant Caliche

Slightly Micaceous

Lithified by silt, clay \& $\mathrm{CaCO}_{3}$ 


\section{DEPTH}

(FT.)

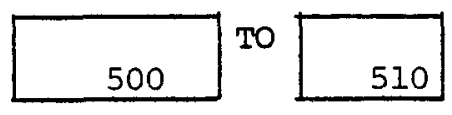

ROCK CLASSIFICATION

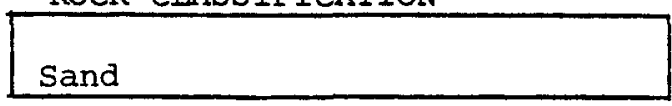

- SIZE RANGE SPHERICITY ROUNDNESS

BASALT $\frac{\geq \text { gravel }}{\text { sand }}$
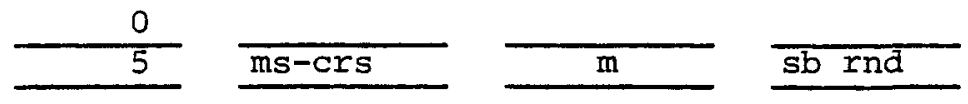

OTHER $\geq$ gravel sand
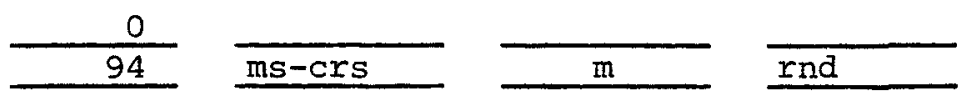

SILT AND CLAY 1

REMARKS

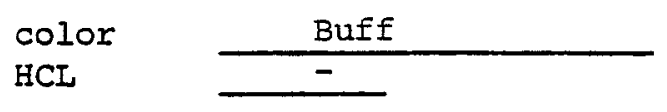

Slightly Micaceous Frosted Quartz

Mature

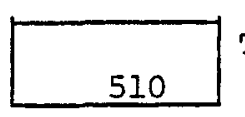

TO

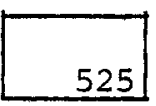

ROCK CLASSIFICATION

(FT.)

525

sandy Gravel

8 SIZE RANGE SPHERICITY ROUNDNESS

BASALT $\geq$ gravel
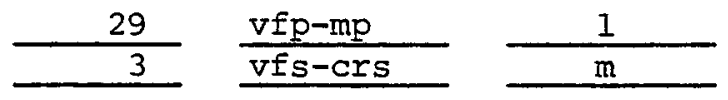

rnd

OTHER $\geq$ gravel
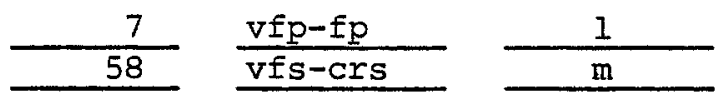

$\frac{\mathrm{w} \text { rnd }}{\text { sb rnd }}$

SILT AND CLAY 3

REMARKS

$$
\begin{aligned}
& \text { color } \\
& \text { HCL }
\end{aligned}
$$

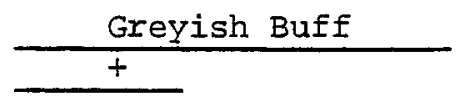

Slightly Micaceous

Unfrosted Quartz

Immature 


\section{DEPTH}

(FT.)

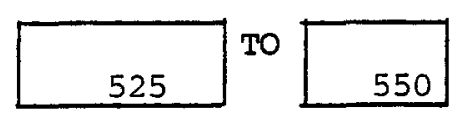

\section{\% SIZE RANGE SPHERICITY ROUNDNESS}

BASALT $\underset{\substack{\text { sand } \\ \text { gravel }}}{\text { Band }}$
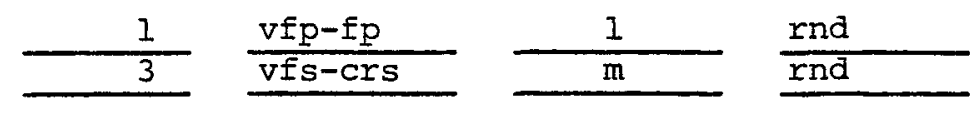

OTHER $\underset{\text { sand }}{\geq \text { gravel }}$
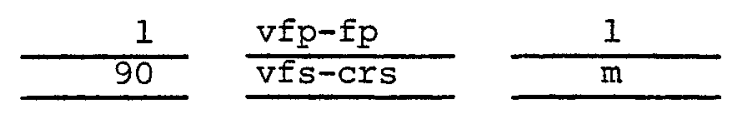

5

SIIT AND CLAY

REMARKS

$$
\begin{aligned}
& \text { color } \\
& \text { HCL }
\end{aligned}
$$

Yellowish Buff

Slightly Micaceous Unfrosted Quartz

Mature

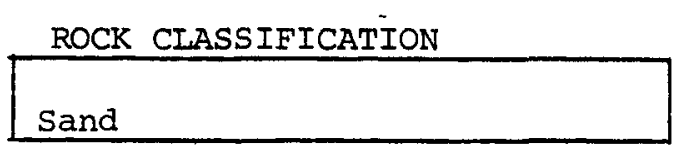




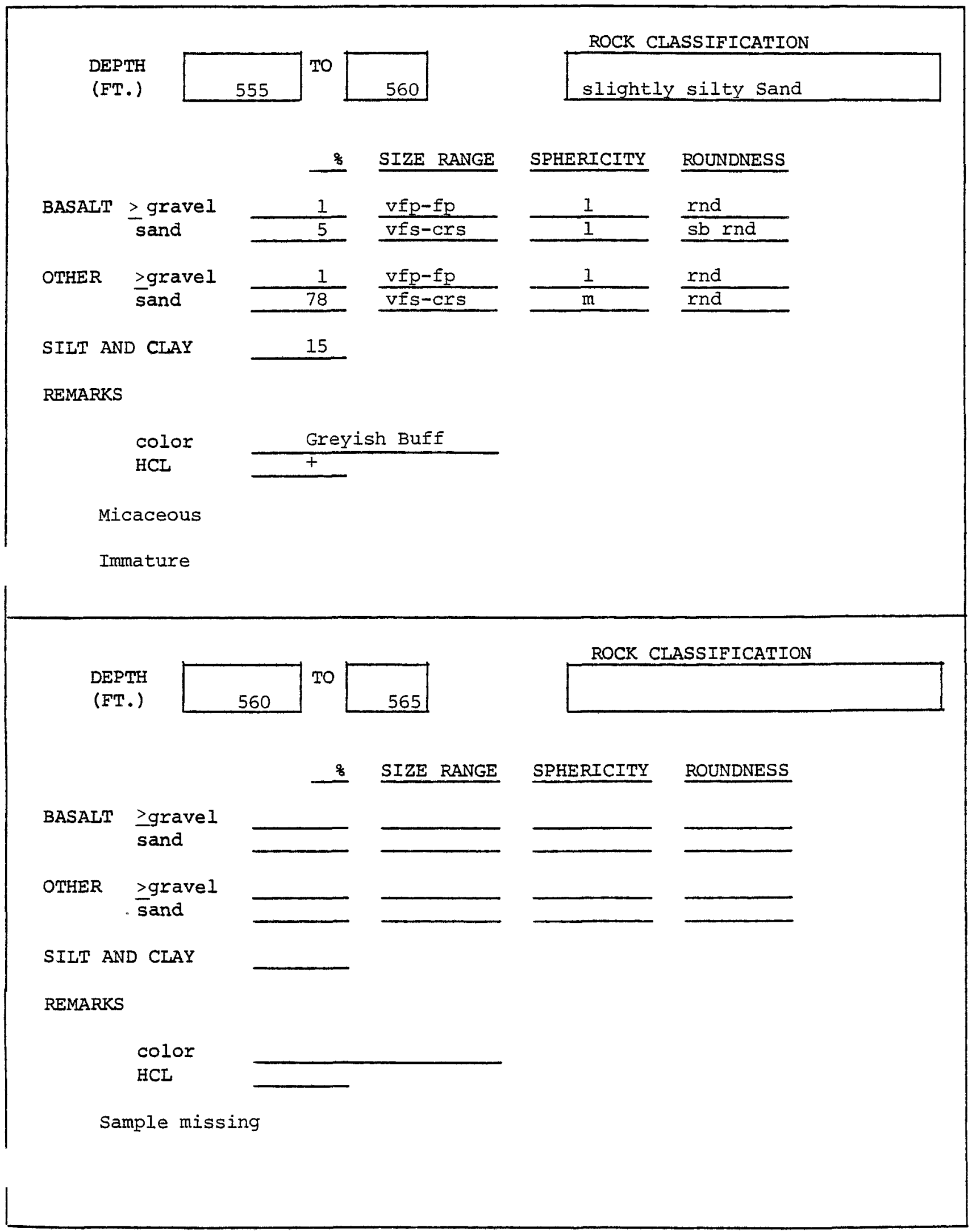


ROCK CLASSIFICATION

DEPTH

(FT.)

silty sandy Gravel

\section{\% SIZE RANGE SPHERICITY ROUNDNESS}

BASALT $\frac{\geq \text { gravel }}{\text { sand }}$

$\frac{20}{3} \frac{\text { vfp-fp }}{\text { vfs-vers }}$

$\frac{m}{1} \quad \frac{\text { rnd }}{\text { sb rnd }}$

OTHER $\geq$ gravel

12 vfp-mp

$1 \quad$ rnd sand
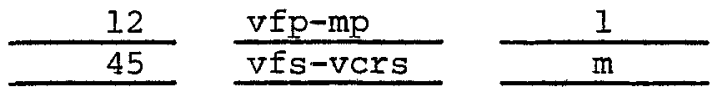

sb rnd

SILT AND CLAY 20

REMARKS

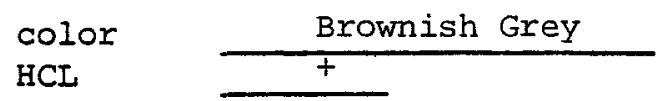

Abundant Caliche (pebbles \& sand)

Slightly Micaceous

Unfrosted Quartz

Lithified by silt, clay \& $\mathrm{CaCO}_{3}$

Immature

DEPTH

(FT.)

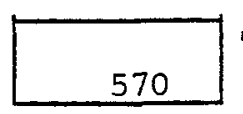

TO

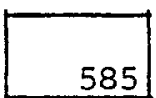

ROCK CIASSIFICATION

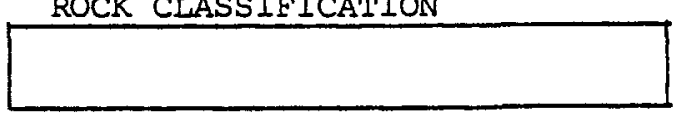

\section{$\%$ SIZE RANGE SPHERICITY ROUNDNESS}

BASALT >gravel sand

OTHER >gravel sand
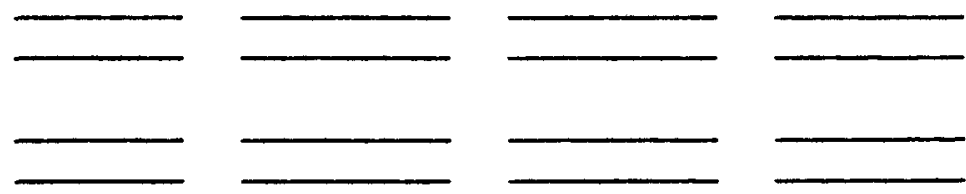

SILT AND CLAY

REMARKS

color

HCL

Sample missing 
DEPTH

(ET.)
TO

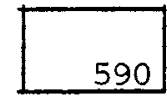

ROCK CLASSIFICATION

Sand \& Basalt Flow

\section{\% SIZE RANGE SPHERICITY ROUNDNESS}

BASALT $\frac{\text { gravel }}{\text { sand }}$

$\frac{82}{8} \quad \frac{v f p-m p}{v f s-v e r s}$

$\frac{\mathrm{m}}{\mathrm{m}} \frac{\mathrm{sb} \mathrm{rnd}-\mathrm{v}}{\mathrm{sb} \text { rnd }}$ ang

OTHER $\geq$ gravel
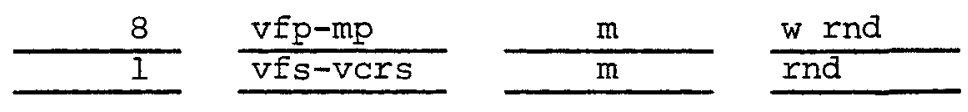

SILT AND CLAY

1

REMARKS

$$
\begin{aligned}
& \text { color } \\
& \text { HCL }
\end{aligned}
$$

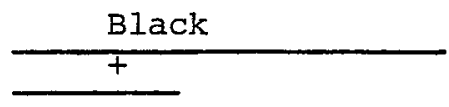

Abundant Caliche (pebbles \& sand)

Frosted Quartz

Immature

DEPTH

(FT.)

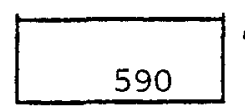

To

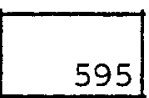

ROCK CLASSIFICATION

Sand \& Basalt Flow

\% SIZE RANGE SPHERICITY ROUNDNESS

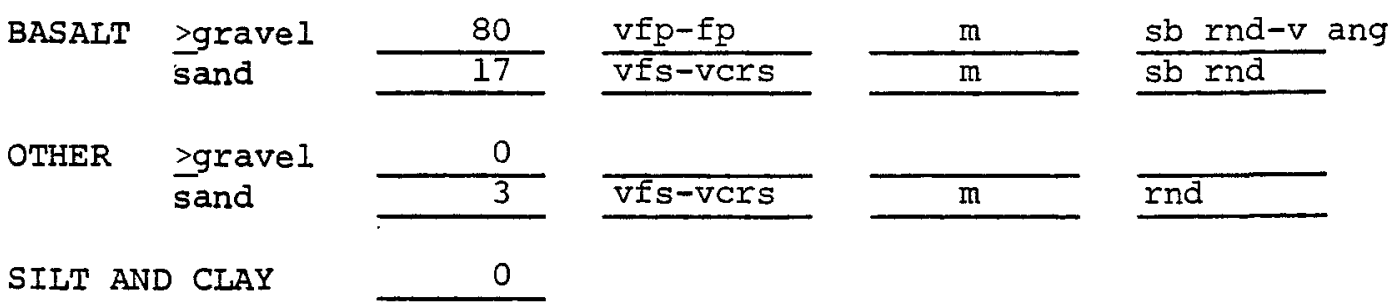

REMARKS

color

Black

HCL

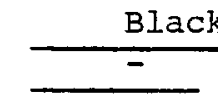

Immature 


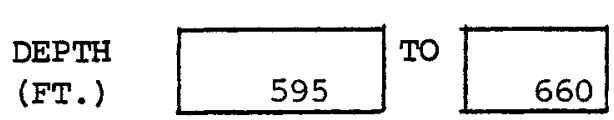

ROCK CIASSIFICATION

\section{\% SIZE RANGE SPHERICITY ROUNDNESS}

BASALT $\geq$ gravel sand

OTHER $\geq$ gravel sand

SIIT AND CLAY

REMARKS

color HCL

Sample missing

Total Drilled Depth reported as $660^{\prime}$

\section{DEPTH}

(FT.)

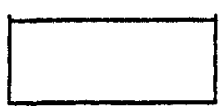

TO

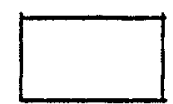

ROCK CIASSIFICATION

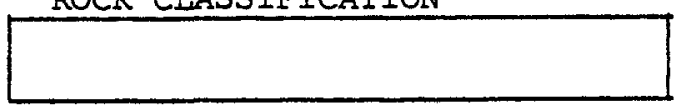

\section{\% SIZE RANGE SPHERICITY ROUNDNESS}

BASALT $\geq \underset{\text { sand }}{\text { gravel }}$
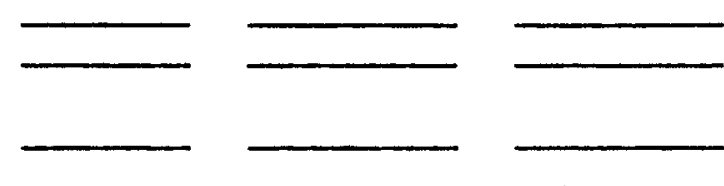

OTHER $\geq$ gravel sand

SIIT AND CLAY

REMARKS

color

HCL 

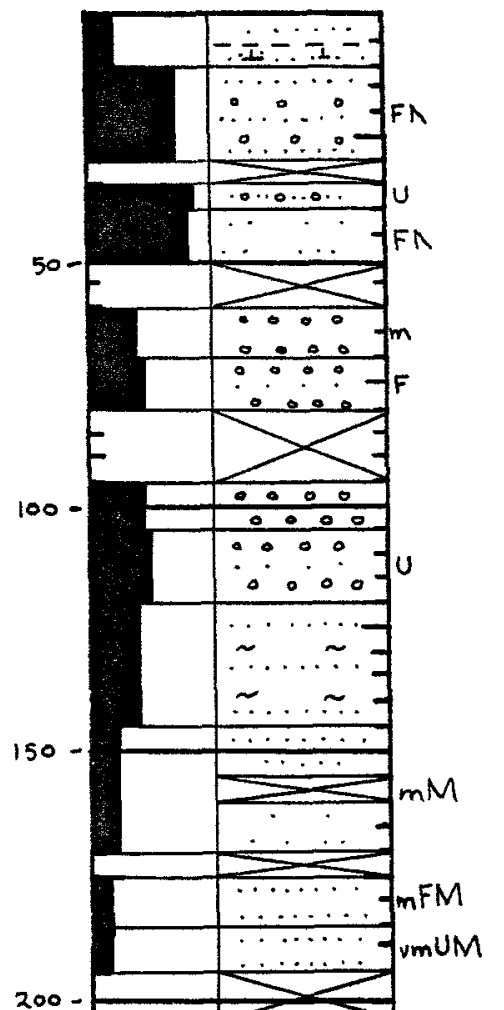

200

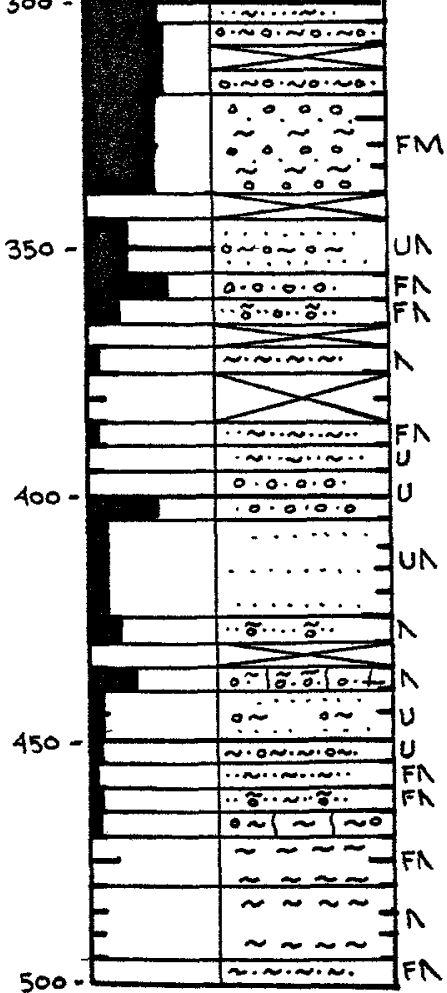

Sample Description

greyish buff

brownish grey

black

black

greyish buff

greyish black

dark grey

greyish brown

greyish $\tan$

reddish $\tan$

brownish $\tan$

buff grey

greyish butf

$\tan$

$\tan$

$\tan$ white

$\tan$

brownish tan

greyish brown

light brown

light brown

brown

greyish brown

light grey

greyish butf

greyish buff

buff

reddish $\tan$

greyish $\tan$

greyish $\tan$

greenish tan;TRACE DIATOMITE

$\tan$ taARE DIATOMITE

reddish tan

brownish $\tan$

$\tan$

light grey
Plant Coordinates

$\mathrm{NO} 30507$

W030678

Altitude 527

Other Data used in Interpretation

(1) Driller's Log

(2) E-Log

(3) Sieve analyses in MCHenry, 1957, p.39-40 


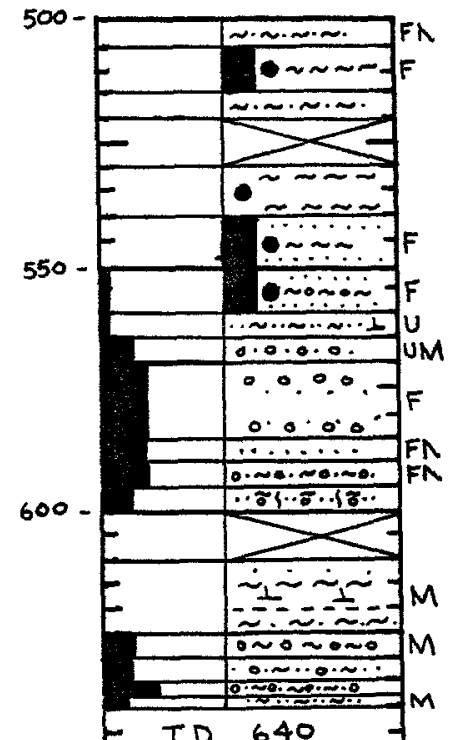

650
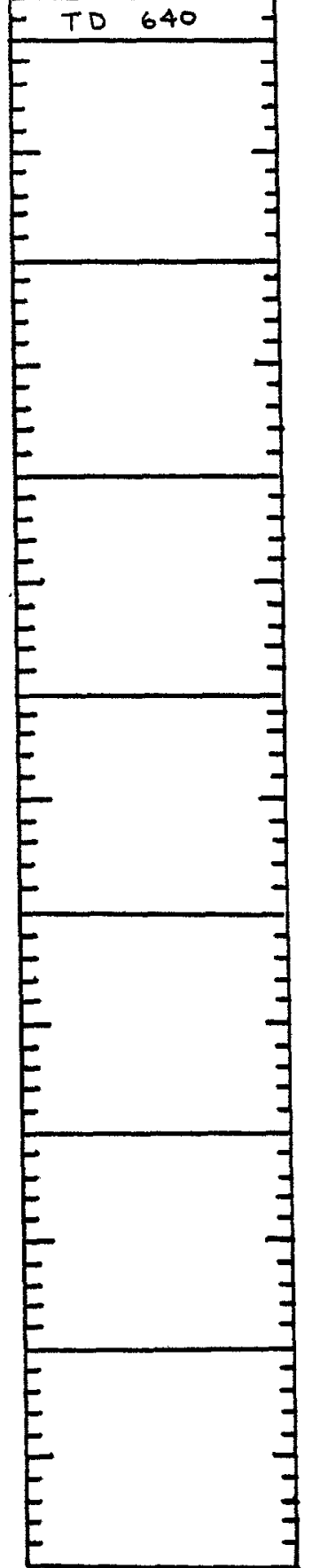

Sample Description

light grey

greyish $\tan$

Altitude

reddish $\tan$

reddish brown

reddish brown

reddish $\tan$

light grey

greyish buff

greyish buff

dark grey

brownish grey

brownish $\tan$

brownish $\tan$

brownish tan

greyish $\tan$
Plant Coordinates

Other Data used in Interpretation 


\section{DEPTH}

(FT.)

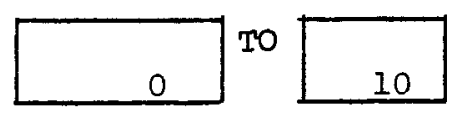

\% SIZE RANGE SPHERICITY ROUNDNESS

BASALT $\geq \underset{\text { sand }}{\text { gravel }}$
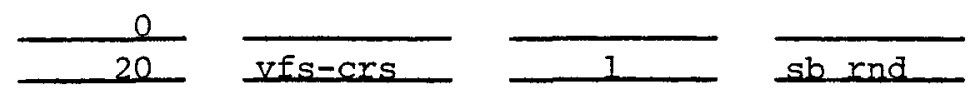

OTHER $\geq$ gravel sand
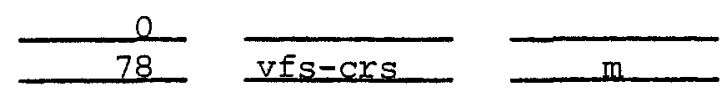

2

SILT AND CLAY

REMARKS

$$
\text { color }
$$

HCL

Greyish Buff

$-\left(0^{\prime}-5^{\prime}\right),+\left(5^{\prime}-10^{\prime}\right)$

Slightly Micaceous

Frosted Quartz

Mature

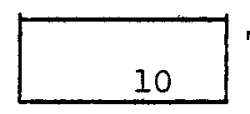

TO

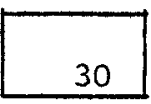

ROCK CLASSIFICATION

(FT.)

10

ROCK CLASSIFICATION

Sand 
ROCK CLASSIFICATION

DEPTH

(FT.)

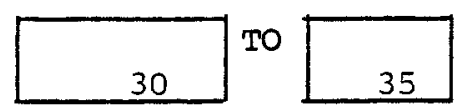

\% SIZE RANGE SPHERICITY ROUNDNESS

BASALT > gravel sand

OTHER $\geq$ gravel sand

SILT AND CLAY

REMARKS

color

HCL

Sample missing
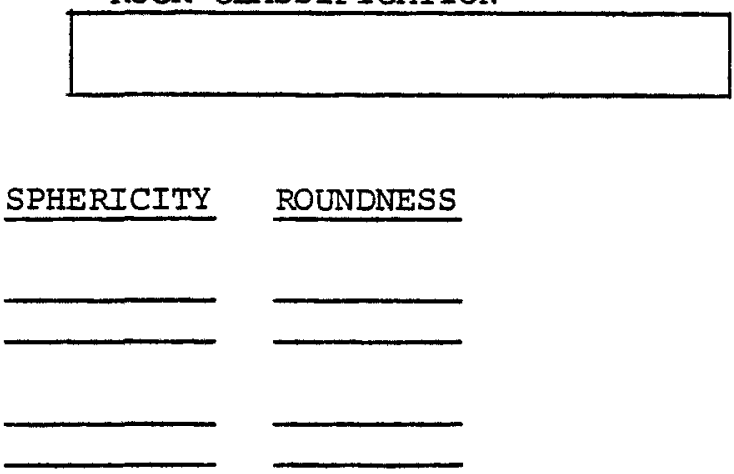

\section{DEPTH}

(ET.)

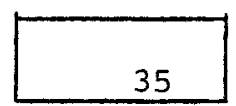

TO

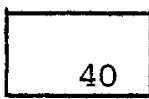

ROCK CLASSIFICATION

gravelly sand

\section{\& SIZE RANGE SPHERICITY ROUNDNESS}

\begin{tabular}{|c|c|c|c|c|c|}
\hline \multirow[t]{2}{*}{ BASALT } & $\geq$ gravel & 18 & $v f p-c r p$ & 1 & $w$ rnd \\
\hline & sand & 68 & $\mathrm{~ms}$-vcrs & $\underline{m}$ & sb rnd \\
\hline \multirow[t]{2}{*}{ OTHER } & $>$ gravel & 2 & $v f p-m p$ & 1 & $w$ rnd \\
\hline & sand & 12 & ms-vcrs & $\mathrm{m}$ & rnd \\
\hline
\end{tabular}

SILT AND CLAY

REMARKS

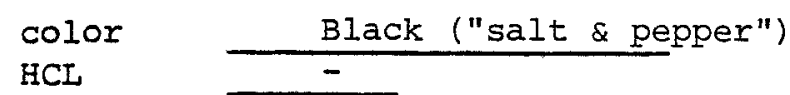

Unfrosted Quartz

Immature 


\section{DEPTH}

(FT.)

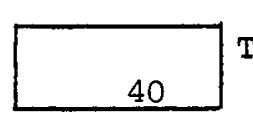

TO

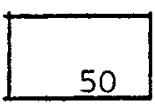

ROCK CIASSIFICATION

Sand

\% SIZE RANGE SPHERICITY ROUNDNESS

BASALT $\underset{\substack{\text { sand } \\ \text { gravel }}}{\text { sand }}$
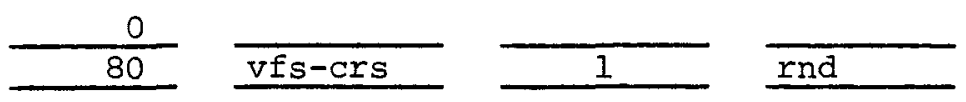

OTHER $\geq$ gravel sand
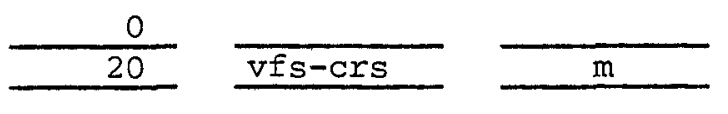

rnd

SILT AND CLAY

0

REMARKS

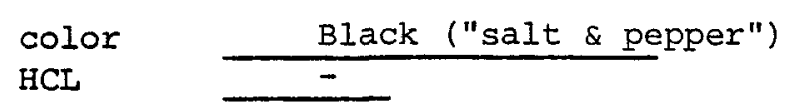

Slightly Micaceous Frosted quartz

Mature

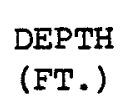

(FT.)

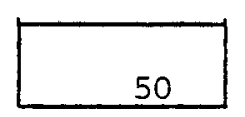

To

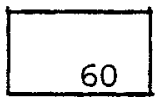

ROCK CLASSIFICATION

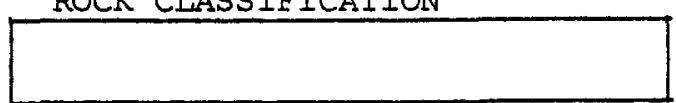

\section{S SIZE RANGE SPHERICITY ROUNDNESS}

BASALT >gravel sand

OTHER Igravel sand

SILT AND CLAY

REMARKS

color HCL

Sample missing 


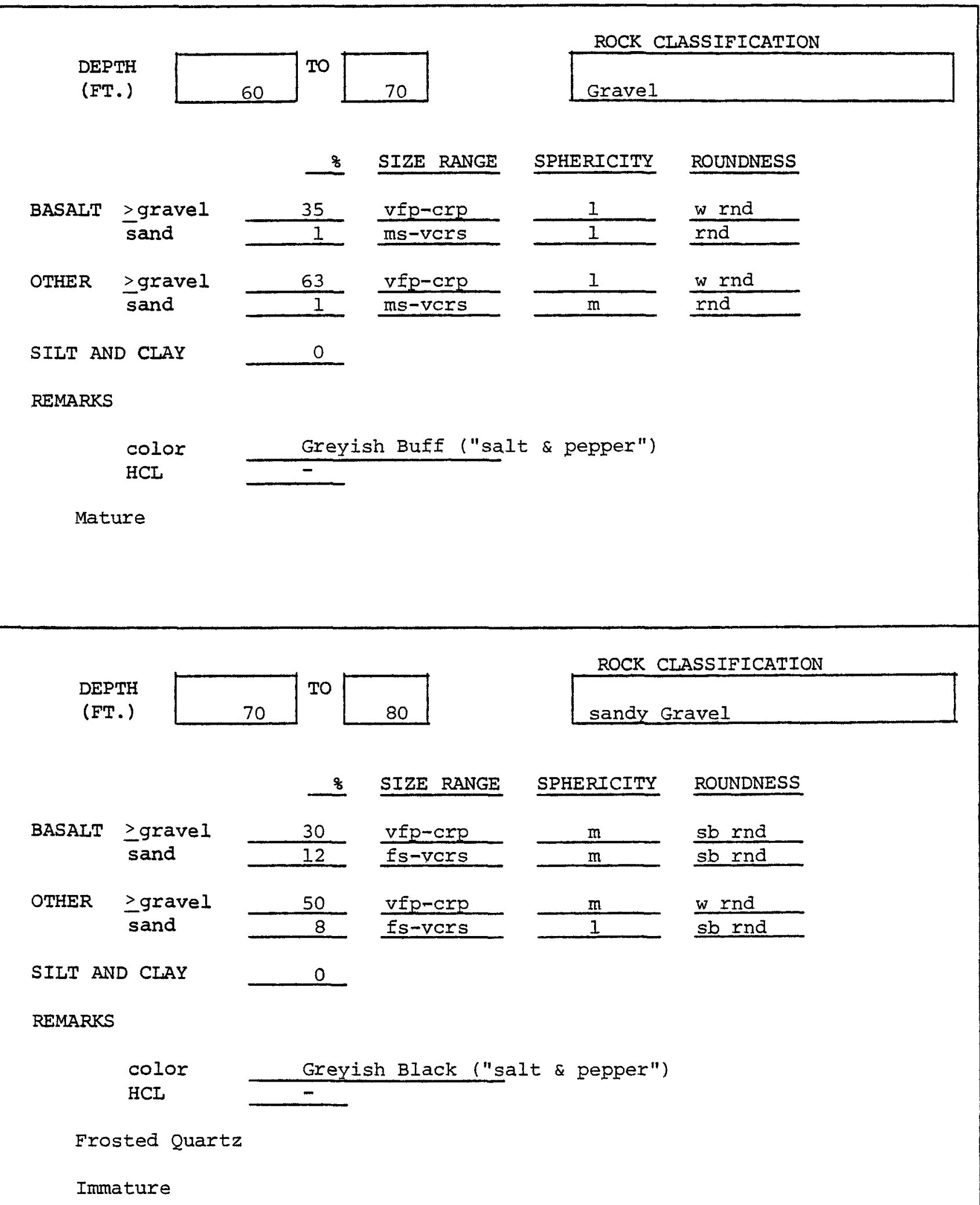

Immature 


\section{DEPTH}

(FT.)

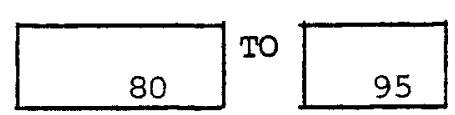

BASALT >gravel sand

OTHER >gravel sand

SILT AND CLAY

REMARKS

$$
\text { color }
$$

HCL

Sample missing

\section{\% SIZE RANGE SPHERICITY ROUNDNESS}

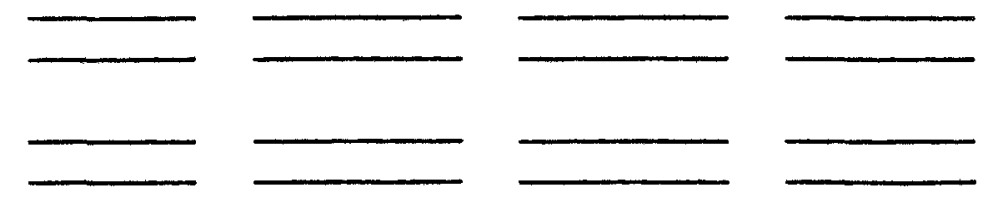

ROCK CIAASSIFICATION

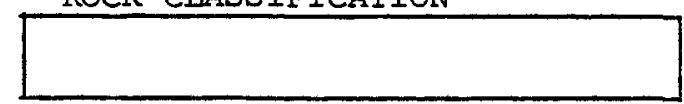




\section{DEPTH}

(FT.)

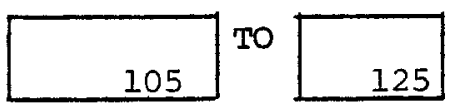

S SIZE RANGE

SPHERICITY ROUNDNESS

BASALT >gravel
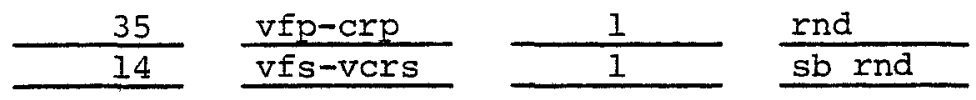

OTHER >gravel sand
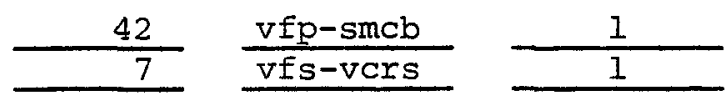

$w$ rnd

rnd

SILT AND CLAY

\section{2}

REMARKS

color

Greyish Brown

HCL

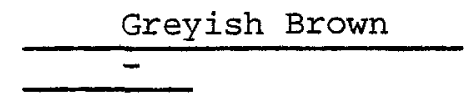

Unfrosted Quartz

Immature

DEPTH

(FT.)

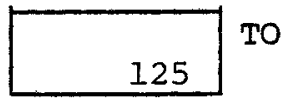

145
ROCK CLASSIFICATION

slightly silty sand

을 SIZE RANGE SPHERICITY ROUNDNESS

BASALT > gravel sand

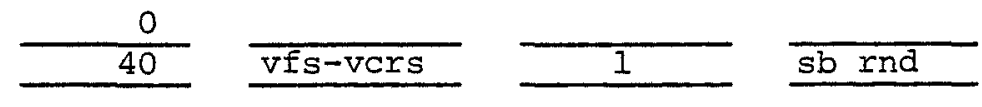

OTHER \gravel
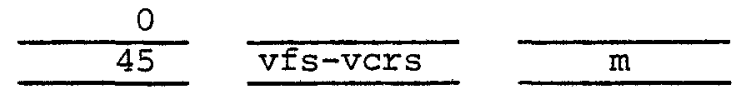

rnd

SILT AND CLAY

\section{5}

REMARKS

color Greyish Tan

$\mathrm{HCL}$ $-$

Sieved (?)

Immature 
DEPTH

(FT.)

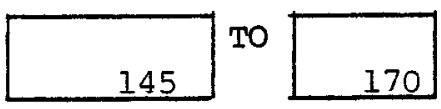

ROCK CLASSIFICATION

Sand

\section{\% SIZE RANGE SPHERICITY ROUNDNESS}

BASAIT $\frac{\geq \text { gravel }}{\text { sand }}$
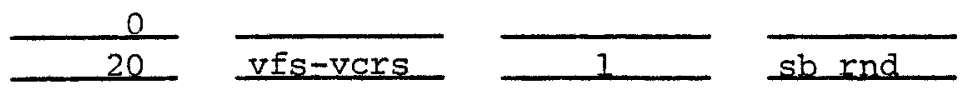

OTHER > gravel sand
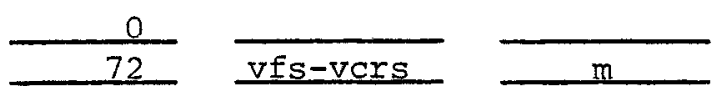

rnd

SILT AND CIAY

8

REMARKS

color

HCL

Micaceous

Sieved (?)

Sample interval $155^{\prime}-160^{\prime}$ missing

Mature

DEPTH

(FT.)

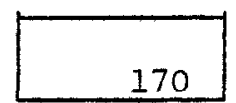

TO

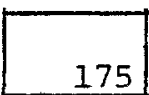

ROCK CLASSIFICATION

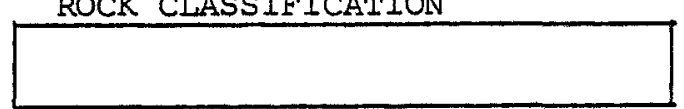

\% SIZE RANGE SPHERICITY ROUNDNESS

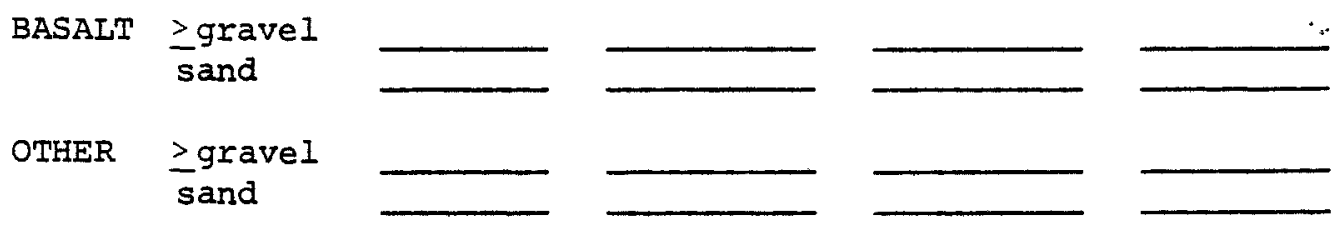

SILT AND CLAY

REMARKS

color

HCL

Sample missing 


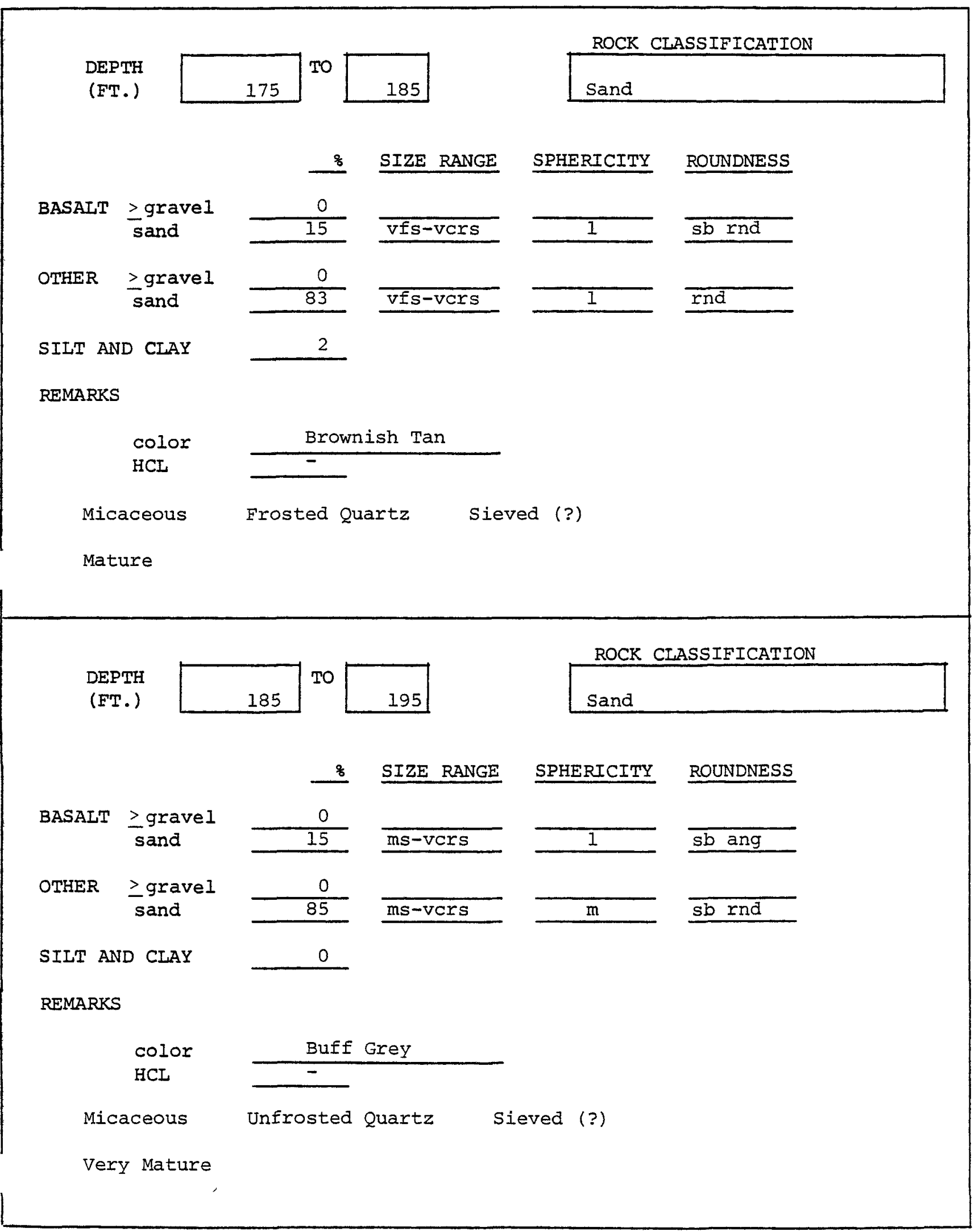




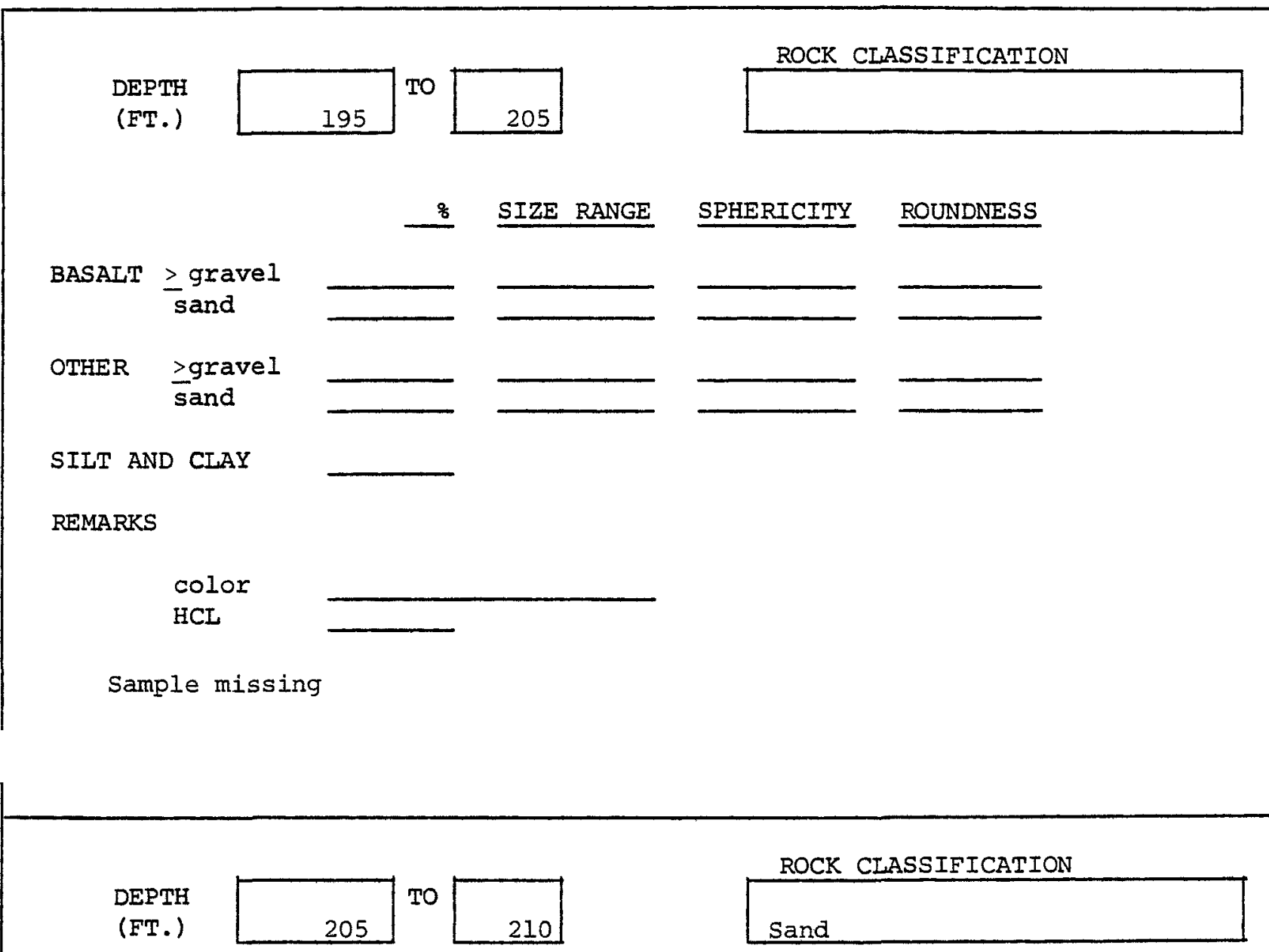

S SIZE RANGE SPHERICITY ROUNDNESS

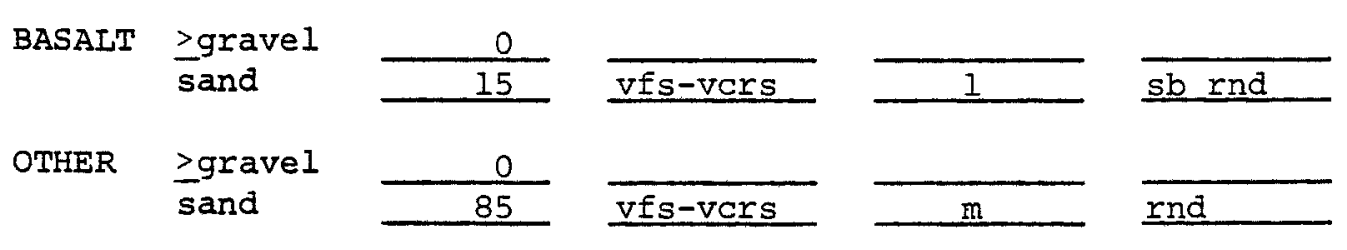

SILT AND CIAY

REMARKS

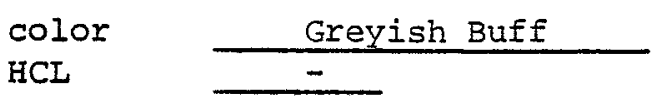

Slightly Micaceous Frosted quartz Sieved (?)

Mature 


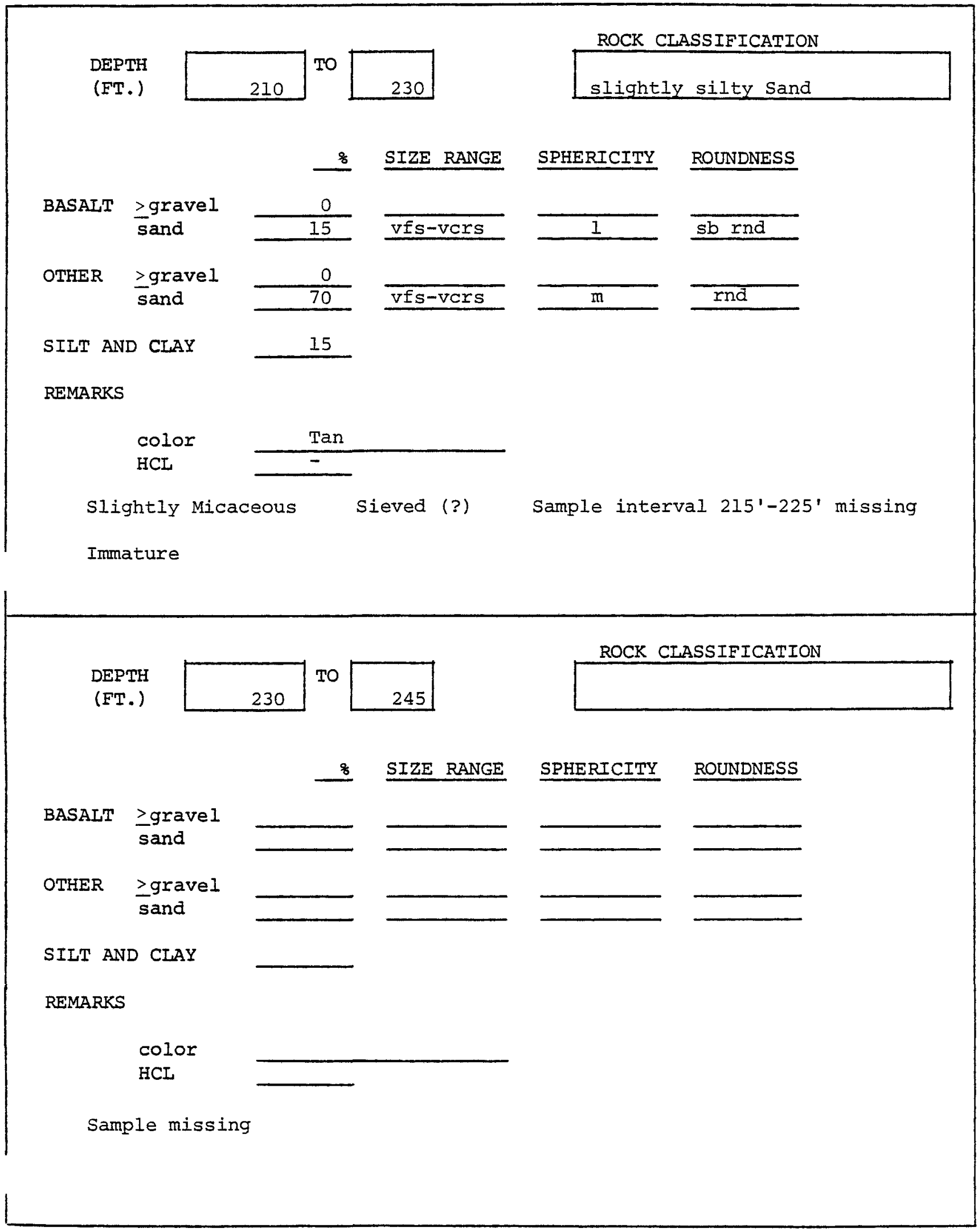

W. K. Summers \& Associates 

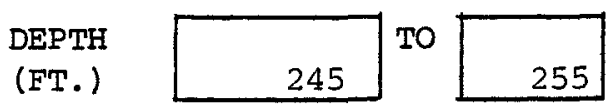

ROCK CLASSIFICATION

Sand

\section{\% SIZE RANGE SPHERICITY ROUNDNESS}

BASALT >gravel sand
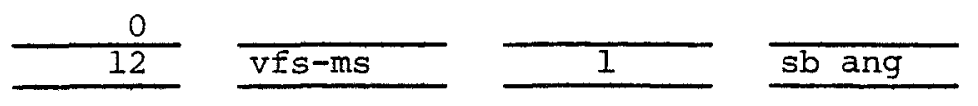

OTHER $\geq$ gravel sand
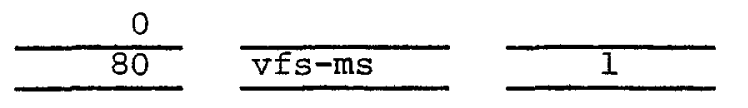

$\mathrm{sb}$ rnd

SILT AND CLAY

8

REMARKS

color

HCI

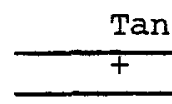

Caliche (sand present) Sieved (?)

Mature

\section{DEPTH}

(FT.)

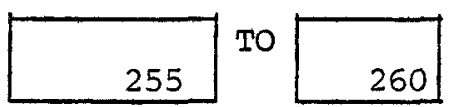

ROCK CLASSIFICATION

silty sand

$\frac{\%}{6}$ SIZE RANGE SPHERICITY ROUNDNESS

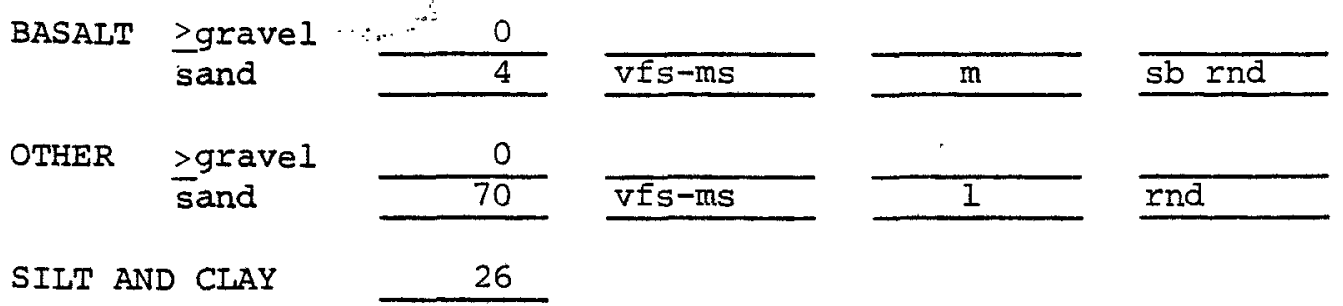

REMARKS

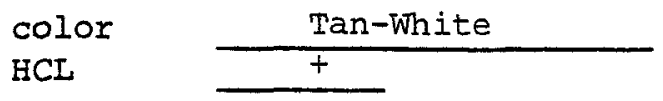

Abundant Caliche

Imnature 
DEPTH

(FT.)

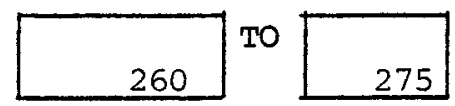

ROCK CLASSIFICATION

slightly silty sand

8 SIZE RANGE SPHERICITY ROUNDNESS BASALT $\underset{\text { sand }}{\geq \text { gravel }}$
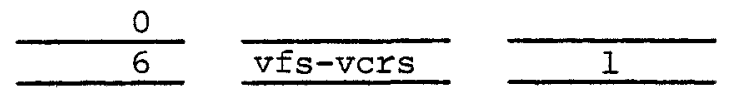

sb rnd

OTHER $\geq$ gravel
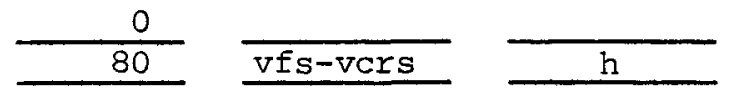

rnd

SILT AND CLAY 14

REMARKS

color

HCL

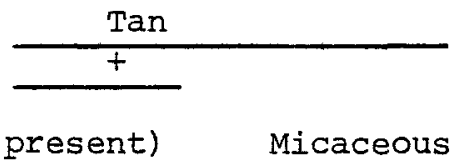

Frosted Quartz

Sieved (?)

Immature

DEPTH

(FT.)

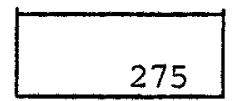

TO

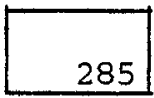

ROCK CLASSIFICATION

silty sand

\% SIZE RANGE SPHERICITY ROUNDNESS

BASALT > gravel sand
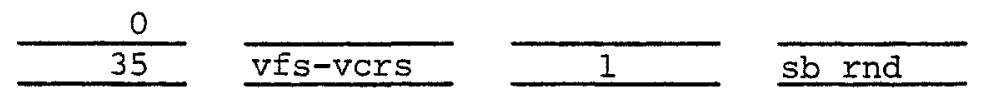

OTHER >graveI sand
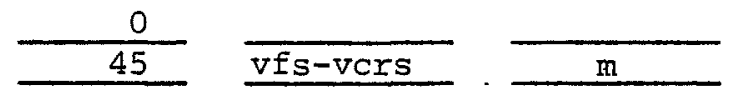

$\overline{s b \text { rnd }}$

SILT AND CLAY

20

REMARKS

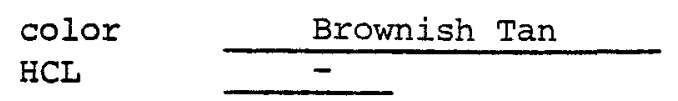

Slightly Micaceous

Immature 
DEPTH

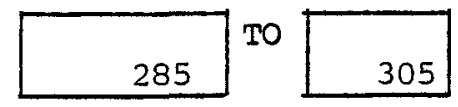

ROCK CIAASSIFICATION

slightly silty sand

\section{\% SIZE RANGE SPHERICITY ROUNDNESS}

BASALT $\geq$ gravel sand
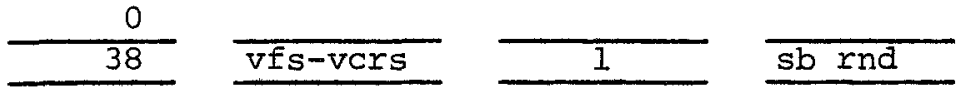

OTHER $\geq$ gravel sand
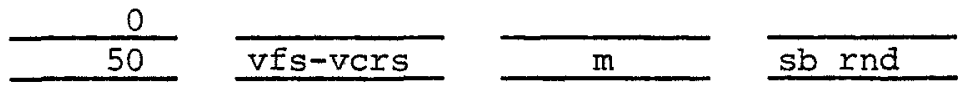

SIIT AND CLAY

\section{2}

REMARKS

color

Greyish Brown

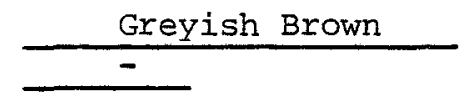

Slightly Micaceous Unfrosted quartz

Immature

\section{DEPTH}

(FT.)

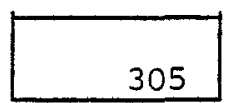

TO

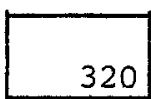

ROCK CLASSIFICATION

silty sandy Gravel

\section{\% SIZE RANGE SPHERICITY ROUNDNESS}

\begin{tabular}{|c|c|c|c|c|c|}
\hline \multirow[t]{2}{*}{ BASALT } & \multirow{2}{*}{$\begin{array}{l}\text { Zgravel } \\
\text { sand }\end{array}$} & 30 & $v f p-m p$ & 1 & rnd \\
\hline & & 30 & vfs-vers & 1 & $\mathrm{sb}$ ang \\
\hline OTHER & Zgravel & 4 & $v f p-m p$ & $\mathrm{~m}$ & rnd \\
\hline & sand & 23 & vfs-vers & $m$ & rnd \\
\hline
\end{tabular}

SILT AND CLAY

13

REMARKS

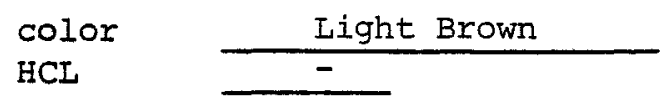

Slightly Micaceous Sample interval 310'-315' sieved (?)

Immature 


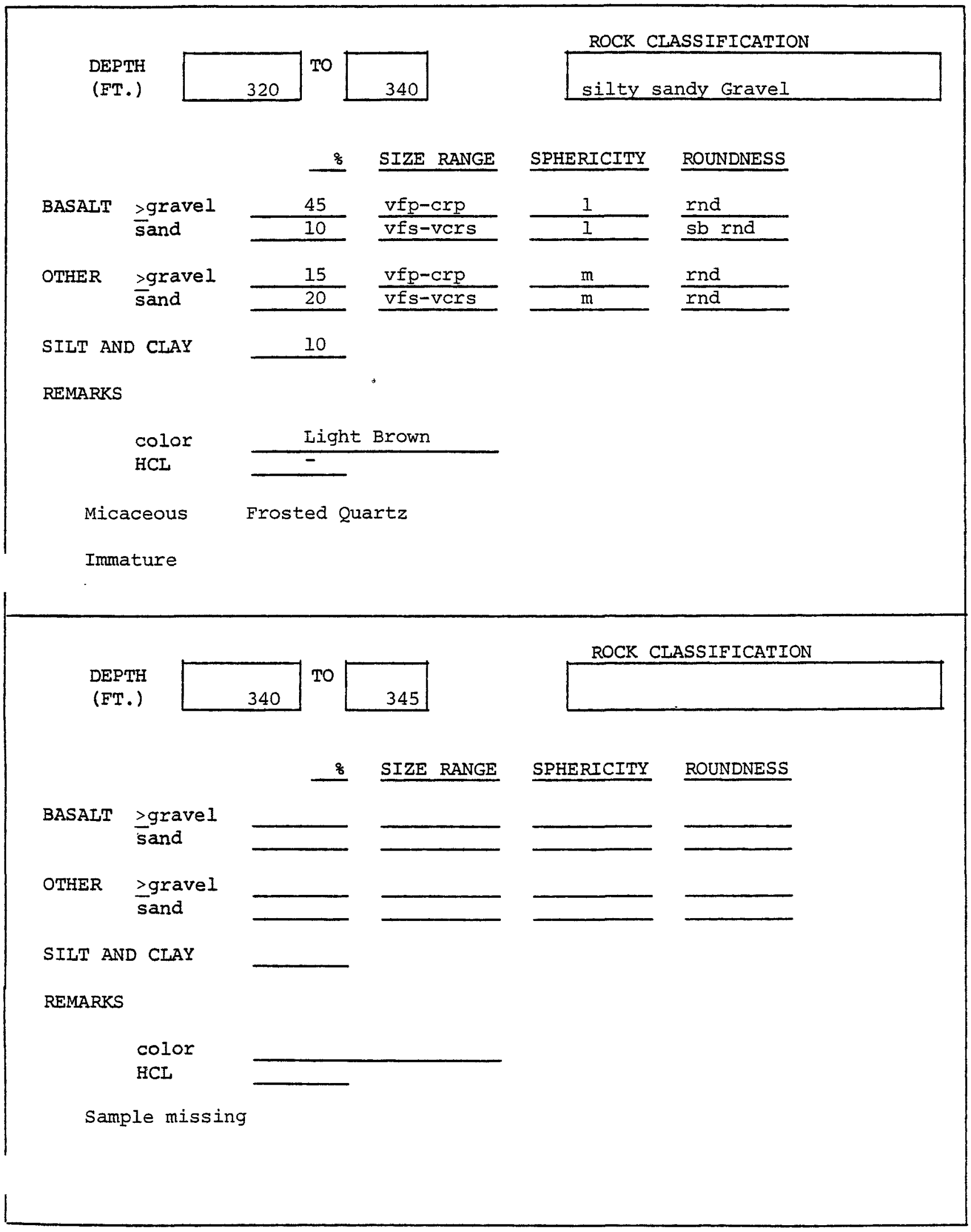

W. K. Summers \& Associates 


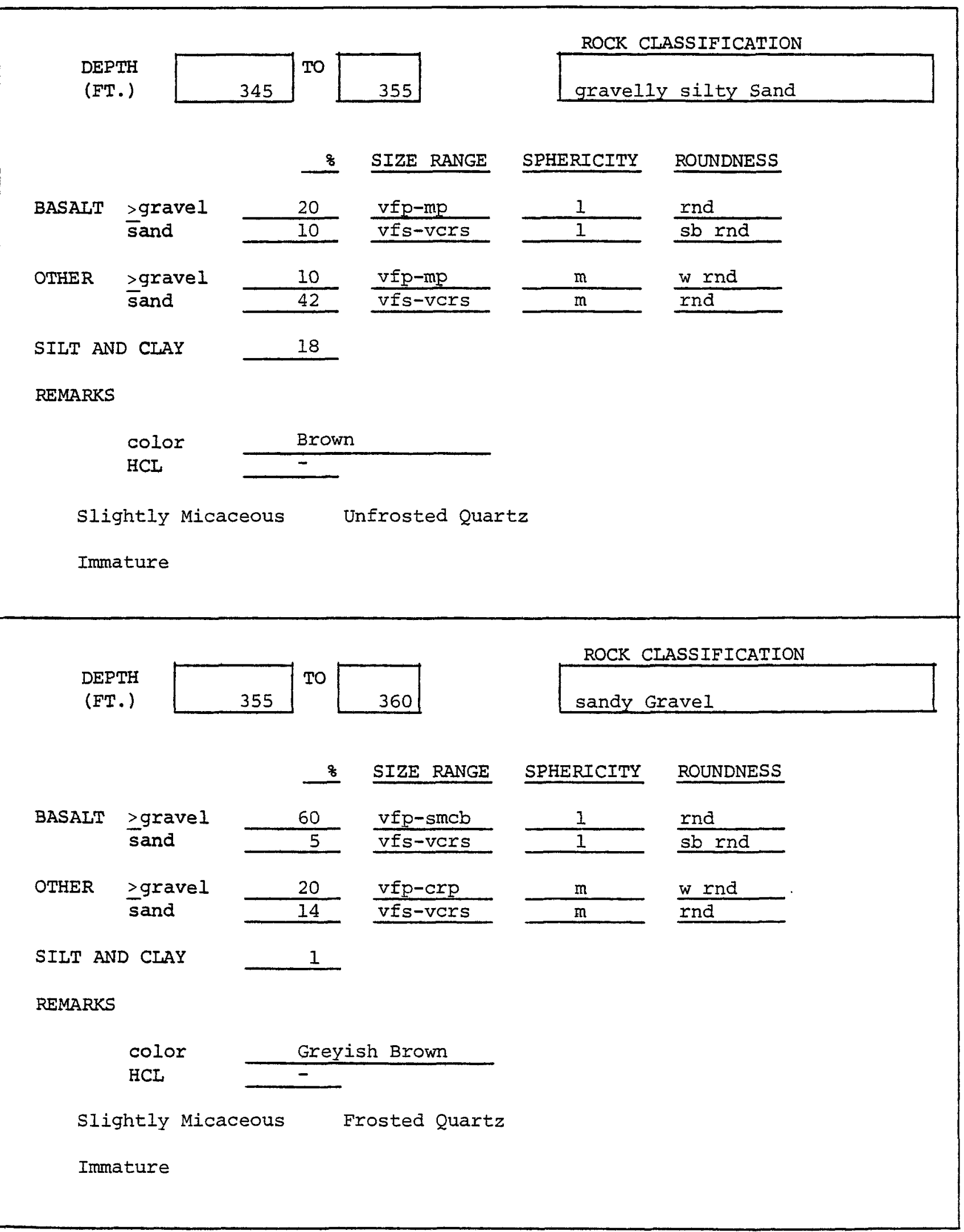




\section{DEPTH}

(FT.)

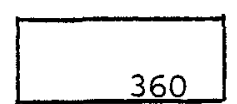

TO

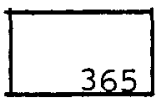

ROCK CLASSIFICATION

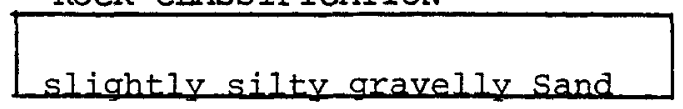

\% SIZE RANGE SPHERICITY ROUNDNESS

BASALT $\frac{\text { gravel }}{\text { sand }}$
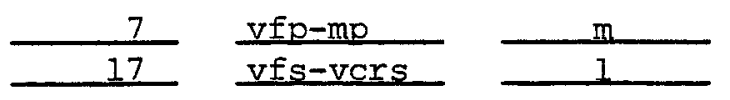

rnd

OTHER > gravel sand
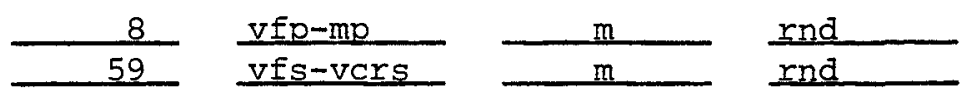

SILT AND CLAY

9

REMARKS

$$
\text { color }
$$

HCL

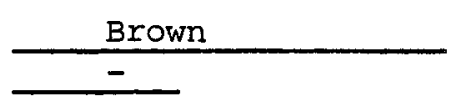

Slightly Micaceous

Frosted Quartz

Immature

DEPTH

(FT.)

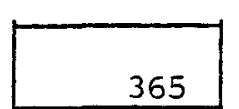

TO

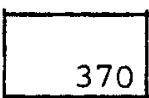

ROCK CLASSIFICATION

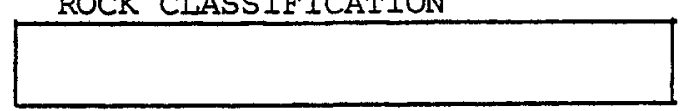

\% SIZE RANGE SPHERICITY ROUNDNESS

BASALT $\geq$ gravel sand

OTHER $\geq$ gravel
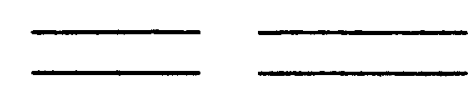

SILT AND CLAY

REMARKS

$$
\begin{aligned}
& \text { color } \\
& \text { HCL }
\end{aligned}
$$

Sample missing 


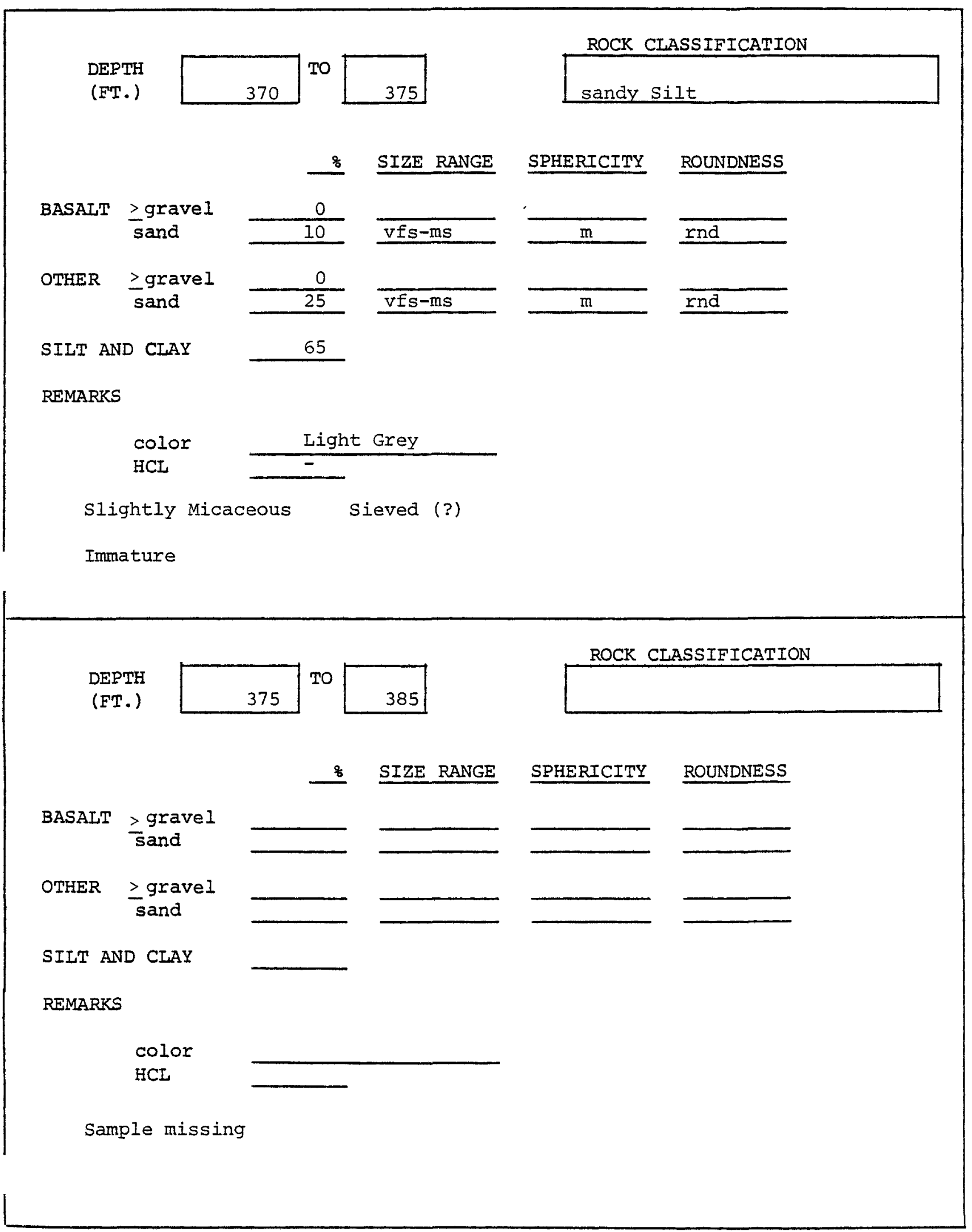



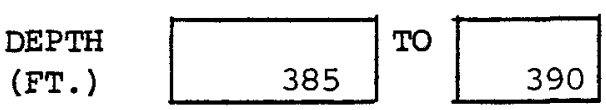

ROCK CLASSIFICATION

(FT.)

\section{5}

\section{\% SIZE RANGE SPHERICITY ROUNDNESS}

BASALT $\geq$ gravel
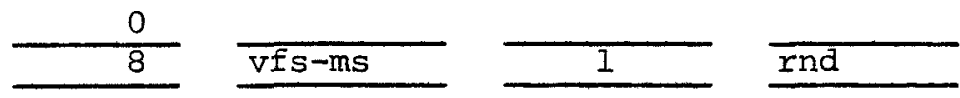

OTHER >gravel
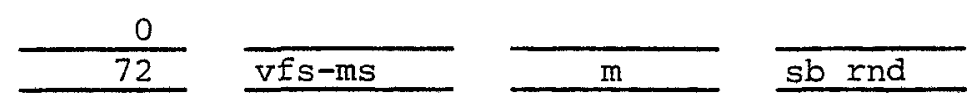

SILT AND CLAY 20

REMARKS

color

HCL

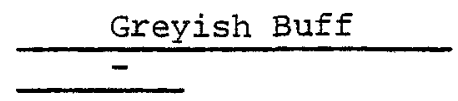

slightly Micaceous

Frosted Quartz

Sieved (?)

Immature

DEPTH

(FT. )

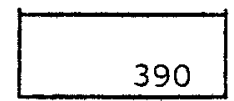

TO

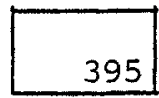

ROCK CIASSIFICATION

gravelly sand

S SIZE RANGE SPHERICITY ROUNDNESS

BASALT

>gravel sand

$\frac{6}{4} \quad \frac{v f p-m p}{v f s-m s}$

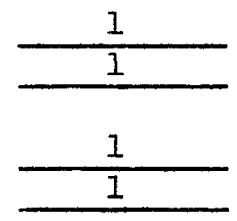
sand

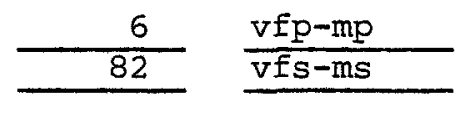

rnd $\frac{\text { rnd }}{\text { sb rnd }}$

OTHER >gravel

2

SILT AND CLAY

2

REMARKS

Color
$\mathrm{HCL}$

Unfrosted Quartz

Immature 


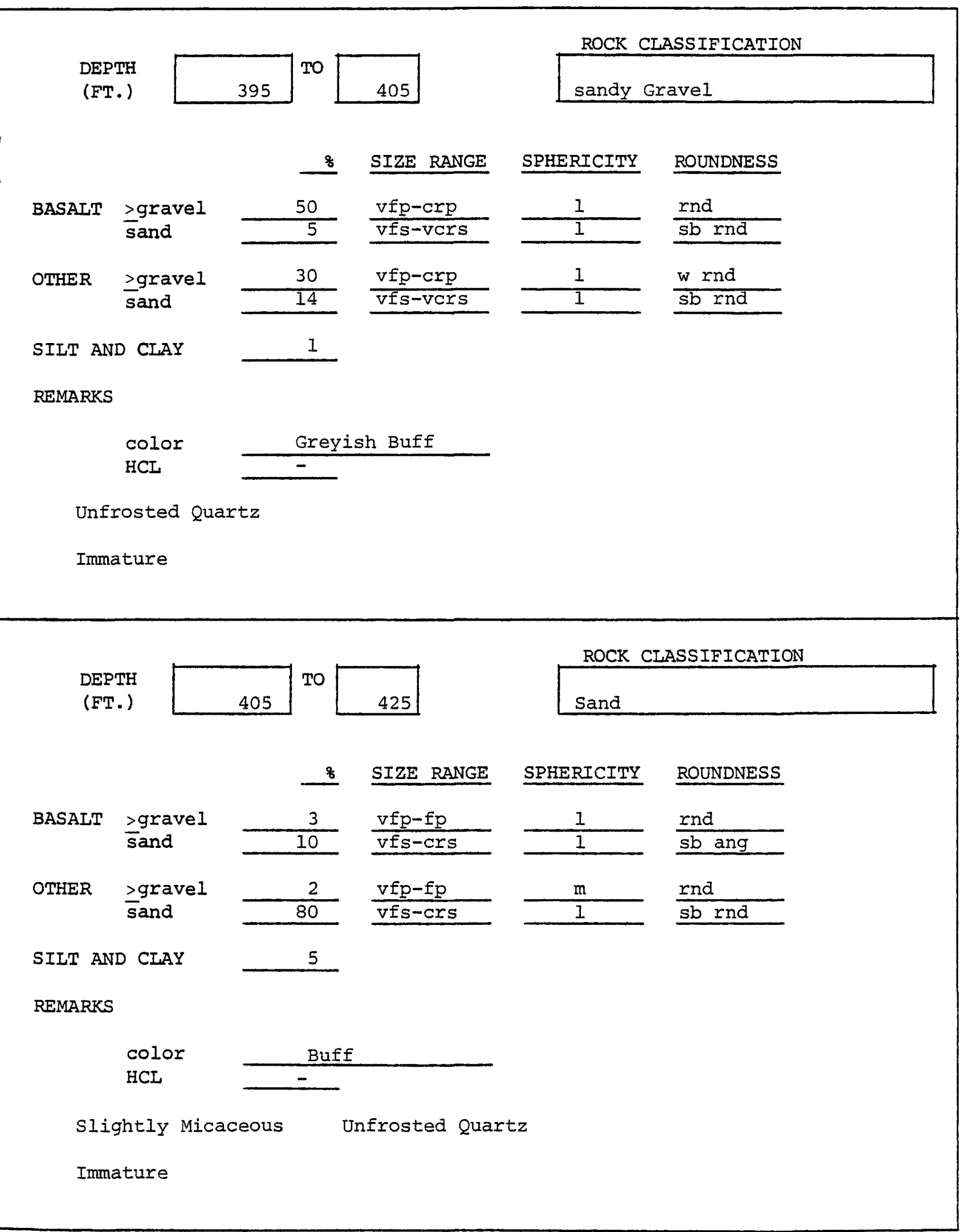


ROCK CLASSIFICATION

DEPTH

(FT.)

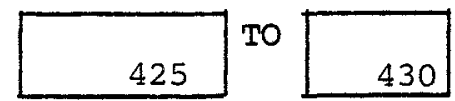

slightly silty slightly gravelly sand

\% SIZE RANGE SPHERICITY ROUNDNESS

BASAIT > gravel sand

OTHER >gravel sand

SILT AND CIAY

REMARKS

color

HCL
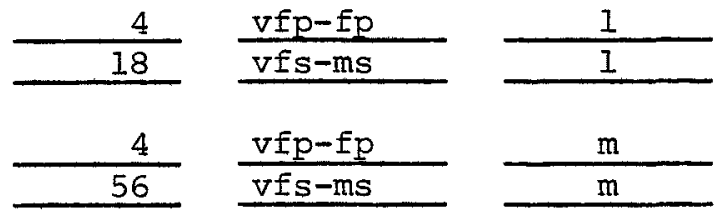

Slightly Micaceous

Immature

DEPTH

(FT.)

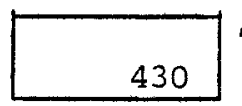

TO

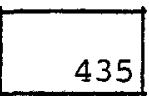

ROCK CLASSIEICATION

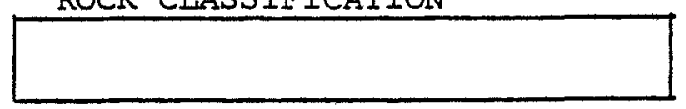

\% SIZE RANGE SPHERICITY ROUNDNESS

BASALT $\geq$ gravel sand

OTHER >gravel sand

SILT AND CLAY

REMARKS

color

HCL

Sample missing 


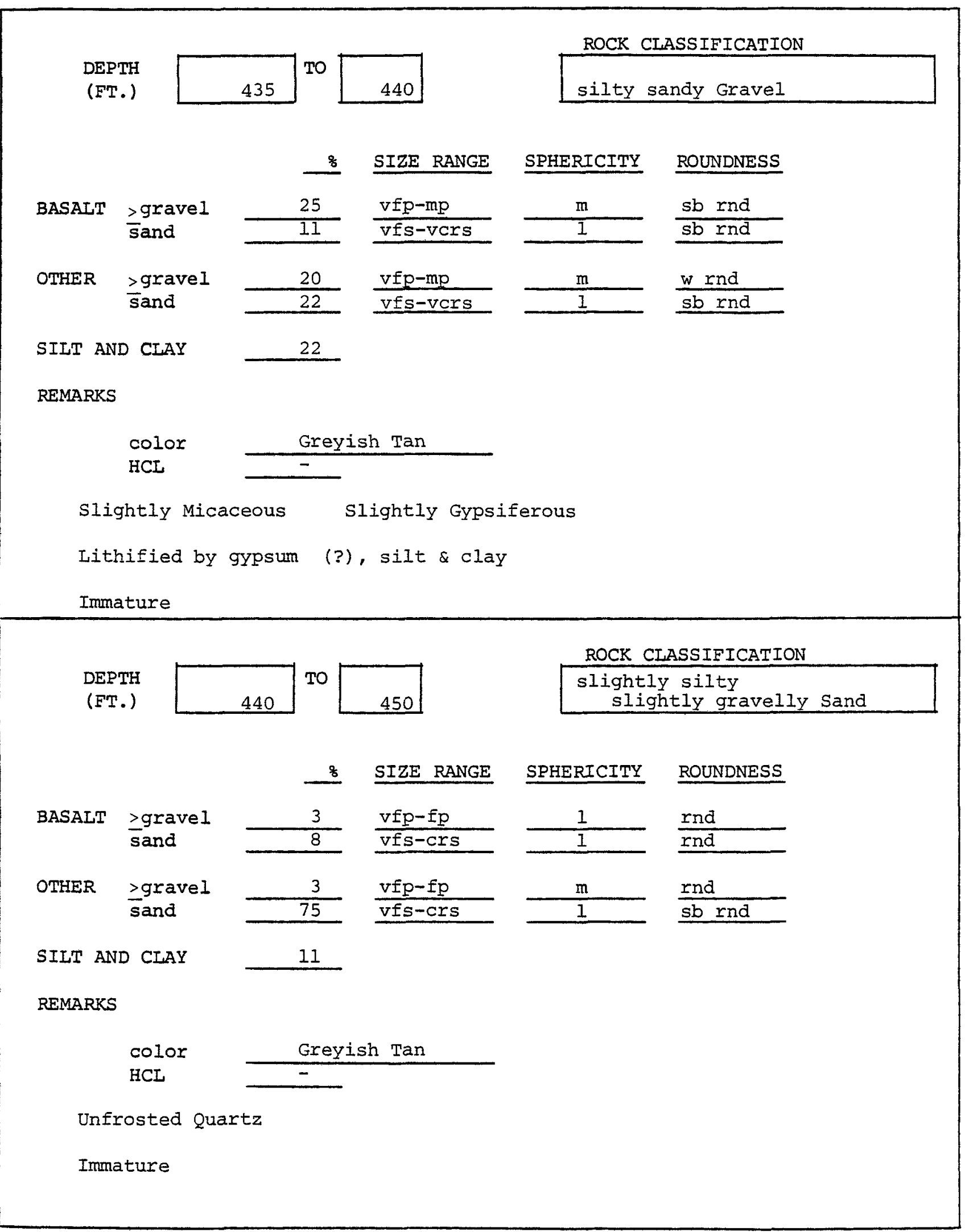


DEPTH
(FT.)

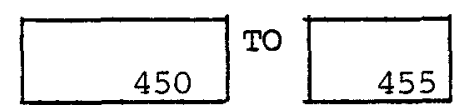

ROCK CLASSIFICATION

slightly gravelly sandy silt

S SIZE RANGE SPHERICITY ROUNDNESS

BASAIT $\geq$ gravel sand

OTHER $\geq$ gravel sand

SIIT AND CIAY
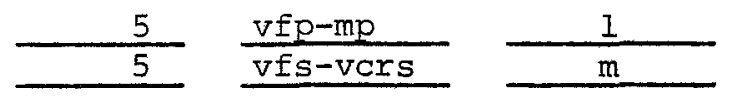

$\frac{w \text { rnd }}{\text { sb rnd }}$
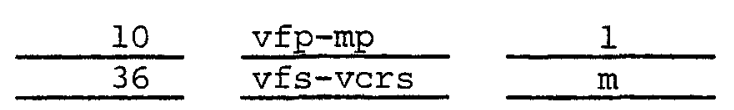

$\frac{w r n d}{s b r n d}$

REMARKS

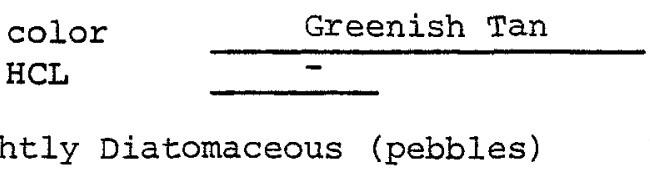

Slightly Diatomaceous (pebbles) Unfrosted Quartz Immature

\section{DEPTH}

(FT.)

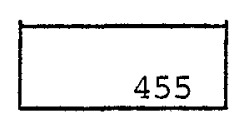

TO

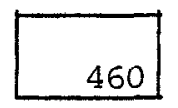

ROCK CLASSIFICATION

silty Sand

\section{SIZE RANGE SPHERICITY ROUNDNESS}

BASALT $\underset{\text { sand }}{\geq \text { gravel }}$
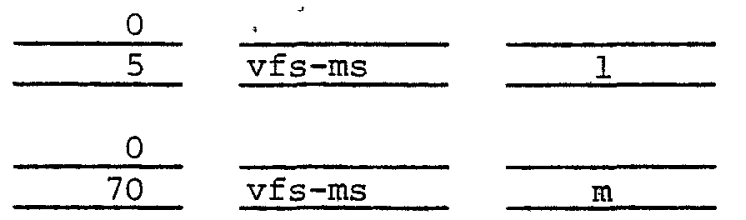

rnd

OTHER > gravel sand

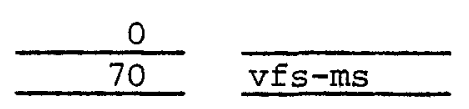

25

SILT AND CLAY

REMARKS

$$
\begin{aligned}
& \text { COIOr } \\
& \text { HCL }
\end{aligned}
$$

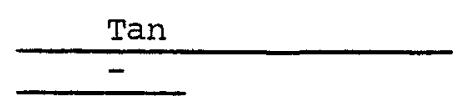

Slightly Micaceous

Frosted Quartz

Sieved (?)

Immature 
DEPTH

(FT.)

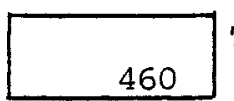

TO

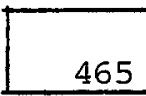

ROCK CLASSIFICATION

slightly gravelly silty sand

$\frac{\%}{6}$ SIZE RANGE SPHERICITY ROUNDNESS

BASALT $\geq$ gravel
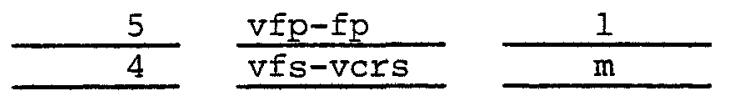

sb rnd

OTHER $\underset{\text { sand }}{\geq \text { gravel }}$
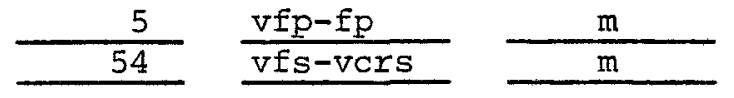

$\underline{\text { sb rnd }}$

SILT AND CLAY

\section{2}

REMARKS

color

HCL

Tan

Slightly Diatomaceous (pebbles)

Slightly Micaceous

Frosted Quartz

Immature

DEPTH

(FT.)

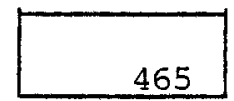

TO

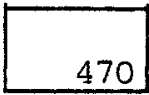

ROCK CIASSIFICATION

slightly gravelly silt

\% SIZE RANGE SPHERICITY ROUNDNESS

\begin{tabular}{|c|c|c|c|c|c|}
\hline BASALT & $\begin{array}{l}\text { >gravel } \\
\text { sand }\end{array}$ & $\frac{4}{3}$ & $\frac{v f p-m p}{m s-v e r s}$ & $\frac{1}{m}$ & $\frac{\mathrm{rnd}}{\mathrm{sb} \text { rnd }}$ \\
\hline OTHER & $>$ gravel & 4 & $v f p-m p$ & 1 & rnd \\
\hline & sand & 15 & $\mathrm{~ms}-\mathrm{vers}$ & $\mathfrak{m}$ & sb rnd \\
\hline
\end{tabular}

SILT AND CLAY

74

REMARKS

color

Reddish Tan

$\mathrm{HCL}$

-

Lithified by silt \& clay

Immature 
DEPTH

(FT.)

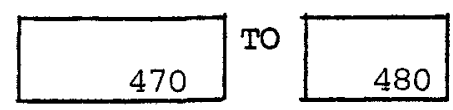

ROCK CLASSIFICATION

Silt

\% SIZE RANGE SPHERICITY ROUNDNESS

BASALT $\geq$ gravel

$$
\text { sand }
$$
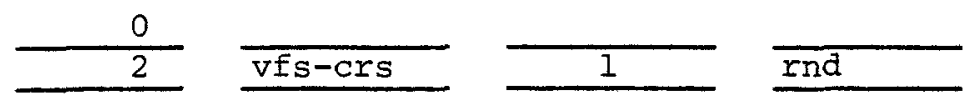

OTHER $\geq$ gravel
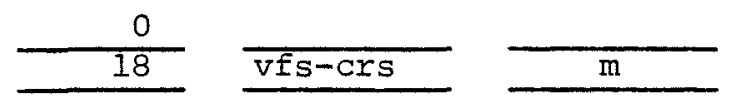

sb rnd

SILT AND CLAY

$$
80
$$

REMARKS

color

HCL

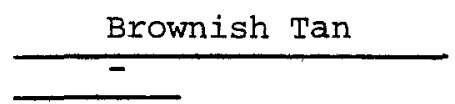

Slightly Micaceous

Frosted Quartz

Immature

DEPTH

(FT.)

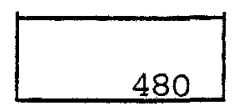

TO

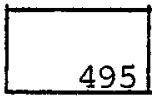

ROCK CIASSIFICATION

Silt

\section{\% SIZE RANGE SPHERICITY ROUNDNESS}

BASAIT Igravel sand

OTHER $\geq$ gravel sand

SIIT AND CLAY

REMARKS

color

HCL
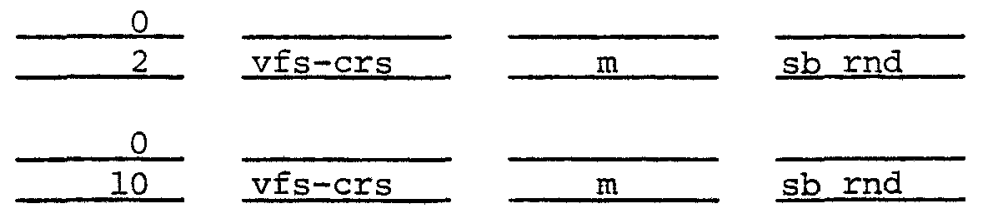

Slightly Micaceous Sample interval $480^{\prime}-485^{\prime}$ sieved (?)

Immature 
DEPTH

(FT.)

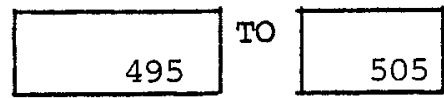

ROCK CLASSIFICATION

sandy silt

\section{SIZE RANGE SPHERICITY ROUNDNESS}

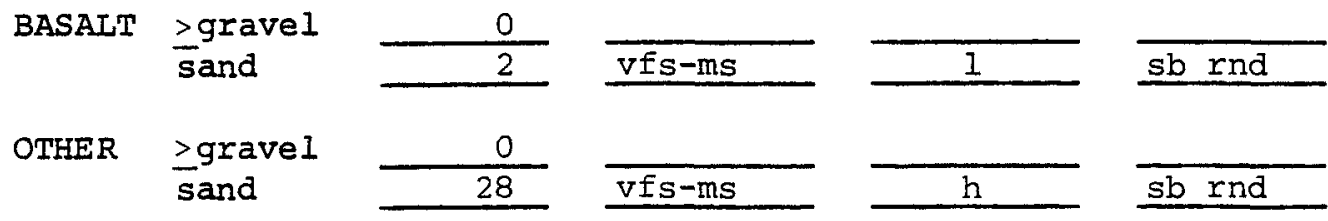

SILT AND CLAY

\section{0}

REMARKS

color

Light Grey

HCL

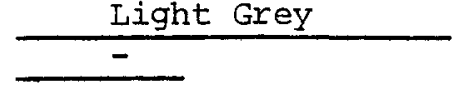

Slightly Micaceous Frosted Quartz

Sample interval 500'-505' sieved (?)

Immature

DEPTH

(FT.)

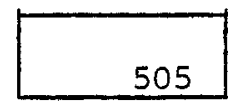

TO

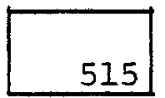

ROCK CIASSIFICATION

Silt

\section{\% SIZE RANGE SPHERICITY ROUNDNESS}

BASALT Igravel sand
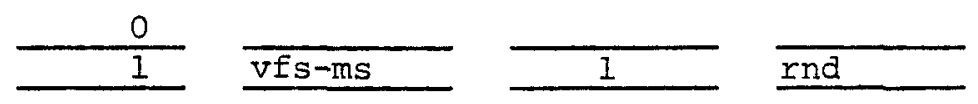

OTHER Igravel sand
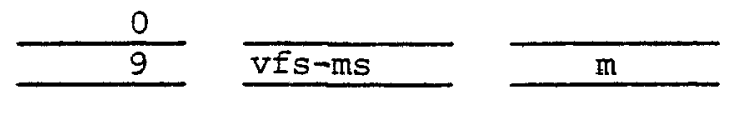

sb rnd

SILT AND CLAY

90

REMARKS

color

HCL

Greyish Tan

-

Caliche (pebbles \& sand present between 505' \& 510') Frosted Quartz Immature 


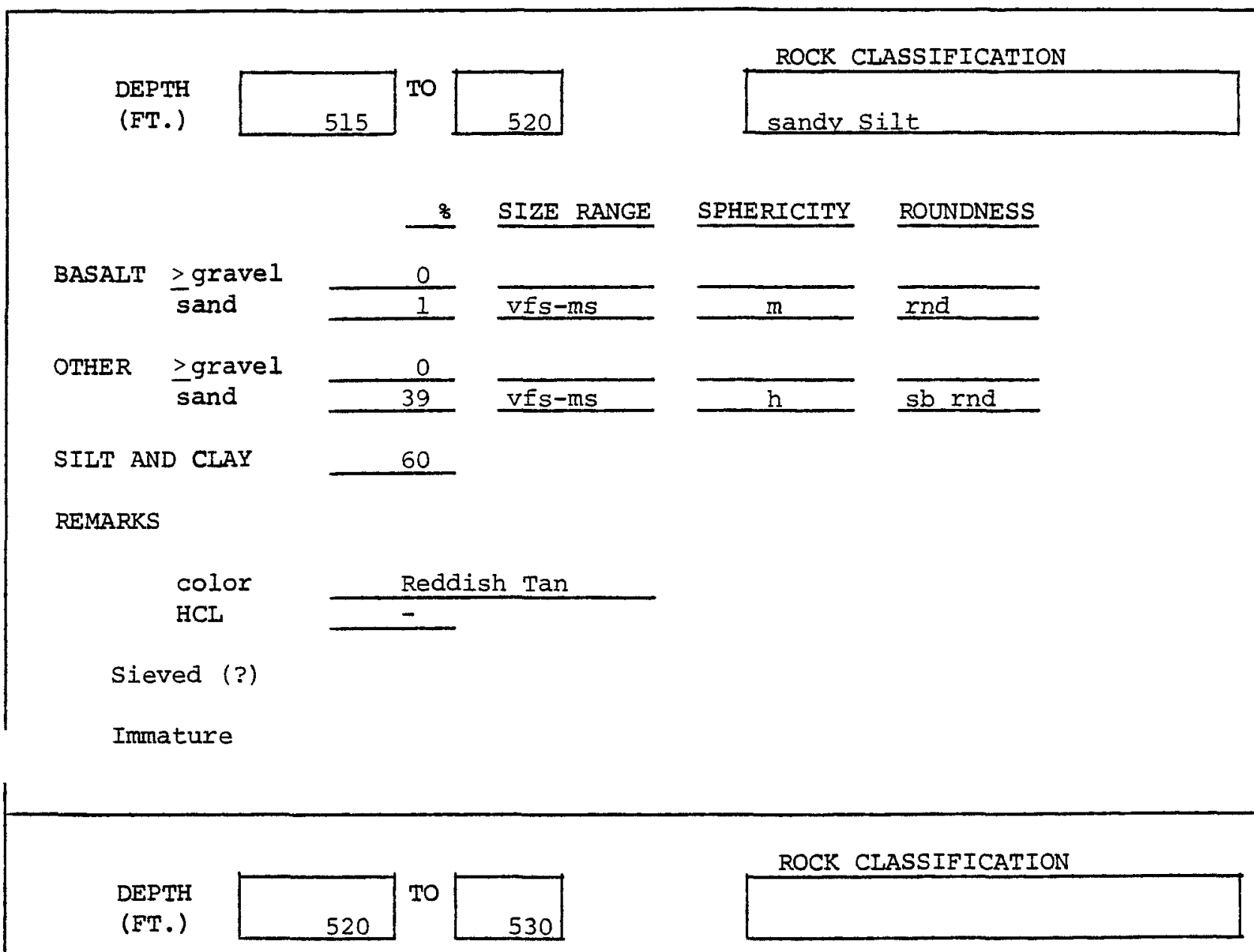

\% SIZE RANGE SPHERICITY ROUNDNESS

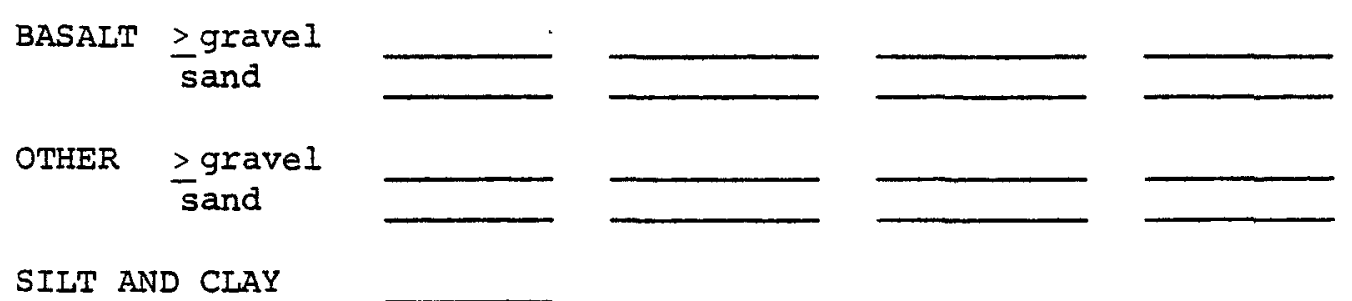

REMARKS

color

HCL

Sample missing 
DEPTH

(FT.)

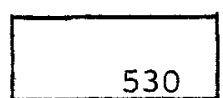

To
ROCK CLASSIFICATION

Silt

\section{\& SIZE RANGE SPHERICITY ROUNDNESS}

BASALT >gravel sand
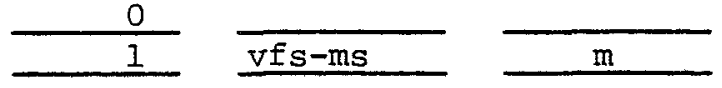

rnd

OTHER $\quad$ gravel
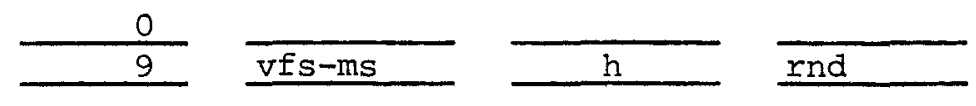

SILT AND CIAYY

$$
90
$$

REMARKS

color

HCL Reddish Tan

$-$

Caliche (pebbles present)

Inmature

DEPTH

(ET.)

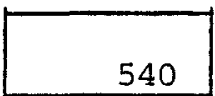

TO

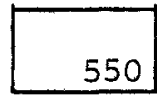

ROCK CIAASSIFICATION

silty sand

\section{S SIZE RANGE SPHERICITY ROUNDNESS}

BASALT >gravel sand
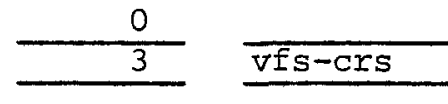

OTHER \gravel sand

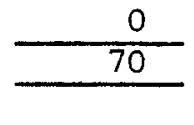

$\overline{\text { vfs-crs }}$ 27

SIIT AND CIAY

REMARKS

color

HCL

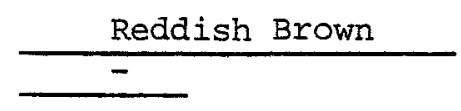

Caliche (pebbles \& sand present)

Frosted Quartz

Sample interval $545^{\prime}-550^{\prime}$ sieved (?) 


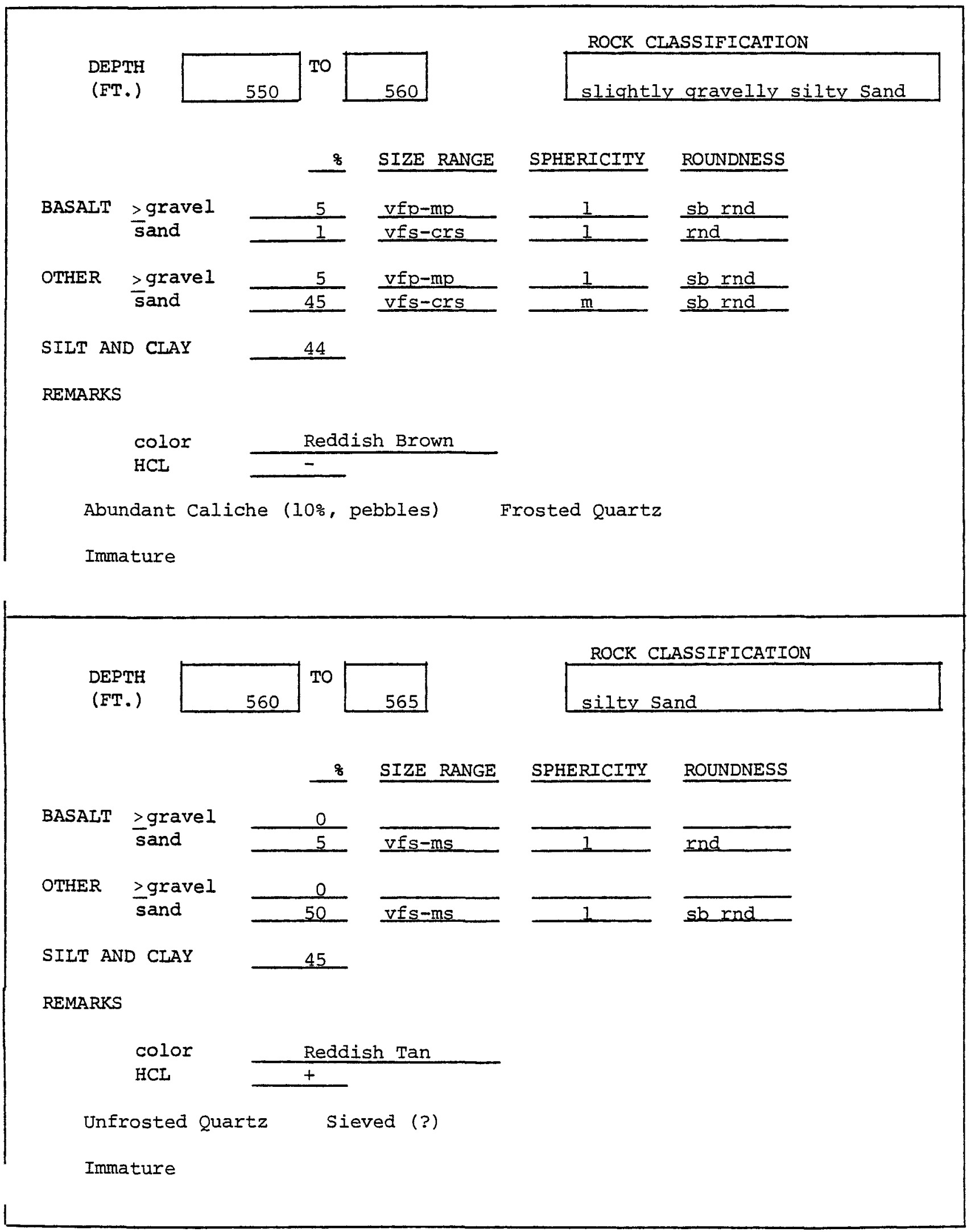


DEPTH

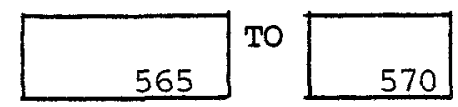

ROCK CLASSIFICATION

sandy Gravel

\section{\% SIZE RANGE SPHERICITY ROUNDNESS}

\begin{tabular}{|c|c|c|c|c|c|}
\hline BASAIT & $\begin{array}{l}\geq \text { gravel } \\
\text { sand }\end{array}$ & $\frac{20}{8}$ & $\frac{\text { vfp-crp }}{\text { vfs-vers }}$ & $\frac{m}{m}$ & $\frac{\text { sb rnd }}{\text { rnd }}$ \\
\hline OTHER & $\geq$ gravel & 47 & $v f p-c r p$ & $I$ & $w$ rnd \\
\hline & sand & 23 & vfs-vers & 1 & sb rnd \\
\hline
\end{tabular}

SILT AND CLAY

REMARKS

color

Light Grey

HCL

Micaceous Unfrosted Quartz

Immature

DEPTH

(FT.)

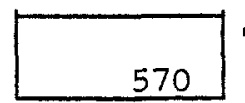

TO

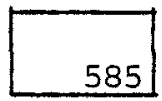

ROCK CIASSIFICATION

sandy Gravel

\section{\% SIZE RANGE SPHERICITY ROUNDNESS}

BASALT $\begin{gathered}\geq \text { gravel } \\ \text { sand }\end{gathered}$
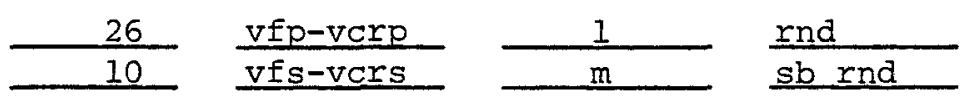

OTHER $\geq$ gravel sand

$44 \quad$ vfp-smab 18 vfs-vers

$\frac{1}{1} \frac{w \text { rnd }}{\text { sb rnd }}$

SILT AND CLAY 2

REMARKS

$$
\text { color }
$$

HCL

Greyish Buff

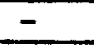

Frosted Quartz

Immature 
DEPTH

(FT.)

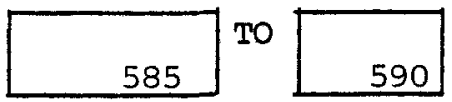

S SIZE RANGE SPHERICITY ROUNDNESS

BASALT $\geq$ gravel sand
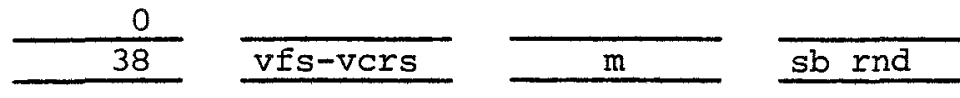

OTHER >gravel sand

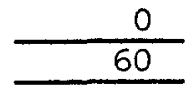

vfs-vers

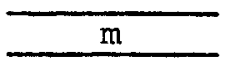

SIIT AND CLAY

2

REMARKS

color

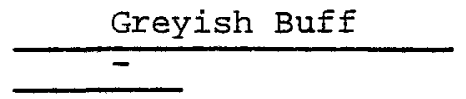

Slightly Micaceous

Frosted Quartz

Sieved (?)

Mature

DEPTH

(FT.)

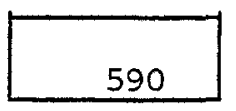

TO

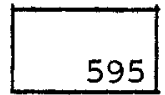

ROCK CLASSIFICATION

silty sandy Gravel

\section{\% SIZE RANGE SPHERICITY ROUNDNESS}

\begin{tabular}{|c|c|c|c|c|c|}
\hline BASALT & $\begin{array}{l}\text { >gravel } \\
\text { sand }\end{array}$ & $\frac{10}{6}$ & $\frac{v f p-m p}{v f s-v c r s}$ & $\frac{1}{\mathrm{~m}}$ & $\begin{array}{l}w \text { rnd } \\
\text { sb rnd }\end{array}$ \\
\hline OTHER & $\begin{array}{l}\text { > gravel } \\
\text { sand }\end{array}$ & $\frac{70}{11}$ & $\frac{v f p-s m c b}{v f s-v c r s}$ & $\frac{1}{m}$ & $\frac{w \text { rnd }}{s b \text { rnd }}$ \\
\hline
\end{tabular}

SIIT AND CLAY

3

REMARKS

COIOr
HCL

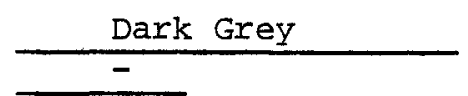

Slightly Micaceous Frosted quartz

Immature 


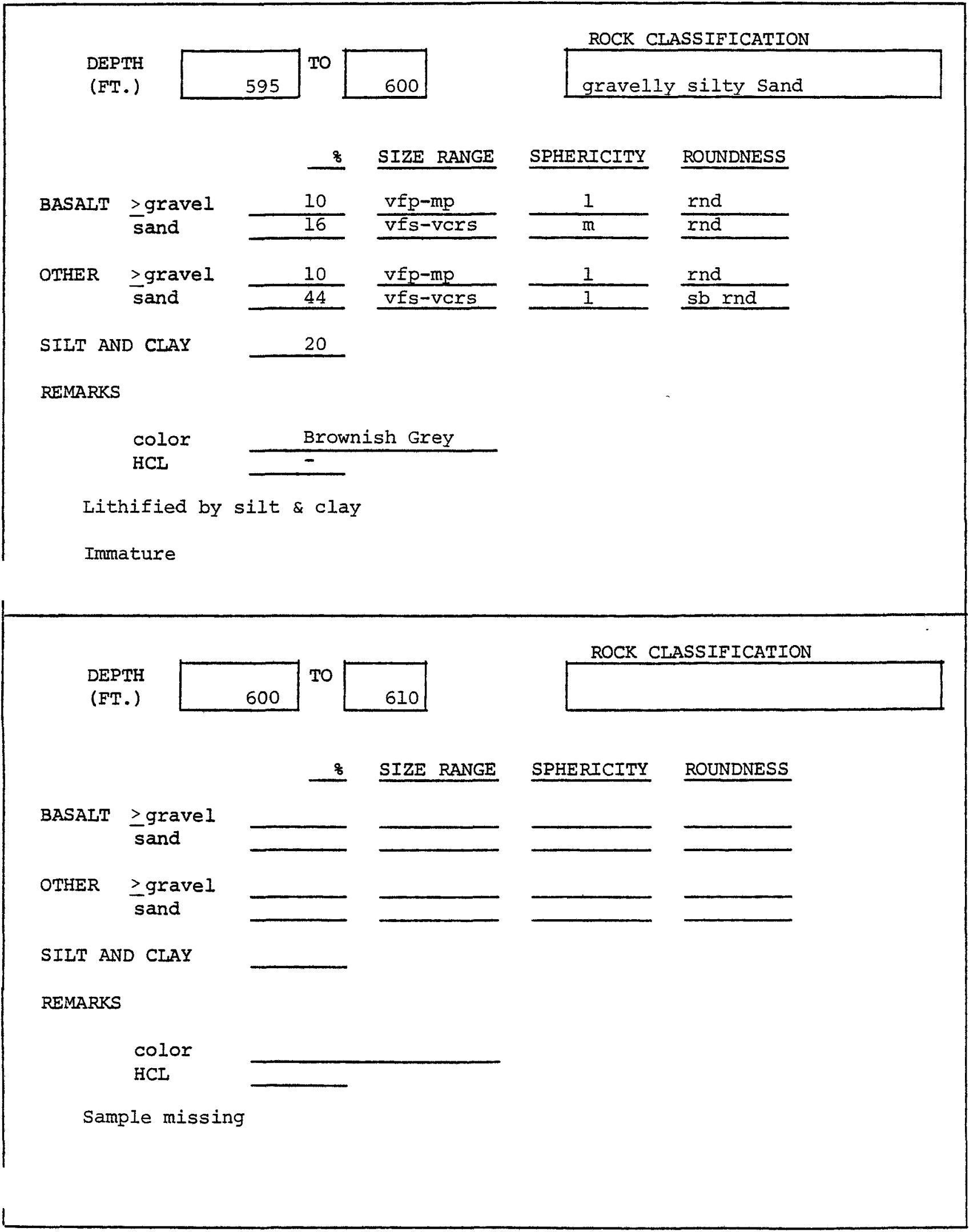


DEPTH
(FT.)

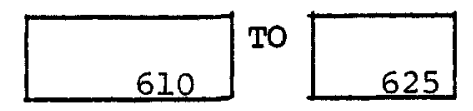

ROCK CLASSIFICATION

sandy Silt

\section{SIZE RANGE SPHERICITY ROUNDNESS}

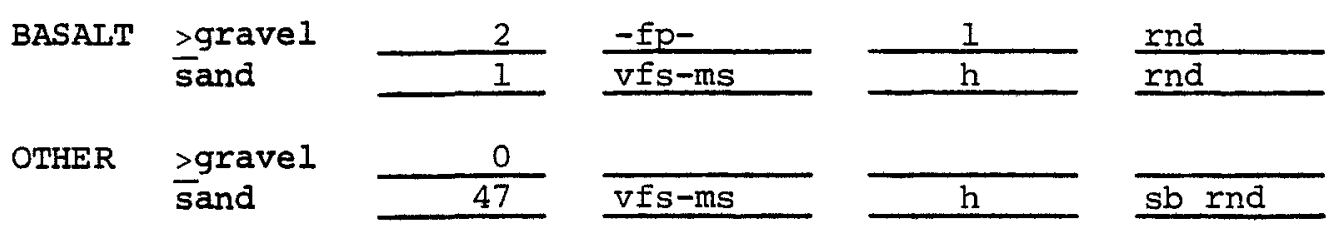

SILT AND CLAY 50

REMARKS

color
HCL

Micaceous

Immature

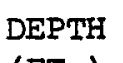

(FT.)

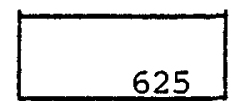

TO

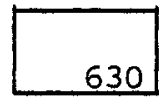

ROCK CIASSIFICATION

silty Gravel

SIZE RANGE SPHERICITY ROUNDNESS

BASALT $\frac{\text { zgravel }}{\text { sand }}$
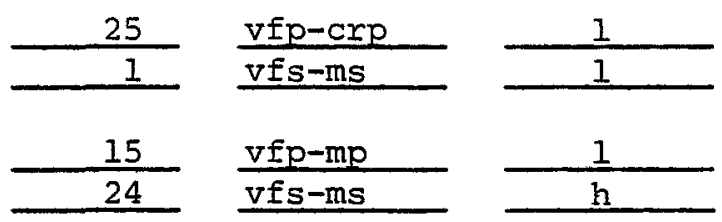

rnd

OTHER >gravel sand

$\frac{15}{24} \quad \frac{v f p-m p}{v f s-m s}$

35

SILT AND CIAY

REMARKS

$$
\begin{aligned}
& \text { color } \\
& \text { HCL }
\end{aligned}
$$

Brownish Tan

$-$

Micaceous

Immature 
ROCK CLASSIFICATION

slightly silty

slightly gravelly sand

(FT.)

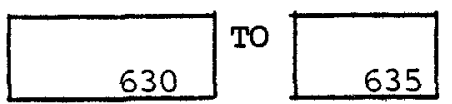

SIZE RANGE SPHERICITY ROUNDNESS

\begin{tabular}{|c|c|c|c|c|c|}
\hline BASALT & $\begin{array}{l}\geq \text { grave } 1 \\
\text { sand }\end{array}$ & $\frac{8}{18}$ & $\frac{v f p-m p}{v f s-c r s}$ & $\frac{1}{1}$ & $\frac{\text { sb rnd }}{\text { rnd }}$ \\
\hline \multirow{2}{*}{ OTHER } & > gravel & 0 & & & \\
\hline & sand & 56 & vfs-crs & $\mathrm{m}$ & $\mathrm{sb}$ rnd \\
\hline SILT AN & CLAY & 18 & & & \\
\hline
\end{tabular}

REMARKS

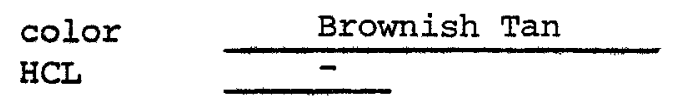

Immature
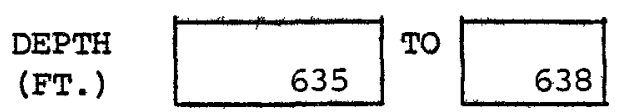

ROCK CLASSIFICATION

silty sandy Gravel

\% SIZE RANGE SPHERICITY ROUNDNESS

\begin{tabular}{|c|c|c|c|c|c|}
\hline BASALT & $\begin{array}{l}\geq \text { gravel } \\
\text { sand }\end{array}$ & $\frac{35}{6}$ & $\frac{\text { vfp-crp }}{\text { vfs-vors }}$ & $\frac{1}{1}$ & $\frac{w \text { rnd }}{\text { rnd }}$ \\
\hline OTHER & Zgravel & 40 & $v f p-c r p$ & $\mathrm{~m}$ & w rnd \\
\hline & sand & 15 & vfs-vers & m & sb rnd \\
\hline
\end{tabular}

SILT AND CLAY

4

REMARKS

color

HCL

Greyish Tan

$-$

Slightly Micaceous Unfrosted Quartz

Immature

W K. Summers \& Associdtes 


\section{DEPTH}

(FT.)

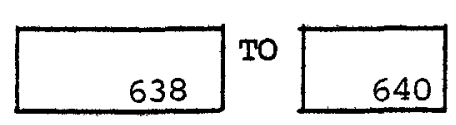

640
ROCK CLASSIFICATION

silty sand

\section{\& SIZE RANGE SPHERICITY ROUNDNESS}

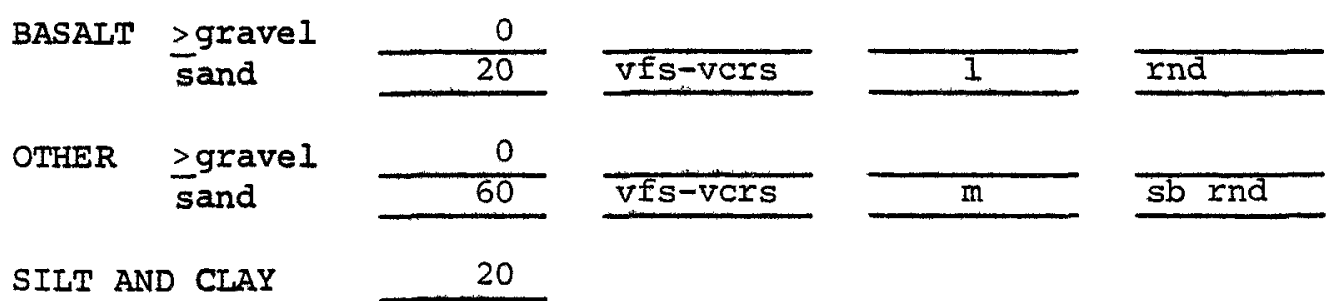

REMARKS

color

HCL

Greyish Tan

Micaceous Unfrosted Quartz

Immature

Total Drilled Depth reported as $640^{\prime}$

DEPTH

(FT.)

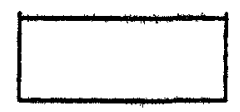

$\mathrm{TO}$

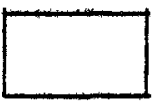

ROCKK CIASSIFICATION

\% SIZE RANGE SPHERICITY ROUNDNESS

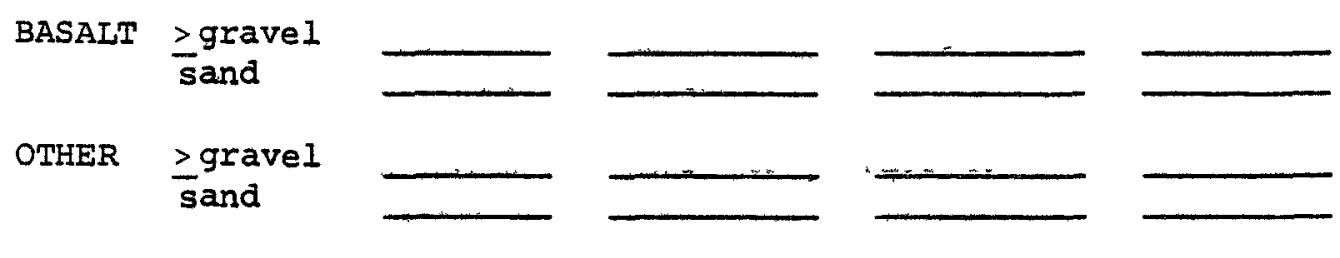

SILT AND CLAY

REMARKS

color

HCL 